
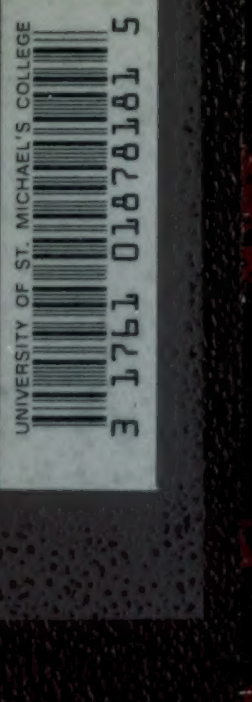

8

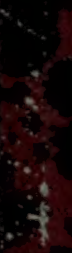




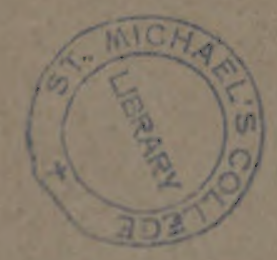




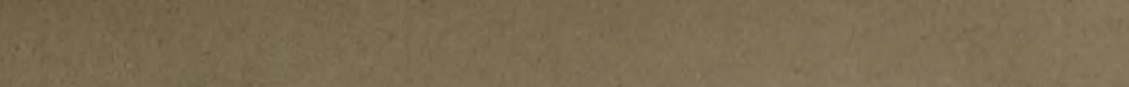

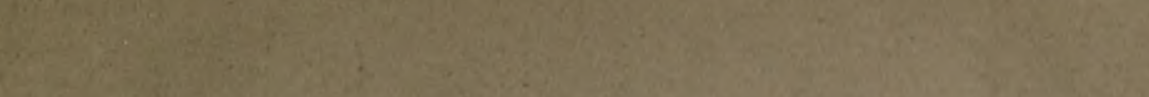
SW.

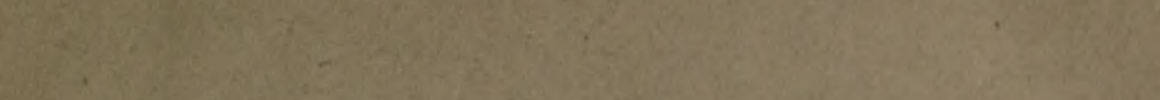

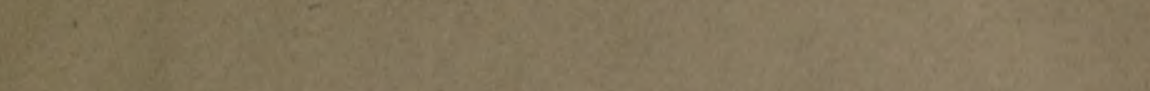

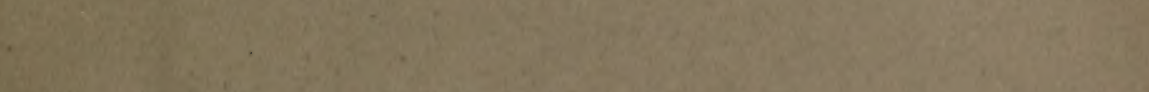

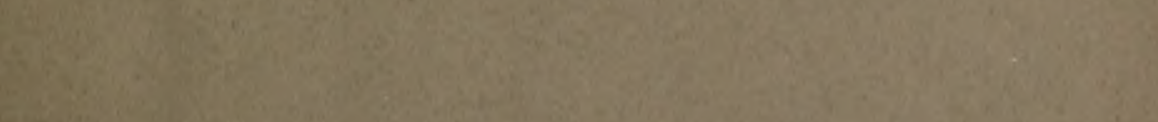

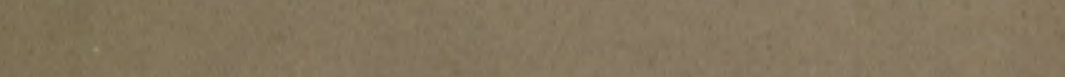

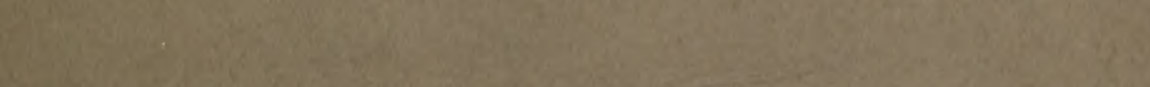

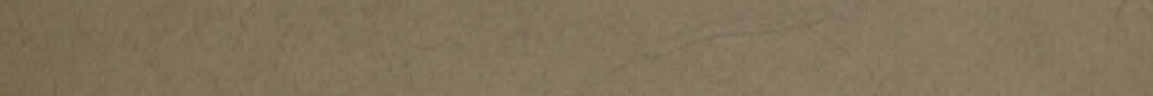

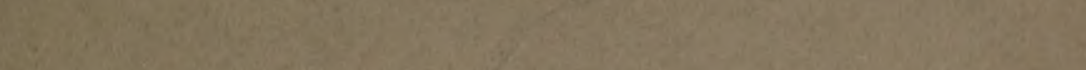

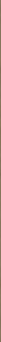

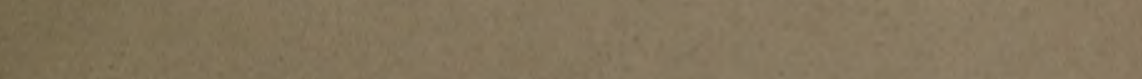

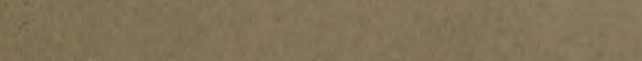

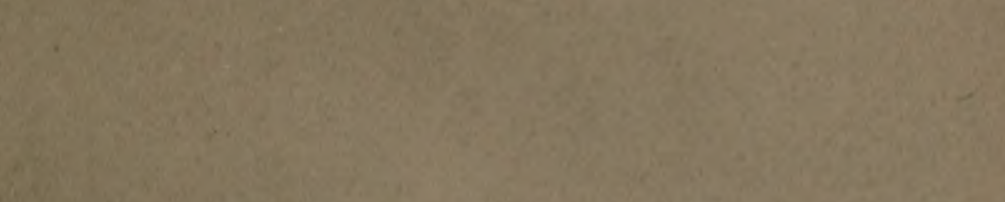

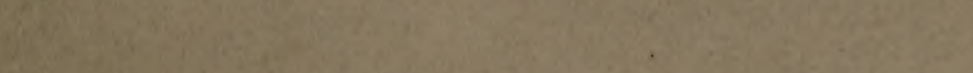

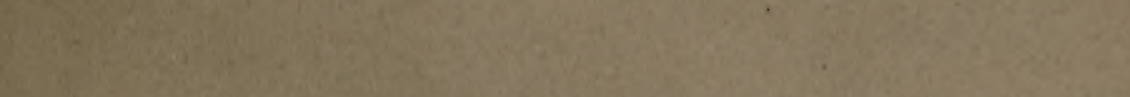

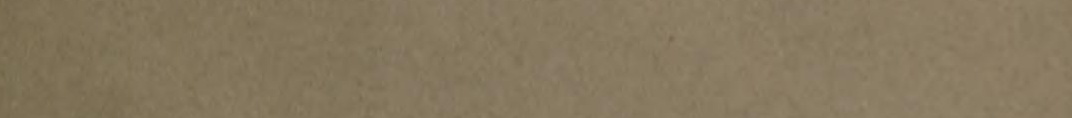

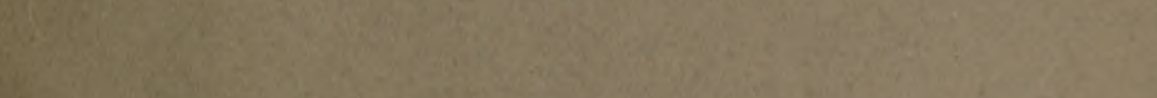

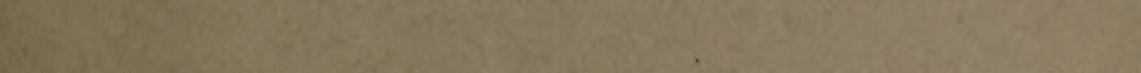

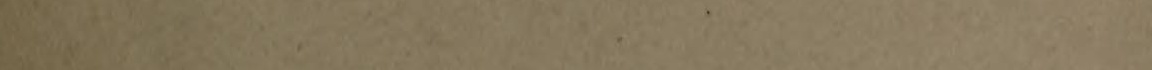
s.

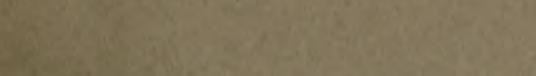

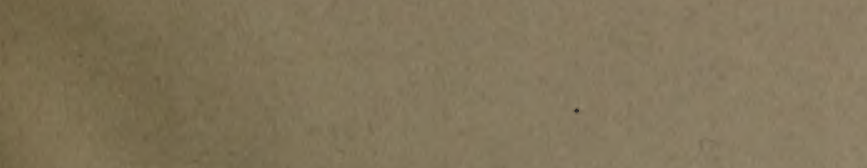

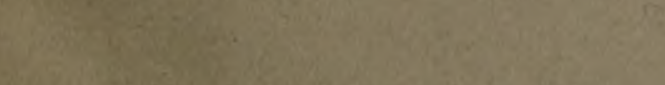





\section{La Philosophie de l'Inconnaissable}

\section{LA THÉORIE DE L'ÉVOLUTION}




\section{IMPRIMATUR}

Lovanii die $4^{\text {a }}$ Junii 1907.

AD. HebBelynck

Rect. Magn. Univ. Lov.

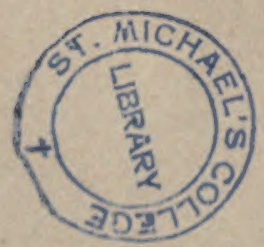




\section{LA PHILOSOPHIE DE L'INCONNAISSABLE.}

La Théorie

\section{de l'Evolution}

ÉTUdE CRITIQUE

SUR LES "PREMIERS PRINCIPES "

DE HERBERT SPENCER

PAR

Le Chanoine Jacques LAMinne

Professeur à l'Université Catholique de Louvain.

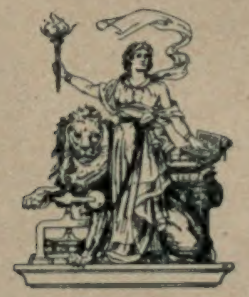

BRUXELLES

LIBRAIRIE ALBERT DEWIT

53, Rue Royale.

$1 \overline{807}$ 
Digitized by the Internet Archive in 2009 with funding from University of Ottawa 


\section{AVANT-PROPOS}

La sécurité qu'offre une maison à ceux qui l'habitent dépend avant tout de la solidité de ses fondements. La mauvaise qualité des pierres qui en forment la base ne permet pas de porter un jugement sur les matériaux qu'on a employés dans le reste de la construction. Il est possible qu'ils soient excellents; il est possible aussi que les diffërents appartements soient parfaitement agencés et que, considérés en eux-mèmes, ils soient en tout conformes aux lois de l'architecture. Néanmoins si tout repose sur des fondements ruineux, l'ensemble risque fort de s'écrouler et la maison n'est pas un refuge sûr pour ceux qui s'y abritent.

Un système philosophique est comme une demeure pour les intelligences. Elles y vivent, s'y meuvent, s'y reposent; leur activité s'y adapte, de même que notre vie matérielle est réglée dans une certaine mesure par la maison que nous habitons.

Il importe qu'avant de s'installer dans un semblabe asile notre intelligence en fasse le tour, s'assure de sa stabilité et ne s'expose pas à devoir le quitter après y avoir élu domicile ou à être enveloppé dans sa ruine.

Le défaut des fondements n'est pas toujours radical ni sans remède. Une partie moins solide peut ètre remplacée et pourvu qu'on se décide à apporter à l'édifice les modifications nécessaires, une meilleure disposition des assises peut parfois prévenir un effondrement général.

Nous nous proposons de faire la critique des bases de la philosophie de Herbert Spencer. Cette tâche nous es 
beaucoup facilitée par le fait que Spencer a consacré un volume spécial à établir les premiers principes. C'est cet ouvrage fondamental que nous étudierons. Cependant, comme il est nécessaire pour l'intelligence parfaite des principes de les envisager dans leurs applications et quoique Spencer prenne toujours soin d'illustrer largement les considérations théoriques par des exemples concrets, nous aurons à tenir compte des autres ouvrages philosophiques de Spencer, notamment de ses Principes de Biologie, de Psychologie, de Sociologie et de Morale.

Si l'on compare au point de vue intellectuel notre époque aux précédentes, par exemple au XVII ${ }^{\text {me }}$ siècle, au XIII'me siècle ou à l'âge d'or de la philosophie grecque, on trouvera certainement que nous l'emportons de beaucoup pour l'étendue et la sûreté des connaissances concrètes, mais on pourra peut-être nous reprocher avec raison un manque d'esprit synthétique.

Depuis l'essor merveilleux des sciences naturelles, depuis les progrès rapides des sciences historiques, l'arbre du savoir humain s'est ramifiè en une infinité de branches. La plupart des travailleur's de l'esprit ont choisi une partie restreinte du chanup de la vérité afin de l'explorer avec un soin minutieux. Un très petit nombre cherche à l'embrasser dans son entier, à s'élever de manière à obtenir une vue d'ensemble. C'est cependant à cela que doit viser la philosophie.

Il est vrai de dire qu'au fur et à mesure des progrès des sciences particulières, la synthèse devient plus difficile. Autrefois lorsque l'ensemble des connaissances humaines n'était pas trop étendu pour qu'un homme de génie pût se l'assimiler tout entier,ou du moins posséder de chacune de ses parties une science personnelle étendue, les conceptions synthétiques pouvaient naitre dans un plus grand nombre d'esprits et aussi s'adapter plus facilement aux faits connus. A notre époque aucune intelligence humaine n'est capable de s'assimiler même la majeure partie de 
nos connaissances et les principes ont bien plus de chances qu'autrefois de rencontrer des fats qui semblent les contredire. Il ust cependant indispensable, si l'on veut concevoir une theorite génerale de l'Lnivers, que l'on connaisse avec exactitude au moins les resultals greneraux de toutes les sciences. Cela mòne est aujourdluui le risultat d'un travail long et aride. Ensuite il faut une intelligences suttisanment puissante pour ordomnel ces combatissances de maniere a en déduir les conclusions ot a saisil les frincignes auxquels obéissent la multitude des faits particouliers.

Yous almettons volontiers que tous les phrenomenes quelque variés qu'ils soient sont gouvernes pal un petit nombre de lois grinirales. Quelle que soit l'idee que nous nous lassions de l'Cuivers, nous sommes portes it rovir" que sa complexité cache une unite mofonde at l'expériencep que nous en avons contirme cette vue à priori. Mais il faut d'autre part que nous nous gandions ders grmeralisations hatives et que nous ne transformions pas a la lingime pu principe universel une loi dont nuus avons constati l'exat'titude dans une categrorie restreinte de plunomines. Il y a dans l'Lnivers uniti et variete et il importe de sitisil le point precis ou l'unite ersse et ou la variele commences.

Lon prut dire que sans synthèse il n'y a pas de scipnce proprement dite. Persomme ne domme le nom de science à la connaissance isolée de l'un ou l'autre fatit particulires. Pour mériter ce titre, la comnaissance doit grouper un grand nombre de fiats de maniere a sitisir une verrite generale qui ! trouve son application. Lorsqu on est ainsi entre en possession de quelques veritis genriales, on peut les grouprer alles-memes de facon à en faire jaillir des principes plus étendus encore. La synthrise sera d'atltant plus elevée que l'on sera arrive a la comnaissance de propositions plus universelles.

Lorsque Yewton, apres aroir étudie les mourements des planetes autour du soleil aussi bien que la chute des corps terrestres, décourrit que tous ces phénomines sont reglés par la loi de la gravitation, il concut une syntlitse: mignitique. De mème, si ctudiant les phénomines ther- 
miques nous arrivons ḋ la conclusion que la chaleur est un mouvement vibratoire des particules matérielles, nous faisons une synthèse en déduisant une proposition générale d'une multitude de faits particuliers. Si, en outre, comparant avec celle-là d'autres conclusions semblables concernant les phénomènes lumineux, acoustiques, électriques, chimiques, nous imonçons une proposition plus générale encore, en disant que tous les phénomènes matériels sont des phénomènes de mouvement, nous aurons fait une synthèse plus élevée.

Toute science a donc pour but de faire des synthèses et de les faire aussi hautes que possible dans les limites de nos investigations. Toute science possède ainsi sous le nom de lois un certain nombre d'énoncés généraux qui représentent le travail de synthèse qu'elle a réalisé. On conçoit qu'il est possible de rapprocher les unes des autres les vérités gènèales découvertes dans chaque science, de chercher à y clécouvrir des principes encore plus généraux et de tendre ainsi vers le plus haut degré de généralisation auquel l'esprit puisse prétendre. Tel est le ròle de la philosophie.

Cependant la méthode qui consiste à découvrir les vèrités générales par la comparaison des faits particuliers ou de vérités moins générales n'est pas la seule possible. Certains principes burent sont évidents par eux-mèmes sitns qu'il soit nécessaire de les vẻritier par l'experience; d'autres peuvent se déduire par l'analyse des notions qu'ils contiennent. Il en est ainsi des vérités mathématiques at en général de toutes colles qui sont la conclusion de raisonnements à priori.

Autant it convient dans l'emploi de cette méthode dont on a souvent abuse, de n’avancer que pas à pas et a coup sirr, antant il serait diraisonnable de la condamner absolument et de repousser ses resultats sams examen.

On peut, ainsi que le fait Sproncer (1), concevoir la tache de la philosophie comme double. Il faut d'abord itablie

1) Premiers Principues. Trad. Cazelles. Paris 1871 1. 141. \$. 38. - F'irst Principles London 190.4 p. $10 \%$. 
et expliquer les princijes géneraux, ensuite montrer leurs applications dans les diffirentes categrories de phrmonines.

On distinguera ainsi une philusophice génerale et une philosophie spéciale. C'est la première qui est l'ohjue du traité que nous examinons.

L'esprit philosophique n'd jamais fait defaut frarmi los peuples civilises. Des synthèses grinerales ont etr édities aux diffërentes époques; elles correspondent ì unf tendance bien accentuee de notre esprit. Souvent elles ne sont pas définitives. Les raisonnements qui les etablissent peuvent ne pas ètre péremptoires ; les faits que l'on invougut pour les appuyer peruvent aroir ete mal observes; ou bien mème la sỵthèse n’a été proposere qu'a titre d'hypothise provisoire.

Cne logicue plus rigrourense, une meilleure combissince des phenomienes, la diecouverte de liats nouveaux preurent amener le renversement de la construction synthetigure ou nécessiter des retouches plus ou moins considerebles.

De mime que l'histoire ders peruples s'imcarne somvent dans des monuments en luines, ainsi en pet-il de l'histoire des idfes. L'homme ne se lasisse pas décourager par l'insuccès de ses devanciers et il a raison. Mime s'il ne rrussit pas a bitil un systeme qui demernre, au moins l'exprerience qu'il a tentre seral ntile à d'autres ot il est fresifue impossible qu'il ne reste rien du fruit dr son travail. Ou bien ce sont des parties de l'edifice qu'on pourla conserver, ou lien l'accumulation de materiaux precieux et abondants facilitera la tache des travailleurs suivants, ou bien les fondements quoique insuflisants contiennent quelque pierre angulaire sur laquelle on pourra batir dans la suite en toute confiance.

Spencer divise son ourrage en deux parties. La premiere a jour titre L'Inconnaissable, la seconte Le Connaissable. Celle-ci a pour objet principal lat therorie génerale de l'Évolution. 
Quoiqu'elles se complètent l'une l'autre, il n'y a cependant pas entre elles un lien de dépendance tel que la seconde ait pour fondement la première. Beaucoup de lecteur's des Premiers Principes s'y sont trompés, comme le remarque Spencer lui-mème dans le Post-scriptum qu'il a ajouté a la Première Partie dans l'édition de 1900. En ayant pris conuaissance ef jugeant inadmissibles les idées qui y sont développées, ils ont cru inutile de pousser plus avant. En réalité la seconcle Partie est dans une large mesure indépendante de la Première.

Yous sommes farmi les lecteurs qui disapprouvent celle-ci et l'étude que nous y consacrerons ne sera guère qu'une réfutation, notre disaccord avec spencer ètant radical. Yous croyons au contraire que l'idée maîtresse de la seconde Partie est juste, de sorte que, à notre tour, nous prions les lecteur's qui tiennent pour vraie la philosophie de l'Inconnaissable - nullement propre a spencer - " que nos arguments n'auraient point éhranlés, de bien vouloir néanmoins nous suive dans la seconde partie de ce travail.

Les Premiers Principes (First Principles) parturent pour la première fois en $186 t^{2}$. Cing annees plus tard sprencere en fit paraitre une seconde eddition dans laguelle la seconde partie, Le Comnaissuble, etait complètement transformée. La principale raison des changerments est donnere dans une note an \$199 (1) dont nous aurons loceasion de parter. Spencere y explique que lidlie mattresse de son oxure, le conept d'rolution, arait subi dans son esprit me modification assez importante.

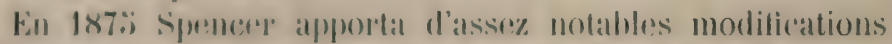
anx chapiteres IV, $\mathrm{V}$ or $\mathrm{V}$ de la Seconde Partie. Enfin dans

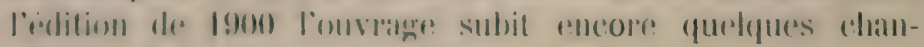
gements d'importance moindre.

II a partu deux tratuctions frangaises: l'une faite sur la 2 indition (Les Premiers Principes par Herbert spencer, truluil de l'. Inglais pur. M. E. Caselles, Paris 18\% I), l'aulre sur la $G^{m m}$ indition (Le's Premiers Principes par Herbent

(1) P. P. P. 3660, - F. P. p. 270 . 
Spencer, truduit sur la sixieme édition anglaise par .M. Guymiot. Paris 1902). Nous citons la premiere qui est lat plus repandue (P. P.) et nous y renvogons ainsi qu'a l'édition anglaise de 1904 (F. P.). Nous arons evidemment tinu compte des modifications que Spencer a apporties a son texte.

Spencer a étudié dans les parties spreciales de sa Philosophie Synthétique, l'évolution des lormes vivantes (Principes de Biologie), de l'activite psychique (Principes de' Psychologie), dr l'organisation suciale (Principes de suciologie). de la conduite et des idées morales (Principess de Morule). Nous avons utilisé ce qui, dans ces etude's, nous semb) ait pouvoir contribuer a l'intelligence des principes géneraux de l'évolution developpes dans les premiers principes. Nous ne nous sommes done occupés des causes particulieres à chayue genre d'érolution ou de l'interprétution des phénomènes spéciaux, que pour expliquer ou délimiter l'application des théries générales. Coest dans cer lout at anssi a cause de l'importance sperciale du sujet, que nous arons consacre un paragraphe anx causes particulieres de l'evolution organique. Nous l'avons fait arec d'autant moins scrupule que nous avons ainsi té amene a parter de la sélection naturelle comme eause générule des phenomines évolutifs.

Parmi les travaus qui ont été publies sur la Philosophie Synthétique on sur. les Premiers Principes, less uns s'attachent a développrer des arpuritiations d'ensemble; les autres se borment it etudier l'un ou l'autre point particuliers. En général, leur fetendue n'est pas en rapport avere l'importances de l'ceurre sipencerienne. Exception doit etre faite pour Ch. Renouvier qui a publie dans la Critique Philosophique (188:3-8(i) des étules suivies sur les Premiers Principes. Yous arons ete heureux de nous trouver d'accord avec l'illustre philosophe frangais dans un grand nombre de critiques qu'il adresse at spencer. Mais nous pensons qu'il a trop sévèrement jugé sá philosophie dans sa partie principale et constructive (la théorie de l'évolution) et qu'elle ne mérite pas la condamnation qu'il prononce. Quant it la théorie de I'Inconnaissable, si très sourent 
nous sommes d'accord avec les appréciations de Renouvier, notre point de vue diffère cependant notablement du sien. Le philosophe français soutient la thèse de la relativité de nos connaissances qui est une des bases, et mème la principale, de la doctrine de l'Inconnaissable, et que nous n'admettons pas dans le sens dans lequel le criticisme la défend.

Il y a donc place pour une étude des premiers principes de la philosophie spencérienne, dans laquelle ils soient examinés tels que Spencer les a présentés, c'est-à-dire dans leur agencement logique, avec les fondements sur lesquels ils s'appuient et la portée qu'il leur a donnée. C'est le travail que nous avons tâché de faire. 
La Philosophie

de I'Inconnaissable 



\section{PREMIERE PARTIE}

\section{LA PHILOSOPHIE DE L'INCONNAISSABLE}

CIIIPITRE I.

La thèse. - Esquisse de la démonstration. L'objet commun de la Religion et de la Science.

a thèse. Il y a, d'après spencer, certaines questions auxquelles l'esprit de l'homme ne pourra jamais donner de réponse. Tenter de le faire c'est s'engager dans des contradictions inévitables, c'est accomplir une tảche sterile. La cause première de tout ce que nous connaissons, la force universelle qui produit les phénomènes, la réalité fondamentale cachee sous ceux-ci, c'est l'Inconnaissable. Le Connaissable, ce sont les phènomènes eux-mèmes et les relations qui les unissent. Au sujet de l'Inconnaissuble, Spencer professe qu'il existe et qu'il est la raison dernière de tous ce que nous apercevons; mais qu'il nous est impossible de rien savoir au sujet de sa nature. II n'y a donc plus rien à en dire, son existence etant une fois admise. C'est à l'etablir que se bornera la première partie du traité.

La seconde partie s'occupera des principes généraux qui gouvernent les phénomènes, puisque ceux-ci constituent à proprement parler l'objet des sciences particulières et de la philosophie. 
On concoit que chacune de ces deux divisions est très importante, quoique pour des raisons differentes. Il est inutile d'y insister en ce qui concerne la seconde; mais quant ¿ la première, sa portee considérable consiste d'abord dans les nigations qu'elle contient, les limites qu'elle assigne a notre intelligence, la prétendue incapacite de celle-ci i connaitre ce que spencer appelle l'Inconnaissable, c'est-à-dire la cause primordiale et mème plus généralement la nature intime des choses. Elle résulte aussi de ce que l'existence de cet Inconnaissable s'y trouve affirmée comme cause première et realite fondamentale.

On n'a pas manqué de faire observer que la théorie de Spencer est contradictoire, puisque, au sujet du prétendu Inconnaissable, nous connaissons qu'il existe et qu'il est la cause de tout ce que nous percevons. Néanmoins, si sachant que la Cause Première existe, on ne connait rien de son essence, si sachant qu'elle est, on ignore ce qu'elle est, ne mérite-t-elle pas le nom d'Inconnaissuble? Lui attribuer d'ètre cause de l'Ćnivers, n'est qu'une relation qui n'affirme rien sur sa nature.

Il reste à voir si en demolissant les doctrines qui disent quelque chose sur la nature de la Cause Premiere, on ne renverse pas en meme temps l'alfirmation de son existence. Nous en jugerons dans la suite.

On peut donc se separer de spencer en enseignant que l'esprit humain est capable d'acpurir des rommissances diterminies au sujet de la Caluse Première, de demontrer qu'elle posside certains calactères et que d'atutres ne peurent pas lui itre attribues; ou bien encorr, en proclamant qu'en dehors du monde où nous viruns, des realities changeantes aver lespuelles l'experience nous mot en relation, il nexiste rien, il n'y a rient a ronnatere ot far consequent pien d'inconnatssable. L'ecole spribilualiste defend la premiere position, landis que la serende est oceupore pal le! matrialisme proplement

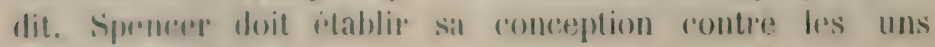
et les autres. 
squisse de la lémonstra-

tion.

Esquissons a grands traits la premine partie dres Premiers principes, alin d'embrasser d'un coup d'ril l'ans'mble que nous aurons ensuite à examiner all letail.

A. La Religion et la scrence, etant lingitimess lane of l'autre, loivent elle d'accord sur quelque proposition thes abstraite. (Chapitre $\mathbf{1})$

B. Toutes les religions, quelque contradicloires que? soient leurs dogmes, sentemlent pour alfirmer l'existenere d'une cause surnaturelle des phemomidnes. A mesure

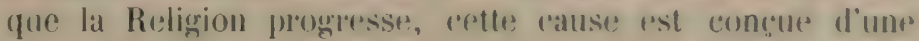
faron de plus en plus indélorminese; ancune notion deter-

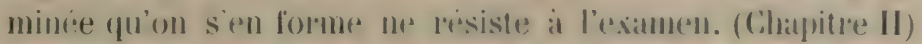

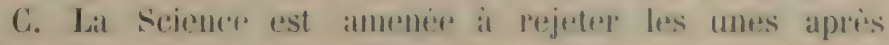
les autres comme insullisantes les canses particuliemes assigneres anx phenomènes at a recomnatre lexistromer d'une cause primordiale dont if lui est impussible de comprendre la nature. (Chapitre III)

1). Yotre intelligence perat connatre te phénomène, mais la réalite fondamentale tle nonmene) que les fhenomentes

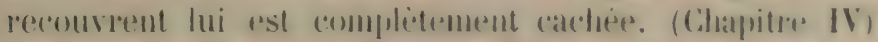

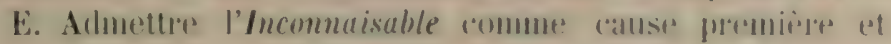

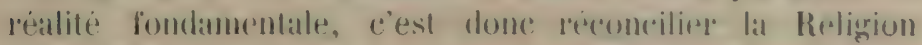

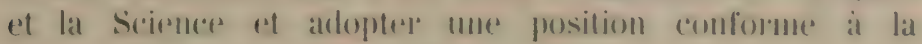
nature de notre esprit. (Chapitre V)

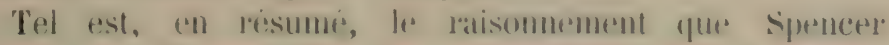

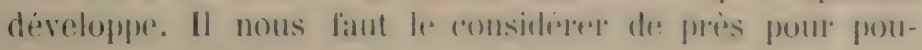
voir juger de sa solidité.

L'objet

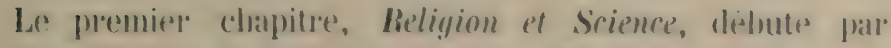

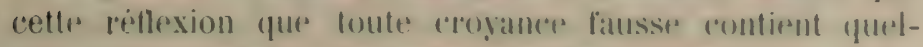
que part de verite, de sorte que dans les oprinions oppo-

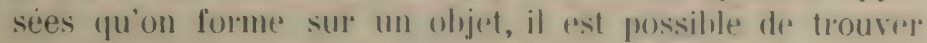

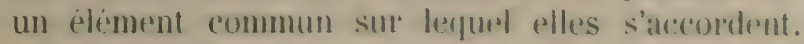

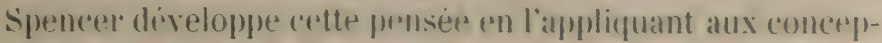
tions variers que les pupples ont eutes de l'alutorite de leurs

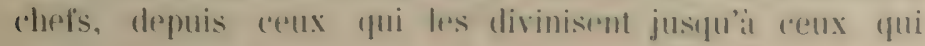


restreignent leur pouvoir dans des limites très etroites. Tous s'accordent pour leur reconnaitre une autorité véritable.

Cette rmaryue est juste et importante. Une proposition peut-ètre fausse parce fu'elle aftirme trop ou trop peu. Celui qui dit que la chimie moderne compte cinquante corps simples, se trompe; de mème celui qui pense qu'elle en compte cent, et leurs eroyances sont opposees. Mais ils admettent l'un et l'autre que la chimie admet des corps simples dont te nombre n'est pas inférieur ¿i cincpuante ni supérieur à cent, et en cela ils ne se trompent pas.

D'autres fois la proposition est lausst en elle-mème, mais elle contient implicitement ou elle suppose une autre proposition qui est varaie. Ainsi, il est probahlement faux que le tombeau de Neron se trouve à l'endroit qui, a Rome, est designé comme tel. Mais il est vrai cqu'un empereur de ce nom a régné it Rome, ce que la croyance susdite au sujet de son tombeau suppose.

D'autres fois encore l'opinion fausse est une interprétation erronce de faits veritables. La theorie du phlogistique elait fausse, mais les faits qu'elle prétendait expliqués étaient réels.

Ainsi il pourra se faire que la diversite des croyances répandues parmi les hommes sur un objet qui les interesse tous, reconvere une affirmation unanime dans laquelle ils saccordent et qui pourra invoquer en sa favenr la consentement miversel. Pour la dégagrer, il fandrat faire abstaction des formes particuliores et opposces que l'idee fondamentale revert che\% les differents pruples, colserver, comme le dit Spencer, ce qui reste apres l'elimination de: ces élements discordants et trouver prour ce residu une expression abstrate qui demenre vatie dans toutes ses moditioations divergentes. " (1)

Ide conflit entre la Religrion et la sciuence n'excelut done: pas la prossibiliti d'un acrord entro elles sur quelque vérité fondamentale.

(1) P. P. P. 10 - F. P. P. 8. 


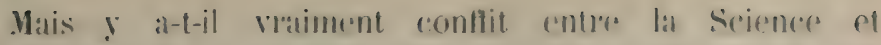
la Religgion? Il va sans dire que cee confllt n'est pas

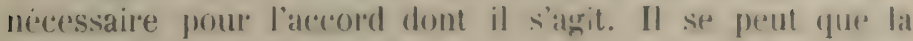
Religion et la Srience s'entendrent sur tous tes objets qui les interessent l'une et l'autre. Il se preut anssi fue? s'occupant d'objets dispanates, colles ne troment jas l'ureatsion de se contredire.

spencer alfirme l'existence du contlit: "De Lous les antagonismes qui s'elevent entre las hommes, le plus ancirn, le plus prolond, le plus grave et lis plus généralement recomnu, est colui de la Religgion et de la scoience. Il a commencé quand la découverte des lois les plus simples des choses les plus communes imposa une limite an listichisme moiversel qui avait justgue lit regrne sur les esprits. On le reflouse partout dans toutre l'elen-

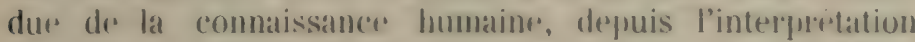
des plus simples faits dee lat meranique juspu'ans phenomènes les plus compliquers der l'histoire de's mations: il a ses ravines dans les profonderurs des lablitudes intellerturelles des dillirents ordres d'esprits. En vutre, les idées contradictoires stur la hature et la vire que ces habitudes intellectuelles produisent sejarement, influenerent en bien ou en mal les sentiments et la conduite des frommers, " (1)

Il importe de ne pas faire de l'histoire à priori. Inans le passige que nous venons de citer, "Relogion "s signitie, semble-t-il, l'ensemble dess manifestations de l'esprit l'eligrieux existant a une ipoque quelconque; "Science" " designe sans doute l'ensemble des enseignements scientifiques géneralement admis par les savants au mème moment.

Que le fétichisme ait éli, dans le principe, universel et (qu'il ait ite ensuite corrige par la découverte des " lois les plus simples ", ce sont deux aftirmations fort sujettes à caution. Spencer semble avoir plus tard renoncé à la première. Car si, commr il dit l'avoir recommu, le fetichisme n'est pas une forme primitive de

(1). P. P. p. 10. \$3, - F. P. p. 8. 
superstition, mais une forme lérivée du culte des morts, on the voit pas pourquoi clle se serait réalisse partout et les faits ne le demontrent certainrment pas. (1)

Lne interprétation religieuse des laits materiels et des événements de l'histoire ne contrerdit pas nicessiarement leur interprétation scientifique. La raligion vénire la Cause Première; les scirnces physiques et historiques recherchent avant tout les causes secondes. Ces deux ordres de causes ne s'excluent pas.

Spencer semble ne pas se rendre compte de cette vérite. Pour lui, la coexistence "des deux modes d'explications des épidemies, celui qui leur assigne pour causes des conditions mauvaises et celui qui en fait les ministres de la rengeance divine s est une contradiction (2). C'en rst encore une d'attribuer à la vengeances de Diru une mort dont les causes naturelles sont apparentes. (3) La distinction (fue nous vellons de rappeler fait évallouir cette contradiction.

II est rai que la Science comprend aussi la Philosophie qui poursuit la commaissance des causes supremes. Dirat-on que d'une facon genérale, la Philosophir ot la Religrion sont en lutte? Elles ont pu être en désaccorl parfois. Par exemple, la religion pä̈enne n'utait pas d'accord, an temps de Platon on mieux encore au temps de Ciceron, aver les rnseignements généralement admis par les philosophes.

Plus tard, lopposition entre la Philosophie et la Religron n'exsiste plus. La philosophie du moyen-ige? fut chrétronne dans son ensemble. Lat situation n'est plus la momr depuis deux siècles ; mais aussi, depuis lors, on cherchreat ren vain it preciser les enseignements grencralement admis palp les philosophes. Bon nombre d'entre

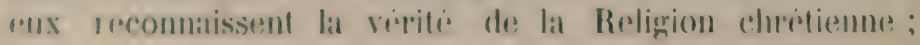

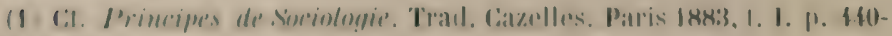
111 \& 103.

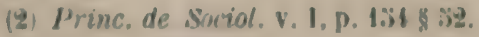

(3) Jlvid. p. 32: $813 \%$. 
quant aux antres ils nont a lui opprosere atucume doretrine positive sur larquelle ils soient d'accord.

En res qui concerne less scienress particulieres, molanment les sciemess historigues, il est vati fues la resligion

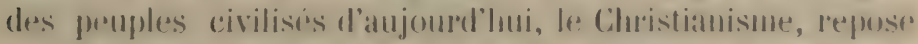
sur des lats listoriquess all sujet despuels cortains sitvants peuvent professer des ophinions inconciliables arme la verite de cette poligion elle-meme. If y a la matiom a controverse entre les savants, mais ancoune lutte pentre? la Religion d'une pant et la sciences de latuter. Loin de lutter contre la science, il semble plubit que less a theologiens "s'evertuent, aujourl'hui plus que jamais, at laire ressortir l'arrord des dogmes avere les vistestes serentitigures.

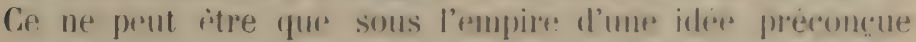

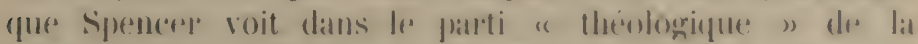

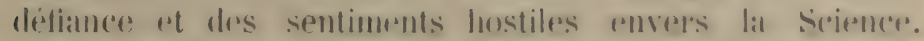

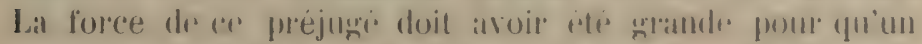

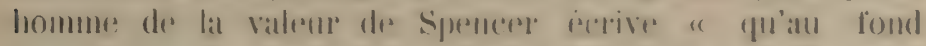

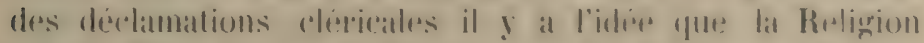

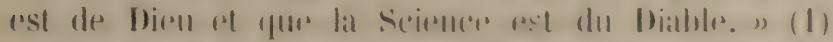

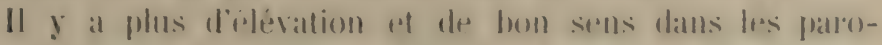

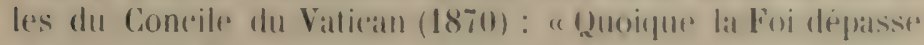

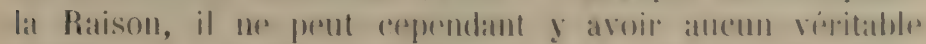

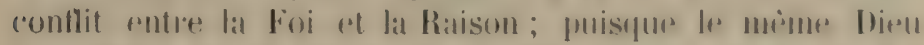
qui revele fes mystions de la Fui et qui donne la Foi

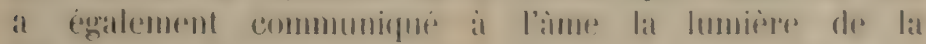
Raison. " (2)

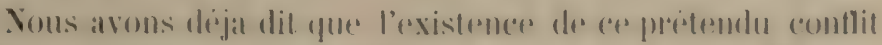

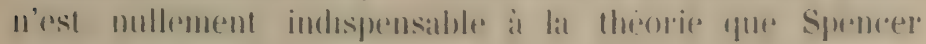
veut ètablir ici.

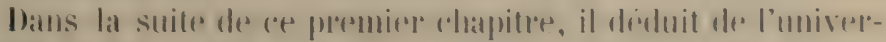
salite of do la temateite du semtiment roligrims la ronelu-

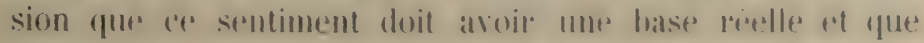

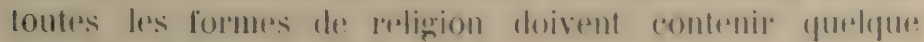
vérite profonde.

1) P. P., p. $20 \$ 6$ - F. P. p. 13.

(2) Constil. de Fide Cathol. c. IV 
If montre ensuite la science augmentant peu a peu ses conguêtes et selevant des faits particuliers aux généralisations de plus en plus hautes. Elle aussi est incontestablement legitime. Il doit y aroir accord fondamental la Science et la Religion. 
(II.IPITRE 11.

\section{LES IDEES DERNIERES DE LA RELIGION}

Imagination et intelligence. - L'origine de l'Univers. La nature de l'Univers.

agination et elligence.

Lorsque nous formulons te jugement suivant: La terre est sphérique (nous choisissons a dessein 1111 premple: dont spencer so sert lui-méme an debut de ce deuxieme chapitre), qu'y a-t-il dans le (hamp) de la conscirences? d'y puis d'aloord distinguer une representation imatginaliver, anditive on visuelle, de la phlatse que je viens d'ecrire. Jy trouve ensuite la liguration fgalement imaginative d'un globe terrestre semblable at cerux que j'ai dejal vus, ou bisol de la surface sur laquelle je malche confusément prolongrese en surlace spherrique. Si je ne fais attention quand imtges sensibles, mal conscirnce pour'a n'en découvrir aucune autre.

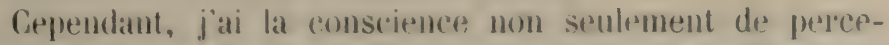
voir la proposition susdite comme une suite de sons, mais encore den comprendere le sens; et il est clair que ce sens liest que très imparfaitement requésenté par l'image de la Trare que je me forme. D'une part, en effet, elle pèche par defaut : la forme spherique n'y est ni netle ni complite; le globe terrestre que je me 
ligure représente peut-ètre aussi bien une planète quelconque que la demeure de l'homme. D'autre part elle piche par excès: l'imagination donne nécessairement à la sphère certaines dimensions détrminées, trop petites d'aillrurs lorsqu'on les applique à la Terre et qui ne sont, en tous cas, aucumement désignees lorsque j’affirme que la terre est spherique. Toute representation imaginative ou phantasme, neétant que la reproduction allaiblie de pereptions sensibles, est concrete et individualisee, tandis yur nos jugements contiennent des idies générales et abstraites.

de veux bien qu'on donne, arec spencer, au phantasme de la Terre le nom de " ennception symbolique n. Mais, si elle est un symbole et que je l'apprécie comme tolle, il faut que j'aie la connassance de' la chose dont alle est fe symbole. Cette derniere ronnaissance n'est pas une image sensible; rependant elle n'en existe pas moins, de mime que le jugement qu'exprime la froposition: La Terre est sphérique. D'apres Spencer, la refresentation imaginative très imparfaite serait la seule notion que nous ayous actuellement de la Terre. Yous nous sepvirions de telles images comme si elles irtirnt rejelles parre que nous savons "qu'elles peuvent itre completeres ") c'est-it-dire corrigees, el qu'elles "srevent d'acheminement à des conclusions dont la validiti a pour pirere de touche la correspondance arec l'obselvation ).

Sans doute, nous pouvons "complinter" m l'image sensible furr mous nous faisons de la Trerre, mais, encore une fois, crest graice a l'idere non spossible que nous en possé-

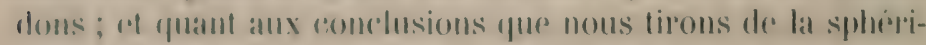

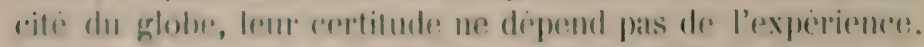

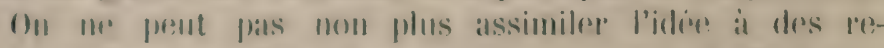
presentations sensibles inconscientes, comme lo font corp-

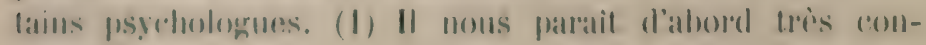

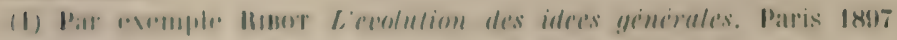
P.p. 14\%, si. 
tristable que des representations alctuelles puissant ichapper à la conscirence lorspue l'altrention est attirese sur notre sifuation psychique. Gnand dome nous farlems on entendons parler de choses sensibles an conpurenant les mots mais sams former l'image fot fule: nous avous vitguement conscience de pourvir la reporduire, ce n'est pats (que l'image existe dans l'inconseient, mais birnl yu'elle existe en puissance.

Quoi qu'il en suit de cotle image inconsolenter on en puissance, relle ne, pent pas itre confondur avere l'idere abstraite. De celle-ci nous en arons conscience et satls ollo tout jugement ou raisommenent est impossible. Sous aroms

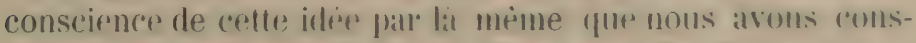

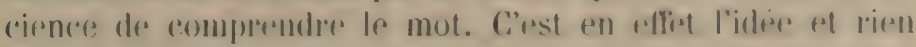

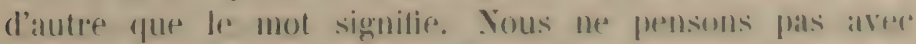
des mots, ni aver des sigmes, mais aver des iders.

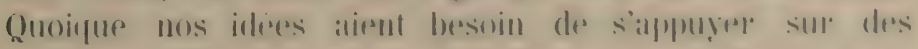
phantasmes: pepresentations reshles ou symbuliegurs, mols

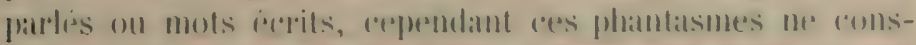

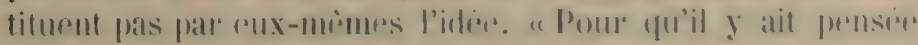

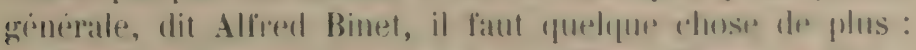

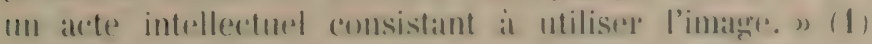

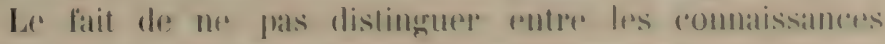
rationnelles ( idlies, jugroments, maisomurments ) at les ro. presentations imagrinatives qui les arerempagment nest pas. chez spencere, l'ellid d'une distratetion, mais bien une' theorie yui, supposise iri, est reprise all rommenterment

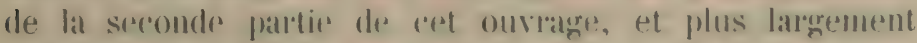
developpere dans lis Principes de Psychologie. Cette ("ontfusion est imadmissible, nous renons de le roire ret nous aurons l'oceasion de nous m ronvainere feneore dius la suite.

Les phantasmes fui adrompangent nos idées et nos raisomnements sont souvent - rien liest plus vaia - la cause d'urreurs, parce qut: nous leur attribuons une: (eon-

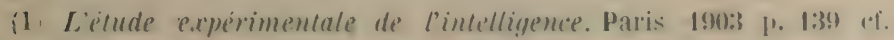

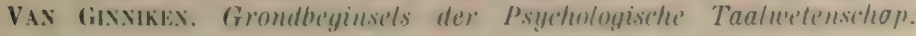
Leuvensche Bydragen 1904 p. 31 sq. 
formiti aver la rialite qüils mi: possedent pas. En ce sens on prut dire qu'ils ont besoin d'ètre "completés"; et lorspue la similitude entre rux el les objets ne peut flas etre verifie directement, les conclusions quon tireratit de cette similitude frisumée ont, sans aucun doute, besoin d'etre contrilees par l'exprience. Mais, de ce que ces phantasmes sont imparfaits ou irréels, on ne peut rien conclure au sujet des idées dont ils sont les symboles.

Les remarques précédentes étaient nécessaires tant pour corriger les réflexions que spencer place en tète de son second chapitre que pour permettre de porter un jugement sur les raisonnements que cte chapitre contient.

Toules les religions consistant essentiellement dans la croyance à un Ethe Souverain, cause première de l'Lnivers, voici les deux propositions qur: spencer so propose de démontrer:

1. Il faut admetle" " une puissance dont l'Luivers est la manifestation. " (1)

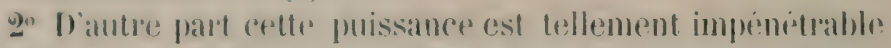
qu'aucume hypothese particulière sur sal mature ne resiste à l'examen.

Dans le developpenment de ces thises qur spencer Jui-mimr ajpelle "la plus importante de toutes less etudes" (2) on doit sattembe a trouver la plus gramde precision ex une extrême rigueur log̣ique.

Yous disons sans hesiter qur ces qualitios no s'y rencomtrent pas. Syentere est souvent dilfus. Sa pensée ne:

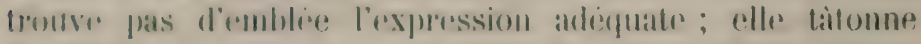

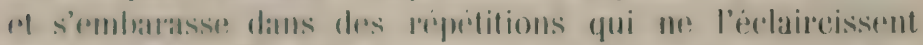

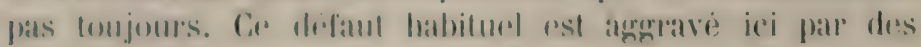

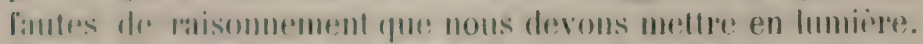

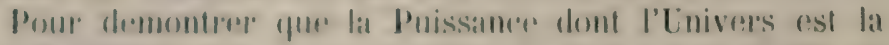
manifestalion rest incomnaissuble, il laul of il sulfit de

(1) P. P. p. 18 11- F. P. p. 84:

(2) P. P. p. 21 \& $\mathrm{k}-$ F. P. p. 17. 
faires roil quancums affirmation concernant sad nature ne peut étre prouvée. On conclura alors legritimement que nous ne pouvons avoir au sujor de sa nature anceune conuaissance.

sprencer va plus toin el fretrond montres qu'aucume hyjolliese sur sil hature n'est concerable, que "les iblements de ces lypotheses ur: peurent point itre reunis dans la conseience of que nous me pouvons nous les ti-

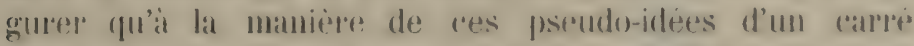
fluide on d'une substance momale, c'est-it-dires, "all we? dererehat jamais it en fatre des idies rrelles n (1); qur loute hypothèse semblable a contient des conceptions symboligues illeggitimes el illusoires " (2), "contient une

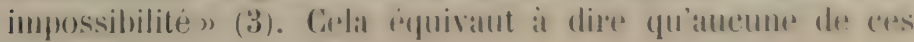
hypotheses n'est vraie.

Sprencere, all surplus, daus sus Principess de Psycholugie domne l'inconcerubilite commo le criterium supreme four distingure le vala du faux : valie est la proposition dont lat contladictoire est inconcerable. "de me puis conceroir"

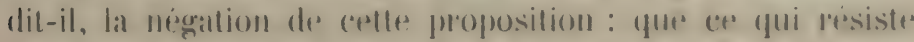

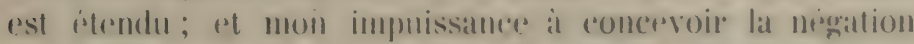
me montre que toujours aver lo sujet ( questyue choses (lo lesistant) coexiste invariablement le predicat (l'elendue)....

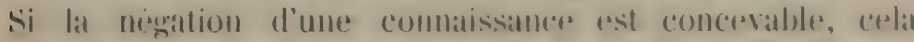

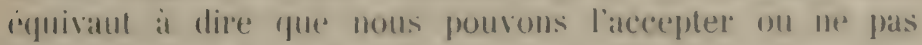

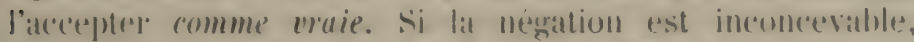
nous sommess obligess de l'accerpter." (4)

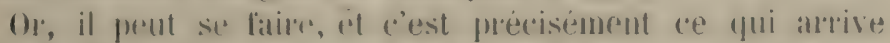

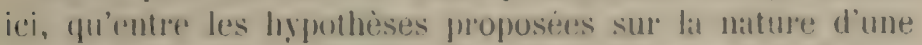
chose il n'y ail flats de milien. Datns ce cas il falldra romelure en bomme logrigue que leur ubjet est non semlement incommaissable mais inexistant. Examinons la chose en detail.

(1) P. P. p. $37 \$ 11-$ F. P. p. 20.

(2) Ibid

(3) P. P. p. $36 \$ 11-$ F. P. p. 26.

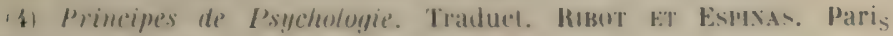
187i) 1. II p. 12: \$ 179 . 
si nous ne roulons pas mettre fin a toute recherche scientifipue ou mème à tout travail de l'esprit, nous derons armettre que toute chose, et par conserguent aussi l'existence do l'Ĺnivers, a sa raison suffisante, son explication. "Yous pourons, dit Spencer, fare trois suppositions intelligibles verbalement sur l'origine de l'Lnivers. Nous pouvons dire qu'il existe par lui-mème, ou qu'il se cré lui-mèmo, ou (qu'il est créé par une puissance extrieure. Il n'est pas necessaire de rechercher ici bquelle de: ces trois suppositions est la plus croyable. Cette question se résoud en détnitive en une question plus haute, à saroil si l'une d'elles est concerable au rai sens du mot. Examinons-les l'une après l'autre. » (1)

La question est évidemment mal posce. Il lly a pas trois hypotheses, mais seulement deux: l'Lnivers a sa raison d'itre en lui-mème ou en dehors de: lui. Spencer va reconnaitre qu’il en est ainsi. Il dit en effer : " I)'abord il est clatr que pour nous les mots d'existence par soi veulent dire une existence indipromlante d'une autre, qui n'est pas produite par une autre.) (2) s’il en est ainsi "l'hypothese de la creation frar soi ) se tromse éliminese.

Spencer continue: "En excluant ainsi l'idée d'une rause?

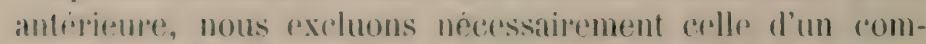
menterment; all admattre l'ilese d'un commencement, admettre qu’il fut un temps ou l'resistenes llavait pas

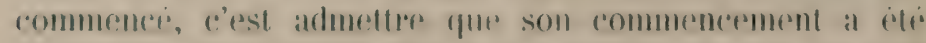

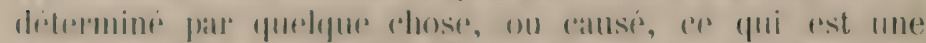
contratiefion. Dono l'existenere pate soli signifio l'existences

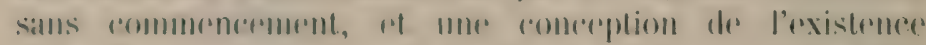

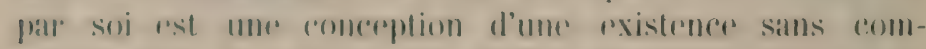

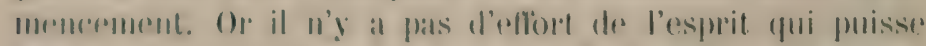

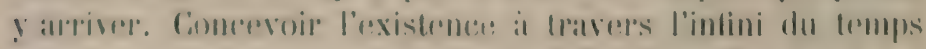

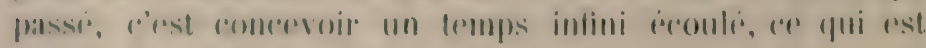
une impossibilite. " (3)

1) P. P. p. 31 \$ $11=$ F. P. p. 22.

(2) P. P. p $32 \& 11-$ F. P. p. 22

(8) P. P, ए, 32 \& $11-$ F, P, P. 93. 
Si concevoir signilis se perprosinter par l'imagination, il n'y a point dre doute quiune telle replessentation ne soit impossilsh. Mais il s'aghit irei l'me conception par l'intelligence.

Au lixu du mot temps mellons durée qui est plus ugtoneral et peut s'appliqum aux choses immotables. Il n'y a pas lien, dans ces dernieres, à cause meme dre leur inmutabilite de distingues les passé du fulur, ni ditfirentes parties du passe ou du futur. Cela etant, peut-on conce-

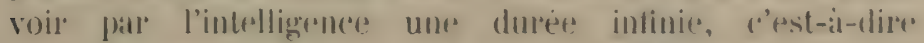
salls bornes, sans commencement ni fin? Je reponds oui sans hesiter : cettre conception no contirnt ancoune contradiction, elle n'est pas " un symbole illexitimes et illusoire " mais une notion parfaitement coherente at dont tous less éléments sont intelligibles.

Autre chose est des savoir s'il all est de mine pour

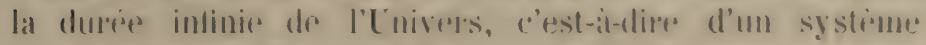
en mouvement. On perul, a motre avis, demontres que lat doure intinie d'un tel strstemes est ume notion contradic:-

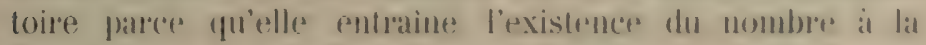

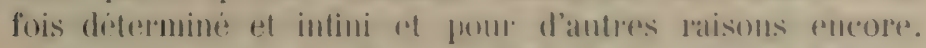

La serule notion de churise, qui fatit alsstratetion du changere-

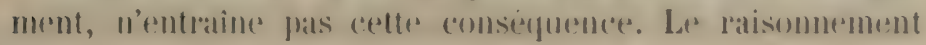
de Spencer est donc inefticace.

spencer poursuit : "Ajoutons à cela qute l'existence par soi fuitelle concerables, elle ne pourrail en atucun seus expliquel l'Cnivers. (On ne peut pas dire que l'existroner d'un objet, à un moment domni, devisme plus concevable paree quion a diecourert qu'il existait me heure, un jour, un all auparavant; et si son existence a i $\%$ moment ne devimt gas le moins du monde plus intelligible par le fait de son existence durant me periode anterieure linie, il n'y a pas d'accumulation de periodes, même poussèe à l'intini, qui puisse la rendres plus intelligible. Aussi, non seculement la throries athriste est inconcevable, mais ne le fùt-elle pas, elle ne serat pas pour cela me solution. Laflirmation que l'movers existe par soi ne fail pas faire un pas an dedi do la comnassinnce 
de son existence presente, et par consequent nous laisse en présence d'une alfirmation nouvelle du mème mystipe., (1)

La theorie atheiste, Spencer l'oublic, affirme non seulement que l'Univers n'a pas eu de commencement, mais en outre qu'il existe par lui-même ce fui est " une explication. » On peut évidemment ne pas l'adopter, mais plle n'est pas une simple constatation de l'existence de l'Univer's.

La critique que fait spencer de la seconde hypothèse, "la creation par soi ", ne donn" lieu a aucune observation importante. Il est facile de montrer qu'elle est "inconcevable ". "Les termes de cett" hypothese, dit Spencer, ne représentent pas des choses réelles, mais sngrgèrent seulement les symboles les plus vagues et les moins susceptibles d'interprétation ") (2). Inconcerable (notons-le encore une fois) signitie donc bien pour spencer: non conforme à la réalité, c'est-à-dire : faux

"Il reste à examiner l'hypothèse géneralement admise du theisme, la création par un pouvoir extérieur. Dans les plus grossières croyances comme dans la cosmogronie qui a depuis longtemps cours parmi nous, on suppose que le ciel et la terre ont été fiats 'n quelque sorte commo un meuble faronne de main d'ourrier.... En supposant que le soleil, les planites, les satellites et toutes les choses que ces corps contirnnent, ont été formés d'unc manière semblable par le Grand Irtiste, nous supposons seulement qu'il a dispose dans l'ordre que nous voyons presentement certains ilements prexistants. Mais d'ou viennent des eliments preexistants? Lat similitule ne nous les fait pas comprendre, et tant quelle ne le fait pas, alle ast sans valuar. Lat production de la matiere tiree de rien, voili le vrai mystère. Cette similitude pas plus qu'une autre ne? nous renol capables de la concevoir, at nous ravons que faire d'un symbole qui ne nous donne pas ce pouvoir. " (3)

(1) P. P. p. 2 \& $11-$ F. P. p. 28.

(2) P. P. p. 398 12. - P. P. p. 24.

(3) P. P. p.p. 34-3.; $11-$ F. P, p.p. 24-25. 
Evidemment, l'hypothese theiste ensrigne que les matciriaux de l'Lnivers, aussi bient que l'Tnivers lui-meme, ont été produits par Diru. Personne ne pretend expliquer la création far l'activité de lartisan. Gu'elle soit un mystrare, cela peut avoir un serls varii. Mais la question est dre savoir si elle est inconcerable, cest-idire, si elle "st une notion incoherente ou contradictoire. Spencer n'essaip pas de l'etablir, mais passant it une alutre consideration, il poursuit ainsi : "L'insulfisantop de bat theorie thejste de la creation devient encore plus manileste fuand on passe des objets matériels it cr yui les contiput, yuand all lien de la matiere on examine l'espace. Nexistàt-il rien qu'un vide incommonsurable, il findrail encore l'explinguer. L'me

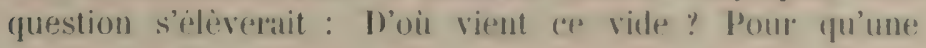
theorie de la creation fiit ampletre, alle devarat repondre que l'respace a ete linit de la meme mamiere que la

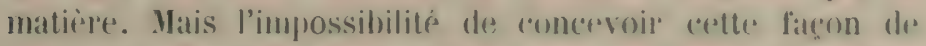
creer l'espace est si manifiete que fressonne nose l'allirmer. En ellet, si l'esplater a ele creed, it n'existait pas auparavant; or, il n'y a pas d'elfert d'esprit qui puisser

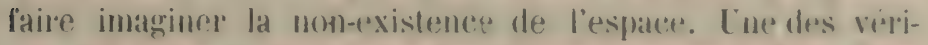
lis qui nous sont le plus familieres, c'est que l'iden d'un espace nous envelopplant de toutrs parts ne peut fals un seral instant etre bannies de la pernsies. Ton seulement nous sommes forces de premser l'espaces comme pressent partout, mais nous sommes incapables d'en enonceroir l'absence, soit dans le passé, soit dans l’avenir. Si la non-existence de l'espare est alsolument inconcevahle, il en résulte que la création de l'espace est inconcevable. ") (1)

Ce raisomement dépend entièrement de lat supposition que tout itre eréc a en un commencement, ce qui n'est pas évident et aurait donc hesoin d'ètre démontré. Les opinions des philosophes sont partigres sur c'e point. Si l'espace, tout en citant crèr, at existe toujour's, l'argument ne tient plus.

En outre, et ceci est plus important, Spencer ne s'est-il

(1) P. P. p.p. $35-36 \$ 11-$ F. P. p. 25. 
pas aperegu que son argumentation suppose une notion fausse de l'espace et n'a de force que contre ceux qui l'admettent? Nous n'ignorons pas les idees tris diflerenles qui ont èté émises en cette matièrt. Pour ce qui nous concerne, il nous ast impossible d'admettre que l'espace alsolu est autre chose que l'étendue des corps consue d'une manière abstraite, c'est-i-dire, indépendamment de leurs dimensions individuelles et de leurs autres propriétés. II n'y a donc, d'après cela, d'espace réel que dans les colps existants. L'espace réel n'est pas quelque chose de distinct des corps et qui les contient, il est constitué par l'étendue des corps eux-mêmes.

Si des corps sont distants sins qu'il y ait entre eux un corps intermediaire, on dit fü'il y a entre eux un espace vide, ce qui revient à dire qu'il n'y a rien. (On domne, il est rrai, a cet espace vide dos limites et des dimensions, ce qui semble indiquer qu’il est réel; mais if est facile de se convaincre que ces limites et cos dimensions no sont réelles que dans les rorps qui bornent l'espace vide et que toute l'objectivité de l'nspace vide consiste dans les distances mutuelles de ces corps.

En dehor's de tout corps, l'espace vide est le pur neant et il n'y a donc pas a st demander d'ou il vient, ni far qui il a ete crés. Quant à la representation imaginaltive par lanfuelle nous nous figurons au delia des colps romme une étendue indétinie, elle n'est qu'une reproduction confuse d'impressions visuelles et clle n'est valment d'ancun usage daus la question acturlle.

()n est d'antant plus itonne dre rencontrer ici une argommentation dams lanpedle on considere l'espace comme une realite indipendante des corps que spencer lui-meme definit l'espaces ot le temps comme des abstractions: "Lat comerention abstrate do toutes les sempences, dit-il, est

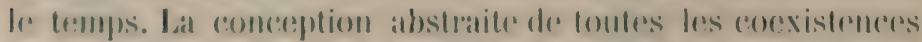

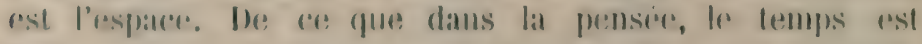

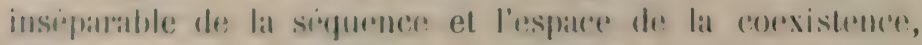

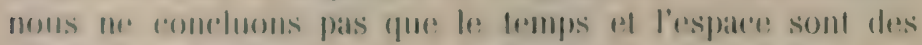

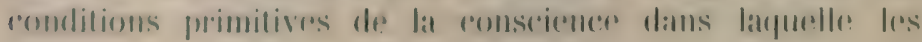




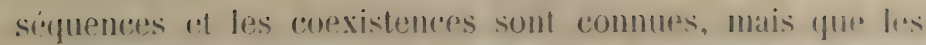
conceptions de temps et despares sont froduites commes d'autres abstraits sont produits par les antres conerets...n (1) Est-ce qu'une alsstratction a une existence en dellors des concrets: Jat mature humaine se trouve-terlle ent dehors des individus humains? Diss lor's dos raisommements sur

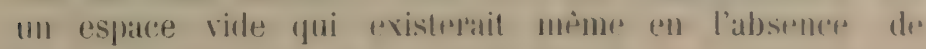
corps, manquent entièrement de base.

"Entin, poursuit icei stremeer, all supposant mèmes yur.

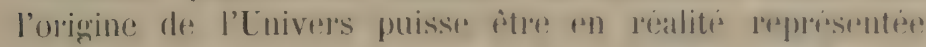
datns la frensere comme le produit deme puissantere exte-

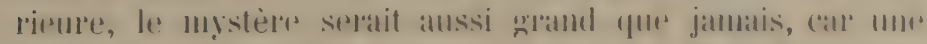
question se poserait meore: llou vient l'existrme d'un pouvoir extérieur"

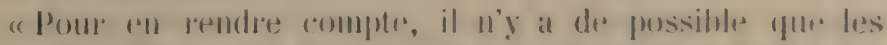

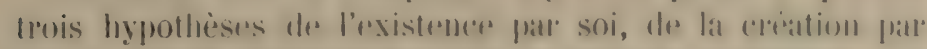

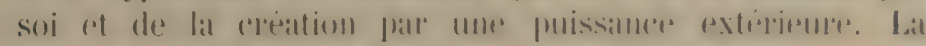

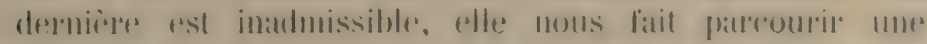

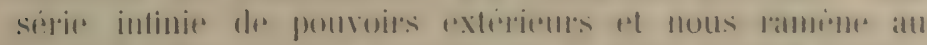

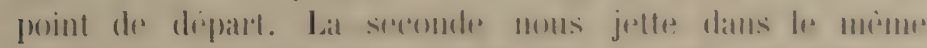

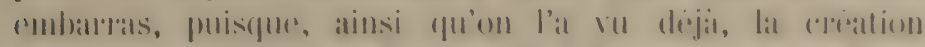

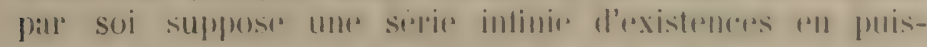

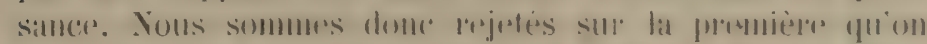

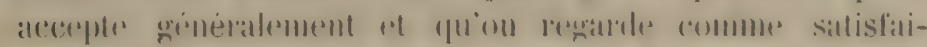

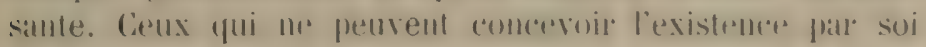

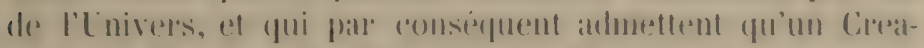

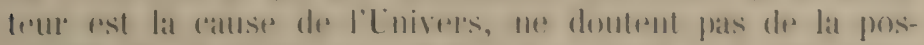

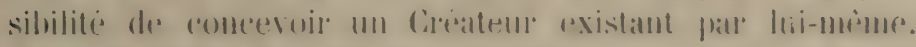

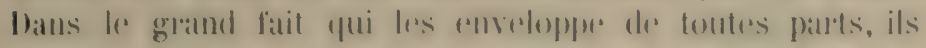

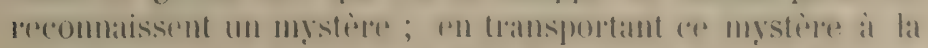

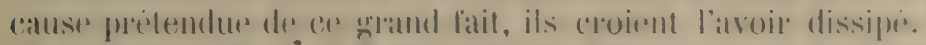

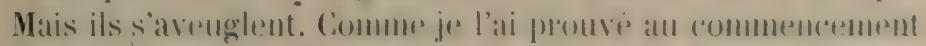
de mat discussion, lexistence par soi est rigouremsement

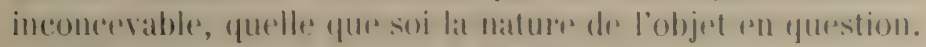

"Qhuiconque reconnait qure la throrie alluiste est insou-

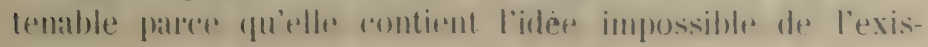

(1) P. " 1. 17:3\$17 F. P. 1. 128. 
tence par soi, doit forcément admeltre que l'hỵpothèso du theisme est aussi insoutenable parce qu'elle contient la même impossibilitè. " (1)

Qur le lecteur reuille bien se rapporter at ce que nous arons dit plus haut. L'inconcevabilite de l'existence par soi n'a ité prouve d'aucune fason. La theisme n'admet jas que l'Lniver's existe par lui-meme, parce qu'il a tous les caractères de la contingence: il est rariable, il a eu Im commencement, il est limité. On est done obligé de chereher la raison sulfisante de son existence dams un Createme existant par lui-mème, immuable, éternel, intini. Nous ne royons pas ce que cette doctrine a d'inconcerable ou d'incohérent.

Il est done facile d'rxpliquer pourquoi les theistes jugent inconcevable l'existence par soi de l'Lnivers et admettent néanmoins l'existence par soi du Créateur. La raison se trouve dans les differences entre l'un et l'autre que nous venons d'indigurer. Si l'on n'admet pas rette raison, il faut montrer (qu'elle n'est pas valable. Lat chose en vaut la preine et sprencer insiste partois longurement sur des matiores beancoup plus simples. S'itonner de (e) que les thristes fassint all point de voe de l'exislence far soi une distinction ratloale entre l'btre intini a immuable et l'Lnivers qui n'rst ni immuable ni intini, c'est l'eftet d'une distraction qu'on pent difficilement excuser.

nature de l'Univers.
Simencere abordes les enseignements de la Religion sure ce quill appelle la nature de l'Cnivers. "si, laissant l'origime dre l'lnivers, nous en voulons connatere la nalure, les mimes diflubllis insurmontables se deressent devant nous, ou plutril re sont les mimes diflicultes sous des formes nouvolles. Nous noms tromvons d'une part obligies for latre cerlatines suppositions, of d'antre part nous

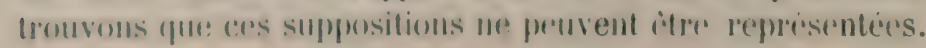

(1) P. P. P. $38-36$ \& $11-$ F.P. pp. 25-26. 


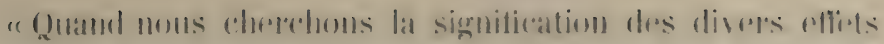
fmoduits sur mos sems, fuamd nous demandens comment,

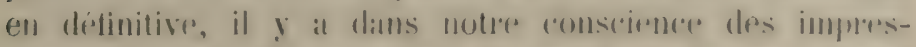

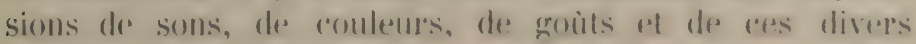

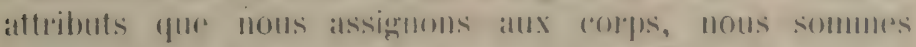

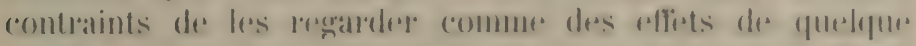

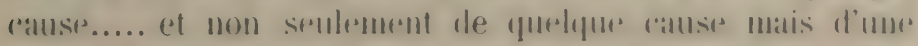

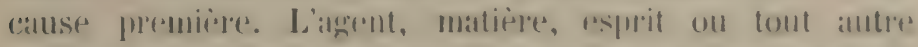

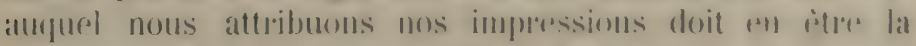

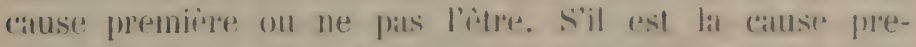
miere, tout est fini. S'it me l'est pas, il fant qu’il y ait drerrière lui unf antre cause qua devienure alors lat cause réelle de l'effet.....

"Mais si nous voulons farme un fass dre plus, si noms

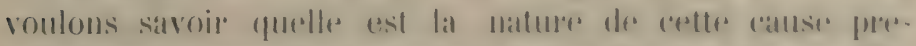

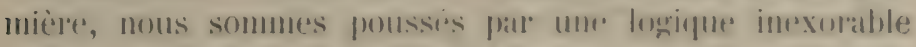

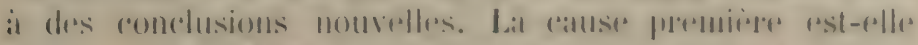
tinie ou infinie? si nous disoms linie, nous nous rmball-

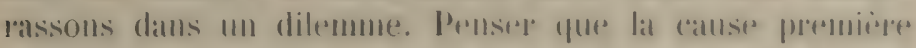

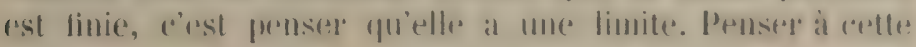

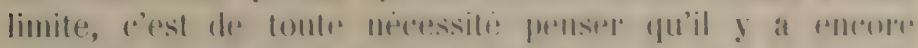
qunelyque dhose an delat; il est alssolmment impossible de

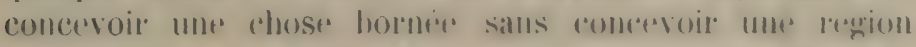
qui l'entomer de tous cotes. Qute dirons-nous de colte

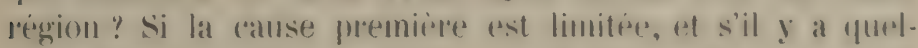
que chose an dehors d'elle, a'e quedque doose are doit

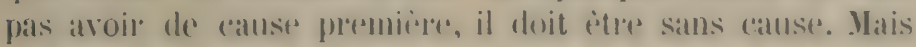

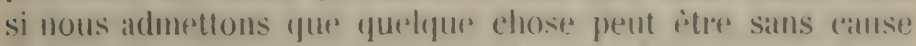
il n'y a pas de rason de supposere qu'une chese. quelconcule ait une cause. si an dehors de cette region tinie

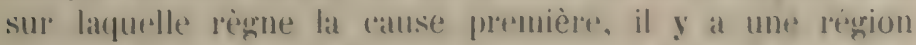
que nous sommes foreses de rexarder comme intinie, sur

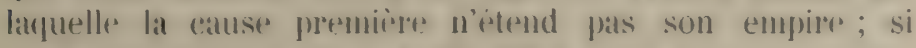
nous admettuns qu’il y a un intini sams cause, enveloppant le fini cause, nous abandomons implicitement l'hypothèse de la causalite. Il est donc impossible de considerer la cause première comme linie. Mais si elle ne peut ìtre finie, il faut qu'elle soit infinie. 
"Il y a une antre conclusion quion ne pent éviter (guand on raisomes sur la eause première. Il faut yu'olle soit indépendante.... Mais pensep que lil cause jremièr est totalement indépendante, c'est pruser qu'elle existe en dehor's de toute autre existence; rar si la prissnce d'une autre existence est nécessaire, la cause premire doit dejondre partiellement de cette autre rxistence, et ne peut plus être la cause première. Cre n'est pas tout; non seulement la rause première cloit avoir ume forme d'existence sans relation necressaire avec toute autre forme d'existence, mais elle ne peut avoir aucune relation necessaire au declans d'elle. Il ne peut rien y avoir en elle qui détermine le changement, ni rien qui lempriche. Car s’il y a quelque chose an alle qui lui imposte ces nécessites et ces restrictions, ce quelque chose doit ètre une cause superrieure à la cause première, ce qui est absurde.

"Ainsi la cause première doit itre dans tous les sens parfaite, complete, totale, renfirmant en elle tout pouvoir et siderant an dessus de tonte loi. Ou, pour nous servir de l'expression resue, elle doit itre absolue.

"Ainsi done sur la question de la mature de l'Lnirers, nous nous hemtons a deux conclusions inévitables. Ises oljets at les actions qui nous entourent, non moins que les phenomines de notre propre conseience, nous foreent dr rechercher une catuse; une fois cette recherche commencere, nous ne pouvons nous arreter nulle part avant d'arriver a l'hypothese de la cause premiere; et nous

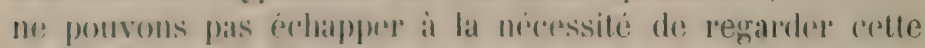
(ants: premiere comme intinie et absolue. Il n'y a pas moyen d'edripluel aux arguntents qui nous imposent ees conséquences " (1)

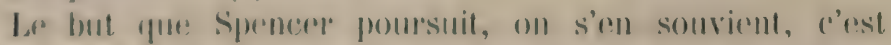
di. montrop quancume des hypolheses quon peut faire?

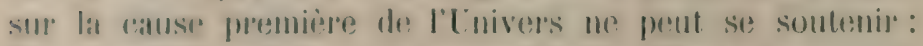

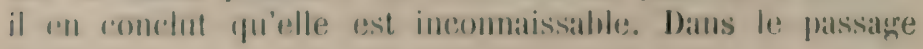

(1) P. P. P.P. $28-40$ \& 12 - F. P. P.p. 27-29. 


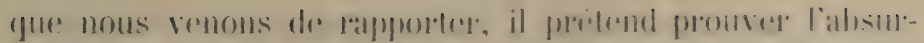

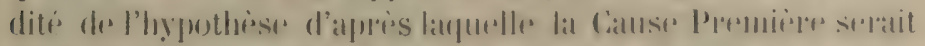

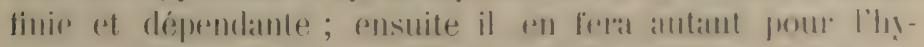

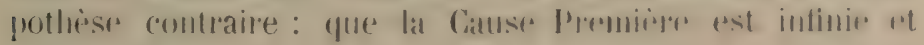
indépendante.

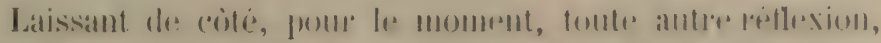

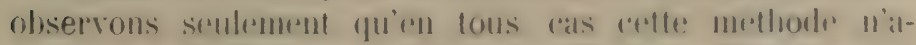

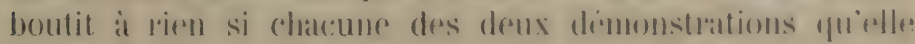
contiont n'est pas absolument rigourense. Examimons la premirire; nous lavons ditie in extenso. Yous allons

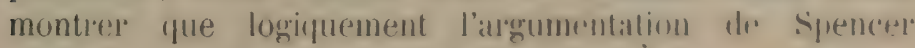

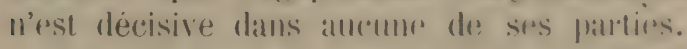

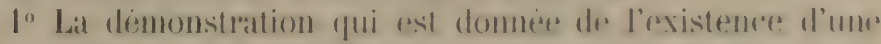
Callses Premiere suppose l'impossililitio on, si l'on lout,

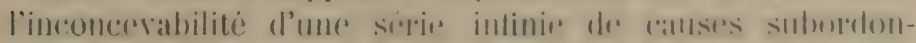

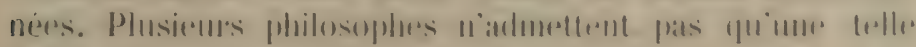

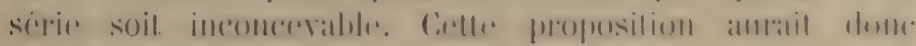
lesoin d'etre dimontree.

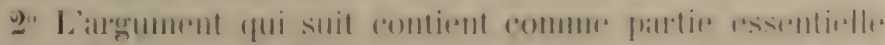

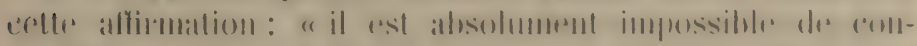

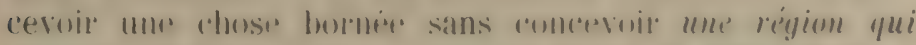

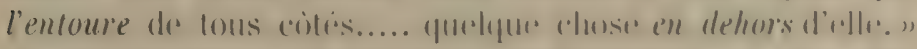

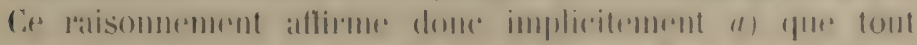

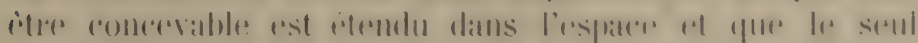

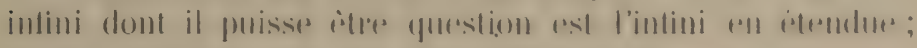
b) que l'espace vide est une chose réelle.

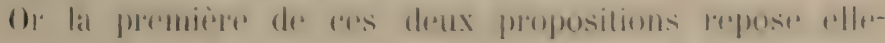

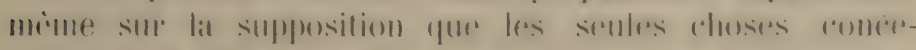

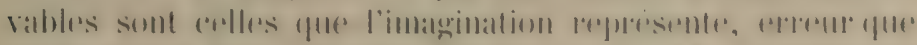

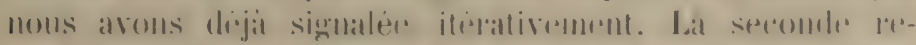

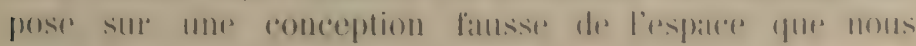
avons écartée plus haut.

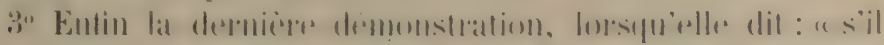

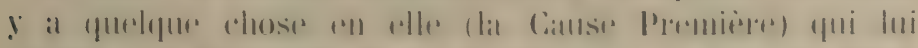

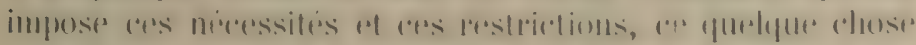

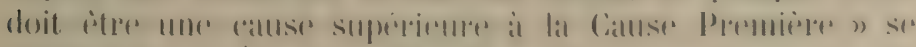

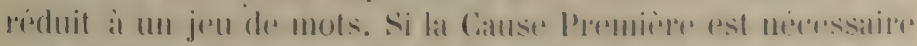

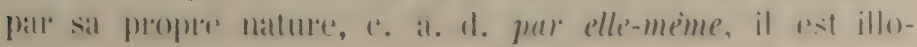




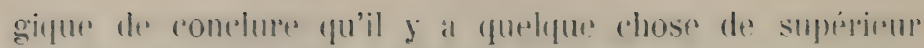
al elle, ou que la nécessité lui est imposée.

Aucume partie dra la demonstration yui doit établir l’inlinite of le caractere absolu de la Cause Premiere n'est rigomrense. Chatpur rasommenent contirnt comme partles essentielle une proposition fausse on une affirmation qui exigerait une démonstration.

Voyons, maintenant, la contre-partie. La facon dont spencere l'introduit appolle une observation. "II est, ditil, it peine besoin de dire aux lecterus qui mont suivi jusqu’ici combien, ces raisonnements rt les resultats auxquels ils aboutissent sont illusoires. si je ne craignais de fatiguer lou patience sans utilité, je n'aumais pas de peinf a prouver une les edements du raisonnement, de? meme qune ses conclusions, he sont que des conclusions symboligues de londere illegitime. Toutofois, an liesu de respeter la refulation que j'ai rmployere ci-dessus, il vaut minus

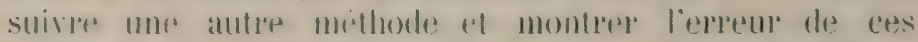

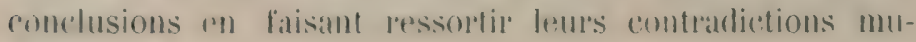
tuelles. $)$

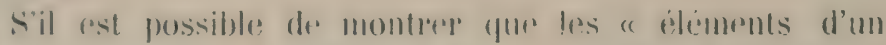
ratsonmement... ne? sont que des eonceptions symboligurs dr. l'ordre illegitime m, on so demande? comment il est

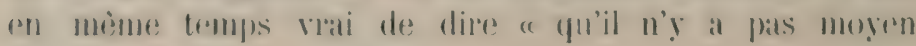
d'echapper à ces arguments. "

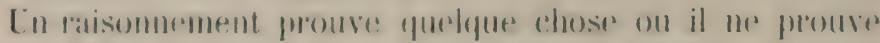

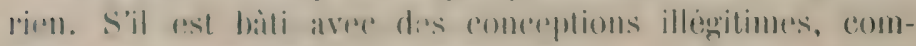

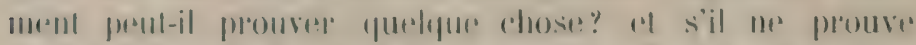

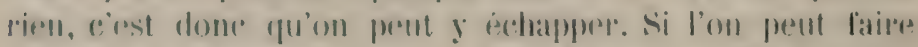

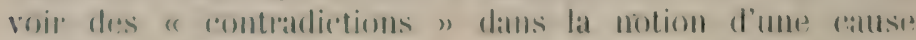

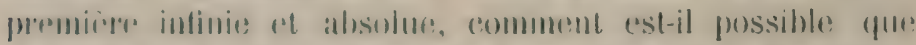

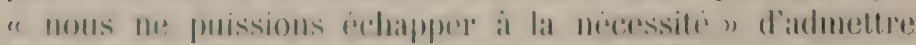

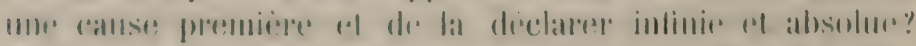

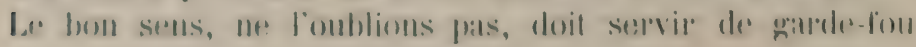
sux spéculations mitaphlıysirgues.

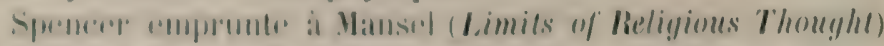

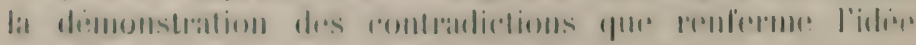

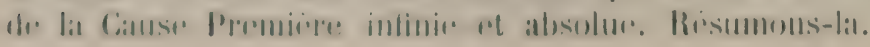


1" Labsolu ne prut pas ètre causcr, lidere de caluse jenfermant une relation.

Qo La Cause Première doit ètre libre ent frall conséquent eonsciente. Or, gui dit conscirne dit relation et nire l'absolu.

3" "Comment.... la puissance infinie peut-elle toute: chose, tandis qut: la bontie intinife est incapable dre faire le mal? Comment la justicer infinie innlige-t-elle les dere-

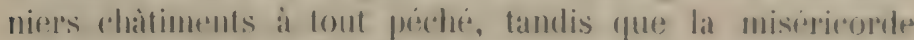
intinie pardonne all compable? Comment la sauresse intinie

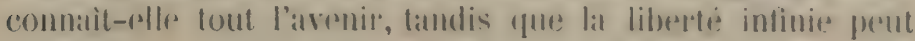
tout faire et tout eviter? Comment l'existrunce du naal

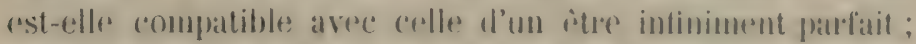
cal si Dien vent lo mal, il nest pas intiniment hon; et

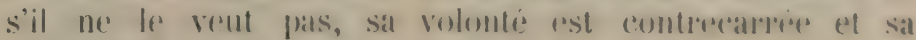
sphère d'action limitée? „) (1)

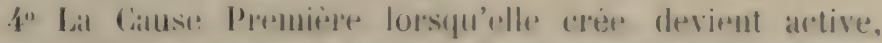
alle allgmente en perfertion; elle netait fonc fas intinie.

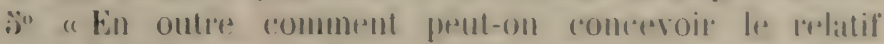

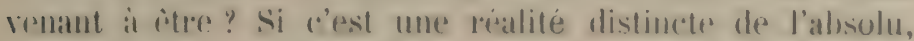

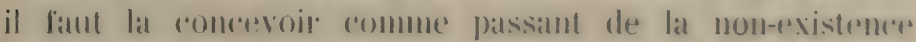

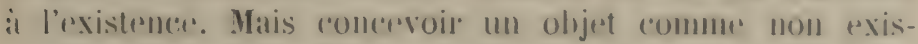

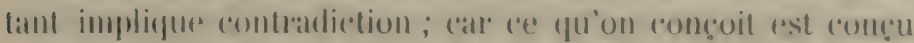
comme objet de pensie dans et par la conception. Nous pourons ne pas penser un objet, mais si nous le pensons, nous me pouvons faire alltement que de le penser amme" existant. 》 (2)

Trille est la demomstration de Manses. Cenx qui croimt notre intellignence! capalble de commaître quelque chose all sujel de la Caluse Premieres - et c'est l'immonse majorite des pensedurs - ont rémni a'es comnaissances dans des ourages ou elles sont déduites avec tonte la rigguene possible et coordonnées.

Parmi les oljets auxquels l'resprit humain s'est appli-

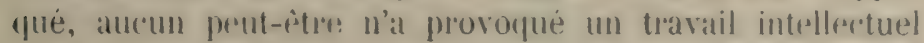
plus intense. Toutes les yuestions s'y rapplortant ont itte

(1) P. P. P. 43. \&13-F. P. 31.

(2) P. P. p. 44. \$ $13-$ F. P. p. 32. 
souleries, résolues parlois dans des sens dilfërents, toutes les difficultes on eté aprrours et examinees. A lire le passage de Mansel que nous venons de risumer, il semble fur lui-meme of spencer avec lui ignorent completemont res travaux, puisque Ies contradictions yu’ils décourrent dans la conception d'une cause première intinie ef absolue ont ite l'objet d'études approfondies et de solutions qu'il est an tous cas impossible de trouver superticielles. On a Ie droit de juger ers solutions insuffisantes, mais on lla pas de droit de les ignorer complefrment lorspu'on veut écrire sur ces matières.

Il est évidemment permis aujourd'hui de ne pas admettro la thénie transformiste, mais l'anteur qui prétendrait refuler cotte hypothèse en lui opposant certains faits sions truir aluem compte de l'interperetation qu'en ont domnee les transformistes, montrerait qu'il n'est fas au courant de la question qu'il trate. L'èrivain que spencer oppose aux théologiens merite d'itre juge ansi. Yous nous contenterons d'indiquer très brievement les defauts de ses raisonnements.

L. dernier n'est évidemmant yu’un jeu de mots. A qui ferat-(on croire qu'il est impossible de concevoir tule

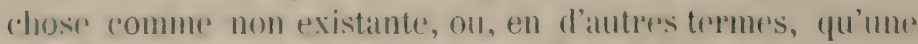

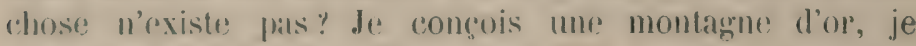
coucois anssi fu'elle n'existr pas. Je romgois donc une montagne d'or coinme non existante.

Largument precident contient ceste alfirmation : fonte

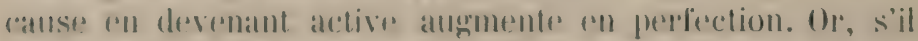

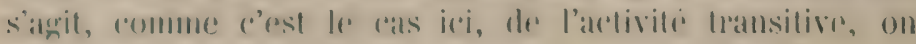

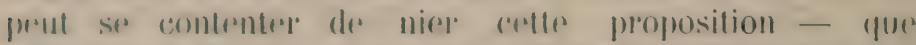

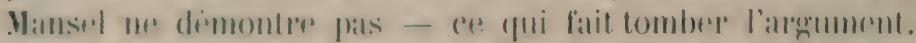

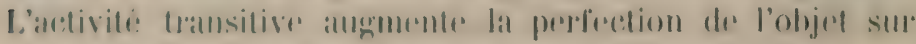

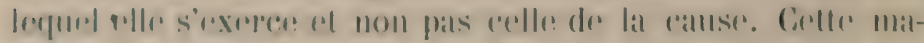

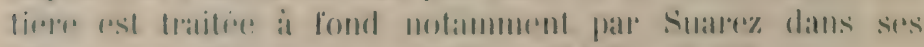
Jiscussims Metuphysiques (Disp. Xi, VIII. S. IV).

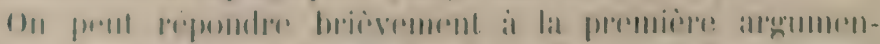

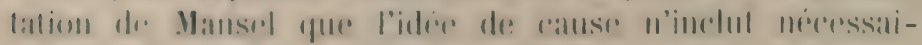

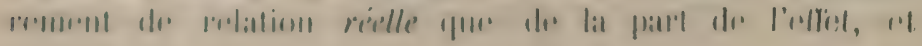


qu'ainsi les reflets de la Cause Premiere dependent diolle

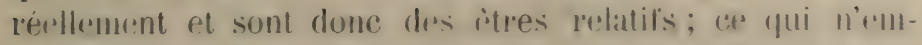
piche pas la Callse Premiere d'ittre absolue. On perent lire le developmement de cefte theorie, far axemple, dans lat Somme théologique de st Thomas d'Aquin (p. I. If. XIII. a. VII.) at lemdroit ois il explipue comment f'on attri-

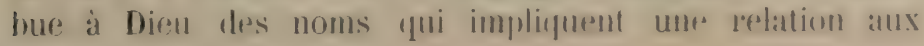
créatures.

La connaissance que bien a des creatures une comstithe fas davantage une relation reolle de lat part de bieu, parce que les créatures sont commues par lui en rertu meme de la prerfertion intinise der l'intelligenese divime. Guant is la comnaissane que Dien a de lui-meme, enthe

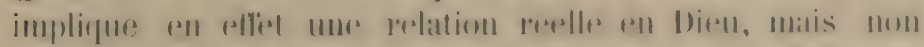

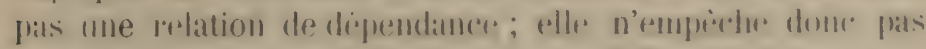

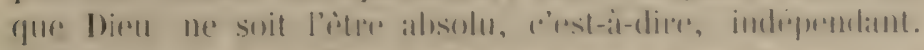

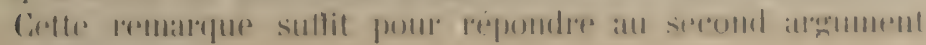
de Mansel.

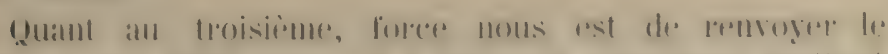

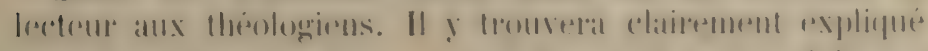

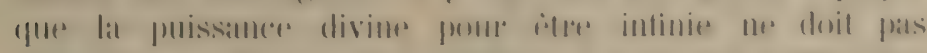

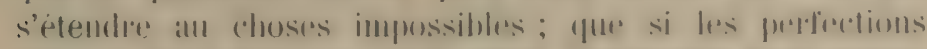
divimes sont intinies, lents allits exterients sont nides-

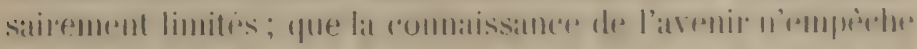

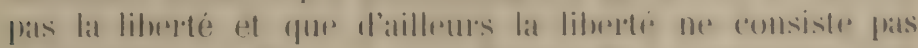

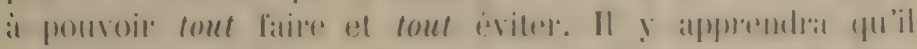

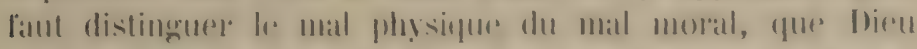

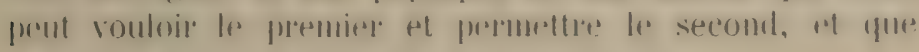

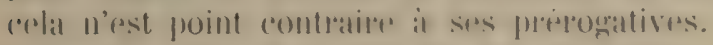

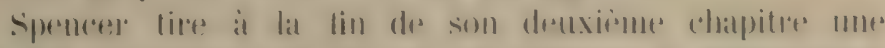
double conclusion: I" Toutes les retigions of mime lathr-

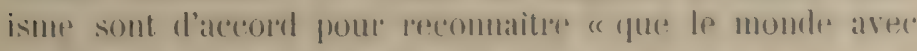
tout re qu'il contient el tont are qui l'entoure mist un mystère qui veut une explication."

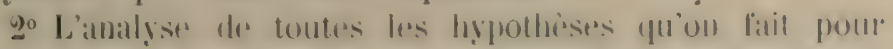

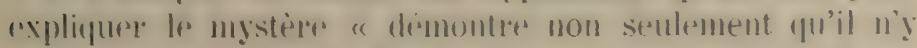
a pas d'hypothèse sulfisante, mais fu'on me preut pats mème en concevoir. » 
Il he nous semble fas que tous les partisans de l'athioisme admettront la premiere partie de cofte conclusion. Quant à la soconde, on a vil ce qưil faut en penser. Ajoutons que les idters fondamentales de la Religion no se présentent pas précisément comme des hypothèses faites pour expliquer le mystère de l'existence du monde, mais plutot comme des conclusions déduites de cette existence. 


\section{LES IDEES DERIIERES DE LA SCIENGE}

\section{L'espace et le temps. - La matière. - Le mouvement. - La force. - La conscience.}

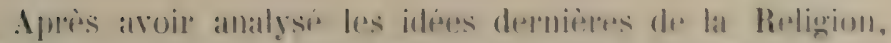

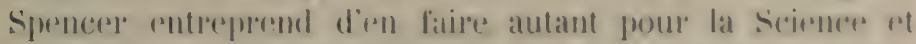

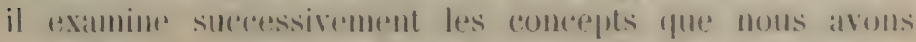

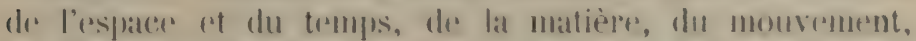

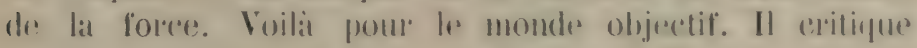

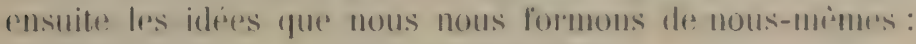
de notre durée, de notre personnalité.

Toutes res commaissances, lant ohjectives que subjectives,

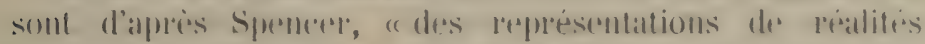
incomprehensibles. "si celat signitiat fue motre commass-

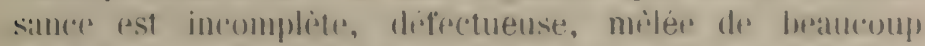

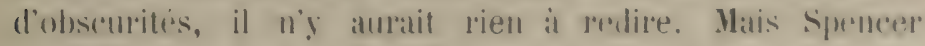
rent demontrer fow nos idees fondamemtales sont radicalement insontemables, contradictoiles et pall conseryutent irreselles. II en conclut que le mystere de l'Inconnaissuble su dresse impenritrable devant la scienere comme devant la Religion. 
espace et le 0 n se ligmre a tort lespace et le temps comme des temps.

realites subsistant ell eltes-mèmes, distinctes dles corps yui occupent l'espalce et qui se meurent dans le temps. Cotte conception se rattache a la philosophie kantienne. II est rai que Kant considere le temps ot lespace comne des elements subjectifs de la connaissance. Mais fall lia mème il les distingue radicalement de toutes les proprictes corporelles th a contribue ainsi. a les laire consilerer comme indejendants des choses concrètes.

Yous tenons pour vaie l'opinion d'après laquelle le temps ainsi que l'espace sont des formalites abstraites qui u'ont d'existence que dans les colpos en tant qu'ttondus, ou dans les choses contingentes en tant que soumises à des dhangements sucerssils. De meme que toutes les notions abstrates, celles d'espare et de temps sont objertives en tant que realiseses dans less objots concrets et ne sont pas objectives dans lamp abstration mème.

En dehors des choses itendues il n'y at pas d'esprace ren ni rell dehors des choses qui durent de temps répl. si l'on dit qu'au delì des corpss l'espare s'etend a l'inlini, (w)al signitie simplement tu'il pomerait exister des corps all deria de ceux yui existent de fait et gu’il n'y a pats des limiles it cetter possibilité. Et si l'on dit qu'alsant le

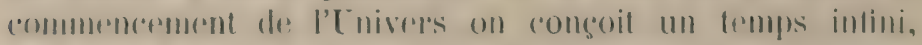

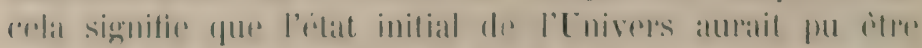

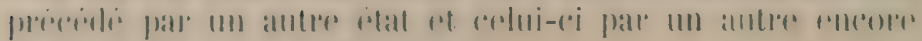
et alusi de suite indefiniment.

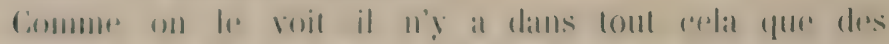

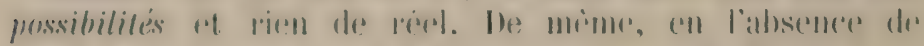

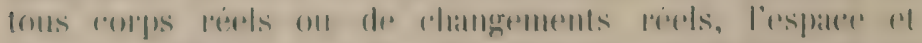

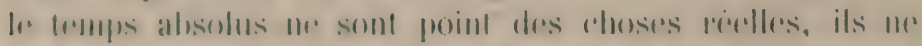

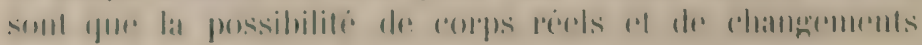
suceessifs.

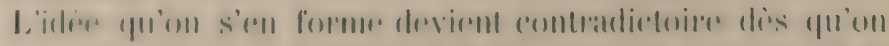

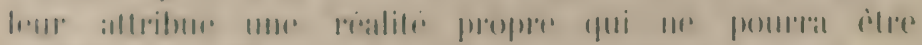
qu'intiute

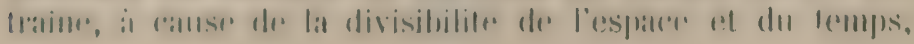




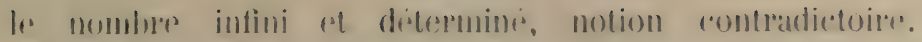
" Les idtees de comprosition d'un tout en ses parties a de l'illimitation reselle dre ses farties, dit tres bien Remenrital. sont lormellement contradictuires l'ume de l'autre. " (1)

Liespace et le trmps sont dome des ratites sombentent dans lo sells que? nous avons dit, et comme realitis its

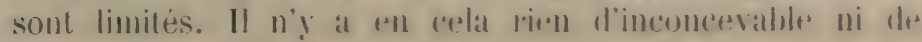
contradictoire.

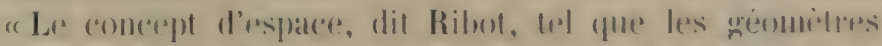

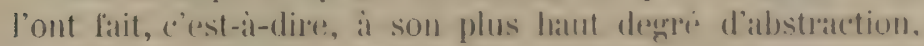

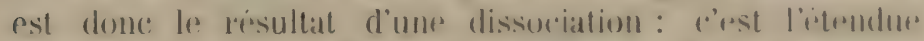
virlee de toutes ses qualites constitutives sauf les dimmsions necessaires qui la detremiment. Ce scelemal (ren

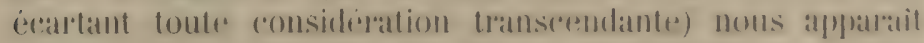

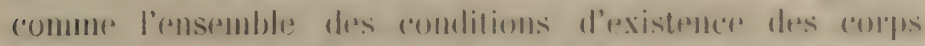

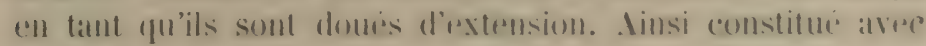

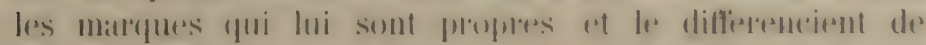

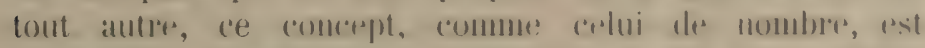
susceptible d'applitations multiples, at de plus, d'atro sans limiles assignables dans tontess les directions, on, suivant l'expression (a)

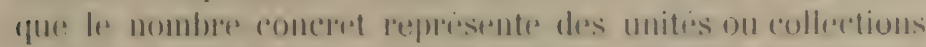

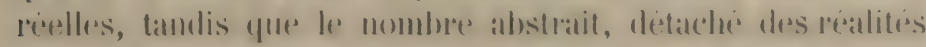
discontinues, permet une numédation salls liu, de mime

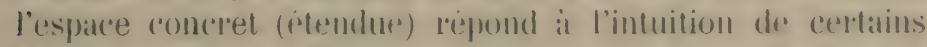
corps, tandis que l'espace alstrat, pur concept nun reepresentah) " (dans l'imagrination) " sinon fall des mots, comporte me extension salls bornes.... Le Leplate n'est

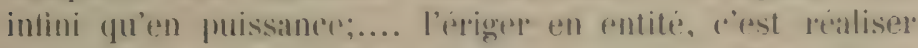
une abstrattion, c'est, ì un concept tout subjectif, attribue'r indiment une valeur objective. » (2)

spencer repousse avec raison la theorie Kantirnme qui fait de l'espacer et du tempse derox formess subjectives de la sensibilite. Dans les Principes de Psychologie. (3) il

(1) Critique philosophique, 1885 vol. 1 p. 409.

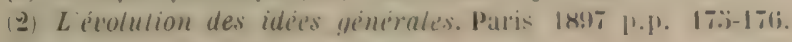

13) vol. 11 p.p. 363. sq. \$ 399 
reprend la refutation de: ectte doctrine et s'y itund longrument. "Ia conscience, dit spencer, alfirme directement que le temps et l'espace ne sont pas au derlans de l'esprit, mais hors de l'esprit. ") (1)

Lil distinction que Renouvier oppose at Spencer pour difendre la theorie kantienne ne tient pas. " L'espace det le temps, (lit-il, appartiennent au moi, selon Kant, en (') sems quils appartiennent ì la representation, laquelle est relative au inoi, est, comme on dit. dans le moi; mais ils appartiennent au non-moi, objectivement, en ce sens qu’ils se rapportent à sa forme objective, douné dans la représentation, qu'ils en sont inscparables, et qu'au surplus les objets sont réels, comme tout le contenu de l'experience dont ils font partie. » (2)

Si en disant d'un element de la connaissance sensible qu'il est une forme subjective de la sensibilité, on ne reut rien dire si re n'est « qu’il appartient it la reporésentation, laquelle est dans le moi s il laut en dire autant de tout le "contenu de l'expérience " at toute l'expérinere sensible sera constituce par des formes subjeclives. Toute la différence qu'il y a cotre l'espace ot le temps ot d'antres attributs sensibles, c'est que toul corps oceupe IIn (retain esplace et dure un certain temps, tandis que d'autres qualites sont accidentelles; mais, yuoi fu'en dise Kant, cela n'est pas une raison pour considerer l'espace at le temps comme des formes subjectives.

Malheureustement, 'll aloptant la théorie de la relativité de nos connatissances, comme nous de rerrons plus loin, sipencer lange l'espaler dans l'orde phenomenal comme. distinct de l'ordre ontologrigue, el ainsi, comme le remancque atrer raison s. Tolver Preston, (3) son oppinion

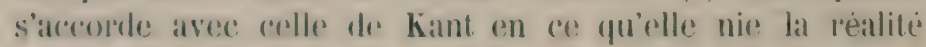
objective de l'espace.

(1) P.P. p. S1. - F. P. p. 37 \& 15

(2) Crilinue Philosophinue. 1883 t. I p. 40;

3) rommarisum af somme biems of spenter and hanl. Mind. Avril $11 \% 00$ p. 231. 


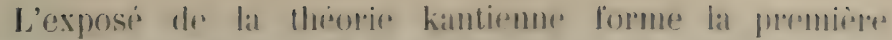
partios de la Critique de lie Raison pure et portro le nom

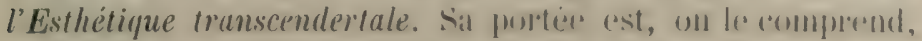
tries considerable; mais les alleruments stor lespueds allo s'appuie u'ont, a motre avis, allemer valeur.

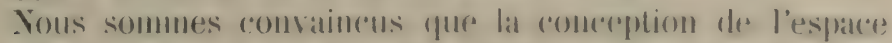

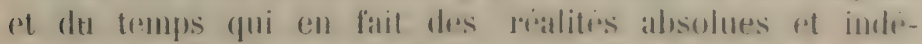
pendantes des choses farticulieres rest lausser. (Or, Kant suppose you nous concerons nexpssairement ansi le temps ot l'respace et il en romelut qu'etant absotument independantes do l'exprepiencer, oes motions sont des intuitions à priori, des lormes subjertires dre llotro sensi-

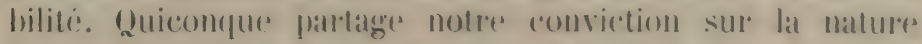

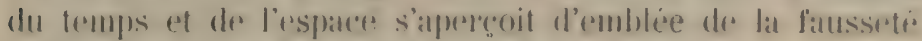
des propositions sulf lespuelles hant sapmuir.

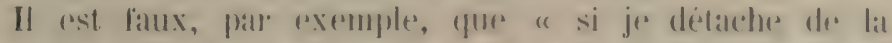

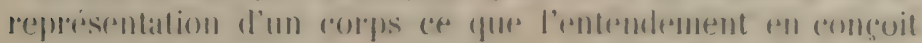

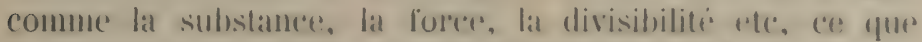

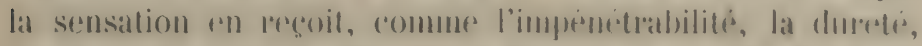

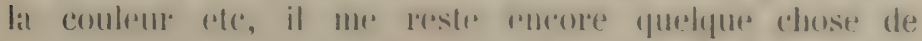

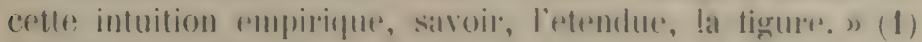

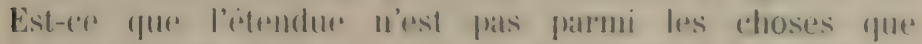

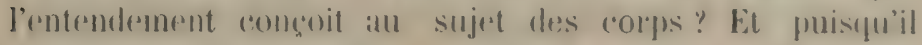
s'ageit iei de la sensibilite, si jenleve toutre qualite sensible furelle representation de lat tignere fourrais-je avoir? Par quelle faculte so reral cettre representation?

Il est faux que " pour yur ju puisse me lepresenter les choses comme retriemos les unes atux atutres, lat representation de l'espare doit dejat ible posere en principe. " (2) L'idere d'espace est all contraire derivie de la prerefetion des ohjets comme etendus, c'est-d-dire, comme extérieurs les uns aux autres.

II est faux encore que "la simultaneite ou la succession ne tomberaient mime pas sous lobservation si la repré-

(1) Critiene de la liaison mure. Ire Partie \$1. Trad. Tissol et capit. Paris 1864 , vol 1, p. 60.

2) Ibid $\$$ II p. 63 . 
sentation du temps ne leur servait de fondement a priori. " (1)

I'idep de temps est dérivé de la perception do choses qui so succident. Comme toutes nos idies generales, celles d'espace et le temps sont acquises par l'abstraction. (que notre esprit exerce sur les perceptions sensitives. Les hases de la théorie Kantirnne sont ruineuses. Ainsi que: Syencer le lait très bien remarquer, d'une part, sedon Kant, nous avons nexessairement la conception de? l'esplace et du temps comme de choses objectives, d'autre part, nous decons admettre qu'ils ne sont que des formes subjectives at rien d'objectif. Cela est contralictoire, et surtout, cerla est arbitraire et conduit directrment au scepticisme.

a matière. "Ia matière, dit spencer, est divisible à l’intini ou alle ne l'est pas. ") (2) On se convainc lacilement (qu" cettr seconde supposition n'est pas tenable, pourvu qu’il soit question, bien entendu, de divisions absolument ou theoriquement possibles, at non pas de divisions qu'on puisse pratiquement exisuter. Toute partic de l'etendue quelgyur petite quon la suppose est alle-mrime étendue: ot par conséquent divisible. Dès lors l'être etendu est divisible it l'intini, c'est-ibdire, indéfiniment.

I'apres spencer, ce rasisonmement sulfit pour dithlir gu'on ne prout flas ronceroir la divisibilite de la mattière commo limiter, mais il pretend que la conclusion qu'on 'n tire, erst-it-dire, sa divisibilite indetinie est egaloment insoutruable: "Si nous disons que la matiore mat divisilue it l'infini, nous nous engageons dans une supposition que nous ne pourons nous ligurer. Sous pouvons

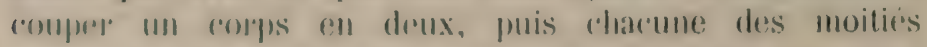

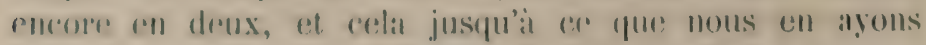

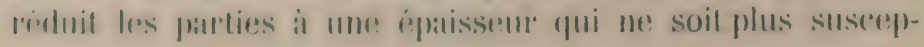

(1) Iticl is po 70.

2) P. P. D. $32816-$ F. P. p. 38 . 
tible d'une division phỵsique, et puis apres nous pouvons encore continuer la mime operation sans tin. Mas ce n'est pas la concevoir la divisibiliti intinie de la mation'te, c'est seulement se former une conception symboliqur qu'on ne peut, en la developplant rendre reelle, et qui n'a pas d'autre moyen dre verilication. En rralite, concevoir la divisibilité intinite de la matière, c'est suivere mentalement les divisiuns a l'intini, mais if faudrait four cela un temps infini. » (1)

Celle argumentation suppose qure pour conceroir un corps comms: indeliniment divisible, on doit se tigurer séparement chacume des divisions qu'on peut y opripre. Autant vaudrait dire: pour ancevoir que tous les hommes sont mortels, je dois mes figurer séparement la mort de chaque individu. Persome n’admettra une telle pxigences. de comprends fort bien op que jo dis quand j'allirmes qu'a diviser un corps en parties de plus en plus pestites il n'y a pas de tin, sans que jaite besoin de me repredsenter frar l'imagination chacune des divisions, (") qui, en

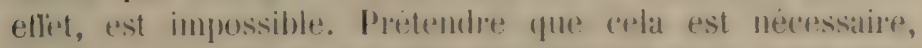
et que sans cela je n’ai flas l'intelligence de cer yu'est cetter divisibilite motefinie, e'est unr alfirmation paladoxale. On y est anuenti par la confusion que nous avons dejä signalée putre les iders rationmelles ot les representations concrètes de l'imagination.

spencer examine ensuitr. les diflempntes hypotheses qu'on peut faire sur la ronstitution do la matière et les troure toutes insoutenables.

Un morceau de metal pourrait ettre conscu d'ahord comme un corps plein, c'rest-a-dire, n'ayant point de poress ni d'interstices qurlconquiss entre ses atomes. "Cette affirmation, dit spencer, nous jetterait dans des diflicultres inextricables. Si la matière intait absolument solide, comme on le suppose, elle serait alsolument incompressible, - ce qui n'est pas - puisquon ne peut concevoir la compressibilité ou, l'unr manière implicite, le rappro-

(1).P. P., p. $32-53316-$ F. P., p. 38. 
chement des parties constitutives, s'il n'y a pas entre elles un espace inoccupé. Et ce n'est pas tout. D'après un principe de mécanique, si un corps, mu avec une certaine vitesse donnée, frappe un corps de mème dimension au repos, en sorte que les deux corps se meuvent ensemble, leur vitesse commune ne sera plus que la moitié de la vitesse initiale. Or, d'après une loi dont la négation est inconcevable, le passage d'une grandeur à une autre ne peut se faire que par tous les degrés intermédiaires aux deux grandeurs. Par exemple, dans It. cas qui nous occupe, un corps en mouvement avec une vitesse représentee par 4, ne peut, par un choc, se réduire à une vitesse représentée par 2, sans passer par toutes les vitesses comprises entre 4 et 2. Mais si la matière était vraiment solide, si les unités qui la composent étaient vraiment incompressibles et en contact absolu, cette loi de continuité (c'est le nom qu'elle porte) serait violée dans tous les cas de collision. Car, elant domnées deux unités élémentaires, si l'une qui se ment avec une vitesse representie par 4 frappe l'autre qui est au repos, l'unité qui frappe doit subir instantanément une diminution de vitesse qui tombe à 2 ; il faut qu'elle passe de la vitesse 4 à la vitesse 2, sans qu’il s'écoule un laps de temps quelconque et sams passer par les vitesses intermédiaires; il faut qu'elle se meuve au mime moment avec les vitesses 4 at 2 , ce qui est impossible. ")

Rrumarquons d'ahord que l'hypothese du corps "plein " nentraine pas cello de son incompressibilite. Presque tons les anciens ont admis les corps pleins et n'ont pas trouve que cette ider fut incompatible avec le fait qu'ils se comprimfnt. Nous avons aujourd'hui de bonnes raisons pour aroire que la compressibilite de la matiere provient de: sa discontinuite. Mais il n'y pas de connexion rigourense entre ces deux idcers.

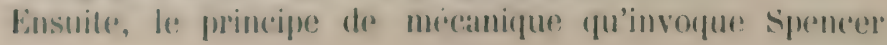

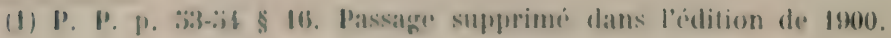


au sujet du choc de deux corps de même " dimension ", (il famdrait dire : de mimo masse, n'at dapplication ques s'il s'agrit de corps nom élastiques. Il suflit done, from

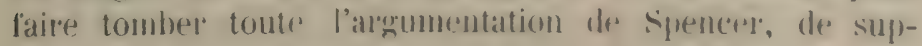
poser les corgs elastipurs, (* qui est parfaitrment rome-

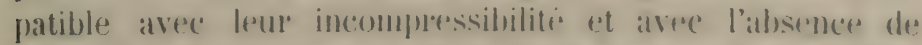

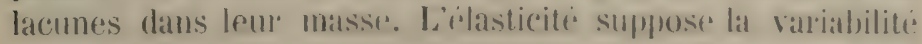
de la forme du corpes, mais pas neressidirement die son volume. Or, dans le choc des corps elastiques, less vatriations dlu mourement unt lies, non fas instantanement, mais au fur et a mesurr de la deformation et de la reformation (s'il est permis d'employer ("re mot) des corps élastiques.

Entin, (que " le passigene d'une granderur à une autre ne jeut se faire rue par fous les degres intermediaires entre les deux grandeurs ", a'eet mup aflirmation qup nous avous souvent pencontree, mais dont nous n'avous jamais v'l une bonne demonstration. Yous crop̣ons que, de fait, le jassige du repos au mouventent, ou vicrversa, ainsi que les changements de mourement se font toujours en prassant frar tous les degres intermediatires, et cela parce que nous sommes convaincus que les corps ne se touchent jamais rellement, mais ayissent les uns sur les autres à distance. Liacceleration positive ou negative que produisent leurs forces attractives ou répulsives étant proportionnelles au temps pendant lequel elles agissent ot dépendant de la distance, varie d'une façon continue comme ces deux facteurs. Mais il s'agit ici, non de la maniere dont ces phenomines se passent, mais de: ce qu'on peut concevoir. Śpencer déclare que la négation de la loi de continuité est inconcevable. Yous avouons franchement ne pas apercevoir l'alsurdite que celte négation contient.

Il esquisse aussi, à la fin du passage que nous venons de rapporter, une dimonstration de cette loi. Mais elle n'a évidemment aucune valeur. La voici : si un corps passe sans intermédiaire de la vitesse 4 ì la vitesse 2, " il faut qu'il se' meuve, au mime moment, avec les 
vitesses 4 et 2 n. Cette conséquence ne tient pas. L moment (instant) dont il s'agit est celui du changement de vitesse. Or, la fonction qui donne la vitesse du corps n'est évidemment pas continue au point où se fait, par hypothèse, le changement le vitesse instantane; dès lors, cette fonction ne donne, pour ce point et pour cet inslant, aucune vitesse déterminée. I"expression de la vitesse $v=\frac{e}{t}$ est indeterminè si $e=0$ et $t=0$ : c'est ce qui arrive lorsqu'on considère un instant. La vitesse à un instant donné n'a une valeur déterminée que si cet instant est pris dans un mouvement continu ou qui varie d'une manière continue; mais elle n'a aucune raleur déterminée à l'instant où se produit une variation brusque ou instantanée, hypothèse que la mécanique n'exclut nullement.

Le lecteur eprouvera probablement quelque étonnement à voir spencer négliger ces notions élementaires. Quoiqu'il en soit, les critiques qu'il élève contre la première hypothèse relative à la constitution de la matière sont sans aucune portée.

La conception attribuee par spencer it Newton, mais qui lui est beaucoup antérieure, et d'après laquelle les corps sont composés d'atomes ettendus qui ne se touchent pas, est repoussée comme reproduisant en petit l'hypothèse précedente des corps " pleins ".

Tous venons de montrer que celle-ci résiste fort bien aux objections qui sont dirigrees contre elle.

Il y a entin le dynamisme de Boscovich qui concoit la matire comme composer de centres de forces sans étendue. Tous croyons, avec spencer, que cette hypothroso est inadmissible. "Admettre que des forces centaales peuvent resider en des points, je me dis pas intiniment petits, mais qui n'oceupent aucun espace, si petit (qu'il soit; des points qui n'ont pas d'autre rolation que leur position, mais qui noont rien pour la marquer ; des peints que rien me distingue de's antres points eireonvoisins qui ne sont pas des rentres de force, ceest faire 
une hypothèse tout a fait hors de la porfies de l'esprit humain $»(1)$.

On pourrat ajouter que priduire les corpsis at des

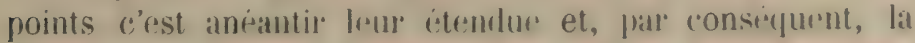
realite de l'espace. L'imagrination, si nous n'y jurnons garde, risqure de nous induire en erreur. Elle parvient à situer dans l'espace des points parre quelle leur attribue nécessairement une etendue ricelle? quoligute tròs petite. Mais l'intelligence nous dit que les point ne prout occuper aurune place et ne peut, fall cousidpuent, par lui-mème, avoir aucune situation dans l'espace, flare'e yue, dans l'espace, il n'a aucune réalité.

Admettrons-nous, comme le reut spencer, ques la throrie de Boscovich est allirmex ingulicitrment par colle de Newton, et que celle-ci doit done subir la minte condamnation que celle-lit? " Lon disciple: dr Boscovich, dit

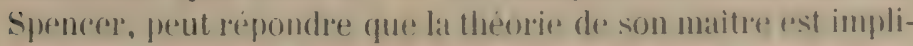
quie dans celle de Newton, et qu'on ne pent s'y soustatare.

"Qnuest-ce, dira-t-il, qui maintient ensemble les parties de ress atomes derniers? - Cne fores: de colnésion, répondrat l'adversaire. - Mais, ajoutera le premier, quand une fores sutlisantr anra rompu les atomes derniers, qu'est-ce (qui retiendra ensemble les parties des morceanx? - Le Newtonien répondra encore : une force de cohésion. - On peut imarniner, dira encore l'autle. que latome soit encore reduit a des parties aussi pertites par lap)fort à lui, qu'il l'est, par rapport à une masse tangible; qu'est-ce qui donne alors à ces parties la propricti fle resister ot d'occuper l'espace? A cotte eternollo yuestion, il n'y a jamais d'autro réponse que la forte de cohésion. Chu'on aille, jusqu'ou l'on voullar, jusqu'd ces ques l'étendue des parties soit moindre que tout ce yu'on puisse imagincre, on ne pourla jas fiviter d'admettre des forces qui supportent l'itemlue. Yous ne fouvons nous arriter que dans la conception des centres de forre sans fitendur.op(2)

(1) P. P., p. $36, \$ 16 .-$ T. P., p. 39.

(2) P. P., p. 36-37. \&16. 
Il n'est pas difficile de faire roir le défaut de cette démonstration. Admeltons que les parties de l'atome sont maintenues ensemble par une veritable fores, on en conclura que cette force réside dans toute partie de l'atome quelcque petite qu'on l'magine, mais non pas qu'elle réside dans un point mathématique. Il "ll est ainsi d'ailleurs de toutes les propriétés de l'atome étendu. La division donnera toujour's des particules coherentes et itendues elles-mèmes. On peut ponsser la division indéfiniment, jamais on n'arrivera i diviser l'atome en points mathématiques; jamais clone on ne sera forcé d'admettre que lit force de coliésion réside dans un point, ce qui est une contradiction manifeste.

La conception yui represente la matière comme composée d'atomes etendus, séparés et agissant les uns sur les aluters par des forces mécaniques, e'est-it-dire motrices, est la mieux en harmonie avec la commissance que nous avons des êtres matériels.

Le Ln corps que nous royons se mouroir d'un mouvemouvement. ment propre est en outre entraine par fo mouvement de rotation de la Terre, et par la translation de celle-ci autour du soleil, et jar le mouvement d'ensemble: du systeme solaire vers la constellation d'Hercule et pentètre encorr pat d'autres mouvements inconnus. Nous ne savons done jamais quelle est en réalite la trajoctoire suivie fatr ee coms dans l'espace. Il pourtait meme se faire, it la rigurur, que la resultante do tous ces mouvements füt, it mu moment dommi, le repos absolu. Il faut conclure de li, ce qui rst encore évident par ailleurs, quir nos sens pereopent semlement le mouvement relatif, cerest-itdire, le deplacement des corps les uns par rapport

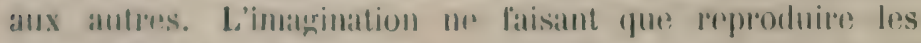
prerepelions sensitives, me represente pas autrement les mouvements doul les roptes sont animes.

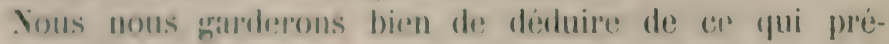


cide, comme le fail spencer, "combien nos idtes du mouvement sont decerantes. " (1) Les connaissances que l'expérience sensible nous fournit sont rxactes; il n'y a pas d'erreur si nous nous contentous d'altirmer ce qu'elles rapporte, c'est-it-dire, Ir deplacement relatif des corps.

De ce que nos sens ne pergoivent le mouvement que comme un changenent dans la situation relative des mobiles, il ne suit pas du tout que le nouvement ne soit point autre chose qu'une telle relation. L'intelligence qui consoit le mouvement comme l'eflet d'une forces et comme une force lui-mine n'a ancume peins à lui attribuer une existence absolue, c'rst-à-dire indéperndante des corps autres que le mobile. S'il n'existail qu'un spu! corps, quelle contradiction verrait-on à cee que cop colps fint anime de mouvement? Oue nous ne puissions pas nous imaginer un tel mourement parce que l'imagination ne conçoil le mouvement que comme un dejplacement relatif a d'autres corps, cela, a la veriti, ne prouve rien. Si un corps se meut, el que, à un moment donné, tous les autres corps cessent d'exister - ce qui n’a rien d'inconcrvable - prétendrat-on que, par le fait mème, le premier corps s'arrite, et que l'energrie cinétique dont il est le sirges se trouve amnihilée? Notre intelligence voit-elle une contradiction quelconque à ce que cette énergie reste ce qu'elle est?

Quant à la transmission du mouvement d'un corps à un autre, c'ost un fait d'experience. En supposant que nous soyons incapables d'en fournir l'explication, ou de comprendre seulement ce qui se passe lorsque dans le choc des deux corps leurs mouvements se modifient, ce n'est pas une raison de nier le fait ou de le déclarel inconcevable, it moins que chacun ne s'arroge le clroit de qualitier ainsi tout ce qu'il ne comprend pas. Ne pas comprendre une chose n'équivaut pas à comprendre qu'elle est impossible ou contradictoire. Il n'y a donc dans tout

(1) P. P, p. $88 \$ 17-$ F. P., p. 42. 
ce que nous venons de dire au sujet du mouvement, rien qui doive le faire taxer de chose inconcevable, comme le voudrait Spencer.

Voici un dernier raisonnement destiné à ëtablir sa manière de voil : "Nous constatons tous les jours que les objets qu'on lance avec la main ou autrement subissent un rallentissement graduel et finalement sarrêtent; et nous constatons aussi souvent le passage du repos au mouvement par l'application d'une force. Mais nous trouvons qu'il est impossible de se représenter par la pensée ces transitions. En eflet, une violation de la loi de continuité y semble nécessairement impliquée; et nous ne pouvons pas concevoir une violation de cette loi: Un corps royageant avec une vitesse donnée ne peut être ramenè à un etat de repos, ni changer de vitesse, sans passer par toutes les vitesses intermédiaires. ) (1)

spencer a fait observer lui-mème (2) qu'il ne nous est pas possible de constater le repos. La base du raisonnement n'est done pas solide. En outre, il repose tout entier sur la loi de continuité (fui n'a pas été démontrée et qui aurait besoin de l'atre'. supposons-la admise et voyons ce qui suit.

" A premiere vue, il semble que rien n'est plus aisé que de l'imaginer passant do l'un à l'autre de ces ètats successifs. On peut penser que son mouvement diminue insensiblement, jusqu'à devenir infinitesimal ; et beaucoup croiront qu'il est possible de passer par la pensie d'uu mouvement infinitesimal à un mouvement égal à zéro. Mais c'est une erreur. Suivez autant que vous voudrez par la fensée une vitesse qui dícroit, il reste encore quelque vitesse. Prenez la moitié et ensuite la moitic de la somme de mouvement et cela à l'infini, le mouvement existe encore; fot lo mouvement le plus petit est siparé de zéro mouvement par un abime infranchissable. " (3)

(1) P. P., p. $6017-F_{0} \cdot$ P., p. 4 . 17.

(2) P. P., P. 38 \& 17 - F. Po, P. 4 .

(3) P. P., p. $6017-$ F. P., p. 13. 
La faiblesse de ce raisonnement saute aux yeux. I'abord une vitesse quelconque n’est pas séparee de zero par un "abime infranchissable, ", mais pall la diflërence entre zeru et celte vitesse, c'est-d-dire par la valeur de cette vitesse qui peut ìtra aussi petite qü l'on veut.

Ensuite, s'il prenait envie à quelıu'un de réduire de moitié la vitesse d'un mobile, puis d'argir de mème pour la vitesse restante, et ainsi de suite, et cela par des actions non continues de telle sorte que le temps compris entre deux diminutions successives ou bien soit constant ou bien ait une limite positive différente de zéro, il est clair qu’une telle operation se prolongerait a l'intini et nous ne croyons pas que jamais personne â̂t conçu le projet d'arriter un mobile de cette facon. Mais en rerite est-ce de celit qu'il rst question? La passage continu du mouvenent all repos ne consiste-t-il pas precisément en re que le temps compris entre deux degres successifs de vitesse - Ir second etant la moitie du premier - tend vers ziro comme ces vitesses elles-mimes, et cela plus ou moins rapidement d'apres les conditions du probleme. Tous ces intervalles de temps compris entre deux degrés de vitesse dont le second est la moitie du premier constituent une série infinie mais convergente, d'est-it-dire, ayant une valeur déterminè qui sera le temps nécessaire pour l'extinction complete da mourenuent.

spencer ajoute encore: "De mime qu'une chose, quelque tinue qu'elle soit, est intiniment grande en compalaison de rien; de mème ancore, le mouvement le moins concevable (considérable) est intini en comparaison du repos. »

Il est vrai que le rapport de quelque chose à rien est ingal a l'intini $\frac{a}{o}=\infty$. Mais ce rapport n'exprime pas la distances de $u$ a $o$ et n'a donc rien de commun avec la question que nous examinons.

Dans l'édition de 1900, Spencer s'étend moins longuement sur les considerations que nous venons d'examiner ; d'autre part il ajoute un argument tire de l'impossibi- 
lité de concevoir qu'un corjes en ment un autre. Qu'estce qui a éte transfiré lu premier au second? Ce n'est ni une close ni un attribut. Qu'est-ce donc? (1) Yous répondrons que rien n'a été transféré, à parler exactement. Le premier corps en s’approchant du second y a produit un mourement, le second, par réaction, a détruit ou diminué le mourement du premier. La conception de ce phénomène repose tout entière sur l'idée de causalité qui n'est pas contradictoire.

Spencer n'a done pas démontré que cles efforts que nous faisons pour comprendre la nature intime du mouvement, ne peurent que nous rérluire à choisir entre deux pensées également impossibles. ”

\section{La force.}

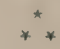

"En soulevant une chaise, dit spencer, nous exergons une force que nous regandons comme exale à la force antagoniste appelée pesanteur de la chaise; et nous ne pouvons penser a l'égalite de ces deux forces sans penser qu'elles sont de meime espèce; puisqu'on ne peut coneevoil l'iggalite qu'entre des choses de mime nature. L'axiome que l'action et la réaction sont égales et s'exercent dans des directions oppostes, axiome dont on donne commumement pour excmple le fait que je viens de mentionner, l'efiort musculaire dirige contre la pesanteur, ne: pent etre concu dans toute autre condition. Et pourtant, all contraire, on ne peut moire que la force qui existe dans la chaise ressemble réllement à la force qui est mesermfr a nos esprits. Nous n'avons pas besoin de faire remarquer que le poids de la chaise produit en nous divers sentiments, suivant fque nous la soutenons avec un seul doight, on avece toute la main, ou aves: la jamber ; et flar conserfurent il est purmis de soutenir que les poids de la chaise ne pouvant ille semblable a tomtes ces semsations, il n'y a fas de raisotr pour qu'il ressemble à alteums. II sulfit de rematequer ques la forre telle que nous la

(1) F. P., P. 42. 
conmaissons etant une impression do notre consciencer, nous ne pouvons concevoir sous la meme forme la foree qui réside dans la chaise, à moins de douer la chaisp de conscience. De sorte qu'il ast absurde dre ferliser yute la force en elle-mime lessemble a la semsation qure nous en avons, et pourtant il est népessaire de lo pensere, pour peu que nous voulions nous la représentro dans la conscience. 》 (1)

Le raisonnement guon vient de lime prpose sur la confusion entre l'effort dont j'ai conscience et la forre deployéc par la contraction des muscles. Yon seulement l'effort varie lorspue des muscles diffirents dejploient la mime force, mais, si le bras est affuibli, par exemple par une longue inaction, l'effort exigh pour un certain deploiement de force sera bien plus grand yu'en temps normal.

Les psychologues discutent la question de savoir si les sentiment de l'effort est d'origine rentraldr et antecedent

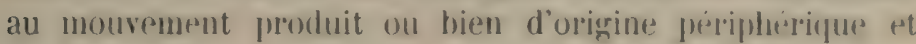
posterienr au mourement musculaire. (2) Cers deux opinions nous parassent pouvoir se concilier. Dans l'effort l'itre est actif; l'elfort lui-même est donc; d'origine intrute et détermine la contraction musculaire, tandis que te sentiment de l'effort comprend outre la conseience de. l'activite deplovere un ensemble d'impressions venant des articulations, des tendons, des muscles, des variations respiratoires etc. Quoiqu'il en soit, anssi hien er que nous appelons l'eflort que ls sentiment de l'aflort sont des actes psychiques qui detrminent ou accompagnent la contraction musculaire, mais ne lui sont pas identiques.

II y a là deux ordres de phénomènes distincts "t superposés, les uns mécaniques, les autres psychiques. L'égalité existe entre le poids de la chaise et la tension du muscle qui agit comme un ressort: forces mécaniques de part et d'autre, égale's en intensité et se faisant ápuilibre.

(1) P. P., p. 61-62 \& $18-$ F. P., p.p. $43-44$.

(2) (f. Riвot. L'enolution des idées générales. p. 204. 
Spencer revient encore à la conception que nous nous formons de la matiere et il pense qu'on ne peut échapper a la nécessité d'admettre que les corps agissent les uns sur les autres a travers l'espace absolument vide, "supposition, dit-il, (pui ne peut ètre représentée. )

L'hypothèse de l'éther ne risout pas la difficulté parce que, ses atomes étant distants, il faudait toujours admettre que leurs actions mutuelles s'exercent à travers l'espace sans intermediaire.

Yous sarons, au moins avec une très grande probalité, que l'action par laquelle le soleil nous éclaire et nous réchauffe est transmise par l'intermédiai!e d'un lluide aurfuel on a donné le nom d'éther. si cette action ne passait par aucun intermédiaire, on ne comprendrait pas qu'elle mette un certain ternp̧s at nous arriver et que, lorque nous regardons le Soleil, nous le voyions à la place où il se trouvait huit minutes environ auparavant.

Iais en ast-il de mime de l'action attractive qui s'exprce entre les astres? Cela est beaucoup moins sur. Les calculs astronomiques supposent que la direction de lat gravitation est la ligne droite qui joint le centre de gravite du soleil au centre de graviti de la Terre. Or crla sruat inexact, étant donné le mouvement de translation de la Torre, si l'attraction itait transmise par le milisu. Cette transmission, "n effet, exigerait un certain temps comme tout phénominte materiel. Il est viai quion pruit supposel ce temps aussi petit qu'on veut de telle sorte que l'reart entre le resultat des calculs et les faits, lout en blant reel, soit trop fable pour pouvoir itre constati. Il landrait, pour cela, adenettre une vitesse de transmission de beatcoup - Laplace disait : all moins cinquante millions de fois - superpieure a celle de la Immiere.

En outro, laction attrative ne connat alleun obstache, rlle n'est sujetlr ni a reflexion, ni a refratetion, ni a ancume Variation dependant du milien. Ces circonstances 
n'indiquent-rlles pas que cette action s'exerce leallement à distances salls se transmettre par le milieu? (1)

Beaucoup de philosophes modernes, lorsqu'il s'agit de lat possibilité de l'action à distance sans intromidiaire, st contentent de la nier sans apporter la preuve de leur noxation. Les anciens niapportitient en grineral d'autre preuve que le timoignage de l'exprerience qu'on peut aujourd'hui invoquer plutot ru sens contraire.

Les arguments a priori que l'on met partois en arant ne sont pas demonstratifs. Yous ne less examimerons pas ici.

Les connatissances que mous arons an sujet des forers élémentaires de la matiene et des circonstances dans lesquelles elles agissent sont trop incomplètes pour qu'on puisser en tiree des conclusions delinitives sur les conditions de leur activiti. Les anciens aropatient que les corps liggers s'ilevent dans l'air ron vertu d'une impulsion ou tendance native vers le hatat. Tous savons aujourd'hui que leur mourement an lieu d'itre dù à uns force simple, n'est qu'ume resultante de l'action combinise de la pression que l'air exerere en vertu de son poids et de. sie fluidite, et du proids du corps qui s'y trouve plongé. Laallatetion universelle, les forces interatumiques sont-elles des forees ilementaires ou des resultanters d'autres forces encore inacerssibles a notre obstration? Il n'est pas possible de repondre à cette question arec certitude, ni de salroir, jal conséqurnt, s'il faut nous représenter les corps comme agissiant it distance ou comne soumis à la condition de transmettre leur action par un milieu matériel.

s'il ne s'agit qur de proposer des hypothises, il est facile d'en inaginer qui s'accordent soit avec l'une soit avec l'autre de ces deux conceptions. L'ether discontinu n'est

(1) ef. Stallo. La malière el la mhysique moderne paris 1891 p. 40 sy. - Rexotrin. Le Persomnalisme suivi d'une Étude sur la

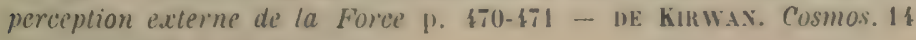
Janvier 190ミ p. 43 sq. 
pas lo seul dont les proprietes puissent se concilier avec les theories seitntitiques. Lord Kelvin considère l'éther comms un fluide continu, non atomique. (1)

spencer n'est done pas fondè à dire : "Nous sommes obliges de conclure que la matière pondérable ou impondẹrable, considérée dans ses masses ou dans ses unitís hypothetiques, agit sur la matière à travers l'espace absolument vide; et pourtant cefte conclusion est positivement inconcevable.» (2) Cette conclusion n'est pas démontrée inconcevable et, en outre, les phénomènes commus ne l'imposent pas nécessairement à l'esprit.

spencer fait encore à la conception atomique que nous avons indiquée comme la plus satisfaisante, l'objection suivante: "En outre, la lumière, la chaleur, la gr'avitation et toutes les forces qui rayonnent d'un. centre varient en raison inverse du carré des distances; et les physiciens dans leurs recherches supposent que les unites de matière agissent l'une sur l'autre d'après la même loi, et ils sont bien obligés de faire cette hypothese, puisque cette loi n'est pas simplement empirique, mais qu'slle: peut se déduire mathématiquement des relations d'espace et que sa negation est inconcevable. Mais dans une masse de matière en équilibre interne, que va-t-il arriver? Les attractions et les répulsions des atomes constituats sont neutralisees. En vertu de cette neuIralisation, les atomes restent à leur's distances actuelles, et lat masse de matiere ne se dilate ni ne se contracte. Mais si les forces avec lesquelles deux atomes adjacents s'attirent et se repoussent mutuellement varient à la fois en raison inverse du carre des distances, ce qui doit itrr, at si les atomes sont en équiliture a leurs distancess acturlles, il faut nexcessirement qu'ils soient en équilibre a toutes les autres distances.

"Supprens que les atomes soient séparés par un intervalle double, lends attrations ot leurs ripulsions seront

(1) Vinetheenth century, 1903, vol. I, p. 1006.

(2) P. P., P. 68-64. \& 18. - F. P., p. is. 
les unes et les autres leduites an quant do lour valeur actuelle. Supposons yu’ils soirut rapploches de la moitie de leur slistance, leurs attractions, leurs rejulsions spront chacune quadrupler. II an resulte que cotte matiere prend avec la mème lacilitis toutes Ins densites et nr pent offir de resistance a des agrents extririurs. Ainsi done, mous sommes obligies de dire que ces forces moliculaires antagonistes ne varient pas foutes les deux en raison inverse du carme des distances, ore qui est inconcervable; ou en-

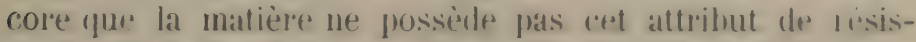
tance par lerpuel nous la distinguons dre l'espare vide, ce qui est absurde. ”

$1^{\circ}$ Tous crogons diflicilement qu'il se trouve un plı̣sicien assez mal avisé pour doter a la fois les atomes de forces d'attaction et de repulsion variant suivant la meme loi. si $a$ at $r$ designent l'intensitio de les forees a l'unite de distancer, flles auront pour valene is unr distance queelconque $d, \frac{a}{d^{2}}$ et $\frac{r}{d^{2}}$.

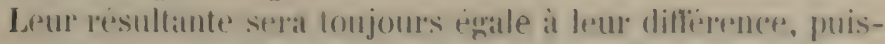
qu'elles sont diamidalement opposies: $\frac{a}{t^{2}}-\frac{r}{d^{2}}$ $\frac{a-v}{d^{2}}$

Celat signitie que l'hypotheses des desux forces revient à fon supposer une serule ayaut à l'unite de distance pour valeur $a-r$, et variant elle-mime en raison inverse du carré de la distance.

Cette force, $d^{2}$ etant toujours positif, sera nulle si $a=r$; elle sera attractive a toute distance si $a>r$ et répulsive à toute distance si $a<r$.

spencer suppost une masse de matière en équilibre. Il faut nexessairement pour cela que $a=r$. II attribue donc aux physiciens l'hỵpothèse que les atones sont doués d'une force d'attraction et d'une lorce de répulsion toujours égales et diametralement opposies, se neutralisant par conséfuent toujours exactement et ne pouvant produire aucun eflet! Yous ne rroyons pas qu'il y ait jamais eu un physicien capable d'imaginer une chose si manifestement inutile. 
20 Boscovich supposait ses atomes doues de forces attratives et repulsires, celles-ci variant suivant une puissance de la distance plus élevée que celles-là. La physique mathematique démontre que l'intensité des forces qui consistent dans un mouvement ondulatoire dı milieu et qui rayoment donc reellement à partir d'un centre, varie nexessairement en raison inverse du carré de la distance; mais quant aux autres forces, par exemple, la gravitation, dont on ne connait pas avec certitude la nature, la physique mathematique n'a rien à y voir. L'expérience seule peut nous renseigner sur la loi de leur variation.

$3^{\circ}$ si nous adoptons la constitution atomique de la matiere, il laut admettre que les atomes des corjes solides et liquides s'attirent, puisque ces corps sont tous plus ou moins coherents. D'autre part, tout en se laissant comprimer, ce qui indique que leurs atomes sont distants, ils opposent une grande résistance à la compression. Il existe donc entre ces atomes des forces répulsives, e.t celles-ci augmentent plus rapiclement que les forces attractives lorsque la distance diminue.

Beaucoup de physiciens considerent la matière comme composée d'atomes ponderables entoures d'atmosphères d'ethir, fluide impondinable. I'apmès cette conception, tandis que le's atomes de matiere ponderable exercent une action attractive les uns sur les autres et aussi sur les atomes d'ether, ces derniers au contraire se repoussent. (In est disposé à considérel l'attraction mutuelle des atomes ponderables comme n'etant pas differente de la gravitation et comme variant, dès lors, en raison inverse du carri de la distance. La mime variation anrait lieu pour l'allaction excecép par les atomes ponderables sur fes atomes d'ether, tandis que la répulsion do ces der-

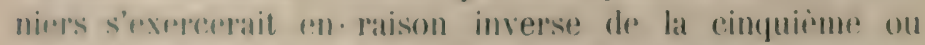
dre la sisieme puissaner de la distance. (1) Ceette repulsion entre les atomes deblore variant on rason inverse de la sisirme pmissancer te lear distance est exigre par la

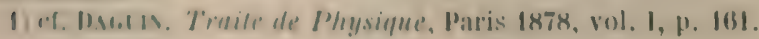


théronte ondulatoire de la lumiene lorsquon lient comple des phenomènes de trunsmission el d'interferences observés par Fresnel.

Cous n'aflirmerons neimmoins pas l'existence d'une telle forere, puisque la theorio des ondulations, telle quar la concevait fresmel, se trouve aujourd'hui battur en lreshe et menacer d'ette remplacer frar une autre (1). Mais il ressont du moins de ar que nous renons de dire que les physiciens ne considirent pas du tout les forces commo. variant necessairement en raison inverse du carres de la distance.

Less recontes dreourertes qui ont ette latites dans le domaine de la plussiquer moditieront salns doute nos ideres au sujet de la constitution te lat matiore. Il serait prema-

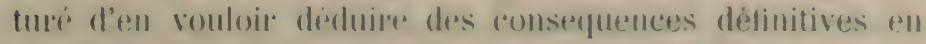
("re moment. Cie que nous alvons dit suffit pour nomtrep

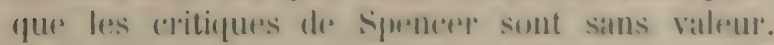

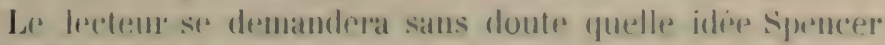

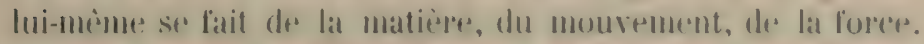

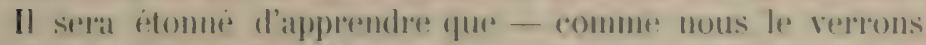
plus-tand - cese ideres ne sont pas fort diflerentes de refless equi sont ici ballues si violemment en breche. Cette contratiction est ceensement resolue fane la thérere de la

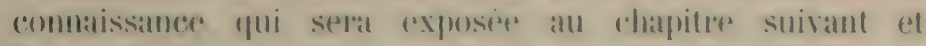
d'apmes lapuelle nos concepts ont pour objut le phenomène et non lat realite. Nous devons avourer qu’il nous est improssible de complendre comment une therorit quelcomplue premet d'alopter des conceptions quion a, par ailleurs, jugres remplies d'impossilbilites. Comment, par exemple, peut-il ètre " lexgitime " a de considérer la malière comme compusfe d'atomess etendus et résistants » (2) alors que rette meme ligpotheses a cite auparavant tratée d" "absurdite» (\$). Pous croyuns d’ailleurs avoir fait voir

1. Ci. Lonkxz. Eutres scientifigues l'evues of anmolies par H. VAlantiner. Copenhague $1896,1.1, \mathrm{p}, 19 \mathrm{i}$.

(2) P. P. p. $177 \$ 18-$ P. P. p. 131 .

(3) P. P. p. $57 \S 16-$ F. P. p. 40. 
clairement quion n'a aucune bonne raison de la juger ainsi.

Ia conscience Les dats de conscience constituent une chaîne qui ne peut pas etre infinie. Cependant, dit Spencer, nous ne pouvons pas non plus la concevoir comme tinie. Nous devrions pour cela en connaitre le commencement et la fin. Or le commencement "s'évanouit dans une obscurité profonde " (1) et quant à la lin, si nous la connaissions, elle cesserait par là mème d'ètre fin, puisqu'elle ne prut être comme que par un acte de conscience nouvaru.

Cet argument ne paraitra pas très fort, si l'on réflechit yur pour conceroir la chaine des actes de conseience comme finire, il suffit de concevoir qu'elle a un eommencement et une lin, sans qu'il soit necessaire de conceroir in individuo l'acte qui constitue ce commencement ou cette tin. Cette distinction s'impose; nous n'y insisterons pas.

"I'risentons, dit Spencer, lat dilliculte sous more atutre forme : Si un changement incessint d'etat est la condition sous laquelle la conscience existe, fuand le dermier dat supposit est atteint par l'achivement des dats précédents, le clangement a cesse; done la conscience a cesse; done l'état supposes n'est point un etal de conscience, done il ne peut pas y avoir de dernier état de conserences" (2).

on ne prout evidemment dire sans contradiction que to dernim batt de conscience "est alteint par l'athèvement des etats probodents $n$. Cokta reviendrait a alfirmer que le dernire chat de conscience n'est que la non-existence des

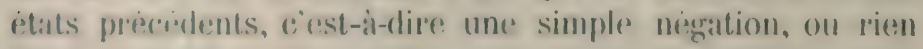
du tont. Mais anssi, qui dira une choses si manifestement. absurde?

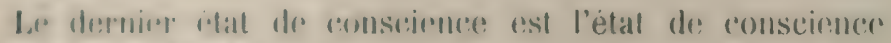

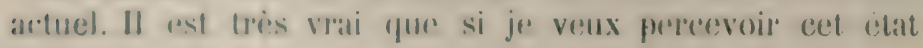

(1) P. P. P. 63 \& $19-$ F. P. P. 46.

(2) P.P. 646.137 \& $20-$ F. P. P. 46. 
de conscience frar riflexion sur moi-mênne, il se produit un nouvel etat de conselenore et qu'tinsi l'autre resse d'être le dernitr. Mais qu'y a-t-il en rela d'absurde ou

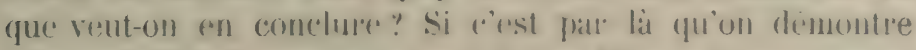
l'impossibilite dre concevoir une chaine d'etats de conscience qui ait une tim, noms avouons ne pas saisir la prortée de l'argument.

Cerle datume dess etals dr conscience constitur-terlle, à elle seule, la persomnalite, ou bien plutot tire-t-elle son unite d'un sujet dent les itate de conscience ne sont que des modilications successives? Snus avons à choisir entre ces deux hypothieses. spencer rejette la premiere comme etant rontralirtoire of en opposition aver la conscience elle-mème.

II prononce lat mème eondaumation strr la seconde, parer que "lat rondition fomdamentale de toute conscience étant l'antithe'se du sujet et de l'objet " (1), il "st impossible de se concevoir soi-même.

Il ne faut pas, répetons-te encore une lois, que la metaphỵsique contredise to hon sens. Elle est jugrés part le fait meme. Aroms-nous oui ou non conseienee de nos actes? si l'allirmative est incontestable, toute theorie de la conscience doit tenir (ompte de ce fatit (2). Silns doute, la conserence exigre une certaine distinction entre le sujet et l'olijet. Mais ne sultit-il pas d'une distinction de raison? Ne peut-on concevoir qu'un être soit présent à lui-mime, c'est-it-dire se connaisse lui-mème tout entier?

Cette identite réelle du sujet et de l'objet n'est d'ailleurs qu'imparfaitement rualisfe dans l'homme chez qui la comnaissance au moỵen de laquelle il perçoit ses propres états d'àme est un acte distinct de ceux qu'il percoit, quoique posé par le même sujet. Il n'y a encore une fois dans cela rien d'absurde ni d'inconcevable.

(1) P. P. p. $69 \S 90$ - F. P. p. 48

(2) Ci. Renouvier, op. cit, p. 410. 
Lamalyose dos detes dernieres de la Religion et de la Scirmer sarrète ici. Le lecteur jugera si spencer y a trouvi une base solide pour la doctrine de l'Inconnaissable telle quil la concroit. Nous croyons avoir montre que les idees fondamentales de la Religion et de la science ne. sont pas sirieusement entamees par sa critique.

Est-ce à dire qu'il n’y a pas de mystère au fond des choses et que toutes les questions qui se dressent devant l'esprit peuvent recevoir une solution satisfaisante? Non, certes : Au cours de ceftr étude nous en avons rencontré nous-mème plusieurs auxquelles l'on domme des reponses opposées sans yu’il soit possible de déciler layuelle doit itre choisie. Plus les recherches de l'esprit penetrent audela des faits farticuliers que l'observation nous fait connaitre, plus aussi ces recherrhes deviennent laborieuses, pleines d'incertitudes et d'obseurites. En ce sens, nous sommes entoures de toutes parts par l’incomnu, ou, par rapport à nous, par l'inconnaissable.

Mais ce qu'il faut repousser, cest la theorie d'après lacpuelle notre intelligence serait logiqurement poussie à des allirmations que par ailleurs on demontre absurdes.

C"est en vain fuon cherche a tracer une ligne de démarcation entrs le terrain sur lequel notre intelligence peut s'engager avec contiance et celui ou elle s'embarrasse fitalement dans des contradictions. Nous n'avons quiune intelligence et les lois fondamentales de? la logique sont les mêmes en toute matière.

Muntrer que nofre esprit affirme nécessairement des choses impossibles et que les principes de la logicue ronduisent a dess resultats contradictoires, c'pst ouvrir la porte toute latge au scepticisme, quel que soil l'objet du raisonnement.

spencere, nous l'avous dit, enseigne que l'inconcevabilite "est l'indice de la faussetio ". Il rapporte l'objection que W. Hamilton lui fait a ces sujet. "Il est imencesable, dit relui-ei at spencer l'admet alrec lui, comme nous l'avons lus, ugur l'espace est lini; il est de mime inconcevable quil est intini. "Pourtant il doit stre l'un ou l'autre?

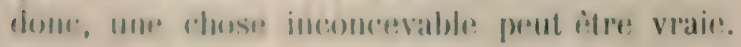


Qure repond spencer" "line chose, dit-i doil exister ou he pas exister: il n'y a pras une troisieme possibilite. Maintenaut, tant que certte loi est considerie comme une loi de la penseje daus ses rappurts avece l'existence phénomenale, elle ne peut àtre mise en cloute. Mais sir W. Hamilton étend cette loi en dohors lles limites de la pensése, et tire une conclusion positive touchant l'existence noumenale.» (1)

J'ahord il n'y aucunte raison de dire qut la remarque de Hamilton s'applique à l'existence nouminalu. L'esplace phenomenal - lo seul que mous commassions - est-il fini ou infini ? s'il est l'un ou, l'autre comment cela ext-il néanmoins inconcevable? Et il n'est ni l'un ni l'autre r'est done qu’il y a turr "troisièmr possibilité " dans l'ordre phénoménal.

Ensuite, que le principe dre contradiction n'ait dre valeur que dans l'orelre phenomenal, cela se rattache at lat theorie de spencer dapres laguelle tonte rentitude a son origine dans l'expérience. Mais il est chair que ca juincipe ne soutfre aucume restriction. S'il ne s'applique pas à l'ordre noumenal, cest donc qur le nouments oul l'Incommaissable peut a la fois existrol ou birn ni exister ni ne pas exister. Let ne De quel droit syencer aflirme-t-il donc qu'il existe?

Pous allons voir an chapitre suivant que la theorie qui sépare l'ordre phemomenal de la reaglité ou du noumine est inarlnissible, ot que ce n’est pas davintage sur ce fondement que lat doctrine de l'Inconnaissable peut ètre bàtie.

(1) Principes de Psychol. vol. II p. $142 \$ 132$. 



\section{LA RELATIVITE DE TOLTE CONVASSANCE}

La question et la solution. - Influence de Kant. - Opinion des philosophes. - M. Brunetière et la relativité des connaissances. - La relativité des connaissances et les théories physiques. - L'inexplicable terme de toute explication. - L'inconcevabilité de l'absolu. - La correspondance entre la conscience et la réalité. - L'existence réelle du noumène. - L'être abstrait et l'être illimité.

question validites connaisces et sa lution.

Lorsquion parle lle la "relativitè " de toute connaissance, il importe d'éviter un malentendu. Lacte de la connaissance est essentiollement relatif ; il est, en eflet, dans son acception la plus générale une representation psychique de quelque chose.

La connaissance est un acte primitif, sui generis. Nous en avons conseience et il est inutile de vouloir, par des définitions, rendre plus claire l'idée que nous en possédons. On preut analy̧ser les connaissances, en rechercher et en distinguer les parties, les decomposer en leur's éléments. Ces éléments eux-mèmes seront des connaissances simples. Lorsque nous les avons distinguées, nous nous trouvons devant une chose incomplexe sur laquelle l'analyse ne peut plus s'exercer.

Toute representation n'est pas une connaissance: une 
photographie d'un monument n'en est pas une connaissance quoiqu'elle le représente. Nous avons dit: representation psychique pour indiquer qu'elle a lieu dans l'àme ef yue nous pourons ell avoir conscience, sans pretendre caracteriser autrement sa nature par ce mot.

Personne, croyons-nous, ne contestera que la connaissance ne soit un phénomène psychique, par conséquent subjectif; ni qu'elle consiste dans une représentation de quelque chose, de rquelque objet. Elle est donc, dans ce sens, objective.

Toute connaissance a dès lor's une double relation; l'une au sujet dont elle est une modalité, l'autre à l'objet qu'elle représente. A ce double point de vue, toutes nos connaissances sont essentiellement relatives. Cela parait. incontestable.

Mais assitot se présente une autre question. Qur dironsnous au sujet des choses qui sont représenteses par nos ronnaissances? Sont-elles réelles ou ne le sont sont-elles fas ? Ont-elles une existence independante de nos connaissances ou n'ont-elles pas d'autre objectivité que d'etre connues? Tel tableau leprésente un homme; l'homme existe independamment du tableau. Tel autre tableau represente un centaure; le centaure n'existe pas, n'a jamais existi: r'est une representation sans réalité.

Lne reflexion attentive permet de distinguer en nous deux genres de connaissances, les unes simples représentations de quelque chose, d'autres plus complexes: inonciations ou juyements, soit aftirmatifs soit negatifs.

Parmi les premicres, les unes representent des objets romprets : perceptions proprement dites on percepts ; les andres reprisentent des objets abstrats; on leur donne sonvent fr nom d'idées ou de concepts. Les simples letIn'soulations me contiennent en elles-mimes l'alfirmation d'aneume realiti. Quelques unes sont démbers de toute: reatite, far exemple, les revess. Quant anx idies abstrates aurun philosophe moderne - is notre comaissance ne. len attribue dobjertivitie an dehors des choses roneriefes ampunlles rolles provent s'appliquer. 


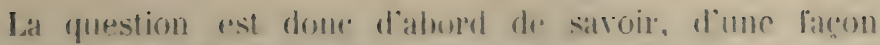

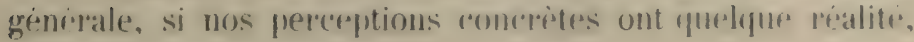
et dans quelle mesure.

Berkeley déniat toutr valeur oljectior anx sensations. Ijautres, parmi lespuels sprencer, admettent une realite des choses percurs, mais nient pur coptte raglite soit telles que nous la fercerons. Do lit refle these : nous ronnaissons les choses non point telles qu'elles somt, mais seruIement telles qu'elles nous apparaissent. Et l'on soms-entend qu'elles sont ou du moins qu'elles formratent itre autrement qu'elles n'apparaissent.

Qur nous commaissons les choses telles fu'elle's nous applarassent, cela est rai flar detinition, fuisque cette apparence est prodeisement la reporesentation que nous

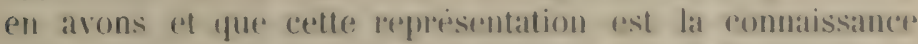
elle-mème. Mais cque reetle afplarence pst diflerente de la

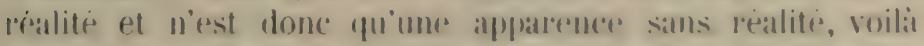

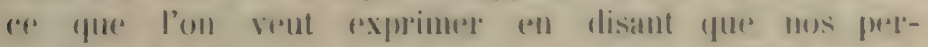
ereptions des choses ne sont que relatives. Tel ast le

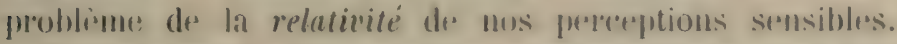

Contrairment allx simples representations, nos jugements aflirment on nient. Timdis qu’il n'y at dans celles-la ni virite, ni crrems, It jugement, all contraire,

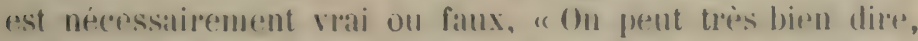
ainsi s'exprime Kant, (1) que les sens ne ste trompent point, non farce qu'ils jugent toujours justr, mais parce qu'ils ne jugrent pas du tout. La verite ef l'erreur.... ne se trouvent que dans le jugement. »

Il y a des jugements affirmant ou niant des fidts ronrorts. Quplques uns de ces fats sont subjectifs cot ronnus par la conscience: je pense, je veux.

Descartes dans son doute mithodique universel s'est arrèté devant l'evidence irresistible de ces jugements et a pretendu en faire la base de sil philosophie.

1) Critique de la raison pure. Dialectimue' transcerdantule. Introduction. Traduction Tissot. Paris 1864 vol ll p. 2. 


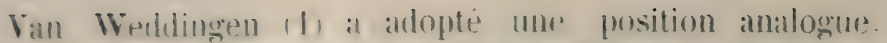
Elle nous farait entachè d'inconserqurnee: des qu'on

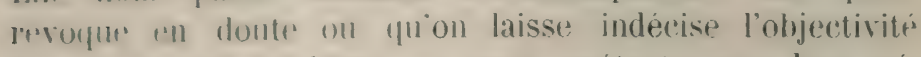
dres jugrments ividents, - sous pretexte que leur megattion pourrait itre également evidente à une intelligence atutrment confomese on que nous sommes peut-citre les jounts doun tre superieur qui prend plassir a nous tromper. - il ny a ancune raison de nt pas y comprendro latlirmation des faits de conscience ou de l'existence du sujet.

Nos jugements peuvent egalement avoir pour objet des faits manifestés par la sensibilite et exterieurs à la consciemcer. Ainsi j’athirme ou je nie l'existence des corps que je percois.

Il y a enfin des jugements abstraits tels que ceux-ci: Les itres intelligents sont doues do liberte: Tout op yui change a eu un commencement.

Lat question do l'ohjectivite de nos commaissances peut être posier au sujet de nos jugements. On perut se demander si nos jugements abstraits ont une valeur objective et dans quelle mesure; si nos aftirmations au sujet des whjets extrepers a la conscience ou meme an sujet des faits de consciencer ont quelque réalite. Quf ces jugrments soient des apparences par lit mème qu'ils sont des comnaissancers, cela, encore une fois, ne fait pas de dente: lat question est de savoir s'ils ne sont que des apparences. C̈est ce qur l'on entend quand on dit que

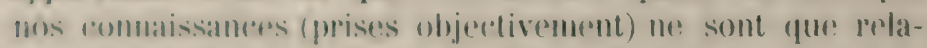
tives, e'est-it-dire que lear objet n'existe que dans la counaissancet.

On thallend fas de nous qur nous exposions, mime

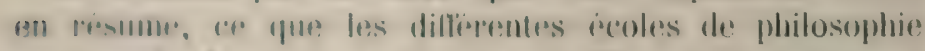

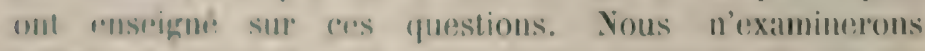

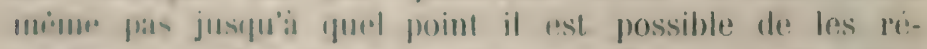

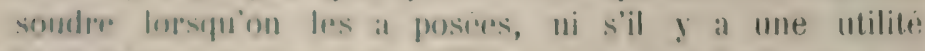

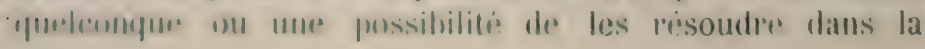

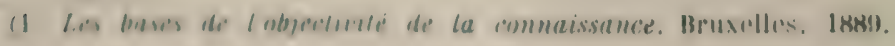




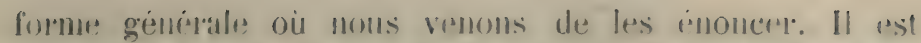
evident que nies ou mettre en doute la rabalite de boutres nos contaissances an hloc ou mime simplement en faim abstration, cest s'anlever pour toujours la possibilite dre

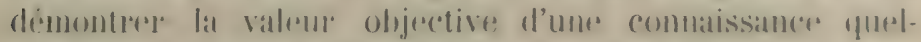

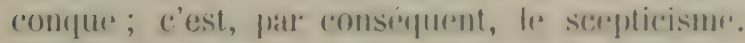

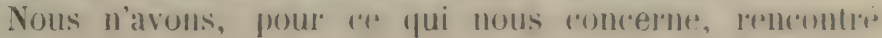
jamais aucume mison serieuse de limiter a priori l'objes:tivite de nos jugements. Il mous a tonjours farto alssi yu’il n'y a pas lien de distingure entre l'evidence d'un jugre-

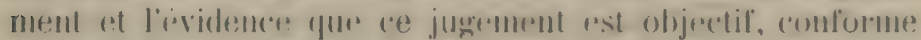

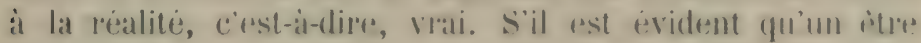
changeant a eu un commencement, it est evident yur colte aflirmation est conforme il la realité, puisyur aette ronformite est precisement as qure le jugpment allirme. Tout jugement evident, qui s'impose a liesprit soit direstement,

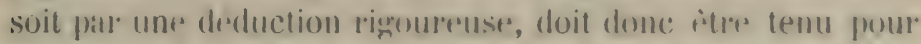

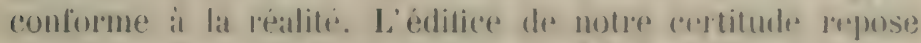
ainsi sur la réalite indeniable des fats concelse et sul la necossiti des jugemonts alostrats obtemus fall l'analyser des idées générales.

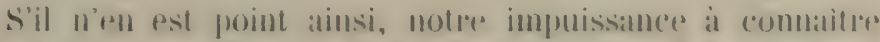
la realite est complite of sans remede at, quoinulon diste, la connaissance de la realite est la seule qui importre. Quant à nos pereeptions semsibles, nous pouvons par le raisonnement determime le dergres dobjectivite qu'il faut Ieur atuibure, et distingurer less pures apparences des sensations réelles.

co de Autrefois la seepticisme, l'Averroïsme et te Pominalisme ont plus ou moins niet l'objectivite des connaissances humanes. Mais e'est à proprement farlep Kant qui a éte l'initiatrur des spriculations modernes à ce sujet. (1)

1. Le's relations entre l'idéalisme moderne dont Kant est lo chef el la philosophie de Destartes sont bien mises en lumiere frar A.

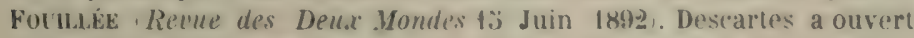
la voie a Kant pluthe far les problimes qu'il souleve fur par les réponses qu'il y donne. 
lat phunat des philosophes qui sont venus apres kant ont dans une certaine mesure subi son influence, mème crux qui rejoussent presque toutes ses doctrines.

Kant se tlattait d'avoir trouvé la méthode véritable de der lit mextaphysique ot prédit les conquêtes détinitives yur cette science va des lors réaliser. "Si le lecteur tolle est la conchusion de la Critique de la Raison pure - a ru la complaisance et la patience de la (la méthode (rritipue) suivre avec moi, il peut voir maintenant si dans le cas où il voudrait bien contribuer à convertir ce sentier en route royale, ce qu'un grand nombre de siècles n'ont pu mener i bonne lin jusqu'ici ne pourrait pas itre accompli arant même que celui où nous vivons soit icoule, à savoir, de satisfaire complètement la raison humaine en une matière dont elle s'est constamment occupée aves ardeur jusqu'ici, mais toujours inutilement" (1). La mime confiance dans les résultats merveilleux de la nouvall. methode est exprimée dans la préface de l'ouviage. (ir ces previsions ne sont pas réalisees malgré l'influence ronsidrable que kant a exercée, et cela senl suffirait pour demontrer qu'il a manque son but.

" Muant i ce qui regarde l'accord de ses partisans dans leurs assertions, dit-il, (2) lat methaphysique en rist l'autant plus èloignere qu'elle semble n'ètre pour eux yu'une arine exclusivement destinée à des jeux bhablis pour devoloppor les forces at dans laquelle ancum des champions n'a pu ou se rendre maître du plus petit

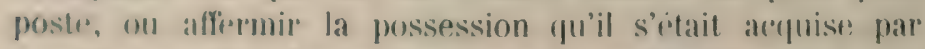
la virtuire. Nul doute done gue la methode suivio jusqu'ice par la metaphlỵsig̨ue n'ait éte qu'un pur latomnement dro simples couerefts. ") Le jugement severe que Kant

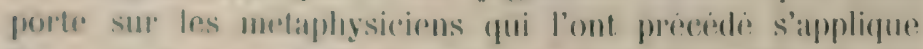
atre plus de verite a cenx qui l'ont suivi et qui

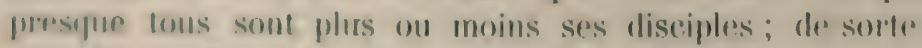
you. lat comdammation prononere par le philosophe de

(1) (op. elt, vol II p. 434.

(2) Jold vol. I D. I. 
Koenigsberg sur la mithode suivire avant lui restombe de toul son poids sur la siemne fropme. La distinction du noumene et da phénomene a engendre les throrios les plus variers of less plus contralictodres depuis le solipsisme de Fichte jusqua l'idralisme radieal des Hocgrol. Mais on cherche an vain quelle rongurte détinitive alle a fait réaliser par la métaphyssique.

Il est raai qure sous l'intluence de Kant beanuenup de philosophes ont abaudonne les conceptions metaphysiques fui elatent universellement alduises antrafois: mais on ne voit pass ore gu'ils ont mis a la plater at la nouvelle methode semble atroir servi plutiot al detruire qua'a édifier.

Lr Kantisme devait logripurement almutir it l'idealismes transerendental. "Kant, dit Cle. Renouvirer, ne pent renversere lat metaphysigue qu'en lui opposant lat fossibilite

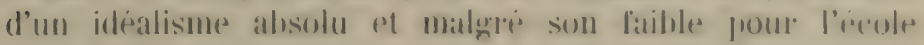

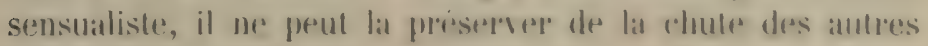
philosophies. " (1)

Or, l'idealisme transerendental a commer point de: dipgart

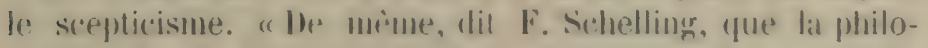
sophire de lat nature, attentior seulement a ree qui est

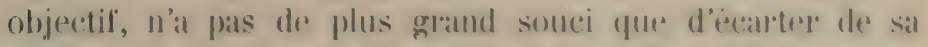
science tout mélange de subjuetif ; ansi an contraire la

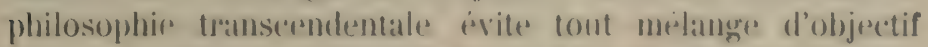
daus le principe subjectif du savoir. Le moyen de s'en debarrasser (das Ausscheidungsmittel) est le scepticisme alssolu, non lo demi-sceptiorisme qui ne s’attaque qu'aux préjugés communs de l'humanité, mais lo scepticisme: radical qui so dresse non contres dos prejugés particuliers, mais contre le préjugé londamental dont la ruine entràne celle des autres. Calr outre les prejugis antiticiels et adventices, il $y$ en a de beaucoup plus profonds qui dérivent, non pas de l'iducation on de l'art, mais de la nature elle-mème, qui pour tous, a l'excreption des philosophes, tirnment lieu de principes de tout saroir, et qui,

(1) Ifanuel de Philosophie molerne. Paris 1K19 [. 361. 
pour le penseru original luimeine, servent de pierre de touche pour toute vérité.» (1)

Yotre comviction est yue si l'on prend la seepticisme alsolu rommer point de lejpart, il est impossible d'en surtir et de faire logiguement un serul pas daus lat sperculation philosophique.

mit-on admettre que jusqu'a présent on n'a pas lormuli un systeme satisfitisant de metaphysique, ce ne serail pas unte raison d'abandonner l'utude de cette science, ni de declarer l'esprit de l'homme incapable d'une "connassinnce absolue "), c'est-à-llire d'une ronception des choses conforme à la réalité.

Opinion des philosophes.
Il est absolument faux que les phus grands philosophes aient ete de tous temps flaccord pour proclatmer l'ineaparite de notre esprit à saisir la rialite, ('omme spremerer le dit ici après W. Hannilton. Le's exemples mumess que cite ce dernicr sulfisent pour nous iditier: Protitgorats (le scrptirpue) Aristote (qui a refuti Protagoras), Saint Augustin, Boèce, Albert le Grand etc.

Ces philosophes nont admis pour la plupart la relativite de la romnaissaner que dims le sens itabli plus haut: toute connaissince comme telle est relative i l'objet connu et celte relation est réelle dans notre esprit. Parr lit-mime, lobjer comn at une relation a notre connaissaner, relation fui consiste precisement it etre connu, et "on ce sems, tout ce qui est connu est relatif. Cette dermiere relation nest point une relation rielle dans lobjet de la comnaissance, car itre connu ou ne pas inter romnu n'atyporte a l'objet ancune modification.

lin ce sens dome, encore une fois, toutes nos connaissames smot relatives at nous me commaissons que le relatif. Lne connaissance alosolue (non relative) est une

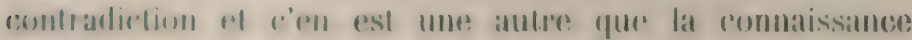

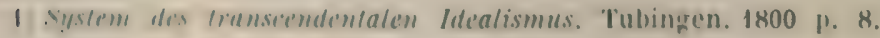




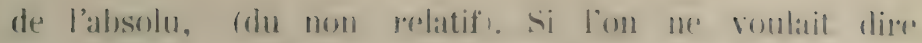
quer rela, il serait oistal d'insister sur des dhoses atussi élémentaires.

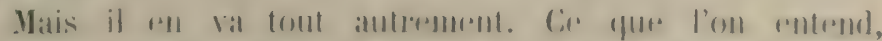

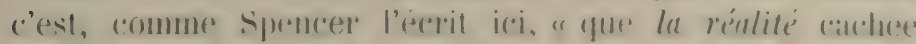

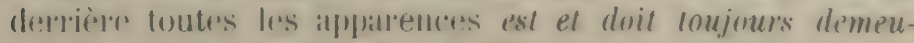

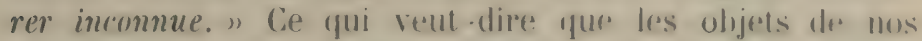
connaissances n'ont aucune réalite, sont do pures appa-

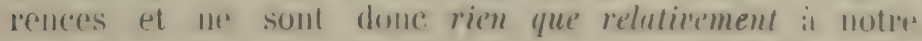
esprit. On allmet ume rialitio (illiegitimement, comme nous le demontrerons), nom gas celle qui rst representere pare nos eommaissitnces, matis une realite " radsere y que nos

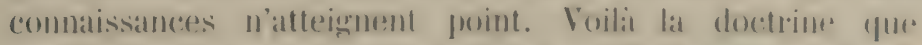

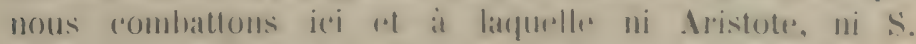

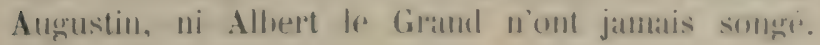

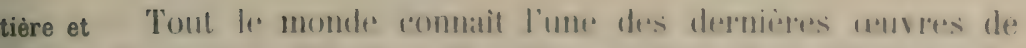

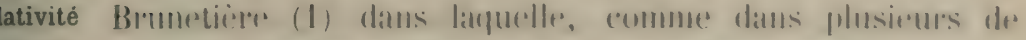
onnais- ses frecerdentes publications, il se montre frattisint de lal aces. theorite de la relativite de nos rommassances. Comme

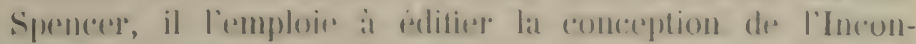
natissable, objet de lat Religion. si noms me devions y voir qu'une "utilisation du Positivismen nous pourrous passer ontre. Mais l'eminent exrivain affirme torp ouvertement ses preferences, ef l'aluturite qui s'attalde a son nom est trop considerable pour yu'on ne soit point emu de l'appui qu'il fournit a cette doctrine. I lire les pages qu'il y consalere, on a l'impression d'une lutte rontinurlle entre lo rohuste hon sens de lauteur el cefte mauvaise métaphysique.

"Je voudrais bien savoir, excrit-il, (2) quel est le malade ou le matuvais plaisant, et je devatis dire le fou,

(1) Sur les chemins de la C'royance. L'ilisation du P'ositivisme. Paris. $190 \mathrm{~s}$.

(2) Op. cit p. 25 . 


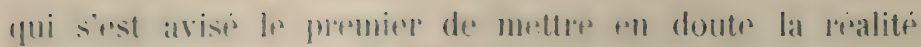

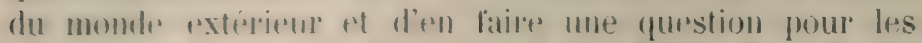
philosophles. " Fort bien. Lal question est en rffet résolue fatl le sans commun et, aroyons-nous, frat la philosophie. Mais il est egaldement clair que qui soulève la question do la relutinté de nos comnassances, soulever par la meme la question de l'axistencer réplle des choses que nous commaissons. Et Iorsque M. Brunetière ajoute: "Nous ne pouvons nous demander si quelque chose existe qu'a la condition d'etre deux : nous, qui nous le demandons, et, hors de nous, quelque chose dont nous Ir demandons ", il n'est pas moins manifeste que cette réflexion n'est qu'un paralogisme.

Brunetiere dit encore: "Tout se passe.... comme si If monde extéripur était ce que nous croyous quiil est. ) (1) Nous comprendrions quapres cela il conclue : il est donc vaiment re que nous aroyons qu'il est. Au lieu de cela l'autrur, après aroir indiqué les solutions possibles de la question, ajoute: "Mais de cees trois solutions, quelle que soit celle que l'on ulopte, et pour quelques lasisons qure cer soit, le monde exterirur n'en continue pas moins à itre tout ce qu'il est pour nons. ” Et ici nous ne comprenons plus. Logigurment il ent fallu dire: n'eu continur pas moins it detre pour nous fout ce qu'il rst pour nous. Mais une telle alfirmation paraitrait a hon droit inutile.

Voici drailleurs les trois solutions dont il s'agit : "Ou

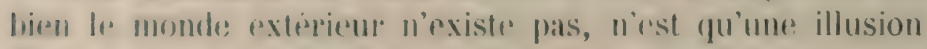
de nos serts, un rève quon ferait l's yeux ourerts, une frojection des lois de notre intelligence a travers l'espace ou lo temps, - a crest la premierre solution. On birn le monde exterient existre of les impressions que nous en

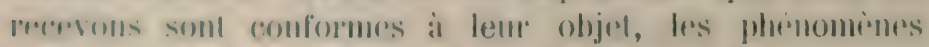

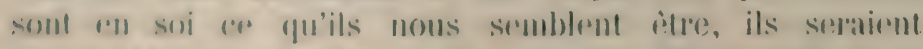
moreme lout re qu'ils somt si mous n'existions fats mous-

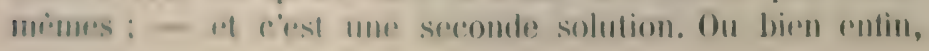

(1) Op. ctt p. 181. 
le monde extérieur axiste, mais entre l'iter que la constitution de notre esprit nous permet d'un prendre et la réalité de ce qu'il est en son fond, il n'y a pas de rapport à nous connu, de communicution certaine, de ressemblance ou d'analogie, - et c'est la troisirme solution. ") (1)

Cette troisième solution est celle de Brunetière at aussi nous le verrons, celle de: spencer. Tous moyons qu'ellp ne diffères de la premiere que par une inconsérquence, et nous essayerons bientot d'stablir ce jugement.

En attendant, roici ou, de nowveau, le bon sens de

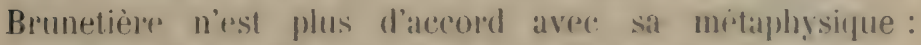
"Quand d'un grland, iserit-il, il sort un thère, on d'un ceuf un poulet, il est possible que ni le poulet, ni l'uruf, ni le chène, ni le grland nte soirnt en soi substantieflement ce qu'ils nous semblent être; mais ce qui est corlatin c'est que le poule n'est pas un chrine et que la diersité de nos perceptions a sa conse en dehors de nons - je veux dire sa raison d'itre - et elle la dans la dicersité du poulet et du cheme." II semblor birn que voilat allirmes

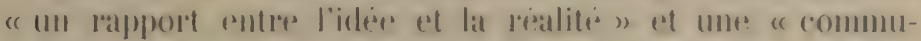

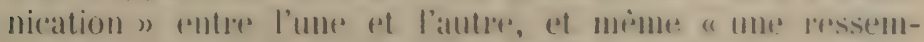

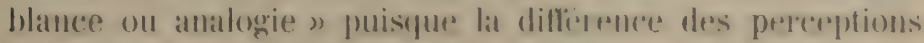
cormespond it la ditheremere des rialites. Lat these de la pure relutivite de notre comnaissancer parait compromise.

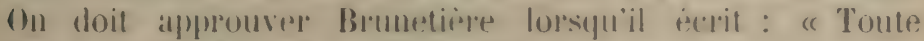
scrence, quand on l'approfondit n'est en somme qu'un sys-

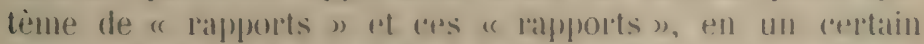
sens, ne sont eux-mintes que des signes n. (2) El plus loin : " lat science n’est qu'un systime de rapports ou de signes. ” (3) En rollet, les rommaissances qui constituent la science sont, prises subjertivement, fes signes formels des realites qu'plles reprisentent, at les rapplorts on relations ne provent exister que s'its sont fondes dans lat réalite.

Mais lorsque l'antrur, fitlile an relativisme, ajoute: " ce qu'expriment ces signes nous ne le sarons pas plus

(1) Ibid p. 159 cest nous qui soulignons.

(2) Ibid p. 1 19.

(3) Ibid p. 1:34. 
que nous ne sarons ce qu'expriment les caractères d'une langue inconnue n, nous faisons observer que les carac-. tères d'une langue ne sont des signes que pour ceux qui les comprenment, et que si personne ne les comprend ou ne les a jamais compris ce ne sont plus des signes.

Que nos connaissances sont relatives cela signifie, nous dit Brunetière, (1) « en premier lieu que nos connaissances dépendent de la nature de nos moyens de connaitre, et, par exemple, que si nous avions, tant que nous sommes, d'autres sens, ou six sens au lieu de cinq, ou le cràne fait d'une autre sorte, le monde extérieur nous apparaitrait peut-ètre sous un aspect assez different de celui que nous lui prêtons; - et on peut bien le dire, mais ils (?) n'en savent rien. »

Sans doute, ils n'en savent rien, mais entin, on peut le dire, et Brunetiere lui-mème ne dit pas autre chose lorsqu'il athirme que "quelques verites très génerales demeureront eternellement les mèmes; ce sont celles qui n'expriment pas tant les lois de la nature des choses que la constitution de lesprit humain." (2)

Dr. sorte que si nous allirmons que deux et deux font quatre, c'est que nous avons le cràne conformé d'une certaine façon; "s'il etait fait l'aube sorte", nous allirmerions pent-ritre avec la mime conviction que drux at deux font cinq. Et comme d'ailleurs co jugement ne srrat " qu'une pure representation, n'atteignant aucunemurnt la chose en soi m, il n'y aurait pas lien de s'en émonvoir beancoup. Evidemment le bon sens de Brunetière n'admet pas ces conchusions, mais nous ne voyons jas comment il y échappe.

Lo relativisme II serail injuste de confondere avere la these radieale

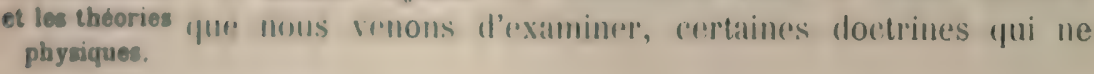

(1) Ibid p. 13.

(2) Ibid p. Ist. 
nient pas d'une facon geneneale l'objectivite de nos rentnaissunces, mais qui la contestent four un gremper parti-

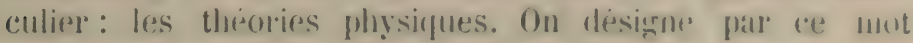
non pas precisement less fats tols yue lexprerifnce les decourre, mais plutot des ensembles de lois at d'hypothios's qui ne sont pas l'objet direct de l'exprerience et qui no provent pas non plus se déluire des faiti par un raisonnement rigoureux.

Ce n'est pas it dire que nous soyons disposes a admettre la relativite ou le callactire purement symbolique drs connaissances de celte sorte. Autre chose est, fall extmpltr.

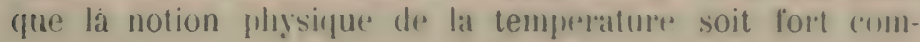
plese et que pour la mesturer an moyen du thermomime il faille s'appuyer implicitrument sur lun grand nombre d. throries phlus ou moins rotaines et de faits flus ou moins exactement rialises; - autre chose est gute aplle notion et cette mesure soient de purs symboles, de simples signes de lat realiti. Sims doute les denrres du thermomitre sont des nombre conventionnels, mais lat dilatation du merecure qui, en sommes, sert de me-ill\% at la tempriature, n’est pas un pur symbole de ceelle-ci, mais bien un phénomine connexp. Donc la notion de trinperalture dont on se sert en physique n'est pas unr notion purement symbolique.

II en est de mime des lois physiques. Que nos methodes d'observation ne soient pas susceptibles d'un exactitude absolue, cela prouve à la vérité qu'une loi malliématiquement précise ne peut pas ètre le résultat des observations seules, mais cela ne prouve aucunement que nous n'avons pas le droit d'énoneer une telle loi. Cee n'est d'ailleurs pas seulement par la précision de son enonce' mais encore far son caractère général, que la loi physique depasse l'exprience, celle-ci etant nicessairement restreinte a des cas particuliers. Et nous ne dirous pas pour cela que la loi physique est approche et particulière, mais bien que nous n'avons pas le moyen d'en verifier directement la rigoureuse exactitude et l'absolue 
généralite. Ainsi nous n'avons aucun moyen de contròler l'exactitude mathématique de la loi d'attraction. S'ensuit-il que cette loi n'est qu'une loi approchee et qu'il faille dire que les corps s'attirent à peu près en raison inverse du carré des distances mais non point exactement? On nous dit qu'une infinité d'autres lois s'accorderaient avec les faits aussi bien que la loi Newton. Soit. Mais ces lois differentes qui les a énoncées? Et à supposer qu'on en inonce l'une ou l'autre, tout ce que l'on pourrait prétendre, c'est que l'expérience seule ne permet pas de choisir entre elles, mais non pas que l'une d'elles - la loi de Newton - n'est qu'approchée.

Yème si l'on accorde qu'une loi n'est qu'approchée, il ne s'ensuit pas qu'elle est șrmbolique et qu'elle n'a avec la realite d'autre relation que celle, par exemple, d'un drapeau avec une nation.

Il y a des lois approchées, par exemple la loi de Mariotte. Atin de lui laisser sa forme précise sans contredire les faits, on dit qu'elle ne se veritie rigoureusement que pour les gaz parfaits, et l'on veut dire par lì qu'à mesure qu'un gad s'éloigne - pall l'alugmentation de la température et la diminution de la pression - de son point de liquélaction, il rérifie toujours plus exactemeut la loi de Mariotte, sans cependant qu'on puisse allimer qu'il la veritie jamais avec une exactitude absolue. Dans ce cas on pourrait dire avec quelque ririte que l'enonce de la loi est une formule symbolique, parce que comventionnellement on l'a adoptée pour remplacer un inonce diflerent, plus exact mais plus long. Cependant, mime dans ce cas, le mot symbolique ne serait pats fort hroremsement choisi, car les laits récls signities par le symbole fusurnt laire croire quil existe des gaz pour fresquels la loi de Mariotte se virities a la lettre. Quoiqu'il en soit, ce nest que par un abus de langrage que l'on peout appeser symboligue une reprisentation vaie de In rialiti sous pretexte quelle pst incomplide. D'apres Doulurm, less lois de la physigue sont provisoires parce qum. symboligumes at il ajoute qualles sont provisoires 
"en ce que les symboles sur lesquels elles fortent sont trop simples pour représenter completement lat realiti. n (1) A ce compte toutes nos connaissances sont symbuliques et il n'y a pas lieu de faire une distinction comme le voudrait l'auteur que nous citons entre les lois physiques et les lois de soms commun. Avint la découverte des rayons $\mathrm{X}$, c’était une loi de sens commun qu'il est impossible de voir a travers une plandle. Inpuis lors il a bien fallu reconnatre que cette lois " ne represente pas complitement la realite. " Mais personne n'en conclura qu'elle est symbolique.

Quant aux hyquotheses scientiticjues, il est la plupart du temps facile de demontrer qu'elles ne fommissent pas une explication (omplete ni dedinitior des phenomenes. Mais faut-il aflimer pour cola qu'elles sont des constructions spmblighues, uniquesuent destinees a nous fiuciliter la chassifuation des phennmènes ot sans ancun lien de ressemblame aver la realite? Nest-il pas plus conforme it l'intention de leurs auteurs at plus ratisomatble de les consideren comme des essais d'explication dont la conformite avere les ohjots devient plus probable d mesure quangmento le numbre des lats qui les confirment? Mime les partisans du symbolisme lecoulent devant l'affirmation absolues de lenr theses. Iat theorir physinge, nous dit-on, tend a se transformer ell une classitication naturelle et on entend par la que "les liens ideanx etablis pale la ratison rontre des conceptions alsitrates correspondent a des rapplorts reels entre les btres con(rets ou ces abstrations mentrent corps. ) (2)

"Plus la theorir physigure se preftectionne, dit Inuhrm, plus nous pressentons que l'ordre logrique dans lequel elle rangre les lois rxprerimentates est le rellet d'un ordres ontologique, plus nous supgomons que les rapports quelle chablit entre les domners des l'nbservation rorrespondent

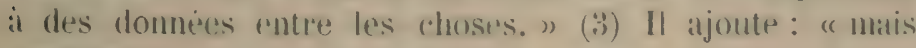

1) La Therorie physinue. Paris $1906 \%$ \% 288.

(2) DUнем. op. cil. p. 33.

(3) Ibid. p. 38. 
et ne peuvent pas itre absolues, soit par l'analyse des produits de lat penseres soit par celle de l'opération de la pensere. ") (1) Nous allons suive notre philpsoplar dans cette double analyse.

Deux ou trois exemples sont longuement developpés pour nous faire comprendre ceci: Le travail de l'esprit qui cherche des explications de plus en plus approfondies de

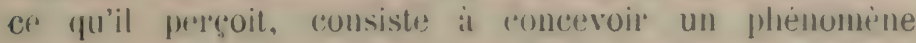
comme un cas particulier d'un fait genéral et colui-ci comme faisant partie d'un fait flus gineral encoles et ainsi de suite. "Nous avons pris pour point de dejart les phrinomines particuliers, nous les avons rapportis a des groupes de phenomenes de plus en plus larges, et en les y rapportant, mous sommes arrives d des solutions qui nous semblent d'autant plus profonde's ques l'operation a ele poussie plus avant. Dommer des explications encore plus prolondes, co serait seulrment fare de nouveaus pas dans la même direction. "n (2)

Comme il est impossible que d'explieation en explication on aille a l'intini, il faudra bien s'arreter à unp proposition qu'on n'expliqus plus et qui, par conséquent, " ne peut-ithe comprise. Done de toute nécessité, l'explication doit nous mettre en lace de l'inexplicable. Le mot " compremdre " duit changug de sens avant que le fait ultime puisse être compris."

supposons un instant que tout te raisonnement de spences soit irrefrochable, pourons-mous en conclure que nous ne commaissons aucune realitio ell elle-mene et que nos commaissances sont purement pelatives? Il y a longtemps quion a dit que nous ne commaissons le tout de rien ; ef cest a froprement farler la conclusion a laquelle minent les considerations que nous avons résumers. Mais de la il la these que spencere s'est charge de clémontrer, il y a très loin of nous ne voyons pas mente très clai-

(1) P. P. p. $73 \$ 22-$ F. P. p. $\$ 1$.

(2) P. P. P. $77 \& 23-$ F. P. P. 533 .

(3) P. P. P. 78 $23-$ F. P. P. S4. 
rement le lien qui rattache l'une à l'autre cette proposition: Tos connaissances ne représent aucune réalité et cette autre: Toute explication nous met en face d'une dernière raison que nous ne pouvons plus expliquer.

On ne: peut s'empècher de trouver ètrange qu'après awoir consacré quatre pages à exposer une chose parfaitement claire (quoique peut-être pas rigoureusement exacte) à savoir qu'une explication consiste à ramener un fait particulier ¿ une loi générale, Spencer ne consacre pas mime une ligne à déduire le quod erat demonstrandum.

Il eut èté également opportun de distinger deux cas qui se présentent lorsqu'on pousse les explications.

Dans le premier on se trouve en dernière analyse devant un fait particulier ou général qui demanderait une explication qu'on ne sait pas donner parce qu'on l'ignore.

Dans le second on arrive en lin de compte à une proposition dont la nécessite se manifeste a l'esprit de telle sorte qu’il n'y a plus rien à expliquer. Choisissons des exemples parmi ceux que spencer lui-mème développe.

Qu'un muscle se contracte sous l'inlluence des nerfs c'est un fait dont personne jusqu'ici n'a donne d'explication. spencer le rapproche du raccourcissement d'une pile d'efectro-aimants sous l'action du courant. Mais cette explication n'en est pas une et nous n'avons ici qu'a confesser notre ignorance. (1) la connatissance que nous avons d'un muscle no nous permet pas de comprendre qu'il perut. ou qu'il doit se contracter.

Il en va tout autrement lorsqu'il s'agit d'expliquer que lo. jou does crites angmente le volume de la cage thoracicqure. Les roites forment des angles aigus avec l'epine dorsale; lorsigubles se relixent elles sont porties an avint de sorte que le diametre antero-postirieur de la poitrine, angmupule, les andres restant sensiblement constants. Ide

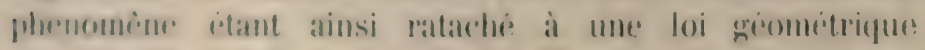

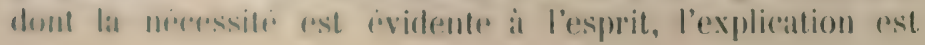

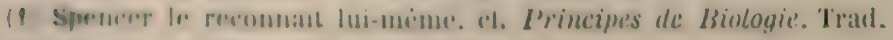
liaktikks. Paris is40, vol I, p: (et) \& 21. 
terminee et il n'y a plus rien it expliquer. Il en est de mime chaque fois qu'un principe mathimatique, ou plus géneralement, un principe analytique quelconque domes l'explication cherches. Je m'etonne peruteêtre de ce que je puis diviser exactement fal trois un nombre charque fois que la somme de ses chiffres permet cette division. Mais dès qu'on m'a montre que ce lait est la consíquence necessaire des verites mathématiques los plus ilementaires et les plus evidentes mon resprit est saltisfait. Il est faux de dire qu'an hout de cette explication je me trouve devant l'inexplicable.

Il n'est pas inrxplicable que drux et trois font ciny. Je vois au contraire qur cela est absolument necessaire et que foute explication pour me le hare comprendre serat supreflue. si je fais roir qu'une proposition perut itre ramenese a cette vériti: grenfrale : deux quantites ingales at une troisieme sont igales entro alles, protendrat-on que si ce principe n'est pas lui-mrime ramente a un principe plus general rncore, on se trouve devant l'inexplicable? Et si lon ne peut pas dire reda seriensement, que reste-t-il de l'argument de spencer?

Il y a encore une antre distinction il faire si l'on admet l'existence des causes libres. Cone serre de causes subordonnies qui s'expliquent l'une par l'autre prout avoir son point de défart dans un acte librement posé. Or, IIn acte de rethe sorte a une latison suffisante, mais n'at point de rason nécessidre. On a donc, dans re cas, a expliquere comment l'efrit a pu itre produit, c'est-it-clire, pourquoi l'acte a pu intre posé, mais non pas qu’il a dù être posée nécessairement.

Cne serie d'évènements peut aroir son point de départ dans le fait que jaa fait l'amone à un fauve. L'explication de cet acte est satisfaisante dis que j’indique les raisons qui ont pu m'émouvoir à céder à la prière de cee malheureux, et on ne doit pas attendre que je rattache ma conduite a une loi dont elle serait la conséquence nécessaire. Nous pouvons conclure encore une fois, après ces remarques, que l'analyse de spencer est en defaut. 
inconcevabilitè de l'Absolu.

$\therefore *$

Passons a la seconde partie de la lemonstration. "Sir W. Hamillon, dit spencer, a donné it la démonstration du caractere nécessairement relatif de nofre connassince comme conséquence de la nature de notre intelligence, la forme la plus rigomeuse qu'elle ait jamais re!ne." (1) Et après l'avoir rapportée spencer ajoute: "Bien qur: cefte demonstration paraisse claire et décisive (quand on l'etudie arec soin, elle r'st exprimee en termes si abstraits que la plupart des lecteurs auront peine à la comprondre. M. Mansel en a clonne, dans son livre intitulti limits of Religious Thought ume démonstration plus familière....." (2) Suit la dèmonstration de M. Mansel.

Yous n'avons pas besoin de nous y arrèter longtemps pas flus qu'a celle de W. Hamilton. Ca's deux auteur's cherchent is prouver d'aborel que nous ne pouvons pas connaitre l'Infini. Or cela n'est pas en question ici. Ensuite ils dimontrent notre impuissance it connaitre l'. Ibsolu farrer fue toute connaissance consiste dans une relation entre le sujet at l'objet de la connaissance. "Ln second caratetirer de la conseitence, dit Mansel, e'est qu'elle n'est possible que sous forme de relation. Il faut un sujet ou une feresonne: consciente at un objet on unr. chose dont Ir sujet soit conscient. It he peut y avoir de connaissance sans l'union de ces deux lacteurs.... De mime il est ivident que la pereception de l'absolu implique contratdiction. Pour que mous aỵns conscience de l'absolu en tant quabsolu, il fitut que mous comnaissions qu'un objet dommi en relation dans notre conscience est identicpue asme un objut qui, dans sal propre? mature, existe sans

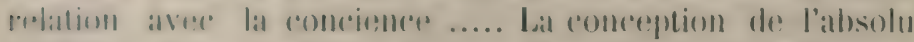

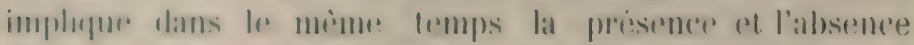

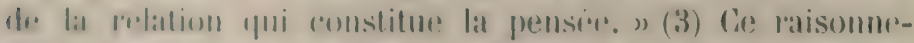

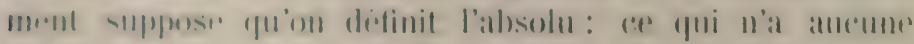

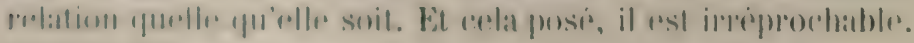

(1) P. P. P. 78 \& 24 - F. P. P. is .

(2) P. P. P. 81 \& $24-$ F. P. D. 83 .

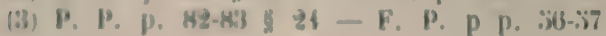


De mim: W. Hamilton érrit : “ La conscience lirst possible que par l'antitheses du sujet et de l'objet de: la pensée......" (1)

Cela est, en effet, "clail et décisif " mais celal n'est point ell question comme oll s'en convaincra en rolisant re que nous avons écrit 1). 78. La vare question est hunt authe et les arguments, tels que spencer les présente, pichent par ignorances de ce qui est en question : ignoratio elenchi.

spencer contirme te raisonnement d'Hamilton et do Mansel. Son mremier angument sepose sur une throrie de la connaissance que nous n'examintrons pas prour le? moment. Voici comment il l'applique at la question presente : "Une cognition du ripl, en tant que distingué du phrenomenal, doit, si plle pxiste, se conformer il la loi de la cognition ron greneral. Iat canse premièr" l'intini, l’alssolu, pour itre commus, doivent itre classés. Pour qu'ils soient

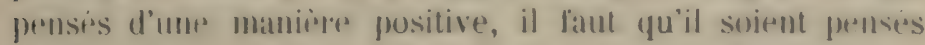

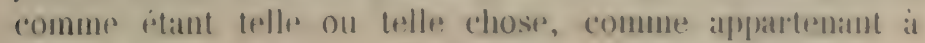
lelle: on telle espines. Prurent-ils être semblables an esprece à quelque (dhost dout fes sens nous ont domme l'rxpritience? Evidemment non. Entre ce qui cares et ces qui est crea, il lant qu’il y ait une distinction qui s'alive all dessus des destinctions yui stegarent les differentes divisions du corer. Cere qui ast sans cause ne peut blo assimile a ce qui rest antuse : il y a motre les deus, dans les termes mentes, mor opposition madicales. L'intini ne peut atre mis

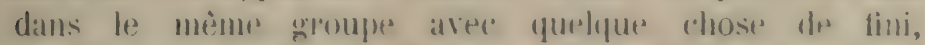
puisfualors il serait resalde comme non infini. Il est impossible de ranger l'absulu et quelque chose de resatil dans la meime catergorie, lant quon delinira l'alssulu: ("e qui n’a pas de relation nécessaire. ” (2)

si l'on definit ansi l'Absolu, il se confond avere l'Inlini, car tout itre tini a tune restation nexessaire avec l'Intini qui est sa cause. Sent l'Intini n'al atucume relation nieses-

(1) P. P. p. $79 \$ 21-$ F. P. p. 34

(2) P. P. $8586 \$ 24-$ F. P. p 88 
saire arec quelque itre distinct de lui. Mais l'Absolu ainsi dètini n’est pas la mème chose que le réel; et si l'on détinit le relatif pall opposition à l'Absolu: ce qui a des relations nécessaires, il n'est pas la mime chose que le phénoménal.

Larmumentation de spencer repose tout entière sur la confusion entre le réel et l'Intini. Nulle part cependant il n'a essayé de demontrer que seul l'Intini est réel. Si meme l'Intini ne pouvait pas itre conmu, il n'en suivrait pas qu'on ne peut connaître aucune réalité.

- Il est faux d'ailleurs que nous ne pouvons avoir de l'Infini aucune connaissancr. Quoiqu'il ne puisse, en effet, ètre rangé dans aucune espéce connue par l'expérience, nous pouvons le connaitre par des notions analogiques et négatives. Yous pourons savoir qu’il existe, qu’il est sans bornes, qu'il posside toute perfection, qu’il est la cause de tous les etres ; qu'il est intelligent, lihre, juste, quoique sans doute d'une manière intiniment superieure à ce que nous voyons autour de nous.

Par cette contaissance que nous arons de lui, l'ḱtre Infini a une relation a notre intelligence, relation de raison, relation non nécessuire et qui n'empèche done pas qu’il ne soit l'Etre: Absolu, d'après la detinition qu'en donne spencer.

La correspondance entre la conscience et la réalité.
Le secomd argument est tire de la matme de la vie. Nous ne ferons pas davantage ici la critique de la notion yure spremere developpes à ce sujet atiu de ne pas nous exarter de: lat furstion. Admettons aedte notion provisoirement ot royons re quon prétend an tires. "Si lone la vie, dit l'autrom, dans foules ses manifestations, y

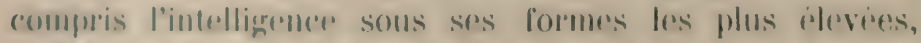

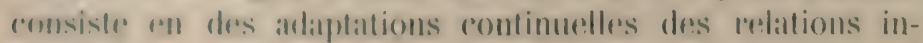

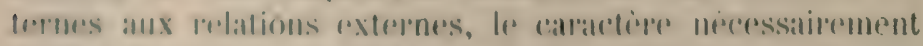
mattil de molm connatissance devient evident.... Si tout

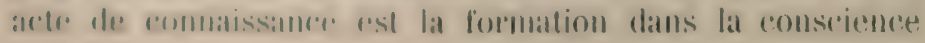


d'une relation parallèle a une relation dans le miliou, la relativité de la connaissance est évidente of devient une banalité. Si pensir c'est établir des rolations, mulle pensee ne peut exprimer plus que des relations. " (1)

En admettant que tout acte de connaissance consiste dans une relation parallèle à une relation dans le milipu, pourvu qu'on admette aussi que la relation dans la conscience est l'image psychique de la relation dans le milieu. on pourra appleter nos connaissances relatives parce cfur. nous connaissons des relations, mas non jas dans le sens que nous ne connaissons aucune realiti ; d'autant plus qu'on ne peut connaître des relations existant dans le milieu, qu'id la condition de comnaitre an meme lemps les choses reelles entro lesquelles ces mations existent.

Mais, dit spencer, lat connaisiance des malites mous serait, an tous cas, inutile : "Soirot $x$ et y des propribles uniformement unies dans un uhjet pxlerieur. et $a$ at $b$ les effets qu'elles poodusent sur motre ronscience: supposons que tandis que la froprigeti $x$ produit en nous l'étal mental indilferent $a$, lat jropuriets y produise l'état montal pénible b (correspontant it une liesion plụsigur), lout ete quil nous faut pour nos besoins, cest de savoir que $x$ etant uniformement aceompagne de $y$ all drehors, a alecompagnera uniformentent $b$ an dedatns; ens sorte que lorsyue, jar la juesence de $x$, a sera produit dans lat ronscience, $b$ ou plutiot l'idée de $b$ le suivara et excitara less mouvements fral lesquels l'eltet de y prent itle evité. Lat seule chose que nous ayons hesoin de saroir, c'est que $a$ at $b$ et la relation qui less unit correspondent toujours a $x$ et a $y$ et a la relation qui les unit. Il ne nous importe nullement de savoir si $a$ et $b$ sont semblables a $x$ et à $y$, ou s’ils ne le sont pas. Lenr parfaite identite ne nous fait pas gagner un iota, et lem dissemblance totale ne nous cause aucun dommagr. " ( (2)

Il faut donc que je sache : lo qu'a chatque apparition

(1) P. P. p. $91 \$ 25-$ F. P. p. 62

12) P. P. p. $91-92 \$ 23-$ F. P. p. 63 
des états de conscience $a$ et $b$ correspondent deux realites $x$ et $y$; 20 que $y$ accompagne toujours $x$; 30 que y donne lieu i certains effets qui sont evites par dis mouvements détermines par $b$. Voila qui est accoldé et il n'est donc pas vrai de dire que nos comnaissauces sensihles ne nous apprennent rien au sujet de la réalité. d'ajoute que lorsur'il s'agit de determiner des mouvements correspondant à la situation $x, y$, on suppose d'une façon generale que $a$ et $b$ en fournissent une image ticlele. C'est ce que Brunetière exprime en disant: "Toul se passe.... comme si le monde extérieur était ce que nous croyons qu'il est. ”

spencer développe plus longruement l'idée qu'il se fait de la correspondance qui existe entre le phénomène et le noumene dans un passage des Principes de Psychologie. Linterial qu'il presente nous rxeusera de? le citer in entier malgré sa longueur.

"Soit .............. la surfate d'un cylindre; soit un cube situe en face de lui et supposons que d'un point quelconque au dela du cube rayomnent des ligness comme celles dont chacune passe par les angles du cube, et aussi d'autres par tous les points qui forment les borls du rube. Ces lignes interceptees par la surface courbe, formeront du cube une image par projection.... (1) On prut observer ici.... que les longururs, les rapports, les directions, etc. des lignes de l'image projetre sont entitroment dilfirents des lignes du solide; que les angles aussi, pris absolument comme dans leurs relations resprectives, sont diflérents; et que les surlaces le sont aussi, dans leurs tigures comme dans leurs directions relatives. Mais an outre on pent voir que les lignes qui sont droiles dans le cube sont courbes dans l'image, et que les surfaces planes de l'un sont representees par

- I Spremer joint a son texte une tigrure yur nous jugeons inutile

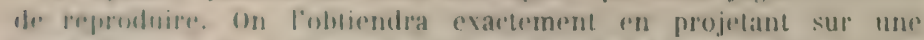

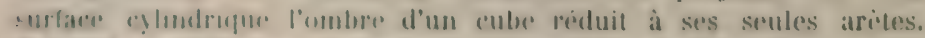

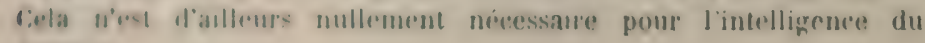
passage. 
des surfaces rourbes de l'autre. Bien fulus cerst un fait a noter que le's lois de variation de's lignes de l'inage

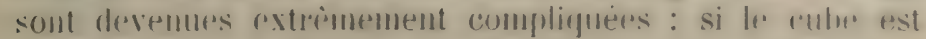
mi lateralement dre lancon que l'imagre projetre tombe bien phos avant sur lasurface en rebait du cylindre, qurelgues-

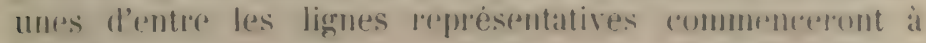
sallongrel bien plus que les aulers; at mime les pallties le's plus eloignees de chaque ligne s'allongeromt "nd des

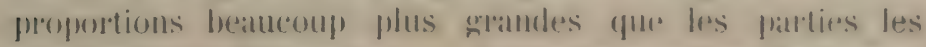
plus proches. Nianmoins.... if ! a un systimme de correspondances absolument defini entre les deax objers. Si l'on pose comme tixes le cylindre, les dimensions du cuhe

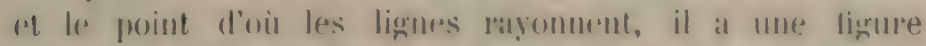
domner contespondante sur le cylimdre; et nul diangement

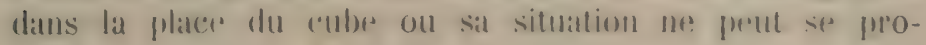
duire sans qu'un changerment exactement correspmendant

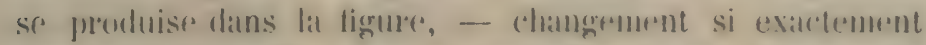
correspondant gure, frar la nouvelle tigure, la nouvelle

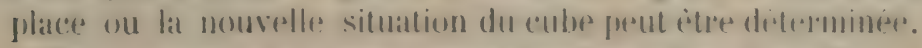

Xous atous alnsi tume symbolisation dans latpuelle, ni les eltements du symbole, ni leurs redeations, ni les lois suivant lesffurlles ces relations varient, ne sont le moins du monde semblables aux elements, aux relations deres elements, aux lois suivant lesquelles eres relations varient

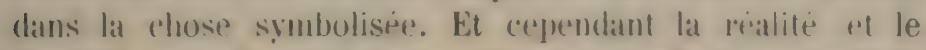

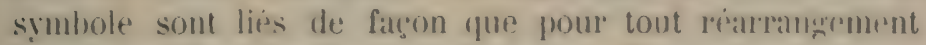
possible du plexus qui constitue l'un, il y a tun rearrangement equivalent dans le plexus qui constitue l'antw.

L'analogie qu'on peut en tirer est si évidente qu'il est à peine besoin de la faire ressortir en letail. Le cube c'est l'objet de la perception, la surfater du cylindre cest le champ de réception de la conscirnce ; la tignars projeter du culve c'est l'etat de conscience que nous apyrelons perception de l'objet.....

Mais ce (que nous avons un interêt capital a remarquer, c'est que, en nous représentant ainsi la matière par un diagramme, nous obtenons une idée distincte des relations qui existent entre les differentes hypothèses qui ont été 
discutees. Le réalisme grossier admet que les lignes, les angles et les aires de la surface courbe sont réellement les mimes que les lignes, les angles, les aires du cube. Lidéalisme observant combien tous ces diver's éléments de la figure projetée changent en eux-mèmes et daus leur's relations reciproques, quand un simple changement de place ou de situation se presente dans le cube, conclut que, romme il n'y a rien dans la figure qui ressemble à quoi que ce soit dans le cube, rien de pareil à un cube ne doit ìtre regardé comme la cause, et que les seules existences sont la figure et la surface qui la porte. Le réalisme hỵothétique acceptant ces prémisses en tant qu'elles affirment le désaccord de la figure et du cube, prètend que néanmoins l'existence du cube doit être admise; qu'elle ne peut ìtre affirmée comme un fait, mais doit être acceptee ì titre d'hypothese néressaire. Le scepticisme, poussant plus loin encore te criticisme idealiste, soutient que non seulement il n'y a rien dans la tigure qui etablisse l'existence de quelque chose produisant la figure, mais que de plus il n'y a rien qui etablisse l'existence d'une surlace contenant la figure; et que, bien qu'il $y$ ait une tendance naturelle à croire à l'existence du cube, nous pouvons raisomablement douter si l'un et l'autre existent réellement, tandis que l'idéalisme absolu, poussant jusqu'ia ses dernieres limites l'argumentation scepticque, assure qur la tigure seule existe, et qu'il n'y a rien de parril ni an cube, ni a la surface. Et maintenant, rejetant toutes ces hypoteses contraires dans leur ensemble, le realisme transforme prend un element a chatene d'elles. II allirme une connexion entre le cube et son imiage projetiese, ce yui concilie ce qu'il y a de valai dans le realisme arece or qu'il y a de vrai dans l'antirealisme. Avec 1. realisme nrossier, il s'accorde al allirmer l'existence du culs: comme marque d'un caractire de certitude originelle ; mais il difliere entierement de lui en affirmant gu'il n'y a aucume parente de nature entre le cube et sa projoction. II reunit l'idéalisme, le seepticisme et les raalisme ligpotetique, en atfirmant que la projection ne 
contient pas un élément, un rapport, une loi qui soit semblable à aucun élément, rapport ou loi du cube réel ; mais il affirme contre l'idéalisme que l'argument sur lequel cette conclusion repose est impossible en l'absence de ce cube ; il affirme contre le scepticisme que l'argument ne nécessite pas seulement un cube corrélatif à l'image, mais encore une aire réceptive pour cette image, tandis qu'il blàme le réalisme hypotétique d'admettre à l'etat d'hypothèses ce que les arguments eux-mèmes supposent comme des faits. Finalement, bien qu'il ait un point de ressemblance avec l'idealisme alssolu, parce qu'il reconnait avec lui que la figure mrojotie ne peut renfermer le moindre trait soit du cube réel duquel elle est projetée, soit de la surfice réelle sur laquelle elle est projetie, cependant il en diffère extrèment en déclarant que l'exislence de ces deux réalités est impliquée d'une manière plus certaine que celle de la tigure, puisque l'existence de la figure n'est rendure possible que par la lenor. ") (1)

Lá comparaison est ingenieuse. Faisons remarquer cependant cerei : rim ne nous dit a priori que nos facultis sont comme des surfaces eylindrigueses déformant les projections phutot que comme des surfaces planes, ou mieux encore comme des miroirs idéaux représentant l'objet axactement, tel qu'il est. A posteriori tout contirme cette dernière hypothèse.

Ensuite, l'existence de la figure (vasy'n'implique /'existence d'une surface s'étendant au delat des limites de la tigure, ni celle d'un cubr, car rien n'emprèche qu'une projection ne soit trateie directement. C'est l'illéalisme absolu seul qui est logique.

Que si l'on admet l'existence du culpe comme canse de la projection, on preut salns doute signaler des différences entre les deux, mais on preut aussi indiquer des analogies: l'un et l'autre ont des dimensions définies, se trouvent du mème cùte du cylindre, ont huit points formés par des intersections de lignes, ne subissent de

(1) Principes de Psychologic T. II p. šl6 sq̣. $\$ 473$. 
changements que simultanément etc. De sorte que connaissant la figure et sachant qu'elle est. la projection d'un objet extèrieur, ce dernier n'est déjà plus inconnaissable. On se demande aussi comment il vérifie les appellations d'infini et d'inconditionné.

Entin si l'on connaissait les dimensions du cylindre et la methode de projection, il serait possible de déterminer la forme de l'objet. Or en supposant que ces dimensions et cette méthode ne puissent pas ètre connues directement, on peut faire des hypothèses en se réservant de les verritier. C'est ainsi qu'on a supposé que l'objet qui se projette sur la conscience sous forme de couleur est un mouvement vibratoire de l'éther.

Il ne serait pas diflicile d'amener spencer a admettre ce (qu’il appelle "le réalisme grossier") et qui n'est autre que le réalisme du bon sens.

L'existence réelle du noumène. si l'on affirme d'une maniere: absolue que toutes nos connaissances sont relatives et n'ont pour objet que le phénomène, aucune done n'a pour objet lit realité ou la chose en soi. Cellr-ei nous est donc complitrment inconnue. Yous ne savons donc pas si elle existe. Et comme ce qui existe ne peut exister qu'en soi, nous ignorons donc s'il existe quelque chose !

sproncer admet que cotte conclusion est logique et que ceprendant elle est erronéce. Celle contradiction amrait clû l'avertir de: la lansiete de la throrie d'ou elle découle. Au liru dre la rejeler, il y apporte dess restrictions.

"Tant que noms ne quittons pas lie cotis purement logiques

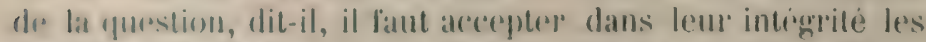
propositious rilioes plus haut (que nous ne pourons alfimer

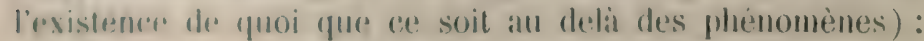
on nre peut le contester. Mais des que nous considerons un

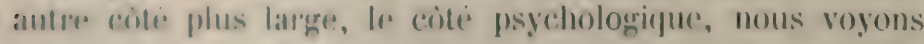
que res propositions axpriment imparfatement la verite;

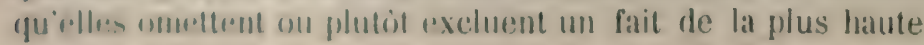


importance. Preceisons. A còte de la conscience définie dont la logrique formule les lois, il y a aussi une conscience indéfinic qui ne peut itre formulere. A criti des pensips complètes at des pensees incompletes qui, bien yu’incomplètes, sont encore sisceptibles de recevoir leur complemmont, il y a des pensées qu'il est impossible de completer at qui n'en sont pas moins reelles, farce qu'olles sont des aflections normales de l'intelligence $\varkappa_{0}$.(1)

Certe distinction est obscure et on la jugrema con outre arbitlaire. Gu'est-ce qur res prensées incompletes qu’il est impossible de completer, at surtout qu'est are qui lear' vatut le priviligre d'attrindre la realite au drola des phemomines ?

Nous ne pouroms admettre qume la conscience indetinie de l'Alssolu se trouve impliquere dans la these qui allime l'impossibilite de le connattre. Pourquoi "dire que nous ne pouvons connaître l’Abscolu ", c’est-il " alfirmes implicitement qu'il ! a un Alssolu "(2)? s'if n'y en a pas, faudrat-il dires yuon peut le connatre? Ois a-t-on frouve que l'Alssolu existe? Et pomrquoi " quand nous nions que nous ayous le pouroir de connailde l'essence de l'Absolu " ("n admettuns-nous " tacitemont l'existence " (3)?

Jous ne recomaltrons pas davantage yute "l'apparences est inintelligrible sans "la réalite » (4); car il $y$ a des appoarences sans réalite correspondante. Et soil est evident " que la demonstration de l'impossibilité d'une représentation definire de l'Absolu suppose invariab!ement une representation indetinie de l'Absolu » (ij); - car on ne peut raisonner sur l’Alısolu sans enll avoir quelque idée; rien ne nous dit cependant que cette idée correspond à un objet reel (6).

(1) P. P. p. $93 \$ 26-$ F. P. pp. 6468.

(2) P. P. p. $93 \$ 26-$ F. P. pp. $64-65$.

(3) P. P. p. $93 \$ 26-$ F. P. pp. $64-65$.

(4) P. P. p. $94 \$ 26-$ F. P. p. 65.

(5) P. P. p, $94 \$ 26-$ F. P. p. 68.

(6. Renouvier refute longuement l'argumentation par laquelle Spencer prétend démontrer l'existence réelle de l'Inconnaissable ( $L a$ C'ritique Philosophique. 188: t. I. pp. 412 squ. Partisan lui-mème de la relativité 
Spencer fait tres-bien voir, contre les auteurs qu'il a suivis dans cette discussion, que l'idée de l'illimité n'est pas une pure négation. "Notre notion du limité se compose premièrement d'une conception d'une certaine espèce d'être et secondement d'une conception des limites sous lesquelles alle est connue. Dans son antithèse, la notion de l'illimite, la conception des limites est abolie, mais non celle d'une certaine espèce d'ètre » (1). II aurait pu ajouter qu'en outre, la nègation dess limites, étant la négation d'une négation, est oljectivement positive quoique conçue négativement par notre esprit.

Mais il ne suffit pas d'expliquer commemt nous avons la conception de l'Absolu pour justifier dı mème coup la conviction que l'Alssolu existe. L'impossibilité de prouver' l'existence réelle de l'Intini au moyen de la notion que l'esprit en possècle a été parfaitement mise en lumiere par Kant (2). Aucume notion ne contient ent elle-mème la preuve de la realite de son objet. Seule la constatation d'un fail nous permet d'atteindre la realite.

C'est pourquoi toute theorie qui concoit les faits que nous connaissons comme des phénomènes sans réalité creuse un abime infranchissable entre celle-ci el notre esprit. On a beau constater "notre ferme croyance à la realitis objective, crovance que la critique métaphysique ne peut ébranler un soul moment. ") (3) si notre ferme croyances est illisgitime, il faut y renoncer, quelque ferme qu’plle soit. Que si on la croit légitime, c'est done la critique metaphysique qui a tort. Vouloir les concilier c'est travailfrer valn ef rest en outre déconsiderer la philosophie.

des emmaisaneres. le philosuphe franças sattache a faire voir uque la

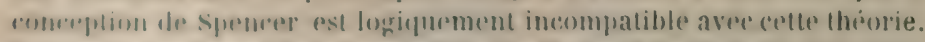

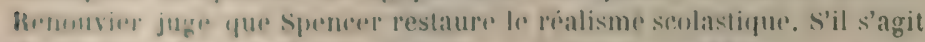

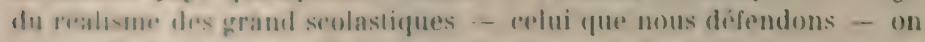
a fur se ronvanore qu'il n'a rion de commun avec la doctrine de l'Inommaissable.

(1) P. P. P. $0586-$ P. P. p. 66.

(2) Inal. Irans. I. II c. III s. IV Trad. Tissot. vol. II p. $214 \mathrm{siq}$.

18) P. P. P. 99 \& 2 B - F. P. p. 70. 
Spencer, dans le Post-Scriptum ajouté en 1900 à la Premiere Partie des Premiurs Principes, fait un nouveau et inutile effort pour se debarrasser de ce dilemme. "L'intulligence, dit-il, n'ètant organiscee que pour attrindre les phenomènes, elle nous enveloppe dans le non-sers lorsque nous essayons de nous en servil pour une chose situee au delà du phénomène. " (1) S’il ell est ainsi, dironsnous, nous devons nous garder de cet usage de notre intelligence, nous aurons soin de ne formuler aucune affirmation sur une telle chose et nous nous abstiendrons soigneustment de dire qu'elle exista. Rien ne sert d'ajouter que " far la nature meme de notre esprit nous sommes forces continuellement d'attribuer les effets que nous connaissons a une cause que nous ne commassons pas, de regarder les manifestations dont nous sommes conscients comme impliquant quelque chose de manifeste" ; que " nous trouvons impossible de penSer au monde comme constitue d'apparences et d'exclure toute idee d'une realite dont il y a des apparences. ") (2) Tout cela fù-il vrai qu'un moment de reflexion suffirait pour nous alvertir de notre erreur. Et si l'on pret-ndait que néanmoins la conviction de la realité demeurera dans l'esprit, nous nous trouverions done avoir deux convictions contratictoires. De quel droit prefèterons-nous la steconde à la première? Qute si nous n'en préférons aucune et que nous enseignons à la fois l'une et l'autre, rappetons-nous que dire des choses contradictoires revient a ne rien dire du tout....

Lorsquil s'agit d'expliquer la grenèses de la conception de l'illimite, spencere tombe dans une confusion qui nous fournit la clef de celle qu'il fait, nous lavons vu, entre le réel et l'infini.

(1) F. P. p. 94.

(2) Ibill. 
Autre chose est le concept dans lequel nous nions les limitess, c'est-i-dire le concept d'un être dont la réalité s'étend au delí. de toute limite; autre chose est la notion de l'êtr's dans laquelle on fait abstraction de toule limite ou de toute determination et qui n'est autre chosp que le concept indéterminé d'être en général. Ce dernisy peut s'appliquer à tout être quelconque, tandis que le premier ne se véritie que de l'Étre Intini et ne peut point s'appliquer aux ètres limités.

Quiconque confond la notion d'itre illimite avec la notion d'ètre pll général, ne distinguera pas davantage la réalité en général, d'avec l'existence réelle de l'être illimité ou absolu. Pour Spencer illimité est synonymé d'informe (1) et d'indéterminé et c'est encore une conséquence de la même confusion. L'Ètre Intini n'est en lui-mème ni informe ni indetermine, quoique la notion que nous en avons soit en grande partie négative of par conséfuent peu distincte. Il ne se confond pas avee toute réalite, quoiqu'il en soit lit source; mais il est par sa perfection mème distinct de tout ètre fini, sans que cette distinction entraine une limitation quelconque. Etant la "aust promière de tous les ètres, il possède pan lui-mìmr, d'une manièr: éminente, toutes les perfections qu'il leur a communiquées.

Ia notion abstraite d'ètre en greniral ne correspond a aucume realite determinere, mais s'applique dans son inclefermination a toute realite, linies ou intinis, all Createme (nu La crature. Or, e'est cette idce alstraite dont spencer exprose la genese dans l'esprit, au lien d'expliquer comment sy forme l'idere do l'Intini ou dre l'Alssolu. "Cettr conception, dit-il,.... ceest l'alsstrait de toutes les pensios, illexs on romerptions. Ce qui lene est commun it toutes,

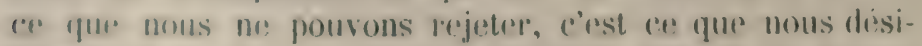
groms fate le mom commun d'existence. Separe de dhacun

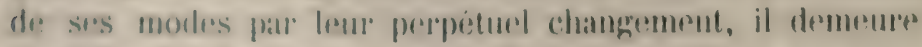
(r)m

(1) P. P. P. $100890-$ P. P. P. 70. 
constant sous tous les modes, une conception indéfinie de l'existence isolée de ses apparences. " (1)

C'est bien la notion générale et indèterminée d'ètre, et non pas la notion d'ètre alssolu (sans relation nécessaire) ou infini (sans limites). De cette notion gínérale pas plus que de n'importe qurelle autre notion abstraite, on ne peut tirer l'affirmation d'aucune réalité quelle qu'elle soit. La tentative de sprencer pour sortir du cercle des phénomènes ou apparences où il s'est enfermé échoue fatalement. La critique transcendentale conduit logiquement à l'idéalisme et celui-ci aboutit non seulenent à la négation de toute réaliti extérieure, ce que l'on a appelé le solipsisme, mais plutót à la nigation ou mieux au doute universel. (2)

(1) P. P. p, $101 \S 26-$ F. P. p. 71.

(2) ef. Balfotr. The Foundations of Belief. Iondres 1901 [p. $143 \mathrm{~s} 1$. 

CHAPITRE V.

\section{L'ACCORD DE LA RELIGION \& DE LA SCIENCE}

Notre connaissance de l'Absolu - Prétendue inconséquence de la Religion - L'évolution religieuse et l'influence de la Science - L'évolution scientifique et les idées religieuses - L'Inconnaissable.

Spencer a debute dans celte premiere partie de son ouvrage par affirmer l'existence d'un desaccord séculaire entre la Religion et la sicience et il a lait entrevoir la possibilité d'une réconciliation. Il s'est ensuite appliquè à applanir les voies en vue d'un rapprochement. Ce travail préparatoire consiste, nous l'avons vu, à battre en brèche les notions fondamentales tant scientifiques que religieuses, au point de vue de leur valeur objective. Spencer a prétendu nous faire voir que des contradictions insolubles naissent à chaque pas, si l'on considiere ces notions comme représentant la réalité. Ensuite il a trouvé la contirmation de cette conclusion clans la thèse drs la relativité de nos connaissances, d'après laquelle celles-ci n'atteignent pas la chose en soi. Cependant il ne s'est pas ralliè à l'idéalisme absolu qui est l'aboutissement logique de cette théorie, mais a prétendu justifier l'affirmation d'après laquelie il existe une réalité qui est la cause de 
tous les phénomènes et qui nous est elle-même complètement inconnue.

Gràce il cette inconséquence il a, croit-il, trouvé un terrain d'entente entre la Religion et la Science. Que la Sciencr renonce à considérer ses enseignements comme lexpression de la réalité et reconnaisse qu'ils ne c'ontcernent que le phénomène. Que la Religion maintienne l'affirmation d'une réalité fondamentale, mais aroue qu'elle est inconnaissable.

Balfour critique spirituellement ce partage qui abandonne à la science tout le connaissable sous prétexte qu'il n'est que relatif et laisse à la Religion l'Absolu, mais en le déclarant inconnaissable. (1) En réalité si les notions dernières de la Science et de la Religion sont inconcerables, les deux ordres de connaissances se trourent également compromis.

Yous arons tìchí de défendre les idées fondamentales de la science et de la Religion en montrant qu'elles ne sont pas contradictoires en elles-mèmes, comme on le jretend, et que rien n'empèche de les armettre comme expression de la réalité. Yous avons combattu la thèse relativiste et nous avons fait roir l'illogisme de la restriction que spencer pretend y apporter. Nous ne considerons done pas comme admissibles les termes de la reconciliation que spencer propose et dont nous avons nie la nécessité. Est-ce à dire qu'ils doivent être repoussés sans restriction? qu'il n'y a dans l'alfirmation qui doit, d'après spencer, rallier tous les suffrages, aucune part de veriti? C"est ce qui nous reste i examiner.

Si nous admettons que nos sensations contròlées par la mison permettent d'obtenir une ronnaissance du monde conforme a lat rialite, que notre conscience de nous-meimes aussi nous meet en rapport aver la chose en soi et non avec de purs phenonemes, nous sommes amenes a admettre des realities liniers, rontingentes, soumises an changement, ayant, en un mot, des camacteres opposés à ceux

(1) The Foundarions of Belief. p. $27 \%$ si. 
que nous attribuons a la Cause Premiere. Celle-ci est donc distincte de l'univers que nous habitons et dont nous faisons partie.

Aucunes de nos facultes cognitives n'atteint en lui-meme l'Etre Intini. Ia certitude que nous avous de sun pxistence est lo fruit du raisommement et la connaissance que nous arons de sa nature rest formée d'eliments empruntris aux êtres dont nous sommes entourés.

Or, il est évident, et tous les philosophess spiritualistres, depus Platon et Aristote, l'ont reconnu, que nous ne ponvons ansi acqueril do l'Ethr Intini qu'une connaissance extrèmement imparfatr. Combien diffirente doit itre sa nature de tout ce que nous royons! Dans les choses qui nous entourant, i'est un changement continuel; la vit nous apparait comme un perprotuel mourement; tandis que l'Etre fortui est alsolument immuahle farce que infini et parce que nécessaire.

Nous ne ponvons admettre en lui nulle compusition; son essencer absolument simple inquivaut cependant à lous les degres de perfoction qui en emanent.

Tout terme exprimant uns perfection qui se manifeste a notre experience, ne pent s'appliquer a Diru que frar analogie. Les commaissances les plus claires que nous avons de l'Etre Absolu consistent a niep les imperfections que nous apererevons dans les êtres limites. Mais lorsque nous voulons nous former une idfee positive de sa nature, nous constatons qu'elle est resouverte pour nous d'un voile impenetrable. Si on veut appeler cet Ètre l'Inconnaissable, nous n'y arons point de repurnance. "II habite, dit St-Paul, des splendeurs inaccessibles » (1).

Mais de meme que pal le raisomement nous comnaissons l'existence de l'Étre Intini, de mème nous ne pourons echapprer a la nécessite de lui refusfr tous les caracteres incompalibles arec celte intiniti; nous sommes forces de

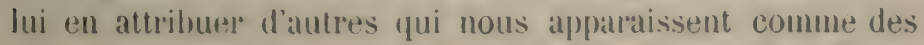
perfections pures, quoique d'ailleurs nous ignorions la

(1) Ire Ep. à Tim. VI. 16. 
manière propre dont elles s'y trouvent réalisées. Ne pouvonsnous pas affìmer que le Premier Ėtre possède l'intelligence, pourvu que nous n'entendions pas assimiler son intelligence a la nòtre? qu'il a cette indépendance que nous appelons lit liberte: quoique, encore une fois, sans doute, d'une autre facon que nous?

Lne certaine similitude, lointaine autant qu'on le veut, mais réelle, existe nécessairement entre la cause totale et l'effet qu'elle produit. Dès lor's, la connaissance des créatures doit ètre un mogen d'obtrnir au sujet du Createur des notions vraies quoique très-imparfaites. Proclamer ces notions, ce n'est point prétendre que notre connaissance de l'Intini est adéquate à son objet, ce n'est pas nier que cet objet dépasse notre entendement.

Prétendue inconséquence de la Religion.

Spencer en reconnaissant à la Religion le mérite d'avoir toujours maintenu la croyance en quelque chose de: superieur aux phénomènes et qui est élevé aut-dessus de nos connaissances, lui reproche d'ètre inconséquente avec elle-mème. "Elle a toujours, écrit-il, fait profession de posséder quelque connaissance de ce qui s’élève au-dessus de la connaissance, et par là elle a contredit st's propres enseignements. ") (1) Cette contradiction n'existe pas en réalité. Ciest plutòt Spencer qui se contredit lorsqu'il enseigne que nous ne pouvons rien connaitre an sujet de l'Infini et qu'en meme temps il en affirme l'existence et proclame qu'il est " un pouvoir (qui agit sur nous ) et (qui se manifeste par les phénomènes (2).

Tous pourrions faire remarfuer aussi que les religions ont generalement fait profession de tenir leurs enseignements d'une atutre sourer que de l'évolution maturelle de mos commassances. Ce point de vue qu'on ne peut flas nogliger dans me atude complete do cette question a rechappri a Spracer. Tous ne nous y arreterous pas.

(1) P. P. p. 107. $28 .-$ F. P. p. 71.

(8) P. P. p. $105827-$ F. P. p. 73. 
Il est très vrai que nous sommes exposés à ne pas nous contenter d'avoir au sujet de la Divinité des connaissances pleines de lacunes et d'incertitudes, et que voulant les préciser nous courons risque de concevoir J'Etre Infini sous des formes qui ne peuvent point lui convenir.

L'anthropomorphisme des Religions païennes est trop connu pour qu'il soit nécessaile d'en donner le detail. Platon s'élève avec véhémence cuntre ces conceptions et nous ne voyous pas qu'elles aient été admises dans aucune des écoles de philosophie de la Grices. "(Vand un poëte viendra nous farler ainsi des dieux, dit-il, nous refuserons avec indignation de l'nontrendre "(1) Toutes les fables qui rapportent les milamorphoses des dieux sont absurdes. "Dien est parfait avere tout ere qui tient it sa nature. Ainsi bieu est l'itre le moins susceptible de recevoir plusieurs formes. " (2)

Cela n'empeche pas que ciny sificles après Platon ces superstitions etaient encore universellement rejandues. Ce ne fut point la Philosophie mais bien le Christianisme qui les fit disparatre parmi les penples covilises, on sait au prix de quelles luttes.

Spencer reproche an outre ¿i "la Religeion " d'avoir" manqué de sincerite en continuant it drfendre des conceptions demontrees insoutenables. Ce reproche ust trop vague. Le manque de sincerite ne se congoit fas dans une chose abstrate ou dans me institution, mais seulement dans ceux qui la ropresentent. Et l'on peut sans doute fare ce reproche à ceux qui representaient le paganisme dans les premiers siecles de notre ère.

Mais ne peut-on pas imputer la meme faute et avec plus de raison à "la science") de ce temps, puisque ses représentants contredisaient par leur conduite religieuse leur's convictions et abusaient de leur autorite sous toutes ses formes pour maintenir la foule dans la superstition?

(1) Rémublique. Trad. Cocsis Paris $18: 33$ vol. IX p. $1 \geqslant 1$.

(2) Ibid p. 11 . 
Snencer fait encore un troisième reproche a la Religion, cest d'etre un peu sceptique: "Dans la foi la plus pieuse, dit-il, nous le voyons d'ordinaire, il y a un noỵa de scepticisme; et ce noyau de scepticisme est la cause de l'effroi qu'inspire à la Religion la science. Obligée d'abandonner une à une les superstitions qu'elle defendait autrefois opiniàtrement, et royant chaque jour ses plus chères croyances de plus en plus ébranlées, la Religrion laisse percer la crainte qu'un jour no vienne ou toutes les choses seront expliquées, rérélant ainsi qliau fond elle doute de l'incompréhersibilité réelle de la cruse incomprehensible dont elle a conscience. s) (1)

Si jamais on a pu craindre ou espererer qu'on était sur le point de "tout expliquer " ce n'est certes pas a notre époque. Le progris des sciences nous a rendus, sous ce rapport, modestes. Quant all Christianisme, il fait profession d'enseigner plusieurs choses impossibles à expliquer et il n'a aucune crainte ni aucun espoir de voir cette situation modifiée.

'évolution ligieuse et fluence de Science.
Sproncer esquisse en quelques traits l'evolution religieuse au point dr vue que nous envisagreons. Depuis le létichisme, pretend-il, jusquatax fornes les plus clevees de la resligion, la Cause promière a chi concue de plus en phus comme envoloppee de mystiere. "L'histoire religiruse n'est all fond que ta sirie des phases de la disparilion des dogmes positil's qui otaient le mystère du inystère. " (2)

Yous arouons ne pas reonnaitre cette loi dans l'histoire intinimont complexe des idees religienses. Si nous considerous l'evolution du Christianisme, nous voyons que ses dogrumes so sont, an contraire, précisés toujours davantage, surtunt prendanl les premirrs siectes de son existence. On

(1) P. P. p. p. 107, $108 \& 28-$ F. P. p. 78.

2) P. P. \%. $106826-$ F. P. P. 74. 
lui reproche plutot d'en avoir ajoute a son rnserignement ¿que d'en avoir abandonné. Cola ne l'empreche jas, a la vérite, de reconnatre que Dieu est l'atre immflahle et mysterieux far axellence. Mais si l'on voulait, à cet regard, considerer le Christianisme comme le terme d'une evolution, il resterait a montrer dans l'histoire des peuples un acheminement vers cefle ronception religipuse. Yous royons pour beaucoup) de raisons (pue ceest une entreppise impossible.

L'histoile de l'humantis n'est point, hidas: crelle d'un frogrès continu, moins encore en fait de religrion qu'a d'antres points de vire. C"est dare que nous ne saturions

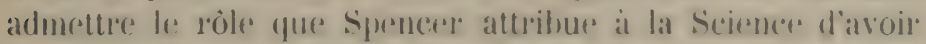

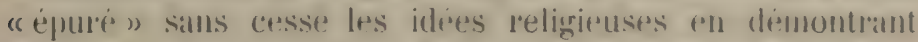
l'alsurditir des conceptions determineses de la Catuse P'remiere.

" Lat religgion ignore, dit-il, lat dette immense qu'tlle a

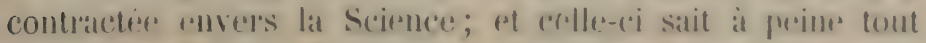

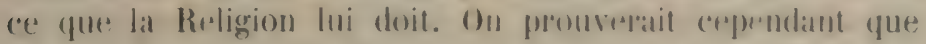
tous les dexpes de developpement parcomrus par la Religrion,

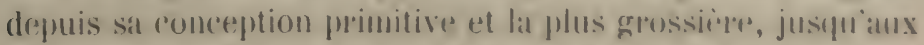
ideres relativement obrees qu'elle grofesse aujourd'hui, alle

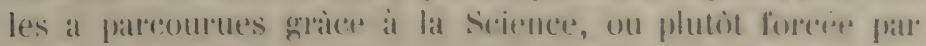
la sidence. De nos jours encore, lat science ne la presset-elle pas de silvancer dauss lo mente sens? ") (1)

La science dont-il s'apit ici doit atre principalement

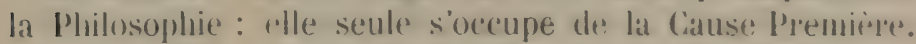
Il n'est, helas ! que trop evident que la Religgion ne doit a la Philosophie aucue reconnaissance. Lad Philosophie greçue s'est trouvée pendant des sircles en contradiction avec la religion reghante; qu'a-t-elle tenté pour la modifier et quelle influence a-t-elle exercè?

Si elle s'est trouver mieux d'accord avec le Christiatnisme, il serait cependant dillicile de dire en quoi elle a "épuré " la conception de la Divinité de cette religion; et si on parle de la philosophie contemporaine, y a-t-il, en dehors de l'école catholique, une métaphysique sur laquelle deux philosophes soicnt d'accord?

P. P. p. $108 \$ 29-$ F. P. p. 76. 
Le seul exemple que donne spencer de cette " épuIation " progressive que réalise la science n'est point emprunté ¿ la Philosophie et n'est pas très heureux. Le voici : "Autrefois on regardait le soleil comme le char d'un dieu; on le croyait trainé par des chevaux. Nous n'avons pas à rechercher jusqu'à quel point on idéalisait l'idée qu'on exprimait si grossièrement. Il suffit de remarquer qu'en expliquant ainsi le mourement apparent du soleil par une puissance semblable à des forces terrestres et risibles, on rabaissait une merveille de tous les jours au niveau des intelligences les plus vulgaires. Quand, plusieurs siècles après, Képler décourrit que les planètes tournent autour du soleil suivant des ellipses et qu'elles décrivent des aires égales en des temps égaux, il conclut que dans chaque planète il devait y avoir un esprit pour en guicler les mouvements. Nous royons par cet exemple comment les progrès de la science ont fait disparaitre l'idee d'une traction mécanique grossiere comme celle qui domait autrefois le mouvement au soleil; nous voyous ensuitr que lorsque à cette iclée grossière on substitua celle d'ume force indefinie et moins facile à concevoir, on crut encore necessaire de supposer qu'un agent personnel était la caust de: l'irregularité régulière du mouvement. Quand entin on prouva que les révolutions planefaires ave leurs variations et leurs pertubations obeissent a um loi universelle, quand les esprits directeurs congus par kippler furent mis de coté, et qu'à leur place on installa la forces de la gravitation, le changement fut en realite l'abolition d'une puissance qu'on pouvait se figurer et l'avènement d'une: puissance qu'on ne pouvait pas se tigurer. Car si la loi dre la gravitation tombe sous les prises de notre entemdiment, il est imposible de se faire une idee d'une forre de gravitation. Tewton lui-meme avouait que colte form est incomprehensible sans l'entremise d'un ether; mais mous avons vil que lhypothise do ret ither ne nous fait pas avancer d'un pas. " (1)

(1) P. P. P. $100-110 \$ 20-$ F. P. p. $76-77$. 
Il serait peut-ètre téméraire d'aftirmer que la conception dı soleil traint par des chevaux a éti jamais antre chose qu'une liction poètique. On peut dire que jamais les hommes n'ont doute de la rexgularitis de lat marche de cert astre. Si neammonins ils ont continue a le considiper comme le char d'un dien, c'est done que spencer a tort lorstupil écrit: "Quand l'expérimes ent pronves que certains changements familims ampirent toujomrs dams lat meme sucerssion, la comerption dime personnalite speciale dont lat volonte gouverne ces dhangements temdit a siefticer de l'esprit ») (1).

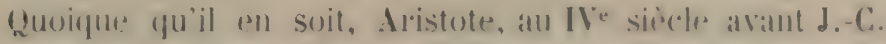

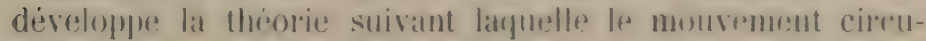

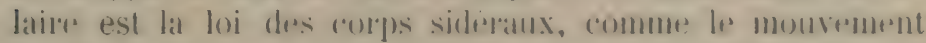
en ligne droite vers le rentre on vers la peripherio est

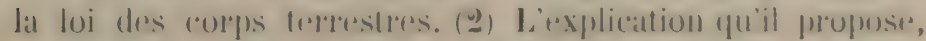

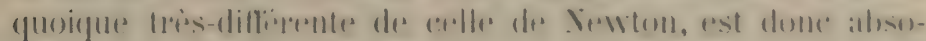

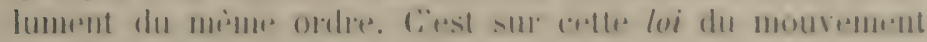

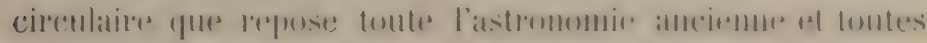

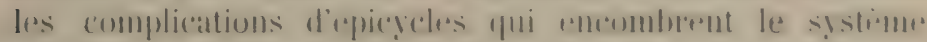

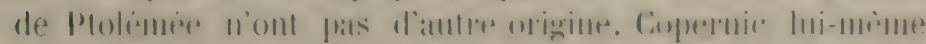

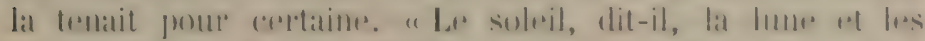

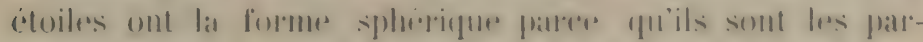

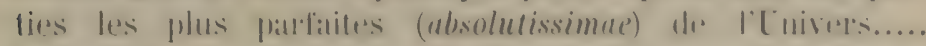

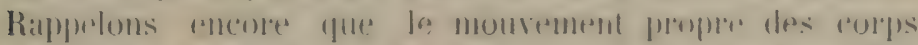

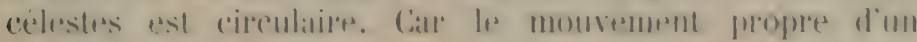

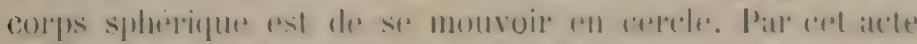

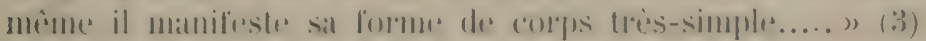

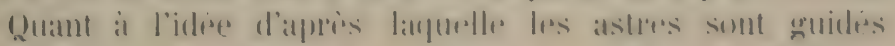
par des esprits, spencere an ighore, stmble-t-il, l'origines

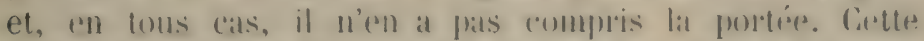

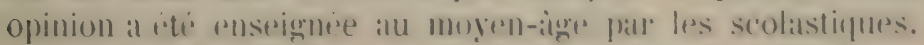

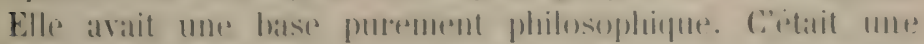

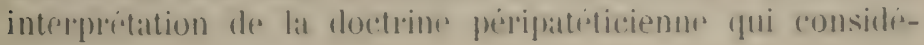

(1) P. P. p. $109 \$ 29-$ F. P. p. 76.

(2) IIspi oủpavòu, 1. 1. c. 11.

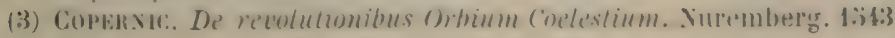
p.p. $1-2$. 
rait le Ciel comme un ètre vivant et lui attribuait une nature supirieure à celle des corps sublunaires. Elle n'empèchait nullement ses partisans d'adopter l'explication qu'Arislote donne de la forme des mouvements astraux.

Ce n'est pas la découverte de Newton mais plutòt l'hypothès copernicienne qui, ruinant de fond fon comble la phrsique d'Aristote, fit abandonner les théories qui s'y rattachaient.

Kejpler, loin d'avoir inventé la théorie des esprits directeurs, enseigne, dans son principal ouvage, que la cause des mouvements planétaires est une force résidant dans le soleil et soumise à des lois géométriques. (1)

II est vaai (qu'ailleurs, repoussant l'opinion de ceux qui enchàssent les astres dans des sphères solides de peur qu'ils ne s'écartent de leurs routes, Képler dit: "Admettons que le monde tout entier soit pourvu d'une àme qui entraine tout ce que nous royons d'étoiles et de comètes et cela avec la vitesse que requiert la distance au soleil et l'intensite de la lorce à cet endroit (et ibi fortitudo virtutis). Admettons encore que chacque planète a une àme particulière, grace à laupelle l'astre parcourt son orbite, et on aura le mème résultat tout en supprimant les sphipes. ") (2) Mais Kipler ne fait ici que se servir d'une idée courante. En outre, il met en note: i Entendez par àme du monde l'image immatérielle du soleril s'etendant comme la lumièr et vous aturez on deux mots le resume de ina physique céleste. " Cette itme et ces esprits n'etaient done plus pour lui que des métaphores.

Imuhern a montre comment la lheorie de Newton est sortir far une évolution continurelle des ideres d'Aristote of qu'elle ast en somme de ncime nature. Il observe que Kefpler a formuli une doctrine de la gravité qui se rap)proche brancoup de crelle du gramd astronome anglais. (l)

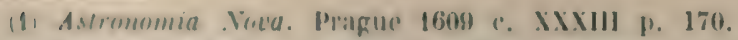

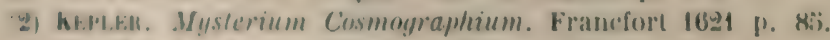

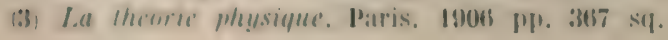


L'hypothise neivtoniemne constitue sans nul doute un grand progrès, mais au point de vue de la "concevabilité " elle est absolument sur la meme ligne que celle d'Aristote. Que les corps aient la propriete de s'attirer, oll de se diriger ver's un point central, ou de tourner' autour d'un point, l'une choses n'est ni plus ni moins inconcevathle que l'autre et l'on voit que le progrès scientitique n'est pas toujours un acheminement vers "l'inconcevatilite" des explications.

\section{olution} tifique et idées riouses.

La science - nous prenons ici re mot datns sat signiticattion générale - s'occupe de rechercher ce qu'on apprile les causes des phenomenes, r'est-ì-dire, les agronts yui déterminent rertaines rategories de fats, ou, si l'on veut, les lois auxquelles ils obeissent. Ces lois ou ces causes sont de plus en plus générales at mesure qu'elles stetemlent a un plus grand nomlre de phenomènes. Il en resulte une subordination on vertu de latquelle: certaines lois ne sont que des conséquences ou des cus particuliers de lois superieures, certaines causes no sont elles-memes que des manifestations d'une catust plus profonde et dont l'action est plus étendue.

Dans ses recherches, la seience va du comprosé au simple, du particulie"l an grineral. Elle voit d'abord les phénomènes, puis leurs causes immediatrs, puis seulement leurs causes lointaines. Elle a étudie les phènomènes du son et a tixé les lois qui les régissent, flle s'est appliquée aux phenomènes de la lumire et a rexonnu les lois qui les gouvernent, avant de savoir que les uns et les autres consistent dans un mouvement ondulatoire et obeissent, dès lors, à certaines lois communes.

Ainsi le progrès scientifique, lorsqu'il ne consiste pas simplement à substituer une interprétation meilleure à une autre du mème ordre, amène l'esprit à reconnaitre des causes ou des lois de plus en plus générales et, en ce sens, de plus en plus abstraites. 
Yais nous ne voyons pas de raison d'affirmer avec spencer que " dres causes de plus en plus abstraites sont des causes de plus en plus inconcevables, " (1) ni, par conserpuent, que " la conception la plus abstraite vers layuclle la science s'avance graduellement est celle qui se confond avec l'inconcevable et l'inintelligible, par suite de la suppression de tous les éléments concrets de la pensée. » (2) Nous avouons ne pas comprendre ce que c'est qu'une pensée abstraite à éléments concrets, ni comment une iclie abstraite est possible si seuls les éléments concrets jouissent de la concevabilité.

Voyons la justification historique de cette manière d'envisager le progrès scientitique. "A ses débuts, dit spencer, quand la science eut commencé à enseigner les relations constantes des phénomènes, et par suite discrédité la croyance aux personmalités distinctes quon regrardait comme leurs causes, elle leur substitua la croyance à des puissances causales qui, si elles n'etaient pas personnelles, etaient au moins concretes. Quand on disait (que: cretains faits montraient que lu nuture a horreur du vide, yuand on expliguait la propricté de l'or par une entite appesce l'aureité, quand on attribuait les phénomenes de la vie à un principe vitul, on établissait un morl. d'interpretation dlu fait, qui, s"il était en opposition avec le morle redigrieux, paree qu'il altribuait ce fait à d’autres puissances, n'en était pas moins inscientificye, parce fu’il faisait profession de comnaitre ee sur quoi rien n'ilat comnu. Lat science a abandomne ces puissances metaphy-

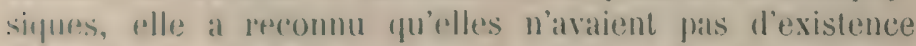
independante, gu'elles nétairat que des combinaisons touta-fail particulieres des auses génerales; en consépuence, clle a plus réromment attribue de vastes groupes de phe-

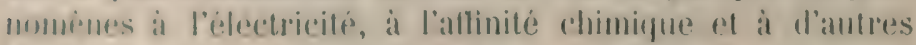

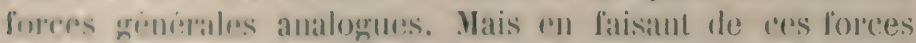

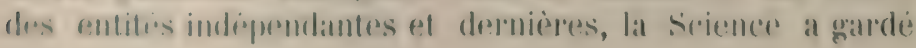

(1) P. P. p. 110 \& $20-$ F. P. P. 77.

(2) P. P. P. 110 \& $29-$ P. P. P. 77. 
en somme la mime attitude qu'auparavant. En explipuant ainsi tous les phinomines, y compris ceux de la vie et de la pensée, non sfulement elle a perseveri dans son antagonisme apparent avec la Religion, parce qu'elle a eu recours à des puissances d'une espèce radicalement dillërente, mais encore ello est resté inscientitique, parce qu'elle s'est donne sans le dire, l'air de savoir qurlque chose de la nature de ces puissances. A present, il est vrai, les savants les plus avancés abandonnent ('ps dernieres conceptions comme leurs predecesseurs avaient abandonné les fremières. I.e magnétisme, la chaleur, la lumiere, qu'on avait quelque temps regardes comme autant d'imponderables distincts, commencent anjourd'hui a n'etre plus pour les physiciens que des modes diflipents de manifestation d'une force unireaselle. Les physiciens cessent donc de se tigurer certte force romme romprobemsible. w (1)

Le passage gum nous venons de citro reflete les idtess

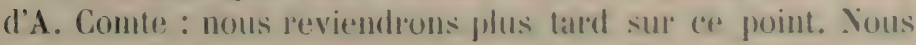

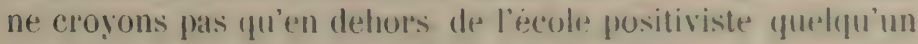
dit jamais considere les inleses seipulitipues, allsyurlles Spencer lat allusion, romme opposies aux idees religieuses. Il est evident que cette opposition n'existe pas; ce qui Ir prouve péremptoirement ce sont les comvictions repligienses de la plupart des savants yui ont realisé les progrès seientifiques.

l'autre part, l'électriciti, l'alfiniti chimicpur sont-elles plus ou moins inconcevables que l'horrerur du vide, l'aurité "t le principe vital, - alsstatation faite, bien entende, de la valeur scientitique de ces ronerptions? Et si tous les phenomènes matiolels sont. pour crtams physiciens, "les inodes diffients de manifestation d'une force unirerselle "), n'est-il pas varai que plusiens pretendent determiner la nature de cette force et disent qua exlle ast le mouvement (2) ou plus generalement qu'elles se riduit a des foress mecaniques? En quoi le monvement ou des foress attactives

(1) P. P. p. 111 \& $29-$ F. P. p. 78 .

12) Cf. SECCH. L'Unité des forces physiques. 
ef ripulsires sont-ils plus inconcerables que l'affinité chimique ou que l'horreur du vide?

"L'interpretation d'un phénomène, dit encore Spencer, ('st devernue meilleure lorsque, d'une part, elle a rejeté une cause relativement concevable daus sa nature, mais inconnue quant à l'ordre de ses actions, et que, d'autre part, elle en a adopté une connue quant à l'ordre de ses actions, mais relativement inconcevable dans sa nature » (1) Cette dernière formule est un peu mitigée mais elle n'est pas plus satisfaisante. Quand le système de Copernic prit la place du șistime de Ptolémée, quand la théorie des ondulations prévalut sur la lliéorie de l'émission, quand l'hypothèse atomique remplaça la doctrine des équivalents, l'interprétation des phenomènes devint incontestablement meilleure. Pourtant on se demande en vain en quoi la cause fulon a rejelée etait plus concevable dans sa nature yur colle yui lui a ste substitue, et pourynoi l'on pouvait dire yur la premiere ctait "inconnue quant à l'ordre de ses actions" taudis que la seconde ('st connue sous ce rapport.

s'il y a en une époqur où l'on attribuait tous les phinomenes a des agents surnaturels, c'est qualors la sirence n'existait pas. Lesprit seientificue consiste it attribuer les faits maturels aux agents naturels ce qui - rst-il besoin de le dire? - ne les soustrait pas a l'influence de la Cause Première.

liexistener d'evenements surnaturels - c'est-it-dire. dieterminis par les ayents surnaturels a l'exclusion des agents maturels - est une question de fait qu'aucun grincipe seientiligue me- permet de résoudse ni dans un sens ni dans l'autre. Spencer ne prejuge-t-il pas la question lorsigu"il berit: "A mesure que la science solive vers son apugrase, lous les faits imexplicatules of en apparence surmalurels rentront dans la catigorie des faits explicables at naturels n? (2) Puisque "en mime temps on anequiert

(1) P. P. P. 112 $30-$ F. P. p. 79.

(8) P. P. P. 118 \& $80-$ F. P. p. 79. 
la certitude que tous les faits explicables et naturels sont à leur origine première inexplicables et surnaturels ", (1) la question se réduira donc a savoir a quel point de l'espace et du temps se trouve cette origine. Car, rien ne nous dit que tous les faits ont une orighine simultanér.

Quant à la réalité de la Cause Première Intinie et Alsolue, elle n'est pas contestie par la science philusophique, du moins celle qui al lapprobation de spenceret la notre; mais all contraire elle y troure sa démonstration.

En principe il n'y a done aucune opposition pntre la Science et la Religion. II pourra y avoir contradiction entre la science et les dogmes que certaines religions proclament. C'est encore une fois une question de fait a examiner en detail et fu'on ne peut pas résoudre à priori.

Le progrès scientifique dans les derniers siectes at mème aupararant s'est surtout realise dans le domaine des sciences d'observation qui n'at guère de territoire commun avec les croyances religieuses, do sorte que tes contlits n'y sont pas beaucoup i craindre. Les allirmations que contiennent les dogmes religieux sont plutit sur le terrain philosophique. Yous arons dija dit re que nous pensons de la pretendue opposition entre la science philosophique et la Religion. La premiere condition pour qu'il y eùt contlit entre les enseignements de la Religion et ceux de la Philosophie serait que les philosophes eussent une doctrine commune qu'on pùt proposer comme l'enseignement de cette science. Or', c'est précisement ce qui leur fait le plus défaut, surtout à notre eporgue.

Il peut y avoir et il y a contlit entre certains dogmes religieux et certains philosophes, mais non point entre la Religion et la Philosophie. II nous est impossible de voir dans l'histoire des idées ni "que la Religion a toujour's étí forcee par la science d'abandonner l'un après l'autre ses dogmes ", (2) ni que la sicience ait été

(1) Ibid.

(2) P. P. p. 111\$30. Ce passage a étés supprimé dans l'édition de 1900. 
"pressée.... par la critique de la Religion qui mettait souvent en question ses hypothèses " et ainsi " obligée de renoncer aux efforts qu'elle avait faits pour enfermer l'inconnaissable dans les limites de la connaissance positive. » (2)

nconnaissable.

Toute cette theorie de l'antagonisme entre la Religion et la sicience et de leur épuration réciproque est une construction qui manque de bases objectives.

Yon point certes qu'on ne puisse affirmer, ce qui est la thèse de Spencer, l'accord fondamental de l'une et de l'autre. C'est notre conviction que, recherchant les causes, la Science aboutit logiquement a la Cause Première et rejoint ainsi la Religion. Yous croyons que cet accord ne consiste flas uniquement en ce que l'une et l'autre reconnaissent un mystere au fond des choses. Lne telle aftirmation est si vague que l'accord qui en résulte est à peine plus que negatif. Yous croyons que, sans dissiper le mystere, la Religion enseigne sur la Cause Première certaines choses que la science approuve et d'autres qu'elle ne contredit point.

Pas plus que spencer nous he pouvons admettre qu'il $y$ ait une obligation morale de proclamer un Dieu personnel et infini s'il a été demontré d'ailleurs qu'un tel objet est de toutes manières hors des atteintes de notre esprit. Spencer, nous l'arons ru, se séparant en cela de Mansel ot de Hamilton qu'il avait suivis jusqu'alor's, tient que nous attrignons l'Intini atu moyen d'une conscience indéfinie.

Qu'i I'Etre Infini et Absolu on ne prisse pas appliquer sans restriction des dénominations empruntees aux choses qui nous entourent, c'est a que les theologiens ont enseigne de tous tomps, ainsi que nous l'avons fait remarquer. IIs are feront aucume difficulte pour approuser spencer lorspu'il exrit : "lans l'idere qu'on se fait d'une cause

(1) Shid. 
ultime, on ne reste pas pris dans une altcrnative embarrassante, on la depasse. Crox qui s'arritent a cette alternative supposent a tort qu'il faut choisir entre une frersonmalité et quelque chose de moins qu'une personnalité: tandis que c'est entre une persomnalite et quelque chose de superieur qu'il faut choisir. Ve peut-il pas y avoir un mode d'existence aussi superieur à l'Intelligence et il la Volonte, que ces modes sont supérieurs an monvement mécanique? Nous sommes, il est vaci, incapables de concevoir ce morle supérieur d'existence. Mais ce n'est pas une raison de le révoquer en doute; cest bien plutot le contraire.... Nen résulte-t-il fas que la cause ultime ne. peut en rien dite conçue par nous, parce qu'plle est, en tout, plus que ce pui perut ètre conşu? Fit, par consépuent, n'avons-nous fas raison de nous garder de lui assigner des attributs, qurels qu'ils soient, patr le motif qure ces attributs, dérives comme ils le sont de notre frepure nature, ne l'élèvent pas, mais la ravalent?» (1)

Dans l'Ecole on cite volontions le texte da josudoDenys (2) décharant qu'il laut phutot nier qu'alfirmer de Dien les predicats juste, sage, bon, ete. si on leur attribue une signification identique à celle: qu’ils ont lorspurils sont appliqués aux ètres linis; et cela non pas parce que l'ktre Infini manque de cers perfoctions, mais parce qu'il les dépasse. Or, c'est précisément dans cre dernir'l sens que nous employons ces attributs. "Nous commaissons au sujet de Dieu, dit $s$. Thomas d'Aquin, (3) son existence et ce qui lui convient nécessitrement comme Cause Premiere dipassant tous ses effets. Yous comnaissons done de lui certe relation aux creatures, qu'il est leur cause à toutes, et la difference entro les créatures et lui, c'est-iidire, qu'il n'est lui-mème rien de ce qu'il a causé, et on doit nier de lui ces choses non point par defatut, mais parce qu'il le's dépasse (quia superexcedit). »

Des qu'on affirme l'existence d'une chose on est bien

(1) P. P. p. $116 \$ 31-$ F. P, p. 80.

(2) Calor. Hieraich. c. II.

(3) Sum. Theol. p. I. 1. XII a. 12. Mlan. I878 vol. I col. 702. 
oblig̣t de la déterminer par certains caractères, sinon latfirmation de son existence n'a plus aucune signification. spencer lui-mème, comme nous l'avons déjà fait remar'puer plusieur's fois, admet que l'Être Intini est absolu et cause première des phénomènes. Or ce dernier caractere comporte une relation de causalité; et puisque d'après Spencer absolu signifie: qui n'a pas de relation necessaire, il suit donc que l'Ètre Intini, cause première des phénomènes, n’en est pas nécessairement cause. Que disons-nous de plus lorsque nous lui attribuons la tibertè? Et la liberté se concoit-elle sans intelligence ou sans une perfection plus haute que l'intelligence et analogue? Nous disons done que l'Être Intini est personnel en ce sens qu'il n'est point une cause aveugle ou fatale, mais sans vouloir le moins du monde rabaisser sa personnalité à la nôtre.

Tout comme spencer nous pepoussons les conceptions anthropomorphiques de la Divinité. Si l'on prite à Dieu des sentiments de rexret, de colère on d'autres émotions, ce ne peut être légitimement que par métaphore. La chose signifibe par ces images n'est autre que les eflets de la puissance divine qui sont semblables à ceux determines par ces sentiments chez l'homme. Toute attribution qui implique la sucerssion, le changement, ou une imperfection quelconque ne peut avoir a l'égard de l'Ètre Intini que la valeur d'une tigure de langage. Mass la liberte et l'intelligence ne doivent pas ètre rangées dans cette catégorie. II est vai que lat maniere dont ces activités s'exercent dans l'homme emporte des changements et des imperfeotions; mais ces imperfactions ne leur sont point essentielles, et si nous ne commaissons pas d'ume fagon fositive lat forme qu'elles revitent en Dieu, du moins nous prouvons par voir de négation éarter les défectuosites de l'intelligences of de lat volonte humainess et attribuer avec virite à lleu une connaissance sans vicissitudes et sans lormes ot mor volonte parlate, independante et immuable.

Dans un chappitre des Institutions Ecclesiastiques intitule: 
Passé el avenir de la Religion, (1) Spencer rofuss? d'attribuer a Diesu la volontes et l'intelligences. "Celui yni conroit, dit-il, une volonté autre que la siemne la congeroit on fonction de la sienne, la seule yu’il comnaisses, puistue, toutes les autres ne sont pour lui que des thrses l'indurtion. Mais la volonté, telle que chatque homme la peregoit en lui-même, suppos" un motif. un désil moteur d'un certain genre. » (2)

Sous ne pouvons en elliet ancevoir la rolonte divine que par analogie avec la notre, ('e qui n'muphedur pas que nous rien ieartions les imperfections qui caractripisent cette dernière. Parmi cess imprerlections, il fall sigonaler laction qu'exerce sur la volontes humaine, par l'intermediatres de l'intrlligences, to but qui meut, qui altirr. Pour lat volonte divine, te but est un simph. terme, (3) ce qui exchut on ellet lout "desilp " et en ce sens implique "l'indifference " mais n'exclut pas la destrmaination de la volonté.

"De plus, ajoute spencer " la volonté, en tant gu'elle implique un désir motenr, connote quelqun tin ronsideréte comme but a atteindre, et cesse d'othe des quar le but ast

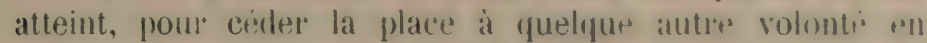
relation avec une autre fin. C'est-it-dipe que la volonte. comme l'émotion, suppose nécessairement une strite d'actes de conscience. L'ides d'une volonte divine, derives de l'idée d'une volonte humainr, impliquas: comme crolle-ci une localisation dans l'espace et le, temps. La volontio d'une lin exclut de la conscience, pour un instamt, la volonté d'autres fins, ce qui la rend incompatible avec une activite omniprésente travaillant en mème temps à reaslisty une infìnité de fins » (4).

Ce que spencer décrit est bien ce qui sir passo dans

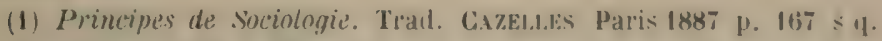

(2) Ibid. p. $206 \& 638$.

(3) ef. S. Thomas. Suma Théol. 1. 1 11. 19 a :3. Milan 187 vol. I col. 787.

(4) Ibid. 
l'homme. Mais cela est-il essentiel à la volonté comme telle? Ine volonte infiniment parfaite veut à la fois toutes les fins qu'clle a décidé de réaliser. Qu'importe que ces fins se réalisent successivement en dehors d'elle? En elle il n'y a aucune succession, on ne peut y distinguer ni passé ni futur. La volonté éternelle est une et simple et en vertu de cet acte unique, la série indéfinie de ses effets se déroule dans le temps, sans cesse.... Nous ne voyons pas que cette conception contienne quelque chose de contradictoire qui nous empèche de l'adopter.

"L'intelligence, nous dit ensuite spencer, la seule que nous puissions conceroir, suppose des ètres indépendants et objectifs pour elle. L'intelligence se compose de changements dont le point de depart est une force extérieure, c'est-it-dire d'implessions engendrés par des choses existant en dehor's de la conscience et d'idfes dérivees dr ces impressions. Parler d'une intelligence qui existe en l"alsencer de ces forces exterieures, e'est employer un mot denue de sems. Il faut conclure que la cause fremière, considérére comme intellignente, doit subir sans "थsis l'impression des forees objectives et independantes;

- el si lon objecteque ces lorees sont drenenues objectives et inclipendantes par un acte de creation, et quavant cet acte elles retaient renfermées dans la cause premiere, on pent repondre que, dans ce cas, la cause première ne pouvait, avant cette creation, rien rencontrer qui rngendrat en olle les changrenents qui constituent l'intelligenere, et par conséfuent

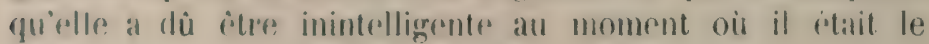
plus neressaire quelle fiot intelligente. Il est done evident quer l'intelligence attribuee à Dien ne répond a rien de

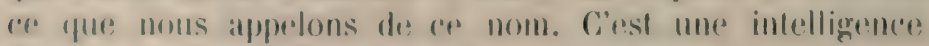
virlere de tout ce qui constilue l'intelligences s) (1).

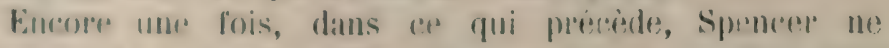

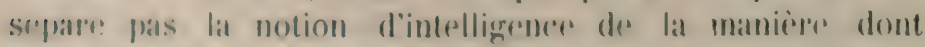

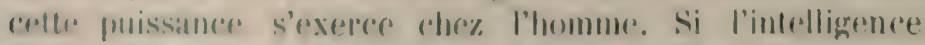

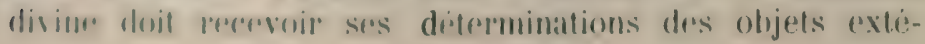


rieurs de mème que l'esprit humain, il est bien clair qu'elle en drpend, qu'elle ne preut lrur être anterirure et que Dient manfurarait d'intelligences au moment ou il en aturait to plus hesoin, e'est-it-lire, atu moment oil cess oljets doivent etre croves par lui. La science de l'homme est en effert produite soit directement, soit indirectement, par l'action que les oljets matritels exerrent sur ses organes. Mais rien n'emprohe de conceroir unn comnaissance des choses qui soit indejendinte de ces impressions. Qu'est-ce done qui defond de se representer la sciemere divine comme existant par celle-mene en vertu de l'inlinie perfection de l'ktres Afsolu? Il y a priscisiduent entue

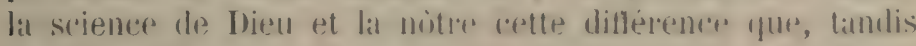

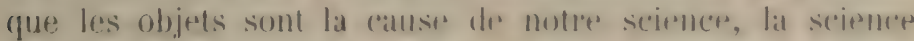
de Dien all rontraire est la callse de oljepts. (l) II est

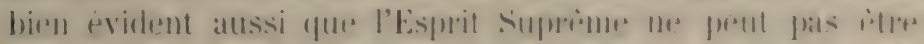

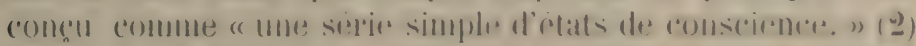
Lacte de l'Intelligremes divine est uniqque; matis anmme

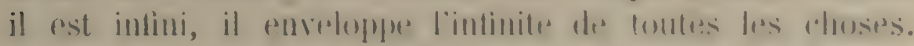

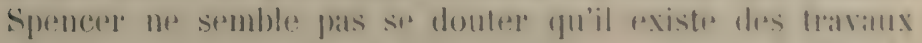

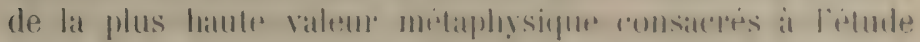

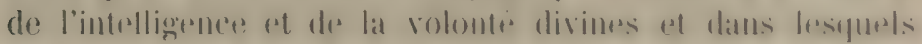

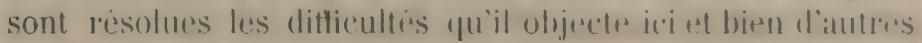
plus redoulables. Il est rexpertable gure le philosophe

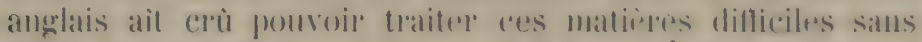

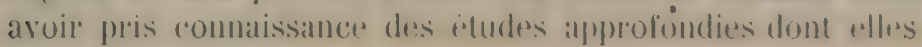
ont fait l'objet.

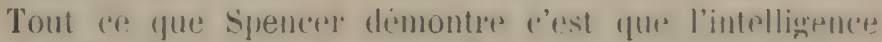
et la volonte n'existent pas en Ident alve les imperfections qui les canacterisent cher l'lumme. A ce sujet il me prent pas $y$ avoir de doute. Dess lors, d'apres la doctrine expresse des philosophes spiritualistes que nous avons rapportée, si par intelligences et volonti ont entend des facultés telles qu'elles sont rialisees an nous, il liaut les

1) S. Thonas, Summa Theol. 1. I (1. 11 a. 8 Milan 1878 vol I col 738 .

(2) H. Splicen. Essais. Trad. Burdean. Paris 1879 t. III p. 33:3. 
nier de Dieu plutot que de les affirmer. Mais puisque aussi bien les perfections divines sont quelque chose de plus élevé et qui contient en les depassant toutes les pripogatives des itres finis, nous pouvons, sams erreur et faute de mieux, appliquer à Dieu les dénominations empruntées à ce qu'il y a de plus élevé dans les créatures, les imperfections qui s'y rencontrent n'étant d'ailleurs pas nécessairement impliquées dans ces dénominations.

Spencer ne donne-t-il pas à l'Ètre Infini le nom de Force? "Il est une virite, dit-il, qui doit devenir toujours plus limineuse : c'est (qu'il existe un Être inscrutable plartout manifeste, dont on ne peut conceroir le commencement ni la fin. Au milicu des mystères qui deviennent d'autant plus obscurs qu'on les fouille plus mofondement prar la pensér, se dresse une certitude ahsolue, à savoir que nous sommes toujours en présence de la Force intinie et éternelle, d'ou prociedent toutes choses. " (1)

Comment spencer justifiera--il cette maniere de parler si ce llest de la façon dont nous-mème l'avons fait quand nous avons appele Dieu : Intelligence et Volonte?

si nous fouvions croire qu'ainsi nous sommes d'accord avec sprencer, il ne nous resterait qu'a demander pourquoi crtle doctrine nous est proposie comme la conséfuence des cliapitres précédents.

Spencer accuse les théologiens de manquer "d'humilité » quand ils pretendent porter la lumière dans les mystères iusondables do la Divinité et rabaissent Diru à notre mesure. II peut y avoir du vari dans ce reproche et certainement l'anteur dont il cite des extraits le merite. Mais il n'est pas moins ivident qu'un Ètre réllement inconnaissable, de la miture, de la prensée, de la volonté duquel nous n'avons ancune idlee ne peut pas itre l'objet d'une religion froprement dite. (2) Il ne faut donc point décourager les

(1) Principes de Sociologie. ibid. p. $21:$ \& 860.

'. CI. Vonizkamb. Het Agnosticisme tan H. Spencer Assen 1896.

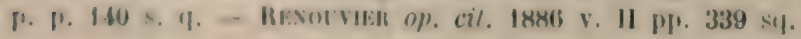


eforts de l'intellgence modeste qui chrorche is s'alphnocher du terme final de toute investigation philosophique at ne point oublier que les thrologirens pretendent avoir pour guides dans leurs recherches des lumires qui ure sont point empruntées à la raison, Si c’est la rérite ou illusion

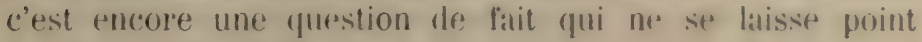
écarter par une fin de non-recevoir.

In des plus illustres disciples de larwin et son rollaborateur, J. G. Romanes, quoinut professant en philosophie de's tendancers agnostipurs, erolit: " 11 m'at tomjours semblè que la doctrine: de I'Incommaissable en tant quelle dilliele de la doctrine de l'Incomm est hautement antiphilosuphigure.

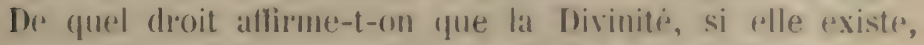
ne perut pas demain reveler le lait de son existences, et colat d'une farcon indubitablo - a toute lat race humaine? Ou, s'il y a un Dien, qui peut dire arrec certitude qu’il n’y a pas une vie future dans langelle tout individu aurad la presuse evidente du theisme? Ciest mes attitude philosophique legitime de pretendre quadetuedlement on ne voit ancune presure du theisme ; mais dire que certainement la ratce humaine n'aura jamais cette preture test une attitude très antiphilosophi(que »(1).

si Dien peut réveler demain, ajoutons-nous, il a pu le faire hier" c'est donc une question it examiner.

Les dernieres fages du chapitre yue nous etudions et qui est lui-mims le dernier de la première partie dos Premiers Principes - developpent une sorte de conclusion pratique: Le's formes de religion qui se sont succedie's sont des etapes vers la Religion de l'inconnaissable. Ce progres ne doit point ètre brusque sous peine d'enlever a l'homme une religrion qu'il comprend pour lui en presenter une qu'il est incapable de comprendre et qui n'cxercera donc aucune influence sur sa vie. La tolérance doit ètre la riggle, mais aussi la liberté ou le devoir pour celui qui est en possession de la virite plus pure de contribuer, en la faisant connaitre, à l'évolution religieuse.

(1) Mind, Motion and Monism. Londres 1896 [. 117. 
Il est cert:in, dirons-nous, qu'une intelligence peu dévelop)pée s'assimile diflicilement les idées abstraites et qu'il est souvent nécessaire, Jorsqu'on veut les faire saisir par les enfants ou les foules, de les incarner, pour ainsi dire, dans quelque symbole qui s'adresse à l'imagination. Mais il ne faudrait point en conclure que tous ces symboles ont la mème valeur, ni surtout que toutes les idées qu'ils enveloppent sont également vraies.

Le mogrès religieux ne consiste donc pas seulement a s'élever du symbole à l'idée abstraite, mais encore à échanger un symbole contre un autre plus convenable, et surtout à remplacer l'rreur par la vérité - qu'elles soient conçues d'une manière abstraite ou sous forme symbolique.

$I$ cet equard il ne faut puint aflirmer que " d'une manière generale, la religion resue a une époume et chez un peuple domne a toujours ate l'explession la plus rapprochres de la virite que ce mime feruple, a cette eqpoque, etait (aprable de conceroir. " (1) Tous les degrés de civilisation yue l'histoire commait sont complatibles arec des idées fansses arec des idees vales tho matiere religieuse conme m toutr autre matiere. Et comme la Religion a l'influence lat plus considerable sur loute la vie de l'homme, (\% stra toujours un grand service à lui rendre que de rombattre l'errem religireuse pour lui substiturer la verité. Mais pour se chatrger de cottr mission, il faut itre sir de pouroir distinguer entre la virite et l'mereur ; et l'homme: morleste en voudra sans doute aroir dans ces matienes dilliriles d'antres garanties que sa contance dans les sperulations metaphysiques anxquelles il s'est livré.

1. P. P. P. 1:3 \$ :32-F. P. P. Xit. 


\section{PARTIE \\ LA THÉORIE UE L'EVOLCTION}

C:HAPITIE I

\section{LES DONNÉES}

Le connaissable. - L'évolution comme objet de la philosophie. - La vérité. - Le moi et le non-moi. - L'espace et le temps. - La matière. - Le mouvement. La force.

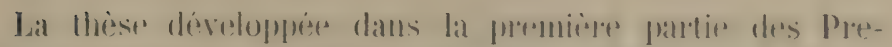
miers Principes, quoigu'clle contienne l'allirmation d'une

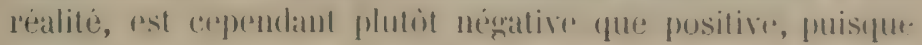
cette realite nous ast domme comme the pouvant idre conmur. Le connaissable, conséguemment, n'est point rexpl mais seulement phénoménal.

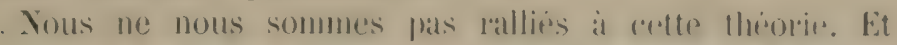
perut-itre le lecterur jugereat-il qu'un dissentiment si fondatmental rend impossible doremavant toute entente sur un objel queleongue des specoulations philosophingues. En un sems il en est ainsi. Mluelle que soit, an eflet, lat chose

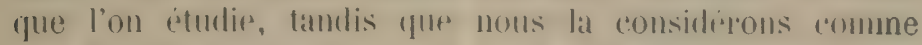
une réalite existant hors dre nous et indépendante de no-

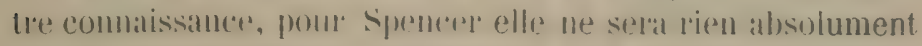
(qu'une representation manifestat une realite inconnur. 
Et il semble hien qu'il ne faille pas raisonner de même dans les deux hypothèses. Cependant il n'est point impossible d'étudier l'Univer's que nos connaissances représentent et de découvrir les lois suivant lesquelles les phénomènes s'ordonnent dans le temps et dans l'espace, en faisant abstraction de leur réalité ou du moins sans résoudre explicitement cette question. On pourra toujours, lorsqu'il le faudra, signaler les divergences qui résultent du point de vue différent auquel on s'est placé. " C'est à tort, dit R. Ferro, que quelques uns ont considéré la partie négative des Premiers Principes comme la quintessence de la philosophie spencérienne; la doctrine de l'inconnaissable n'en est pas mène une partie essentielle, mais forme seulement une espèce.d'introduction. " (1)

0. Gaupp énonce le mime jugement (2) et il semble bien que ce soit la manière de voir de spencer lui-mème. " Les questions dont nous allons nous occuper, dit-il dans le Post-scriptum qu'il a ajouté à la Première Partie, sont independantros des questions discutees jusqu’a présent; el le lecteur peut rejeter rn tout ou en partie ce qui precede et rester néanmoins libre d'accepter en tout ou en partie ce qui va suivre.......

"Une description de: la Transformation des Choses telle qu'elle est donnir dans les pages qui suivent est simplement une relation ordonnee des faits, et l'interpretation de corux-ci n'est autre chose que la constatation des conformites ultimes qu'ils présentent, des lois auxquelles ils obrissent. Le lecteur est-il athee? L'exposition de ces faits et le ces lois n'apportera pas de confirmation à son opunion et ne la détruira pas davantage. Est-il pan-

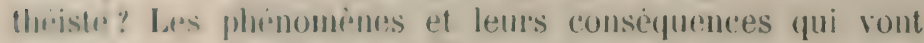
inre decrits ne lui imposeront rien qui implique le rejet de relle: opinion. Pronsert-il (que: Dien est immanent en foules dooses depuis la concentration des nebuleuses jus-

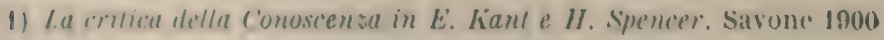
p. 62 .

18) Herberl sipencer, Stultgart 1900 p. 75 . 
qu'aux pensées des poètes? La theorie que nous allons développer ne contient aucune desapprobation de cette manière de voir. Croit-il à l'existence d'une Divinité qui a donné des lois immuables à l'Lnivers? Il ne trouvera rien qui ne s'accorde avec sa crovance dans une exposition de ces lois et une description de leurs effets. „ (1)

Néanmoins il résulte de notre désaccord avec spencer que nous ne pouvons pas considerer les phénomines qui nous entourent comme constituant à eux seuls tout le connaissable, et nous n'avons pas dès lor's conservé à cette Seconde Partie le titre : Le Connaissable que Spencer lui a donné.

Nous pouvons donc suivre spencer dans celte itude, adopter ou repousser les résultats auxquels il aboutit, sans que, en general, nos conclusions soient influencres par la clésapprobation que nous avous prononcée sur la therore de l'Inconnaissable. Plus d'unt fois on retrousered celle-ci dans ce qui nous leste à examiner et nous aurons parfois l'occasion de confirmer ere que nous avons dit déjà. En général, cependant, nous ne reviendrons pas sur les considérations emises et le lecteur voudra bien s'y rapporter.

La conception que spencer developpe dans crite seconde partie est grandiose et originale. Après avoir ètabli quelques notions préliminaires, il etudie les principres genéraux qui dominent l'Lnivers et less ramène à un seul: la persistance de la Force. Ensuite, constatant que l'histoire de tout itre est comprise entre son commencement et sa fin et se compose d'une série de changrements (l'evolution) qui se termine par sa destruction (la dissolution), il cherche quelle est la loi générale de ce procès. Entin il tente de faire voir que les lois de l'évolution et de la dissolution sont une conséquence de la persistance de la Force. 'Tous les phénomènes de l'Univers et l'évolution de l'Univers lui-mème se trouvent ainsi

(1) F. P. p. 98. Post-script to Part I. 
ramenes a un principe unique, ee qui est le but de la Philosophie.

\section{'évolution} mme objet de la hilosophie.

Les proyrès des sciences d'observation ont attiré davantagre l'attention de l'homme sur cetle vérité : que chaque étre parcourt durant son existence une série d’états successil's depuis son origine jusqüa sa destruction. Cette évolution se manifeste avec le plus d'évidence chez les itres vivants qui, avant d'atteindre la forme arlulte, traversent me longue suite de formes embryonmaires, qui, mène à l'àge adulte, se transforment encore d'une manière sensible, passent par la jeunesse, par l’àge mùr, par la vieillesse, jusqu'a ce que la mort les lasse rentrer dans le règne inorganique.

En réalite aucume partie de l'Lnirers n'est à labri du elangement. Toutes, justuatax cristatux les plus durs, après avoir pris maissance par le jeu des lonces materielles, sont soumises à des modilications perttètre insensibles mais reelles qui aboutissent finalement i leur destruction. Yous savons que l'histoire de notre golobe rablise igalemont ce programme et qu'il en ast ainsi de tous les astres qui roulent dans le ciel.

Il ny a pas non plus de diflerence essentielle sous ce rapport entre les arures de.la nature el les arures de l'homme. Comme les premieres, celles-ei ont ume histoire

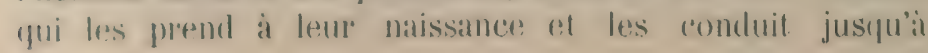
leur ruine.

C.r diest pas seulrement pour les individus que l'évolulion existre, mais encore pour les grompes d'individus soit qu'ils se surerident dans be templs, soil yu’ils se rémissent

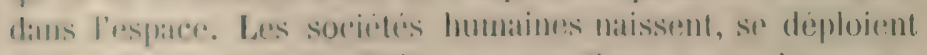

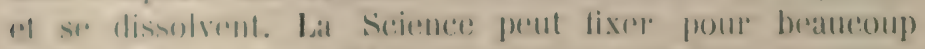

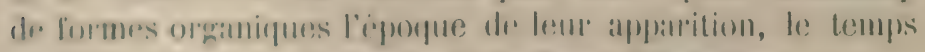

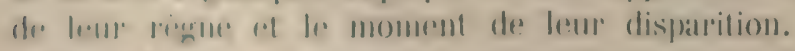

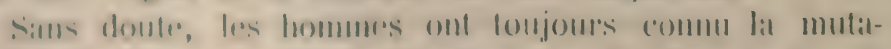

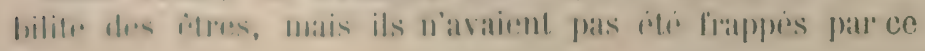


fait que tous, tant qu'ils sont, individus ou grompenents, fareourent une carriere plus ou moins complexp at ils

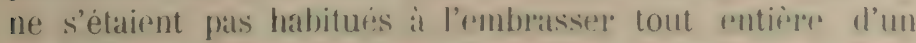
coup d'eeil. L'evolution des itre vivants etait considerente autrefois comme se réduisant it la croissinnere on at lat

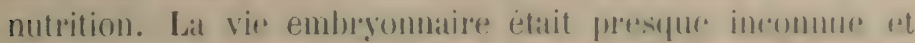
l'on n'avait pas remartpue que si pour beancoup d'orrat-

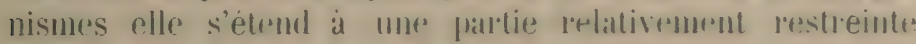
de lenr rxistence, pour d'antres c'est le rontratre yqui so

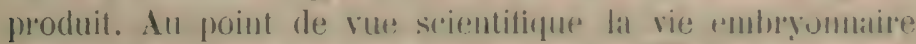

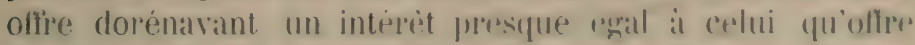

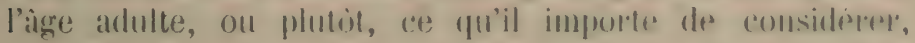

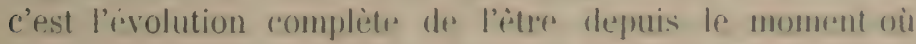

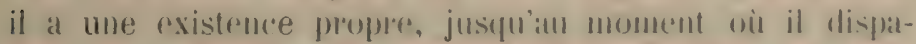
rait. Eit il en est des ethes collectifs romme des fores individuels.

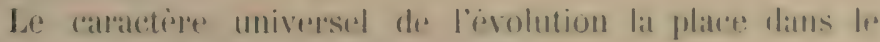
domaine de la metaphysique.

Lia philosophie comme l'expose spencere dams le premien

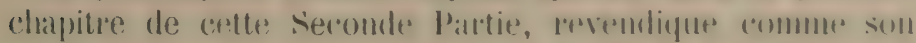
objet propure les prineipes yui se veritient a la lovis pour

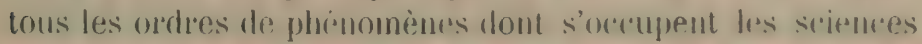
particulières. Elle est, dans la mesure dlu pussible, lat

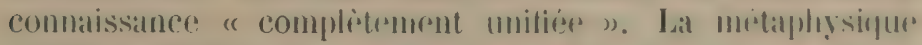
génerale etudie les vérités les flus hautes en ellesmimes, tandis que la metaphysigue spexdiald compormol l'interpratation des principaux grompes de phrmomimes all moyren de ces verites. Cotte detinition comprems, sinon la philosophie toute entiere, du moins tout lo domaines de la philosophie réelle.

Rien to permet d'allimer a priori gute les canses de l'évolution sont partout les mimes, ni qu'plles prourent ditres lamenees a une cause grinerale. Mats quelque diffremtes qu'on les suppose, il est possible on mime probable,

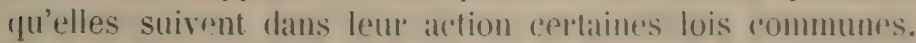
Il appartient au philosophe de les rechercher.

L'éturle de l'evolution applartient lone sitns aucun doute à la Philosophie, et méme, si l'on songe (fur c'est par les 
changements auxquels elles sont soumises que les choses acquierent leur nature et leurs propriétés, cette étude a une portée qui s'étend à la philosophie tout entière des êtres qui sont l'objet de notre expérience.

D'autre part une étude qui envisage toutes les formes de l'évolution doit se proposer uniquement comme but de découvrir des lois très générales.

La description d'un groupe d'animaux ou de plantes comprend des caractères d'autant plus universels et par lia mème plus simples qu'il s'agit d'un groupe plus vaste. La présence de ces caractires dans tous les groupes n'empèche pas la présence d'autres caractères moins universels qui introduisent dans ce groupe des subdivisions, lesquelles vont se subdivisant elles-mèmes jusqu'à l'individu. Celui-ci se distingue également de tous les autres par certaines notes. dinsi en est-il des lois qui gouvernent les phènomènes. Si l'érolution d'une société politique et celle d'une socirté commerciale suivent certaines lois communes, chacune cependant a en outre des lois particulieres de diveloppement. Celles-ci ne contredisent pas les lois générales et mème leur sont subordonnées; elles n'en sont pas pourtant de simples corollaires, mais resultent de la nature propre de chaque societe. A plus forte rason, les lois très génerales qui s’appliquent a tous les genres d'evolution ne peuvent pas prétendre les gouverner à elles seules, a l'exclusion des lois particulières.

Cette remarque est de la plus haute importance, et si Syencer ne la fat pas ici c'est qu'plle se justifie d'ellemime. Il écrit ailleurs : "Nous ne devons pas nous attendre à ce que la loi generale du progrès (de l'evolution) nous fomrnisse me explication immediate le telle ou telle forme dre fmogres fort pru semblables entre elles : elant t’n rapplort avec des ordies de faits tres divers, elle n'a de rapport particulise aves aucun orde de faits sprecial $)(1)$.

En outre, il importe de pemarquer quee les lois, quelles

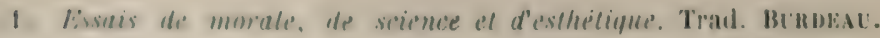
Paris 1699 p. 12. 
qu'elles soient, générales ou particulières, ne suffisent pas pour expliquer les phénomènes dans leur forme dèterminée. II faut tenir compte des agents et des circonstances. In problème de mécanique n'est point détini si l'on connait les lois suivant lesquelles agissent les uns sur les autres les êtres materiels; il faut en outre dire quels sont les corps particuliers que le problèmr envisage, quel est lour nombre, leur situation, leur masse, les mouvements dont ils sont animés, les forces extérieures à l'action desquelles ils sont soumis. Pour decrire les mouvements des planètes, il ne sullit pas de connaitre la loi de la gravitation qui est commune a tous les systèmes, mais il faut en outre savoir les masses relatives du soleil et des planètes, le nombre de celles-ci, leur situation a un moment donné et en général tous les elements caractéristiques du système solaire.

L'histoire interieure d'une socièté ne dépend pas seulement des lois qui fixent les droits et les devoirs de chaque classe de citoyens, mais encore, - indépendamment de la liberté, - du nombre et de la qualité des hommes que chaque classe comprend et toutes les circonstances dans lesquelles ils agissent.

Ainsi en est-il, d'une manière gènèrale, dans la question qui nous occupe. La marche des phénomènes qui constituent une évolution quelconque est déterminée non pas uniquement par les lois qui la régissent, mais bien aussi par les éléments qui y interviennent et surtout par la situation que l'on prend comme point de départ.

Néanmoins, dans les phénomènes, les lois sont ce qu'il y a de plus général et de relativement immuable. Elles sont par excellence l'objet de l'étude scientifique, parce que seules elles confèrent à la science ce caractère d'universalité et de nécessité qui est son apanage.

Avant d'étudier l'érolution en elle-mème, il est indispensable de considerer les éléments qui y interviennent et les lois auxquelles ils se conforment. Tel sera l'objet de ce chapitre et du suivant. Nous allons dorénavant nous trouver fréquemment d'accord avec Spencer. Lorsque 
nous n'approurerons pas ses idies, nous aurons tonjours soin, comme nous l'avons fait jusqu'ici, de justifier notre appreciation et d'opposel à l'opinion de Spencer celle que nous croyons deroir prefèrer. Quant aux doctrines que nous adoptrions, nous ticherons de les confirmer de notre mirux et de les compléter, si possible. Nous n'avons pas pu, dans ce dernier cas, eviter toujours un ('ntrelacement de nos propres idées avec les enseignements de spencer. L'inconvénient qui en résulte est minime et la lecture des Premiers Principes suffirait en tous cas pour y remédier.

Avant d'ahorder l'examen des éléments de l'évolution, spencer commence par proposer des notions de la vérité et de la distinction entre le monde objectif et subjectif qu'il nous faut brièvement examiner.

On apprelle commumément vruic la comnaissance qui alfirmo ce qui est ou nit' re qui n'est pas, fausse all contraire celle qui affirme ce qui n'est pas ou nie ce qui est. Le hon sens fait donc consister la verrite dans la conformiti: entre la connaissance prise objectivement et la réalité: adaequatio intellectus et rei.

si l'on professe que nos connaissances n'atteignent pas la rabliti, cette definition de la verite doit ètre abandonnor. "Exclus, dit spencer, comme nous le sommes de tont re qui dépasse le relatif, la verite, dans sa forme ba plus obrere, ne peut etre pour nous rien de plus que la concordance parfaite dans tout le clamp de notre experirner, entre les reprisentations des choses que nous appeloms inleales at les presentationsdes choses que nous appelons rorelles. si, quand nous decouvgons qu’une propositon n'est fas vatire, nous voulons dire tout simplement que nous avous deconvert une diflerence entere une chose atterduce

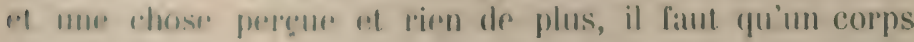
de romelusions, dans lequel il ne se mresente jamais 


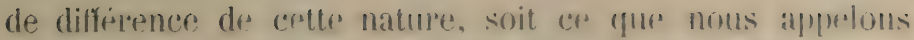
un corps de conclusions entirement vais. ") (I)

Il ne faut point oublier gule si on fait cousistrs lit vénité d'une proposition illéale dans sal concondance alréc une exprepience, e'est farce quon suppose qur l'rexprience manifeste la roalite. Si les " presentations de aboses. que nous appelons reelles ") nre manifestent riful de reel, toute raison d'apperler vales les representations qui sont d'accord avec elles disparaît.

En outre, autre chose est lat fausseté d'unf proposition,

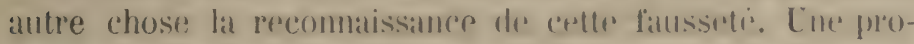

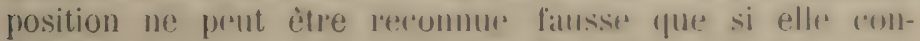
tredit une autre comaissance de l'esprit, mais rient l'mul-

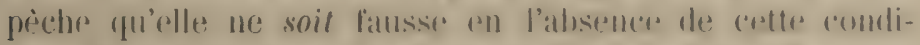

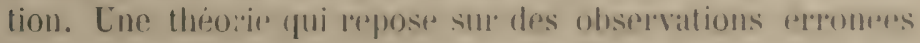
forme un corps de doctrine " vil it he: se flresente atu-

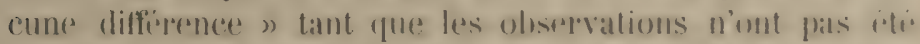

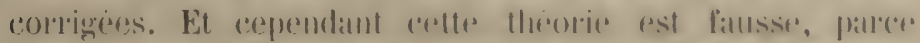
qu'elle neest pas d'accord anere la réslitr. On dima perut-

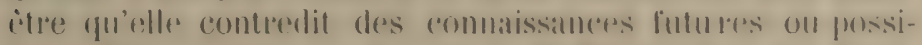

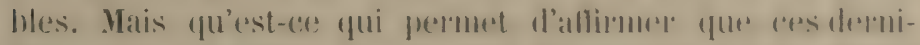

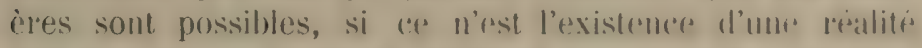
avec larmolle les premienes observations ne sont pas d'aceorel?

La détinition de la virite que domme Kant, plus ggennlable (pue celle de spencere, n'échappe pas a la mime critique : "Laceord avee les lois de l'entendement, dit-il, constitue le formel de toute veriti. ") (2) Vais procistment les lois de l'entendement reposent sul l'hỵpothrise de la réalité des objets que l'entendement repuresemte.

Cie qu’il faut accorder is spencere c'rst qu'une proposition n'est jugée fausse gut lorspu'elle contredit une autre proposition qu'on juge vrair, et qu'au contratre, toute proposition est jugée vraie qui non semlentent ne: ronIredit pas mais s'accorde positivement avec une autre connaissance admise comme telle.

(1) P. P. p. $146 \S 40$ - F. P. p. 109.

(2) Dialect. transcendant. Introd. Ed. Tissort. vol. II. P. y. 
Spencer fait remarquer, non sans raison, que nos connaissances les plus générales se supposent souvent les unes les autres, de sorte qu'il est difficile d'en retenir une en faisant abstraction du reste et qu'on s'expose, lorsquon veut les justifier, à commettre un cercle vicieux en s'appuyant sur des propositions qui contienuent déjà implicitement celle qu'on veut démontrer. Il est donc préférable d'admettre d'abord provisoirement ces connaissances générales. Elles se trouveront justifièes par les conclusions auxquelles elles mènent et qui ne sont jamais démenties par l'expérience ni contradictoires entre elles.

On pourrait ajouter que ces affirmations générales: il existe quelque chose; il existe plusieurs choses; certaines choses different et d'autres se ressemblent, et ainsi de suite, s'imposent à l'esprit comme absolument évidentes et qu'il est inutile de chercher par des analyses subtiles ou des raisonnements à obtenir plus que l'évidence complète.

L'evidence de la verité d'une proposition n'est point autre chose que l'évidence de la proposition elle-mème; et puisqur, en fin de compte, l'évidence est, dans nos connaissances, le résultat au delà duquel on ne peut rien espérer, des qu'une proposition se manifeste comme évidente, il est absurde de se demander s'il est bien sùr qu'elle exprime la vérite. L'analyse doit plutòt s'attacher ¿̀ demêler dans la conscience ce qui est évident de ce qui ne l'est pas et à l'rxprimer convenablement.

Parmi ces postulats provisoires, se trouve, d'apris spencer la veracite du "lemoignage que rend la conscience lor'squielle alfirme que cortains de: ses etats sont semblables ou dissemblables » (1).

Spencerer ratend par états de conscience toute notion d'un objel. Diss lors, exprimer que des ptats de conscience sont sumblables on dissemblables c'est formuler un jugroment allirmatif on negatif. si ces jugements ne contiennemt que ce quee la consciences voit, on less appelle

(1) P. P. p. $147 \$ 11$ F. P. P. 110. 
évidents. Que leur vérite doit être admise sans qu'il soit nécessaire ni possible de la démontrer, c'est précisément ce que nous venous de dire. "On ne peut pas prouver qu'elle (la conscience) est mensongère en ceci qui est son acte primordial, puisque.... la preure implique une acceptation redoublée de cet acte primordial» (1).

moi on-moi.

Une des premières et des plus fondannentales différences que la conscience affirme est celle du moi et du non-moi, du sujet et de l'objet.

Cette distinction, d'après Spencer, est hasée sur l'existence d'une double classe d'etats de conscience, les uns forts, les autres faibles. Les premiers sont les sensations proprement dites, les autres sont désignées par Spencer sous le nom d'idées. Cette dernière catégorie comprend donc toutes les comnaissances qui ne sont pas des sensations; elle réunit et mème confond des choses fort dillërentes: les representations concrètes de l'imagination, les concepts alstraits, les jugements. L'épithete faible convient bien aux perceptions imaginatives qui ne sont que la reproduction diminuée de sensations précédentes; mais il n'y a pas de raison de l'appliquer aux idées alstraites ni aux jugrments. Aussi sont-ce principalement legs premières que spencer a en vue.

Entre les deux catiggries il decourre des différences qu'il expose longuement et résume comme suit : 1. "Les manifestations de l'un (des ordres) sont vives et celles de l'autre sont faibles. » 2. «Celles de l'un (les manifestations d'un des deux ordres) sont des originaux, tandis que celles de l'autre sont des copies." 3. "Les premières forment entre elles une sèrie ou un courant héterogène qui n'est jamais interrompu, ou, pour parler avec rigueur, dont on ne comnait jamais directement l'interruption. " 4. "Celles du premier ordre adhèrent entre elles

(1) P. P. p. $148 \$ 41-$ F. P. p. 110. 
non stulement dans le sens longitudinal, mais aussi dans le transrersal; celles du second s'missent de la mème manière. " "̈. "Ces adhérences sont indissolubles pour It premine ordere, mais pour le second elles sont pour la plupart très laciles à rompre." 6. "Tandis que les bromes de chaque serie, les parties de chaque rourant, sont unis par des adherences si intimes que lo courant ne pent se diviser, les deux comrants grlissant en réalité cote a cote ne contractent que de faible adherences: le frind comant vif prisiste absolument au faible ot celui-ci peut prespue s'isoler du vif. ” $\bar{T}$. Les conditions sons lespuelles les manifestations de chacun des deux ordres se mésentent appartiennent elles-mèmes à cet ordre; mais si dans l'ordre faible les conditions sont toujours presentes, dans l'ordre vif il arrives sourent que les conditions ne le sont pas, et qu'elles sont quelque part en dehors de la serie. Sept caractires distincts servent done. de signe a ces deux ordros de manifestations et les distinguent l'un de l'autre. » (1)

Quoique ces dillirences suffisent en géneral pour classer. une pererption dans l'une des deux eatignories, elles ne sont ceppendant pas, mime réunies, tellement nettes que ce classement puisse toujours se faire sans incroptitude. Les caractires if et $t i$ sont des ressemblances mutre les deux series phutot que des differences; de meme le?

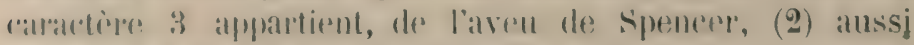

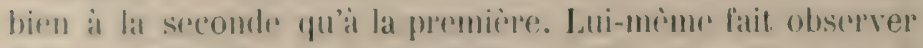

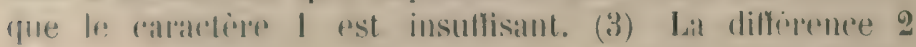

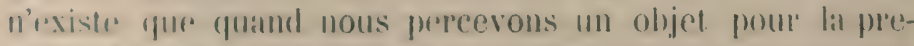

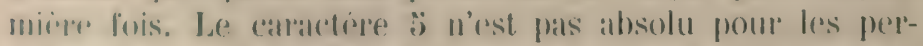

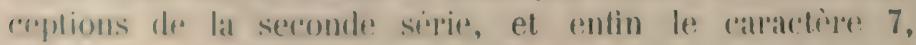
qui mil le principal, ne se verpitie pas toujours on ce qui

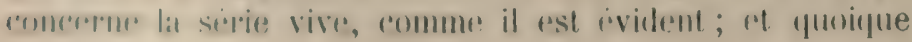

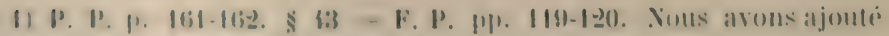
les mumiros au texte de spencer.

(2) P. P. p. ISS \& $43-$ F. P. p. $11: 3$

18) P. P. P. $189848-$ F. P. P. 113 


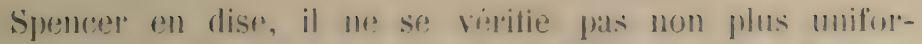

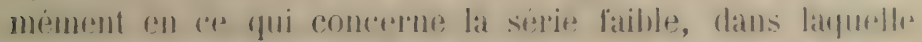
surgisistal triss somvent des illées dom il nous ast impossible de trouver l'origine.

lad division n'est fats nette at antte ladison sullit pumb gue nous ne ba confondions fals arrec la distinction entres

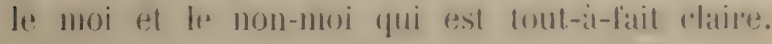

I) aillours, que signitie exartement l'allimation qu' "s en employant les mots moi th non-moi mous voulons dire,

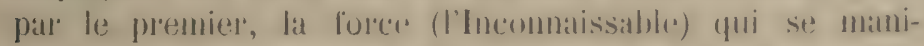

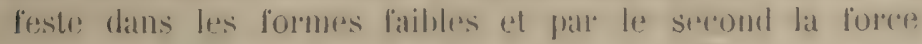
qui se manileste dans les lonmes viress s? Toute commais-

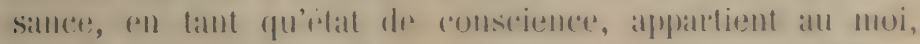

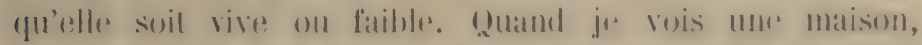

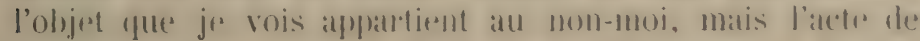

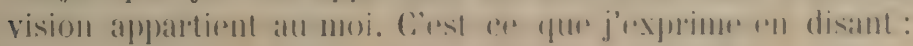
Je vois.

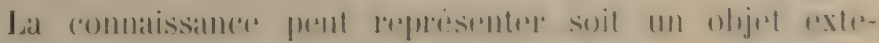

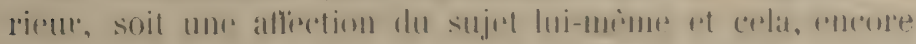

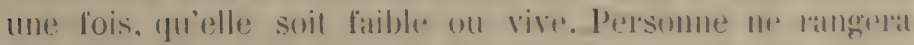

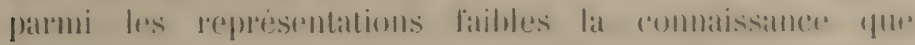

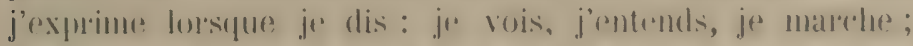
et rependant lexe ohjet ast les moj; l'on range all contraire parmi les representations faibles le sourenir que je conserve d'un palysitger, quoiplue, datss ce cas, l'objet appartienne au non-moi.

Lil distinction "mter le moi et le non-moi ast evidente, et lon pent on geminal separer mos representations en fortes of en lahihles; mais il nous ext impossible d'admeltre que ces deux divisiuns so rorrespondent.

espace Jes etres dont nous avons l'expriritures sont des ibles a temps. materiels; leur évolution implingur des mourements determines par des forres; ello sacromplit dans l'esface el daus le temps. Spences considere done un chapitre it tixer la signitication qu'il faut altalder anx mots: espace, temps, matiere, mourement, force. 011 st 
rappelle que notre auteur à prétendu, dans la Première Partie, montrer l'inconcevabilité des objets qu'ils désignent. " J'ai montré, dit-il, que nous ne connaissons rien de ces formes considérées en elles-mêmes. Cependant comme nous sommes obligés de continuer à employer les mots qui leur servent de signes, il est néanmoins nécessaire de dire le sens que nous leur donnons. ") (1) Le sens d'un mot, c'est précisément la chose dont ce mot est le signe. Si nous ne connaissons rien au sujet de cette chose, comment expliquerons-nous le sens du mot? Nous touchons encore une fois du doigt la contradiction qui se trouve au fond de la théorie de l'Inconnaissable.

Spencer emploie encore inutilement quelques pages à la mettre d'accord avec le bon sens. Elle n'empèche pas, prétend-il, de dire que les objets de nos connaissances sont réels. "Que veut dire le mot réel ?.... Par réalité nous entendons persistance dans la conscience: une persistance, ou bien inconditionnelle comme l'intuition de l'espace, ou bien conditionnelle comme l'intuition d'un corps que nous tenons à la main. Ce qui distingue le ciel tel que nous le concevons, c'est le caractère de la persistance; e'est par ce caractère que nous le sejparons de ce que nous appelons non-reel. Nous distinguons une personne placée devant nous de: l'ideje de cette personne, parce que nous pouvons écarter l'idée de la conscience, tandis que nous ne pouvons pas (') écarter la personne quand nous la regardons. ") (2)

H fatudra d'apres cela juger non-rrelle la personme: dont nous llavons qu' " une idere", un souvenir que nous pourons écarter de la consciencre ; une chose ne sera reelle yuiatant qu'elle persiste dans la conscience, elle cossera d'etre refelle dis qu'elle n'y persiste plus! Nous ne combesterons pas que ce ne soient la des conséquences ligitimes du phénoménalisme, mais ce n'en sont pats moins des propositions absurdes.

(1) P. P. p. 166 \$ $45-$ F. P. p. 122.

(2) P. P. P. 100) $10-$ F. P. p. $125^{\circ}$. 
Spencer nous le savons repousse la theorie kantienne qui lait de l'espace et du temps des formes subjectives de la sensibilité.

Nous arons dejà cité le passage où il definit le temps comme la conception abstraite de toutes les séquences, et l'espace comme la conception alostraite de toutes le's coexistences. Pourvi qu'on comprenne que, dans cette dernière détinition, il s'agit des coexistences d'itres étendus, on peut admettre ces notions.

Spencer pense que notre perception de l'etendue est dépendante du sens musculaire. C'est par l'experrience de tensions musculaires adaptées it la résistance qu'ollirent les contour's de l'objet et d'autres mouvements adlaptes à l'étendue que nait en nous cette perception. Ainsi l'intuition de l'espace aurait son origine dans des experiences de force (il vaudrait mieux dire d'effort).

Cela ne doit pas itre admis sans restriction. Il ne faut point confondre le sens musculaire avece te toucher. si je repose la main sur un objet sans liare aucun effort musculaire, je pergois ta surface etrmulue de cet ohjet par le seul toucher.

La vue aussi perçoit les oljets itendus, du moins en longueur et en largeur sprencer (1) pretend à la verité que lat vue ne pergoit l'étendue qu'en connexion avec des expreriences de mouvement; mais il conlond a notre avis, l'apuréciation des distances et des dimansions qui dépend probablement de ces expériences, alvec la perception de l'tendue. L'image visuelle, comme l'impression de contact, est etendue indéfendamment de toute association. D'autre part, il faut réconnaitre que l'experience des mouvements exceutés par le sujet sentant semble nécessaire pour acquerir la connaissance d'une étendur a trois dimensions qui est, en somme, l'espace víritable.

N'importe quels mouvements conscients de nos membres sont capables de nous donner cette perception. II n'est

(1) Principes de psychologie. Trad. Riвot el Espixas. Paris 1873 vol. II p. $171 \S 326$. 
pas necessaire pour expliquer sa genese de recourir at des eflorts qui rencontrant un obstacle extericur nous rensegrnent sur l'étendue résistante des corps solides. Ia notion d'espace sera donc liée a l'experience de l'effort nécrssaire pour mouvoir nos membres, mais non pas nicrssirement a lisperience d'une force de risistance exterirure. Entin, de cette comnexion d'origine on ne peut jas disduire que la notion d'rspace a pour objet un effort ou mor lorer. Ia représentation yue nous en avons actuellement n'eveille plus an nous aucune image semblable, mais seulement des images visuelles ou tactiles. Puisque foeil peroit des objets situes en dehors les uns les autres, l'imagination poura representer des objets distincts daus des plans diffients, une fois acyuise la notion de profondeur, ef cest hien ainsi gu'actuellement nous royons les objets.

Spremere suppose dams la suite que lidere de temps a

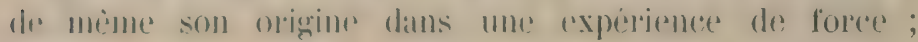

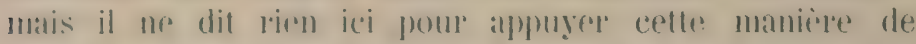
voir. Ia conception abstraite de toutes less síquences ou

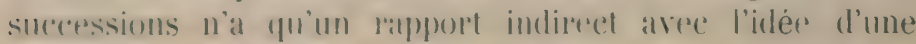

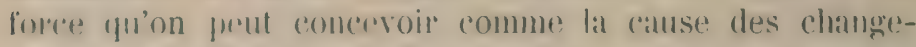
ments impliques daus eres siquences. Dans les Principes de Psychologie (1) Spencer insiste sur l'union étroite yui

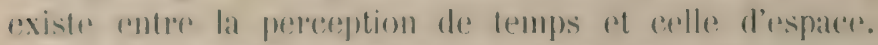

Ces demx nofions sont reptainement tres roisines, mais nous me rovons ceppendant pas de mason d'admellee yue la premiere dejend de la seconde. Tous les dhangements nor sont fas frerens par nous comme de's momvements.

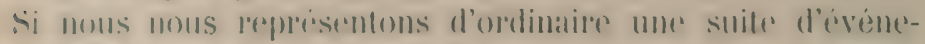
ments comme mor sipe d'objots allignes dans l'esplace.

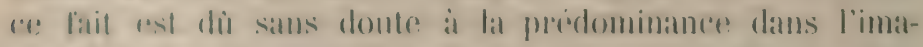
grimation des images visuelles, mais on atuat fort den

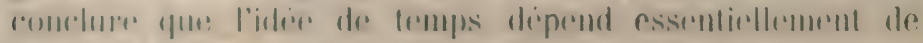

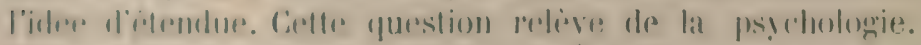

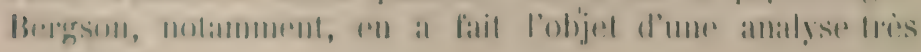


subtile. (1) Il nous sultit ici d'avoir rappelé, d'accord, en somme, avec spencer, que l'espace n'est pas autre chose que l'itendue des corps congue d'une maniripr abstraite, et que le temps dans son acception propre? est la durée des changements continus qui s'y produisent.

atière. "Nous concevons la matièr", dit spencer, conme des positions coexistantes qui opposent de la resistance ; c'est l'illec la plus simple que nous puissions nous en fare; nous la distinguons ansi de notre conception de l'espatces dans larpuelle les positions coexistantes noblirent aucune? résistance. Nons concevons le corps comme hornes far des surfaces rui résistent et comme composés rutiopentent de parties qui résistent. ” (2)

On attribue parfois it la matiere l'impénétrabilité comme caractiere rssentiel et l'idie qu'on s'en lat "st sans doute tres voisine de ce que spencep appelle ici la résistance. Nous prefórons cente derniere dénomination parre qu'elle constate le fait sans impliquer aucune throrie métaphysique.

Des deux caractires essentiels de la matiere, l'etendue et la resistance, lequel faut-il considerer comme primordial? spencer prélend que e'est la résistance, flarce que, dit-il, "les experriences d'ou nous tirons par abstraction notre conception de l'espare ne nous vienment que des impressions do résistance produites sur l'organisme. " (3) Nous venons de dire ce que nous persions de cette atlirmation. Mais fût-il vaai que nous comaissons l'ètendup secondairement, il ne s'ensuivrait pas encore que la résistance est réellement l'attribut primordial de la matière.

Si par résistance on entend l'impénétrabilité proprement

(1) Essai sur les dommés immidiale's de la Conscience. Paris linoti pp. $69 \mathrm{sq}$.

(2) P. P. p. $178 \$ 18 .-$ F. P. p. 129.

(3) P. P. p. $176 \& 48,-$ F. P. p. 130. 
dite, c'est-à-dire l'impossibilite de faire pénétrer un corps dans l'espace actuellement occupe par un autre, on peut soutenir que cette proprieté n'est pas différente de l'utendue concrite. (1) $\mathrm{Si}$, al contraire, l'on veut signitier la répulsion que les corps exercent les uns sur les autres et en rertu de laquelle ils se maintiennent toujours a dislance reelle quoique sourent insensible, ce qui est probahlement le fait, l'exercice de cette force suppose l'existence (lu corjs auquel elle appartient et sa présence à tel endroit. Ainsi considérée la résistance serait done dans la réalité posteriemre à l'étendue.

spencer se represente la matière comme composic "d'atomes étendus et résistants" et admet "un éther universel composé de molécules». (2) C'est, on s'en souvient, la conception incime que nous avons défendue contre les critiques de spencer dans la premire partie de ce travail. Il est rmai que lá elle est presentee comme une rialite, tandis qu'ici elle est "la manilestation d'un mode de l'Inconnaissable uni a la matière par la relation de cause a reflet \%. (3) Mais comment les contradictions qu'on pretendait y decourrir disparaissent-elles par sa transposition de la realiti dans le phemomène? Spencer objectait que si les unites rqui composent la matiore sont etendues, elles seront incompuressibles at gu’alors cla loi de continuite dont la négatlion est inconcevable sera violie dans lons les cas de collision ». (6) Est-ce-pue cettr difficulte n'existe plus pour la matier-phenomene? Les idees de la loi de continuite, de collision, do rompressibilite noont-elles pas pour objet des fhrmonnines? Et, dis lors, le raisonmement fout entier ne sapppliquer-til pas an momble phemominat? Nestere point

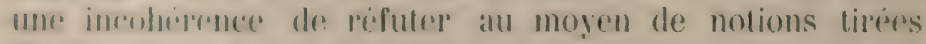

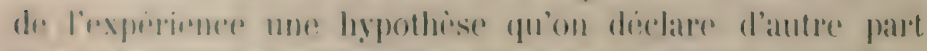

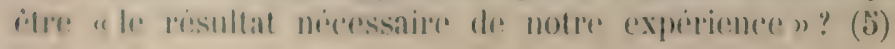

(1) Ci. Brikison op. cil p. 613-197.

(2) P. P. p. $177 \& 48-$ F. P. pp. 131.

(3) Ibid.

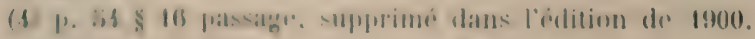

(B) P. P. P. $177848-$ F. P. p. 131 . 
Admettons donc avec spencer l'hypothitis alomingu

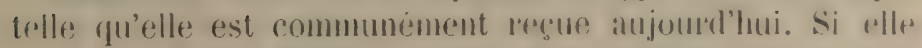
a besoin d'itre pricisee st freut-itre contigere far le pro-

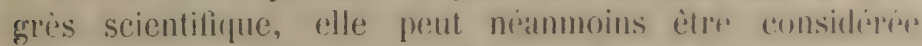
comme aequise definitivement quand a ses donnees essenlielles: des atomes pondrables, des forces, une matiere imponderable ocenjant les intrestices interatominues of les espaces interastratux. Admettons anssi que la mation ne se inanifeste a nous cque par des forees et notamment par la foree de resistancer, fuoigure res loreres nor soirnt pas toujours, ni meme en general, preperes diretement conme telles.

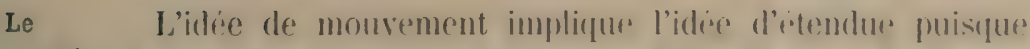
vement. le mourement consiste il parrourir l'etrudue, - de tempse, puisqu'il constiture more sureession de positions, - et de matiere, puisquion ne pent concevoir fo monverment que d'un itre situi dans l'espacer at, par consépurnt, itendu.

Lat perception des mouvements que nous axeroutons est

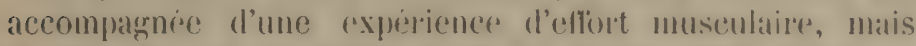
il n'en est pas de mème pour les mourmments yure nous pereevons hors de nous at nous croyons qure la notion de monvement ast indeprondante de cette notion d'eflort, aussi bien que la notion d'espare et de temps.

La conscience que nous avons d'exécutril des nouvemonts peut certainement nous fournir, parr abstraction, l'idée genirale de force, mais cette idee peut se dedure de toute expropience de monvements se produisant rigulierement dins certaines circonstances déterminies. Celat n'umpèche pas d'admettre yur: la perception du mourement est une "manifestation di: force» puisque aussi bien le mouvement est lui même une force. 
La force. Spencer termine l'analyse sommaire de ces idées scientifiques fondamentales par l'examen de la notion de force. Nous avons déjà signalè dans la Première Partie la confusion qu'il fait entre cette notion et la conscience de l'eflort. L'eftort délermine la mise en aurre d'une force appartenant au sujet, et c'est comme phénomène subjectif qu'il est percu par la conscience. S'il sagit de l'effort musculaire, le seul dont spencer fasse mention, il a exclusivement pour objet le mouvement des membres et il est évidemment impossible de l'identifier, par exemple, avec l'attraction qu'un corps exerce sur un autre. L'eftort que percoit la conscience ne peut mème pas se confondre avec la force que deploie le muscle et ne lui est pas proportionnel.

Le vulgaire s'accorde avec les savants pour concevoir la force dans son accejtion générale comme la cause (productrice ou moditicatrice) du mouvement. Tous sommes capables nous-mêmes d'exécuter certains mouvements et nous avons conscience, sous forme d'eflort plus ou moins intense, de l'activité deployée par notre laculté motrice. Mais nous ne nous tigurons pas que dans les gaz qui font eclater une hombe, il se rencontre quelque chose de semblable.

si la force est le " principe des principes " (1), ce n'est certainement pas l'efort dont nous avons conscience. this lors nous ne voyons pas l'importance que spencer attache a nous faire considerer les notions despace, de trmps, de matiore et de mourement comme diriveres de la frerception d'effort ou le mowrement musculaire. Si la mation nous est comnue comme une manifestation de la forces, recla doit sentende avant lout de la foree de resistance, comme nous l'avons dit Mime si cette resis-

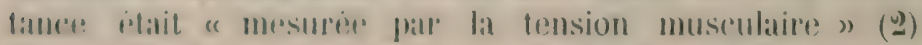

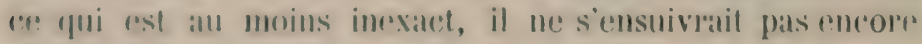
yu'il faut la confondre avec elle.

(1) P. P. 1). $1798850-$ N.P. P. 132.

(2) P. P. 1). 17818 - F. P. P. 198. 
Lespace et le temps, comme te dit tris bien Spencer, (2) ne sont qur les conditions sous lesquelles se presentent la matiere et le mouvement; et puisque lo mouvement aussi bien que la matiere se manifrstent a nous sous forme de force, nous sommes amenes a considérer l'Lnivers en tant quobjet de notre experifence sensible, comme une inanifestation, non point d'eflort, mais de force ; quoique, bien entendu, la semsibilite nt. la pereroive fas sous cette lormalite ginerale, mais birn sous les formes concrètes de couleur, son, résistance, dhaleur, rtc. dont plusieurs ne sont connues cenme forees que grâce au raisonnement.

De meme que la matiore designe l'ensemble de tous les corps, de mème on peut appeler la force l'ensirmbles de toutes les forces de l'Luirers. Il ne fandrait pas conclure de corte maniere de parler qu'il n'y a qu'une espree de corps ou quane espuere dr forees. On at parfois emis lidée que toutes les forces de l'Cnivers mo sont que du suourement. Mais cette conception nre lisiste pas à l'examen et elle est anjourd'hui, cmyous-nous, completement abandonnee. Il y a donc d'antres forces que le mouvement. Nous pouvons en nummer plusieurs: l'attaction universalle, l'elasticite de l'ether at ainsi de suite, mais sans itre certain que ce sont des forces blementaires. Elles ne sont peut-itre que des risultantes de forces plus complexes ou des ais particuliers de forces pulus guénémales. En ce sems il est rai de dire que nous ignorons la nature intime des forees. En outre, la relation de cause qu'elles contiennent, quoique de notion fort simple et imposée par l'experience et par l'analyse, n'en reste pas moins enveloppée d'obscurité.

Dans les formules de méanigur, la force ne tignure yu'en tant qu'elte mst representre gat son effet qui lui sert de mesure ot qui est la quantite de: mouvement: $f=m y$, Ir temps etant 1. On pent amsi laire abstraction de la notion de rause. Mais en philosophlie il n'en est pas de

(1) P. P. p. $179 \$ 50-$ F. P. p. 132. 
mime: le principe de causalite ne permet pas d'admettre des mouvements sans causes et a moins de profess'l l'occisionalisme, il faut admettre que les causes des mourements se trouvent dans les corjs. Telle est la notion de force.

Fous pourons distinguer dans la nature trois catigories de forces: I" les forees mécaniques stricto sensu dont laction consiste i imprimer aux masses corporelles un mourement suivant une ligne determinée par le siegge de la foree et son point d'application. Le monrement frroluit par une telle force so combine évidemment arec les mourements determine's par d'autres forces, mais il est en lui-meme absolument determini. Il est probable que loutes les foress de la mature inorganique (physicochimiques) appartiennent a rette catigorie.

2. Les forces organiques on plastiques dont l'action consiste a frodures une rertaine forme on organisation on a exereer certatines fonctions en vue de la forme. Pour rialisis lexr effiel prophe, elles doivent imprimer anx masses materiolles des mouvements dillèrents suivant Ins ciremstances dans lestumes elles agissent.

3" Les forces psychiques ou intentionnelles dont l'atelion est grudere pall la romnaissance : perreptions sensibles on commassances rationnelles; dans do dermiel cats nous truons yoinles jonissent de la liberte.

Certe division laisse ouverte la question de la maturer intime de cess forces, aninsi que la question de leur difroremere essentiolle. Le meanisme consiste it aldmeltre

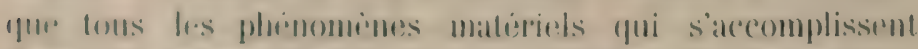
dalls les corpes vivants on nom vivants peuvent sexpli-

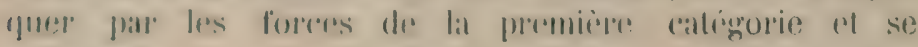

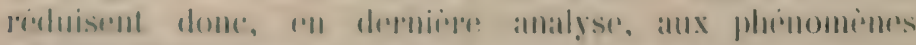

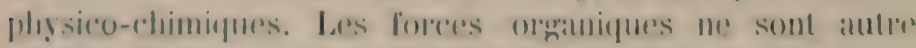

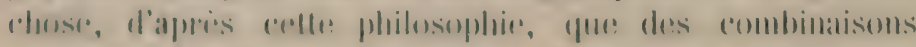

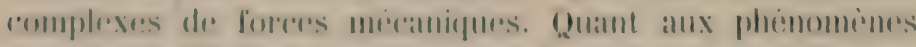

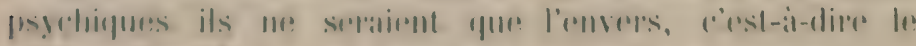

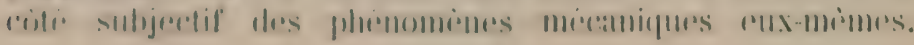


Ce meanisme est alopte par spencer. Tous aurons dans la suite l'occasion de l'apprécier.

s'il est vari que l'lniver's sis prisente at mus comme? une manifestation dre forece, il n'est cepremdant pas possible? de le considerer comme constitué par la force sesale. L'experience nous apprent qute les momement, romms force, comprend dems elements: la masser et la vitesse. Celle-ei n'est qu'une modaliti: du mourenent ot en est inséparable, mais la premirere en est siparable, at, isolere,

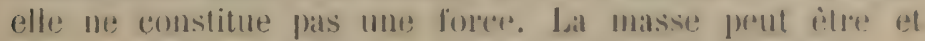

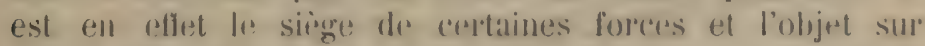
lequel alles soxererent, mais alle ne leme est point bilentique. On detinit parfojis la masse commen lat quantite

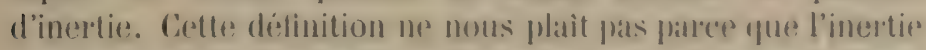
est une propriele negative yui noulmet pas de dentre. Tous prefirons considerer la masse comme un ielinment irrialuetilite?

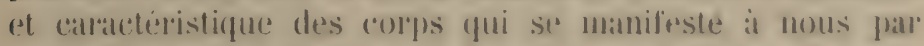
10 fait gu'il laut une? forces plus on moins gramer pour leur communiquer une centaine vitesses, at qui, dis lors, mit

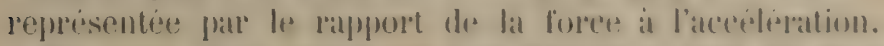

En outre nons atons vil que l'elendue, tont ent se matnilestant par la foree de resistamere, me doit fras ible

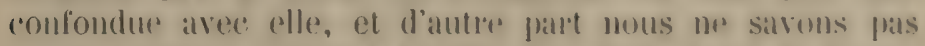
qualles sont les relations de l'etendue leeelle arece lat matsise, ni mime si ces relations existent. Les notions de l'une pt de l'autre sont an tous cas diflerentes. De sorte que dans l'Lnivers materiel, nous rencontrons yuatre cilements distincts: l'étendue, la masse, le mourement at des forces yui ne sont pas du mouvement.

Tous les phenomines materiels comportent des diplatcements de masses corporelles el, en tant qu'objuts dr: l'experience externe, ils se reiluisent a cela. ()n peut done a cet egard les dérorire en termes de matieré et do monvement. Cette manière de les moisager ne préjuge en rien la question de savoir quelle est leur mature intime ou la nature des forees par lespurlles ils sont produits. A plus forte raison ne prejugr-t-1le pas la yuestion de savoir s'il n'y a fras d'autres phemomines que les pheno- 
mènes matériels, inaccessibles ceux-là à l'olsservation externe, du moins en eux-mèmes. mais manifestés dans la conscience. Cependant cette activité immatérielle, qui est exercée d'après nous par la raison et par la volonté de l'homme, n'applartient à la présente étude que par les relations qu'elle a avec les phénomènes matériels ou phénomènes le mouvement. L'étendue, la masse, le mourement, des forces qui peuvent ètre mécaniques, organiques ou plastiques: tels sont donc les éléments de l'évolution.

Quoique le mouvement soit une force en tant que les mouvements des corps se modifient réciproquement, cependant toutes les forces ne sont pas des mouvements: la raison d'ètre de certains mouvements ne se trouve pas uniquement dans d'autres mouvements. Il y a done des rauses de mourement qui ne sont pas du mouvement et qui constituent la catégorie des forces. Nous avons fait remarquer aussi que la masse ne peut se confondre ni avec la force ni avec l'étendue, de sorte que les quatre éléments signalés ici doivent ètre considerés comme irréductibles.

Cela ne veut point dire (qu'ils n'ont entre cux aucune relation, bien au contraire. D'abord ils sont toujours réunis : il n'y a pas dans l'Lnivers de corps immobiles, tous ont In volume, une masse et sont le siege de forces.

Les corps occupent l'espace par leur etendue qui est donc la condition essentielle du mouvement. Colui ${ }^{\text {ei }}$ est linlet de la force, tandis que la masse s'exprime par une relation entre la force et le mouvement. Quoique fo volume apfarent soit variable, on admet généralement fure lo: volume lreel d'un corps est constant tout romme sat masse. Les forces, an contraire, et les mouvements varient sans cesse. 
CHAPITKE H.

\section{LES PRINCIPES}

5. L. Lindestructibilité de la matière comme principe à priori. - L'indestructibilité de la matière comme résultat d'induction. - L'indestructibilité de la matière et la persistance de la force.

destructi- Après aroir examiné les éliments de's phénomines milité de la natière me prino à priori. teriels, spencer etudie los principus generaux qui les grouvernent et qu'il faut supposer comme base dans la recherche de la loi d'évolution.

Le premier est l'indestructibilité de la matière.

Que dans les phenomines il $y$ a des translionmations de matière et jamais de destruction ni de crétition, c'est une verite expérimentale reconnue plus clairemant à mesure que l'on a étudie les litits avec plus de soin. Ellr: est donc certainement une loi fondamentale de l'ordre naturel.

Est-elle une proposition certaine à priori, e'est-it-dirte independante de l'induction consciente et devons-nous dire que la destruction d'une partie de la mattière est "inconcevable»? Line remaryue est ici nécessaire. On ne peut pas concevoir une transformation de la matière abouttissant au non-eitre; ce serait une transformation sans 
terme, co qui est contraclictoire. Il est done rrai que la matiere ne peut pas devenir rien, de mème que rien ne peut pas devenir quelque chose. Toute transformation suppose dewa termes: un point de départ et un point d'arriver. si l'on a en vue les actions transformatrices, telles çu'en exercent les agents naturels, il faut, sams nul doute, attirmer que la matiere est indestructible. J'ajoute que tomle action positive qui se termine a la matière ne peut itre quine transformation, puisqu'elle a la matière comme sujet fassif. On ne peut donc pas conceroir que la matipre, ni une substance quelconque soit purement et simplrment détruite, èest-it-dire réduite à rien, par une action positive.

Mais est-il concevable quime partie quelconque de la matière n'existe pas, qu'ayant existé aujourd'hui elle nexiste plus demain? lat réponse aftimative s'impose. It rasomnement qui amène spencer à la repousser n'a aucune valent. L $\alpha^{2}$ voici $|0|$ qu'il a été remanié en 187;: : Concever l'espace dibamassi de tous les corps excepte un. Haintenant imaginez ce corps restant ne changeant pas de place, mais fondant en rien, tout en restant in place. Vous n'y reussirez pas. L'espace qui etait solide ne peut être conşu comme vide sans déplacement de ce qui le rendait solide. Ce qui est appele l'rafrime incompressibiliti de la matièe est une loi admise? far la pensiate. A quelque petitesse de volume que nous concevions reduite mo portion de matiere, it est impossible de la conceroir commer réduite a rien. Alors que mons pouvons nous rejuresenter ses parties comme rapprorheres, mous ne pourons pas nous representer la quan-

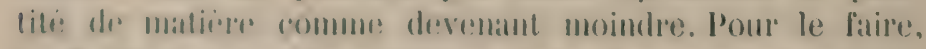
il fimdrail imagioner quelques unes des parties compri-

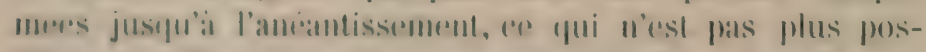

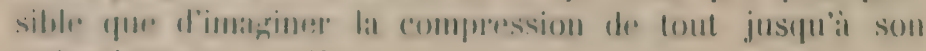
ancantissement. „) (1)

(1) F. P. p. 1334 है : 283 . 
Encore une fois : antre chose est comprendes el autro chost est se representer par l'imaginition. W'ailleur's

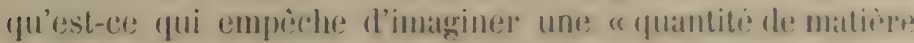
comme devenant moindre s)? C'est un phimomine qum nous constatons tous les jours. Il est rrai qu'anjumbl'

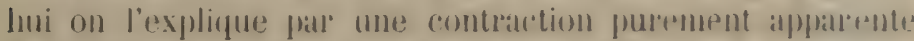
qui consiste dans le "rapprochement des particules" ;

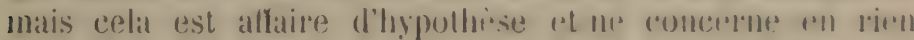
lexperience ni l'imagination. Spencer ne notr-t-il pas

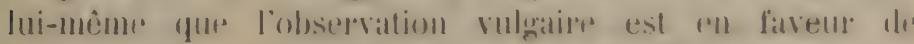

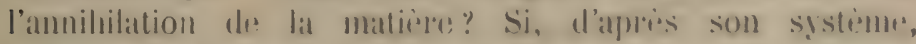

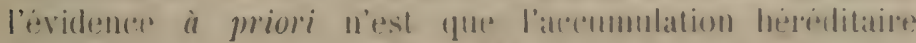
d'experiences innombrables, 11) fumelles sont dome les

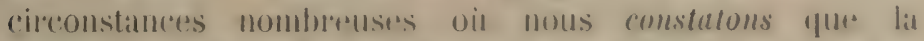

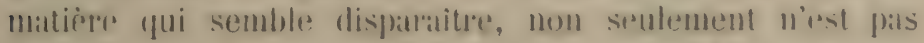

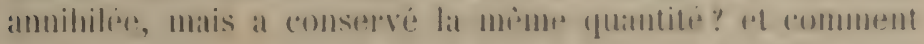
est-il possible que ces experineress qui, on realitio sent

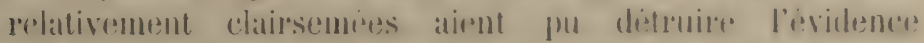

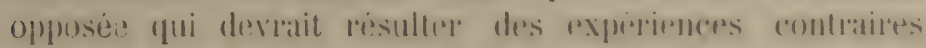

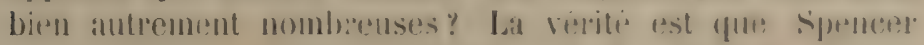

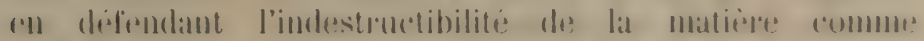

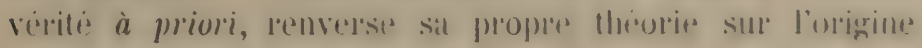
expérimentale de ces vérités.

"I La pertsets, dit-il encorr, est unr position de relations.

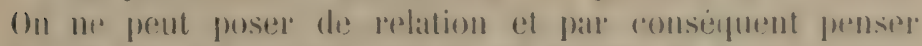

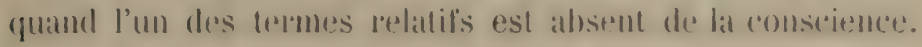
Il est done impossible que quelque chose deviemane reien,

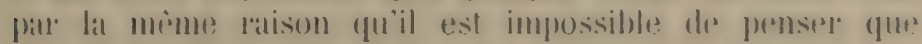

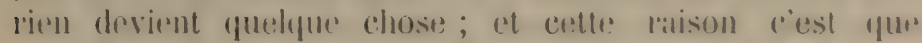

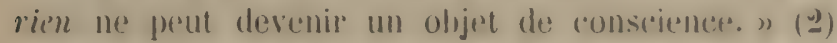

L'expression : "quelpute chose derenant rien "st incorrecte. Line chose qui devient ne perut derenir yow quelgue chose. Ibevenir rien est done, al la rigureme

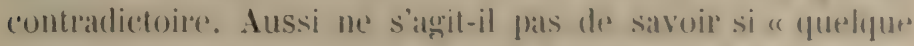

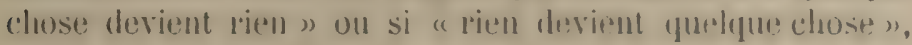

(1) if. ibid note p. 139 .

(2) P. P. p. $186 \S 33-$ F. P. p. 138. 
mais seulement si quelque chose peut cesser ou commencer d'ètre.

En outre rien, comme toute autre négation, est un objet de conscience. Il n'est même que cela puisqu'il n'est point un objet réel. C'est pourquoi on l'appelle, de mème que les autres négations, un étre de raison : il n'a d'objectivite que dans la raison, e'est-it-dire dans l'intelligence ou la conscience. Si le non ètre ou rien n'est pas un objet de conscience comment en parlerait-on?

L'idée qu'on en a n'est pas une pseudo-idée. Les pseudo-idées sont des idées contradictoires; ce ne sont pas vraiment des idées, du moins des idées précises, mais plutòt des mots accollés ou des notions unies non dans l'esprit mais seulement dans le langage. Il n'y a aucune contradiction dans l'idée de non-être. Elle est claire et a une signification déterminée.

On peut done conceroir que la matière existant "ll ce moment n'existe plus au moment suivant et cesse, fal conséquent, d'exister; de mème on peut conceroir que la matière n'existant pas maintenant existe dans la suite, rest-i-dire, commence i exister. Dans ce sens, on peut concevoir que la matière est créée ou est détruite. "Je peux parfaitement me représenter, dit Renouvier, que telle chose rui tout a l'heure n'etait pas, maintenant soit, et telle, autre (fui était ne soit plus; c'est ainsi (fue j'envisige le changement, fuand je pense aux phenomenes qui faraiss'nt ou disparaissent dans l'ordre de l'experéence., ") (1)

L.'Thivers est, d'apress spencer, une manifestation de l'Incennaisable. Cette manifrestation ret nécessaire on libere Gurllu: raison avons-nous d'allirmer yu'elle rest néessabe?

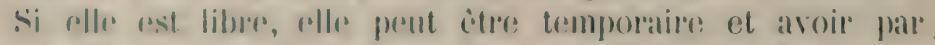

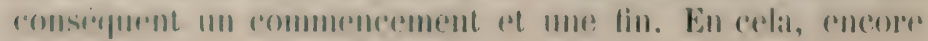
line fois, il n'y a rien d'inconcevable.

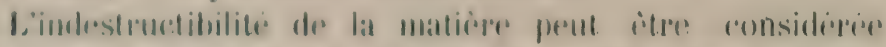

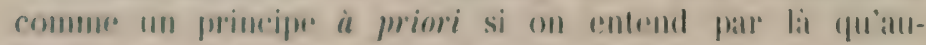

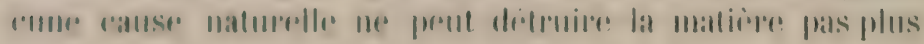

(1) op. cll. 1 स\%8 1 । p. 123 
qu'elle ne peut la creser, mais non pas en ce sons qu'il soit impossible de conceroir la matiere cessant d'exister. Et avant tout, la permanence de la matière et une verite d'ordre expérimental.

destructi- Jusqu'i quel point est-elle le fruit du progrès scientibilité a matière me résultique récemment accompli? sjencer verse manifestement dans l'erreur a ce sujet. Yous n'exaninerons pas jusquäa quel point "le's superstitions primitives on la croyamee a la magir... supposent que sous l'action d'un charme puissant la matière peut être évorgues du non itre on rejetres dans la non existence" (1). Nous avouons navoir aucune lumiere pour confirmer on dementir as ressignement. Mais il est faux que la destructibilitie de la matièn "domine l'enseignement de la theolugie: sur te eommencerment "t la tin du monde" (2); il est laux aussi que la virite contraire " n'a ette mise hor's de donte que dans les temps modermes et seulement far les hommes de science $"(3)$.

Le commencement et la tin du monde n'ont rien à roir arec les lois de la nature et, en outre, persomne n'a jamais cru que l'tuivers est destiné it ètre annihili.

Nous ne connaissons aucun philosophre qui, soecupant des phenomènes naturels, n'ait enseigni que, dans tous, la matiere se transforme: et par considguent ne s'anilhile point. Les idles des atomistes grees dont le poite Lucrèce nous a conservé les enseignemrnts, ne differrent pas en substunce, sur le point que nous cousiderons, des conceptions modernes.

Quant à l'école péripattiticiemne. elle admettait dans la nature des transformations substantielles, mais pas de

(1) P. P. p. $183 \$ 32-$ F. P. p. 134.

(2) P. P. p. $184 \$ 52-$ F. P. p. 134.

13. P. P. P. 187 \$.33, jassage moditio danx l'älition de 1900. Yous: nous oceupons plus loin de cere que ceremaniement rontient de noruveau. 
destruction ni de creation de matière. Il est rai qu'elle idmettait la compressibilité réelle des corps ; mais, quoique spencer ell pense, comprimer un corps ne consiste dans ancune hypothèse " a faire disparaitre certaines de ses parties. ") (1)

Il n'y aturait pas, dans ce cals, de compression. La compression, comme la concevaient les péripatéticiens tout en diminuant le volume réel, respecte la substance; elle n'a done rien à voir avee la destructibilité de la matière.

Les theries de cette école etaient communement admises au moven age et comptent encore aujourd'hui des partisans. Quant a l'atomisme, it a cte repris et perfectionné à partir du XVIIe siècle.

Lat decouverte récente, due aux chimistes et principalement it Lavoisier, ce n'est pas l'indestructibilite de la matière, c'est la permanence du poids, ou, comme on dit mieux aujourd'hui, la permanence de la masse.

spencer comprend dans l'indestructibilite de la matiere lat permanemere de cerlle-ci " soit dans ses masses, soit dans sers atomes. ") (2) La permanence de's atomes est une hypothese conme les atomes eux-mèmes, tandis que la permanence des masses est admise comme un fait. Il n'y a pas davantage liesu de distinguer la prermanence "des quantites et de's poids". Si dans ceet enonce la quantite n'est pas la meme chose que le poids ou la masse, qu'est-elle ? Pas, a coup surr, le volume apparent qui ast variathe, ni le volume risy qui nous rst ineonmu.

Les amcirns novaient aucune idere precise de la masse, mais ils admerttitiont que le poids des corps, qui est une consisfuence de leme masse, peut varier lorstue les rorps subi-sent des lamsformations sous ladtion des ratuses naturelles; of colat, a comp suir, n'est fas inconcevable.

Xianmonins la dhimie moderne enseigne que, de fait, 16 poids des corpss reste constant it travers toutes les

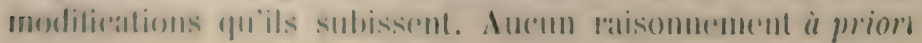

(1) P. P. P. 186 \& :i3-F. P. p. 183.

2) P. P. p. $1883828-$ F. P. p. 134. 
ne peut ancher refte ronclusion, (1) mais serulement l'expérience.

Dans l'édition de 1900 speneere ajoutre a la fin de cee paragraphes la remarrue suivante: "II faut dipe eneore (ju'aturune verification exprerimentales de l'indestructibilitie de. la matiere n'est possible: sans la supposition latcite de cette verite. Call tonte verifiation exige des pesies et

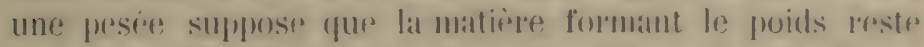
bat mime. " (2) Yous revirndrons birntit sur rettre ohser-

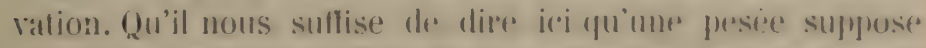
non pas l'indestructibilite de la matiere on lat festsistance dr. lat masso comme principm grinteral, mats seulement lidentite de la masse du corps qui sert de poids prendant le temps de l'expérience.

Yous ne pourous pats admettre lat mamiere dont Renouvier presenter lat question de la permamenere de la masce.

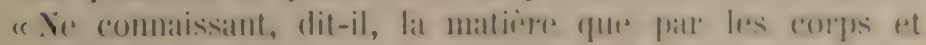

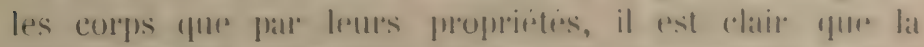

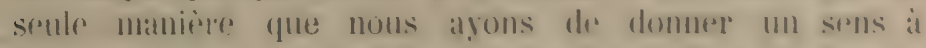

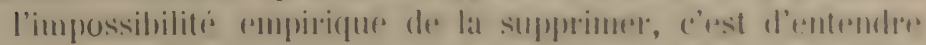

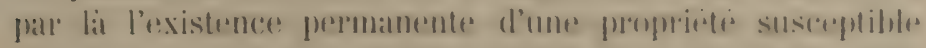
de mesure at qui se trouve constamment la meme pour

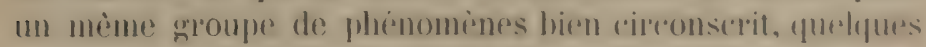
changements qu'eproment d'aillemes les corps qui y

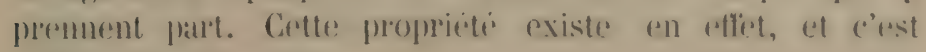
la pesanteur. »

D'abord la pesanteur n'est pas constinte, mais serulenemt la masse. Ensuite, l'impossibilite empoirique de supprimer la matierea a $u n$ sens des que nous concevons yuducume partie de la matiere ne s'amnihile, ee qui n'empeches pas que toutes ses proprietes ne se moditient, y compris lit masse. Entin nous admettons qu'un corps comprend outre ses proprietes multiples et changeantes, un sujet unique

1) cf. J. Wann. Valuralism and Agnosticism. Lundres 1903 p.p. 86 sq.

(2) F. P. p. $138 \$ 33$.

(3) La Critique Philosophique. 1886 vol. I. p. 120. 
et permanent. La permanence de ce sujet à travers les changements qu'il subit, voila l'indestructibilité de la matière, landis que la permanence de la masse n'est que l'invariabilité d'une de ses propriétés.

ndestructi- "Ite fait qu'il nous importe le plus d'observer, dit ilité de la Spencer, c'est la nature des perceptions qui nous fouratière et la nissent perpétuellement des exemples de la permanence rmanence e la force. de la matière, d'ou la science tire la conclusion que la matière est indestructible. Ces perceptions, sous toutes leurs formes, reviemment simplement à ceci que la force exercé par une quantiti donnée de matière est toujours la même. Telle est la preuve sur laquelle se fondent à la fois le sens commun et la science exacte. Quand par exemple nous disons qu'un individu qui existait il y a quelques années existe encore parce que nous l'arons vu hier, notre assertion revient à dire qu'un objet qui, dans un temps passé, a opcré dans notre conscience un certain groupe de changements, existe encore parce qu'un groupe semblable de changements a ete produit de nouveau sur notre conscience: nous regardons la continuation du pouvoir de nous impressionner comme une preuve de la continuation de l'objet.... Tous voyons encore plus clairement que c'est en délinitive par la force que nous mesurons lił matière, dans les cas où la forme de la matirare a change. On remet a un orfère un morceau l'or four en faire un hijou; quand il le rapporte, le hijou parat plus pertit que: Ie morecau d'or; on le met dans une: balance; s'il fait expuilibere à un poids bien moindre qu'auparasant, quand il stait i l'etat brut, on en conclut

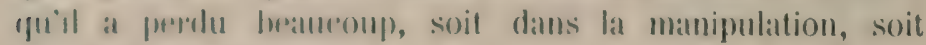
par une soustraction.

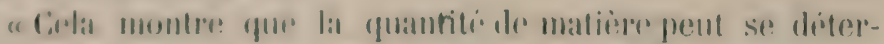

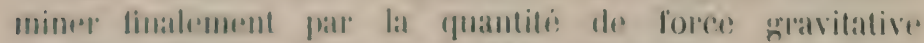

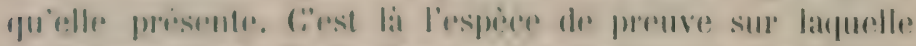

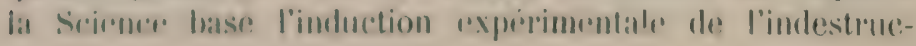
tibiliti de la matiere.... 
" Ainsi done par indestructibilite de la natière nous voulons dire l'indestructibiiité de la force par laquelle la matière nous affecte. De mème que nous n'avons conscience de la matière que par la résistance qu'elle oppose i notre activité musculaire, de mème nous n'avons conscience de la permanence de la matiere que par la persistance de la résistance qui sto manifeste a nous directement ou indirectement. Cette vérité devient manifeste non seulement par l'analyse de la connaissance a posteriori, mais aussi far l'analyse de la connaissance a priori. Car ce dont nous ne pouvons concevoir la diminution par l'eflet de la compression continuée de la matiere, ce n'est pas sa proprieté d'occuper l'espace, c'est son aptitude a r'esister. ") (1)

Il est inexact de dire que la force exercie jar unr quantité domée de matiere est toujours la mema. La pression d'un gaz est une force at varie avec la temprorature; les forces attractives et répulsives varient avec la distance; l'alfinité chimique des corps varie d'ilprès los combinaisons dans lesquelles ils sont engagres.

Dans l'édition de 1900 la phrast: que nous critipuons a élé corrigée. Il y est dit que l'indestructibilite de la matiere se manifoste en ('e que "la force qui est contenue dans une quantite domnere de matiere est toujours la mème dans les mêmes conditions. " (ֻ) Si ces clerniers mots signitient: en l'absence de tout changement, alor's, mais alor's seulement il est vrai de dire que la mème matiere dans les mèmes conditions contient tonjours la mêne force. Mais dans ce as laallimation se réduit it une pure tautologie at il serait a coup stir pueril de prestendre que l'indestructibilite de la maticre se manifeste en ce qu'une quantite de matirie qui ne subit aucun changement conserve la meme lorer. Aussi nous est.il impossible de voir aucune preave de cette indestructibiliti dans le fait que le diamant se reconnait toujours a sa dureti: et à sa rélringence, ce qui est l'exemple ajouté par spencerer.

(1) P. P. p. $189-190 \& 31$

(2) F. P. p. 138 
Il est rrai que la masse ne varie pas quelles que soient les circonstances et quels que soient les changements que sujissent les corps, et que l'invariabilité de la masse se manifeste par l'invariabilité du poids. Mais celle-ci n'indique la permanence d'ancune force, pas mème de la force de gravite. Les variations de la gravite n'affectent en aucune manière le poids. Cela est trop connu pour qu'il soit nécessaire d'y insister.

la masse d'un corps, nous l'avons déjà dit, n'est pas une force, mais bien un rapport entre une force qui agit sur ce corps et l'accélèration qu'elle y produit dans l'unité de temps; ou, si l'on préfè̀re, la masse est une propriété du corps qui a son expression mathematique dans ce rapport.

s'il est vrai que l'attraction de deux corps est enraison directe de leurs masses, il est vrai aussi qu'elle est en raison inverse de leur distance qui est variable, les masses restant les mimes. Autre cliose est dire que la malière est indrestructible dans sa masse, autre chose qu'elle ne peut subir aucun changement. Il est évident au contraire qu'elle en subit, et de très considérables. Ln homme, non prevenu, mis en prisence de l'ozone n'y reconnaitra certainement pas l'oxygène qu'il respire continuellement. Aussi l'ozone exerce-t-il des forces très difrerentes de celles de l'oxygene. Le poids de l'ozone est le mime que celui de l'oxygene qui lui a donne naissance, mais cela, encore une fois, ne prouve la permanence d'aucune force. Pour l'expliquer il suffit de dire que les masses n'ont point varié et que lat graviti est a chacpue instant proportionnelle aux masses quelque changement qu'elle subisse d'ailleurs.

Sans doute, toute masse de matiere oflere toujours une cortaine risistance, mais il serait pueril de pretendre que arte resistance tolle fu'olle se manifeste a l'experience, est loujours la mime. Ia permanence de la masse n'est dene pas manifestee par la permamence de la resistance.

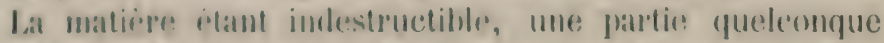
dre la matiope exerce toujours reptanines forees et ollee toujours une replaine resistancer et rest ansi que se manifeste son indestructibilitr. Mais re que spencer a en vue 
c'est de faire admettre que l'inearabilite de la masse ses manifeste far l'ineariabilité de la fores at cela est manifestement faux.

\section{$\$$ II. La conservation du mouvement a priori et devant l'induction.}

In corps en mourement, soustrait a tuute influence extérieure, continur a se momvoir indertiniment rn ligne droite d'un mouverment uniforme. Rien n'empreche d'appeler "contimuite du mouvement n ce uni est exprime par cet enonci.

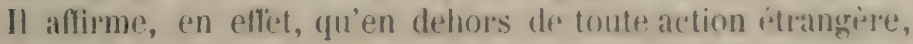
done de sil nature, le monvernent reste inditiniment ce qu'il est.

Cette loi n'est qu'une conséquence du principue plus général d'inertie en vertu dupuel aucun corps ne peut changer son propre etat de repos on de mouvement. Yous devons ces notions an grenire de Gallition.

Spencer a rason de dire quelles etatent ignories des Grees. Aristote qui, parmi les anciens, a le mieux traite les questions physiques, admettait deux sortes de mourements simples dont tous les altites sont composés. l'un naturel au corps, l'autre contre nature ou violent. (1) II pensait que le corps reagit contre ce dernier qui, dès lors, tend à prendre lin. Ln corps lom qui s'elève verticalement est animé d'un mouvement contre nature, parce que le corps lourd tend vers le Centre et cette tendance naturelle ramene bientòt le corps au repos en attendant qu'elle lui imprime un mouvement opposé. Le mourement naturel ou bien est rectiligne et dirige vers un endroit determine, comme par exemple le Centre de la Trere et alor's il s'arrete lorsque cet endroit est atteint; ou bien il est circulaire et n'est diriger vers aucun endroit; c'est le mouvement des corps culestes et celui-la seul est de sa nature perpétuel.

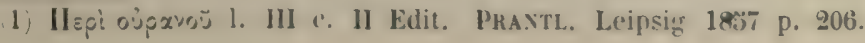


Ces idées d'Aristote ont été pendant longtemps admises universellement. Elles supposent une conception de la gravitis et de la légèreté des corps différente de celle que l'on a aujourd'hui. Yous considérons avee raison la gravité des corps comme la manifestation d'une force générale d'attraction et comme résultant d'une action mutuelle des corps les uns sur les autres; tandis que, pour les anciens, la gravité et la légéreté étaient des tendances inlıérentes aux corps indépendamment de toute action exercée sur eux.

En outre, ils concevaient le mourement comme étant, ou, du moins, pouvant être une tendance vers un endroit déterminé de l'espace, tandis que nous le concevons comme fixé uniquement ell vitesse et en direction.

Il est désormais certain que nos idées en cette matière sont conformes aux faits, tandis que celles des anciens ne le sont pas. Faut-il ajouter que les lois du mouvement telles que nous les formulons sont des principes absolus susceptibles d'une démonstration a priori? C'est ce qu'il serail peut-ètre téméraire d'aftirmer. En tous cas, il est important de ne pas détourner le principe de la continuité du mouvement de son véritable sens en affirmant que le mouvement persiste comme tel en toute hypothèse. II y a bien en mécanique une loi qu'on appelle la conservation de la quantité totale de mouvement et en vertu de laquelle, dans un systeme soustrait aux influences axterrieures, la somme algébrique des quantités de mouvement Jeste constante. On y considère les mouvements de sens oppose comme btant de signe contraire et se neutralisant par conséfuent dans la somme. Celle-ci ne nous apprend donc rien an sujet de la quantite absolue du mouvement el dies lors la loi prexition neen contime en rien la persistance.

Dans la premiere redaction du chapitre que nous etudims. Spencer n'evitail pas la confusion signalie iei. II disait: "Colle loi (de la conthuite du momvement) a étis alsordber de: nos jours par une loi plus comprehensive: fgu. le mourement comme la matiere est indestructible, et fure font ere qui est peerlu par une partie quedconque de 
la matière se transmet à d'autres parties. Bien que cette nouvelle loi semble en désaccord avec les faits qui nous montrent des corps s'arrètant subitement après avoir frappe un objet immolile, elle se concilie avec ces faits depuis qu'on a découvert que le mouvement perdu en apparence se continue sous de nouvelles formes, lesquelles ne sont pourtant pas directement appréciables. ” (1)

L'indestructibilité du mouvement était représentée comme une vérité a priori au mème titre et pour les mèmes raisons que l'indestructibilite de la matière: la destruction du mouvement est une " pseudo-idée ", " une pure forme verbale "; que la réalite relative de ce que nous appelons mouvement ne preut jamais arriver a l'existence ou cesser d'exister, c'est une rériti impliquée dans a nature mème de noth' conscience, cte. (2)

Ces passages ont disparu dans l'édition de 187\%. spencer reconnait que le mouvement peut ètre detruit sans se continuer sous une autre forme de mourement: il en apporte comme exemple te pendule qui s'arrite au bout d. sa course sans que son mouvement soit transmis a d'autres corps.

Par une contradiction difficilement explicable, spencer maintient dans ce chapitre d'autres passages qui renferment l'erreur ancienne d'après laquelle tout mouvement qui disparait est remplace par d'autres formes de mouvement.

Pardant des variations dans le mourement des planetes, il dit: "Nous arrivons alor's à conclure l'indestructibilite du mouvement, non a cause de la vitesse uniforme de la planète, mais à cause de la quantité constante de mouvement manifesté en tenant compte du monuement communiqué aux autres corps cilestes ou transmis par eux $)(3)$. Et plus loin: "Co dont la prensee ne preut pas se defaire cest la force que le mourement indique. Let ('hangement

(1) P. P. p. $193 \$$ :3ij-C'est nous qui soulignons.

(2) Ibid.

(3) P. P. p. $195357-$ F. P. 559 p. 147. 
incrssant de position, considéré à part, peut ètre effacé de la pensée salns difficulte. Nous pouvons facilement imaginer que le ralentissement et l'arrèt sont des résultats de l'action des corpss extérieurs. Mais cela n'est pas possible si l'on ne fait pas abstraction de la force impliquée dans le mouvement. Nous sommes obligés de concevoir celte force comme imprimée sous forme de réaction dans les corps qui causent l'arrêt. Jous sommes forcés de regarder le mouvement qui leur est communiqué, non comme communiqué directement, mais comme un produit de la force communiquée. Nous pouvons par la pensée diminuer la vitesse de l'élément-espace du mourement, en répartissant le moment ou l'élément-force sur une plus grande masse de matiere; mais la quantité de cet ilément-force que mous regardons comme la cause du mourement, est invariable dans la pensée » (1).

Lorsque le mouvement d'un corpss est arrèté par un autre, il y a en effet raction du premier sur l' second; mais c'est une erreur de croire que cettr reaction determine toujours un accroissement de mourement dans le second; rien n'empioclie que le contraire n’ait lien. Dans ce cas loin que la réaction "imprime aucuno force ", wlte diminue la force vive constitue par le mouvement. Ians le fait du pendule, le mouvement apres aroir cesse, roprend; il reste vai néammoins que sal quantiti a varié sans cesse et yutit un moment dommi il est totalement defruit; at si pal un méanisme facile d imaginer on arprite: le pendule an moment ou il a achere mose oseillattion, son monvement peste mul alussi longtemps quon le vout. Il nest mimr gas diflicile de reitre des cas ou le

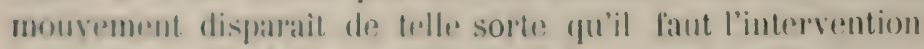

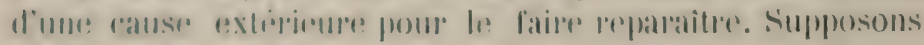

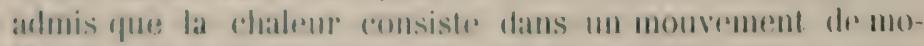

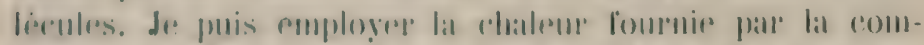

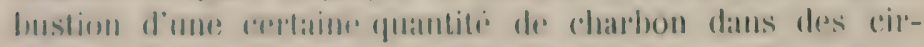

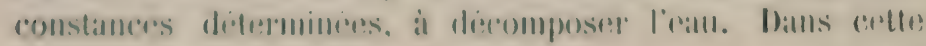

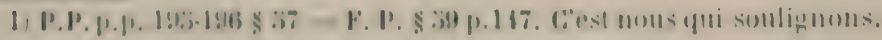


operation une certaine quantitis de mouvement disparait sans être transmise à aucun corps du moins en quantité équivalente, et j'obtiens comme résultat trois gaz: l'anhydride carbonique, l'oxygène et l'hydrogeine. Le monvement perdu reparaitra lorsque l'hydrogène et l'oxygène se combineront de nouveau, ce qui n'a pas lie'u spontitnément, mais exige l'excitation d'une force extérieure.

Les exemples choisis montrent que le mouvement perdu est remplacé par autre chose: soit une certaine position du pendule, soit une certaine constitution chimique. Cette position du pendule et cette constitution chimique peurent, dans certaines circonstances et gratce aux forces de la matière, déterminer la production d'une quantité de mourement exactement égale à colle qui a èté rmployée it les produire, et en ce sens le mouvement a iti: conserve virtuellement. Mais il n'en est pas moins vai que le mouvement, comme tel, a disparu et on me peut pas dire " qu'il se continue sous de nouvelles formes. " Cine position n'est pas une forme de mouvement et une constitution chimique pas davantage. Il n'est donc pas exact de dire que le mouvement est indestructille.

Cette observation, nécessaire pour que le principes de la continnité du mouvement ne soit pas détourne de son veritable sens, prouve ègalement l'inanité du raisonnement par lequel spencer prétendait ériger l'indestructibilité du mouvement à la tauteur d'un principe absolu. L'argunentation est la mème que celle qui sert de base a l'affirmation à priori de l'indestructibilité de la matière. Yous avons dit ce qu'il faut en penser. Spencer $y$ a renoncé en ce qui concerne le mouvement; on se demande pourquoi il a continué à le maintenir dans le chapitre qui traite de l'indestructibilite de la matière. Loin que la destruetion ou la diminution du mouvement soient inconcevables, ces phénomènes doivent être consus a priori comme pouvant avoir leur cause dans la nature. La métaphşsique est donc d'accord avec l'expérience. Le mourement n'est point une réalité subsistant en elle-mème mais une modalité des corps qui se meuvent, de sorte qu'une destruction 
du mouvement n'est qu'une modification accidentelle du mobile et peut ètre réalisée par les causes naturelles.

Le mourement est une forme de force ḋ condition qu'on tienne compte non seulement de la vitesse, mais également de la masse du corps en mouvement. Comme le mouvement est destructible, il faut en dire autant de la force qu'il représente. Lorsqu'il disparait, il est toujours remplacé par une quantité équivalente d'énergie potentielle. Mais celle-ci n'est point une force, ni surtout une force nouvelle; elle est un travail possible et sa valeur depend non seulement des forces mais encore de la situation des corps entre lesquels elles s'exercent. L'énergie potentielle peut donc augmenter sans que la force augmente, ou même lorsque la force diminue.

Spencer n'aime pas le mot: énergie potentielle. Aussi au lieu de s'en tenir aux notions adoptées par les physiciens, il remplace, dans la nouvelle rédaction de ce chapitre, l'indestructibilité du mouvement par la persistance du " principe d'ativité ", qui existe tantôt sous forme visible (mouvement), tantôt sous forme de " tension qu'on peut concevoir comme le produit de son intensité par la distance à laquelle elle agrit. ” (1)

On ne peut que désapprouver hautement l'inexactitude de ces conceptions. 1 $^{\circ}$ Le mot tension qui s'applique convenablement à certaines formes d'energie potentielle, ne convient gutere a d'autres, par exemple, à l'affinité chimi(jur ; 2n Comment concevra-t-on qu'une tension a pour mesure son intensite multipliée par la distance a laquelle ollr agit? Ians le cas des forces attractives, si l'on nuglige Iror variation en fonction de la distance, l'énergie potenlielle augmente, il est vai, avec la distance, mais la tension n'augmente pas. Prenant pour exemple le " principe d'artivite " qui remplace le mouvement perdu par un corps lance en l'air, spenerer nous invite i le considerer comme igguivalent a la tonsion de cordes chastigues d'une cere-

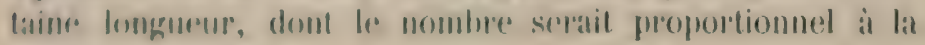

(1) V. P. p. 14:1 17 
distance entre le corps et la terre. Or en tendant des cordes les unes i la suite des autres, la force de tension est proportionnelle au nombre des cordes tendues tandis que la force d'attraction n'augnente pas avec la distance. 30 Dans le cas des forces réjulsives l'energie potentielle est d'autant plus grande que la distance est plus petite.

Contentons-nous de ces remarques. La raison pour laquelle Spencer préfere des notions inexactes, alors que les notions exactes sont à la portée de tout le mondr, c'est qu’il veut sauver absolument le principe de persistance. "Il est inconcevable, dit-il, qu'une force (mouvement) se change en relation d'espace. " Lat verité ('st non pas qu'une fores se change en relation d'espace, mais que le mouvement est detruit et qu'en mème temps il se produit une relation d'espace telle que les forces du syslime preuvent regenérer le mouvemrnt. C"est re qu'on appelle l'énergie potentirlle. On voit qu'ello implique la notion de force et que spencer se trompe une fois de plus en affirmant que les physiriens congoivent l'encrgie potentielle comme constituée par des relations dans l'espace à l'exclusion de l'idée de force.

$\$$ III. Véritable sens du principe de la persistance de la force. - Ce principe est-il un postulatum ? - La force et l'inconnaissable.

ablesens Jetons un coup) d'oeil sur le chemin frareouru. Nous rincipe le la istance force. avons précise le principe de l'indestructibilite de la matière: il atlirme l'invariabilité de la masse. Nous avons vu que cette persistance de la masse n'est liée a aucune persistance de force. Nous avons reconnu que le principe d'inertir? a comme conséquence la continuite du mouvement lorsque lo mobile n'est soumis à l'action d'aucune force exterifure. En dehors de ce cas, nous arons constate que le monvement peut itre ditruil à condition d'ètre remplacé par une situation nouvelle qui est capable d'ren dirterminer la reproduction dans certaines ronditions. I.' $\mathrm{f}^{2}$ 
nergie potentielle etant une quantité qui dépend de facteurs autres que la force, la persistance virtuelle du mourement dans l'energie potentielle n'implique pas un persistance de la force. Enfin nous avons accordé à ces conclusions la valeur de vérités expérimentales, mais non celles de principes a priori.

Il nous faut maintenant examiner le principe de la persistance de la force. Le chapitre que Spencer y consacre a subi des remaniements plus considérables encore que les deux précédents. La plus grande partie a disjaru et quelques considérations nouvelles ont èté ajoutées.

D'après Spencer, la persistance de la force est un principe qui "étant la base de la science ne peut-être établi par la science." (1) Cela ne nous parait pas admissible. Il est question ici de l'étude de la nature. Pour qu'on puisse avec fruit chercher à connaître les lois des phénomènes, il n'est pas nécessaire de supposer que les forces par lesquelles ils sont produits ne varient pas, mais seulement que dans la nature cos forces ne varient que suivant les lois fixes. En d'autres termes, la base des sciences naturelles n'est pas l'invariabilite des fores, mais l'invariabilite des lois. D'elle dépend la certitude de loutes les conclusions scientifiques, entre autres (le) l'invariabilitio de la masse et des lois de la transformation du mouvement.

Et l'on pourrait croire que peut-itre spencer, en parlant de la persistance de la force, na pis autre chose "n vue que l'invariabiliti des lois suivant lesquelles les forces agrissent. Mais dans ce cas il ne dit pas assey, (at) mon semlement les foreres agissent suivant certaines lois fixes, mais all outre loutes leurs variations (ou, si

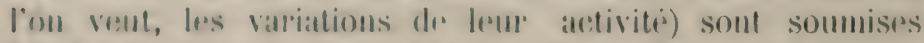

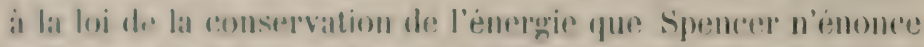
mulle part sems mor forme precise et pui est cependant

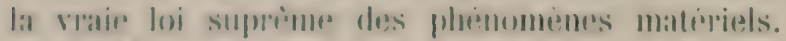

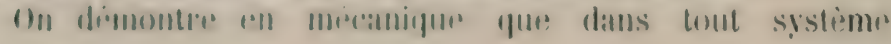

(1) P. P. p. $201 \& 89$. 
soumis uniquement a l'action de forces interienes (attractives ou répulsives) proportionnelles aux masses et ur: rariant qu'aver les distances des corps entre lespurelles elles s'exercent, l'énergie tolale est invariahle. L'inergirs totale est tume somme qui comprend deux esperess ir termes: les uns portent le nom d'énergire on dre force vive et s'expriment en fouction d $t^{2}$ la masse et (te) lat vitesse $\left(\begin{array}{c}\mathrm{mr} r^{2} \\ 2^{-}\end{array}\right)$, les autres s'appellent l'inergie potentiolle ret expriment le travail maximum que peuvent produire toutes les forces du systeme à partip de la position que loun considère jusqu’a l'état d'equilibre stable. Ainsi formule le principe de la conservation de l'energie est un theorême mathématique.

La conservation de l'energie est-elle realisere dans l'Cui-

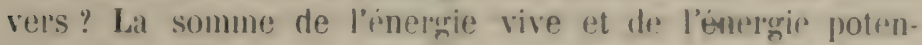
tielle y est-elle constante? Faute de pouroir u primi déterminer la nature des forces qui y agrissent, nous devons recourir à lexpérience pour résoudre cette yuestion. (O), nous connaissons avee me exactitule sullisante les phrinominns d'ordre materied (nous fasons pour le moment abstraction des phenomines vitaux dont nous aurons l'oceasion de parler dans la suite) pour pouvoir athirmes que, de lait, l'energie no: s'y eree point et ne s'y amnihile pas davantage, mais qu'elle se transforme et que ces transformations sont soumises a la loi d'equivalence. Celte alfirmation a la valeur qu'ont les lois physiques en géneral.

Nous pouvons en conclure que les conditions dr la conservation de l'ineregie sont realisées dans l'Cuivers, et yue, par consécfuent, les forces attractives et repulsives qui y)ésident aux transformations de l'énergie sont proportionnelle's aux masses, quelles ne dependent quant i lrur intensité ni du temps, ni de l'orientation de leur direction, ni du mouvement dont les corps sont animis, ni de quare(que circonstance que ce soit autre que la distance des corps entre lesquels elles s'exerernt. Yous sarons que la inasse des corps est invariable; mais leurs distances changent sans cesse et par conséquent aussi les forces qui dépendent de ce facteur. 
Notons encore que certaines forces tout en modifiant la direction du mouvement n'en altèrent pas la quantité ot ne transforment par conséquent pas l'énergie vive en energie potentielle ni inversement. Telle est la force centripete dans le mouvement circulaire. Cette force a un travail nul et elle pourrait disparaittre sans que la quantité d'énergie s'en trouvât altérée.

Le principe de la persistance de la force a donc besoin d'itre interprété dans le sens du principe de la conservation de l'énergie et dans ce sens il est susceptible d'une démonstration.

En remaniant le chapitre que nous étudions, Spencer a introduit, de mème que dans le chapitre précédent, la notion d'énergie actuelle et potentielle; mais la définition exacte de l'une et de l'autre fait toujours défaut et il en résulte une confusion inextricable.

Le nom de force est donni à l'énergie totale. Or on a vu plus haut que l'énergie totale n'est pas une force, et il rst facile de comprendre qu'un système peut contenir des forces qui ne représentent aucune forme d'énergie pour une situation donnée. Il est inutile que nous nous arrêttions a la distinction que fait Spencer. "Dans les deux chapitres précédents, dit-il, nous nous sommes occupés de deux classes fondamentalement diferentes de manileslations de force, - la force par laquelle la matiere nous drmontre qu'elle est existante et la foree par laquelle (1lle nous demontre qu'elle est active. ") (1) La matière IIr frut disnontrer son existence qu'en agissant directoment ou imdirectement sur nos organes et olle demontre? par là mèrne son activité.

lipres spencer, la prersistance de la premiche classe d. Inanifistations do forere correspond i l'indestruetibilite de la matipere, tandis que la persistance de la seconde correspond it la prepsistance du momrement anduel ou fotentiel. S"il rn est ainsi, on ne voit pas pourfuod la

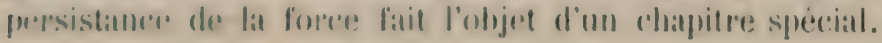

(1) P. P. p. 140 \& 800 . 
Spencer en donne comme motif que les raisonnements établissant l'indestructibilité de la matière et du mouvement supposent la persistance de la force. Dans le développement de cet argument il ne parle que des preures expérimentales. Le paragraphe ou était rappelée la preuve qui prétend établir l'inconcevabilite d'une destruction de la matière ou du mouvement a éte supprimé. Or, ce que dit Spencer au sujet des preuves experimentales ne perut être approuvé, comme nous allons le voir.

jersistanle la force -elle un tulatum?

Il est certain que pour mesurer les grandeur's matirielle's : dimensions, masses, forces, nous nous servons de certaines grandeur's prises pour unitis et que nos mensurations n'ont de valeur que si nos unites restent invariables. Lat question est de savoir si nous avons de bonnes raisons de croire qu'elles ne changent pas - les conditions restant les mêmes - ou si, comme le pretend spencer, leur invariabilite est un postulatum indimontrable.

Il n'est point absurde d'allirmer la persistance de la masse d'un corps que nous ne vogons subir aucun changement et qui n'est soumis a ancune action à laquelle nous puissions attribuer une moditieation de sit masse; surtout si nous constatons en outre que sa masse reste dans un rapport constant avee la masse des autres corps qui ne subissent aucune moditication sensible et qu'il continue à se comporter de la mime manière sous l'action des différentes forces auxquelles on pent le soumettre. Car si l'on pretend que sa masse varie, il laudra admettre que les masses de tous les autres corps varient dans la même proportion quoique ne subissant aucun changement sensible et de même toutes les forces qui agissent sur eux. $\mathrm{Si}$, au contraire, dans les conditions que nous venons d'indiquer, on admet que la masse d'un corjs est invariable, ce corps pourra nous servir pour véritier la masse des autres corps qui subissent des changements sensibles, atin de constater si oui ounon ces changements ont alfecté leur masse. 
Exaninons de près, comme Spencer nous y invite, cette verification dans le cas des changements chimiques. "Dans les recherches, dit spencer, qui conduisent le chimiste à conclure quaucune partie du charbon, disparu pendant la combustion, ne s'est perdue, et que, dans le produit qui en resulte, l'acide carbonique, on retrouve la totalité du charbon, quelle preuve invorue-t-on sans cesse? La fresuve fournie par la balance. En fonction de yuoi s'exfrime le verdict de la balance? En unités de poids, en unites de force gravitative. Et (fuel est le sens du verdict? Que le charbon présente encore autant d'unités de force gravitative qu'il en présentait auparavant. On dit que la (quantité de matière est la mème si le nombre des unités de force qu'elle contrebalance est la mème. Par conséquent, la validité de la conclusion dépend entièrement de la constance des unités de force. Si la force avec laquelle la parcelle de métal qui représente l'unité de poids lend vers la terre a varié, l'expérence de l'indestructibilité de la matière est vicieuse. Tout revient au principe ou à la supposition que la gravitation des poids est persistante; mais de cette persistance, nous n'arons et ne pourons avoir aucune preuve. » (1) D'abord, ce raisonnement confond l'unité de poids aree l'unité de force gravitative. Lat definition du gramme ne change: pas quelles que soient les rariations de la gravite. La mène masse de matière a toujours le mime poids, tant que la gravité, quoique variant, reste proportionnelle aux masses des diflèrents corps sur lesquels elle s'exeree. Ia determination des masses au moyen du poids supposse donc la constance de la loi d'apres lapuefle la gravitation est a chayue insfant rn mason directe des masses, mais ne suppose mullement la comstance de lat force de gravite.

Ensuite, il est vari que la determination de la masse does romps que l'on prise suppose l'invariahilite de la masse des comps yoion emploie comme monites de poids. Mais la masse niest pas whe force. Ce n'est done en ameun cas 
la persistance de la force quu est postulee. Il'aillours, s'il l'est flas "inconcevahle " que la masse des fieces de mital emplovées varie, nous n'avons cependant atcune raison de l'admettre, d'autant plus que cette supposition toute gratuite en entlaincrait une autle : c'est quo' le carbone et loxygene auraient en se combinant subi la mime moditication dans leur masse que lo morceau de metal qui a servi a les peser avant et apres la combinaison. ()r cela n'a aucune probabilité.

Il est done rad que dans toutes les monsurations on suppose que l'unitio de mesure reste invariable fou ne varir que dans la mème juchortion que le's choses yuton mesure), mais en gineral linvarialilito de cetle mesture se trouve conlirmee far les innombrables conclusions qu'on en a tirees et dont la veritio a ete controlée par des exfreriences indigendantes de cretle mesurr. Il en rest de mère de l'invariabilite des lois naturelles. L'experitence leur sert continuellement de contròle.

les astronomes supposent dans leurs calculs lat persistance des masses of de la loi dattraction qui dit que celle-ci est proportionmelle anx masses et ne varie quavec la distance. Si les resultats de leurs calculs sont toujours verities par l'expérience, c'est done que leur supposition est conforme à la réalite. A mesure que ces rérifications se multiplient, la probalitis augmente et si elle natteint jamais a la certitude absolue cela prouve simplement qu'il y a une diffirence entre une loi experimentale et une conclusion mathématique.

La persistance de la force serait encore, d'après spencer, impliquece dans le principe de l'action et de la reaction. Ce principe peut s'énoncer comme suit : chargue fois qu'un corps A attire ou repousse un corps B, celui-ci attire ou repousse le corps $\mathbf{A}$ avec une force égale.

"Allirmer, dit Spencer, que l'action et la réaction sont égales et opposées, c'est affirmer la persistance de la force. En réalité, l'aflirmation revient à ceci, qu'il ne peut y avoir une force isolie, partant de rien et aboutissant à rien, mais qu'une force manifestee implique une force 
antécédente égale, d'où elle dérive et contre laquelle elle réagit. De plus cette force ne peut disparaître sans résultat; il faut qu'elle se dépense en quelque autre manifestation de force, qui, une fois produite, constitue sa réaction; et ainsi de suite. Il est donc évident que la persistance de la force est une vérité dernière qui ne peut avoir de preuve inductive. " (1)

Il faudrait plutòt conclure le contraire si le raisonnement de spencer était juste, car le principe de l'action et de la réaction n'est pas un postulatum mais une vérité d'expérience. D'ailleurs, lorsque deux corps s'attirent ou se repoussent on peut indifféremment attribuer l'action et la réaction à l'un et à l'autre; il n'y a donc pas de "force antécédente"; en outre, personne ne donne le nom de réaction à la force que peut faire naître la disparition d'une autre force équivalente.

Nous concluons: $1^{\circ}$ Rien ne nous autorise à considérer le principe de la persistance de la force, quelque sens qu'on lui donne, comme un postulat fondamental sur lequel repose tout l'édifice de la science.

20 Ce principe n'est vrai qu'interprété dans le sens précis de la conversation de l'énergie. Spencer ne tente mème pas de montrer que le principe de la conservation de l'énergie est ce postulat universel dont il parle.

3. Le principe de la conservation de l'énergie appliqué à l'Chivers est une vérité d'expirience ayant la même certitude que les autres lois naturelles. Néanmoins l'induction ne permet pas jusqu'à présent d'etendre cette loi aux phémomines organiques. Nous aurons à revenir sur ce point; mais il ast necessaire de constater que spencer n'a cite jusqü pris prent aurun fait, ni fomrni aucun rasonnement uqui permefte de la leur appliquer. Nous verrons que les fmonves expremimentales dommes plus loin ne sont pas demonstratives.

f) Puisqur certaines forces ne produisent ancon travail

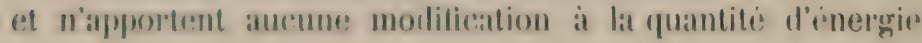

(1) P. P. P. 200-201 \& 89. 
vive ou d'énergie potentielle, le principe de la conservation de l'énergie ne nous apprend rien au sujet de cess forces.

\section{Force et} onnaissable.

Ia force qui persiste n'est autre, d'après spencer, que l'Inconnaissable. De fait, nous ne comnaissons pas les forces naturelles en elles-mèmes, directement, mais seulement par les efluts qu'elles produisent. II en est ainsi igalement de la force musculaire que nous déployons. Nous avons conscience de l'effort, mais non pas de la force que nous mettons en jeu. Spencer confond encore ici ces deux choses. " Yous ne connaissons imméliatement, dit-il, aucunes force, à l'exception de celle dont nous avons conscience pendant nos eflorts musculaires. " (1) Celle exception n'est pas justifies et plle contredit l'assimilation de la fore avec l'Inconnaissable.

Si nous ne connaissons pas directement les forces, nous les connaissons cependant asse'z par leur's reftets pour savoir qu'elles sont limities et changeantes et qu'on ne peut par conséquent pas les identilier avec la Cause Premiere à laquelle le nom d'Inconnaissable a efi l'eservé.

Spencer, dit Berthelot, " pretend relier les deux parties de son weuve par l'idee de force, mais ce n'est qu'a condition de confondre dans ce mot équivonue la force mécanique, simple rapport mathématique entre des changrements physiques, et la force inconnaissable, inconsciente, divine des romantiques, principe commun de l'esprit et de la matière. ” (2)

James Ward a vivement critiqué cette inlentitication. Spencer n’a pu la réaliser, dit-il, qu'en accomplissant " deux ou trois tours de jonglerie philosophique.... Líl persistance daus le sens de permanence est affirmée d'abord au sujet de la Réalité Inconditionnise dont la non-existence est inconcevable, quoique toute connaissance i son sujet

(1) P. P. p. $198 \$ 39$.

(2) Bulletia de la Sociéle française de Philosophie 1904 p. т. 
soit impossible, - puisque connaitte c'est conditionner. Ensuite la persistance, mais dans le sens de constance quantitative, est transférée de la Réalité Inconnaissable à ses manifestations phénoménales, et cela en affirmant à leur sujet un principe (la constance de l'énergie) que l'experrience ne donne pas le droit d'affirmer avec une rigueur absolue......... Nous obtenons l'existence absolue de la Force, avec majuscule, à condition de ne rien connaitre de ce qui la concerne. Ensuite nous obtenons la constance absolue de la force, avec minuscule, à condition d'énoncer des affirmations précises au sujet de l'Inconnaissable. Le saut périlleux intellectuel ainsi accompli rapidement est couvert en assumant que l'existence continuée implique la quantité invariable. ") (1)

On se rappelle que Spencer a identifié avec la force tous les éléments du monde phénomenal; dès lors la persistance de la matière et du mouvement ne sont plus que des corollaires de la persistance de la force. D'autre part, l'Inconnaissable qui se manifeste objectivement dans l'Univers, se manifeste subjectivement dans la conscience; en sorte que la persistance de la conscience dont nous avons l'experience se confond fondamentalement avec la persistance de la force et en mème temps nous impose la nécessité de l'affirmer.

Spencer a supprimé le passage où il développait cette synthese finale. Elle ne manque pas de grandeur dans sa simplicite, mais nous arons fait voir dans les pages précerlentes qu'elle ne résiste pas à l'examen. Ce qui en demente lorsqu'clle a ite passece au creuset est plus modreste mais aussi plus solide : Les phenomènes materiels sont regis far deux grandes lois: la conservation de la masse et la conservation de l'eneruge. Calle-ci comprend lat couservation au moins virtuelle du mouvement, l'invariabiliti des foreres a exaliti de distance et leur intensiti forpentionmello a la masse, ce qui permet de constaller lat fursistance de colle derniore.

(1) Fuluralism and agnosticasm. 11. 11. 219-2901. 
$\S$ IV. - La constance des lois (relations entre les forces).

L'évolution consiste dans une série ininterrompue de changements à travers laquelle persistent les lois. Si nous suivons par la pensée l'évolution du système solaire d'après l'hypothèse de Laplace, depuis la nébuleuse primitive jusqu'à la situation actuelle, si nous considerons la longue série des modifications qu'a dù subir chaque astre et en particulier la terre, avant d'avoir réalisé la solidification de sa surface et tous les détails de sa configuration, nous ne rencontrons pas, pendant cette immense période de temps, deux situations identiques et nous ne pouvons y signaler aucun point d'arrèt. Cependant depuis l'origine tous les phénomènes se sont accomplis suivant des lois qui sont demenries les mèmes jusqu'aujourd'hui.

Nous concevons que dans d'autres parties de l'C'nivers, d'autres systemes dans des conditions diverses, ont subi des évolutions analogues mais non parfaitement semblables, avec des phases plus nombreuses ou plus simples, dans des proportions plus vastes ou moindres. Et nous admettons que toutes ces évolutions ont été régies par les mêmés lois.

Tandis que les événements, les situations concrètes varient d'une époque à l'autre, sont diffierents ici et lii-bas, les lois au contraire sont indépendantes du temps et de l'espace : elles se véritient dans la nature partout et toujours.

Spencer déduit la constance des lois de la persistance de la force : c'est la raison pour laquaclle il en est question à cet endroit. En réalité elle en est indépendante.

D'une manière générale, les phénomènes naturels sont l'effet des forces de la nature. La constance des lois auxquelles se conforment les phénomènes, exige donc que l'action des forces naturelles soit indépendante de l'espace et du temps. La conservation de l'energie dans l'Univers provient de ce que les forces n'y varient pas 
autrement qu'en fonction des distances. La constance des lois naturelles est donc renfermée dans le principe expérimental de la conservation de l'énergie. Elie n'en dépend pas pourtant.

Dès qu'on admet que tout ce qui se passe dans l'ordre naturel a son explication, c'est-i-dire sa cause, dans la nature, et en particulier, que les mèmes causes produisent partout et toujours les mèmes effets, la constance des lois de la nature est garantie, ou plutôt, elle n'est pas au fond autre chose que cela. Elle repose donc en dernière analyse sur le principe de causalite et sur la croyance que les phénomènes ont dans la nature des causes fatales, non douées de liberté. Cette conviction est le fruit de l'expérience sauf en ce qui concerne les actions libres de l'homme, lesquelles ne sont pas déterminées par les lois physiques.

§ V. - La transformabilité équivalente de l'énergie. - Les phénomènes physico-chimiques et les phénomènes psychiques. - Les phénomènes organiques. - Les phénomènes sociaux. - Spencer et le matérialisme. - Matérialité des phénomènes psychiques. - Etude inductive de la loi de transformation. - Son application aux phénomènes organiques. - Son application aux phénomènes psychiques. Son application aux phénomènes sociaux.

La transfor. mation équivalonte de l'ónergie.

Les agents naturels: ('haleur, lumiere, electricite, mouvement visible ele, peurent se transformer l'un en l'antre, on plus exactrment, si on veut se horner a l'aflirmation du fait, ils se substituent l'un a l'autre de telle sorte qu'il y ait eqquivalroner, rest-i-dire, que lat memes quantite d'electricite, par exemple, produise toujours la mème quantiti de chatemr, lacquethe a son tour peut reproduire la quantile d'olectriciti qui lui a donne naissance, et ainsi 
nour les autres. C'rost lá une vériti bien ronnue aujourd'hui et en mime temps une des plus briles conquètes de la science.

Il en résulte que ces divors agrents naturels ont leur équivalent mécanique, et lepresentent, en faisant abstraction de toute hypothèsr, une certaine quantite d'eneregre La quantité totale d'energie leste la mime dans cous ces changements successifs. A cause de cela on les nomme transformations de l'energie plutot que transformations de la force, commo les applelle Spencerr. L'idée est d'ailleurs la même.

Ce fait d'experience apporto un approint solide à l'hypothese, comfirmee frar d'autres faits nombreux, d'après laquelle tous les phenomenes materiols (alstraction faite de la vie) sont exclusivement des phenomines de mouvement determines par les forors inherentes aux ultimes particules de la matirere, et sont explicablues par les lois de la méanique, certaines positions on certains mourevements des particules etant supposis comme point de départ.

Les transformations de l'enerorie ne sont plus dès lor's que des transformations de mouvements, et elles nous deviennent plus intelligibles parce qu'elles rentrent dans la caltegoric des phénomenes que constate l'experience quotidienne. Yous voyons le monrement du vent produire le mouvement des ailes du moulin et ensuite se translormet de mille farons dans les rongrenagres et sous les meules. Dans une multitude de dirconstances pareilles, le monve ment joue sous nos geux le roile d'un l'potere dont les metamorphoses sont sommises a des righles fixes qui nous sont connues et qui nous permettent de les prévoir at l'avance.

Cependant il importe de ne pas confondre l'lygpothèse d'apres laquelle les phenomenes phỵsico-chimiques set réduisent a des phenomenes spatiaux, alve le lait d'experience, c'est-it-dire le remplacement du mourement peregu comme

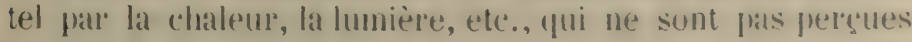
comme des mouvements. 
C'est donc à tort que Renouvier proteste contre la manière dont spencer présente le principe d'équivalence. " On se tiggure, dit-il, que le mouvement s'interrompt et que telle autre chose: chaleur, lumière, etc., en prend la place..... " En réalité " le mouvement des masses arrêté ne doit pas être dit avoir pour produits la chaleur, l'électricité, etc.; il a simplement pour remplaçant un mouvement vibratoire..... Et il est ridicule de dire que le mouvement des masses, quand il est engendré, préexistait comme quelque autre mode de force, puisqu'il préexistait au contraire, dans le sens le plus ordinaire et toujours le même de force, à savoir comme mouvement, seulement avec une distribution différente des parties mobiles et des vitesses. "

Que la lumière et l'électricité sont des mouvements de masses insensibles, ce n'est qu'une lyypothèse - que nous admettons volontiers - mais de la vérité de laquelle ne dépend pas la loi expérimentale de la conservation de l'energie. En outre, il ne faut pas oublier que le mouvement peut cesser et être remphacé par l'énergie potentielle ou énergie de position qui n'est pas un mouvement.

Las phénoaénes physip-chimiques et les hénomènes psychiques.

Après avoir établi le fait de la transformation de l'énergie au moyen d'éxemples nombreux, Spencer inaugure la methode qu'il va suivre jusqu'i la fin de ce traité. Flle consiste a faire l'apptication du principe qu'il expose aux diffirents ordres de phénomenes, depuis la condensation des astres jusfuna l'histoire des societes lumaines. Nous devons faire à ce sujet une remarque importante.

Arec spencer nous avons reconnu qu'une des plus fondamentales differences parmi les objets de nos perceptions est la distinction entre le moi et le non-moi. Nous n'avons pas admis que cartle distinction se confond avec celle que que lon prout fitire entre nos perceptions vives et nos fmereptions faibles. II nous faut ici considerer ce sujet d'un peu plus près.

Latissous de colti, pour le moment, les connaissances alstratios of ne nous occupons que des perceptions concretes 
qui se terminent a des objets individuels. C'est parmi ces dernières qu'existe la séparation qui nous occupe. Les unes, en effet, sont des perceptions de choses extérieures au moi, telle la vision d'une maison blanclie; ou, du moins, si les choses perçues font partie du moi, elles ne sont pas percues comme telles, par exemple, la vision de ma main blanche. L'objet propre de ces perceptions sont des manières d'être que j'attribue aux choses extérieures pour en avoir l'expérience directe. Je dis: Cette maison est blanche, ma main est blanche. J'affirme ce que je vois.

Les autres sont des perceptions de faits intérieur's au moi; telle la conscience que j'ai de voir une maison blanche, d'entendre le son d'une cloche, de désirer goùter d'un fruit. L'objet propre de ces perceptions sont des manières d'être que j'attrilue à moi-même pour en avoir l'expérience directe. Quand je dis : je vois une maison blanche, j'aftirme ce que je ressens.

Il est évident que cette proposition : Je vois une maison blanche, et cette autre: Cette maison est blanche, ne peuvent pas être confondues. Elles ne se véritient même pas toujours simultanément.

Les manières d'être que nous attribuons aux choses extérieures pour en avoir l'expérience directe, sont l'objet des sens qui, à cause de cela mème, portent le nom de sens externes; tandis que les manières d'ètre dont nous n'avons l'expérience directe que relativement à nous-mêmes sont l'objet de la sensibilite interne ou mieux et plus généralement de la conscience stricto sensu.

On dit que la blancheur de la maison consiste en réalité en une certaine disposition de ses motécutes superficielles qui réfléchit les vibrations lumineuses de telle sorte que celles-ci, frappant l'extrémité des fibrilles du nerf optique, me donnent l'impression de blancheur.

Ainsi la cause extérieure de ma perception de blancheur est un mouvement des particules matérielles. Cela peut être vrai ou faux, mais cela n'est point absurde.

On dit aussi que les rayons lumineux ne produisent 
pas autre chose dans les nerfs que des diplacements d'atomes et que ces déplacements d'atomes qui pourraient être provoqués par une autre cause, (par exemple, par un courant électrique) nous donnent l'impression de blancheur; et cela encore est plausible. Mais dire que l'impression ou la perception de blancheur elle-mème n'est qu'un mourement d'atomes, cela est aussi absurde que de prétendre qu'un triangle est la même chose qu'une journee, et nous ne pensons pas que personne ait pu l'affirmer en se rendant compte de ce qu'il disait. Une sensation et un mouvement sont des choses irréductibles; c'est ce que Spencer lui-même affirme en plus d'un endroit. (1)

Quoique la perception de blancheur, toutes les autres sensations et, en général, les faits subjectifs que la conscience proprement dite atteste ne soient percus directement que dans le moi, cependant nous attribuons les ctats d'àme semblables à des ètres qui font partie du non-moi. Non pas, à la vérité, à tous, mais seulement à ceux qui possèdent des organes analogues à nos organes des sens ou chez lesquels nous constatons des mouvements que nous jugeons avec une probabilité plus ou moins grande ètre la manifestation de situations psychiques.

Ainsi nous attribuons à tous les hommes, c'est-à-dire aux itres que nous voyons posseder la même organisalion que nous, des sensations, des désirs, des passions semblables aux notres. Constatant que l'organe de nos improssions visuelles est l'ail, nous attribuons des impressions semblables a tous les animaux chez lesquels moe fartire du rorps posside une configuration analogue. Voyant qur les animaux inferients raggissent far leurs momvenents au moindre contact, nous leur attribuons des phemomines pistelicgues phus ou moins semblables a nos sensations tactiles.

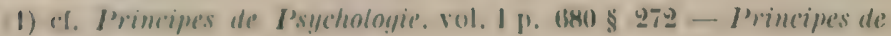
Biologie. vol. 1 p. 119839. 
Ces jugements par analogie sont regardés avec latison comme absolument certains lorsqu'il s'agit d'animaux d'une organisation et d’une activite cxterieure très-roisines des notres. Mais a mesure que certe ressemblance diminue, cette certitude s'evanouit, do telle sorte que pour heaucoup d'organismes nous ne sarons s'il faut leur attribuer ou leur refuser la vie psychique. Les phénomènes qqui constituent celle- $c^{\circ}$ ne sont done pas l'objet de notre expérience immédiate, satuf lor'squ'il s’agit du nous-mimes; nous ne les connaissons chez les allies itres que par une conjecture basie sur l'analogite qu'ils ont avec nous. Par la raison contraire, nous n'attribuons atcun plienomene psychinue aux corps inorganiques. Et cela confirme encore, quoiqu'elle n'ait besoin d'aucune confirmation, la dlistinetion entre ces phenomènes et les mourrments des atomes ou des molecules. Ce's mourements, en rellet, existent dans tous les corps. Ils echappent en geniral à nos sens a cause de leur petilesse et de leur rappidite; partois cependant, ils sont perengs sous furme de chaleur, lumiere etc. En tous cas ils rentrent dans la catrigorie des laits qui, par leur nature et comme tels, sont l'objet de l'experience externe.

D'après Spencer, et c'est sans doute ce qui rest au fond de la pensée de ceux qui ne veulent voir dans la vir psychique que des vibmations norveuses, il est à la verite impossible de confondre un deplacement de corpuscules arec un etat psychipue, mais c'est néanuins la mème réalité qui se manifeste sous ces deux formes: objectivement, ebranlement des nerf's ; subjectivement, sensation de chaleur ou de lumirre. Ce sont, d'apres lui, les deux laces d'une mème chose. "Unoiqu'il nous svit encore impossible, dit-il, de prouver que l'etat de conscience at l'action nerverusezont les fiters interne et extreme du mime changement, cependant cette hypothese s'accorde avec tous le's faits observes. ") (1) Telle qu'elle vient d'itre formulie, cette conception e'st inadmissible. D'abord, si

(1) Principes de Psychologie. vol, I p. 127-128 \$ 
les mouvements atomiques ont un revers qui est un état psychique, ils doivent l'avoir partout ou ils se produisent. Le rayon de lumière qui frappe la rétine d'un cadavre ébranle sans doute les fibrilles nerveuses, mais n'y produit aucune sensation. On répondra que ce mouvement n'est pas le même que celui produit pendant la vie, et nous admettrons cela quoiqu'il soit peut-être difficile de l'etablir. Mais quelle raison imaginera-t-on pour expliquer que certains mourements atomiques sont par leur revers des états psychiques et que d'autres ne le sont pas? Il est probable que les modifications produites sur les nerfs par les excitations périphériques sont d'ordre chimique, analogues par conséquent à celles que la lumière produit sur une plaque photographique. Pourquoi y a-t-il sensation dans le premier cas et pas dans le second? Pourquoi dans l'animal vivant y a-t-il sensation lorsque l'ébranlement arrive jusqu'aux centres cérébraux et pas sensation lorsqu'il s'arrète aux centres de la moëlle épinière? Ce sont des atomes semblables qui vibrent ou se déplacent de part et d'autre; tout au plus leurs dispositions ou leurs trajectoires diffèrent-elles dans quelques détails. Est-ce là que nous chercherons la raison de l'état psychique qui se réalise d'un côté et qui fait défaut de l'autre?

Si le cerveau d'un animal n'est pas autre chose qu'un amas d'atomes disposés de telle ou de telle sorte, comment concevoir qu'un rayon de lumière y éveille la vie psychique dont lui-même est dépourvu? Lor'sque frappant un melange d'hydrogène et de chlore, ce rayon provoque la combinatison de gaz et l'explosion du récipient, l'eflet produit est dans le mème ordre que la cause : ce ne sont ugur des mourentents de part et d'autre. Mais il n'en est phus ainsi lorsque l'effet produit est une sensation de lumière.

Qun: faut-il done dire? C'est qu'rividemment la sensation est lo: fitit d'une astiviti sui generis existant dans le cervean vivant; que l'élnaulement des nerfs est, non pas l'ondroit d'un phenomine dont elle serait l'envers, mais bien l'aceompagnement nécessaire, la condition, la détermination intrinseque du fait psychique. 
L'impossibilité d'identifier celui-ci avec le phénomène physico-chimique résulte encore du degré d'unité ques le premier possède et que le second ne possède pas. Ou se passe la sensation? C'est un problème difficile à résoudre ; mais on admet généralement qu'elle a son siège dans les centres nerveux. Tant que la sensation existe, il $y$ a dans ces organes des phénomènes matériels complexes, juxtaposés dans l'espace et se succédant dans le temps, mais ne réalisant aucune intégration qui en ferait une action unique. Tout autrement en est-il de l'état psychique correspondant qui possède, lui, une unité véritable: e'est une seule sensation qu'un seul individu eprouve tout entière.

On a parfois voulu explicfuer la sênsibilité des animaux en attribuant une sensibilite rudimentaire à chayue atome. Hypothèse gratuite et, en outre, inutile. Si mème, dans l'animal, chaque atome sentait, cela ne suffirait pas pour que l'animal sente. L'organisme comprendrait une multitude d'ètres sentants et de sensations, mais on n'aurait pas encore le principe de la sensation unique de l'animal.

Ensuite, si l'acte psychique n'est que le rever's du phénomène psychique, non seulement il doit y avoir correspondance exacte entre l'acte psychique consideré dans sa totalité et les mouvements de la substance nerveuse, mais il faut qu'à chaque partie élémentaire de ces mouvements, à chaque atome qui y prend part et i chaque partie du mouvement qui l'anime, voire mème à chaque dimension de l'espact qu'ils occupent, à leur masse, à leur situation et ainsi de suite, corresponde un élement distinct de phénomène psychique. Or un tel parallélisme est manifestement impossible à établir (1).

Enfin, c'est une chose évidente, admise par le bon sens et par la philosophie, que la vie psychique exerce une influence réelle sur les mouvements de l'animal. En effet, elle ne comprend pas seulement des actes de perception dans lesquels l'être vivant est passif et ne fait autre

(1) Cente dernitre consideration est developpée par A. E Tar.or. Mind and Body in recent Psycology. Mind. Octobre 1904. p.p. $483 \mathrm{sq}$. 
chose que ressentir les impressions qui viennent de l'extérieur. Les connaissances qui sont ainsi acquises éveillent des désir's, déterminent des réactions intentionnelles. Les mouvements de l'animal ne sont point comme ceux d'une machine, ils sont guidés par la connaissance et par les tendances qu'elle fait naitre.

si les lois physico-chimiques et les forces inhérentes aux atomes suffisent pour expliquer toutes les manifestations raterieures de la vie, le ròle dles facultés animales est tout i fait superflu. Il sera faux de dire que l'animal s'empare de sa nourriture et la dévore parce que la faim le pousse ou qu'il a eu l'expérience du goùt agréable de la viande. Il faudra admettre qu'en réalité et en dernière analyse ce sont les forces attractives et rejulsives des atomes qui déterminent à elles-seules tout ce que l'on considère comme la manifestation de la sensibilité tet de l'intelligence. Lorsque Virgile écrivait l'Enëide, sa plume itait donc conduite exclusivement par les affinites chimirues et les propriétés physiques des tissus musculaires et nerveux, et d'une manière générale, toutes les aurres du grénie de l'homme se seraient réalisées absolument de meme si l'intelligence de l'homme n'avait jamais existé, puisque dès l'origine et avant qu'un citre vivant existat sur notre globe jusqu'aujourl'hui, tous les mourements de tous les atomes ont toujours èté déterminés par les forces physico-chimiques, et qu'il est inconcevable qu'ure situation yuelcongue d'atomes entraine par elle-méme l'intelligence.

Cette conception se tronve done reclue par lo propre argument de Sprencer ajoute dans l'edition de le000 at l'ondeoit des Iremiers Principes que nons efudions. II remouse l'opinion de Huxley suivant lequel la sensation n'est yüun phemomine concomitant de l'action nerveuse of n'est

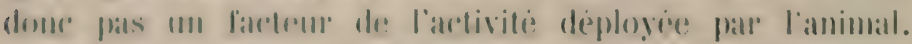

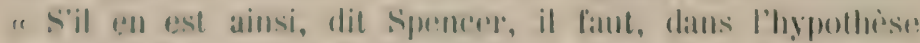

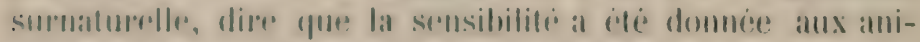

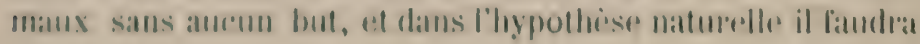


admettre (qu'elle a pris naissance pour ne rien produire ) (1).

Nous dirons de mème: si la sensation n'est qur l'enwer's du mouvement moléculaire, tout se passe donc extérieurement comme si cet enver's n'existait pas. Comment expliquer dès lors l'origine ou l'existence de la sensibiliti puisqu'elle ne joue aucun rôle dans la série des phénomèness objectifs qui constituent la vie de l'animal?

Nous avouons ne pas parvenir à concilier l'ophinion de Spencer d'après laquelle la sensation n'est que l'envers de l'ébranlement nerveux avec celle qui attribue à la sensation un role elfectif et qu'il adopte ici. Le passage suivant qui apparait aussi pour la première fois dans l'édition dp 1900 , semble d'ailleurs indiquer un changement dans ses idès.

"Qne la sensation soit seulement 1 an accompagnement de certaines actions nervases, ou qu'bllp soit, comme nous venons de conclure, un factemr dans cess actions, la connexion entre les deux rest inscrutable. Si nous supposons que la chose qui est le siène de la semsation est une réalite immaterielle qui n'est pas enveloppee dans ces actions nerveuses mais qui rst néammoins aflecters? par elles de manière a produine la sensation, alors nous sommes obligis d'amettre que des changements materiels - des mouvements moléculaires - produisent des changements dans une chose ou il n'y rien qui puisse ètre mis en mouvement; et nous ne pourons pas conceroir cela. Si, d'autre part, nous concevons une relation entre cette chose capable de conscience et rertains changements nerveux de telle sorte que les sensations qui y naissent déterminent par ce moyen des mouvements musculaires, nous rencontrons la même difficulti par son còté opposé. Nous aurons à almettre une réalité immatérielle - daus laquelle il n'existe pas de mouvement moléculaire - capable d'influencer des mouvements moléculaires : nous devons lui attribuer le pouvoir de produre des ellints qui, pour autant que nos connaissances s'etendent, peuvent unique-

(1) F. P. p. 176. ef. Romaxes, Mind Motion and Monism. Londres. $1896 \mathrm{p} .71$ 
ment ètre produits par des forces matérielles. De sorte que cette alternative est en dernière analyse inconcevable.

"La seule supposition avant de la consistance est que la sensation est inherente à l'éther qui pénètre tout. Nous savons qu'il peut subir l'action des molécules de matière en mouvement et que de son còté il peut agir sur les mourements des molécules, témoin l'action de la lumière sur la rétine. D'après cette hypothèse nous pouvons admettre que l'éther qui penètre non seulement par tout l'espace mais encore dans toute matière, est capable d'être affecte par les changements nerveux dans certaines conditions et dans certaines parties du système nerveux de manière à concevoir des sensations et que réciproquement il est capable dans ces conditions d'influencer les changements nerveux. Mais si nous adoptons cette explication, nous devons admettre que le pouvoir de sentir est universel, et que l'évolution de la sensibilité dans l'éther n'a lieu que dans des conditions très complexes qui se réalisent dans certains centres nerveux. Néanmoins ce n'est là qu'un semblant d'explication, puisque nous ne savons pas ce qu'est l'éther et puisque de l'aveu de ceux qui sont les plus capables d'en juger, aucune des hypothèses imaginées ne rend compte de toutes ses proprietes. " (1)

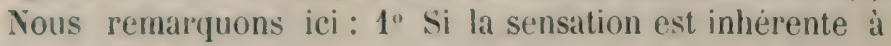
l'ither, elle n'est donc pas à proprement parler "le mème changement "que l'action nerveuse.

20 La vibration nerveuse determine-t-elle dans l'ether des mouvements qui a leur tour se transmettent aux nerfs d'apres les lois de la mécanique? Si oui, quel est le rôle de la sensation? Si non, comment est-il vrai de dire que ce qui se manifeste à notre conscience sous forme de sensation de de pensie est la mème chose que ce qui se manifeste exterieurement sous forme de matiere et de mouvement ? (2)

3o Nous ne concevons aucune raison d'attribuer la

(1) F. P. P. 177

(2) Cf. Irinc de Biol. vol. I p. S98. Appendice 
sensibilité à l'éther plutôt qu'à la substance vivante pondèra ble des nerfs. Il y a au contraire de bonnes raisons pour ne pas attribuer à l'éther la sensibilité ; car elle appartient évidemment aux seuls animaux, de manière à ètre une fonction propre a chaque animal, puisque chaque animal constitue un individu sentant distinct de tous les autres.

4. Comme nous allons le voir, la doctrine spiritualiste qui enseigne l'existence d'un principe de vie distinct de la matière, n'exige nullement que le mouvement dess atomes affecte une substance immaterielle. La difficulté des relations effectives entre l'esprit at la inatière n'existe que pour l'homme dont l'àme est sp̧irituelle. Les philosophes spiritualistes et notamnent l'école péripatiticienne ont donné à cette difficulte des solutions très acceptatbles et certainement moins inconcevalles que co que sprencer admet au sujet de l'éther.

II $y$ a, a notre avis, corretation entre la nature de l'animal et l'activite psychique qui le caracterise. Qu'on reunisse tous les atomes nécessaires pour former un organisme et qu'on parvienne it les mettre exactement dans la disposition voulue, pouvons-nous concevoir que ce seul arrangement d'atomes constituera la vie, la sensibilite? Non. Il faudra done en outre que l'organisme ainsi constitué soit animé, qu'il possède son principe de vie s'unissant intimement avec les atomes de manière i constituer une seule substance qui sera le corps vivant. De même, pouvons-nous concevoir qu'un ébranlement d'une certaine forme constitue une sensation? Pas davantage. Il faut que ce soit l'ébranlement des nerfs de l'animal virant, il faut que cet ébranlement soit comme animé par l'exercice de l'activité psychique. Comme il n'y a pas d'animal sans corps, ainsi il n'y a pas de sensation sans ébranlement nerveux. Mais de mème que le corps seul ne constitue pas l'animal et que sa vie n'est pas simplement l'enver's de la matière qui apparât aux yeux, mais consiste dans un principe substantiel propre, ainsi l'ébranlement nerveux n'est pas a lui seul l'acte de la sensation. Et il en est de même des autres activités psychiques. 
On comprend par ce que nous avons dit que nous considérons la vie psychique comme une force, cest-ádire comme une cause de mouvement. On pourra admettre en général que les mouvements qui ont leur siège dans les nerfs centripetes, qu'ils soient ou ne soient pas accompagués de sensations, sont absolument déterminés par l'agent excitateur. Mais une fois l'ébranlement transmis aux centres, l'animal réagit et il faut dès lor's admettre que dans cette réaction les mourements des atomes dépendent de l'activité pșychique. De savoir où son influence s'exerce, c'est une question secondaire; mais puisque la réponse a l'excitation part des centres nerveux, c'est lia, en tous cas, que cette intervention se produit, ce qui n'emp̧êche pas qu'elle n'aît lieu encore ailleurs.

Les phénomènes physicochimiques et les phénomènes organiques.
Si maintenant nous limitons nos considerations aux faits qui constituent le non-moi, nous devons encore nous garder de juger a priori quils sont tous de même nature, que tous obéissent aux mèmes lois. Nous devons aussi nous abstenir de résoudre ces questions à la légère et d'après un coup d'œil superticiel.

Quoiqu'il soit peut-être difficile d’indiquer les limites précises qui séparent les corps vivants des corps non vivants, cependant ces deux catégories d'êtres materiels se distingurnt clairement l'une de l'autre dans leur ensemble. Jes corps vivants possedent, d'une maniere? groncrale, tontes les propriétes des corps inorganiques; mais ils manifestent en outre une activite propre a laquelle on a domnt le mom de vie organique ef qui existe avec des diflerrolues relativement pru importantes dhez les animaux et chez les plantes.

Colle antiviti est-elle due tout entiore et exclusivement anx forres mrmentaires des atomes qui constituent

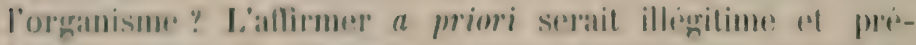

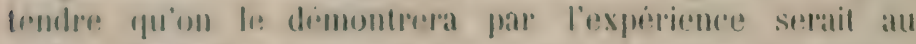
moins limiratre. In contraire, depuis spencer, la science, 
Sans itre arrivé ¿ lessoudre délinitivement cettr question, a néanmoins fait du chemin vel's la solution opposée. De plus en phus les biologristes renoncent à explinuer les phenomenes vitaux par les proprietés physico-chiminues de la matire of inclinent a restaurer la force vitale. (1)

Co qui a déconsideré cette dernière, c'est le róle excessil et alloitraile qu'on lui attribuait en l'invorquant comme le deus ex machina change fois fu'on ne parvenait pas i rendre compte d'un phrinomine biologique. On a té alisi amene à attribuer a l'activitis propres du principe vitul des phenomenes qui ont pu itre réalises plus tard dans les laboratoires. En outre, far l'exageration d'une idese juste an fond, on eoncevait la force vitale comme etant constamment en lutte avec les forces physico-chimiques; on considerait meme conte lutte comme constituant la vie dans ce qu'elle a d'essentiel.

On ne poura recourir de plein droit a la force vitales dans les explications seientiliques, yue lorsquon aura ditini Sil nature et les hormes de son intluence. En attrudant, plusieurs biologistes s'applipuent à demontrer qu'elle existe? et (qu'il faut renoncel a interpreter mecaniquement les phénomènes or'ganiques.

Le seul moyen de demontrer positivement qu’il ne faut pas admettre de force vitale, ce serait, ou bien d'expliquer les phénomènes organiques au moven des forces de la matiore brute, ou hien de montrer celle-ci engendrant dans le fait un etres vivant. On est alujourd'hui plus éloigné que jumais de pouroir satislaire à l'une ou à l'autre de ces conditions.

En 1872, Spencer répondait à Martineall que l'abime sépamant la vie organique du monderorganique va se comblant sans cesse. (2) Cette appréciation ne peut plus se soutenil. spencer declare avoir omis la partie de sa synthèse dans

(1) if. Gritiont. Le montement antimécaniciste en Biologie. Revue des Questions Scientitiques. Octobre 1903.

12. Contemporary Review. Juin 18т2. Article repris dans Essais. Trad. BLRdeAU Paris 1879 T. III p. 20. 
laquelle aurait dù être traitee la question de l'évolution inorganique. Le dernier chapitre de cet ourrage, dit-il, " traiterait de l'évolution de la matière inorganique, l'étape qui précède l'évolution des formes vivantes. Aỵant toujours présente à ma pensée la matière de ce chapitre qui n'a pas eté écrit, je me suis quelquefois exprimé comme si le lecteur l'avait devant les yeux et je me suis exposé par là à voir quelques unes de mes propositions mal comprises. »(1)

J. Ward (2) raille spirituellement Spencer à ce sujet. Qui est le plus à plaindre du lecteur exposé à mal comprendre Spencer depuis trente ans que manquent ces deux volumes, ou du philosophe lui-même qui gros de cet ourrage qu'il n'écrit pas doit se résigner à voir ses doctrines détigurées? En attendant les biologistes ont peiné dur et sans résultat pour resoudre le problème qui reste un ingstère pour tout le monde excepte pour spencer.

Celui-ci ne doute pas que les forces atomiques ne suffisent à produire a la longue des formes vivantes. Dans la leltre-appendice gui se trouve à la fin du premier volume des Principes ode Biologie, l'organisation caractéristique de l'itre vivant est attribue aux lois d'équilibre qui determineraient la configuration de l'organisme, comme elles determinent celles du cristal. La complication plus grande du premier serait due uniquement it la complexité extrême des molecules constituant les tissus.

La mème doctrine se trouve indiquée éralement dans le corps de l'ouvrage. "La forme de chaque organisme, dit spencer, est déterminée par une particularité dans la constitution des ses unités, et ces unités ont une structure speciale dans laquelle alles tendent it s'arranger comme en ont les unites de matiere inorganique.... Lne plante ou un animal d'une espèce quelconque se compose d'unités sprecciales dans chacune desqualles reside une aptitude intrinseinue is s'agreger dans la forme de cette espèce: c'est

(1) Principes de Biol. vol I p. 378. Appendice.

(2) Naluralism and Agnosticism, p. 263. 
ainsi que dans les atomes d'un sel reside mure aptitude

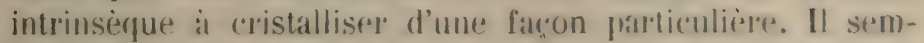
ble diflicile de concevoir yu'il puisse ell retre alinsi; mais nous voyons qu'il en est ainsi m (1).

Le: fait, c'est que l'ètre vivant posicile lo: pouvoir de réaliser une structure deterninee, at que dans les organismes relativement infrieurs une partip meme assoz pethe peut regenerer le tout. Mais on ne peut aflirmer commes fait ni la production de cette structure flall des unités physioloyiques qui seraient un "intermediatre entre les unités chimiques et les unites morphologiques (cellules) ) (2), ni l'assimilation des forces qui produisent l'organisme à celles qui produisent le cristal.

Un cristal se distingue d'un organisme de bien de's fatgons. Dabord une rondition de sat structure c'est l'homogénéité de sal composition. C'est parce qu’il est complosé de molécules identiques on tresi-roisines que sa forme régulière est possihle: le cristal est essentiellement homogène. Or', les organismes sont caracterist's frar une heterogénéite très grande. Cente circonstance s'opplose à la conception d'unites physsiologicuess qui seraient caracteristiques de l'organisme et qui devarient donc se trouver identiques dans tous les tissus malgre les differences de structure et de composition chimique.

Tout au plus pourrait-on admettre que ces unites se rencontrent dans le's tissus, mais rien constituent qu'une petite partie, ce qui augmente d'autant la dilliculte d'expliquer comment l'organisation tout entièrce est déterminée par leur structure caracteristique. De ce qu'un morceau de la feuille du begonia mise en terre sutlit pour reproduire la plante, on conclut logriquement que les principes déterminants de l'organisation de cette plante se trouvent dans les parties qu'on en detache. Mais ce fait ne nous donne atteun renseignement sur la nature de ces principes ni sur les forces qu'ils mettent en jeu pour produire ce résultat. Qu’il soit dû exclusivement ¿̀ la

(1) Princ. de Biol. vol. I p. 217-218 §65.

(2) Ibid. p $221 \$ 66$. 
stucture dinites physiologiques comme la forme du cristal est due a la structure de ses molécules, c'est ce yu'on ne peut conclure yu'en admettant a priori l'identiti des foreses organiques avec les forees de la matière brutr. Yous pourons expliquer au moyen des lois de la mécanique comment la forme des cristaux est une consequence de la structure de leurs molécules. Les théories cristallographiques permettent de déterminer a priori quelles sont les formes que peut revitir la matière cristallisée. Rien de pareil n'existe pour les formes vivantes. L'invaisemblance qu'il y a à ce que la forme d'une plante ou d'un animal puisse jamais être dèduite, au moyen des lois de la mécanique, de la structure de ses unités phỵsiologiques qui, en toute hypothèse, ne constituent qu'une petite partie de sa masie, rend au moins tries probable l'existence de forces autres que celles qu'on rencontre dans les cristaux.

Ensuite, la forme cristalline est essentiellement une situation d'équilibre, tandis que l'organisme ne présente pas dans son ensemble une situation d'équilibre proprement dit, mais seulement d'équilibre mobile qui implique des changements continuels.

En troisième lieu, quoiqu'une mème substance chimique puisse aflecter des formes cristallines difterentes, cependant celles-ci sont toutes dérivees d'une forme primitive géometriquement diterminée et dépendant de la structure de la molécule. Elles sont toujours d'une grande simplicité. Larsque les molicules se compliquent, nous ne voyons pas les formes cristallines affecter une complication correlative. Ies formes restent simples, mais deviennent de plus en plus dithiciles a réaliser, de sorte que pour les corps tris complexes de la chimie organique le phinomine de la cristallisation n'existe plus. L'organisme an comtraire est largement plastique; sat forme n'a rien d.e geometrigue ot elle est extrimement compliquese.

Entin, IIn cristal n'est a aurrun degroi 111 organisme. La lorme de celui-ci est correlative a des fonctions qu'il doit resprom, landis yue la forme d'un cristal n'est 
relative at ancume fonclion, mais srulement a la structures des molécules.

Pour que la conception dre spencere lut admissible, il faudrat expliquer comment unte contigutation exigre pal lexpuilibre dres moites physiologiques est en memr temps adaptée a l'exercice de l'activite vitale. Premons exemple: concret. D'apres spenerer, (1) la circulation de lat sève dans des directions disermineses a ru pontre résultat.

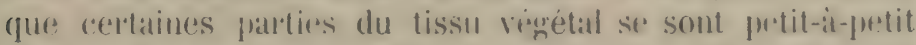

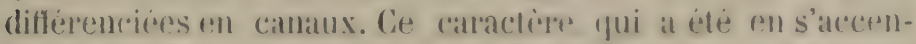
fuant dans les genorations suressives graich a l'herédite est devenue une partir essentiplle de la structure des vergetaux superieurs. Si nous almettons rette explication, nous pouvons compremalre comment une structure sprecifique s'est formere en vure d'une fonction. Mats comment concevons-nous que cettr structure est exigee en mime

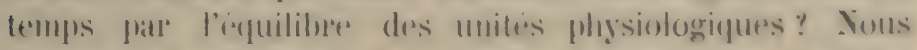

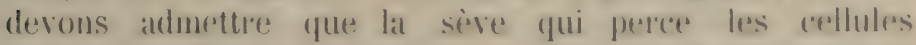

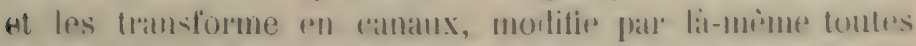
les unites phǫsologripues du vougrtal de manière que la forme deefuilibre suit doremavant un tissu vasculater. Suprosant fune la circulation de lat sive exerce tume inlluence sur la struchure des unites physiologigures, quelles relation roncevons-nous entre le clecusement dre allans et une modification de ces unitis telle yur leur situation d'inguilibre represente une strutare vasculare? Et ceperndant

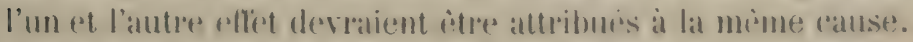
Pour fontes aes raisons il est impossible de fonsiderer lorgandsme fomme le résultat do la cristallisation d'une substance organipate d'aillems's impossible a definir.

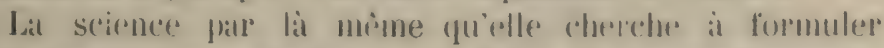
des propositions genemales est fiate de rapprochements. Mais pour que ces rapplochements soient ligitimes, il fiut yu’ils ne: s'arretent point it la surface. si cretaines similitudes peuvent parfois sugremer utilement drs gineralisations qu'une itude plus approbondie viendra ronfirmer, il faut se rappelel anssi qưil n'est pas moins

(1) Princ. de Biol. vol. \$11 p. 306-308 \$280. 
impurtant, an point de vue scientifique, de reconnaitre les ditlërences des phénomènes que leurs ressemblances. Il peut etre interessint de rapprocher l'organisation des itres vivants de la structure cristalline en tant que dans l'une pt dans l'autre il y a une forme caracterisant une especer, de comparer la cicatrisation des cristaux avec la régenteration des tissus vivants, la nutrition de ceux-ci avec laugmentation des corps cristallins dans les solutions, la multiplication des plantes et des animaux avec la production des cristaux dans les solutions sursaturées ou dans les liquicles à l'etat de surfusion par le contact d'une particule eristalline. (1) Mais au fond, ce ne sont la que des analogies lointaines et plùtot faites pour frapper l'imagination que pour satisfaire l'esprit.

Quelle utilité scientifique peut-il y avoir at comparer la naissance des planetes par séparation d'avee la masse centrale dans un systime astronomique en formation, avec la multiplieation des cellules par bourgeonnement, comme le fait G. H. Darwin (2) ? Au inoven d'interpretations ingénieuses il est possible, sans doute, do faire la "physiologie des mélaux ", de décrire leur fatigue et ses remides, leur patholonie, leurs empoisonnements, leur sensibilite, teur mort. (3) Mais n'est-ce pas faire de la science un peu fantaisiste?

En tous was, ces assimilations ne font en riph avancer la question de l'explication de la vie organique au moyen des forcens de la matiene brute et ce llest pas au moyen de considrations aussi superlinielles fuon parviendrat jamais is la risondre. "les hiologistes moderne's, exrit bord kelvin, en arrivent, je pense, une fois de plus a la croyance certaine gu'il y a quelgue chose atu delit des

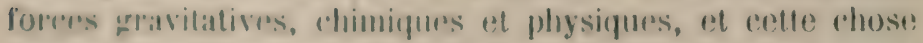
incommere est un frincipe vital.... II y a quarante ans

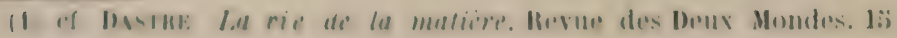
(3) 1801.

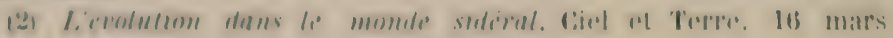
19013 ค. 27

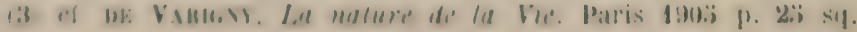


je demandai à Liebig, en me promenant arec lui à la campagne, s'il croyait que l'herbe et les tleur's que nous voyions autour dr nous croissaient en vertu des seules forers chimiques. Il me répondit: non, pas plus que je ne puis croire qu'un traité de botanique qui les décrit puisse être engendré par ces forces. " (1)

hénomé- Ce que nous venons de dire des faits d'ordre organisociaux. que et psychique doit s'appliquer a plus forte raison à l'évolution des sociélés humaines. Nous disons: a plus forte raison, parce que critte évolution ne dépend pas seulement de ces mimes phénomines, mais en outre d'un farteur que nous n'avons pas mentionne jusqu'ici : la librerté de l'homme.

Les faits sociaux ne sont pas des phenomenes primitils ou elenentaires. De mème que la socicté rst composese d'individus, de mème l'activite sociale n'est yue la somme organisée des activités individuelles. Celles-ci étant irréductibles aux forces de la matiere organique, il faut en dire autant de celles-lá.

Spencer, nous l'avons déjii dit, confond, dans l'ordre jsychique, les phénomènes de la sensibilité avec ceux de la vie intelligente et nie la liberté. Mais il $y$ a de bomnes raisons de ne pas le suivre dans ces théories. II est donc impossible de ronsidérer les faits sociaux comme n'étant qu'une transformation de l'énergie physico-chinique.

Yous n'aurons pas de difliculté à inontrer l'insuftisance des arguments sur lesquels spencer tente d'appuyer l'ilentité fonciere de tous les faits à quelque ordre qu'ils appartiennent.

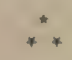

natéria- Le lecteur peut juger par l'exposé que nous pn faisons isme Spencer. de ce que la philosophie de spencer est en fait. La

(1) Ninetheenth Century 1903 vol I p. 1069 
question de savoir comment il faut l'appeler est de moindre importance. Nous ne nous y arrêterons pas longtemps. Mais puisque nous parlons ici de la manière dont spencer interprite les différents ordres de phénomènes, c'est le moment d'en dire un mot.

spencel proteste à plusieurs reprises, et mème vivement, contre la qualification de matérialiste donnée à sa philosophie. (1) Le matérialisme n'est pas la négation de l'esprit, - cela serait trop absurde, - mais il est l'affirmation de l'existence d'une seule réalité, celle que nous connaissons par l'expérience sensible, la matière, et d'une seule espèce de phénomènes, les mouvements des particules matérielles, qui constituent la pensée comme ils constituent la chaleur et la lumière.

spencer admet que cla réalité manifestee hors de la conscience sous les formes de matière et de mourement est lá mème que celle qui se manifeste dans la conscience sous forme de sentiment et de penséen (2); "qu'il y a une seule et mème realité ultime qui se manifeste à nous subjectivement et objectivement. " (3) Cotte these, c'est le monisme. Or le monisme de spencer est mécanique, c'est-a-dire matérialiste. Il tient que tous les efrets de l'Inconnaissable "se réduisent à une seule espice d'uflets, ) et toutes les lois auxquelles ces effets olveissent "à une seule espèce de loi." (4) Or ces lois sont les lois du mouvement, et res effets sont des mouvements dans le sens propre, e'est-it-dire des plumomìnes niateriels.

Gur repond spencer is ce raisonnement? « L’interprétation dre lous les phenominos, dit-il, en fonction de matière, dre monvement et de force noest rien de fulus que la rroduction do nos idires symbolipues complexes à des symbole's fulus simples, ret, lorsque l'épuation it etri réduite

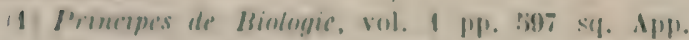

(2) Princ. de Biol. vol I p. S88. App.

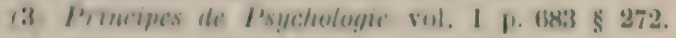

(1) Princ, de Biol, vol, I p. 599 App. 
a sid plus simple exponsion, les symboles n'en sunt pas moins des symboles. Par suitr, les raisonnements qu'on peut suivere dans less pares précedentes ne fommissent aucun appui à aucunf des hypotheses rivales sur lat nature ultime des choses. Ils n'impliguent pas plus le materialisme que lo: spiritualisme el pas plus le spirilualisme que lo: materialismo. Tout amsument qui sembls militer en faveur d'une de ces hypotheses est alussitit nutrat-

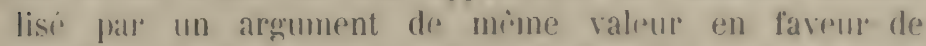
l'autre. Le materialistr voyant que pall une diduction necessaire de la loi de corrélation, ce qui existe dans la conscience sous forme de sentiment feent st transformer en un éfuivalent méandifue, et frar conséyurent en éguivilent de toutes les antres forces manifestires pall la matière, peut croire dimontree lat materialitis des phenomrines de conscirnce. Mais le sprititualiste partant de la mime donnee, pent somtenir alvec lat mème anterite que si les forees defplopires farr lat mathiere ne sont connaissables que soms la fomme de ces mimes fopuivalents de conscience qualles moduisent, il lant ron conclume que cos forces, fuand elles existent hors de lat conscience, sont de la meime nature que lorsquidles existent daus la conseience ; rt qu'ansi se justitie la conception spritualiste, d'apres latquelle le monde axterieur consiste dans quelque chose d'essentiellement identique avec a pue nous appetons l'esprit. Evidemment le principe de la corrélation et de l'épuivalence les lorces du monde interieur et du monde exterien peu t servir a les assimiler les unes aux autres, selon que? nous fartons de' l'un on de l'autre. Maris ceux qui comprennent bien la doctrine de cel ouvaiges verront quateun de ces deux termes ne doit ètre pris comme fondement. Bien que la relation du suject et de l'objet nous oblige a cos conceptions antithriques de l'Fsprit et de la Matière, l'une est tout autant que l'autre le signte de la Realiti inconnue qui les supporte l'une et l'autre. » (1)

(1) P. P. p. $398-399 \& 194$ - F. P. p.p. $445 \%-446$. 
Spencer caracterise très-inexactement la théorie spiritualiste. Celle-ci n'enseigne pas l'identite du monde extérieur avec l'esprit, mais bien la distinction entre l'esprit et la matière, entre les phénomènes materiels et les phénomènes spirituels.

Cette distinction n'est pas iquivalente à celle qui sépare le sujet et l'objet. L'esprit n'existe pas seulement dans le sujet et tous les phénomènes subjectifs ne sont pas des phénomènes spirituels. Le spiritualisme professe donc qu'outre les êtres matériels que perooit l'expérience sensible, il y a des ètres spirituels qui échappent à la sensibilité. De ce nombre est la Cause Première et, d'une façon gimerale, les substances douées d'intelligence at de liberte. Chacune de celles-ci a conscience de sa propre activité et dans ce sens seulement, les phénomènes intellectuels pervent etre appeles subjectifs. Mais pour chacune aussi l'activite des autres substances applartient au monde objectif au mème titre que less phénomènes malériels.

"L'interpretation de tous lis phenomènes", y compris les phénomènes intellectuels, "en fonction de matière, de mouvement et de force ", c'est bien le matérialisme. It ne trouve aucume justification en ce que prétendument "ce qui existe dans la conscience sous forme de sentiment peut se transformer en un equivalent de mouvement mecanique s; car une telle transformation n'a pas lieu. Daus l'homme lonte activité subjective comporte un certain mouvement organique, mais cette circonstance ne permet pas de les identifier.

La connaissance rant un phemomène subjectif, les objets

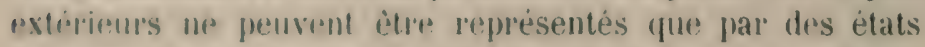
de conscience, ce qui n'est pas la meme chose que de les represeruter "soms forme d'etats de conscience" et ne domme dome aurun droit de les assimiler a lesprit.

Yous nu punvons, dapmes spencer, nous representer, dans l'ordre phenomenal, la cause des cotats de eonscience yue comme ume de colle's " pui se mamilestent dans des existronees que nous rangeons dans la catherorie de la matiere. 
soit notre propure corps, soit les olpjets muvirommants. " (1) Encore uno fois, c'est bien te materialisme.

Toute la reponse de spencer revient a faire observer. que, d'après lui, nos connaissances ne representent fas

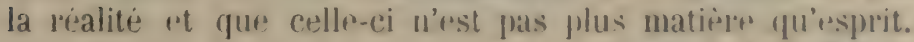
C'est à cela aussi que se rriduit sa réplique à d. Ward. A l'objection (fu'il n'est pas possible de tirer d'unc thiorie ce quielle ne contient point, ni dr desluire la vie et l'esprit d'elements mécaniques, temps, espace, masse, (2)

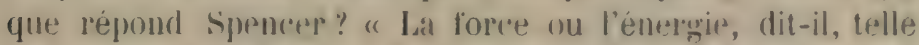
que la theorir: mécanique la compremu, ne peut pas ètre cette cause ultime d'ou toutes chosiss procident ; il y a autant de raison d'appeler cette cause dernimpe spirituelle qur do l'appreler materielle. "(3) Cette rejonse est insulfisante. Lal philosophic de spencer est méaniste parce quelle pretrend expliquer l'origine de la vie nt de l'esprit et rendre comples de leur nature, telle que mous la connaissons, au mogen de l'exolution méanigue de la matiere. Pru importe, après cera, yu’il y ajoute la these idéaliste de la riblativite de nos connaissances qui implingue notre ingnorances des la realitis. Ine philosophlie dre la vie et de l'esprit est caracterisere par la manisere dont elles interprite l'uns ct l'autre dans l'ordre phénominal.

Il est parfaitement légitime de: sulstituer, dans l'interporitation des laits matiorels, des lois micaniques a des ayents immateriels, d'explinger les faits biologrigurs par des lois biologiques. Macpherson se trompe lorspunil pritemd tirer de la une justific:ation de spencer : " Lorspur, dit-il, Newton mit sa conception de la gravitation a la place de la théorie angélique des mouvements planetaires, il introdusit dans l'edude du monde un istement mécinigue verritiable en termess de force. Cela sulfit-il pour que Newton soit materialiste? Lorsque Darwis substitua a la theorie sporitualistre des créations particulières la conception

(1) Princ. de Psychol. vol. I p. 682 \& 272.

(2) Naturalism and Agnosticism. p. p. $243-946$.

(3) Forthnithly Reriew: 1899 novembre p. 898. 
tion dynamifure d'une lutte contre les organismes pour une quantite determince de force nécessaire à la sustentation do la vie, etait-il necessairement un matrialiste? Or. ce que spencer a fait, e'est simplement de fondre les genfralisations de la sience en une seule géneralisation universelle. » (1)

sprencer a en effet tichè d'englober toutes les synthèses frarticulières dans une synthèse générale, et nous verrons dans futel sens cette entreprise est ligitime et jusqu’d (quel point il a riussi. Mais il fait davantage; il a affirme l'identiti essentielle de tous les phénomènes, et comme les premier's dans l'ordre d'érolution sont les phénomènes. purement mécaniques, il a pritendu que tous les autres ne sont que des combinaisons de ceux-lád. C'est ainsi que la philosophie de spencer fut interpretée dès l'apparition des Premiers Principes. D'après M. Spencer, écrit Laugrel "'l 186if, la loi de: la metanorphose yui s'applique à toutes les foress physiques, unit aussi ces dernieres aux furers mentales.... si tant de physiologristes hesitent a ranger la force vilale au meme niveau que les forees plysico-chiminues, l'hesitation n'est-rlle pas plus légitimes encore, s'il sagit d’y flacer atusi cette puissance mystiprouse yni fait notres grandeme of notre dignite ?.... On resenle involonlairement devant l'assimilation la phus lointaine du phinomine subtil ot transcendant de la pensese aux jheinomenes grossiers du monde matériel. " (2) Langel cite lo passage oi spencer se diefend d'ètre materialiste. "A la favent dre ces reserves et de ces explications, dit-il, on sera prut-itre moins dispose it repousser sans examen une theorie. qui assimile aux autres forces matumellos les forres vitales (d montales. o) (3) II ajoute gu'en tous cas une lolle assimilation ne peut pas ifre laits: an nom de lat phlosophie

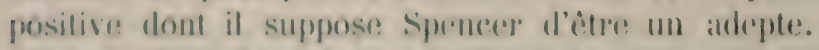

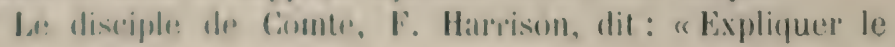

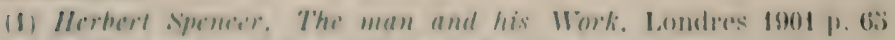

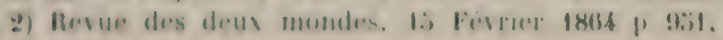

(3) Jbid P. Mist. 
progriss social ret moral all langage de physigue in de mecanique, cela, dans l'école? ou j'ai ete forme, rst reconnu pour etre du matiobalisme dans le serus strict de ce mot, ce qui consiste à latisonner sur l'àme de l'homne comme si ses fonctions etaient simplement celles des unitris matérielles ». (1).

La philosophir des Premiers Principes, dit Renonvier. "cest du gemre matrialiste el non jas atutre"

Nous no calomnions donc pas spencere en disant que sa philosophir est miscaniste at materialiste. (2)

Śrialité les omènes taux.

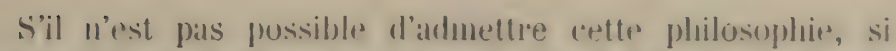
nous devons considerer l'antiviti des corps bruts, la vie olganique (at moins tres probablement), les faits psychiques (certainement), comme: autant de categories diflerentes de mature et irréductibles, nous ne nions pas pour cerla que tous ces phénomenes ne puissent etre soumis a certaines lois communes, surtout si ces lois sont très génèrales, telles que le sont nicessairement les fremiers principes. Lat solution de ce probleme n'est point prejugrés par les remarques que nous avons faites.

Une chose incontestahles ef qui a éte de lous temps l'occasion de l'erreur matérialistr, c'est que, dans les etres corporels - les seuls que nous connaissons par l'rxpéricnce - aucun phenomène n'est complètrment indéfendant de la matière, ni de ses forees elementaires.

Toute activité des êtres vivants ou bien consiste dans des mouvements, ou bien exige ces mouvements comme condition nécessaire, ou bien les entrañe comme conséquence - qu'il s’agisse de mouvements visibles ou invisibles. I a vie organique comprend des déplacements de matière et des transformations de forces physico-chimiques; la vie

(1) The Herbert spencer Lecture. oxford 190:3 [0. $2 . ;$.

(2) op. cit. 1886. v. 11 1. 33̈t. ¿'. PH. B. Revue de Thiologie et de philosophie. Janvier 1904 p. 77. 
sensilive exure des moivements atomigues comme condition intrinsèque et determine des mouvements visibles; la vie intrllectuelle de l'homme a pour base la sensibilité et a sous sa dependance - par la volonté - heaucoup de mourements du corps; toute action psychique a sa répercussion nécessaire dans l'organisme.

Wriest-ce que le développement de l'embryon et de lasdulte, qu'est-ce que la nutrition, la respiration, la circulation et tous les autres phénomenes phỵsiologiques si ce n'est un ensemble compliqué de mouvements visibles ef invisibles, de transports de materiaux, de transformations chimiques, une mise en ourre continuelle de toutes les forces atomiques?

Toute sensation ne requiert-elle pas l'ébranlement des nerfs correspondants et la transmission de cel ebranlement au cerveau? Les circonstances qui entravent l'activité nerveuse des centres cerebraux entravent l'exercice de la sensibilite; tout ce qui stimule celle-là renforce celle-ci. Lévolution de la sensibilité et celle du șstème nerveux sont parallèles.

Quoique les idées génèrales et toutes les formes de l'intelligence proprement dite aitut des caracteres de simplicitè et d'immatérialité qui, à notre avis, ne permettent pas de les attribuer à des facultes organiques, néanmoins tout exercice de l'intelligence est accompagne de représentations sensibles correspondantes, de sorte qu'il n'y a aucun acte psychique que n'accompagne un deploiement d'activite nerveuse. Toute la vie de l'ètre virant est donc intimement liere aux forces de la matiere.

(In sait combien l'histoire des peuples dépend des conditions matririlles dans lesquelles ils vivent. Elle est comme une resultante des agrents naturels at de l'activité humaine et a ce double point de vate elle se trouve soit dir..riment soit indirectement sous l'influence des forces matericlles.

Par conseyfuent, s'il est rertain que toutes ces classes de phenomines ayant leur nature propre ont aussi leur lois distimetes, il rien est pas moins ivident que dependant, 
comme nons venons de lo dirre, des phénomenes purement materiels, ils sont inglement soumis dans une certaine mesure aux lois yui gromverment ces derniers.

Les fitres dont sorecupe la philosophlie et dont nous avons entrepris d'étudiel !'evolution, sont des itres corporess, ce qui ne reat pas dire qu'ils me sont que matière, ni surtout qu’ils ne sont que des agrigrats d'atomes, mais semement qu’ils sont malièe et agregats d'atomes. Lem artiviti est toujom's malerielle frar yuelque côte. c'est-it-dire qu'elle implique toujours des redistributions de matiere et de momvenral. Whes lors, leme évolution est nécessabirement sommise aux lois grempartes qui s'applingent à la matiere et au mouvement comme tels, pour ne pas parler des lois metaphysigures commumes i toms les ètres.

Il ne lant pas conceroir la matiere ot la vie, meme la vie intellectuelle de l'homme, comme ders choses sepalrées, mais all contraire comme substantiellement unies. de sorte que c'est reellement la vie qui est soumise aux lois de la matiere a raison de son coute materiel. Caest précisement à ce point de vure fure nous etudions iri l'évolution.

ude tive de oi de ormaon.

Les remarques précedentes etant laites nous punvons reprendre l’étude de la loi de translormation et d'equiràlence dans les dilfrents ordres de faits. Les experiences qui ont fremis de l'enoncer ont eu puur oljet des series de phenomenes nettement limitees et accessibles dans toutes leurs parties. Si l'on considere l'ensemble des transformations de l'énergie dans l'L'nivers, ou mème dans leur totalite les mrincipales classes de faits ou elles s'accomplissent, il est evidemment impossible de constater l'équivalence avec rigueur. "Le plus que nous puissions espérer, dit Spencer, c'est d'établir une corrélation qualitative, vaguement quantitative, et quantitative seulement en ce qu'elle établit une proportion convenable entre les causes et les effets. " (1)

(1) P. P. p. $217 \& 67-$ F. P. p. 164 
L'hỵpothèse d'une néhuleuse comme point de départ de la furmation du systeme solaire implique que l'attraction qui précipe à l'origine toutes les particules materielles vers le centre commun de gravité, est, avec le mourement rotatoire, la cause des états successif's jal lespuels le système tout entier et les astres qui le composent ont passé jusquaujourd'hui. Cette attraction des particules largement disstminées représente une énorme quantité d'énergie 'potentielle.

Le mourement rotatoire étant donné, la concentration doit avoir déterminé une rotation de vitesse angulaire croissante. La rotation elle-mème a déterminé la formation de centres secondaires d'attraction autour desquels la matiere s'est condensée tout en continuant a tomrner autour du centre commun.

bans cette première transformation de l'énergie nous constatons que les particules matérielles qui, šétant approches davantage du centre ont raisemblablement enmagasine plus de vitesse dans leur chute, possèdent un mourement de translation plus rapide que celles qui sont resties tres eloignees. Jupiter dont la distance au soleil est un peru plus de cing fois celle de la Terre n'accomplit sa révolution qu'en douze annces. Neptune met cent soixante cing ans a parcourir une orbite trente fois plus longue que l'orbite terrestre.

Pour la mème raison, les planites qui contiennent plus de mative auront un mouvement de rotation sur ellesmemes plus rapide, les particules ayant parcouru en moypme dr plus grandes distances pour s'y réunir. Ainsi dupiter entraine en dix heures autour de son axe un volume 1414 fois plus grand que celui de la Terre.

Lat condensation a pu ingalement produire l'énergie vibratoir sous formr de chaleur at de lumiere. Cette energie s'est repandue dans l'espace of les astres se sont refroidis plus ou moins bapodement suivant l'importance de leur masse. Tandis que le Soleil est encore incandescent aucune. planite n'ast anturellemont lumineuse. Tout an moins la surfare des petites planides siest soliditiee, tandis que 
celle de Jupiter, par exemple, est probablement encore liquide. Le refrodissement a permis a la matiere de se condenser davantage et a determine ainsi a la surface de la Terre les dipressions, les ridements de l'écorce, tunt le travail tectonique. La chaleur conservere à rinterieur du globe se manifeste par les iruptions des volcans et les phénomènes accessuires, tandis que la chateur emmagasinée dans le soleil, produisant les vents qui lancent les flots contre les rivages et la vapeur d'eau qui retombe en pluie et ruisselle a la surface du continent, est l'origine du travail géologique accompli par les agents extérieurs à l'écorce.

Tous ces phenomènes dans leur complication intinie at qui sous d'autres formes se realisent dans les autres astres, ne sont que des transformations de l'energic contenue dans la nébuleuse primitive.

La loi d'équivalence n'est que vaguement apergue dans ces phénomènes qui échappent a nos mesures; mais quel exemple grandiose de l'aptitude de l'énergite méanique a se revitir de mille formes diverses!

Remarquons-le cependant: les faits quapres spencel' nous avons decrits dans leur's grandes lignes at en rectitiant quelques détails, ne? sont pas produits par l'action d'une seule force. Ia gravitation universelles suffit pour que les particules de matire se précipitent vers le centre; mais elle seule ne peut pas expliquer l'origin. des mouvements de rotation ni de l'énergie vibratoire. Celle-ci n'est pas possible sans elasticite ou sans forces répulsives.

Les phénomenes de ragonnement exigent - Spencer a adopté lui-même cette conception - une matière impondérable possédant des forces caractéristiqurs.

Les allinites chimiques qui ont joué un si grand róle dans la formation de l'écorce terrestre ne sont-elles qu'unr? forme particulière de la force gravitative? On n'oserait pas l'affirmer. Sommes-nous autorisés à attribuer à la force gravitative les phénumènes d'électricité, de magnétisme et d'autres qui sont encore pleines de mystère pour nos 
Jhỵsiciens? Pas davantage. Peut-être pourrait-on dire que la concentration de la maticre par la force d'attraction est une conditlon nicessaire pour l'action des autres forces, mais il serait difficile de preciser cette affirmation.

Il est bon de le répeter encore: il ne faut pas qu'it force de parler de "la matiere " et de "la force " nous nous tigurions qu'il n'y a qu'une sorte d'etres étendus et que toutes les forces se reduisent en derniere analyse it une seule. L'unite ne doit pas nous empêcher de voir la variété.

La loi d'équivalence et les phénomènes organiques.
Cette remarque est plus nécessaire lorsqu'il s'agit des phénomènes organiques dans lesquels spencer nous montre une autre application de la transformation de l'energie.

Que les animaux et les plantes ne créent pas de toutes pièces l'energie qu'ils déploient, mais l'empruntent, d'une faron générale, au milieu dans lequel ils rivent, cest ce que personne aujourd'hui ne contestera ef r'est ce que demontrent les faits relatés par spencer. Tant it l'animal gu'à la plante l'energie doit ctre fournie sous forme d'aliments, de chaleur, de lumière. Sans la lumière du soleil il n'y a pas d'elaboration chlorophỵlliemne, pas de vie regetale et, comme consiofuence, pas de vie animate.

Dans certaines limites, la vie dans les deux rèonnes est d'autant plus intense ot plus luxuriante que l'inergio exterieure lui est plus ahondamment fournir. Mais faut-il conclure de la que les organismes ne mettent point en jen d'autres forces que celles gurils trouvent dans te milien? Evidemment la conclusion depasserait les prémisses.

Nons avons ru plus haut les raisons gu'il y a d'admettre des forces diflerentes de celles de lat matiere brute,

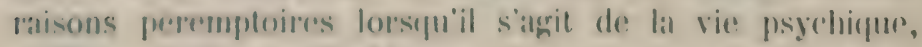

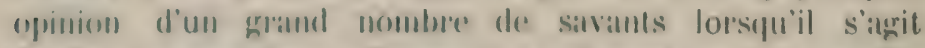
de lat vie organique. On peut se demander dans quelle mesure lessistence ale cees forces est conciliable areer la 
conservalion de l'enoryir. Ce n'est fas salls raison qu'en

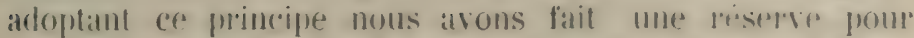

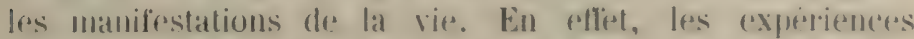
fintes jusqu'aujourd'lui sul les vivants sont loin d'itle aussi mombrouses of allsisi exactes fute celles qui ont en pour objet la matière inorganique.

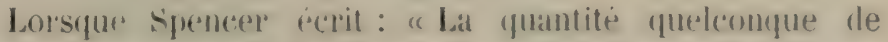
forere quan organisme depense sous une former est corerélative ot éfuivalente a tme forre que cet organisme a

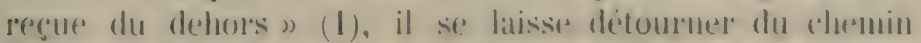
sù de l'exprerience par des considerations a priori que

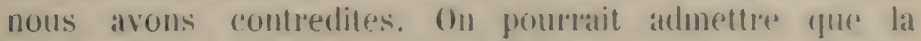
force vitale produit dams l'organisme de lesgrodes valdations d'entergie sans se tronver en contranliction atver liexprerience.

Le foile d'une force dans le mouverment n'est pas tenjours en rapport area la quantite d'energite qua'elle produit,

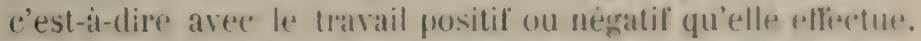
Le conducteru d'une antomobile la lait isoluer a sa guise et joute donc dans les mouventents de la machine un ròle preponderant. Le travail qu’il fournit est pourtant insignifiant. Ainsi les forers vilites pesurent gouverner l'activite de l'ette vivant en developpant tris peu d'entegre.

Les forces peurent meme diriger le mourement salns executer aucun travail, lorsque leur direction est constamment perpendiculaire a celle du mouvement, par cxemple la lorce centripite dans le mourement circulaire. C'est ce qui a amené certains auteurs à listinguer, dans la question qui nous occupe, la qualite du monvement, c'est à dire, sa direction, et Sa quantite yui ne depend que de la vitesse et de la massp.

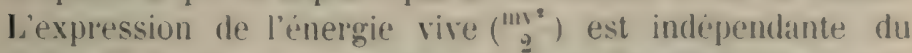
signe de r, et, par conséquent, de la direction du munvement. Si des expériences plus précises nous apprennent que dans les phenomines vitaux il n'y a aucune perte ni aucun gain d'energie, il faudra en conclure que lá force vitale, soit psychique soit organique, a le pouvoir de moditier

(1) Princ. de Biol. vol. I p. $68 \S 23$. 
la direction du mouvement sans altérer la quantité d'énergie vise ni la quantité d'énergie potentielle.

De fait c'est précisément ce ròle de principe directeur qui est assigné à la force vitale par les biologistes qui l'admettent, et c'est sous cette forme que nous concevons l'intervention des facteur's psychiques dans les mouvements de l'animal. Lorsque celui-ci contracte ses muscles et exécute les mouvements les plus compliqués, il déploie l'énergie qui lui a été fournie sous forme de nourriture; mais qu'il marche plutòt dans une direction que dans une autre, que ses mouvements expriment plutôt la joie que la colère ou la tristesse, c'est ce qu'on ne peut point attribuer au seul jeu des force atomiques, ainsi que nous l'avons vu.

Spencer n'est donc pas justifie à dire: "Les forces manifestées dans les actions vitales, végétales ou animales, se dicluisent.... d'une manière.... évidente de la chaleur solaire ». (1) Nous sommes impuissants à faire celte déduction. "Rien ne nous permet, dit ailleur's spencer, de suivre la manière d'après laquelle le mouvement moléculaire se transforme chez les animaux en mouvement de masse. Nous ne pouvons ramener à des causes connues l'action rythmique d'un disque de méduse.... nous ne sommes jas mieux en etat de dire comment le mouvement insensible transmis par un nerf donne naissance à un mouvement sensible dans un muscle.....) (2) Ce qui est vrai, c'est que l'énergie déployee par les êtres virauts a son origine si pas unique, au moins principale, dans le: rayomement solaire. Mais les transformations de cette infergie: soprent sous l'action de principes directeurs, ainsi que nous l'avons expliqué.

l.ps phrnomènes organiques dejendent réellement de: la conservation de la matiere et, au moins dans une large mesure, de: la conservation de l'inerugie. Mais ces lois semules ne sullisent pas a les expliquer. Prenons pour

(1) P. P. p. 222 \& $70-$ F. P. p. 108.

(2) Princ, de Biol vol. I p. 06 \& 22. 
exemple le phenomene de la crossance. Coeteris puribus, l'augmentation du volume d'un organisme depend de lat quantite de matire assimilable qu'il a a sal disposition; Alle varie, en tous cas selon lexces de la nutrition sur la depense. Or la dépense est réglée par la quantitie d'energre deployee, do sorte que la croissance sera ralcentio

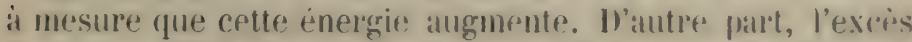
de la mutrition pourra itre de prines abord d'autant plus considerable en valeur absolue, gute l'organisme posside unc plus grande masse en commeneant sal vie. Beameoup de circonstances de la rooissance se trousent donc rexpliqueses par la conservation de lat matiese et de l'énergerie, mais non point tontes. Xi l'organisation, ni l'adaptation de l'atre vivant à son milien n'almettent une augmentation indetinite de volume. Pour contribuer a lat conssamer, la nomriture doit ponvoir itre assimilese ot distribuer dams les diflerentes parties de lamimal on de la plantre late grenee de vie que mènent les parasites des animanx sulperients on les lappins qui habitent des terriers, ne leme

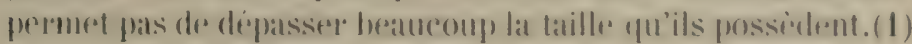

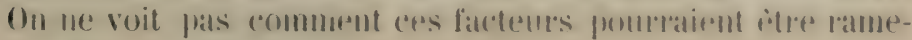
nès aux précérlents.

Vient ensuite lat tausformatton des foreres matrapelles en " forces mentales. ) (2) Soms des nom sifencer comprend tous les etats de conscience.

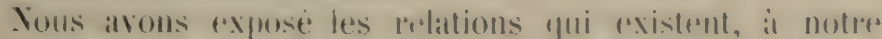

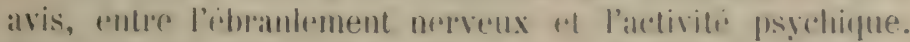

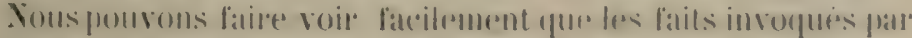

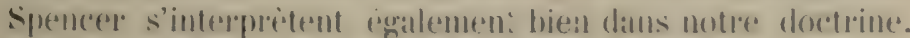

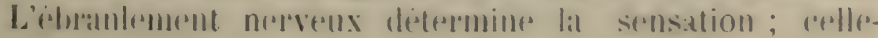
(ai seral, grosso molo, d'autant plus intense que l'buran-

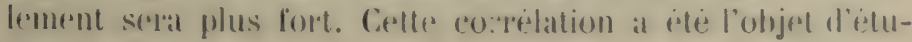
des pricises.

(1) ef. Principes de Biol. vol. 1 p. 127 sq. \$ 43.

(2) P. P. p 226 \& $71-$ F P. p. $1 \% 0$. 
des hommes pux-menes. " (1) En d'antres termes, lievolulion sociale est la résultante de l'artivite physico-chimigure, organique et joschique que nous aroms deja eludiests an point de vite de la transformation de l'onergios.

Nous avons sempenent a insister un peu sur l'intervention de la liberte. Si nous avions almis sams restriction la conception de spencer, si nous considerions lous les phinomenes vitaux comme risultant de la transformation de l'enerpice miscaniques sans l'intervention d'aucun antres facteur, il n'y aumit evidemment pas lien de parter de liberte. Mais d'autre part, si l'on a trouve plausible la manière dont nous avons exposte nous-mime lat relation entre l'activite vitale et la loi dénuivalences, la these de la liberti ne doit sonlever ancme dithiculte nowralle.

Nuns ane voulons pas dire que toute influence directricte capable dagrir sur les atomes et de moditier lemes moure-

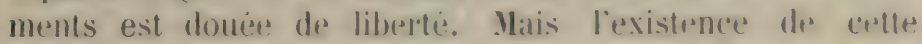
fmerogative est une puestion qui interpesse lat metaphlysique

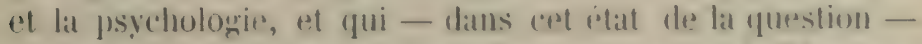

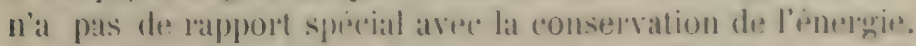

Il est bon des latere oloservere ausci que l'existenese de la liberte nempriche pas que les actions humaines, considerées dans lrur ronsemble, ne suient sommises non serulement a des lois qui expriment ce quilles doivent itre au point de vole moral, mais encore a des lois qui expriment ce qu'elles sont rn realiti. Evidemment, si on appligue ces lois anx cas particuliers, les conchusions auxquelles elles comblusent n'ont plus quime probabilitis plus ou moins grandr, lorsqu’il s'agit d'une matière sur latpuelle la liberte peut sexpress. Catte probabilite yui existe, pour les cas particuliess correspond al une influence plus ou moins forte à latpuelle la volonté peut résistre, mais a latuelle elle ciolera de fait d'autant plus frérpummant que cette intluence est plus inpueriense. Il en l'isulte: une? direction generale qui peut fournir l'énoncé d'une loi ayant une certitude veritable quoique de natures praticulieres of

(1) P. P. p. $293872-$ F. P. p. 178. 
qu'on ne peut pas confondre arec la certitude physique ou métaphysique.

L'activite deplovè par une societé dépend évidemment du nombre de ses membres. Elle dépend aussi dans une large mesure des ressources matérielles. L'importance de la situation économique a été peut-ètre exagèrée par certains historiens; elle est certainement considérable. Or, la plus grande partie de la richesse matérielle, qu'elle consiste en charbon ou en énergie mécanique (chutes ('eau, etc.) ou surtout en récoltes, est empruntée au rayonnement solaire; celle qui est représentée par les metaux, les pierres précieuses on utiles, n'a pas, en somme, une origine difterente, puisque, en tous cas, ces minéraux résultent de la condensation de la matière qui est également la cause de la chaleur et de la lumière du soleil.

On comprencl encore que la richesse matérielle facilite à l'homme la vie du corps; elle lui permet ainsi de consacrer plus de travail a la vie de l'intelligence. Et si l'on se rappelle que cette activite intellectuelle dépend, par son cote materiel, de l'energie qu'elle emprunte au dehors, il est impossible de ne pas ètre lrappé d'admiration devant le spectacle majestueux de cette unité qui relie les uns aux autres les phenomènes de tout ordre.

spencer reconnait qu'il est impossible de déduire de lexpérience le mécanisme outré qu'il défend. "La doetrine coutrnue dans ce chapitre, dit-il, reneontrera plus d'un incredule si on la donne pour une induction. Plusieurs do crux aux yeux de qui la transformation des forces est maintenent demontrie, pour les forees phigsigues an moins, diront prout-ître que l'on n'a pas pousse assez loin les recherches pour avoir le droit d'athirmer l'épuivaIence. Pour les forees dites vitates, mentales ot sociales, ils IIf veront dans nos preuses rien gui dimontre d'une mantiare dereisive la transformation rt, moins eneore,

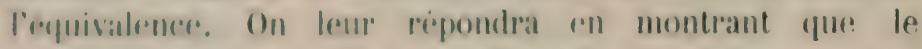

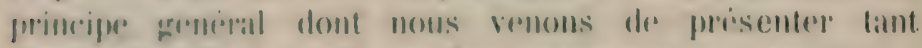
drexemples divers qui all fomt comprendre tontes les 
formes, est un corollaire nécessare de la persistance de la force. " (1)

C'est done sur des considerations a priori que repose la généralisation excessive que nous avons comballue et que nous avons tente de réduire a ses justes proplortions. Cellesci sont assez vastes pour offrir un beall coup)-d'ceil a l'intelligence, un large champ a la spéculation philusophicpue; et surtout, elles ne depassent pas la base solide des faits. Quant au principe a priori de la prersistance de la force, cre que nous en arons dit plus haut nous dispense de nous $\mathbf{y}$ arrêter davantage.

\$ VI. Les lois du mouvement. - La tendance du mouvement à suivre une ligne déja parcourue - Le mouvement suivant la résultante des forces. - Les lois de la direction du mouvement dans l'activité vitale.

lois du vement.

Tous les phenomines physico-dimigures comprernant des momements visibles of invisibles, doivent pobbablement s'expliqure par l'existence de forces attractives et répulsives entre les élements materiols. C'est lat conception quablopte Spencere tout en la drelarant inconervable. "si la proposition, dit-il, que la pression (attraction) et la tension (ripulsion) coexistent partout est intelligible verbalement, nous ne pouvons.... pas nous tignrer ) (ciest-it-dire, dans le langage de sprencer, ancevoir) " une unitè irreductible de matière attirant une autre unité de matière et la repoussant en mème temps. Cependant nous sommes forcés de conserver" cette dernière croyincer. On ne prot roncevoir la matiere que comme manifestant dfes forces d'attraction et de répulsion » (2).

Ine chose inconcevable est done la sfule chose roncevable! Tel est le genre de conclusions auxpuelles aboutit la doctrine de la relativite de nos connaissances.

(1) p. p. $236-237 \S 73$ - F. P. p. 180.

(2) P. P. p. $210 \$ 74-$ F. P. 1812. 
In element materiel ne peut pas a la fois attirer et repoussel un autre élement; mais ce n'est pas la non plus unr conséquence de l'pxistence de forces attractives et répulsives. Yous arons indique (1) une idée plausible qu'on peut se fair de celle-ci et qui n'entraine pas cette contradiction.

L'action simultanée de tontes les forces attractives et répulsives, auxquelles viennent s'ajouter les forces vitales dans les corps organiques, se combine a chaque instant arec le mouvement existant compte en fonction de la masse et de la vitesse (mv). Ainsi toute particule matérielle a son mouvement déterminé en vilesse et en direction. Xe considerant que cette dernière, on trouve qu'elle est sommise il certaines lois. Spencer les résume comme suit: "Nous avons a remarquer comment chaque mouvement s'oprere le long de la ligne de plus grande traction " (lorsqu'il ast drermini par des forces d'attraction) " ou de plus faible resistaner ") (lorsqu'il est déterminé par des forcess de répulsion) " on de leur résultante; - comment It) (ommencement d'un mouvement sme une certaine ligne devient une cause de mouvement le long de cette ligne; - comment, miammoins, le changement des rolations aver les forces exterifures finit devier de cette ligne; it comment le drage de deviation saldroit toutes les fois qu'une nouvalls intluence vient s'ajontel a celles qui s'exerçaient déjà »" (2).

a cendance lu mouveont a suivre une ligne déjầ sarcourue

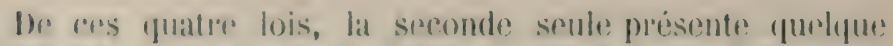

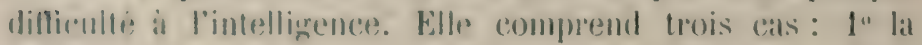

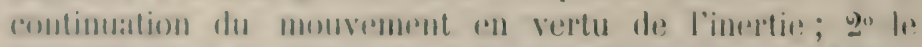

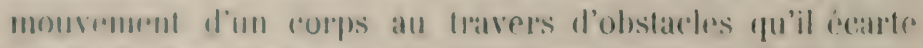

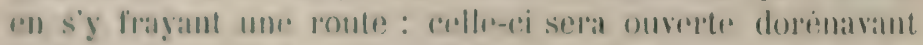

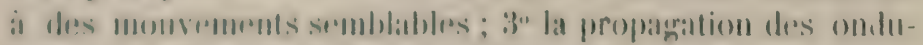

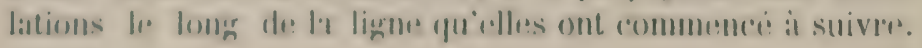

(1) v. pl. haut p 64 .

(2) P. P. P. $241-$ F. P. D. $18487 \%$. 
Le premier de ces thois cas inclut mon swolement la continuation du mouvemement dams un mime mobile, mais aussi le fait de sal rommunication a d'antres mobiles par le choc. Inis lors le premicel et he serond as ane domment, semble-t-il, lieu a aucme objeretion, surtout si l'ontient compte des autres lois yui prevolent les morlitications de. la ligne de monvement par des forces axtidreurs. Parmis celles-ci il faudrat ranger les reactions aventuelles du

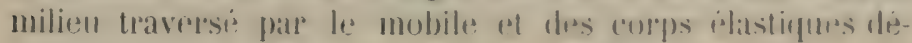
formes par les choes.

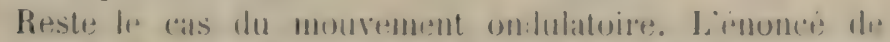
Spencer est ambigur. " latablissement des ondulations sur certaines ligntes, dit-il, detromine leur continnation le

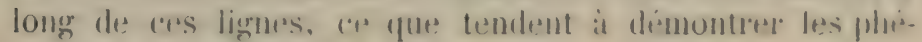
nomènes magnétiques. ग) (1)

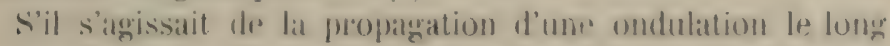

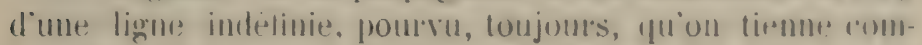

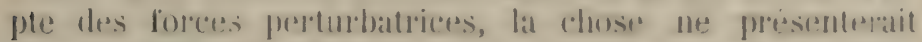
pas de difliculte. Mais sprencer entemel que la transmis-

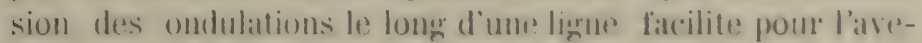

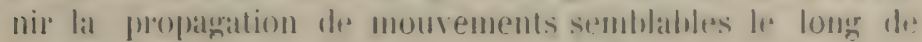

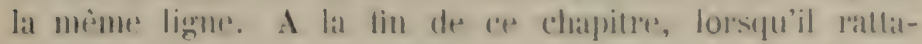
che les lois de la direction du mourement a la pressis-

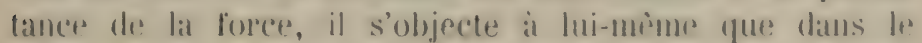

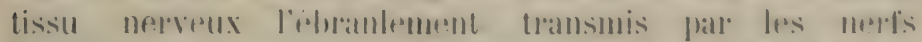
produit des changements chimigues qui les rendent moins

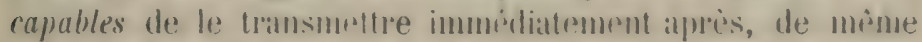

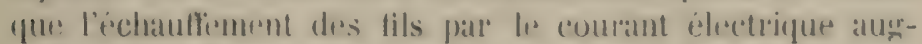

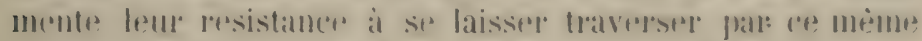
courant. Il reppond: "Lat vatare furestion est pourtant de

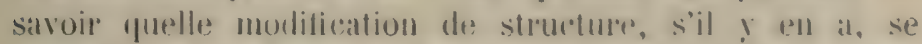
produit dans la matione traversere, en dehors des forens

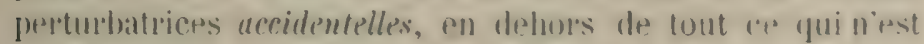
pas la résistance? nécessare de la matiopen a salvoir la résistance qui résulte de l'inertite de ses unites. Si nous bornons notre attention à cette partie du momement yui,

(1) P P. p. 243 \& 7 F. P. p. $18 ;$ 
échappant à la transformation, continue son cours, nous pouvons diduire du principe de la persistance de la force que la partic de ce mouvement restant qui se perd à changer les positions des unites, doit les laisser dans un elat qui leur permet bien moins de gèner un mouvement subsequent dans la mème direction. " (1)

spencer ne prouve pas cette derniere aftirmation et il ne montre pas davantage comment elle suit du principe de la persistance de la force.

Dans l'aimantation des barres neutres, il se produit, d'apre's l'hypothese aujourd'hui admise, une orientation des elements magnetiques, mais il ne s'agit pas, dans ce aas, de la transmission d'un mouvement ondulatoire. On pourait citer le fait que l'étincelle électrique passe plus facilement au travers d'un gaz lorsfu'elle l'a traverses une prenière fois. Le phenomène s'explique par l'ionisation du gaz au moyen de la première decharge. D'autre paut le passage du courant électrique daus un milieu grazenx ionise y détermine une polarisation domant lieu a tune force électromotrice contraire. (2) On poursait protterter, produre d'antres exemples qui trouveraient de mime lemr interpretation dans des circonstances accidentelles. Mais il n'y a ancane loi ni aucune throrie physipue yni contirme d’une maniere generale l'alfirmation que sfuencer imet ici. Nous remons tout a l'heure furelle flace importante elle tient dans sal Psychologie. (On ne

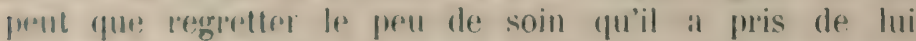
tromser une formule acceptable et de lui dommer une base experimentale solicle.

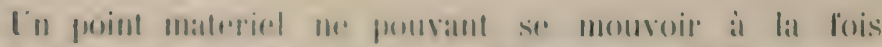
yue sur ume semle limme, soll mourement se lasil, pall

(1) P P. p. 207 है 81 - F. P. P. 19!1

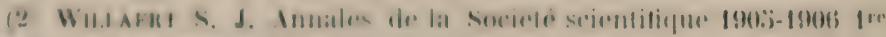

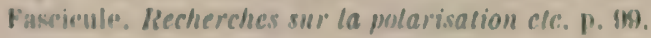


délinition, suivant la résultante de toutes les forces auxquelles il est soumis. Le caleul determine la direction de: sa trajectoire et, si ellr n'est pas une ligne droite, sit forme.

$\mathrm{Si}$ deux forces ont une action diametralement opposere, c'est la plus grande qui l'mporte et le mobile suit la ligne de plus forte traction ou de plus faible résistance. El ce ne sont la encore, en somme, que des définitions, puisqu'on mesure les forces par l'action qu'elles produisent sur le mobile. Dans tous les autres cas, le inobile ne suit la direction d'aucune force en particulier, mais seulement de leur resultante. Pour la determiner on estime l'intensite des forces individuelles par le mouvement qu'elles sont capables de produire dians le mobile lorsqu'elles agissent seules et la resultante se determine d'après le théoreme du paraltélogramme.

Dans catte determination, on doit évidemment tenir compte des nouvelles forces qui interviennent it un moment quelconque, ainsi que des résistances qui the sont, en somme, que des forces répulsives.

De sorte que la direction du mouvement est toujours determinee par des lois qui s'etablissent à priori. D'appès Spencer, leur démonstration implique la persistance de la force. "La force résultante, dit-il, résultat de la nentralisation de toutes les autres, doit mouvoir le corps dans la direction sur laquelle elle agit. Aftirmer le contraire c'est affirmer qu'une force preut se dépenser sans effet, sans engendrer une force équivalente, ce qui impli. querait qu'une force peut cesser d'itre, c'est-d-dire une negration de la persistance de la force. ") (1)

Ce raisonnement n'est qu'un sophisme. La force résultante n'existe pas. Elle n'est qu'une liction. Ce qui est réel c'est le mouvement résultant de l'action simultanée de plusieurs forces. On appelle force résultante celle qui serait capable de produire i elle seule le mouvement résultant.

Pour que les forces produisent un mouvement résultant, il faut qu'elles agissent; si elles n'agissent pas te

(1) P. P. p. $264 \$ 81$ F. P. P. 107. 
ne sont plus des forees, all sins meranique de ce mot. On suppose done comme donnés du problime lexistence de certaines forces et non la persistance d'ume force quelconque. Si une on plusieurs forees ressent d'agir, c'est-dithe d'exister, les donnees du probleme changent, mais aucune loi ne se trouve contredite.

En realite dans la throrie de la composition des forces telle gu'on l'entend ici, on suppose la continuite du mouvement. (1) Colutr-ci, d'après Spencer, n'est qu'un cas frarticulier de la persistance de la force. Nous la considerons, au contrarie, comme une conserfuence de l'inertie. Or le principe d'inertie est plus general que la loi de la conservation de l'énergic (seul sens admissible du principe de la conservation de la force), et il en est independant. Aussi, tandis que, a notre avis, la conservation de l'energie dans l'Lnivers ne peut pas se dimontrer' a priori, nous inclinons a croire que le principe d'inertie est susceptible d'une telle démonstration.

Il baut bipn remardquer anssi que la direction du mouvement n'est determine par les forces que pour autant qu'on $\mathbf{y}$ comprend le mouvement lui-mème. Sir une surface accidentre la ligne de plus forte pente (appelons pente la résultante, en chaque point, de la gravité et de la resistance de la surface) sera en grineral sinueuse. Ln corps place au sommet de cette ligne et abandonné it laction de la gravite ne suiva pas cette ligne, mais s'en fecartera continuellement en vortu de la vitesse acyuise. tandis que son poids tend a l'y ramener.

Thl est done dans sa rigueur le mrincipe de la direction du mourement. Il a lieu à chaque instant suivant la résultante du monvement existant deja ef de toutes les forces qui agissent sur lo mobile. C'est le seul principe ayant une portée tout à fait générale.

Coramoins on a sourent plus spercialement en vue le ras ou il est possible de comparel des resistances ou

(2) cl. plus haut p. 16:3. 
des trations qui s'elablissent suivant des directions dillirentes. Lorsquan gaiz est comprime dans un réropient, lat

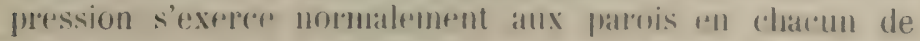
leurs proints at ast equilibere partout par une resistance diametralement opposie. Si les partois codent a lat pression, re sera à l'endroit où la resistance est moindre et le jet de gaz se fera suivant la ligne de moindere lésistance.

Lorsque des tractions sexererent sur un mohile dans des dirertions opplosieses, éest suivant la dirretion de lat

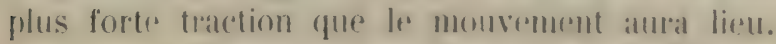

Lal loi qui rent que le mouvement se fasse suivant la ligne de moindre resistancere ou de plus forte traction a done une signitication vatie, mats restreinte. Lorsque des pressions on des tratctions egrales se produisent dans differents sens at se trouvent rell lutte dans chatpue direction avere des resistanees differentes, c'est suivant lat lierne dre mointre resistance que de mouventent se probluira de preferencor. Que si les resistances sunt ingales do lontes parts tandis que les pressions ou les tractions different, le mourentent se fera suivant la ligne de plus forte traction ou pression.

ois de la ction du vement l'activité itale.

Lon peut trumer dans la maturr des cas nomberux ou se viritie soit le principe greneral du monvement resultant, soit ce's lois restreintes. Le mouvement des planètes autour du soleil est unc application continuelle de la composition de la force d'attaction avec le mouvement acquis. Leur orbite, elliptique en vertu de l'attraction solaine, subit de legires perturbations a cause de l'artion variable des autres astres du système.

Les mouvements des itres vivants dependent des forces physico-chimiques et en outre des forces d'ordre physiologiqur, sensitif et intellectuel. Ils saccomplissent donc suivant la resultante de toutes ces actions réunies. Cette conclusion n'a besoin d'aucune autre demonstration. Les mouvements des membres de l'animal suivent a chaque instant la resultante de la force de gravite et de l'effort 
musculaire deployé. La croissance des arbres se fait suivant la résultante de la pesanteur et des forces internes mises en weuvre par le végétal.

Souvent, pour ne pas dire la plupart du temps, les forces inherentes au milieu agissent sur l'évolution des organismes par l'intermediaire des forces vitales dont elles déterminent la réaction. Cette évolution dépend ainsi du milieu de deux manières: directement par des mouvements délerminés ou modifiés dans le sens ou s'exercent les forces du milicu; indirectement par la réaction des forces organiques à l'action du milieu. L'influence de la gravité (géotropisme) et celle de la lumière (héliotropisme) est particulierement importante à chacun de ces deux points de vue.

La forme individuelle d'un organisme est aussi la résultante d'intluences heréditaires nombreuses qui s'exercent sur son développement.

S'il est difficile, en cette maticre, de préciser les exemples, cela provient, non pas de ce que le principe soil douteux, mais de ce que nous ne commaissons que très imparfaitement les forces spéciales aux ètres vivants et les lois suivant lesquelles elles agissent.

On pourra leur appliquer également - sans prétendre l'employer à resoudre tous les problemes de direction la loi d'après laquelle le mouvement se fait suivant la ligne de moindre resistance ou de plus forte traction.

Tres souvent les forces vitales ont a lutter contre des ressistances extérieures d'ordre purement mécanique. Elles frodluisent alor's leur monvement suivant la ligne ou cette resistance est la moindre. C'est ainsi que l'arlue qui chreche a etendre sus racines de toutes parts dans le sol, les glisse dans les interstices, les conduit autour des obstareses, les pousse, en somme, suivant les lignes de moindre résistance.

be vol flun oisean anferme dans une cagere finira par se produire au travers de la seate ouverture quon y a laissee.

Ces exrmples quoil serait aise de multiplier ne sullisent pas a faire compremble la los. Pour en saisir toute la 
portée, il faut remarquer que les forces vivantes rencontrent des résistances d'ordre physiologique, sensitif ou - intellectuel fort différentes des résistances mécaniques, mais qui agissent suivant la mème loi. Des tendances héréditaires, des instincts, un sentiment de frayeur, des objets repoussants, des difficultés prevues ou soupconnées peuvent constituer autant d'obstacles à l'action des forces de mème ordre et fournir des applications de la loi de direction du mouvement. Sauf l'exercice de la liberté par l'homme, cette direction sera determinée d'une manière aussi nécessaire que lorsque seules les forces physico-chimiques sont en jeu; et nous pouvons voir que souvent elle coïncide avec la ligne de moindre résistance ou de plus lorte traction. Ln animal entouré d'ennemis fuit du còté ou le danger parait moindre; l'ètre intelligent qui rent atteindre un but, choisit pour y arriver les moyens les plus faciles; l'homme se rapproche de ceux de ses semblables qui lui inspirent le plus de sympithie ou le moins de répulsion.

On peut ainsi, comme on le voit. étendre heancoup la signification de la loi. Il n'est pas da tout nécessaire pour cela de considerer, avec spencer, tous les phénomènes comme purement méaniques. Lne mime loi generale peut s'appliquer i des forces de nature dillerente. Sa signification s'adapte d'elle-mème aux cas varies qu'elle gouverne. Une interpretation rigoureusement mecanique ne convient pas à tous les ordres de phenomenes.

spencer fait de vains eflorts, jar exemple, pour rendre compte, au moyen d'explications purement mécaniques, de la transmission de l'intlux nerveux, depuis l'impression sur l'extrémiti des nerts centripètes jusqu"a la réponse qui se produit à l'extrémité des nerfs centrifuges. Il avoue que l'entreprise est ardue et ajoute un peu naïvement: "Les difticultés sont telles qu'il n'est pas possible de faire plus que d'indiquer brièvement les preuves qu'on pourrait domner si l'espace le purmettait. ") (1)

(1) P. P. p. $292 \S 79 .-$ F. P. p. 190. 
L'espace n'est jamais mieux employé qu'à expliquer des choses difficiles, et au surplus, le style de Spencer ne peut faire soupconner à personne qu'il se trouve à l'é-。 troit.

Il suffit que nous examinions la première partie de cet essai d'explication mécanique de l'action nerveuse: tout le reste en dépend. "Supposez, dit spencer, que les diverses forces qui règnent dans un organisme soient préalablement en équilibre. Si une partie de cet organisme devient le siège d'une nouvelle force, ajoutée ou dégagcee, c'est de là que la force, rencontrant des résistances des forces ambiantes plus petites, commencera un mouvement vers une autre partie de l'organisme. S'il y a dans une autre partie de l'organisme un point où se dépense une force, c'est-à-dire un point qui soit diminué d'une force qu'il possédait auparavant, au lieu d'être augmenté d'une force qu'il ne possédait pas, et qui par ce fait devienne un point où la reaction contre les forces ambiantes soit moindre, le mouvement qui s'opère entre le premier et le dernier de ces points se fera inanifestement dans le sens de la plus faible résistance. 0r, une sensation implique l'addition ou le degagement d'une force dans la partie de l'organisme qui en est le siege, tandisqu'un mouvement mécanique implique une depense ou une perte de force dans la partie de l'organisme qui en est le siège. Il en résulte que si, comme nous le constatons, le inouvement se propage d'ordinaire de ces parties d'un organisme auquel le monde extérieur ajoute des forces sous formes d'impressions nerveuses, à ces autres parties de l'urganisme qui réagissent sur le monde extérieur par les contractions musculaires, il ne fait quobéir à la loi que nous avons énoncée. " (1)

Yinsistons pas sur le manque d'exactitude qui depare ce raisonmement, ni sur les objections de moindre importance qu'il suggrire. Observons d'abord que le point essentiel, ceest a dire ta transmission de l'ebrantement

(1) P. P. P. $252 \div 0,-$ P. P. p. 190. 
de l'endroit où il est produic à celui où l'organisme "depense une force," n'est pas du tout elucide. In muscle ou un tissu analogue qui sp contracte execute un travail au dépens de l'énergie potentirlle qu'il contenait sous forme chimique. En quoi cette circonstance constituet-elle une résistance moindre a la transmission d'un mouvement analogue ḋ un ébranlement nerveux? C'est ce qu'il faudrait dire tout d'abord et c'e que spencer ne dit pas. Qu'un muscle qui se contracte "devient un poini où la réaction contre les forces extérieures est moindre, ") e'est ce qu'il est impossible de comprendre. Quelles sont ces forces ambiantes, en quoi consiste cette reaction et pourquoi diminue-t-elle? Il ne nous est donné aucun rensegnement à ce sujet.

spencer repète le passage que nous critiquons au commencement du chapitre des Principes de Psychologie qui traite de la génèse des nerf's, (1) sans lui donner aucun développement. L'endroit des Principes de Biologie anupel il renvoie part de l'affirmation que dans une mitsse de matière organique, un ébranlement moleculairs doit se transmettre davantage suivant certaines lignes, mais il n'est pas question de la circonstance que ces lignes sont dirigées vers l'endroit ou le protoplasme réagit contre une force extérieure, ce qu'il s'agit précisément d'expliquer ici.

Ensuite, la transmission de l'ébranlement dans l'organisme suivant certaines lignes n'explique l'établissement de directions constantes nécessaires à l'activité nerveuse, que si on admet le principe critique par nous plus haut, à satvoir que le passage d'une ondulation le long d'une ligne facilite la transmission d'ondulations semblables le long de celte même ligne. si cela n'est pas adnis - et nous arons vu qu'il n'y a aucune raison de l'admettre - l'interprétation mécanique de la vie animale s'écroule.

spencer essaie, dans l'ouvrage que nous venons de citer, (2) d'etablir ce prineipe pour ce qui concerne l'activité

(1) vol. 1 p. อัง๋3-อัวิ4 \$ 223.

(2) Princ. de Biol, vol. Il p. 406 sq. \$ 302. 
nerveuse, en montrant comment on pourrait concevoir la diftérenciation du tissu nerveux dans la masse du protoplasme. L'explication est longue et un peu confuse. Voici comment on peut la résumer:

$1^{\circ}$ L'excitation nerveuse est un ébranlement qui produit une moditication chimique des molécules colloïdes du protoplasme. Il s'y propage à cause de l'instabilité chimique de ces molécules.

20 Cet ébranlement « se diffusera plus loin sur certaines lignes que sur d'autres, si les éléments du protoplasme ne sont pas dispersés d'une facon tout à fait homogène et si certains d'entre eux se transforment isomériquement plus aisément ou avec moins de dépense de mouvement que d'autres. »

$3^{\circ}$ "La même influence qui tend à propager les transformations isomèriques, tend aussi à former de nouvelles molécules du mème genre au dépens des éléments adjacents. "

$4^{\circ}$ Done une onde de perturbation moléculaire diffusée d'un centre et voyageant le long d'une ligne autour de laquelle se trouvent le plus grand nombre de molécules susceptibles de subir facilement la transformation isomérique, aura pour effet, en mème temps, de différencier davantage cette ligne et de rendre son caractère plus prononcé qu'il n'était.

$5^{\circ}$ Il faut supposer que les molécules considérées reprennent leur structure primitive dès que l'onde a passè.

Contentons-nous de signaler les défauts essentiels de ce raisonnement un négligeant les remarques accessoires.

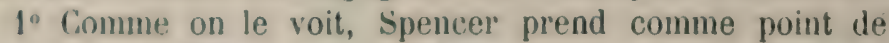
départ une diflèrenciation existant déjà. Si les élèments les plus facilement transformables du protoplasme sont distribues dans sa masse d'une laçon quelconque, il n'y aura aucune difference appréciable entre la ficon dont l'ébranlesnent se transmettra dans une direction ct dans une autre. Afin qu'il se transmette davantage suivant une direction deterninose, il faut présupposer une consentration des molecules instables le long de cette ligne. Or, en somme, 
c'est précisement une concentration de cette sorte qu'il s'agit d'expliquer.

2u Après avoir suppose une concentration de molécules aisement transformables le long d'une ligne, spencer ne s'occupe plus que de ce qui se passe sur cette ligne et néglige absolument de considérer les autres directions, sur lesquelles cependant se produiront les mèmes phénomènes que sur la direction privilegiée quoique à un degré moindre. Cette omission est nécessaire pour faire concevoir la structure du systeme nerveux qui affecte la forme de cordons linéaires minces et allongés, mais clle est injustifiable logiquement.

$3^{\circ}$ La tendance des inolécules à produire autour d'elles des molécules semblables est indepentante d'un ébranlement qui en modifiera la structure. Cette tendance aura donc pleinement produit son effet avant tout ifranlement. Dès lors on ne voit pas du tout en yuoi celui-ci contribue à la diflerenciation de protoplasme. Il est done impossible de trouver dans l'explication de spencer un appui quelconque pour la conclusion qu'il formule en ces Lermes: " toute repétition de l'agitation aidera à accroitre, à intégrer, à définir folus complètement le cours du mouvement moléculaire, c'est à dire, prolongera sit partie la plus éloignée tout en rendant sa partie la plus prochaine plus perméable. »

Le systeme nerveux, comme toute autre partif de l'organisme, se développe frar l'exercice at nous n'avons ancune rason d'exclure ce facteur de son ixolution. Mais il s'agit ici de savoir si l'excitation elle-mentest rapable d'en expliquer l'origine et si celle-ci est un eximple d'un mouvement ondulatoire creant les voies suivant lesquelies il se propagrera. C'est ce que spenter ne parvient pas à montrer.

Entin, si on concedait à spencer tout ce qu'il dit dans le passage des Premiers Principes à l'oceasion duyuel nous avons exaniné cette question, il en résulterait qu'il s'tablit dans l'organisme des trajets par lesquels se transmet l'energie vibratoire des impressions exterieures, 
mais loin que la contraction musculaire doive ètre considérée, d'aprés celte explication, comme le résultat de ce phénomène, elle y ligure comme en étant la cause.

Nous avons montré auparavant qu'a priori on ne peut pas admettre une explication de l'activité nerveuse qui prétend négliger le côté subjectil (1). Nous voyons ici combien est insuffisant l'essai qu'en tente spencer.

Si on donne à loi de la direction du nouvement la signification plus large que nous avons indiquée, on peut l'etendre aux mouvements produitș par les forces vitales tout en maintenant la nature particulière de celles-ci.

Rien même n'empêche de la généraliser encore davantage et de l'appliquer aux collections d'hommes et d'animaux.

Le pouvoir qu'ont les espèces vivantes de se multiplier et par suite de s'étendre sur des espraces de plus en plus considirables, la proprièté aussiqu'elles manifestent de se transformer peu a peu suivant des directions divergentes et ramitices sont des forces veritables puisqu'elles sont causes de mouvement.

L'expansion rencontre des obstacles de toute sorte tant physiques (hautes chaines de montagnes, océans, continents) que physiologiques (manque de nourriture appropriee, climat trop (haud ou trop froid, trop sec ou trop humide, organismes ennemis etc.). Le mouvement se produira dans la direction de lat moindre résistance, cest-à-dire des moindres difticultés.

La transformation des esperces est constamment limitee par les conditions d'existence et la concurrence vitale, de sorte qu'elte ne se fait que suivant certaines directions privilegièes quii sont rgalement des lignes de moindre résistance.

De meme les societes humaines nous presentent de nombremx monvements qui seffectuent d'apres ce principe, lant daus les eflorts quelles font pour s'etemdre ou se deplacer ques dans les phimomines interieurs yuon y observer. Limgouement qui se manifeste pour certaines ralriores, l'allluence vers les centres industriels, l'ècou- 
lement des marchandises, les lignes suivant lesquelles se transportent les choses et les hommes, le mouvement des capitaux sont autant de phénomènes dans lesquels on reconnaitra aisément la loi de la moindre résistance ou de la plus forte traction.

Il faut donc lui reconnaitre la valeur d'un énoncé général utile à l'interprétation d'un grand nombre de pliénomènes de diffërents ordres. Mais il serait déraisonnable d'en vouloir exagérer l'importance jusqu'à y vouloir découvrir l'explication de tous les mouvements fint-ce d'un ordre déterminé. Le raisonnement de James Hinton cité par spencer est aussi faux qu'il est tranchant. "La forme organique, dit-il, est le résultat du mouvement. Le mouvement prend la direction de la moindre resistance. Par conséquent, la forme organique est le résultat du mouvement, dans la direction de la moindre résistance. ") (1)

Au lieu de se borner à noter quelques faits généraux dans lesquels la croissance des organismes est réellement influencée par les résistances qu'elle rencontre, qu'on essaie de rendre compte, an moyen de cette loi, des diff̈erences de forme que présentent les ètres vivants dans le mème milieu. Ou bien l'on se convaincra de son insuflisance, ou bien on sera amené à lui attribuer celte signitication génerale que chaque élément matériel suit la résultante de toutes les forces qui agissent sur lui. Ce. dernier principe est utile pour l'étude des mouvements, mais il ne nous apprend rien au sujet de la nature ni du mode d'action des forces qui y entrent en jeu.

\section{§II. Rythme du mouvement. - Justification de la loi du rythme. - Exceptions.}

thme du Des chapitres des Premier's Principes étudiés jusqu'a préuvement. sent, le plus interessant, le plus suggestif et qui prête le inoins le flane à la critique est celui qui est intitulé: Rythme du mouvement.

(1) P. P. p. $248 \S 78$ - F. P. p. 188. 
Le mouvement est rythmique, c'est à dire, les changements que subissent les êtres sont périodiques et les ramènent après des temps égaux dans des situations semblables. Spencer s'attache principalement à montrer l'universalité de cette loi. Pour en comprendre la signification il nous sera utile d'analyser brièvement un cas très simple.

Ln rỵthme élémentaire est constitué par le mouvement du pendule. Nous y remarquons les chose:s suivantes : un système en équilibre sous l'action de la gravité et de la résistance du fil suspenseur, une cause quelconque écartant la masse suspendue de sa position d'équilibre, la pesanteur ramenant le pendule à sa situation primitive en lui imprimant un mouvement accéléré, la position d'équilibre dépassée en sens contraire, par suite de la vitesse acquise, d'une quanté égale au déplacement primitif, le retour en arrière, le va-et-vient indéfini.

Des phases analogues se produisent dans un nombre immense de phénomènes. Depuis la précession des équinoxes qui après $2 \$ 920$ ans ramène au même point de l'éclyptique l'intersection de celle-ci avec l'équateur, jusqu'aux vibrations lumineuses qui s'acomplissent par centaines de milliards en un millième de seconde, nous connaissons une infinité de mouvements rythmiques s'accomplissant dans une période de temps plus ou moins longue. Les phenomènes de gravitation, de son, de chaleur, de lumière, beaucoup de phénomènes d'électricité consistent dans des mouvernents rythmiques. Les planètes se balancent d'un coté à l'autre de leurs orhites, les corps concoivent et transmettent des mouvements vibratoires plus ou unoins rapiles qui affectent les organes de la sensibilité.

Le mouvement des ragues a la surface de l'océan, l'agitation des feuilles, le balancenent des trones d'arbre, lo's plis ondules des drapraux sont des mourements rythmicyues provocques par le vent. Lui-mime est lie à l'exitence des grandes vagurs largement rythmiques de l'atmosphere el des vibutions sonores animent toute la masse de l'air qu'il transporte. 
La rotation de la terre autour de son axe ramène régulièrement le jour et la nuit, son mouvement autour du soleil engendre les saisons tandis que la lune soulève et abaisse les eaux de l'océan.

La périodicité des jours et des saisons produit des mouvements rythıniques dans la vie des plante's et des animaux. Le sommeil et la veille, les gains et les pertes périodiques d'énergie, la courbe de l'existence, la matière alternativement saisie par les forces vitales et abandonnée au monde inorganique. les générations qui se succèdent cornme les feuilles que le printemps fait éclore et dont l'automne emporte les débris, les espèces qui naissent, se developpent et meurent - les vibrations des nerfs, les contractions des muscles, les periodes d'ardeur et de fatigue, d'excitation et d'abattement, de trouble et de calme, d'attention et distraction - les fluctuations du marche, l'abondance et la rarete des objets, les crises industrielles, les alternatives de gutrre te de paix, de progrès et de décarlence, les périodes d'enthousiasme et de réaction, tous ces phénomènes de l'ordre le plus varie ont un caractère rythmique plus ou moins régulier mais assez évident pour qu'il soit inutile d'y insister.

II arrive frequemment que plusieurs rythmes se superposent. Ils peuvent alors être plus ou moins facilement distingués l'un de l'autre dans le mouvement qui résulte de leur combinaison. Parfois ils ne se cachent pas du tout parce que leurs périodes sont très différentes, ou bien parce qu'elles sont subordonnées, c'est-it-dire que le second rythme est une modification périodique du mouvement qui constitue le premier, et ainsi de suite pour les autres.

Dans un concert les repos alternent avec les morceaux de musique. On a choisi ceux-ci de manière à faire entendre un morceau d'allure vive après un morceau d'un mouvement plus lent. Dans chaque morceau les forte et les piano alternent, les différentes parties des phrases musicales se repondent; dans chaque mesure les temps forts et les temps faibles reviennent $\dot{a}$ intervalles égraux et 
entin chaque note est constituée par des ondulations de l'air. Nous n'avons pas de difficulté a reconnaitre tous ces rythmes simultanés et ils nous procurent une jouissance parce que l'activite rythmée convient à nos facultés psychiques.

Lorsqu'on examine da la loupe la ligne ondulée que trace la pointe du phonographe, on distingue les éléments vibratoires des trois ou quatre sons que l'instrument a enregistrés simultanément. Notre oreille les isole plus facilement que notre œil.

Les mouvements alternatifs assez irréguliers du baromètre cachent complìtement, à première vue, deux ondulations diurnes régulières qui se superposent plus ou moins elles-mèmes et ne peuvent ître reconnues que gràce à de minutieuses observations.

Il résulte de cette coexistence de rythmes différents que généralement le mouvement rythmique ne ramène pas des phases exactement pareilles. Lne saison est constitue principalement par la situation de la terre par rapport au soleil, mais elle dépend aussi des fluctuations de la pression atmosphérique. de certaines périodes alternatices peu connues d'humidite et de sécheresse, de variations a rythme très lent dans les courants marins, dans la ligne des rivages et de bien d'autres farteurs. De telle sorte que jamais deux hivers ou deux étis ne so ressemblent complètement. Malgré ces irrégularités, dans nos contrées les saisons se distinguent aisément l'une de lautre; il n'en est pas de même dans des régions plus voisines de l'íquateur, ou leur rythme, moins marqué a cause de la latitude, disparait parfois complètement dans les changements accidentels du temps.

Le: caractire oscillatoire du mouvement peut étre, on le voit, plus ou moins manileste et plus ou moins complexe. Il est extrimement interssint d'en rechercher l'existence dans les phenomines les plus divers. II correspond certainenant a une grande loi qui grouverne lous les btros materiels, et mous rroyons que c'est à spencer que revient l'honneur de l'avoir mise en lumiers dans toute son étendue. 
Les objections que Renouvier oppose à cette loi ne sont pas bien considérables. "En fait, dit-il, la chute d'un grave, la trajectoire d'un corps sous une impulsion passant par son centre de gravité, la révolution d'une splhere autour d'un de ses dianctres, sont-ce des mourements rythmiques? Sensiblement, non; insensiblement on peut le concevoir à l'aide d'hypothèses précises sur la composition chimique, sur la loi de communication du mouvement, sur la résistance des milieux, sur la variation des actions en fonction des distances. On ne voit pas que M. S. se voit occupé de ces petites conditions du problème qu'il voulat résoudre. Une faute capitale d'une autre espèce c'est d'avoir généralise la notion du rythune an point d'en fare disparaitre cet élément essentiel, la régularité, la loi numérique, ” (1)

La chute d'un grave est un mouverment rythmique de sa nature, comme le mouvement du pendule. si le rythme n'y apparait pas, c'est parce que le mouvement du corps qui se dirige vers le centre de gravité de la Terme est arritio à sa surface at transformé en un autre mourement rythmique: la chaleur. Cette transformation se rattache a une categorie d'exceptions a la loi, comme nous le verrons.

Quant à la trajection d'un corps sous la seule impulsion d'une force qui passe par son centre de gravité, s’il s'agit d'une force continue, le mouvement sera en general alternatif ; s'il s'agit d'une force instantanée, on peut rejondre qu'un tel mouvement n'existe pas dans la nature.

La rotation d'une sphere est un mouvement rythmique en ce sens que clacun de ses points se trouve alternativement d'un coté et de l'autre de l'axe du mouvement.

Le mouvement rythmique n'a jamais une régulariti mathématique. Il est sensiblement régulier lorsqu'il est principalement déterminé par des forces peu complexes. Ainsi une corde tendue qu'on éloigne de sa position d'équilibre par une impulsion unique y revient par des

11) Examen des Premiers Principes de $H$. Spencer. La critique philosophique 1886 vol. II pp. 247-218. 
mouvements sensiblement réguliers. Au contraire les alternatives de prospérité et de décadence d'une nation dépendent de causes très nombreuses et d'intensité très variable, de sorte que la régularité du mouvement de va-etvient n'existe plus. Elle n'existe pas non plus dans le mouvement des flots ou dans celui des épis sous le soufle du vent. Ne pas vouloir qu'on les appelle rythmiques parce qu'ils ne sont pas réguliers, cela nous parait abusif et en tous cas c'est une question de mots. Renouvier trouve que c'est une banalite que de coustater l'universalité du rythme dans le sens large. Nous ne saurions être de son avis.

Iustification de la loi du rythme.

Spencer considère le rythme du mouvement comme une conséquence de la persistance de la force. De fait, en l'absence de la force, le mouvement se poursuit indéfiniment dans la mème direction. Le rythme suppose aussi l'inertie en vertu de laquelle le mouvement se poursuit au dela de la position d'équilibre. Enfin il est une des formes de la conservation de l'énergie.

I)'après Spencer, tout mouvement, dans la nature, est necessairement rythmique. "Tout transport dans l'espace, dit-il, doit altérer la proportion des forces en jeu, augmenter ou diminuer la prepondérance d'une force sur l'autre, emprecher l'uniformite du mouvement. Si le mouvement ne peut ètre uniforme, en l'absence d'accélération ou de retardation continuees pendant un temps intini et a travers un espace infini lresultats quion ne freut concevoir), il n'y a fras d'autre alternative que le rythme, ")

(1) pourat reprocher a col argument de ne pas tenir compte du mouvement qui disparait comme: tel, e'est a dire, qui so transforme en inergrio potentielle. Mais comme l'energie: potentis le peut risgenerer le mouvement

(1) P. 273 \& 82 F. P. p. 204. 
qui lui a donné naissance ou un autre équivalent c'est un cas de transformation alternative qui rentre dans la loi.

Une objection plus grave résulte des mouvements qui se transforment en d'autres de telle sorte que la transformation inverse ne soit plus possible. Spencer fait remarquer à propos d'un cas semblable (1) que la nouvelle formo dr? mouvement est elle-mème rytlmique. Mais cela ne résout pas la dilliculte, surtout si la transformation dont il s'agit n'est pas accidentelle mais normale.

Entin l'argument ne mentionne explicitement qu'un seul élément du rythme, la vitesse du mouvement (accéliration et retard). Le rythme peut resulter aussi de la direction du mouvement. Il implique aussi une certaine periodicité et il consiste, en general, dans des écarts alternatifs par rapport à une situation moyenne qui peut itre une position d'équilibre réalisable ou non.

Nous croyons que c'est dans cette derniere condition du mouvement rythmique qu'on peut trouver la raison de son universalité.

En effet, tout itre individuel, naturel ou artiticiel, cor" respond à une situation d'équilibre stable autour de laquelle il oscille parce que des forces exterieures tendent continuellement à l'en écarter, tandis que les forces interieures l'y ramènent. D’une faron plus générale, les forces naturelles sollicitent !es corps ver's des situations d'équilibre stablp et déterminent, par conséquent, des mouvements de ra-et-vient autour de cette situation.

In organisme, un éditice, une société sont des exemples d'êtres individuels. Le premier comprend une certaine quantité d'énergie potentielle grice à laquelle il exerce ses fonctions, ou encore une certaine disposition intérieure et extérieure des parties، La réaction contre les agents externes épuise cette énergie et il la répare par la nutrition sous toutes ses formes; de lá l'oscillation entre les périodes de travail et de repos, de désassimilation prédominante et d'assimilation prédominante. Les agents exté-

(1) P. P. p. $271 \$ 82-$ F. P. p. 202. 
rieurs tendent a alterer la disposition de ses parties, d'où les alternatives de maladie et de santé, de lésion et de reconstitution des organes. Le rythme respiratoire correspond à l'action alternative d'éliminer l'excès d'oxygène combiné et d'absorher l'oxygène libre qui fait défaut.

L'édifice est constitué par une certaine disposition des matiriaux. Le vent, les mouvements du sol lui impriment de légères deviations qui déterminent des mouvements oscillatoires tant qu'elles ne dépassınt pas la limite d'élasticité de la construction.

L'équilibre social comprend l'égalité des besoins et des ressources, une liberté suffisante des citoyens sous l'empire des lois, certains moyens de défense et d'attaque, une activité convenable dans les différentes branches gui contribuent is la prosperite de la nation. Lorsque des excès ou des defaillances se produisent d'une part ou d'autre, la societe reagit par ses forces conservatrices et il en resulte pncore une fois un mowrement d'oscillation autour de la situation moyenne qui n'est jamais réalisée exactement.

Les forces d'attraction en attirant vers le centre, c'est à dire vers la situation d'équilibre, les atomes de la nébuleuse primitive ont determiné des monvements oscillatoires des planètes autour de cette position.

Tout mouvement ondulatoire soit visible, soit invisible se fait autour d'une situation d'equilibre, qu'elle soit représentie par la surface unie de la mer, par la densité des corps que traverse le son ou par la tension des cordes de violon sur le chevalet.

l'existence d'une situation d'équilibre vers laquelle se dirige le mouvement est la cause de son caractère oscillatoire, parce que la mobile dépasse la position d'équilibre en vertu de la vitesse acquise par l'action des forces qui l'y sollicitent.

Le besoin d'unc denrée dans une societe se manifeste par une hausse de prix. Colle circonstance determine une angmentation croissante de la production, de telle sorte quapres un crotain temps, non semlement les besoins 
sont satisfaits, mais If marché est encombré. Immédiatement les prix baissent, la production se rallentit et tombe bientòt en-dessous des besoins, ce qui ramène loute la seric de ces phemomènes économiques.

Le travail epuise l'activite nerveuse et amène If sommeil réparateur. La réparation achevée determine lo reveil et la reprise du travail. Cette sire physiologique comprend deux oscillations correspondantes: travail et r'epos, depense et gain d'energie nerveuse. Elles se font autour d'une position moyenne, d'ailleurs irrealisatole, dans laqquelle le travail ne depenserait qu'une quantité d'entergie qui pourrait continuellement ètre réparée.

ptions loi du hme.

La consideration precedente, en faisant connaitre les conditions du mouvement oscillatoir", nous permet aussi d'indiquer les cas ou elles nte sont pas realisées et qui constituent, par conséquent, des exceptions à la loi.

la premiere de ces conditions est que les forces qui maintiennent la situation d'équilibre soient capables de ramener en arrière lo mouvement qui s'en écarte, ou, en d'autres termes, que la limite d'élasticité du système ne soit pas depassée. Cn organisme trop fortement ébranlé ou trop atliabli ou trop mutilé périt; une maison trop secouée par le vent s'écroule, une société attaquée par des ennemis trop puissants, troublèe pas des agitations interieures excessives, se disloque et se dissout. Dans cescas le retour en arrière ne se fait pas et il n'y a pas de mouvement alternatif.

Tant que l'archet se promène légèrement sur la corde du violon, celle-ci vibre autour de sa position d'équilibre, mais un effort trop violent rompt la corde; les vibrations cessent et aucun mouvement oscillatoire ne correspond à la rupture.

La seconde condition est que la situation d'équilibre vers laquelle marche le mouvement puisse ètre dépassée. Si par sa nature elle constitue un état final, le mouvement 
au delà est. impossible, et par consèquent aussi le mouvement en retour et l'oscillation rythmique. Dans ces cas le terme était atteint, le mouvement cessera. Sans doute, il ne sera pas réduit à rien, il sera transformé. Mais il n'existera plus sous sa forme spécifique et il n’aura pas possédé le caractère oscillatoire.

Le Soleil rayonne sa chaleur' à travers l'espace. Ce mouvement tend à établir l'équilibre de température entre cet astre et le milieu où il se meut. Cet équilibre étantréalisé, il ne se produit plus aucune communication de chaleur: c'est une situation de sa nature définitive qui ne comporte aucune oscillation de température. Le mouvement d'échaulfement progressif s'est transformé en vibrations caloritiques uniformément distribuées.

Telle est aussi la transformation générale du mouvement visible en mouvement vibratoire. lci la transformation inverse est possible et domne lieu a des oscillations, mais elle n'est jamais complète : le mouvement visible tend à dispararitre tout entier et à être remplacé par du mouvement invisible. Telle est est la situation ver's laquelle l'Univers marche ; elle est, de sa nature, définitive. "On ne rencontre dans la nature, dit Auerbach, que des procès qui ne sont pas ou ne sont qu'imparfaitement réversibles. Ce qui est passé est passé et l'énergie dissipée ne peut plus itre rassemblée de nouveau, du moins completement. C'est comme si une autorité supérieure levait un impòt sur chaque phénomène naturel, et quand on essaie d'éluder cet impòt par l'introduction de procédés forcés et inverses, l'agent du fisc s'attache à leur suite. " (1).

C'est ce qu'on appelle la dégradation de l'énergie ou l'accroissement de l'entropie. L'énerrerie de l'Ĺniver's reste constante mais elle tend a se transformer en chaleur qui tend elle-mime a se répandre uniformiment. Elle devient ainsi intransformable en une autre formo d'inergie, de sorte quen derniere analyse l'Univers tend vers une situation oi ausune transformation d'energie n'anra plus lieu.

(1) Due Wellherrin und ihre sihallen. Jena 19002 p. 3:3. 
Ce sera le repos complet dans une température uniforme (1).

Cette situation comporte, il est rai, des oscillations moléculaires, et sous ce rapport la loi sera toujours vérifiée; mais le mouvement ver's cet etat n'est pas un mouvement rythmique.

Dans la dernière édition des Premiers principes, Spencer reconnait que la disparition du mouvement visible dans certaines circonstances constitue une restriction a la loi du rythme. Il fait remarquer que le mouvement invisible, par lequel il est remplace, est lui-mime rythmiques. (2) Nous venons de dire que cela n'emprelie pas l'exception d'exister.

Spencer en signale une antre dans lexistence des comires non périodiques, et conclut quil faut entendre la loi d'un systime lerme (3). Cat cas peut se lamenter aux pruptures d'equilibres dont nous arons parli.

(1) ef. ibid. p. 41.

(2) F. P. p. 216.

(3) Ibid. 



\section{L'ÉVOLUTION}

Récapitulation. - Evolution, dissolution et loi d'évolution d'après Spencer. - La redistribution principale : l'intégration. - La perte du mouvement. - Confirmation inductive de la loi d'intégration. - Les forces qui agissent dans l'intégration. - L'évolution composée. Les conditions. - La différenciation. - Confirmation inductive de la loi de différenciation. - La définition et la coordination des parties. - La redistribution du mouvement. - Les forces qui agissent dans les redistributions secondaires. - La loi d'évolution amendẻe.

capitulation.

La métaphysique de spencer est nécessairement incomplète. Par sa thérie de l'Inconnaissable qui repose sur la these kantienne de la relativite de nos connaissances, spencer s'est interdit toute recherche sur la Cause Première et sur la réalite des êtres que nous percevons comme corps (par la sensibilité) ou comme esprit (par la conscience réflexive). Il affirme cependant l'identité de la réalité qui correspond à ce qui est perçu objectivernent comme corps et de la rialité qui correspond à ce qui est perç subjectivement comme esprit. Et cela est à la fois une thèse metaphysique que nous avons 
jugree fausse ot une inconséquence, car on ne peut affirmer l'identité de choses inconnaissables.

spencer confond aussi la réalité qui se manifeste sous forme de corps et d'esprit avec la Cause Première; et c'est là encore une fois une thèse métaphỵsique condamnable et une inconséquence du mème genre.

La doctrine que nous avons exposée dans ces derniers chapitres et que nous allons récapituler avec les corrections que nous y avons apportées ne concerne donc que le monde corporel, comme il se manifeste à l'expérience. Pour autant qu'elle est une philosophie, et nous sommes d'avis qu'on ne peut pas lui refuser celte dénomination, elle est une philosophie des corps et plus particulierement du mouvement. Cependant les propositions qu'elle énonce ont rapport à la vie et à l'intelligence - prise dans son acception la plus large - mais seulement en tant que l'activite de celles-ci est liee à la matière el au mouvement.

Voici la doctrine que nous avons adoptée: L'Lnivers corporel comprend quatre éléments irréductibles : l'étendue, la masse, le mouvement, les forces.

Le premier principe général est la conservation de la matière quant à la masse; c'est ce que Spencer appelle l'indestructibilité de la matière.

Le second, qui est l'indestructibilité du mouvement, comprend le principe de l'inertie. Au surplus, pour être universel, il doit s'entendre d'une persistance du mouvemênt qui peut n'être que virtuelle.

Le troisième principe, qui est appelé par spencer la persistance de la force, doit s'interpreter comme signifiant la conservation de l'énergie. Ce principe est certainement applicable aux phenomenes inorganiques, mais sa veritication exacte dans les phénomènes vitaux est douteuse.

la meme restriction doit s'appliquer an principe de la Iransformation equivalente des forces, ou mieux, de l'éneryie.

La persistance de la force, en y comprenant sa consépuncere qui est la persistance des relations entre les lorers, inplique encore un principe géneral qui est la constance des lois de la nature. 
Le principe do la direction du- mouvement a une signification tout à fait gènérale quand il affirme que le mouvement se fait suivant la résultante de toutes les forces. Il a une signification moins universelle, mais donnant lieu à des applications nombresuses tet interessantes, forqu'il énonce que te mouvement se fait suivant la ligne de moindre resistance ou de plus forte traction.

Entin le principe d'après lequel tout mourement est rythmique ou se transforme en mouvement rythmique semble se vérifier, de la manière dont nous l'arons expliqué, dans tous les phénomènes materiels. Tel est le chemin parcouru jusqu'à présent; tels sont les résultats obtenus.

trolution, Nous ahordons maintentant une atutre seripe de considesolution et d'évolution rations. Apre's avoir passe en revue les dommes dre l'evolution et les lois les plus grinerales qui less regissent, il faut étudier l'évolution elle-mine. Il ne sullit fras d'indiquer les éléments dont se compose l'Lnivers at les principes qui le gouvernent, il est nécessaire de rechercher en outre de quelle manice les isements concoment a procluire les phenomènes et quel est le rosultat des principes que l'on a etablis.

Après avoir fait l'analyse, il laut etudier la șnthese; non point la synthese spéciale a chatque ètre, mais une synthèse generale que realisent tous les itres. Il faut tronser ou du moins chereher une formule i larputle toute existence se conforme et qui se virritie lle loutes les existences réunies. "Chaque ohjut, dit spencer, non moins que l'agregat de lous les objuts, subit à chaupue instant quédque changement d'etat.... La futestion qui se pose est: quel principe dynamique vari de la métamorphose considerée dans sa totalite et dans ses details, exprime ces relations toujours changeantes? ) (I)

(1) P. P. p. $297 \$ 92-$ F. P. p. 221 
Spencer entreprend done une géneralisation de grande envergure. Il sera indispensable que nous nous écartions de la methode que nous arons suivie jusqu'à prisent et qui a consiste is suive spencer et à mereer notre eritique parallèlement au développement de ses idlees. Ini il est nécessaire que nous exposions d'abord dans son entier la conception de Spencer en résumant autant que possible les développements considérables qu'il lui domne. Après cela nous en étudierons séparément les dilterentes parties. Voici donc les grandes lignes de sa doctrine:

La masse de la matière ne subit aucun changement. Son étendue ne se manifeste a nous que par la situation relative des corps dans l'espace; les forces, par le mouvement qu'elles produisent; de sorte que les phénomènes materiels peuvent être considerés comme detrominés par la disposition des elements materiels et la forme de leur's mouvements.

Lat loi que l'on cherche est done celle qui exprime la restistributiom continue de la matiere et du mouvement.

Line connaissance complite d'un itre embrasse son origrine pt sit fin et comprend tous les changements qu'il subit entre res deux termes. Que le commencement d'une chost soit on the soit pas l'objet de notre experiencer directe, qur sa tin puisse itre constatie ou seulement privue, que lis chamgements qu'ellr subit s'accomplissent sous nos yemx ou he nous soient connus que pall inderetion, lat sciente que nous en avons, sous purine d'etre

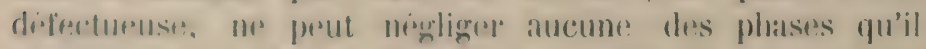
Iraverse. Dans l'idude des itres en particulier anssi bien

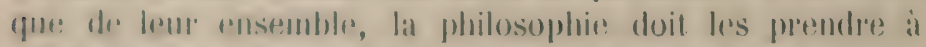
leur origine en les suive jusqua leur destruction.

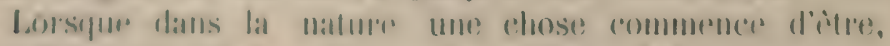
les itements qui la composent existaient dejai anparavant;

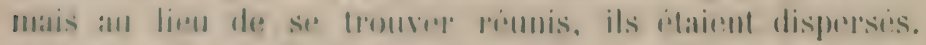
Less atomes nui constiturent l'animal ctaient ripandus daus l'air. dans le sol, dans des virrelaux, dans d'aulres animanx. Len matipriax qui compusent une maison provirment 
des fortets, des carrieres, de gisements de minerais. Pour constituer l'édifice, ils ont été réunis, assemblés.

Le phénomène inverse va se produire lorsque la chose cessera d'avoir une existrnce propre. La substance organique se décompose, les gaz qu'elle contient sont mis en liberté et se répandent dans l'espace; les matières solides sont réduites en poussirire et dispersér's. Les liens qui retenaient ensemble les materiaux d'une conslruction se relachent et se brisent. Les pierres, les poutres se disjoignent et finalement se dispersent.

Avant leur réunion, les éléments, indépendants les uns des autres, étaient animés de mouvements relatifs plus ou moins considirables ; après leur rémnion, ces mouvements sont restreints dins des limites étroites. Le's Cléments reprennent leur liberti lorsque l'itre est ditruit.

De cette maniere simple d'envisager l'existence d'une chose quelconque, spencer deduit la formule suivante: "Le passage d'un étalt dillus, imperceptible " (c'est-a-dire non distinct) " a un etat concentre, prerceptible, est une intégration de matière et une dissipation concomitante de mouvement; at le passige d'un blat concentre, prerceptible, is un etat diffus, imperecptible est une absorption de mouvement et une desintegration concomitante de matière. " (1)

La première opération porte, fautr de mieux, le nom d'évolution; la seconde est désignée sous le nom de dissolution.

D'après cela, l'évolution est le proce's par lequel un Atre est produit et progresse, la dissolution, le procès par lequel il se détruit et tinit par disparaitre. Néanmoins le mot évolution est employe igalement pour désigner l'ensemble de ces deux procès et nous l'employons nousmème dans ce sens lorsqu'il n'y a pas lieu de les distinguer.

L'état d'un être corporel n'est jamais absolument constant; il subit sans cesse des changements peu considérables peut-être, mais réels, par exemple, dans sa masse et

(1) P. P. p. $301 \S 94-$ F. P. p. 22:; 
dans sa température. Ces changements - sans prétendre par là les définir complètement - sont souvent soit des intégrations de matière avec perte de mouvement, soit des absorptions de mouvement avec désintégration de matière. La première commence par dominer, puis, après des alternatives de prépondérance tantòt de l'une, tantòt de l'autre. la seconde l'emporte détinitivement.

Lorsque l'évolution d'un agrégat se réduit à une intégration de matière avec perte de mouvement on lui donne le nom d'évolution simple. La plupart du temps l'existence d'un être nous offre une complexité beaucoup plus grande. C'est qu'à còté de cette redistribution principale de matière et de mouvement que nous venons de définir, les forces qui agissent sur l'ètre matériel en produisent d'autres concomitantes, intérieures à sa masse, affectant differemment ses diftérentes parties; on a alors l'evolution composée.

Tous les ètres ne présentent pas au mème degré les redistributions secondaires. Elles sont insignifiantes dans les corps fortement intégrés dans lesquels les molécules ne jouissent presque d'aucuné mobilité les unes par rapport aux autres, ou encore dans les cas d'intégration rapide sous l'action d'une force prédominante.

Elles deviennent al contraire très importantes et très complexes dans les êtres dont les parties sont très mobiles, qui contiennent, comme dit spencer, "une grande quantiti- de mouvement actuel, ou potentiel ou les deux a la fois. m (1) Ainsi, les redistributions secondaires s'accomplissent plus aisément dans un agréegat qui subit un ébranJement dî à des mouvements visibles ou vibratoires. La stabilité chimique, par exemple, décroit souvent à mesure que la trompriature angmente; elle décroit aussi à mesure fu'augmente la chaleur absorbee par la combinaison.

Litut fluidre est une situation favorable aux redistributions secondirires, aver cette restriction, que si elles y sont nombreruses of ietendues, elles y manquent de stabilite et

(1) P. P. P. $200899-$ F. P. P. 231. 
n'y lasssent point de traces, l'etat de ces corps rendant impossible toute structure.

Les circonstances les plus favorables i des redistribution qui, étant nombreuses et très complexes, oftr'unt cependant en meme temps une certaine jermanence, sont réalisées par les êtres vivants, lesquels contiennent une grande quantité de mowrement latent et prisentent une cohésion intermediaire entre l'état solide et la fluidité.

Ces conditions sont réalisées au plus haut clegré chez les animaux.

Yous arons ainsi, laissint pour le moment de coté la dissolution, une idée compréhensive mais assez vague de l'èvolution. Elle comporte, dans sá forme complete, deux procès en un certain sens opposés: une intégration d'ensemble avec perte de mouvement, c'est la redistribution primaire; une diffrenciation progressive des parties dues aux redistributions secondaires.

Cette conception obtenue, comme un vient de le voir, par vole deductive, se contirme et se précise par l'induction. Considerant d’alord la redistribution primare, on se convainc que ses conditions sont realisies daus tous les orches de choses: la formation de notre système sideral, la consolidation du globe terrestre, la vie organiquue, la vie sociale, la formation du langauge, les progrès de la science et des arts. Nous retrouvons partout " un changement partant d'une forme moins coherente pour aller à une forme phus coherente par suite de la dissipation du mouvement et de l'intisnration de la matière. "(1)

Passons aux redistributions secondaires. Celles qui sont permanentes produisent des modifications de structure. A mesure qu'elles se multiplient et s'accentuent, elles cléterminent nécessairement une hétérogeneisté croissante des parties. Cette loi se vérilic, comme la précéclente, dans toutes les catergories de phénomenes depuis la formation du système solaire jusqu'à l'évolution des sociéti's et des produits de l'intelligence humaine.

(1) P. P. p. $349 \S 115-$ F. P. p. 263 
Done " partout ou l'érolution est composée, c'est-àdire dans l'immense majorite des cas, tandis qu'il se fait une condensation progressive de l'agregat, soit par un rapprochement plus intime de la matiere dans ses limites, soit par l'annexion d'une plus grande quantité de matiere, soit par les deux procédés, et tandis que les parties plus ou moins distinctes qui risultent de la division at de la subdivision de l'agrégat se contractent chacune de son còté, ces parties deviennent disșemblables, - dissemblables par le volume, la forme, la structure, la composition, par plusieurs de ces caractères ou par tous. La même opération se montre dans l'ensemble et dans les parties. L'ensemble va sintégrant et se diflérenciant des autres ensembles; ct chaque pirtic de l'ensemble s'intigre en mime temps qu'elle se difterencie des autres. " (1)

Nanmoins, si toute évolution composie pst une marche dans le sens de la differenciation, toute differenciation ne constitue pas un progrès de l'evolution, mais au contraire elle est qur!lyutois un commencement de dissolution. Pour quo'il $y$ ait progrès, il litut que la diflierenciation amène non seulement une: " multiplication de parties dissemblables, mais encore un aceroissement de la nettete avec laquelle ces fraties se distinguent les unes des autres. " (2) Lanarchie dans une sociéte se manifeste par des phénomines varies qui accroissent, peut-on dire, l'héterogeneiti; mais elle tend d'atutre part a confondre les denrés de la liérarchie sociale.

Lat mardhe de l'indetini vers le difini caracterisant la dillerenciation evolutive, tel est le caractere fuion retrouse,

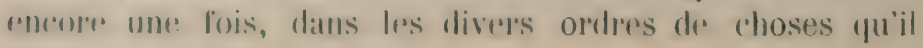
nous est permis d'etudier.

Eufion, la redistribution do la matione implique neressairement une redistribution du mouvement qui n'est pas

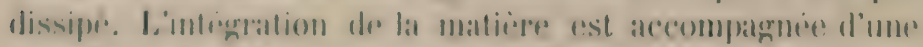

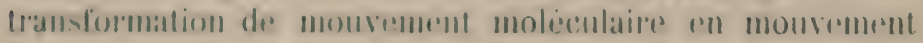

(1) P. P. P. 384 \& 127-F. P. p. 291

(2) P. P. p. 287 है $120-$ F. P. p. 293. 
visible; les mouvements rythmiques des parties se difierencient necessairement avec la diflerenciation des jarties ellesmêmes et deviennent plus detinis a mesure que les patries s'intègrent. Ln examen des dilferentes catégorirs de phenomènes permet de confirmer ces affirmations inductivemunt.

Les considérations qui préèdent conduisent à la formule suivantes à laquelle spencer donnes le nom de loi d'ivolution: "L'évolution est une intingralion de matière accompagnée d'une dissipation de mouvement, pendant laquelle: lat matière passes d'une homogéneitr indefinis, incoherentes is une héteroginéité distinie, colnerente, et pendant layuelle aussi le momement retenu subit unt? transformation analogue. ” (t)

Dans l'exposis suceinet des raisommements sur lespuels celte loi repose nous n'avons fitit qu’indiguer sams aucmu developprement la partie ou se manifeste le mirux l'esput symbetigue de spencere : lexur justilication au moyen d'une induction moverselle. Ayant isole les ideses de spencere de leurs applications concretrs, nous leme avons moleve

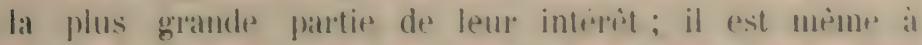
craindre que, malgres les rllorts que nous avons faits pour les exposer dairement, nous ne les ayons remdurs peu intellighibles. La critique que nous allons en farre remediera dans une larger mesure a cet inconvenisent qui était ici inévitable.

Avant de l'aborder éartons une idée liansse. Qunoique incontestablement les diflérents ordres de choses ofhent le spertacle d'un frogrès dans l'evolution, ere serait nianmoins me arreur de croire que ce progres est continu. Beancoup de redistrilutions secondaires qui se foroduisent dans les agrigats au lieu dappartenir an moces ivolutif. sont plutit un acheminement vers la dissolution, au liru de marquer un progriss elles marpuent un recul, ou bien elles ne produisent ni l'un ni l'autre. "()n conscoit ordimairement l'évolution, dit spencer, comme l'ellet d'unts tendance intrinsèque en vertu de laquelle tout devient

(1) P. P. p. $124 \$ 143$ F. P. p. 321. 
supérieur ; c'est s'en faire une idée erronée » (1) Quoiqu'il en soit de l'existence de tendances intrinsèques, il est certain qu'elles n'ont pas pour résultat un progrès continuel et que non seulement beaucoup d'agrégats se dissolvent, mais encore qu'ils présentent souvent dans leur existence des alternatives de progrès et de recul.

spencer applique cette remarque à l'évolution de notre race. "Il y a des raisons, dit-il, qui permetlent de penser que les hommes des types inférieurs existant aujourd'hui, et qui forment des groupes sociaux de l'ordre le plus simple, ne:sont pas des spécimens de l'homme tel qu'il fut dans le principe. Il est probable que la plupart d'entre eux, sinon tous, eurent des ancètres qui étaient parvenus à un état supérieur, et l'on retrouve au nombre de leurs croyances des idées qui ont èté élaborées durant ces etats superieurs. Si la théorie de la dégradation, telle quion la presente d'ordinaire, est insoutenable, la théorie de la progression, dans sa forme la plus absolue, me semble tout aussi insoutenable. Si d'une part on ne peut mettre en harmonic avec les faits la notion qui fait venir l'etat sauvage d'une chute de l'homme à l'ètat de civilisation, d'autre part rien ne nous autorise a penser que les degrés les plus bas de la sauvagerie aient toujours eté aussi bas qu'aujourd'hui. Il est bien possible, at, sulon moi, très probable que le recul ait éte aussi fréquent que le progrès.» (2)

Fous allons maintrnant étudier plus en detail les dillérentes parties de l'évolution.

La redistribution principale.
Examinons d'abord le proces d'intigration. Le caracetime gronial de la greneralisation de spencere ne doit pas

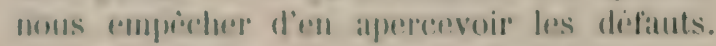

En ne: royant dans tous les phinomenes materiels que

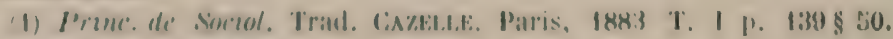

(2) Ibid. p. 133880 . 
des redistributions de matiere et de mouvement, spencer se montre conséquent avec sa conception purement mécanique de l'Lnivers. Dis qu'elle devient exclusive, colle manière d'envisager les choses est illigitime. Elle est en tous cas incomplete, comme nous l'avons déjá montré et comme nous le verrons encore bientòt.

Nous arons, en premier lieu, ì examiner jusqu'à quel point on peut détinir l'existence sensible en disant qu'elle comprend une integration do matière st une dissipation de mouvement (évolution) suivies d'une desinternration de matière avec absorjtion de mouvenent (dissolution).

Lorsqu'on voit dans l'être le resultat de l'intégration, on le considere au point de vue de l'unité qu'il réalise et qui disparait dans la desintégration. L'unite est un caracteres? essentiel de l'stre et se confond avec lui. Tire qu'il y a plusieurs hommes revient a dire qu'il y a plusirurs fois un homme: sans unite, il n'y a ni un ni plusieurs; il n'y a. rien absolument.

Tout ètre, comme tel, est un. Il y a donc autant de modes d'unite qu'il y a de manières d'être. Nous bormant aux existences sensibles dont il est question ici exclusivement, nous trourons au plus bas échelon de l'unité, par exemple, une masse liquide. Elle n'a point d'autre unite que celle qui risulte de la juxtaposition quelconque et de la cohésion de ses molécules. Ln solide ayant une forme déterminée, réguliere, manileste un degré d'unite de plus : l'unite de la contiguration exterieure. Telle masse d'or est en mime temps une houle d'or. Si la forme extérieure est le résultat de la réaction des forees internes de la masse matérielle, comme dans le cristal, cette circontance constituera un nouveau degré d'unité.

Ln édifice suppose un arrangement de multiples parties en vue d'un but; c'est une unité qui est réalisée à un degré plus élevé par une machine dont les parties agencées travaillent de concert pour obtenir un résultat. Une coopération analogue, mais bien plus merveilleuse, se rencontre dans tout organisme virant.

Que si nous considérons les organismes doués de 
sensibilıté, il se manifeste dans leur connaissance une unité nouvelle de l'être sentant ou intelligent, diffèrente peutètre des autres, mais non moins evidente à coup sur. Si la conscience seule persoit cette unité directement, elle peut cependant ètre reconnue par l'observation de l'activité extérieure des animaux et de l'homme, laquelle est inexplicable sans une intelligence ou une sensibilite qui la dirige.

Entin les animaux vivent en troupe; les ê'res intelligents vivent en socièté: nouvelles formes d'existence et, par conséquent, nouvelles formes d'unité.

Yous nous garderons bien de mettre sur la même ligne tous les modes d'unité que nous venons d'énumérer. l'uisrgu'il y a dans la nature des substances, par exemple l'homme, et des accidents, par exemple le mourement, il y aura nécessairement deux modes d'unite radicalement distincts, l'unité de la substance et l'unité réalisée par l'accident.

Totons enfin qu'aucune unité résultant de l'union de principes preexistants n'est primitive, car elle dépend de l'unite de chacun des éléments qui la consiituent.

Ces remarques nous révèlent un double défaut dans le point de départ de spencer. D'abord il n'est pas premier. Toute integration de matière suppose des élements ma-. teriels antérieurement dispersés 11 ne servirait à rien de dire que dans cen èlat de dispersion ces eléments n'etaient pas sensibles. Mème an point de vue de la théorir phrinomenalistr, ces éléments étaient quelque chose; car ils étaient capables de devenir sensibles par leur réunion. Ils avairut donc une existence indirectement ou potentirollement sensible que la métaphysique ne peut pas uegliger. "Votre theorie des choses dit spencer, considerees individurellement ou dans leur totalite est incontestablement incomplite tant ques des parties fuedconques du passi et du futur de: lour existence semsilile restent sans explicalion. "(1) Mr, dans la doctrine gne nous examinons,

(f) P. P. P. 300 / $03-$ F. P. p. 222. 
l'existence des elements matérials qui par leur integration constituent l'existence sensible reste sans aucune rxplication. Crette doctrine est donc incomplete.

Dans l'édition de 1900), Spencer reconnait qu'il an est ainsi ; mais, ajoute-t-il, cola est inevitable. "Cne connaisSance complète du commencement et de la tin des objets individuels ne peut dins beacoup de cas pas itre obtenue; leurs etats initial ot final restent vagues apres que l'investigation a fait gle qu'elle a pu. A plus forte raison, lorsqu’il s'agrit de la totalite des choses, devons-nous conclure que les etats initial et tinal sont en dehors des attrintes de notre: intelligence (beyond the reach of our intelligence). Comme nous ue pourons pas mesturer l'intini ni dans le passe ni daus le futur, il suit gue lat massance et la tin de la totalite des existences stensibles doirent toujours rester des matieres de sfreculation plus ou moins justifier par le rasonnement sur drs dumees etablies, mais toujours de spéculation. » (1)

On ne lit pas ce passage sams quelque etomnement. Si le commencement et la fin des chosiss sont en dehor's des atteintes de notre intelligence, ils ne penvent ètre l'ohjet d'une speculation légritime. En outre, si le passé de l'univers est infini, il na donc pas eu de commencement, de mime que si son avenir est intini il n'aura point de fin. Dans cette hypothest, le commencement et la tin de toutes choses étant nies ne peuvent èvidemment pas être l'objet d'une spéculation.

Nous tenons que l'univers n'aura pras de fin; dans ce sens la question de son élat final n'en n'est pas une. Mais nous sommes convaincu qu'il a eu un commencement, parce qu'on peut démontrer qu'il n'a pas fourni une carrière infinie.

Des lors, la question du pourquoi de son commencement se pose et il appartient à la metaphysique de la résoudre. Enfin, mème si l'on admettait que l'univers n'a pas eu de commencement, il resterait toujours la question du pour-

(1) F. P. p. $224 \S 93$. 
quoi de son existence qui ne peut pas davantage ètre écartée par une fin de non recevoir. Toute formule de redistribution de la matiere et du mourement laisse ouverte la question de l'existence de la matière et du mouvement et ne suffit donc pas pour constituer une philosophie.

Voici maintenant un second défaut de la doctrine spencérienne: elle ne mentionne que l'unité accidentelle résultant de certaines relations entre les éléments matériels. De quelle manière cette unité est réalisée dans les existences sensibles, c'est ce que nous allons examiner tout à l'heure; mais il est nécessaire de constater que pour beaucoup d'êtres elle n'est pas la seule ru'ils possèdent. L'unité de l'homme ne peut pas plus se réduire à l'intégration de la matière que son être n'est constitué tout entier par les atomes. Spencer, nous le savons, prétend quu'il en est ainsi et en cela il est d'accord avec l'école materialiste, mais nous avons repoussé cette opinion et nous trourons ici une nouvelle raison pour ne pas l'admettre, c'est qu'elle ruine l'unité substantielle de l'homme.

Tous les êtres visibles étant composés de parties matérielles, il est indispensable qu'il existe une union entre ces parties pour qu'elles appartiennent à un mème tout. L'indication sommaire que nous avons dornée des differents degrés d'unite que présentent les itres corporels a déja fait comprendre combien variés peurent ètre ces liens qui rattichent les parties les unes aux autres. "L'intérration de la matièren représente des choses assez diflérentes suivant les cas. Tantòt c'est le simple rapprochement les molécules, tantoit c'est la liquefaction d'un gaz ou la solidilication d'un liquide, tantòt une disposition convenable des parties, tantòt leur mutuelle dépendance, soit physigue soit morale. Dans le langage, c'est le racourcissemint des mots par la lusion des syllabes, ou la cohésion de la phasase par les relations qui existent entre ces parties; dams la science, dans l'industrie, dans l'art, partout spencer rementre des phenomines qu'il appelle des integrations. Lemr caractiere commun est yu'ils constituent un achemimement vers un denré superieur d'unite par l'etablissement 
de liens entre des blements auparavant isoles, ou par la consolidation de liens préexistants ou par la fusion de parties précédemment distinctes.

Dans ce sens on peut admettre que la naissance d'un être sensible implique toujours une intigration de matierre, quoique, comme nous l'avons dit, cette intigration de matire ne constiture pas toujours toute l'unite que la nouvelle existence possède.

Les critiques de spencer nont pas manque de faire remarquer la diflérence de signitication de la loi d'intégration ef plus graemalement de toutes les parties de la loi d'évolution, lorsquon les applique aux diffirents ordres de phénomines. Ils en ont conclu que cefte loi n'a aucun sens precis. Cest le rejuroche sur lequel Renouvier insiste le plus. Nous ne croyuns pas qüil soit antirerement fonde. Il va de: soi qu’unr loi tres grencrale qui s'appliqu" a des choses tres diverses doit s'entemdre pour chaqur: ategorie d'objets conformement i leur nature propre; elle doit s'interpreter in subjecta materia. Il ne suffit pas de lui attribuel une signilication vagure quai reste la mime dans toutes les applications, mais yui i earuse même de son imprécision lla plus vamiment aucun sens détermine. Il fant donner a la loi la signitication qu'elle: a dans chaque ordre d'ideres. Le progres social est evidemment un phemomène très dilfërent de lat cristallisittion d'une substance (chimigue. De part et d'autre ceppendant te process consiste dans l'itablissement de liens plus stables et plus réguliers entre les parties. I a loi d'intégration ne consiste donc pas simplement a dire qur " toutes les productions de la nature sont des compositions de phénomenes; " (1) mais plutò à athirmer que dans tous les

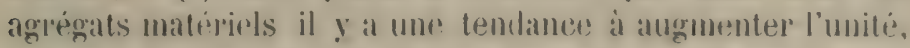
soit par l'adjonction de parties nomveltes, soit par l'union plus élroile des parties proxistantes, tendances qui serä realise diversement suivant les agregats dont il s'agrit.

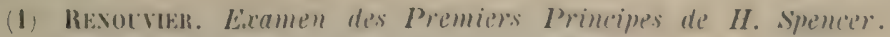
La crilique philosophique. 1886 vol. II p. 2\%3. 
Si cela est vrai, il est intèressant de le constater. Nous ne nions pas que, par suite des interprétations nécessaires de la loi, celle-ci ne possède pas la rigueur des lois mécaniques. Mais cela ne suffit pas pour lui dénier toute valeur et toute signification.

La perte du mouvement.

L'integration de la matière est donnée par spencer comme corrélative de la perte du mouvement. " Les parties constituantes, dit-il, ne peurent s'agréger sans perdre de leur mouvement relatif; et elles ne peuvent se séparer sans recevoir plus de mouvement relatif. Il n'est pas question ici d'un mouvement des éléments d'une masse par rapport à d'autres masses; il n'est question que du mouvement qui les anime les unes par rapport aux autres. Bormant notre attention à ce mouvement interne et à la matière qui le possède, il est un axiome que nous avons ¿ reconnaitre, c'est qu'une consolidation progressive implique une décroissance du mourement interne, el que l'accroissement du mouvement interne implique une déconsolidation progressive. " (1)

Quelques remarques nous paraissent ici nécessaires. Elles sont suggerées par un cas des plus simples. Considérons la congelation de l'eau. Ce phénomène est une intégration de matière. L'état solide comporte une situation stable (dans rertaines limites) des molécules les unes par rapport aux autres et une cohesion assez grande; stabilité at cohésion qui existent à un degré beaucoup moindre dans l'etat liquide. Les liens entre les molécules se sont donc consolidés.

II y a amssi perte du inouvement interienr. I'abord pour que la solidification de l'eau soit possible, le mouvement calorifique a du diminuer. Ensuite, lit soliditication a pour effict de: rendre impossibles les mouvements itendus des molécule's qui s'accomplissent sans cesse daus les liquides et

(1) P. P. P. 30: $94-$ F. P. p. 225 
yui donnent lieu aux phinomenes dre diffusion, d'osmose etr. Laquelle de ces prites de mouvement spuncerat a-t-il en vue? II semble considireer l'inteigration de lat matière coumme la conséquence de la perte de mouvement: " prestr" de mouvemrnt, dit-il, et intrgration consécutive (conserquent). „(1) Cela ne peut ividemment s'entendre que du mouvement caloritique dont la perte a, en eflet, entraine la congrelation, et non pas de la suppression des grandes excursions moleculaires qui est la consedpuence ot non la cause de la solidification.

Quoique la chose soit evidente, il n'est peut-citre pas inutile de faire remarguer que la perte de chaleur est la condition et non pas, à proprement parler, la cause de la congelation de l'eatu. Si les molécules deviemnent adher'sntes et occupent des positions stables, c'est évidemment gratope a l'action des forces interieures de la masse. Cette action attractive etait épuilibrés auparavant par la repulsion résultant des vibrations caloriliyues; leur diminution permet i celte action de devenir preponderante.

On en conclura que lorsque l'integration n'est pas le résultat des forces inhérentes à la matière qui s'intègre, mais doit ètre accomplie par des influences venant du dehors, l'absence de mouvement relatif n'est pas une condition suffisante pour que les parties s'intigrent, quoique l'existence d'un tel monvement puisse faire obstacle a l'intégration. L'absence de mourement relatif des matériaux d'un édifice, quoique nécessaire pour que la construction soit possible, ne suffit pas pour que le batiment s'èlève.

Quant aux mouvements que l'intégration rend impossibles, rien n'autorise à affirmer qu'ils existent dans tous les cas où l'intégration fait défaut. Il est mème évident qu'il n'en est pas ainsi.

On voit donc que l'intégration de la matière n'est pas nécessairement accompagnée de perte de mouvement. Les éléments que la plante absorbe dans le sol n'ont pas plus

(1) P. P. p. $301 \$ 94-$ F. P. p. $22 \%$. 
de mowrement relatif avant qu'apres, et il en est ainsi dans bien des cas. Mime, la soliditication d'une masse liquide i l'état de surfusion est accompagnée d'un accroissement de température et par conséfuent de mouvement.

Il serait plus juste de dire que l'intégration diminue la mobilité, c'est à dire le mouvement relatif possible des éléments intégrés, et cette diminution doit itre considérée non comme antérieure il l'intiggration mais comme sa conséquence.

Cette perto de mobilité correspondra à des choses assez différentes dans les divers ordres de phénomènes. Pour que l'intégration comprenne tous les cas, nous avons dû la concevoir d'une facon très générale, comme correspondant à un accroissement d'unité par la production ou la consolidation de liens entre les éléments; de mime nous interprèterons la perte correspondante de nobilite comme une diminution de leur indépendance relative.

En tenant compte des critiques que nous venons de faire, nous modifierons la formule de Spencer et nous dirons: l'évolution de toute existence sensible comporte une integration de matière pxigeant souvent comme condition une perte de mouvement et entrainant en tous cas une moindre mobilité des béments; la désintigration de la matière, résultant en géneral d'unt: absorption de mouvement et produissant en tous cas une plus grande mobilite des eliments, constitue la dissolution.

Nous n'aurons pas de peine à comprendre que dans l'existence d'un itpe quelconque et plus spécialement d'un bure vivant, les deux procis existent toujours simultancment at qur, jusqu'it la dissolution definitive, cest tantiot l'un tantôt l'autre qui prédomine.

Confirmation inductive de la loi A'intógration.
Au lien de suive l'ordre aloptr par Sprencer el diaborder l'idude dindurtive de levolution composer, nous aroyons

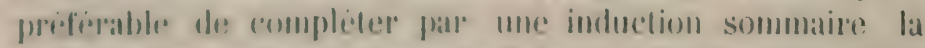

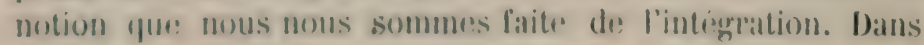


le chapitre que Spencere y consucre, il l'envisage non seulement dans l'etre total, mais exralement dans ses parties, sans cependant s'occuper explicitement do lat division du tout en ses parties qui appratient à l'evolution composée.

Lat redistribution principale est lincilement reconnassable dans la formation du systène solaire d'apuès l'hỵpotese de Laplace', (1) soit qu'on ronsidere l'ensremble, soit qu'on considere les differents astres qui s'y meuvent. On y constate l'integration de matiere consistant en une condensation prosressive rendue possible par la perte du mouvement calorifique. Cente condensation a pour consequence une diminution progressive de la mobilite des molecules grentes de plus en plus dans leur's deplacements par leurs voisines, juspu’a se trouver entiu invariablement unies dans la soliditication. Le procis d'integration du ghobe terrestre a inarche dre pair avec des integrations partielles de l'ecorce, de l'rau, qui ont elles-mimes rendu possibles d'autres phomonemes semblables : la constitution des masses continentales, la sédimentation et ainsi de suite.

si nous passons aux êtres vivants nous voyons sans peine que la mène formule peut leur ìte appliyure: l'evolution organique: comporte une intrigration de matière primitivement disperspe daur l'air, dans l'rau, dans lr sol, dans d'antres organisines. Matis er sont surtout le's infriglattions secondaires des differentes parties qui olfrent ici de l'interet. On peut les suivere dans l'evolution de loindividu. Dans l'embryon, de's organes yui sonl d'abord

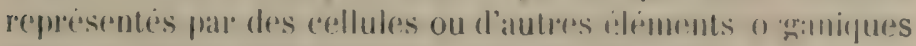
plus ou moins indeprendants, s'integrment pan l'mion de ces

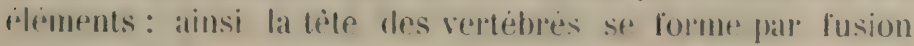
des vertehres anterienures. Co process, surtout rn qui concerne iess os, se poursuit prendant la vit: adtulte, meme jusqu’à la vieillesse.

1) Par hypothise de: Salylare. mons entenduns l'hupothèse nebulaire: nous n'avons pas a choisir entre les difierentes formes sums losquelles elle a élé proposée par les astrunomes. 
Lintegration peut aussi s'observer dans l'évolution de la forme, ou, comme on dit, de l'espece. Au point de: ve anatomique elle présente deux modes principaux. C'est d'abord l'intégration longitudinale qui s'observe, par exemple, chez les articulis dans la reduction progressive des segments depuis les mille-pieds jusqu'aux arachnides. Le groupe des crustacés est particulièrement intéressant sous ce rapport. On y constate a differents degress soit la fusion, soit la réduction du nombre des segments. (1) La fusion des vertèbes chez les animaux superieurs fournit des exemples nombreux de la mème loi.

C'est ensuite l'intégration transversale, gràce à laquelle? des organes doubles s'unissent ou se fusionnent. On f'll a un exemple dans l'évolution des la cavité uterine depuis les montremes ou elle est double, jusqu'aux primates où toute trace de division disparait.

Au point de vure physiologigur, le progres de lintegration consiste dans les rapports plus etroits que l'on obserwe

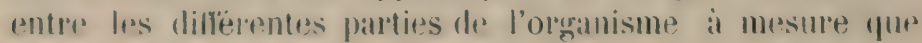
l'évolution de la forme progresse.

Pourva quion donne a l'intégration la signitication large (fue nous avons indiquere plus hatut, on la trouvera encore realisere dans les relations de dipendance que contracfont les diffepents groupres d'organismes animanx of vergetatıx, ou dans les liens yui s'etablissent entre individus de la minn esperee. On ast ainsi aneno a considerer les societes lummaines ef on comstate qualles obéissent, elles aussi, a la loi d'intesgration. Co sont d'abord les fimbilles qui sunissent en tribus, puis les tribus en peuphates, les permplades roll nations: fes mations contractent entre elles

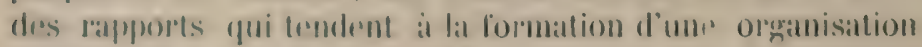

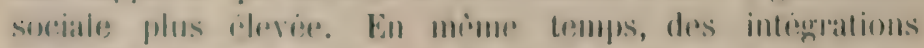

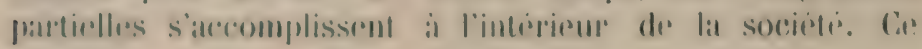

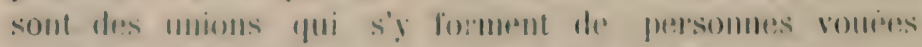

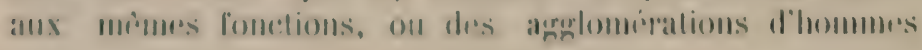

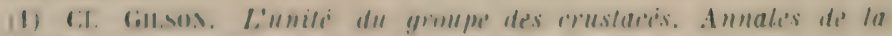

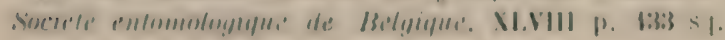


participant sous des formes diverses at une mème industrie. on d'autres organismes secondaires qui naissent et se diveloppent.

La veritication de la loi d'intiggation peut se pussuires également dans les aruves de l'intelligence humainte : le Jangage, la science, les arts. "Sans doute, dit spenceret nous attirons l'attention du lecteur sur cette remarque on ne prest pas dire que l'évolution des produits variés de l'activité lumaine fournit un exemples direct de: l'integration de la matime et de la dissipation du mouvement; ils en sont cependant des exemples indirects. En ellet le progres du langagre, des sciences ot des arts est un proces-verlal objectif de changements subjectifs. Lues changrements de structure de's êtres humains et les alterations concomitantes de structure dans les arregats des itres humains produisent conjontement des chamgements correspondants dans toute's les creations de l'humanites. " (1) Ou du moins, dirons-nous, les modifications dro l'activité intellectuelle individuelle on collective, ne vont pas sinss modifications correspomantes du systeme nerveux ou de l'organisme social. En outre, pour autant que les produits de l'intelligence loumaine sont des etress matioriels, ils peuvent fournir des exemples directs de la loi d'interrration. Le langage manifeste cette loi, soit dans la simplitication des mots par la fusion des syllabes, suit par les moditications des mots tendant à étalilir entre les parties de. la phrase des relations de dépendance muturlle, soit par l'union des propositions en phrases complexes.

Les idees s'integrent par la synthèse et l'on sail que le progrès scientilique, s'il exige la commassance d’un grand nombre de faits, consiste cependant surtout dans des geniralisations yui englohent ces faits et les réduisent à l'uniti. Spencerl rappelle qu'il a énoncé des prineipes yui s’appliquent aux phenomenes de tous les ordres : l'indestructibilite de la matière, la persistance de la force etc. Cela ne sultit pas. "Si la philosophie, telle que nous la concevons, est possible,

(1) P. P. p. $310 \$ 111-$ F. P. p. 150. 
ajoute-t-il, on arrivera nécessairement à une integration universelle. » (1)

Entin dans les arts tant industriels qu'esthétiques, l'intégration se manifeste. Ce sont les diverses formes de travail rassemblées et organisées dans les machines ou dans les ateliers. Ce sont descréations à la fois plus complexes et plus cohérentes dans la peinture, la musique, la littérature...

S'il est facile, dans toutes ces évolutions diversus que nous venons de parcourir avec spencer, de voir en quoi consiste l'intégration et même, du moins pour les premières, l'inlègration de la matière, il est moins aisé d'y reconnaitre? la dissipation du mouvement. Et à urai dire, spencer ne nous y aide pas. En quoi l'integration des cavites utèrines, par exemple, est-elle consécutive à une perte de mouvement? C'est ce qui n'apparait point et il en est de mème pour la plupart des autres cas. Souvent, comme dans l'integration des vertèbres, il est diflicile de décider jusqu'a gurel point la perte de nouvement relatif est une condition on une consequence de la fusion. Il y u'a guère que la condensation des astres ou la corrélation soit incontestable. C'est pour nous une raison de plas de préfirer la formule de la redistribution principate telle que nous l'arons modifiec. Lorsque les syllabes sont fusionnées, elle ne peuvent plus ètre prononcies sépariment; lorsque les mots ont pris des formes essentiellement relatives à d'autres mots, ils n'ont plus de sens employés isolement. Les sciences qui s'integrent reconnaissent par la mèmo des principes communs dont alles ne peuvent plus s'icarter. Plus il y a d'uniti dans une ouvre d'art, plus aussi les différentes parties sont inintelligibles l'une sams l'autre.

orces qui ent dans égration.

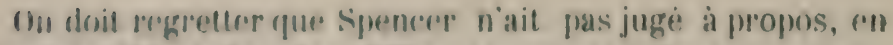

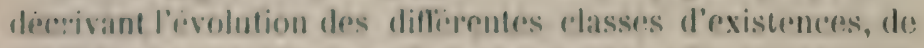

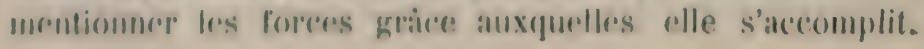

(1) P. P. p. 310 \& $111-$ F. P. p. 961 . 
Nous avons dit, en ellet, qu'en tous cas la ferte de mouvement n'est fu'une condition et non une cause de l'inte. gration. Ces forces sont differentes dans les dilferents cas, "t, à notre avis, irreductibles, quoiqur leur mode d'action soit analogue. Et c'est ce qui nous oblige à consinlerer les differentes ixolutions comme des phenomenes foncierement diffèrents quoique obéissant à la mème loi.

Autre chose est l'attraction qui produit la condensation dro la inatière inorganique, autre chose la vire qui produit l'inlégration dans les ètres organisés, antre dhose l'instinct qui groupe: les animaux et l'intelligrence qui reunit les hommes en socièti. On ne voit jas du tout comment on peut attribuer a la mème cause la fusion des spllabes rot la coherence dess ceuves d'art. Ce qu'il faut dire, quoique "re soit assez vagure, crest que tous les itres pall lo fait de

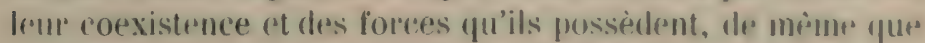
les dillèrentes manil'estations de l'activitis humaine, exel'rent les uns sur les autres des influencess rinciporgues qui

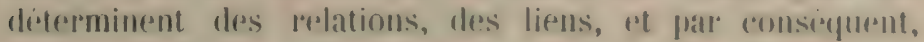
une certaine forme d'integration.

Fons ferons chose ntile et nous anrons des ideres plus prircises, en distinguant les formes d'irolution suivant les forces qui les dirterminent. Parfos cers forees sont inherentes allx chements qui subissent l'integration, "t par constoquent, antriemes a l'existence concrète quelles engendrent. Ainsi l'évolution du systeme solaterest due à l'altraction mutuelle de ses parties; l'origine des socieles humaines est due aux tendances qui poussent les hommes il s'unio pour s'assister of se drefondre. Cefte evolution peut idre appelée spontancé.

Parfois en l'alisence d'une action qu'exercent les elloment les mus sur les aures, reux-ci sont integres dans un itte preexistant, grace aux forces dont il dispose. Lacroissement des organismes aux depens du milieu, l'agrandissement des nations par l'absorption d'autles nations plus faibles nous en fournissent des exempulas. Cette forme d'évolution n’est pas primitive, puisfu'elle suppose comme: condition prealable l'existence de littre ou alle se 
produit. Yous lui donnerons le nom d'évolution naturelle.

L'intégration peut ètre due aussi à des causes qui sont extérieures aux eléments et à l'existence conerète qui résulte de leur réunion. C'est ainsi qu'un édéfice s'élèregràce au travail des ouvrier's et a la direction des architectes; qu'un nid s'assemble par l'instinct des oiseaux, qu'une machine se construit. Cette troisième forme d'érolution sera convenablement appelée artificielle.

Dans certains cas interviennent à la fois des forces intégrantes extérieures et intérieures soit inhérentes aux èlements, soit propres à l'ètre qui évolue. Certaines combinaisons chimiques qui ne s'engendrent pas dans la nature, mais seulement dans les laboratoires des chimistes, sont dues à l'affinité des elements, mais aussi à l'activite du savant qui a mis en présence les éléments dans des condilions tres speciales of compliquees. En accumulant la nourriture aupros thes animaux, en stimulaut leur appetit ou même en les gavant on produit un engraissement dì evidemment a la force assimilatrice de l'organisme, mais aussi aux conditions exceptionnelles et intentionnelles dans lesquelles on l'a place. Souvent on dit de ces resultats fu'ils sont artiticiras. Yous leur domerons plus exactement le nom d'évolution mixte.

Entin il pent se faire que des elements somissent par hasard, cest a dirp sins aucume canse qui tende a cette mnion comme telle, farl' exemple, la disposition des étoiles en ronstellattons. Ces agregats sont newssairement très rares dis quils representent un dergre d’intigration un peu èleve: rar i mesure qu'ils romproment plus d'elements of des relatoms rotre les bements plus nombrenses at plus deforminias, la probabilitio des lesur production diminue ra-

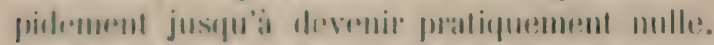

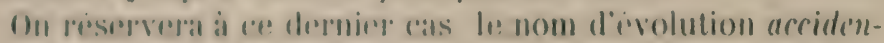

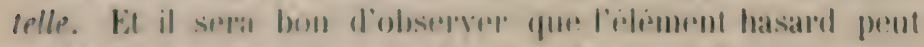
se combine plus ou moins avere les formes d'évolution precridentes.

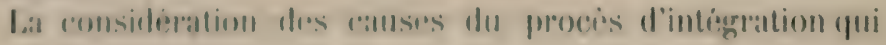


est l'élement fondamental de l'évolution, permet dro concevoir qu'tur agregat soit le siegre de moditialtions multiples, d'origine dillerente et plus on moins opposies. Elle domme la solution de certaines objections quon a faites it lat loi d'évolution.

On a dit, par exemple, que, d'apres's spencer lui-meimr, dans l'organisme social le régime industriol tend à bemplacer" le reggime militaire. Or ce derniep est caracterisé par une centralisation rt unr foree plus grande du Pouroir. Des lor's l'évolution sociale consiste plutit dans un frocers de? desintegration que dans un process d'integration.

La difficulti dispalat, si l'on lat altention aux causes

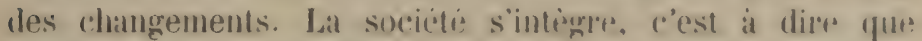

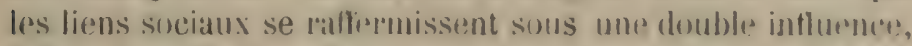

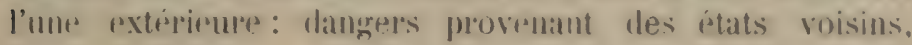

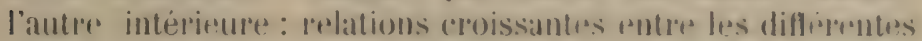
parties de la nation. Lat première de ces canses detepmine.

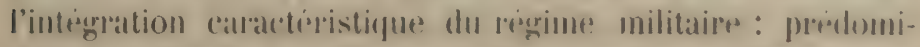

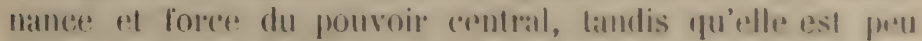

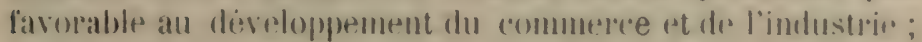

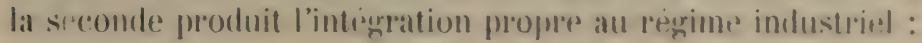
interdeprendance économigue lles rilogens. Or, il est naturel que gratce au progrés de la civilisation, less dangrens provenant dagressions possibles de la part dess preupless voisins diminurnt. Dhis lors, on rongoit ques le proceses d'integration qu'ils determiment samerte el mime soit premplace par une moditication foll sens contraire ot fque lat

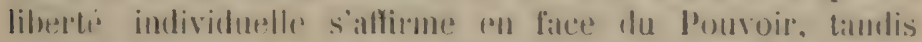

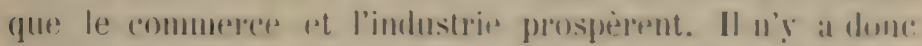

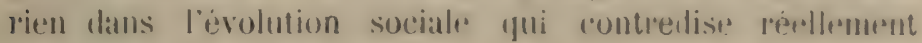
la doctrine de spencer.

Souvent on risireve le non d'énolution à l'ivolution romposie, dont il lint maintonant, aver Spesncer. préciser et developper la connaissance. Apres en avoir exposis

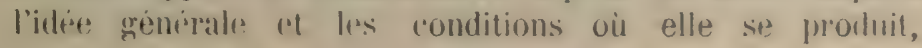


nous l'étudierons d'abord inductivement dans les diffèrentes catégories d'ètre materiels ; ensuite nous aurons à examiner quelles relations elle a avec les principes précerlemment etablis.

L'évolution, nous l'avons dit, est simple lorsqu'elle consiste uniquement dans les mouvements d'integration "t de dissolution. Elle est composée lorsqu'elle est accompagnée. d'autres mouvements. Un type d'evolution simple est, par exemple, la soliditication à laquelle succède la liquefaction (dissolution). L'évolution d'une société, au contraile, parce (qu'elle comprend, outre l'intégration progressive, beaucoup de changements secondaires, est une évolution composée.

nditions 'évolution mposée.

Pour que ces changements concomitants soient possibles, il faut évidemment qu'il existe une certaine independance des eléments les uns par rapport aux autres. bes que la rire commence à se soliditier, ses molecules perdent presune complètement leur indejpendance et l'integhation se proursuit, simple, jusqu’a la durete complete. Dans une socirté, all contraire, les lirns socianx sont loin d'entever anx membres toute leur indépendance, de sorte que des mouvements secondaires s'y produisent facilement. Tous les agrenats ne sont done pas egalement aptes it l'evolufion composise.

"Comment, sf demande spencer, pourpions-nous exprimer crolte diflemence de la manime la plus generale? Quand un atrregat est diflus sur une large ibtendur ou n'est que faiblement integria, rest gu’il contient une grande plantite de monverment atetuel, ou potentiel, ou des derux a la

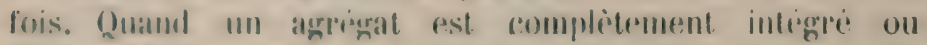

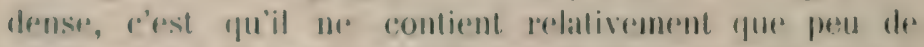

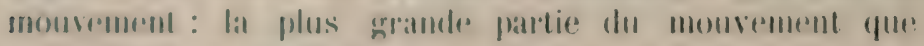

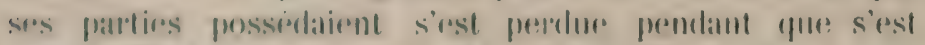

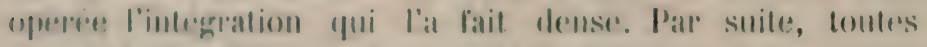

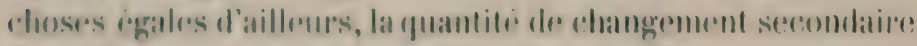

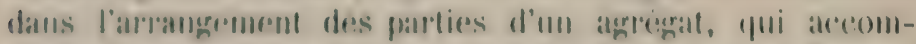

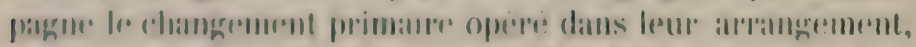

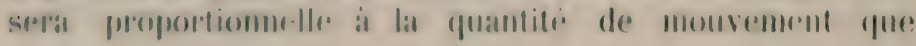


l'agrigat contient. Par suite aussi, toutes choses emales d'ailleurs, la quantité de la redistribution secondaire qui accompagne la lendistribution primaire sera proportionnelle an temps churant lequed le mouvement interne est conservé m。(1)

Il n'y a rien à observer au sujet de la seconde de ces conditions. La premiipres appelle quelques lemarques.

Le mouvement actuel favorise les redistributions secondaires pour autant qu'il maintient l'indépendances des élements ; par exmule, le mouvement thermique est nécessaire it l'existences de l'atat gazenx on liquide.

Ensuite, il est possible que le mouvement (énergie) potentiel atussi bicn que le mouvenent actuel soient transformables en mouvempnts qui constituent des redistributions secondaires. Cala n'est cepremdant pas toujours le cas. Ainsi l'énerge potentirlle constituee par l'itat liquide se transforme en chaleur dans l'acte de solidliteation. Or la solidification elle-mime est un obstacle i l'isolution composéte. Dans l'état liquiele, la temprisature, qui est du mouvement actuel, assure la pussibilité de changesments secondaires; mais l'energie potentielle dont nous vemons de parler ne lavorise en rien cess changements. D'autres part, indépendamment do mouverment acturel ou potentiel, l'agrégat peut présenter unc indejpendance des elements qui rend possibles las readistributions secondaires. Il n'est donc pas vaai que l'aptitude à l'évolution composie est proportionmelle a la quantite de monvement actuel ou potentiel, quoigue l'existence de ce mouventent soit souvent, ou mème généralement, unt condition favorable à cette évolution.

Yous prélerons dire que l'importance des redistributions seconlaires est proprortionnelle à lat mobilité des éléments, en domant a ce mot sa signification la plus genérale. Et notamment, il y a lieu de distinguer une mobilite passive par rapport aux forces extipientes et une mobilite action

(1) P. P. p. $309 \$ 99-$ F. P. p. 931 . 
provenant des fores intericures at du mowrement interne trunsformable que possède l'agrégat.

La correction que nous apportons ici à la conception de Spencer est corrélative à celle que nous avons faite dans la délinition de la redistribution principale où nous arons remplacé "perte de mouvement ) par " perte de mobilite. )

Les exemples que spencer développe s'accordent mieux avec la formule telle que nous l'avons moditiée. On favorise le tassement de petits objets jetés pèle-mèle dans un recipient en les agitant. Les vibrations produites dans les solides au moyen de choes répetes y favorisent les redistributions de molécules en cristaux, ou la disposition régulière qui caracterise les aimants s'il s'agit de barres de fer. Le mouvement thermique favorise en général les réarrangements intérieurs de la masse.

Dans tous ces cas, si l'augmentation de mouvement favorise les redistributions de matière, ce n'est pas que le mouvement communique a la masse y soit utilise luimème. En effet, ces redistributions sont déterminees par la gravité, la magnétisme, l'attraction moléculaire, forces independantes du mourement vibratoire. Mais celui-ci contribue à detacher les éléments les uns des autres, à les rendre indépendants, de manière à permettre à ces forces d'exercer leur action; cela revient ì dire qu'il augmente la mobilité des éléments.

De même, si l'etat liquide on la suspension des solides dans un liquide farorise les redistributions secondaires, er n'est pas parce que l'état liquide représente du mouvernent potentiel (mouvement thermique qui sera mis en libertis dans l'acte de la solidification), mais bien parce que ret rhat rousiste dans une mobilité caractéristiqur des molerules, ou paree qur les éliments solides suspendus daus un lig̣uide sont très mobiles les uns par rapport aux autres. Aussi l'aptitude aux mouvements inlirreurs noummentr pas che\% les liquides en proportion dr. la quantite de chaleur produite par la soliditication (mouvement potentiol quils contiennent), mais seulement arec la fluidite phos ou moins parfaite. 
Spencer dit: "Le mouvement moléculaire qui accompagne la mobilite qui est le propme de l'etat liquicle permet un rearrangronent facile " (1). Mats il pst pvident que si le rearangement est facilite frar le mourement moleculaire, ce n'est qu'indirectement, parce que ce mouvement pend les molécules plus indépendantes en augrmentant leur distance moỵnne, $\mu$, dès lors, fulus mohiles. Cette mobilite depend encore d'autres conditions que de la quantite de chaleur et n'est donc pas proportionnelle à celle-ci.

L'aptitude variable aux changenenents chimiques domme lieu a des observations analogues. La stabilite chimique diminue dans bealleoup de corps arece l'augmentation de tesmperature. Il y a dans ce cas augmentation de motivenent vilratoire, lropuel est sourent atsorbe en fartie par Ite changement chimique qu'il determinr. Yous nous représenterons convenablement les choses ren considerant re monvement comme emplose a rompre ou at aflaiblir des liens dans lesquels Ies atomes se trouvalent engragres, a augmenter, par conséquent, leur mulsilite relative.

L'instabilite des combinaisons d'elements gazerux complares ¿1 la stabilite des corps formes d'élements solides est due, d'après spencer, à ce que "lètat grazrux dr la matière contient relativement plus de mouvement moleculair't, tandis que la forme solide en suppose unt quantité relativement faible. " (2) $0 \mathrm{r}$, si cela est rrai lorsqu'on compare entre eux l'etat gazeux et l'etat solide d'un même corps, ce ne l'est plus l'orsqu'on compare un corps gazeux avec un autre corps solicle. La quantité de mouvement moleculaire que contiennent deux poids égaux de corps différents à ınème temperature est proportionnelle à leur capacite caloritique, et celle-ci n'est pas nécessairement plus élevée dans les gaz gue dans les solides.

D’après la loi de Dulong et Petit les gaz dont le poids atomique est eleve', par exemple le chlore $(33 \%, 3)$, ont une

1. P. P. 1. 317 \$ $102-$ F. P. p. 237.

(2) P. P. p. $314 \$ 101-$ F. P. p. 235. 
capacité calorifique superpieure ì celle des métaux dont le poids atomique est faible, par exemple l'aluminium (20).

D'autre part, les particules des gaz sont évidemment plus mobiles que celles des liquides ou iles solides.

L'application, d'ailleurs, est mal choisie; car d'un cóté il s'en faut de beaucoup que les composís formés par les graz soient toujours les moins stables. L'eau, l'ammoniaque, les hydracides sont des exemples du contraire. D'un autre còté, l'aptitude aux redistributions doit s'expliquer par l'etat actuel des particules dans la combinaison, et non par leur situation antérieure, ì l'état de liberté.

spencer mentionne encore l'instabilité des molécules complexes qui contiennent d'autant plus de mouvement qu'elles comprennent un plus grand nombre d'atomes, et l'instabilite des composés azotés qui absorbent de la chaleur, c'est a dire du mourement, dans leur formation.

La thermochimie nous permet d'interpreter ces phénomènes d'une manière plus générale et aussi plus satisfaisante. Ine réaction chimique comporte rigulièrement un double travail, l'un positif, l'autre nigatif, le premier correspundant à la constitution d'une molecule nouvelle, le second à la destruction d'une molécule préexistante. Ce double travail se traduit par un phenomène thermique? qui reprosente leur diffërence: degagement de chalrur lorsfue Ir travail positif l'emporte sur le travail nigatif, absorption de chaleur dans le cas contraire.

Dans la simple combinaison des éléments, le travail positif est proportionnel a l'alfinité qui existo entre eux, et pal consigfuent a la stabilite du compose. Lorspue ("e Iravail l'emporte sur le travail negatif correspondant it la flestrudion des molicules des ebrments, le composé est exoflermigue ; il a denarge de la chalenr dans sa formation at il represente une.situation relativement stable. Lorsque le travail minatif est plus (onsiderable que le travail fositif. le compose est endothermique : sa formation,

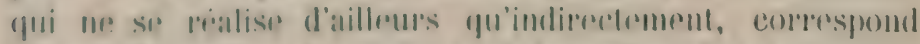
a une alssopption de chaleur ef le compose est instible.

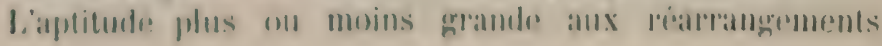


dépend ici de la quantiti de mouvement moléculaire absorbee ou digagre, parce que ces phenomènes thermiques sout l'indice du degré de solidité des liens chimiques dans lesquels les particules materielles sont engagees, c'est a dire de la mobilité chimique qu'elles conservent. Jans les combinaisons azotées ces liens sont souvent très framiles, les redistributions d'atomes s'y produisent donc en ginnral facilement.

Totons erpendant qure cette manière de conceroir less choses, empruntée a la thermochimie, ne vaut pas toujours. Ires faits s’y conforment grenéralement, lorspu’ils se passent a des lemperatures assey basses. Mais curtains composés rxothermiques se dexomposent spontanement dès

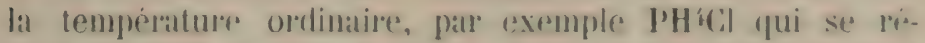
sout en PH: et HCl. Aux tempriatures très alerobst tous les comproses exothermigues so diecomposent spontantement, tandis que les composés andothermiques, qui se dedruisput à des temperalures plus basses, y prennent naissance. Lat méanique chimique moderme permet de prevoir ces rasultats (1). Il est inutile d'y insister pour folnjet qui nous occupe.

(in peut ronclure de ae que nous ranoms de dire quels sont les deres madriels qui presentent des redistributions secondaires importantes. Dans le monde inoryanique, les matsse's liquides et gazenses en realisent fes conditions au plus laut clegre, pourvu que leme rastence ait une

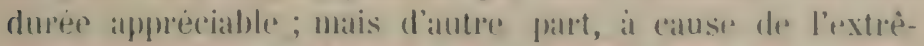
me mohiliti des molicules, les rearlangrments ny ont ancuncestabilite et ny laissent point dre trateres. Moins rapides dans les comps solides qui ont conserve quelque plas-

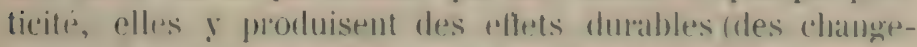
ments de structure) ed alteignent, fal conséquent, en sro superposant, une grande complexité.

Il llest pas dillicile de se comvaineme que les itres

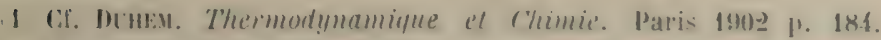

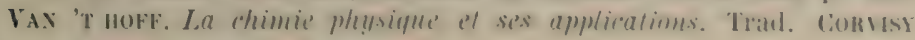
Paris 1903 p. 24. 
vivants et surtout les animaux réalisent au plus haut degré les conditions des réarrangemients stables et par conséquent complexes. D'une part, en effet, la plasticité y est assurée surtout par la présence d'une grande quantité d'eau dont les tissus sont imbibés. La mobilité physique des inolécules y est donc considérable. D'autre part, la mobilité chimique y est extrême. Les parties actives des ètres virants contiennent en grand nombre des composés endothermiques à molécules très complexes, fort instables et représentant beaucoup d'énergie potentielle. Ces caractères sont surtout réalisés par les matières albuninoïdes (azotées) lesquelles se rencontrent en plus grande quantité dans le règne animal.

L'expérience confirme cette vue a priori. Les ètres vivants, surtout les animaux, sont le siège de redistributions secondaires nombreuses et rapides, se traduisant dans des changements de structure, et cela principalement dans les parties à composition chimique complexe, abondamment pourvues d'eau et pendaut l'ige ou ces conditions sont le mieux réalisées.

L'influence de la temperature que nous avons signalée à fropos de la stabilité chimique y est également évidente. Les animaux ont en général une température plus elevèe que les régétaux; les animaux à sang chaud manifestent plus de vie que les animaux à sang froid, ot nous voyons que le froid suspend, pour ainsi dlire, l'activité vitale de heaucoup d'organismes qui restent engourdis pendant l'hiver et se réveillent au printemps. Les mimes remarques s'appliquent aux societes humaines.

L'instabiliti exerssive des relations sociales, telle qu'elle existe dans certaines peuplades primitives, a pour consiquence qur les changements qui s'y produisent n'offrent rien de prermanent, tandis qu'une societe qui presente dejat une certaine stabite d'organisation est un milieu propre is la gentise de veriables modifications de struture. (1) Eilles pourront se produire mème dans les societés

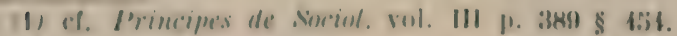


fortements organisers, surtout lorspue les rirconslances auront determine un relichement des liens socianx.

différenciation.

L'évolulion comprosée, surtout si on restreint celle denomination, comme le fait dorinavant spencer, it celle qui comprend des ledistributions secondaires ayant une certaine permanence, u'est flas un phromome alsolument

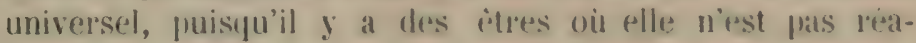
lisee. Néannoins ce procés presente un caractipe de grande generalite et de glande improptance: c'est prérisiment far les changements dont elles offrent lo sprectacle que les existences materiplles sont l'uhjet de nos etudes. (1r, si l'on ne peut pas alfirmer que tous ces changrements sunt ivolutifs en ce sens qu'ils impliquent un chamgerment des structure grand ou petit, neanmoins le plus grand numbre ast dans ce cas.

Cela n'emperehe pas qu'on ne les éludie à d'antres points de vue. Il est ivident, par exemple, yur si toute activité deployee par un être virant laisse certaines traces plus ou moins permanentes dans l'organisme et modifie, far conséguent, plus ou moins sa structure, crpendant, cette activits peut ètre également consideree comme une adaptation momentance it des circonstances intierieures ou extérieures, ou d'une antre manipre quelconque. Yéanmoins le point de vue ivolutif est important ct c'est celui que nous envisageons ici.

Losque, les parties intrigrantes ayant conservé une crertaine mobilité relative, des redistributions secondaires ont lieu, celles-ci seront en général diflèrentes dans les dillérentes régions de la masse totale; le résultat sera une differenciation croissante. Telle est donc la furme sous laquelle se présentera l'évolution composée: en mème temps que se réalise l'intégration, il se produit une différenciation des parties. Celle-ci est regardée avec raison par Spencer comme secondaire, quoique son importance et l'intérèt qu'elle présente masquent souvent celle-là. 
Il faut que l'itle matiriel se ronstitue et qu'il continue a assuror ef i levelopper son rxistence prar lo procès d'integration, arant quon puisse le concevoil comme sommis a des redistributions secondaires qui produisent la diffirenciation.

nfirmation ductive de loi de difrenciation.

Si nous eonsiderons l'évolution hypothélique du système solaire, nous voyons que le mouvement de concentration a èté accompagné de la division de la masse totale. Plusieurs de ces centres secondaires de concentration qui constituent les planetes se sont seindes à leur tour et ont domne naissance a des satellites. Ainsi s'est produitr la dilferenciation de la masse primitivement homogène, et cette différenciation va s'accentuant par les modifications que subissent les planetes et les satellites suivant les conditions interinures at roterieures où elles se trouvent.

Cas moditications ne nous somt guare commes quien re qui roncerne notre globe et leur etude nous met rn face d'une seconde application de la loi génerale. En mime lempls que l'intigration de la Terre se poursuit depuis te moment ou elle extait toute entiere gazeluse, sus diflérentes parties gazeuse, liquide et solide ont acquis une resistence propre. si nous considerons l'iecoree terrestre, nous constatons de mème mue fendance a la differenciation progressive de ses parties constituantes. Lal variete croissante des conches sidimentaires ì mesure? qu'elles sout plus récentes, la délimitation a la fois plus notte et plus emulexe des ontinents of des mel's, les contrastes de plus en plus marques antre les climats ot les saisons dont l'histuire geologinue temoigne, constituent antant de verpitiralloms de la loi drevolution composéer.

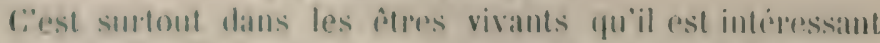
d'en poursuive l'application. Spencer n'a aucune prome i la mellee en lumbere dans l'evolution des individus ol-

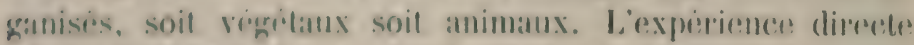

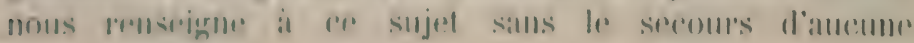




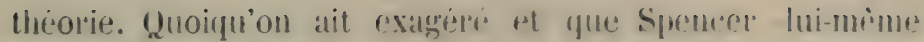
exagere l'homogineiti de la rel!ule mnigue yui sert de point de depart à l'evolution de tout illes vivant, nfialmoins elle nolipe ividemment aturums trace de la struc-

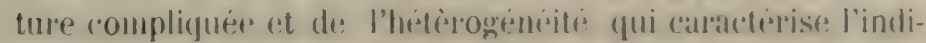
vidu arlulte, surtunt dans les lormes vivantes les plus ilevies. Or, cette structure se dreploie pretit à petit proudant la période embryonnaire et mime dans la suite. Les cellules se multipliont at en meme temps elles presentent des difterences de position, de formes, de composition "hiinique. Les tissus de la plante ou de l'amimal se dilferenodent progressivement. Leur structure se complipure de jolus en plus. Les organes daus lespuess les dithrements tissus s'enchevetrent, s'integrent progressirement et premurnt leme forme caractertistique, de sorter que l'organdesure lotal drevient bientot d'une complexiti extrinte. Lat virilication de.

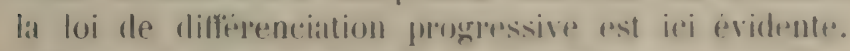

Spencer se dematude si la paleontologie permet de lat recommaitre frome l'revolution de la vie en gremeral. Ibans

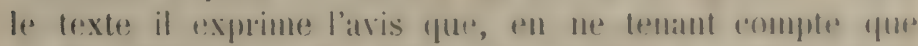

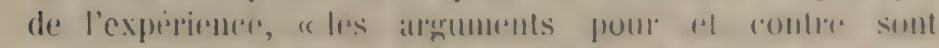

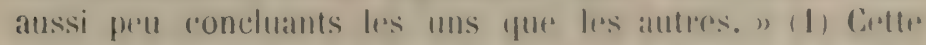

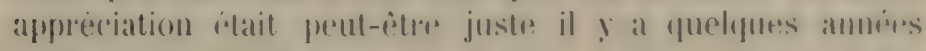
mais anjomd'hui, sans mul doute, on peut tromide des confirmations interessantes do la loi dams la sueression

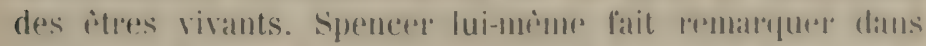

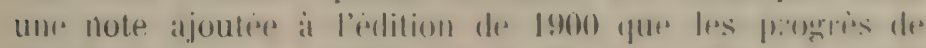

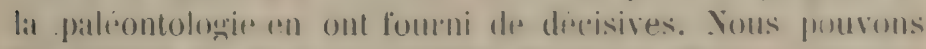

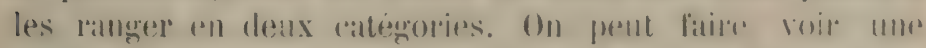

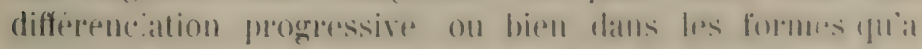

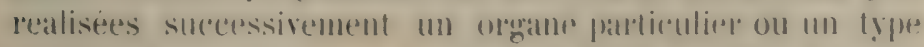
organiques complet, - ou bien daus l'enstemble des inni-

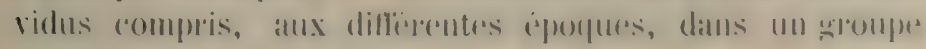
plus ou moins étendu.

Considerons, par axemples, la denture. Les premiers animatux, poissons on peptiles, yui possident des dents,

- (1) P. P. p. 362 \& 120 - F. P. p. $2 \pi 1$. 
les ont toutrs pareilles. Ia différenciation s'accomplit chez les mammifères clont les premiers ont des dents encore fort semblables; mais bientot les diflërentes formes paraissent aver chacune sa denture caracteristique. Ils présentent en gieneral quatre sortes de dents plus on moins distinctes: les incisives, les canines, les prémolaires et les molaires. On peut dire que la differenciation a été en progaressant, quoique inégalement dans les dillërents groupes. Les molaires, par exemple, ont présenté des structures de plus en plus caracteristiques et compliquees ; chez certains manmifires les incisives (èléphants) ou les canines (sangliers) se sont fortement dillerenciess en s'allongeant en lefenses; daus d'autress on peut signaler la differenciation redativement récentr de la carnassière, l'établissement d'un espace vide de dents, etc.

L" cour nous oflie egatement un exemple de differendiallon conisiante. Calvite simple chez les arthropodes, il

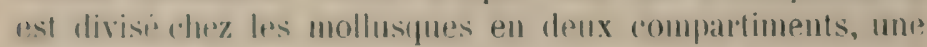
oreillelle at un rentricule, de mime que chez les poissons ynii sont les premines vertibles; diez less reptiles dont

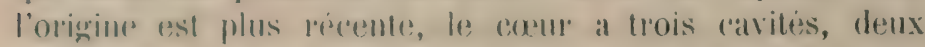
wreilletles et un vontricule; entin chez les vistaux et les mammiliores qui sont les derniers venus, on obsirve les yuatres romprartiments distincts dont chacun a sit fonction spredele.

Dir mime, il est facile desuivere, dans les groupes de? vertebres qui appanassent suce ssivemum, une complieation corrissante du cerveatu, une differponciation dome de plus en plus comsiderables des cet organe, le plus important dre tous. Las premiers mammifioles possedent tous an

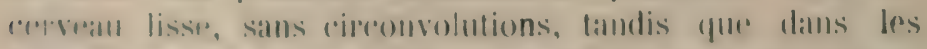

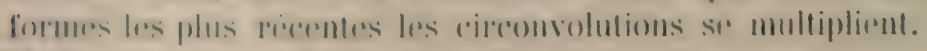
si mons funtens motro allemlon sur des organismes

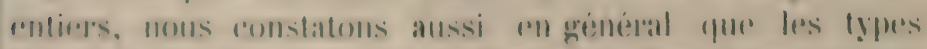

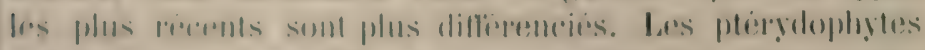

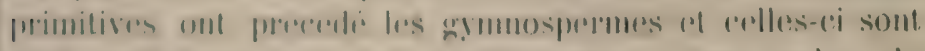

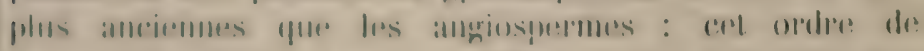

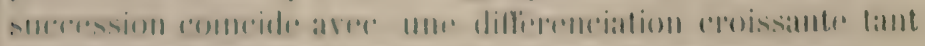

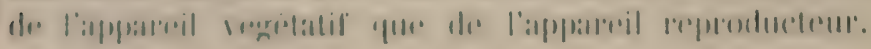


Les poissons cartilagineux sont antericurs aux poissons osseux dans lesquels lo squelette est phus nettement distinct.

Il ne serait pas diflicile de multiplier ces exemples empruntes a lobservation et qui ne supposent aucune théorie particulière.

lorsque, au lieu de considerer l'organisation partielle on totale des individus vivants, nous envisagreons l'ensenblo qu'ils constituent, le problime revient à se demander " si la flore el la faune présentes sont plus hitérogines que les faunes et les flores passices. " (1) Lá preuve negative, cost-à-dire, que dans les rouches les plus anciennes on lla pas, par exemple, rementrit de vertebrés, peut ne pas parailre preremptoire, par ce que les restes de ces animaux ont peut-inte dispartu ou nont pas ancore ite decouverts. Mais, evidemment, fa forer de cette? preme val grandissant a mesure yue les observations se multiplient. Qhoigue nos comnaissancess sur la faune et la llores soirat d'autant plus incomplètes qu'il s'angit l'époques plus eloignees, et que nous ne connaissions rien au sujet des formes rieliment primitires, cependant on ne pent pas contester certaines conclusions genirales qui impliquent une léponse allirmative a la question que nous venons de poser.

En eflet, tandis quaucun embranchement et presifuaucune classe: d'animanx ou de plantes n'ont complètenent disparu, plusients classes au contraire et des embranchements entiers se sont ajoutes a la faune et a la flore dans le cours des siècles; par consiquent, la diversite est allee en croissant. Il est certain que la faune cambrienne the comprenait aucun vertébré, que la faune sillurienne ne comptait aucun reptile, que l'ere secondaire a vu apparaitre les oiseaux et les mammifères. Ta tlore arbonifere ne contenait point d'angiospermes, et comme les premires regetaux terrestres connus appartiennent au déronion on ne s'aventurera gruère en alfirmant qu'ils fai-

(1) P. P. p. $360 \$ 120-$ F. P. p. 971. 
saient defaut à l'époque cambrienne. Il y a donc dans l'rnsemble différenciation progressive.

Ce mème procès s'observe si l'on consillère un groupe restreint d'animanx ou de plantes, surtout un groupe assez bien conuu. Si l'on remonte, par exemple, à l'origine des manmifères, on les trouve représentés par un certain nombre de types ayant des caractères fort semblables tant at point de vue de la taille, que de la conformation des membres, de la denture, du cerveau. Pendant l'ère tertiaire, les groupes se séparent de plus en plus et se subdivisent, de telle sorte que la classe toute enlipre ou mème certains ordres, conme celui des ruminants, se frésentent aujourd'hui avec une variète de formes imnense.

on observe souvent en paléontologie une spécialisation croissante, c'est à dire une adaptation de plus en plus mancquee ou exclusive à un genre de vie détermine. Cet plénomène, se realisant simultamement dans des directions unultiples, motraine nexessairement une différenciation mogressive des formes qui subissent cette adaptation.

Nous sommes convaincus que la diflerenciation progressive est réellement une loi d'évolution des groupes organiqur's, comme elle l'est des organismes eux-mènes. Mais somvent ce procès peut ètre plus ou moins masqué frar des phenomenes concomitants. En meme temps que certains groupes dvoluent, d'autres disparaissant, de sorte que parfois la faume, ou la llore, considérees dans leur ensemble ou dans une de leurs parties, all lien de s'enrichir, s'appausissent et fuansi l'heterogeneite diminue: all lieu de s'aceroitre. Mais ceest la, comme? on roit, un resultal furement apparent an point de vure qui

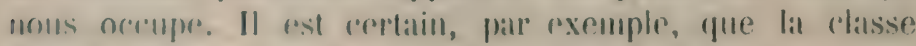

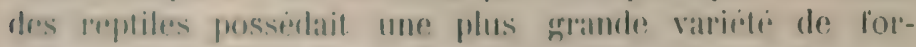

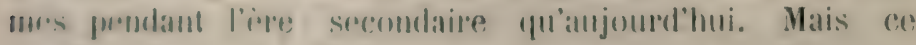

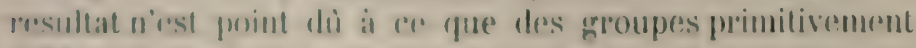

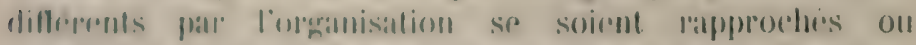
confomblus, mas bien a la disparition de la plupart des obdres

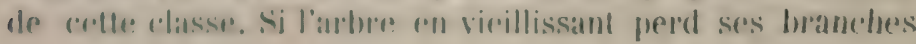


une à une et ne conserve plus pur quelques mameaux sur son trone mulile, cela n'emprofle plas que la loi de sa croissance ne soit une randication divergentro yui tend a multiplier les branches indeliniment.

Il peut se faire aussi qu'un tyje organdiqur an s'adaptant a un gemre de vie sprecial, notamment an parasitisme, perde par atrophire des organes devenus inuliles et presente ainsi une dillerenciation moindre que ses ancritres. Tels les rirripèdes, crustaces parasites, qui ont presipur complètement perdu les appendicess ciphlaliques. Cieux-ci ne sont representes que par des restes de l'antennule el flar de fabbles rudiments de mandibules on de maxilles.

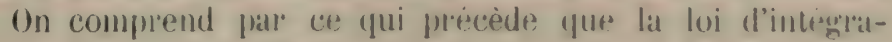
tion et la loi de diflérenciation produisent des lesultats

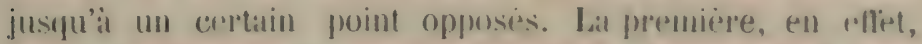
unitie tandis que la seconde multiplie. Cerpendinte il n'y at pas putre elles d'opposition diametrale.

Si l'integration lait parfois disparaitre des dilleperneres en lusionnant des relements distincts, alle ne produit jamatis

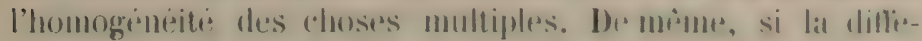

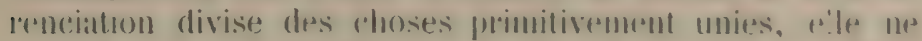
produit pas la multiplicaltion de choses semblables. L'ill-

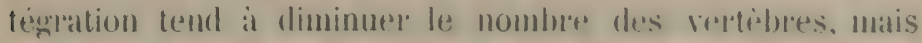

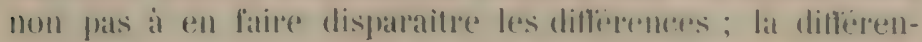
riation divise le eacur en eavites multiples, mais a romdi-

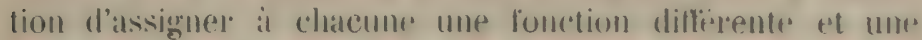

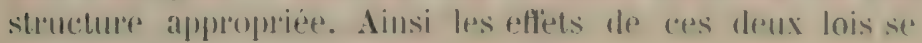
combinent sans se détruire.

spencere signale le progreses de la dillerenciation daus la

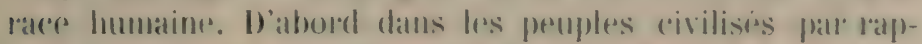
prort all saluvages ret daus l'adulte par lapprort al l'mal'ant,

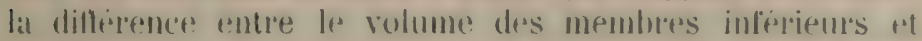

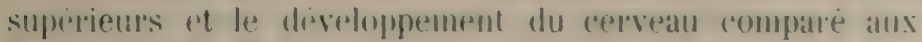
autres parties de la colomme vertibnale sont plus ancentues. En outre, fes baces hmmanes divergent a faldir de la souche dont elles sont issues.

C'est surtout pour les societis lumaines yur Sprucer s’applique à mettro an evidence le process de dillerencia- 
tion progressive. Point de société sans chef : tel est le principe de la hierarchie sociale qui va se compliquant. Dans la suite des temps l'autorité politique et l'autorité religieuse tendent a se séparer, en mème temps que s'établit l'autorite de l'itiquette ou usages sociaux, lesquels dérivent des marques de respect réservées primitivement au chef. En mème temps que le gouvernement politique, le gouvernement religieur et less usages évoluent en organisations de plus en plus complexes, la division du travail produit une diversité croissunte dans la vie économique. Yon seulement les individus, mais mème les localités, les régions, les pays se spécialisent au point le vue de la production.

Les manifestations de l'intelligence humaine présentent te mime spectacle. Dans la langage ce sont les formes multiples i signification preciste dérivées d'une racine rommmne à signification plus grenérale, e'est la formation des langues diltërntes issues d'me mème souche, la multiplication des dialectes, la sejpatation de l'écriture darre la sculpture el la peinture, la diversification des écritures et des alphabets.

Corst encore la sepparation procressive des arts primitivement mois: do la jecinture, de la seculpture et de l'archilecture, de la poissie, de la musique et de la danse ; c'est la variote et la distimetion croissante des genres, la eomplexite gramdissante des manifestations artistiques. Coest rontin la distinction realise entere la litterature et la scionce, motro la science religieuse et la science profane. la division de chacume en 101 grand nombre de lamelues qui vont si" ramiliant de phus en plus. Spencer

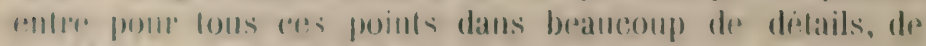
maniere a modtre la chose an plesine lumbere.

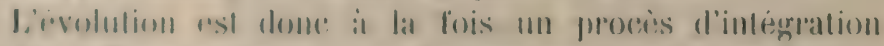

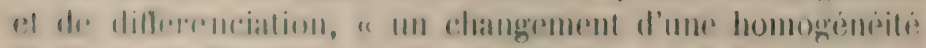

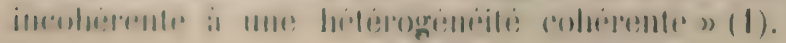

(1) P. P. p. $281 \$ 127-$ F. P. p. 291. 
éfinition coordiation parties.

Si l'evolution composée est caracterisie par unn heterogrnéitri croissantr, peut-on dire que tout progrès d'heterogéneite appartient a l'évolution? Yon, répond Spencer. Ia dissolution, surlout dans ses débuls, est souvent marquée pal un progrès dans l'hétérogénêité. La malanlir qui s’attayue a certaines parties du rorpls, les troubles sociaux qui se? traduisent en manifestations varieses all sont des exemples.

Pour que la differenciation soit érolutive, il faut qu'elle soit subordonnée an procies d'intégration. A mesure que les parties se multiplient ot se diversifient, il faut pu'pllesmèmes et le tout qu'elles roustiturut rontinment a s'integrer.

Le resultat sepa une delimitation de plus en plus delinip des parties et une coordination progressive entre elles, en tant que ellos comstituent te tout. Lal diflerrenciation sera dome caracterise par un progris de l'indetermine an déterminé et de la confusion à l'ordre.

spencer consace un chappitre it etablir inductirement celle froposition. Il insiste surtent, at a motre aris tape exclusivament, sur la distimetion de plus pal plus mampleres des parties at he fail pas sultisamment ressortir le callactere coherent at orlonme de lat complication mogressivo yni résulte de leur diffirenciation.

Il est prespun inutile que nous noms arêtions à la vèrification do fremies point. En pareonrant les differents ordies de choses quo mons arons deja imumeres fulusieurs fois - la formation du sylème sulaire, du grobes terrestre, le divalopprment de longranisme individum,

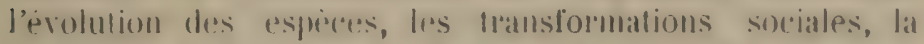
formation progressive du langagere, l'histoire des suremers. des industries of des alts - on ses convaincra farilioment qur les parties qui s'y diflerencient tenulent at so délimiter avec une netteté croissante.

guant all progres de coordination of de suburdinaton

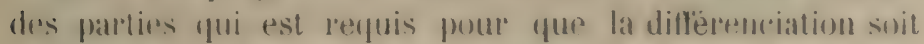

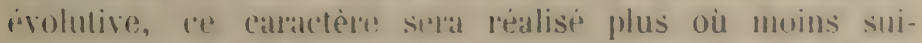
vant le degré d'unitio que presente l'atre qui érolue. Nons en trouvons des rextmpless datls l'organisme virant ret dans l'organisme social. 
Dans les formes inferrieures de la vie, la différenciation des parties est peu considérable et leur dépendance mutuelle est lauble. Aussi royons-nous les dillérentes fonctions exercés presque indifferemment par toutes, de telle surte que si on les coupe en morceaux, charque morceau continue à vivre isolément.

Cette divisibilite des formes inferieures, qui existe à un certain degre dans tous le's regetaux et que l'on observe igalement chez les zoophytes, est un indice d'une integration rolativement faible.

Dans les organismes superieurs a contraire, dans lesquels lat differenciation des parties est tres avancee, loutes les parlies principales ont des relations essentielles les unes arec les autres. Chacune a sa fonction propre et toutes sont parfaitement coordonnees a la vie de l'ensemhle. Aucume partie ne peut vire isolement at l'ablation d'un organe: important entratue souvent at Iref delai la destriction de l'ètre tout entier. Ainsi la diflérenciation a progresse de manière à accoloutre l'unite.

Ciettr verite est alfirme par spencer datss les Principes de Biologie. "Les divisions al les subdivisions de: fonc:lions, dit-il, derenant détinies à mesture qu'olles se multiplient, In: conduisent pas a uno independance de fonclioms de plus en plus complete, comme celat arriverat s'il ne se passait ripm de plus que ere que nous atoms

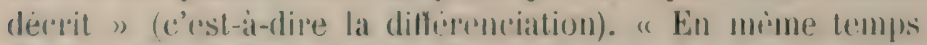

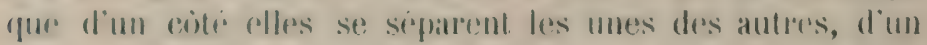
autre eriti elles se combinent les unes avere les autres.

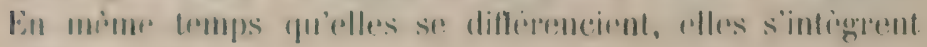
aussi. " (1)

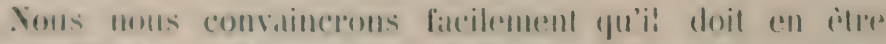

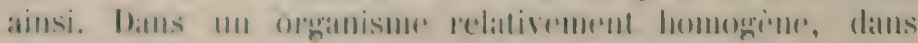

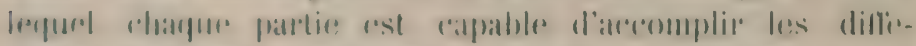

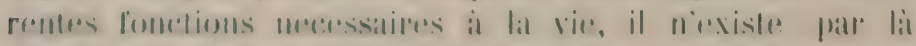

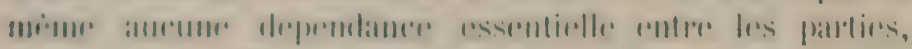

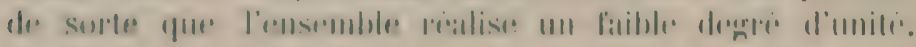

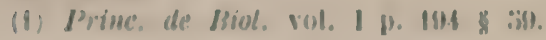


Mais a mesurr qua les partirs so differencient, ollos se sprecialisent et rice-versa, cest it dire que chacume sudiphte de plus en plus axclusivement à une fonction speciale et devient

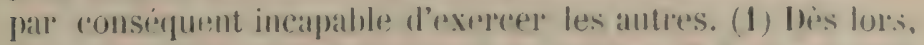
jour yue l'organisme continur a existre, il fat qua chaque organe remplisse sa fonction spreciales non seulement pour lui-mème, mais egalement pour les antres parties. II faut done nécessairement que la dillerenciation evolutive soit accompagnes d'ume integration qui remele les difletentes parties solidaires, et itablisise entre efles les rapports organigures necessiares four l'influence qualles doivent exercer les unes sur les autres.

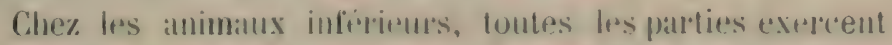

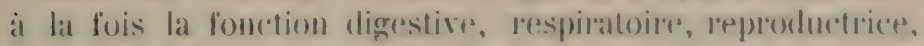

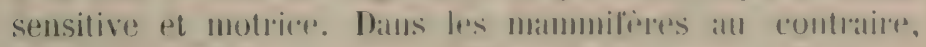

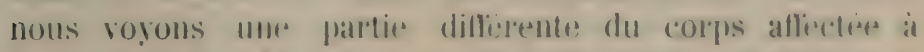
chacume de ase fonctions. Aussi est-il imlispensable pour lat vis de lanimal yur les poumons fommissent non seulement loxygene qui leur est necessiare a mox-mimes, mais allcore celui qui est indisprensable au leste de l'organisme.

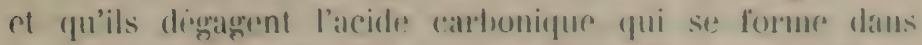
le corps tout entier. Ia rem et les vaissmatux ne fournissent pas seulement a eux-mimes les liquides nomriciers et ne charrient pas seulement lours propres drehets, mais ils emroirnt leurs ramitioations dans tous les ormanes atin de remplir fartout cette double fonction. Les uryants reproducteurs transmettent les caractires de tout l'orgatnisme. Les nerfs qui deviennent innctifs des que la respiration ou la circulation sarrete pendant quelques instants, conferent a toutes less parties par lenrs tibres disséminés le pouvoir de rexpler leur activite d'aprés les impressions exterieures on intérieures. Les muscles de lat poitrine qui recoivent l'oxygrene des poumons communiquent à ceux-ci les momrments indispensables pour leur fonctionnement.

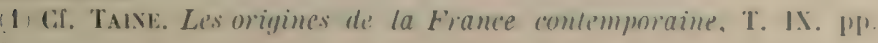
$173, \mathrm{sq}$. 
Ces exemples sulfìsent ponr montrop dans quelle etroite dependance sont les diffërentes parties de l'organisıne et quelle est l'unité admirable qui en résulte.

Yous trourons ici un critère facile pour distinguer la dillèrenciation évolutive de celle qui ne l'est pas. Si la diflerenciation des parties s'établit rn l'alsence des re'iations de dépendance mutuelle et de solidarité, au lieu de contribuer à l'évolution de l'ètre, elle prépare sa séparation en plusieurs agrégats indépendants, c'est à dire sa dissolution. Les tumeurs qui se développent dans le corps non seulement n'apportent aucun aicle aux organes, mais inime entravent leurs fonctions, de sorte qu'elles tendent a détruire l'unité de l'organisme. Aussi la différenciation qui résulte de l'apparition des tumeur's n'est pas érolutive. De mème, si dans une societé naissent des factions qui loin de se preter un mutuel appui ne font que se combattre avec violence, s'il s'y forme des associations qui ont pour but de violer les lois ou d'entraver l'exercice du pouvoir, loin de considerer cette différenciation comme un indice de progres, nous y verrons une menace de ruine.

Si, au contraire, la differenciation accentue la dependance entre les parties, comme celle qui se produit dans les tissus de l'embryon, elle les unit par là même plus étroitement et produit une integration plus parfaite, c'est a dire un progrès d'évolution.

Si los formes vivantes divergent a partir de souches communts, augmentant la variété du monde organique, nous royous en mime temps que celles qui occupent le mime territoire ont rontre elles des relations souvent itroites qui sont posterieures a la differenciation et qui constituent l'unite de la Crialion. Darwin insiste longuement dans son Origine des especes sur ces dependances souvent insoupconnees parce que tres indiroctrs qui existent entre les dillerents grompes d'animanx ef de vegretaux.

De mimu, dams les societes, à mesure que les differentes fonctions sont exerries par des individus dilferents, nous voyons ansisi s'acrotole la dependance entre les parties W. l'organisme: social, tandis yuur dans le's peruplades 
primitives on peut impunement retrancher une partie quetconque sans que la vie sociale en soil troublés; mème les chefs sont lemplaces aisement et sur l'heurs dans les fonctions peu compliquees qui leur sont devolues.

Lne marche semblable s'ohserve dans les manifestations de l'intelligence humaine. Lá transformation du langage yui tend à diversitier de plus an plus les fonctions el les formes correspondantes des mots, at en mèmp trmps pour conséquence de rendre les relations entip les mots de plus en plus nombreuses et determinies.

Lat division du travail yui inultiplie les industries ne les rend pas independantes, bien au contraire; alles sont de plus en plus solidares, soit par les matieres qu'elles emploient, soit par les foreses qu'elles mettent en jern, soit par les objets à la labrication desquels elles concourent. Il n'est pas dilticile de s'apercenor qu'il en est de m户்me des sciences et des arts. Cone scrence qui grogresse tend at se sprecialiser en meme temps qu'elle a de plus an plus besoin des lumières vemues des autres branchess du savoir humain.

Ce caractere qui consistu dans l'acroissement de l'ordre, quoique implicitement contenu dans la " cohésion " progresssive, est assez important pour qu'il soit expressimment signale dans la défintion de l'évolution. Aussi, ajoutant un mot a la formule de spencer, nous dirons que l'ivolution est une marche vers l'hétérogénéiti colsérentedéfinie et ordonuér.

distribu- Jus(pu’à prèsent nous n'avons considèré dans l'èolution ion du avement. que la redistribution de matiere. II est evident qu'elle comporte également une redistribution de mouvement. spencer rapuelle rqu'il a fait consister l'évolution dans une integration de matière avec perte de mouvement. C'est le mouvement dissipé. II ne représente pas la totalité du mouvement de l'agrégat; celui-ci en conserve une partie dont la redistribution appartient à l'évolution. C'est le mouvement retenu. 
Tous avons deux raisons de me pats adopter cette distinction sans réserves. I'abord nous n'arons pas admis qur l'intignation soit necessairement accompagnée de perte de mouvement. Ensuite la redistribution du mouvement dans l'ètre qui évolue ne s’etend pas stulement au mouvement retenu, mais aussi et sourent principalement au mouvement recu du dehors.

C'est ainsi, comme spencer le fait remarquer lui-méme, que la plupart des changements qui s'accomplissent à la surface de la Terre ont leur cause dans le rayonnement solaire pt notamment l'activité déployée par les itres vivants n'a guère d'autre source que celle-li.

L'on se convainc facilement que la redistribution du mourement suil les mêmes lois que la redistribution dr la matiere. Ia premièr est la loi d'intégration. Tandis que la matiere s'intègre, le mourement subit une modification analogue. L'évolution du système solaire romfrorte la naissance de mouvements de masse remplacint le mouvement moléculaire. Une transformation semblable sobserve dans l'évolution de la Terre, et particulierement dans l'evolution des itres organises, surtout des animaux : l'energie mécanique qu'ils deploient existe auparavant sous forme invisible. Dans tous ces eas, ce sont des mouvements d'ensemble qui succirlent i des monvements isolés des particules.

Do mime " dans les societés lumanes, le progrès se fatit toujours daus le sens de l'alosorption des actions individurlles dans les actions des corps d'etats. " (1)

Nons devons faire remarquer quil est indispensahb.

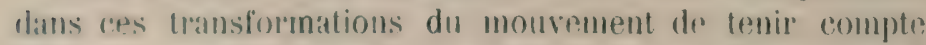
du mouxement (inespre) potentiel et yue spencer eit fait chose opjortume en le: mentionmant ici expmessement.

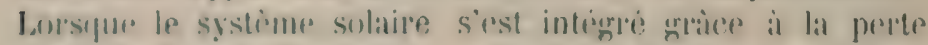

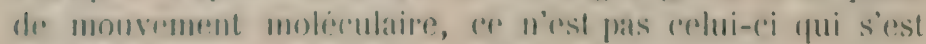
Iransforme an momsement visible. Ce momvement molede-

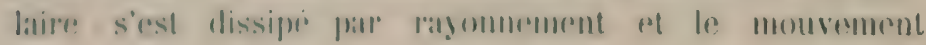

(1) P. P. p. $110 \$ 180$ 
visible est né de l'énergie potentielle résultant des forces attractives de la matière et de la situation des molécules. Encore faut-il observer que Laplace postulait le mouvement rotatoire parce qu'il est impossible d'en expliquer l'origine au moyen de la gravitation.

Le raisonnement que fait spencer n'est pas concluant. " Si l'évolution, dit-il, est un jassage de la matière d'un état diffus à un état agrenge; si, tandis que les unites diffuses perdent une partic du mouvement insensible qui les retenitit il l'itat diffus, les masses cohirentes de ces unités acquièrent des mouvements sensibles les unes par rapport aux autres, il faut que ces mouvements sensibles aimt existe précédenment sous forme de monvement insensible dans l'unité. ») (1)

Les mouvements sensibles que concoit la nébuleuse sont en partie le risultat du mouvement de condensition. Or, la condensation est la transformation de l'anergre potentielle et suppose la dissipation de l'énergrie vilöatoire. Si l'oneruie vilnatoire de la nélunleuse ne se dissipe pas, il lly atura pas de conclensation, ni, par consépuent, de mouvement visible. Cest uniquement gràce à une dissipation d'énergie hors du systeme fur colui-ci peut accomplir son evolution. Il importe pen que l'on considire la chaleur interne de ha nébuleuse comme nume siluation prinstive on comme le résultat de la condensation elle-mime: colle-ci ne prent se poursuine que si la chaleur so dissipe. Nous sommes done en pressnce d'un excmple d'integration accompangere de perte de mouvement et non pas d'une transformation du "mouvement retemu », a moins d'y ('omplendre, comme nous l'ivons dit, l'énergie potentielle.

Le raisonnement que nous aritiquons a éte modifir par spencer dans l'édition de 1900 de la manière suivante: "si l'ivolution est un passage d'un étal dillus it inl etat angegr, les mouvements des corps ciplestes doivent itre callses falp les momvements conserves

(1) P. P. p. $408 \$ 1$ 10. - F. P. p. 308. 
(uncancelled) de leurs éléments qui ćtaient auparavant épars. En mème temps que les mourements moléculaires existant partout, il y avait des mouvements en masse de ces rastes courants de matière nébuleuse qui furent rngendrés pendant le procès de condensation - mouvements en masse dont de grandes parties se dissiperent graduellement sous forme de chaleur en laissant d'autres parties non dissipées. Les mouvements en masse de ces courants nébuleux étaient constitués par les mourements isolés d'innombrables parties gazeuses incohérentes, se mouvant plus ou moins indépendamment; dès lors, lor'sque la condensation en une masse céleste liquide puis solide fut realisée, les mouvements partiellement indépendants de parties incohrentes furent absorbés par le mouvement de l'enstmble, ou en d'autres termes, des mouvements non intégrés devinrent un mourement intégré. » (1)

L'erreur qui faisait deriver les mourements visibles des mouvements invisibles a disparu dans la nouvelle redaction, mais la lransformation de l'énergice potentielle en mouvement actuel n'est pas signalie plus qu'aupararant et c'est une lacune évidente.

Lorspue la mobilité relative des élements d'un ètre matériel diminue, les mouvements que ces elements eflectuent sont nécessairement de plus en plus des mouvements d'ensemble. La perte do mouvement rolatif, au contraile, n'entraine pas nécessairement cette consiquence. C'est encore une ratison d'admettre la moditiontion qur: nons avons apportere a la formule de l'integration.

lievolution composée qui a produit une diflérenceiation croissandr des parties, determinerat de mème une différenciation croissante de lrums mouvements. Que le monvement soit rommunique du dohors on qu'il ait son ori-

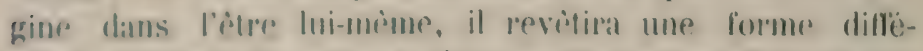
rente suivant ta nature des parties auxpacelles il est transmis. The mime que les proudules de diflerentes longrueurs oscillent difleremument soms l’action do la gravite, atosi,

(1) F, P. p. Boss. 


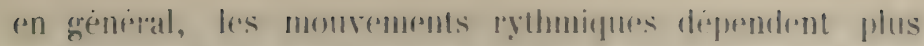

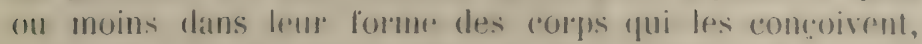

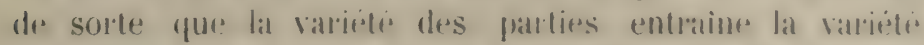
de's momements. El fulus la diflimenciation dres parties

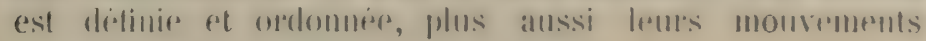
présenteront les mêmes caractères.

Il est it preine besoin dexposer la rontimation inductive de ces condusions. Le monvement confus de lat nebulense primitive a che remplace par des mouvenents nettement distincts of dithements en meme tempsis qute s'integraient les astres de notre systimre.

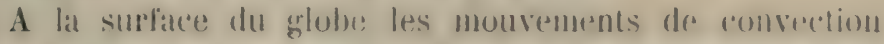
de thides et les mourements de lat tempreathere sont devemus fus distincts et plus renuliers en meme temps

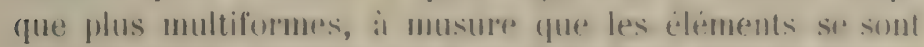

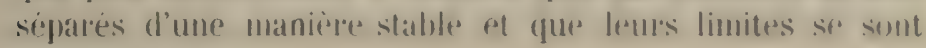
compliquées.

I)ans les olganismes tant individuels que rollectils, il y a intime corrélation entre lat structure des organes at

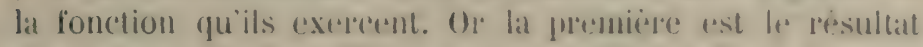
de la redistribution de la matiere, landis qure la seconde n'est antre chose que lo mouvement propre qui leug est attribue; elle est done le resultat dro la redistribution du mouvement.

On peut considerer l'évolution de's organismes an point de vue statique ou plastique; e'est ce que nons avons fait pricédemment; ou bien on pent les envisagre all point do vur dynamique; cest ce que nous faisons en ce nument. P'uisyu'il y a connexion etroite entre l'organe et lat fonction qu'il exeree, la loi d'erolution sera nécessitirement la mème de part et l'autre. En eflet, il n'est pas difficile, lorsqu'on compare 'mtre eux les organismes inferrieurs et supérieurs, de constater chez ces derniers des mourements beaucoup plus diversitits et en mème temps plus détinis et mieux ortonnés. Spencer met en parallible les mouvements peristaltiques du système digestif qui chez les animaux inferieurs s'exécutent uniformement en rythmes mal définis le long du canal tout entier; tandis que chez 
les vertibres superieurs, la bouche, l'osophage, l'estomac, l'intestin, le roctum executent ces mouvements chacun d'une lason diflirente par des rythmes bicu détinis et parfaitement coorlonnes. Il en est de mime si nous considerons les sécretions qui s'y produisent.

Le progres des manifestations intellectuelles chez l'hommre, qu'on considère soit l'individu, soit la race, marque, comme nous l'avons dit, une nultiformité en même temps qu'une précision ef une coordination eroissantes, indices d'une évolution parallèle aussi bien dans la structure du systeme cerébral que dans les fonctions qui s'y exercent.

Les mouvements qui saccomplissent dans une societé ne sont autre chose que l'acomplissement des diffirentes fonctions sociales; ot ces dernieres sont corrélatives i) l'organisation sociale qui va s'intégrant, se diversifiant et se précisant, comme il a été dit.

Lat redistribution du mouvement ast un phinomene qui acrompagne l'évolution composie ; clle ne se rencontre (que chez les ères (pui présentent une mobilité notible des parties et en mime temps une stabilité suftisante pour jrialiser une structure drinie ; Ia dilférenciation du mouvement est défrendante de la differenciation de structure, comme nous. l'avons vu.

lies lors, la redistribution du mouvement a lien principalement ot presque exclusivement dans les organismes, soit individuels, soil collectifs. Ce n'est gurere que chez enx fuion trouve des fonctions proprement dites, si l'on enternd par ce mot des mouvements non seulement diflérents, comme ceux qui se passent, par exemple, dans l'almosphiore, mais encore bien délinis et coordonnes.

11 rist interessant d'observer anssi dis a présent, quoigue spenere doire nous fournir plus tard looceasion

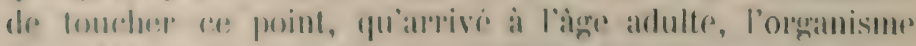

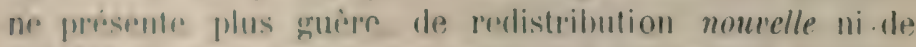
maliepe ni dre monvement; mais que neammoins la redis-

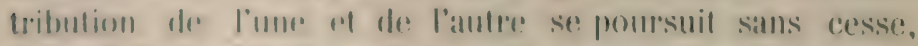

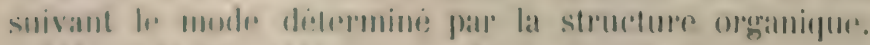

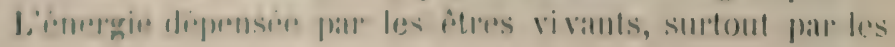


animaux, fist emprunté directrment aux aliments qu’ils se sont assimiles et qui font partie integrante de leurs organes. Pour qu'ils puissent continury a vire, il fiut que la matière qui rst éliminere, après aroir crolé l’ènerrrie qu'elle contenait, soit rmmplacée sams cesse. Il se produit ainsi che\% les animaux, et mime chez les visgritaux quoigue a in moindre degre, une dirculation de matière et de force, cest a dire une redistribution de matière et dre mouremrent qui se distingue de l'évolution proprement dite en ae qualle ne comporte pas de changements de structure appriadiables. Vianmoins a phenomine se rattache itroitement à l'évolution, parce yu'il en est en quelque sorte l'aboutissement. Ce que nous venous de dire de l'organisme vivant, le lecteur le transportera fatcilemfont, mulutis mutundis, it l'organisme social.

forces qui sent dans redistrioutions ondaires.

Si nous reprenons ici la division que nous arons falblie plus haut pour l'integration en consitterant les forees yui la determinent, nous nous apurerevons quil n'y a plus lien de distingura d'antre action yur ceple des forles

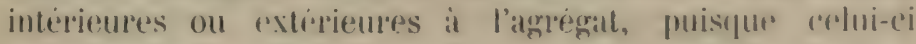
est suppose constitue, lorspu'on rnvisagre les ledistribulions seronduires de matiene et de momvement.

Ces redistributions seront donc maturelles, antiliciolle's ou mixtes suivint qu'olles seront dues il l'influence de's

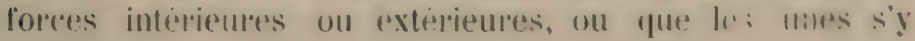
combineront avec les auties.

Dans la ronstruction d'un éditice, on pexut distinguer la redistribution primaire yui compnend l'adaptation ronvenable des parties les unes aux atutres, et les redistribulions secondaires yui eonsistent dans l'applopreiation des differents appartements a l'usage spécial qu'on leur l'esel've on dans l'ornementation de dratail. Le's serondes comme 1a première sont artiticielles.

Natturelles an contrairr, sont, furespur sans restriction, 
les redistributions secondaires d'un organisme physique, ou mime d'une société, aussi bien que leur intégration.

La production des variétios d'une plante cultived constitue au sein de l'espèce une redistribution secondaire qui est antiticielle, du moins en bonne partie, tandis que l'intiogration, rost à dire la maltiplication de l'espece, est naturelle.

si l'on reut aroir une connassance convenable d'une, irolution queleonyur, il est extremement important de demriblel los forees qui interviemnent dans les phénomenes sourent fort compliques dont elle se compost. Xous atlrons l'occasion d’insister encore sur ce point.

a loi d'évolution amendée.
Yous pouvons maintenant comme conchusion de celte

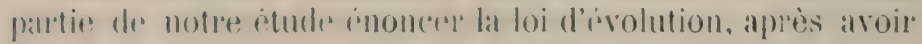

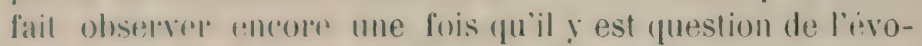
lulion des itpes materiels on tant qu'elle est ume redistriIntion de matiere ot de momroment. La formuld de spencer a the domere plus haul; fes remarques que nous avons

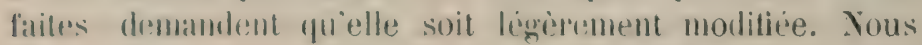
dirons done: L'érolution est une intégration de matière exigeant sourent une gerte de mouvement el entrainant en tous cas une perte de mobilité des éléments intégress; pendant celte intégration la matiere passe d'un élal d'homo-

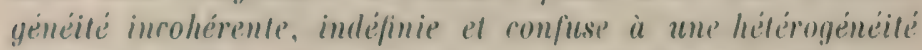
coherente, définie et ordonnese al le momrement subil une. transformation analogue.

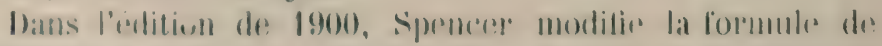

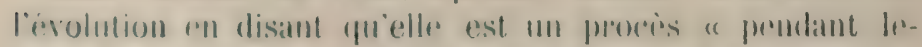

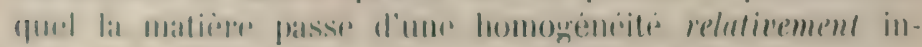

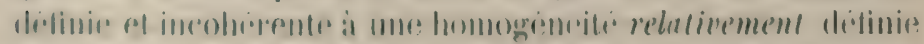

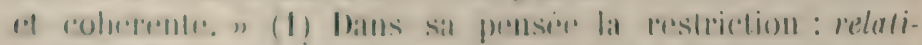

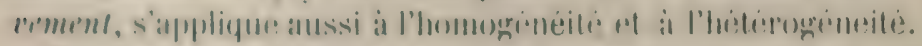

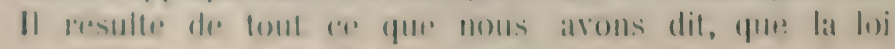


d'ivolution doit s'entendre de cette manière et qu'elle ne peut s'interpreter autrement. Spencer en fait lui-mème la remarque; il n'introduit le correctif que pour exviter des malentendus.

Lne hétérogéneité absolue ou absolument diffinie et cohirente est inintelligible: un agregat peut toujours devenir plus cohérent et défini.

Yous arons déjà dit et nous répèterons encore que la doctrine de l'évolution ne peut pas résoudre la question d'origine première. L'évolution part nécessairement d'une situation determinee de l'agrégat materiel. Il n'y a aucune raison ni a priori ni a porteriori de se representer cet état sous la forme d'une homogénéité absolue, absolument indétinie et incoliérente. L'on ne voit mène pas comment une telle situation pourrait être le point de départ d'une évolution déterminée.

Yous pouvons donc conserver la formule telle que nous l'avons énoncée. 



\section{L'APPLICATION DE LA LOI D'ÉVOLUTION}

L'évolution des organismes. - L'évolution de la vie La loi d'évolution en psychologie - La loi d'évolution et l'origine de la vie psychique - La loi d'évolution en sociologie - La loi d'évolution en morale.

En étudiant les differente's partires de la loi d'avolution, nous avons eu l'oecasion de montrer fomment elless se lealisent dans la nature et dans l'histoire. Cependant, après en avoir obtenu la formule complive, il ne sera pas sans interct de la contirmer et de l'interprèter encore frar unr veritication inductive. Le lecteur aura ansi une perepetion plus claire de sa grande portée. En mème temps nous pourrous signaler à grands tarits les developpements que spencer a donnés à sa throrie génirale dans les difirantes parties de sa Philosophie Synthétique, et nous exanninerons l'un ou l'autre probleme que soulere son application dans les. differentes branches des sciences genérales.

volution des ranismes.

C'est surtout en Biologite que l'évolution a éte dans ces derniers temps et est (ncore anjourd'hui l'objet d'etudes assidues Il ressort des remarpues faite's au cours du précedent chapitre quon peut l'rnvisager d'abord daus le règne vivant tout entiel ou dans une de ses sublivisions 
- ensuite dans une forme virante representie far une sepric linéaire d'animaux ou de plantes issues les uns des autres et considerés géneralement à l'ritat adulte - enfin dans tout individu vivant.

Ces trois procés d'évolution ne sont pas indépendants l'un de l'autre. L'évolution du règne organique n'est que l'ensemble des évolutions de toutes les formes particulieres. Quant à l'évolution de l'individu, les transformations par' lesquelles il passe pour aboutir a la forme adulte rappellent dans leur allure générale les moditications subies flat la forme dans les générations successives.

Ainsi que nous l'arons déjà fait remarquer, l'évolution individuelle a eté forcément reconnue de tous temps, mais c'est i notre époque seulement qu'on en a acquis une connaissance plus exacte on donnant aux stades embryonnaires leur importance réelle.

dnuant a l'évolution des formes et par conséfuent de l'ensemble du rigne organique, si l'on entend par la la série. des apparitions el disparitions de formes vivantes a la surlare du ghobe prondant les lomps grologiques, elle est III litil reconnu par la science moderne et complitement ignore anparavant. L'hypothese qui fait deriver cos formes vivantes les unes des autres par voie de génération a compli: ditns le monde des sciences des defenseurs et des arvorsaires eminents. Si l'on lat abstraction des questions secondatres, all sujot despuelles reggne encore beancoup d'olscentip, et quon envisage seulement la question de

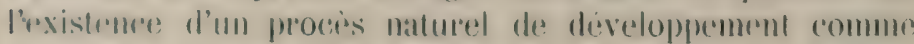

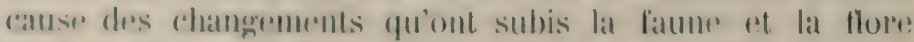

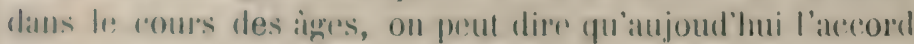

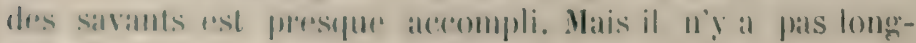
trmps que ce résultat a été obtenu.

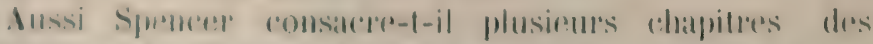
Primeipes ale Biologice al developpere les arguments qui

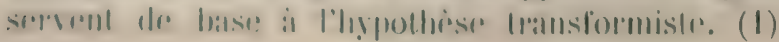

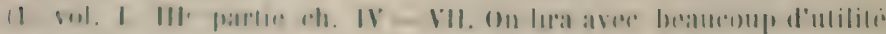

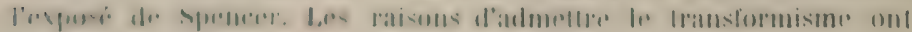


Si on l'admet, on considerena les espreces vivantes fui ont successivement peuple le globe comme issurs det formes primitives ayant more organisation relativement simple. Catte evolution grandiose, drepuis les aldrurs monocrellubaires jusiguans phamerogames ef depuis les protozoaires jusqualux mammifires, repifie parlatioment lat loi formulere a la lin du chapither precedent. C'est ce? que Spencer sapplique it me!tre an lumiere dians le second rolumo des Principes de Biologie. II y groursuit pour les dens resgnes tant an proint de vue de la structure

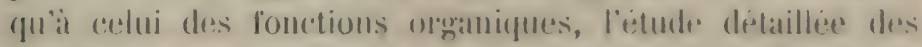
phenomines de developprement que noms avons deja indi-

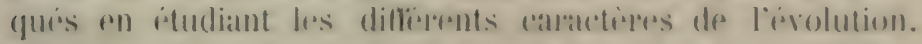
La propagation de la vir i la surface du globe unit dans

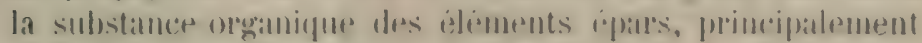

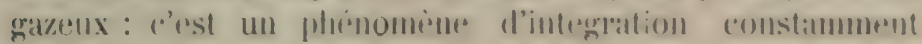

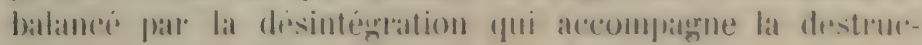
tion des êtres vivants.

Lat multiplication dismenente des formes issures d'une mime sonche, - la emmplexili arenssante de structure

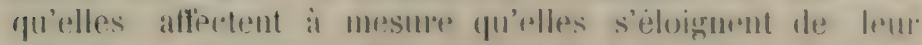

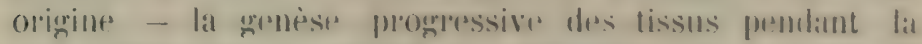

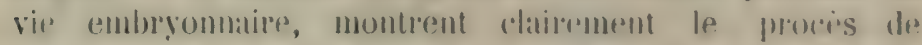
dillerenciation dans le's trois aspreds de lievolution orenatnique que nous avons signalés.

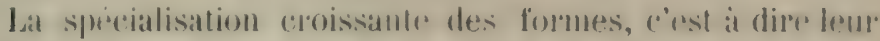

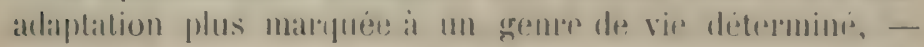

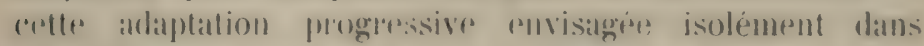

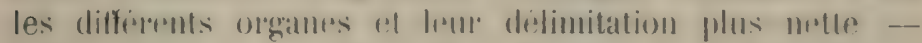

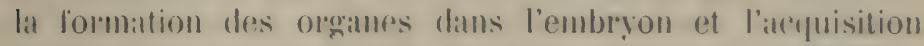

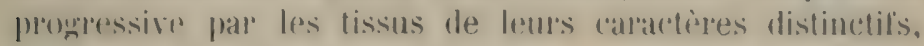
realisent la loi de la detinition.

La dependance muturlle des expeces vivantes, - la condination et la subordination des oronanes fontr ba vio

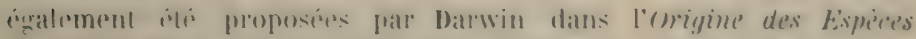

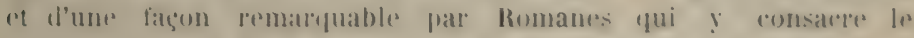

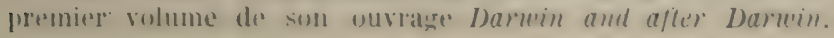


de l'individu et de l'espèce, - l'aptitude croissante de l'individu à la vie à laquelle il est destiné par son origine, satisfont à la condition de cohésion et d'ordre progressifs.

Spencer ne se contente pas dexposer les laits et de montrer qu'ils sont d'accord avec la loi, il tache aussi de rendre compte de l'évolution, d'expliquer les changements que subissent les organismes. A cet égard nos connaissances sont encore très rudimentaires et tout ce 'ju'on peut dire i ce sujet, surtout lorsqu'on entre dans les details, est necessairement fort sujet ì eaution. C'est a peine si les facteur's de l'évolution organique sont connus d'une façon grénérale. Yous nous en occuperons dans la suite.

évolution la vie.

Au lien d'etudier l'evolution de la vie dans ses formes concrètes, on peut aussi l'envisager dans ce qu'elle a d'essentiel. Spencer raprroche la définition de la vie de celle de l'évolution. (1) P'uisque celle-ci ne sobserve flas sentement dans le règne organique, mais qu'il y a, ainsi que nous l'avons vu, l'evolution superorganique des societes rt l'irolution infraorganique de la matière hrute, il ne prut pas itre question d'identifier less deenx phenomentes. Et s'il est vaai que l'ivolution des ètres vivants ne se distingue pass de la vie elle-meme, cependant la vie ne merite le non d'evolution que pour antant qu'elle comprend des dhangements de structure ef de fonction.

La vie a done une detinition propres ; on pent etudier

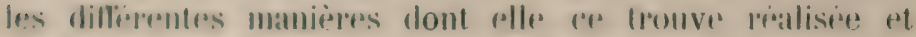
relrowser encore ici la loi de l'évolution.

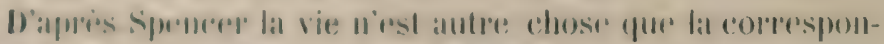
dance aree te miliem, of il s'applieque, dans le premier

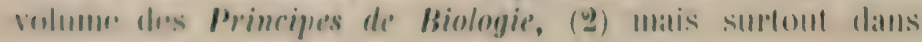

1) Princ, de Biol. vol. I P, 10k \& 36 .

2) Is Partio ch. V in VI. 
les Principes de Psychologie (1) à étullere, dans les dillirents degries de la vie la correspondance progressive alvec le milieu d'apres la loi d'évolution: intégration, différenriation, définition. colrésion el coordination.

Le coe que la vir, est une correspondance, il me suit pas que tel est le concept adeiguat de son essence. Sans doute, fes fonctions vitales sont délrerminers dans leur nature par le lapport quelles ont avec les cireuntances exteripures, mais olles

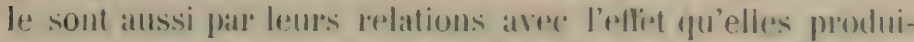
sent dans l'individa vivant. Lat contiguration des olonanes de: la nutrition, far exemple, dépend de la situation et de la forme

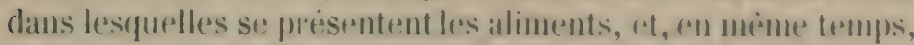
de la necessite d'assurer leur assimilation fall l'organisme.

En definissant la vie comme atant "lat combinatson délinir:

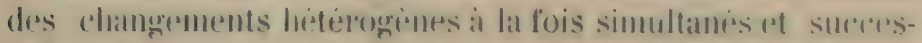

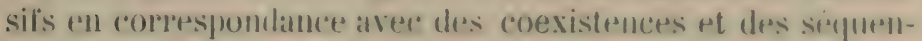
ces externes ", (2) on nrighige de dire i quoi tendent,

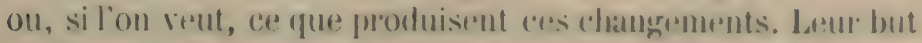

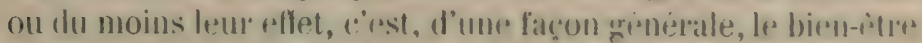
de: l'individu ou de l'espreer. En n'en faisant pas mention, on

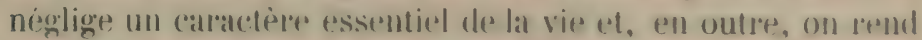
si fas inintelligible, llu moins extrimement vagrese ce qu'il fatul entendre par correspondanor arec le miliru. Comment ron oflet distinguerons-nous les changements adtaptes an milien d'antres qui ne le sont pas? " Notpe surprise, dit Spencer, quand

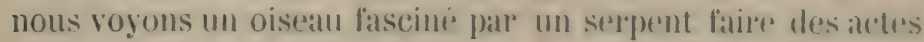
qui tendenta le perdre est une prenwe quorengemeral nous avous obsteve une adaptation des changements vivants anx (flangements survenus dans les cireonstances ambiantes. ) (3) spencer considipe donc comme non adaptiss all milieu les mouvements qui contriburent a la perter de loosean at comme. adaphtes ceux qui auraient pour resultat de le samere. Ce nest

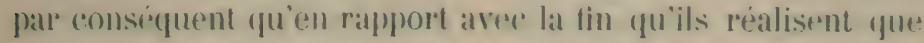

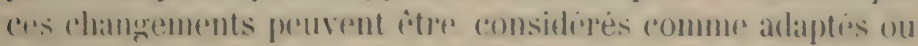

(1) vol II. Synthèse générale.

(2) Princ. de Biol. vol. I p. $89 \$ 97$.

(3) Princ. de Biol. vol. I p. $87 \$ 97$. 


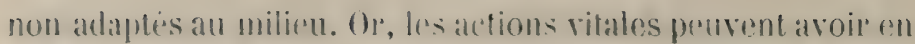
vue l'existence de l'individu, son bien-ètre, l'existence de l'esprece, sa propagation, sa prosperite. Ces résultals peuvent ètre atteints à des degrés diflepents et de diflerentes manières.

Ce point de rue est evid:unment ass ntiel dans laativité des êtres vivants.

Tous croyons aussi que l'immanence de l'activité est le veribable caractère distinctif de la vie, et nous entendons par là que l'ètre vivant, lout rn ayant besoin d'excitation pour agir, se meut néanmoins lui-mème par une activite mopre. "Dans le fait, dit Strasburger, tous les ètres vivants se présentent it nous avec une proprieté determinée qui manque aux colpss non vivants et que nous designons frar le mot irritabilité. Elle se manileste en ce que? des impressions exterieures ou interiemres agissent sur l’organis. the vivant comme des dirchargers (auslësungen) it le déterminent à des actions qu’il exécute par des movens dont il dispose ou qu’il peut se procurer, de la maniere exige frar sa structure al correspondant a ses besoins. ") (1)

On ne peut pas nier que la vie ne soil me correspondance arre le milien. Ce caractipe convient essentiellement ¿ toute la vie intentionnelle, c'est a dire à l'ensemble des mouvements dirigies par des conmaissances sensibles ou intellectuelles et a ces comnaissances elles-mèmes. Iat vir organique inconsciente ou du moins non intentionnelle? manifeste de meme une dependance etroite relativement atux rireonstances dans lesiguelles elle se deploie.

Jes animanx bant en roprespondamer avec le milien par leurs facultes aognitires et motrices, plus cess faculles sont variees, plus alles sont developpees de manieve a

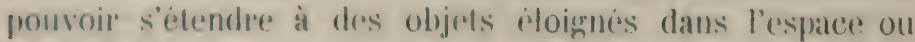
dans le temps, plus aussi la correspondanere de la vie animale avere le milien sera ilendure at varice. Il en sera de memre de la vir oreraniefue a mesure que ces fonctions deviennent plus complexes.

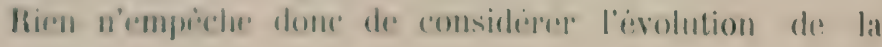

(1) Lelirbuch der Bolanik. Jena 1904, p. 1. 


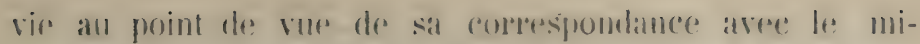

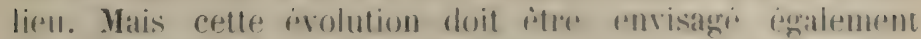

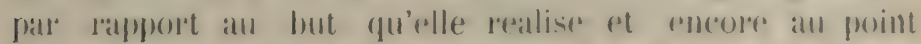

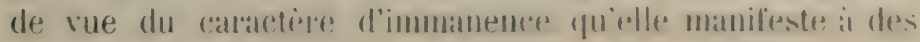
degres differents.

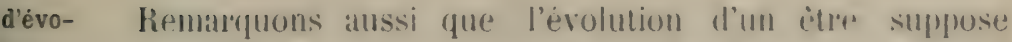
all prealabr son existence et que les lois qui rogissent la premierr me peuvent pas rexplipuer la seconde. Autre chose est le developmerment de re qui existe dejil, atutre chose laaplarition d'un atpre alsolument nouveatle, romme l'est a notre avis la vire animale "ll tant que distinceses de la vie organique.

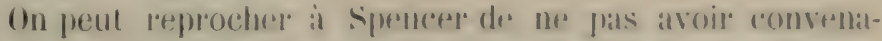
blement distingrue l'une de l'antres. Lon partir des momrements vitaux sont determines fall des connatssanees, tandis que les autres ne le sont pats. Unod qüil soit proulitre difteile de tracer la ligne de dematration matre les dens, paree que la commaissance prent alvoir flus on moins de part a la production d'une action vilale. qu'clle peut n'aroir qu'unce litible intemsite at flissiel

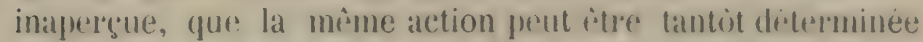
par la commaissance, tantot en etre indépendante, celat n'rmpiche pas la distinction d'exister el d'itre resentiellr. Ia combassance est un phemomene sui generis ineductible aux phimomènes organiques. A moins de lis declarel complètement inutile, il faut admettre qu'elle a une influence reielle sur certains phenomènes vilaux. Ceux-ci se distinguent donc en deux catigrories irreductibles suivant qu'ils sont ou qu'ils ne sont pas gouvernés par la connaissance, e’est à dirc intentionnels.

II nous est impossible d'adopter sur (er froint la manière. de voir de sprencer. "Les deux grandes classes vitales appelees physiologie et psychologie, dit-il, se distinguent en gros l'une de l'autre par ceci : c'est que, tandis que l'une renferme des changrements à la fois simultanés et 
successifs, l'autle ne renferme pue des changements sucessifs. Les phenomenes qui sont l'oljejel de la plyssiologie se présentent sous la forme d'un nombre immense de serries réunies ensemble. Ceux qui sont l'objet de la pisychologie ne sp presentent que sous la forme d'une simple série. En jetant un simple coup d'oeil sur les nombreuses actions dont la continuté constitue la vie du corps en general, on voit qu'elles sont simultanées; que la digestion, la circulation, la respiration, les excrétions et sécrétion, ele., atre leurs nombreuses subdivisions, s'accomplissent it la fois et dans une dependance mutuelle. Et il suffit de la plus conrte réflexion jour voir elarement que les actions qui constituent la pensée se produisent, non ensemble, mais l'une apres l'autre.

Il nien resulte pas cependant qu'il y ait entre ces deux classes un abime infranchissable. Quand même (et nous verrons bientot qu’il y a des raisons d'en douter) la plus haute vite psychique serait absolument distincte de la vie physigne dont nous venons de parter, il r'sterait toujours vrai que la vie pisychique, dans ses phases inferieures, ne s'en distingue pas de celle manière; la distinction ne nait qu'ave le cours du progres vital. Celte dillirenciation et rette intrigration graduelles qui se montrent igalement et dans l'évolution de's structures organiques et daus l'evolution do la correspondance entre leurs actions ef celles de leur milieu, se roit anssi dans la sépanation de cette correspondance? en ses deux grands orderes. Ciest par elles que se sont produitrs les divisions subordomnees de la comenspondancer; far elles aussi (que soest produite refte division fondamentale. » (1)

Si la vir pssybhigue se détinit en disant qu'elle comprend ume serule serie de chamerements, il faudea done que chateme

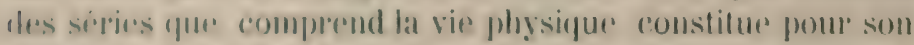

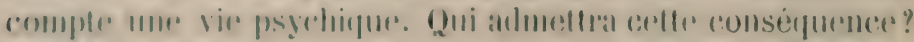

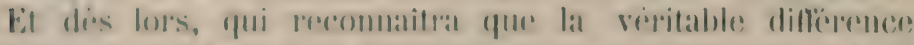

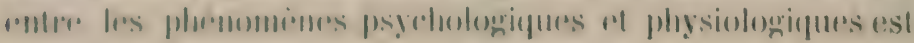

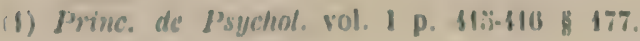


celle: indiquer par spencer? Iui-mème reconnait quelle n'a rien d'absolu. Lat vie psychique? peut jusqu'il un certain point comprendre plusieurs orders de changements simultanés et d'autre part il ne suffit évidemment pas que la vie consiste dans une seule serpir de changements pour qu'elle soit psychique.

'Nous me commaissons la vie jesyluigue des etres distincts de nous qu'indirectement : par la structure de leur système nerveux qui sert d'organe it la sensibilite at par la natur des mourements qu'ils exicutent et que nous considerons comme intentiomnels. Il semble bien que des demx rriteres indiguent, a partir des animaux infriereurs, un accroissement

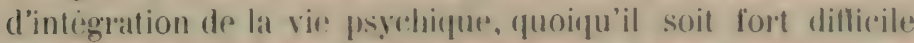
de diere exactement en quoi if consiste. Mais d'une part, le systime nepveux me semble pas itle essenticel a la sansibilite. et d'autre part, les plantes manifestrut une uniti tres grandro dans la coordination de leurs ditherentes fonctions sinns yum la vie psychique existe chez elles.

Nous concluons que la diflerence signalese frar spencer - en admettant qu'elle soit reelle - est d'importance secondaire, tandis que fa diflerence asspntiplle — l'existence ou la non existence de la comnaissance - a ets négligér par lui. Cela etant, il est facile d'uxplinuer qu’il n'a pas apercu entro les deux vies un abime infranchissable. En réalité cet abime existe. La vie psychique et la vie organique, quoique itroitement unies dans l'animal, sont essentiellement difterentes et ne peuvent pas être considérées commo appartenant à un seul procès d'évolution.

Nayant pas pris soin d'envisager la vip psychinue dans ar qu'elle a de rêllement essentiel et caractèristipue, spencer n'a pas accorde: une importante suffisante à la distinction qui existe entre ses deux parties: les connaissances et les mouvements intentionnels. Dans les premieres l'animal est plutòt passif: il recoit les comnaissances du dehors; dans les seconds il est actif: il reagit sur le milieu par ses facultés motrices. Lorsyu'un fitre possédant un wil rudimentaire (1)

(1) Cf. Princ. de Psychol vol. I p. 153 \$190. 
se contracte au moment ou une ombre passe sur cet ceil, la relation externe entre le inouvement d'un corps opaque et un choc qu'il produit correspond, au moins primitivement, non pas a une relation interne, mais à deux : la première entre la percention de l'ombre qui se déplace et le souvenir (perception affaiblic) du choc, la seconde entre le souvenir du choc et le mouvement que ce souvenir determine. Cette distinction est négligie ou du moins n'est pas faite assez netiement dans les difërents chapitres que spencer consacre aux facultés pisychiques et c'est un glave défaut de son analyse.

spencer neglige encore la distinction entre la cunnaissance sensible qui a pour objet les choses concrètes et la connaissance intellectuclle qui comprend les idees alostraites, distinction radicale qui est le fondement de la distinction également essentielle entre l'instinct et la raison. C'est donc à tort que spencer se refuse à admottre cette dernière.(1)

De ce qui precede nous tirons cette conclusion : c'est faute davoir aperẹu le véritable caractère des diverses formes de la vie que spencer a pu les considerer comme comprises entièrement dans un seul procès d'evolution. Celte conception me resiste pas à un examen attentil.

La loi d'èvolution en psychologie.

les Principes de Psychologie de spuncer debutent par la verification de la formule de l'évolution dans le developpement dus systime nerveux (2) depuis sil première apparition dans l'organismo jusqua'i son organisation la plus complète. On pent considerer ceter ivolution soit dans les diflerents stades yue: pareourt l'alre: individuel principalement pendant la vie embryonnaire, soit dams fes differentes formes animales qui pussidem un systeme nerveux depuis les plus inferieures jus-

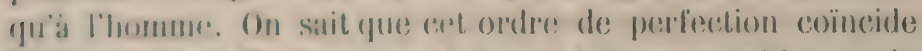
larrement avere l'ordere chronologicue de lear apparition sur le, globe.

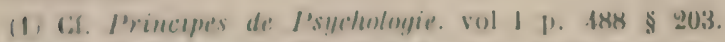

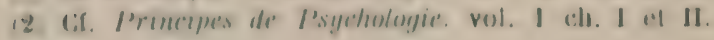


Entre la petite masse centrale ande fils diverements qui

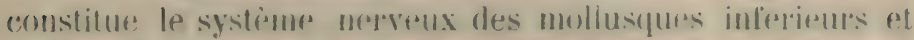
le strstime nerveux de l'homm qui comprend des matises voluminfuses dou partent des tibres inmombrables et qui sont relieres entre elles par une intinite de tilaments, on rencontre tous les intermediaires. L'évolution du șrstème nerveux comporte d'abord une angmentition progressive de son volume, tant en valent absolur quen proprotion avec le volume du corps tout entier.

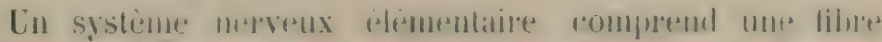
alferente ou sensitive, un gangrion contenant an moins denx cellukes et une fibre ellerente on motrice. I mresure ques le systime so derelopple, les filmes et les gillorlions se multiplient a l’intini. les libmes s'unissant pandint lat phos grande partir de leur trajet pen fatiscaux qui forment les cordons neverex, tes gangrlions s'assemblant en restres folus ou moins voluminemx. Ljallgrmentation de la masse du systeme merveux portesur les fibres nerveruses qui parroutrent les organtes, mais primcipalement sur les contres, et par

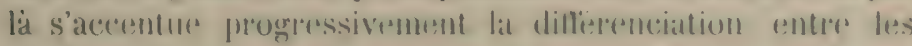
cordons et les inasses centrales. Lorsque le volume de res dernières rest considerable, on ronstate quidles sont

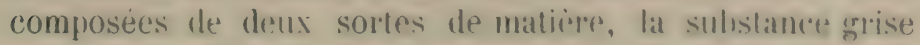
qui constitue les centress fmoperenent dits et la matiere blanche qui romprend les fibres itablissant des relations entre les différentes parties.

Lat diflerenciation se poursuit dans les cordons nervoux par la multiplication des libres, par leur développenent inegral, par les dillérences de structure qu'on observe surtout dans leurs extrémites. Les commaissances yu'on a surce dernier point ont principalement trait aux fibres sensitives. Les organes des sens : de la vue, de l'ouïs, de l'odorat, du goùt, du toucher dans sa signification la plus large, ne diflerent pas seulement par leur structure apparente, mais encore par la configuration intime des fibrilles nerveuses qui viennent y aboutir. Les fibres qui dependent du système sympathique ne sont pas non plus entièrement semblables à celles qui dépendent du système cérébro-spinal. 
Quant aux centres, ils se diltèrencient par leur multiplication, par les divisions qui s'y établissent et dont les circonvolutions cérébrales représentent une forme typique, par la superposition des systèmes qui dépendent les uns des autres, par les diflérences de structure qui s'observent dans les parties centrales: les ganglions du sympathique, la moëlle épinière, la moëlle allongée, le cervelet et le cerveau.

Cette différenciation du système nerveux est détinie cohérente et ordonnée: définie en ce que les centres et les cordons ont une situation et une structure de plus en plus déterminée, en ce que les parties se distinguent de plus en plus neltement les unes des autres; cohérente et ordonnée, en ce que les ganglions disseminés d'abord sans ordre apparent se concentrent dans la suite, se disposent en masses symétriques, superposies les unes aux autres par ordre d'importance, relieses entre elles par des communications nombreuses. Cette disposition ordonnée des centres implique une disposition semblable des fibres qui y aboutissent. Si l'on examine le système nerveux d'un animal inférieur, on n'y perçoit souvent aucune unité, tandis yu'un coup d'oeil jeté sur le système nerveux de l'homme y fait voir toutes les parties régulierement distribuées et convergeant symetriquement vers le cerveau. Une grande partie des fibres et des cellules auxquelles elles aboutissent, n'ont d'autre raison d'être que d'établir des connexions de plus en plus nombreuses entre les éléments du système, de manière à augmenter son unité d'organisation. C'est ainsi que dans les vertébres supérieurs nous voyons s'accroitre rapidement l'importance du fasceau de fibres commissurales qui prlie entre eux les hémisphères cèrébraux.

La redistribution de matiere que vous venons d'esquisser a été accomparnée d'une redistribution parallìle du mouvement, rest-i-dire d'une evolution concomitante des fonclions. La fibre aflirente transmet les impressions de la periphrierie au centre; les ganglions mettent en liberte l'energie qui est dransmise a la peripherie par la fibre efferente. 
Les terminaisons differentes des libres sensitives romespondent it des sensations diffiomentes at transmettent normatement les impressions de difliments anents. Les lihres motrices ditereninent diflérentes activites suivant les or ganes dans lesquels elles se terminent. Quoique nos comnaissameres soient encore tris incomplites, nous savous aujourl'hui yue les difterentes parties des centres mrvalux president a dillerentes fonctions, et si lat question des localisations cierribratles est rncore loin d'être celucidèe, nous avons an contraire des ronnaissances assez completes sur les localisations des fonctions dans les centres inferionurs.

Il se presentr ici une question du plus hatut interit, mais dont la solution est enveloppere de probondes olsceurites. Nous savons que l'abranlement d'une tibere semsitive, quelles qu'en soit d'ailleurs la canse, preduit toujours unt sellsation

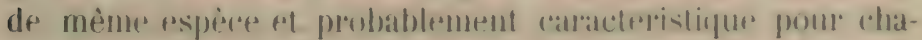
que tibre individuelle. Les medes atuditifs itant excites detereminent toujours une sensation anditive of l'on pense yüa lat

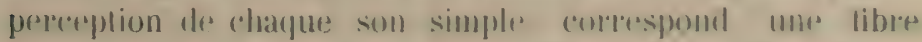

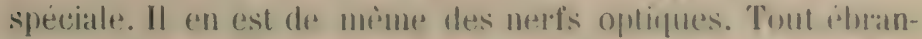
lement y groduit une sensatiun de vision en la motine est organisser de telle sortr que les farties qui pereoivent les couleurs possedrnt des tibrilles dithentes gour ehatpue

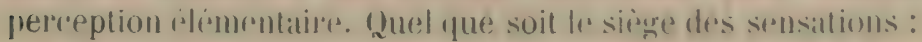

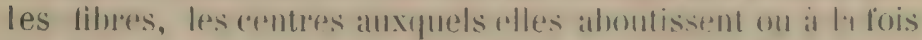

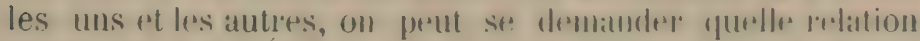
existe ratre lat natlure du monverment produit dats lat masise

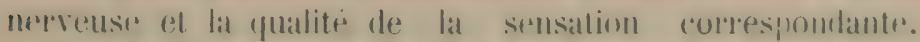

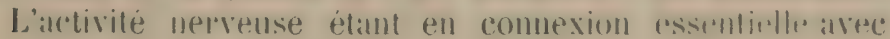
frs sellsations ot les mourements de l'animat, on andmettrat

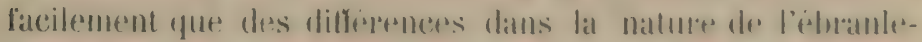

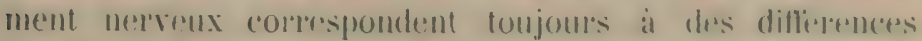

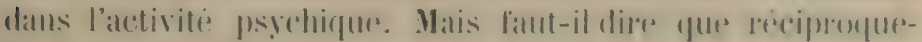

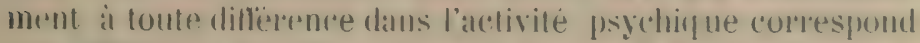

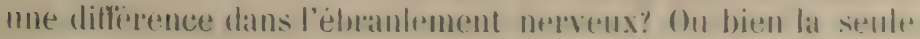
situation d'un nerle on d'un e'entre datls l'organisme peut-elle deteminer sil fonction an point de vue psychique? 
Les etudes qu'on a faites sur le developpement embryonnaire ont amene les biologistes a cette conclusion que la fonction des cellules est détrrminée non seulement par leur structure mais encore par leur position. II n'est pas improbable que ce qui se rialise pour la vie organique ait son pendant dans la vir animale. S'il en fetait ainsi, la différenciation fonctionnelle suivrait encore de plus près la diféerenciation structurale que ne le fait la différenciation du mouvement, puisque, indépendamment de toute diversité de relui-ci, il y anmait une differenciation de fonctions dépendant uniquement de la contiguration de l'organisme; et même la dillérenciation fonctionnelle serait plus profonde que la differenciation des organes. Malheureusement nos comnaissances positives nu nous permettent pas d'aftirmer qu'il en est anisi el c'est la mison pour laquelle nous ne? développerons pas l'argument qu'on pourrat en tires pour

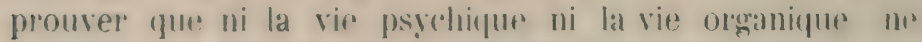
s'expliquent par la seule anefiviti phọsico-chimique des atomes.

En mème temps que les fonctions du systeme nerverux se dilferencient, olles se détinisisent et s'ordomnent. Lat cohesion etroite at la subordination des lanultes dans les animaux superirurs comespondent parfaitement a la structure regulime et cohripente du systeme nerverux. Tamdis que les contres volumineux contiemnent de grandes reserves d'aclivité mer-

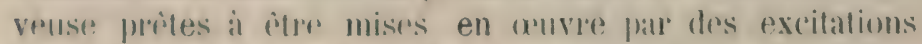
mime tris faibles, les masses de matiere blanche correspondrolt aus innomblables romexions qui existont entre. les actes infiniment varias amxquels le systime nerverux sert d'organe.

La verilication de la loi d'roblution pent so poursuivere dans l'ensemble des fonctions nerveuses ou dans le developpement l'une laculte sperciale. Soit, par exemple, l'aptitude

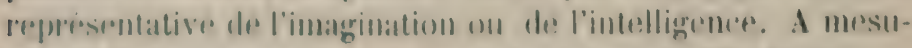

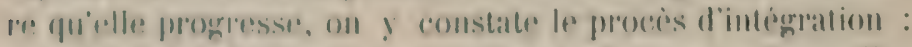

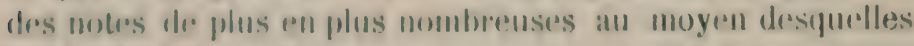

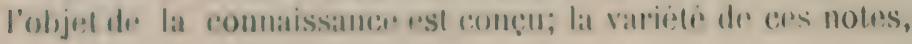


leur nettete et lem cohésion réalisent la loi de la difrerenciation et de la définition ordonnée. (1)

L'évolution des facultés prsychiquess rentres dans la detinition grenerale de l'ivolution (redistribution de matiere ot de mouvoment) en tant qu'elles ont leur sieme dans le systime mereux et que lem artivite componte des mouvements dans ces tissus.

Nous arons la conviction que l'intelligrence n'est pas nécessairement liés a la matière, qu'elle pesut exister en dehors de la matirre et que l'intelligrmes proprement dite qui est capable d'abstraction, de réllexion, le raisonnement, est toujour's une faculté immaterielle. Nous pensons que l'activite de l'intelligence humaine n'est pas essentiellement lies: a la coupripation des oremanes. Peanmoins nos facultes sont solidaires et de fait notre intelligente n'acquiert ses connaissances que gratee a des impressions qui lui viennent de sens, de tella sorte que la sensibilitio lui fournit la matiere sur laquelle elle s'exerce. A cause de cepla toute activité mentalo s'accompagne de représentations sensibles; et quoique ces images soient moins vives et aient des rapports plus vagues avec l'objet de la spéculation a mesure que calle-ci devient flus albstraitr, elles ne s'effacent cependant jamais completement. Il n'est pas possible de confondre la representation intellectuelle, abstraite mais très précise avec la représentation sensible imprecise (2); cependant l'une n'existe pas sans l'autre et a mesure que l'esprit est occupé de choses phus roncrètes, Jes connassances sensitives jouent un roble de plus en plus important.

Dess lors, on congoit que l'evolution intellectuelle jropresment dite soit parallèle an développement des facultés psychiques d'ordre sensitif et par conséquent au développement des centres nerveux qui servent d'organes à ces dernières. Quoique l'acte de l'intelligence ne consiste pas

(1) Cf. Principes de Psychol. vol. 11 p. 237 \& 181.

(2) Ci. Alfined Binet. Étule expérimentale de l'Intelligence. Paris 1903 p. $139 \mathrm{sq}$. 
dans une redistribution de matière et de mourement, il est cependant de fait dans l'honme lié à ces phenomènes materiels et à cet ègard l'intelligence se trouve elle-même soumise à la loi générale de l'évolution.

si nous voulons préciser davantage cette affìmation, nous devons, semble-t-il, distinguer entre l'évolution des aptitudes intellectuelles qui se poursuit dans la race et l'évolution des connaissances intellectuelles qui s'accomplit presque exclusivement dans l'individu. L'opinion de Spencer (1) - et c'est également celle des. Thomas d'Aquin (2) - est que, parmi les sens externes, la perfection du tact exerce une influence preponderante sur les aptitudes intellectuelles. Elles dependent ensuite dans une large mesure de l'imagination et de la mémoire sensitive. La linesse du tact est influencée sans doute principalement par la structure des élements nerveux terminaux, mais l'imagination et la memoire dépendent du développement des centres, de sorte qu'à cet égard le développement de l'intelligrence est dépendant de l'organisation du cerveau. Il est beaucoup plus dificile de difinir les relations qui existent entre l'évolution cérébrale de l'individu et l'évoJution de ses connaissances intellectuelles, à cause du ròle variable que jouent dans celles-ci les images sensibles; mais cette correlation n'en est pas moins reelle, quoique,sans doute, moins rigoureuse que la précédente.

loi d'èvolution Sociologie.
Le: premiel volune des Principes de Socioloyie de sinencer est consacri a l'etude des idees relignieuses principalement dalls les premiers stades de lear developpement. Spencer y latit remarquer la veritication de lat loi d'evolution. Lat forme primilive de la Religrion est, d'apmès lai, le: alulte des morts, les etres qu'on venerait ou qu'on maignait

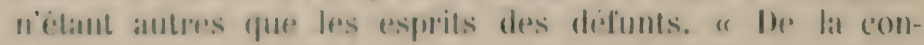

(1) Prince de Psychol, vol I p. $37 \pm$ sq. \& 163.

(2) sum. Theol. p. I q. 76. a. 5. 
ception jadis uniforme, dit-il, de l'esprit revenant sont sonties les diverses conceptions d'itres surnaturels." (1)

D'abord ces croyances sont faibles at incertaines; plus tard, daus les idees des pruples plus avancers, les ditres surnaturels forment un groupre qui a une lendames at s'augmenter. A mesure que le: nomber des divinites s'ancroit, elles deviennent diffërentes les unes des autres, des lonctions differentes leur sont dévolues at plles constiturnt une hirrarchie. "Originellement, dit spenter, les sentes distinctions qui séparaient les doubles les morts m bons et mallvais etaient exelles que l'on trouve chez les vivants; il u'y avait pas non plus d'autle dillipence dans leur puissance. Mais il ne larde pas at se former des idees de diffrencers dans la bonte entre les esprits des palrents et ceux d'antres persumes, de mime qur des diflinences plus prononceres entre des esprits birenveillants qui applartiennent à la mome race ot des esproils malveillants yui appartiennent a d'autres. Des que l'institution do ranges sociallx s'est itablie, il en resulte des differences de rang et de puissance chez les itres surmalurels, yui deviennent de plus en plus prononores i mesure que less legremdes prement de l'pxtension. A la lin ste lorme par artle

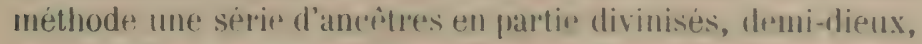
grands dieux, et parmi ces derniers un dien supmème: de mème se forme une hiemarchie de puissances diaboliques. Alors se produsent de nouvelles diffierenciations, colles qui specialisent les fometions et les habitats de ces ètres surnaturels, juscju'it ce que touts mythologrits ait ses agents divins grands at petits, depuis Apollon juspu'a la dryade, depuis Thor jusqu’à une fée. ” (2)

En mème temps, les idéestelatives à la puissance el à l’action des divinites deviennent plus précises et plus cohérentes: "La forme des diferents gemres d'etres surnaturels devient plus délinie; leurs dispositions, leurs puissances, leurs habitudes le deviennent aussi, jusqu’à cequ'entin, dans les inytholo-

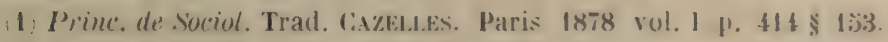

(2) Princ. de Social. v. 1 p. $377-578 \& \geq 07$. 
gries avancees, ils se distinguent par des caractieres d'espè et mime des caracteres individuels, qui sont des attributs nettement pxprimés. Incontestablement, les croyances qui constituent un scistime de superstitions se developpent de la mime manière que toutes les autres choses. Par une opiration d'integration et de diflérenciation continue, elles forment un arrégat qui, en s’accroissant, passe d'une homogénéiti. indétinie, incohérente, ì une hétérogénéité détinie, cohérente. " (1)

La conception que nous renons de résumer est loin de s imposer à l'esprit. Que les idees religieuses aient évolué en se dilferenciant a partir d'une situation uniforme, cela est extrimement vraisemblable et en partie contirme frar l'histoire. Mais, autre chose est qu'on puisse assigner le point de départ de cette erolution. Nous rencontrons frequemment des peuples qui vénèrent comme dieux des ancitres, surtout ceux qu'on considere comme les fondatems de la race. Mais, mème dans la doctrine de l'evolution, ce fait est susceptible de deux "xplications : ou bien, dans le culte des morts, un defunt a joui d'une véneration speciale et croissante jusqu'i se trouver clevé a un rang qui le sépare completement de la foule; ou bien le caractère divin - quelle que soit l'orignine de l'ider de la divinité - a été attribué par extension(intégration) à des persomes pour lesquelles on professait un grand respect. Cette derniope interpretation, spencer me l'ignore pas, rst conforme: a lopinion commme de ceux qui se sont orcupés de centte matiore. Less raisons pour lesquelles il l'abandome IIe sont pas du tout peremptoires: quelques remarques suffi-

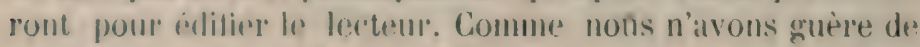
renseignemunts positifs sur l'itat des aroyances avant l'ipo-

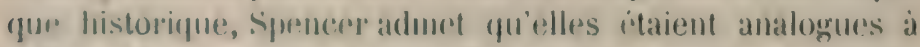
celles quion rementre anjourd'hui chez les peuplos sauvages. ()r, rian ne permand d'aflimmer qu'il en est ainsi et la remarquer faitr par lui-mime, - que c les hommes du type inferiesur existant anjourd'hui.... ne sont pas des speseimens de l'lumme le: qu'il ful dans le principen "el "que?

(1) Ibid.. p. 579 \& 307 . 
la plupart simon tous enrent des ancritres tgui itaient parvenus a un chat suprerien $n$ (1) — incline l'esprit a admettre plutôt le contraire.

spencer proteste (2) contre la these dre Vax Yüller d'apres lequel " flus nous remontons daus le passe, plus nous examinons les premiers germes d'une religion, plus les conceptions de la divinite so montrent pures. "Mais il ne demontre pats que cela est faux. La doctrine de

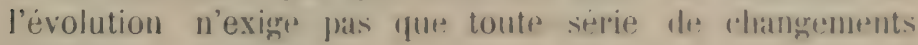
constitue un progrès.

Disons encore que l'inferpretation des croyancess ast une those tres delicate at que, de l'avis des hommes qui ont fréquenté les sauvages, rien n'rst plus difticile que d'obtrnir des rensignements ratcts sur les idées qu’ils prolessent en matière religriensa. II convient iggalement de st: défier des phrinumines de convermence frequents an colle matiore. Ainsi, quoiqu'en prense Sprucer, (3) et malgres quelque apparence superficielle. le coulte des saints dans l'Eglise catholique it me origrine el une signilication tout a fait dilferente dre celle qu'il attribue au culte des morts; d" même l'infaillibilité du Pape n'a rien de commun avec l'inspiration des oractes painns, ni avec ancune, inspriration en general. Il faut aussi ne pas confondre une plaisanterie d'ailleurs grossière aver l'expression d'une croyance. (4)

Lat conception de spencer que nous critiquons ici est sévirement jugrt par Benjamen Kidd: "()n ne peut mìme pas dire que l'essai d'etude des phénomènes religreux fait par Herbert spencer dans sa sociologie soit conçu dans l'esprit de la science de: l'évolution, trolle qure nous la concevons aujourd'hui. Il rst difficile de suivre l'autrur dans sa thérie qui fail sortir les croyances religiesuses du culte des esprits et des anceitres, sans éprouver un

(1) V. plus haut p. 232.

(2) Princ. de sociol. V. 1 p. 612 (App. A).

(3) Ibid., p. $331 \$ 131$ et p. 362 \$ 203.

(4) Cl. ibid., p. 610 (App.A). 
sentiment de desappointement et mine d'impatience en voyant quelles explications triviales et mème insignifiantes il nous offre four l'endre compte du développement de faits sociaux aussi importants. Ses disciples n'ont fait que suivre son exemple..... »(1)

Dans sa discussion avec Spencrer, le disciple de Comte, Harrison, a vivement attaupe la Ghost theory (theorie des esprits) ot la methorle que Sprencer emploie pour l'etablir. (2)

Entin, la loctrine de l'évolution n'enseigne pas qu'il faut considerer la nassance de toute religion comme due uniguement au divoloppement naturel de l'esprit humain. Lexistence d'un ordre surnaturel n'est pas contredit par l'existence de l'ordre naturel. Rien n'empèche d'ailleurs qu'il n'y ait des lois lois communes à l'un et à l'autre et qu'un systène de doctrines ayant une origine surnaturellr ne se developpe, tout en conservant son caractere propre, suivant lat formules grénérale de l'évolution.

Yous sommes donc loin de repousser a priori toute interprétation évolutionniste des idées religieuses. Nous crogons au contraire quilles presentent a cet égard un objet d'étude de plus haut interret. "L'évolution graduelle de la Revilation, dit 111 des plus illustres disciples de Darwin, est en hamonie aver les autres reures de Dien. ") (3) Mais nous sommes couvaincus que la conception developpee dans lo promier volume des Principes de socioloyie, quoique probablement vaie dans beanconp de details, est finsse dans son point de depant; et l'ensomble rast naturellement affecté par cette erreur originelle.

bans lo second volune dres Principes de Sociologie, Speen-

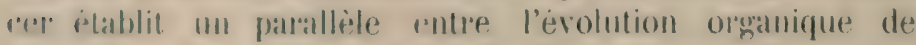
l'animal el l'evolution sociale. (4) Cette comparaison, pal'fois $13 n$ prou forriog dans les details, est rependant d'une

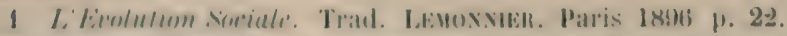

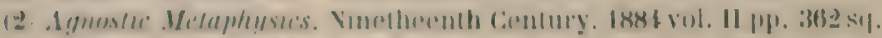

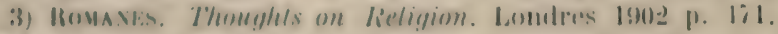

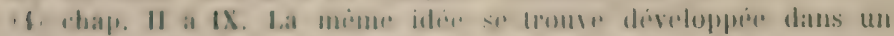

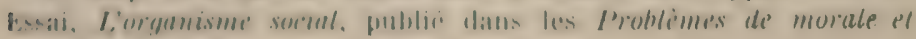
de sociologie. Trad. DE Vamitny. Paris $38 M$. 
vérite diviente dans son ensemble, ef il strouve ainsi contirmé que la mème loi ganérale gouverme les deux ordres de phenomenes. L'analogie la plus frapplante que Spencer signale.c'est l'etablissement progressif et l. diveloppement, dans les societés, de trois apparpils: l'appareil producteur, e'est a dire les diflèrentes formes d'industrie, l'appareil distributeur, c'est a dire les mogens de transport, le commerces et les atutes institutions qui assurent la circulation et la distribution (onsemable des produits de l'industrie, enfin l'appareil regrulaterne, cest à dire It pouvoir sous toutes ses lormes. Ces trois apparrils, en eflet, cormspondent aux organes d'assimilation, an systrime circulatoire et au système nerveux.

De meme que, en suivant le developplement organique dans les animaux depuis les célenteres les plus primitifs juscuaux vertébres, on voil les organes d'assimilation ste former au lepens de l'molorerme, tandis que les systemenerveux et les organes des spus proviennent du développroment de l'exodrime, et que le misodprme fournit le systime circulatoire, - ainsi dans la socirte l'appareil rignlateur a pour origine la niccessite dans laquelle elle str trouve de laire face aux dliflicultes et aux dangers extérieurs, qu'ils proviennent de la nature on des tribus ennemies ; l'appareil producteur au contraire pourvoit aux besoins intérieurs du corps social; entin l'appareil distributeur qui devient nécessaire lorsque la société grandit, etablit les rapports indispensables entre les differents organes des deux appareils précédents.

A mesure que la société se développe par interration, soit que des peuplades voisines s'umissent en vue de se défendre contre des dangers communs, soit qu'une nation plus puissante absorbe par conquète les peuples limitrophes, la loi d'évolution se vérifie également par la diflérenciation que nous venons de considerer et qui se poursuit - dans la société comme dans l'organisme animal - par la complexité croissante de chacun des appareils fondamentaux. Du chef unique qui exerce toutes les fonctions de l'autorité, on passe par un progrès continuel à la division des pouvoirs avec 
toutes les complications qu' elle presente dans nos états constitutionnels. L'industrie s'étend en se diversiliant. Les moyens de communication se multiplient et les fonctions qui président à la distribution des produits industriets deviennent de plus en plus variées.

Cette dilhirenciation est accompangne d'unc ditinition croissantr des droits et des devoirs de chaque organe social et aussi d'une subordination de plus en plus itroite entre les diffirentes parties de la suciété. Le pouvoir gouverne en vie de la delense de la nation; il se trouve à la tête de l'armée et protege aussi l'ordre social contre les agressions venant de l'intérieur. En mème temps, il dirige dans une mesure variable les institutions gui assurent la circulation des choses necessaires a la vie et il exerce son influence sur l'activité productrice de la sociéte. D'autre part ceux qui sont employés au gouvinement ont besoin des produits de l'industrie of du bon fonctionmement des moyens de communication, et entin il a $\mathrm{x}$ iste une defondance mutuelle entre le developperment de ceux-ci et le progrès industriel.

l'évolution - on le voit - est la marche d'une homogreneité indefinie et incohirente vers une hetéroréneite definie of coherente en mème trmps que sre poursuit le procés d'intéglattion.

Le troisième volume des Principes de Sociologie traite de l'érolution des institutions ceiremonielles et surtout des institutions politiques. L'union des individus sous la . pression du besoin ou du danger, - le choix d'un chef trmporaire puis permanent, - la division de la societé naissante en roi, nobles et peuple, - la réunion spontanes on violente de petiles socieles en socictes plus etendues ot les gouvermements locaux subordonnes au pouvoir central, - la sippanation des pouvoirs executif, le grislatif et judiciaire, - la multiplication des organes dr. elateun, - la didinition progressive des relations sociales, des fonctions, des righles de conduite, - la dependance mutuelle el la coordination progressive des dillimentes parties de l'ensemble, - telles sont, dans leurs grandes ligues, les phases par lestguelles passent les institutions 


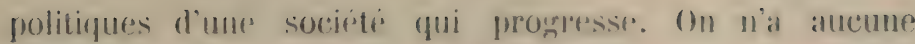
peine a y recombatm les diffrments alments de lat loi d'erolution: intregration - dillidenciation - drefintion coliesion et coordination.

on peut evidemment itre d'un antre atvis que Spencer an sujet des callses conrrites yui ont determine lat transformation de ces institutions et, fatr suite, concevoir d'une

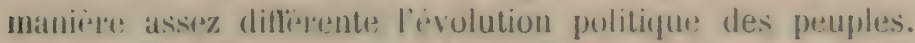
Les lois grenceales de l'évolution, a cause de leor grine-

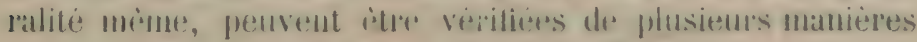

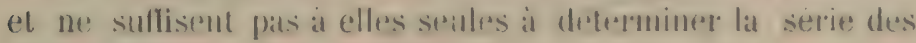
transformations des sociètés.

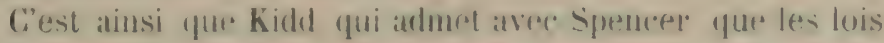
d'evolution biologigue: sapplipuent ryalmutent anx organismes sorians, aboutit at une concepption de l'esolution poli-

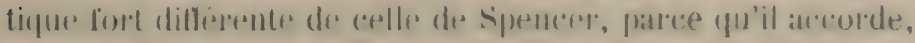

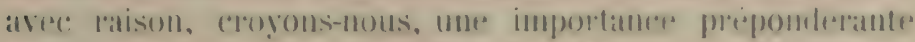

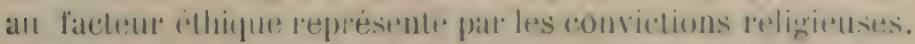

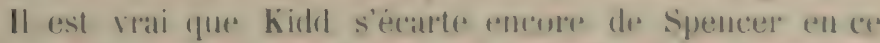
que contranment it celui-ci, il nladmet pas la transmis-

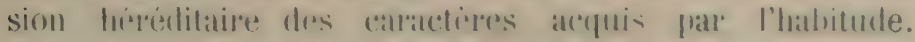

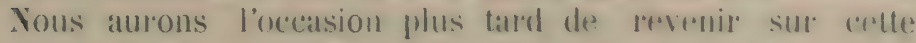
question. Contentons-nous de: dire ici que tout ent itant importante ale point des vue de l'intrerpretation de l'évolution sociale, elle na cependant fras la porter que Kidd lui attribur. "Si nous atvons, dil-il, une tendanne a hériter des resultats de l'education morale et mentales des genérations anterrecures, nous arons le droit d'attender ane societe future toujours en progres, la lutte frour l'existence fittelle supprimer, la population exactement regree sur les moyens de subsistance et l'antagronisme entre l'individu et l'organisme social disparu, comme le predit Horbert spencer. Mais si, comme je le crois, ce sont les theories de Weismann qui sont vraies, s'il ne peut y aroir progres que grace a l'accumulation de variations congenitales, superieures a la condition moyenue el excluant les variations inféricures, si, sans la sélection constante qu'implique cette loi des variations, la tenclance de toute forme 
elevée de la vie est de rétrograder, alor's ltr destin de la race humaine entière est d'itre suspendue à cette rivalité laborieuse, a cette lutte pour la vie qui progresse depuis le commencement. Alor's doit continuer la lutte pour l'existence, avec des conditions plus humaines, mais toujours inévitable jusqu’à la fin. » (1)

Nous verrons dans la suite que la cessation de la sélection naturelle ne semble pas entrainer nécessairement la tendance à rétrograder. En outre Kidd neiglige de considérer ici l'influence exercée sur chaque individu par l'éducation, influence qui peut suppléer à l'absence d'habitudes héréditaires et qui peut mettre en échec la tendance a rétrograder, en admettant qu'elle existe.

Ces divergences d'opinion ou d'interpretation dont on pourrait multiplier les exemples, n'emprehent pas de reconnaitre l'application de la loi genèrale d'évolution dans le développement politique des peuples. C'est en somme dans les phénomènes sociaux pris dans leur acception la plus large que le procès évolutif se manifeste avec le plus d'évidence : ils ont éte, on le sait, l'objet des premiers travaux de Spencer.

Les deux derniers volumes de Principes de Sociologie dans lesquels il est question des institutions ecclésiastiques, professionmelles et industrielles offrent des applications interessantes de la loi d’évolution, principalement le développement des professions issues, d'après Spencer, des fonctions sacerdotales : le medecin, l'artiste, l'orateur, l'hounme de lettres, l'homme de sience, l'homme do loi, l'homme d'enseignement.

lá sifparation graduelle de ces dernières d’avec celles-là, lenr laicisation, pourrait-on dire, ensuite, leurs subdivisions de plus en plus nombreuses, témoignent à la loi de la diflërenciation.

leme sejparation de plus an plus nette est conforme a la loi de la délinition.

Eufin lour developpement croissant, les organisations

(1) Licuolution sociale, p. 187. 
obligatuires on libres qui englobent leniss membres, leur dipendance mutuelle montrent, dans l'onsemble et dans les parties, l'existence des procis d'integration et des coorlination.

On pent y observer anssi de curieux phènomines de convergence ; telle l'union renouvelie, par une alaptation plus complite au but, des fonctions de medecin et do (hi-

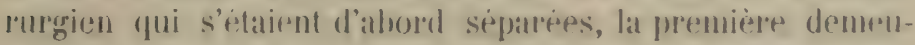
rant plus ou moins une attribution du clergé ou restant sous sil dépendance, la secombe tombant aux mains des barbirers. bes progres de l'une et de l'auter pexigeant la mime

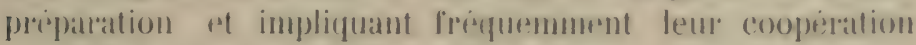
mutuelle, elles se sont trombers rimmies, jusqüi ar que la sprecialisation dess fonctions mirlicales qui s'allirme de plus en plus les sépare de nouveau.

sprencer étudie l'érolution des fonctions écclésiastipues, lelles qu'elles sollt, sous mur forme ou sous une alutre, rommunes a tous les peruples. Confondurs a lorigine

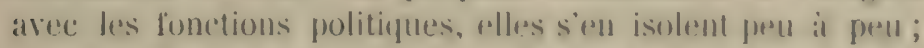
les pritres constituent un corjes social sejpare dont les

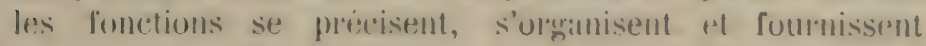
ainsi un exemple de la loi.

En ee qui concerne le Christianisure, fes functions onelesiat-

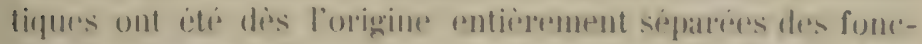

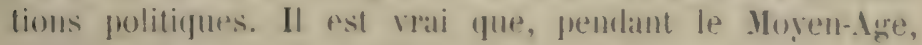
sompent un pouvoir politigue plus ou moins etendu s'est

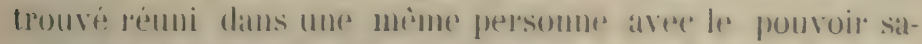
rerelotal, notamment che\% les feteques et les abbes; mais les

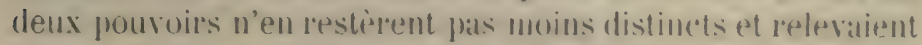
charem d'une autorite somveraine diflerentr. Des sorte pu'il latul considerer cette union on rette ronfusion des chatlgees, non fats comme une situation nomale et uniserselle, mats bien plutrit comme le résultat nullement gienteral de circonstances historiques variables d'un endroit a l'autre. spencer lui attribues une importances exagtiée.

Il est rat que rertains prelats italent ril meme tempss princes temporels; des cleres preuvent avoir pxpece des fonctions puliticues on militaires; mais il 11 'y arat pas d'union 
régulière entre les attributions ecclesiastiques et profanes. Si Richelieu a dirige la politique de la France sous Louis XIII, comme spencer le rappelle, (1) on aurait néanmoins tort d'y roir une relation quelconque entre les fonctions de premier ministre et la dignité cardinalice. Tout ce que l'on peut dire, c'est que ces liens personnels et accidentels entre les fonctions civiles et religieuses sont devenus dans la suite de plus en plus rares et qu'il faut voir dans cette circonstance une application de la loi de ségrégation.

loi d'évolution morale.
Spencer appelle conduite l'ensemble des mouvements d'un individu vivant, en tant qu'ils sont diriges vers un but. Le philosophe anglais donne ainsi a ce mot une signification beaucoup plus large qu'on ne le fait d'ordinaire. Dhabitude on riserve ce terme a l'homme et encore ne s'applique-t-il it proprement parler qu'à ses actes libres. La definition de spencer pourrait it la rigueur s’interpriter dans $c r$ sens restreint. En effet, quoique tous les êtres vivants posent des actes qui sont derigrés vers une fin, cependant tous ne les y dirigent pas eux-mèmes. Pour diriger ses actions il faut en etre maitre, et pour qu'un individu soit maître de ses actions. il faut qu'elles ne soirnt fas rigourrusement diterminees par sa mature et les circonstances oil il se trouve, en un mot, il faut la liberte. Il n'y a pas de place pour la liberte dans fe meranicisme de spencer et c'est une raison de plus pour ur pas admeftre ceflr theorie qui se trouve ansi rol contratition aver la conscience et avec lo sens commun.

Semuls les actess libres sont l'objet de la Morale dans l. sens on l'on emploire habituetlement ce mol. (2) Lat liberte drune action ast toujours supposée, lorsquion la froclane home ou mamaise. Sous navons pas a

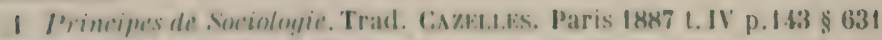

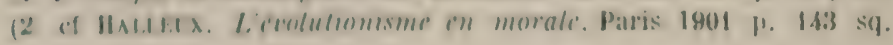


justitier ici cette maniere l'monisagrer les choses ; nous conslatons seulement que, si l'on rexceple quelques philosophles. alle est universelle. II suit de lit que sprencere emploie tous les mots qui ont rapynort à lat monaliti, bien, mul, devoir, droit, etc. dans un serns qui est, atu fond, fout a lait different de la signification qu'on y attache d'ordinaire. Celle-ci a pour base lal liberté, tandis que pour spencer la liberté n'existe pas.

spencer étudie en détail l'évolution de ce qu'il anpelle la conduite dans la premire farie des Prinripus de .Morale qui a paru d'abord sous le titre de The datu of ethics.

Lat loi d'évolution ne se manifeste pas semlementut dans la structure at dans les fonctions qui lui sont corridatives, mais encore dans la maniere dont l'itre expere ses foncetions et se sert de ses organes. Chez les animaux inferierurs la plupart des mouvements sont executes comme all hasard, tantòt utiles, tantot inditlerents, tantòt muisibles:

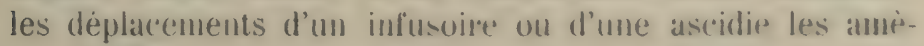
nent indiffiremment en contud avec ume substance gui les nourrit ou avere un animal qui les devore. A mesure quon monte dans l'échelle animale, les actions qui ont un lut leterminé se inultiplient et se précisent : elles tendent an bien de l'individu, ou de l'espece, on encore de la communante a layuelle l'individu appartient. Chez les êtres superiturs on voit de plus en plus un grand nombre d'actes coordonnés en vue de la réalisation d'une fin procise et atteignant cette fin d'une faron sùre. La conduite devient plus coherrente en même teinps que plus hêtérogine et et plus définie.

A cet igard, la conduite de l'homme s'élire intiniment au-dessus de celle des animaux, parce qu'clle est dirigeje non seulement par les impressions, la mémoire sentive et l'instinct, mais encore par l'intelligence, qui, gràce au raisonnement, permet de poursuivre des tins éloignées au moyen d'actions très nombreuses et parfaitement coordonnées. (1)

(1) cf. Les bases de la morale ivolutionniste Traduction française de The dala of Ethics) Paris 1880 pp. ¿ sq. \$3 et lip. 34 su. \$ 24 
La conduite morale de l'homme est caracterisée encore, nous l'avons dit, par la liberté.

On comprend que la liberté est dans la conduite une condition qui farorise la differenciation, soil qu'on compare les individus entre eux, soit qu'on considère le mème individu aux diflerents moments de son existence.

Lorsqu'on constate que Spencer appronve le titre de Morale érolutionniste pour la Première Partie de ses Principes de Morale (1) et déclare: "Cette dernière partie de la tiche (Les Principes de Morale) est celle pour laquelle toutes les parties précédentes ne sont, à mon avis, qu'une préparation. ") (2) - on est fort surpris de voir le peu de place que la throrie de l'évolution tient dans la derniere partie de la Philosophie Synthétique.

spencer s'applique d'abord at etablir l'essence de la moralité. Elle consiste d'après lui dans l'intlence favorable yu'ont les actes sur la vie indiridurlle, sur celle de la prominilure ou sur la societé. Lne telle ronception, quelle que soit sa valeur, (3) est complètement indépendante de la loi d'évolution. II stra possible de montrer l'applieation de cette loi dans la conduite, en tant que celle-ci réalise flus ou noins parfaitrment le hut que la Morale lui assigne. Mais les principes de l'évolution ne donnent aucune lumière pour découtrir les règles de conduite qui résultent de celte conception de la Morale, pas plus qu'ils ne peuvent conduire a la découverte des lois de la Jhysique, de la Chimie ou de l'heredite. Il est impossible d'en deduire quelles sont les actions favombles ou defararables a l'individu, à la famille ou à la societe.

La loi d'èvolution indique, il ast vrai, les conditions grinerales qur doivent réaliser les transformations pour etre progressives. C'est ansi que spencer justitie le

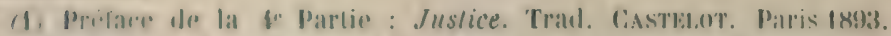

- Prolane do la 1" Partie : Les Bases de la .Morale Evolutiomiste.

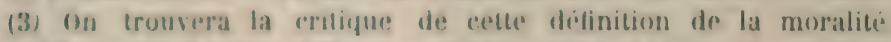

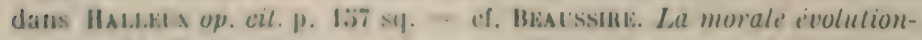

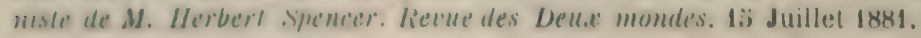


le: principe d'apros lequel l'Etat doit ètre diecentralisitemer

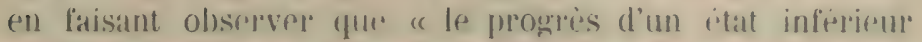

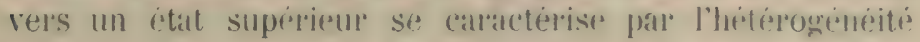
roossante des structures pt la subdivision crossiante des fonctions. "(1) Telle pucore la remarque que la sulsstitulion des gratifications anthitaires (pourboires) anx récompenses fixces par contrat ronstiture me " moditication rétrogrado: " parces que " l'ellet de l'évolution est d'imprimer aux choses un caractimp plus difini. » (2) Mais dr. telles ronchusions sont nfressairement peen nombrenses a cause do la grande greniralite de la loo, de la variabilité et de la complexite des cireonstances qui alccompagnent son application. Spencer s'en est rendu compte lui-mime.

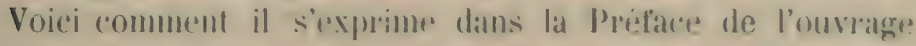
que nous renons de citer : " Lat doctrine de l'roulution n'al

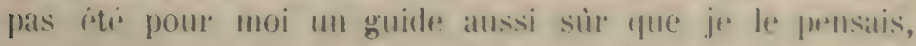
et la phupart do mes conclusions, obtenues far voir

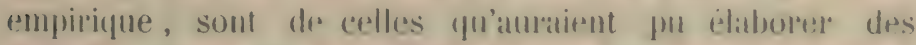
hommes doues de sentiments droits et d'une intelligencer cultivie. En dehors de quelpues sanetions gromelales

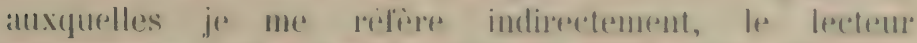

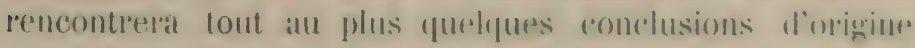
fivolutionnare iparses at particulierement lassembleses dans les derniers dhapiters; elles virmment sajouler and conclusions ordinaires, mais alles en dillement partosis.

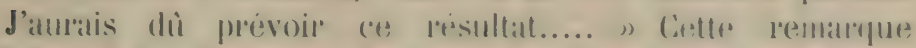

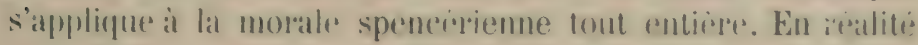
l'utilite de la theorie de l'ivolution pour l'èturde de la Morale dans son ensemble: est tres lestreninte. Cust lat conclusion qui ressont de lia lecture des volumes que sipencer y consacre.

A proprement parlere, il n'y a pats de morale évolutionniste. Il peut se farre que la doctrine gernerale de livo. lution ou certatines doctrines farticulieres, commes celle de

(1) Justice. p. 269 \& 124 .

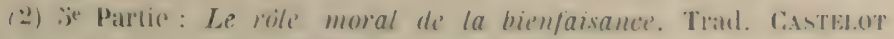
el Maktin S. Llon Paris $189 \%$ p. 6; $\$ 410$. 
la sélection naturelle ou de la transmission hériditare des caractères acquis, fournissent des conclusions se rapportant i la conduite. Mais la doctrine de l'évolution ne peut pas servir de base à la science morale. Celle-ci comprend essentiellement et arant tout une conception de la moraliti. Yous venons de remarquer que la conception spencérienne est indépendante de toute doctrine évolutionniste. Yous ne pensons pas qu'on puisse démontrer, comme spencer le prétend, que l'action plus favorable à l'individu et a l'especer tinira par apporter la plus grande quantite de jouissance, gràce à l'évolution. Mais quand cela serait, la question de l'essence de la moraliti ne serait pas pour cela résolue.

Lia science molale comporte ensuite l'application du concept de moralité aux dillérentes manières d'aunir. Quelles sont les actions qui favorisent la vie de l'individu et du groupe? Lat doctrine de l'évolution pourra dans certains cas donner des lumières pour resomdre cettr question, mais la plupart du temps la solution en sera independante. Il suffit dre lire les volumes que Spencer consacre it itablir les règles de conduite pour s'en convaincre.

On a dit que la loi morale, telle que spencer la formule, n'est que la loi d'adaptation transportee? sur le terrain sucial. (1) Cie rapprochement de la morale spencerienme avec la theorie de l'évolution ne nous parait pas heureux. Qu ne peut pas confondre une loi biologique qui inonces or qui est, avec une loi morale qui énonce ce qui doit ou clerrait itre et qui souvent riest pas. Ensuite, agir en vue du bien dre la socichi n'est pas la mème chose que s'adapurer a lat vie sociale dans le sens que le mot adaptution a en hiologre. Cin ètre vivant s'adapte au milien par los

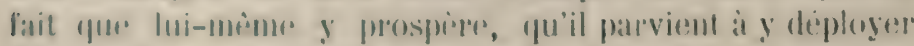
son activite de maniere a favoriser son propore bien-itre.

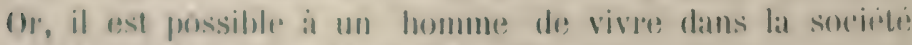

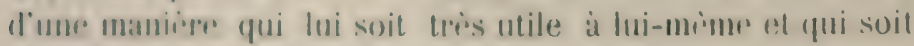

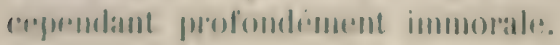


Salvadori reconnait d'ailleurs que te principe de justice de Spencer consiste dans l'union d'un élément biologrique, l'individuation, avec un element moral, la solidarite humaine.

II ne faut pas non plus confondre la question de l'rssence de la inoralité avec celle de l'orignine de l'idée ou du sentiment de moralite ou d'obligation morale chez l'homme. Comme toutes les idees fmmaines, l'idere de moralité est sommise a la loi d'évolution; quant à savoir si c'est grace à l'évolution el dans quelle mesure que l'idee de moralité s'est developpée de fait, c'est une question historique qui est differente de l'autre. Sans doute, pour étudier la question de l'origine des sentiments moraux, il faut savoir d'ahord, an moins d'une façon confuse, en quoi la moralitis consiste, et la question de sa nature pourlat recevoir a son tour des échircissements par l'étude de son origine et de son évolution. Les deux questions ne sont donc pas indipendantes, mais elles sunt distinctes; et de ce que les idees morales sont soumises à l'évolution, on ne preut pas conclure qu'il $y$ a une morale isolutionniste, pas plus qu'il n'y a une géometrie evolutionniste parce qu'il y a une evolution de la geumetrie.

spencer pense que l'idée d'obligation implique trois facteurs : l'autoriti, la coërcition et l'opinion publicque; (2) il tache d'expliquer l'origine de chacun de ces elements et comment il s'est imposé à la conscience par voie héréditaire, conformément à ses théories sur les facteur's de l'évolution. Mais ce n'est pas au moyen de la doctrine de l'évolution qu'il établit le concept de l'obligation morale qui est le point de départ de cette explication.

Vidari distingue trois sortes d'élements dans la ductrine

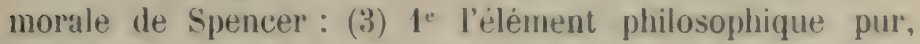
c'est à dire la conception utilitaire, hédoniste, yui confond en dernière analyse la moralite avec le hien de l'individu et la

\section{(1) Ibid.}

12. La morale des diffërents peuple's. Paris 189:3 p. 30 sı.

(3) Rosmini e Spencer. Milan. 1899 p. 136. 
jouissance, 2e l'èlement scientifiçue, c'est à dire la doctrine de l'colution, 3e l'élément national, e'est à dire le sens pratique et la tendance individualiste du peuple anglais.

De ces trois elements, le premier est incontestablement le principal, ou mème, c'est le seul vainnent fondamental et qui détermine le caractere de la morale spencérienne. Le lien qu'établit le savant italien les entre principes de morale et la doctrine de l'évolution nous parait assez artificiel. "Darwin, dit-il, avait fait roir que les sentiments moraux sont un produit nécessaire de la sélection naturelle, loi grace ¿ laquelle la vie non seulement est conservée, mais se développe continuellement; et comme en mime temps on démontrait conformément à la doctrine ancienne et traditionnelle que le fait primordial d'ou découle le sentiment moral est la sympathie et, au fond, l'égoïsme ou la recherche? du plaisir, on etablissait ainsi scientitiquement un rapport entre la conservation et le développement de la-vie d'une part et la félicité de l'autre ».(1) Et plus loin : "L'évolution de la conduite (l'après spencer) nous montre comment a son degré le plus éleve elle doit avoir pour effet necessaire d'assurer a la fois la plus grande totalité de la vie dans l'individu, dans sa descendance et dans ses compagnons sociaux. Et puisque, ell vertu du principe mis en relief par Darwin et acerpte par toute l'école naturaliste, il y a un rapport direct, comme nous l'avons vu précédemment,entre la conservation et le diveloppement de la vie d'une part et d'antre part le plaisir et la félicite, il s'ensuit que l'évolution de la conduite assurant la fulus grande tolaliti de la vir, assure en mime temps comme eflet nexessaire le plaisib le plus complexe qui romprend les glatisirs da: l'individu, de la descendance et des rempagnons sociaux $\eta_{0}(2)$

On voit que la base de toute la théoric est toujours

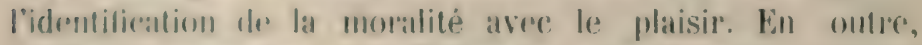

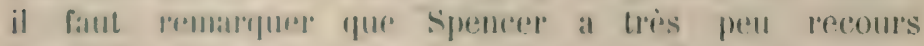
a la selerelion maturelle pour expliquer lorigine: des idees

(1) ibid. p, 189

12) ibial. p. 167. 
morales, mais plutòt à l'experioner et aux habitudes intellecturlles, alfectives et pratiques qui en risultent, transmises par l'hérédite. Ce llest donc que fla un abus de langage qu'on parle de morale érolutionniste. "Le prétendu rattachement de la Sociolomie et des la Morale aux sciences naturelles, dit avec raison, Angrot des Rotumrs, est factice et plus scientitique en apparence yu’en réalitr." (1)

(1) Réforme Sociale. 1904 vol. 1. p. 183. 



\section{LES CAUSES DE L'EVOLUTION}

\section{§ I. LES CACSES GEVERALES.}

Le point de départ. - L'instabilitè de l'homogène. - Forces qui différencient l'homogène. -. L'instabilité de l'homogène et la persistance de la force. - La multiplication des effets. - Confirmation inductive et justification a priori de ce principe. - Convergence. - La ségrégation. Récapitulation.

Les elements primitifs et essentiels de lévolution sont départ. Ia matière et la forces. En outre, l'évolution supposte la matière et la force en certaine quantite et dans une cor"taine situation déterminées.

Lnue, du moins dans l'Lnirers à nous connu, la matière et la force se trourrut en quantité limitée, relat est evident, non serulement en ce sens que notre investigation est necessilirement limitée daus l'espace, mais encore parce que, dans ces limites mèmes, la masse de la matiere et l'intensite de la force pouratent itre plus grandes yu'elles ne le sont en réalité.

Lorsque nous avons esquissé l'évolution du systemente solaire, nuus sommes partis d'une situation ou la matière des planites ot du soleil se trouvait tout entière a l'etat de nibuleuse, et nous avons admis implicitement que la formation des autres systèmes stellaires a ete analoggue. 
Yais rien ne nous dit que cet etat nibulrux est primitif. Spencer hasarde plus loin quelques considerations destinces à nous faire concevoir quelle pourrait aroir été son origine. Yous les examinerons à leur place. Mais il semble qu'en faisant l'interprétation de l'évolution, il fallut rechercher tout d'abord la raison d'itre de la situation (qu'on prend comme, point de départ et des cléments essentiels qui y interviennent.

Il ne serait pas légitime de se retrancher derrière la théorie de l'Inconnaissible pour échapper à cette obligation. II siagit ici de phénomenes qui ont leur raison d'ètre comme tous les phénomènes naturels. Connaissatunt la structure de notre globe, nous en rendons compte alu moyen d'une situation antérieure clans laquelle il aurait été complètement fluide et nous remontons it une autre siluation où sa substance était confondue dans la nébuleuse primitive. Yous considerons comme indubitable que la masse et la situation des astres de notre systeme sont la consequence rigoureuse de la masse, de la forme ot des forces de cette nobulruse. Il n'y a aucune raison de ne fas poursuive cette recherche des causes; ou, si l'on rroit avoir des raisons de s'arreter, il fiut les dire. Spencer ne fait ni l'un ni l'autre et e'est mne lacume de sil mrtitphysique.

L'etude de l'évolution que nous poursuivons ici n'est

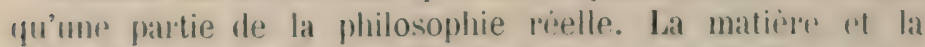
fores en certains quantite dans une situation déferminée no perment exister que par lo fail d'une Cause Première,

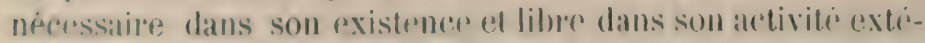

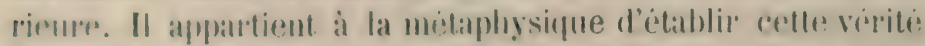

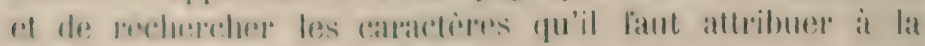
Cause Prembere. Dans la premiere partie do re travail nous arous repondu anx objoctions que spencer sombere

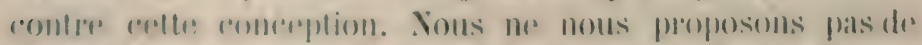
la dromontrer positionment pats plus gure les autres theses de la philosophie spirilualiste.

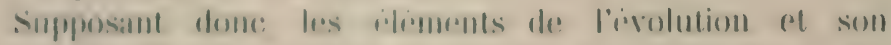

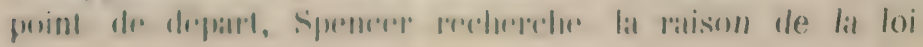




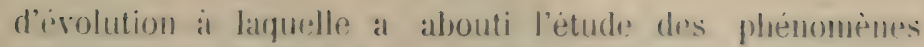

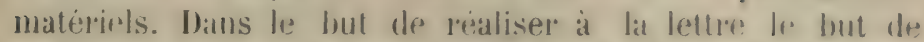
la philosophir qui ast de réduire a l'unite les connassances acquises, il pretend fares sortir la loi d'irolution du primeipe unique des persistance de: la forer.

si nous arous en raison en contrestant que les lois les plus gendrales qui rigissent les phimoménes matriels puissent àtre ranmences a cot unique principe, la tentative

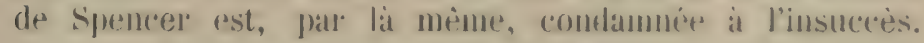
II est neammoins necessaire de lexamione en ditail, d'abord pour avoir une connassance complete de sa synthise, ronsuite et surtout, farce que nous arons ansi l'oceasion d'examinep jusqun"il quel proint il mous est possible de lournir l'explication der l'erolution, alpres en avoir constaté l'existence.

Par manien de prenotion, sprencer distingure dillierentes

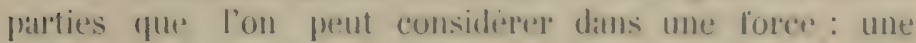
parties eflective; a'est conlle qui actuellement froduit un cllet; celle yui n'y contribue pas s'appelle non effective. Suivant que l'eflet est une modifiralion permanente de structure: on min simple elnamlement rythmique, la loree est dite effective d'une fason permunente on effective temporairement. Enlin la moditication de structure qui ne comporte qu'un riarangentent chimique des atomes est dite insensible, tandis qu'on appelleril sensible colle qui entratue un deplatement relatif alpuriciable comme tol des parties matérielles.$$
\therefore
$$

astabilité de omogène.

Ln chapitre est ensuite consacre par Spencer à établir ce qu'il nomme l'instabilité de l'homogène.

On rend raison de l'évolution en aftirmant d'abord que l'évolution est inévitable el on frécise en disant qu'elle entıaine nécessairement une augrmentation d'héterogénéité. 
Telle est la portee de ce principe, ou du moins, cest ce que tendent à prouver les considerations qui l'appuient.

L'inonce de Spencer: "L'homogéneité est une condition d'écuilibre instable " ne nous parait pas trés hrureusement choisi.

Quoiqu'un tout plus ou moins homogène soit destinè à perdre son homogénéité sous l'influence des forces qui agissent dilféremment sur ses différentes parties, l'homogénèité n'est cependant ni la cause, ni la condition des modications qu'il subit. Car s'il était hétérogène, il serait aussi bien atteint par ces actions modificatrices et son ixpuilibre n'en serait donc pas plus stable.

Ce: qui est rrai, c'est que tont changement d'un agrégat n'accentue pas nécessairement son hétérogénéité, et qu'il y a d'autant plus de chances qu'un changement produise cet effet que l'homogéneité y existe à un plus haut degrè. De sorte que plus un agriugat est. homogène, plus il est exposé à perdre son homogénéité.

si cest cela que spencer a voulu dire, il l'eùt exprime plus clairement et plus exactement en disant, non pas que l'homogreneite rend l'é(quilibre instable, ce qui est faux, mais que l'homogéneite est d'aulant plus instable qu'elle est plus grande.

Dans l'appendice a qui ligure à la tin de l'édition de 1900 , spencer declane que son principe signifie seulement la tendance qu'ont tous les agregats a devenir moins homogines. C'ist en rl’et ce que signilie à première vue: instabilité de lhomogène. Nous croyons ne pas nous ecarter de l'idere de spencer en ajoutant, comme nous venons de le faire, yuc plus langregat est homogène plus il y a dre chances que celfe homogeneite diminur.

bans la mature telle: qu'elle est constiture, l'homogineite parfaite n'est pas possible pour un agregat limite - le sesul dont mous admettions la possibilite. Cne sphère

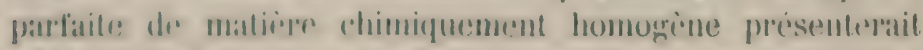
mecrsitirement, de la surface an centre, un alecroissement de densité do à l'attraction.

Si l'lumogeneite fatriate etait possible, foule: modilication 
qui ne s'étendait flas de la mème manimer à toute la mass' détruirait cette homogriniste, tandis yu’un agregrat très heterogine pent subir des changements, sans que son heterogenéiti s'en trouve angmentep. De sorte qu'un ensemble serat d'autant plus expose à perdre son homogeneite que colle-ci se rapproche davantage de l'homogrineité parfaite.

En outre, dans tout agringat hetiroggrine, il $y$ a une

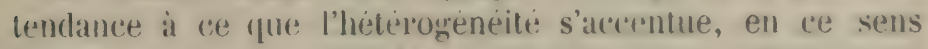
que les parties considéres isolément itant rolativement homogines, elles tendent it perdre leur homogeneiti. Elles y sont cependant d'autant moins exposées qu'elles sont fulus petites, parce qu'slles oflrent ainsi moins de difliorencess de situation far mapnort aux forers qui agissent sur elles.

Entin, en l'atsence d'homogrineite parfate, il $y$ a lonjours une certaine différence dans l'artion que chanue fartie subit de la part des antres, de sorte que ce ne sont pas seulement tes forces extriranes qui contribuent a produre l'heterogeneiti, mais anssi les forres interieures de l'agrégat.

Le principe de l'instabilite de l'homogrine, interpretis comme nous venons de le farre, ne soulève pas d'objections notables et le lecteur a compris, sans alleun doutc, comment il contribure à l'interpmetation de: lat loi d'évolution.

Il y a cependant encore une remarque a liare. On peut imagriner facilement qu'un agrégat matériel soit soustrait a l'action de toute force exterieure, soit que ces forces n'existent pas, soit qu'elles se fassent équilibre ; et en outre que toutes les forces intérieures se neutralisent. Dans cotte situation qui est compratible avec un haut degré d'homogineite, ancune modification ni par conséquent ancune évolution ne se produira.

Il peut aussi se faire qu'un agregat se trouve à un tel degre d'integration que les forces interieures et exterieures n'exercent sur lui aucune influence appreciable. Telle est probablement la situation actuelle de la Lane. 
Il faut conclure de là qu'un itat de la matière qui soit farorable a une évolution longue ef complexe, tel que relui de la nébuleuse primitive, est contingent et que l'explication de l'Lnivers ne sera jamais achevée, tant qu'il r'rll seral pas rendu compte.

Le point de dipart logique de l'évolution de l'Lnivers dans la théorie mécaniste ne peut ètre qu'un état d'homogénéité absolue. Cet état est aussi bien contingent qu'un autre quelconque. Mais en outre, comme le dit avec raison J. Ward, "une telle homogénéité est essentiellement stable; ainsi le premier pas dans le schema d'évolution (de l'Lnivers) congu par spencer devient impossible parce que dans son zile pour être radical, l'auteur a éliminé toute source de différence. Ou s’il ne l'a point fait, il n'a pas réalisé son entreprise; il ne: commence pas au commencement, mais bien avec des atomes possirlant d'une façon indélinie un grand nombre de potentiatlites et distribues suivant une contiguration specifique.... De fait spencer commence avec l'hypothese nihulaire, tout ce qui precerle cette situation étant couvert alloitement par la formule qui n’a rien de scientifique ni de philosophicpur: homogénéité indétnie, incohérente. ” (1)

Quoi qu’il en soit de cette dernière appréciation, la remarque est juste et les reflexions que nous venons de faire combirment ot complètent celles que nous arons exprimees au délout de ce chapitre. L'homogène, ainsi que le fait ressortir Renouvier, est, dans le système de spencer, un point de depart arbitraire et imprécis et qui s'accorde mal avec ses conceptions mécaniques et avec sa croyance à l'éler nite de l'Univers. (2).

Forces qui différencient I'homogene.
Suivanl son habitude, spencer montre l'application de la loi

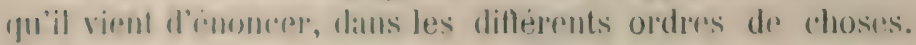

(1) Naluralism and Agnosticism. vol. 1 p. 22:\%.

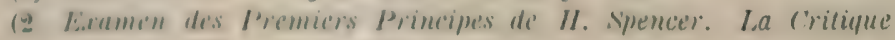
I'hilosophique 18K8 1. $11 \mathrm{pp} .821 \mathrm{~s}(\mathrm{j}$. 
Il n'y a cette fois pas beaucoup d'utilite ni d'intérèt a lo suivre dans ses développements. Le progrès de l'homogìne vers l'hètêrogène a déji été étudié inductivement à propos de l'évolution composée qui consiste, comme on le sait, dans une diferenciation progressive. Or, surncer ne contirme pas ici sa loi autrement qu'en montrant une homogénéit: relative fasant place à une hétérogéneité relative. C'est donc, au fond, le développement do la mème pensée, sauf yu’il insiste davantage sur les forces exterienres ou intrireures sous l'action despuelles l'homogrinéite disparait. Cela nous doune l'occasion de faire oloserver que le role des unes et des autres n'est pas toujours lacile à dinliniter.

De ce? que certaines firconstances exterienres provorguent dans un agrégat des modifications corréntives, il ne faut pas conclure d'emblee que ces moditications sont exclusiroment ou même principalement le résultat dres forces exterieures.

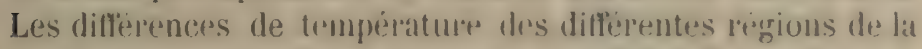
Terre sont presque exclusirement imputahles il l'artion qu'ererce sur olles lo Soleil, d'apres leur exposition an rayonnement de cet astre; mais il n'en est plus de mime des dillérences quon obscerve daus la faune ou la flore des refurions chaudes, froides ou tempérées.

Cotle remarque s'applique mène aux dillerences des tissus qui correspondent manilestement, dins l'elre vivant, à des dilferences de situation. La moditication, par exemple, dre la couche rexterienure de cellules qui sobserve des les premiers stades du drveloppement d'un emberyon ret qui depend done certainement de l'action des forces ambiantes, ne doit pas pour cela ètre considire comme leur eftet exclusif.

Dans bien des rirconstances, fes lorees auxquelles, a

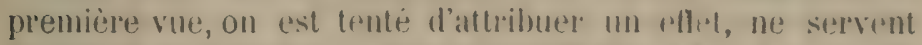
en réalité (fu'it mettre en mourement d'autres forces anxyurelles le resultat doit atre principalement attribue. Nous avons déjit attiré l'attention sur ce point quand, distinguant ditlèrentes catreguries d'évolutions d'après les forces qui les déterminent. nous signalions les cas ou les forces interieures et exterieures interviemmont simultaniment.

On peut exprimer la mène chose en disant que la diflërence 


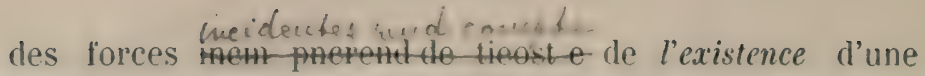
difference entre les tissus, mais n'explique souvent que dans une faible mesure la nature de cette difference.

En outre, une difference entre les parties du corps sur lesquelles agrissent des forces differentes ne peut pas toujours ètre considérée comme due ì l'artion de ces forces. Pour que cette interpretation soit acceptable, il faut qu'on puisse, d'apres les connaissances que nous avons, considerer les caractires dans lesquels la difference se manifeste comme l'effet des forces où l'on constate une variation. Ainsi il semble raisonnable d'attribuer à l'action plus intense de la lumière les couleurs plus vives qui distinguent les parties superieures du corps dres animaux, comparées aux parties infèrieures. Nous savons, en elleet, que l'éclairement favorise la coloration des ormanismes. Cette explication est donc plausible quoiqu'elle ne s'impose pas.

Mais il serait absurde d'attribure it la meme cause, ou en general a des inffuences dillerentes du milien, d'autres diflëeren-

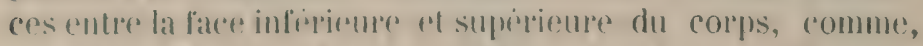
far exemple, dhe lis mammifires, la présence des mammelles.

Surtout, il est illegitime d'attribuer a une difference de forces une dilferenciation de l'organisme, guand celle-ci est une condition prealable de colle-lä. Spencer dit dans les Principes

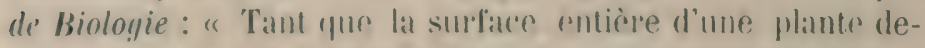
mene dans lesmemes rapportsavere le miliev, comme dans un protororrus ou un voleox, elfe dememe miforme ; mais quand il y a ume surface fixe ol une surfiare lihre, ces surfares etant sommisers a des actions dissemblables, devirmment dissemblables. Ce risullat est visible mimr dans ume algure collubaire, des quielle se fixe; on le voul dans la distinclion des deax surlateres

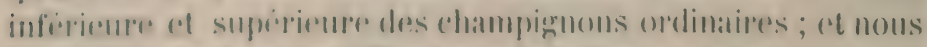

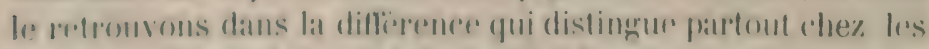

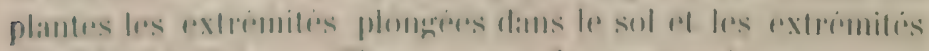

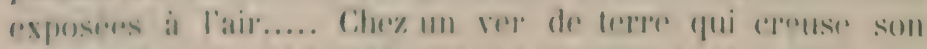

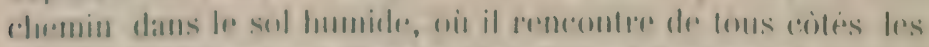
menes conditions, ou cher un lionia haigne egalement partout

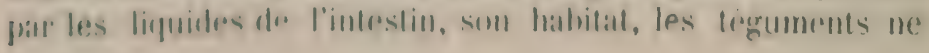


diffërent pas les uns des autres d'une fagron appréciable; mais chez les animaux qui n'ont pas autour d'rux les mèmes intluences, par exemple ceux qui rampent ot ceenx dont le corps est en partie enfermé, on troure des dissemblances de trimuments qui correspondent it des dissemblances de conditions. Le disque du limagon a une surface interiente gui nirst pas uniforme avec lat surface de son corpis tournere en haut, ert corllesci n'est pas non plus uniforme partunt dans sat partie decouverte et dans cerlle qui est protegee par lat compille. Ches les animanx articules, il y a ordinairement une distinetion entre les faces ventrale et dorsale;.... $)$ (1)

Lat diflitence des actions qui sexcerent sur la fine venttrale et dorsale d'un animal supposent pue cers facess soment

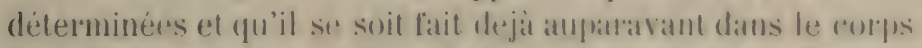
une diflerenciation qui lixe le dessus at le dessons. On prent concevoir que: cettr differenciation itant realisere dims cte quelle a d'essintiel, d'antres distemblameres secondaires resultent de l'exposition ingegale dres parties anx forres

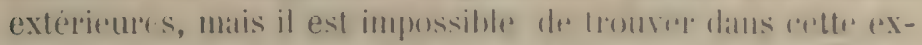

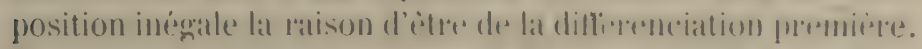

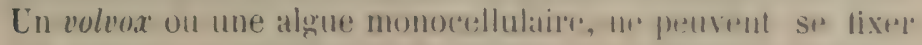

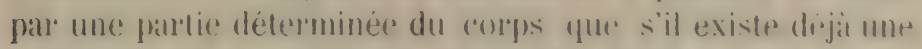
dilfërenciation superticielle; ("itr s'il n'en riste ancune, cess or-

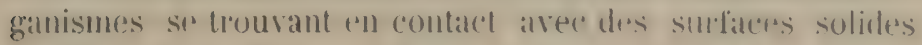
tantôt par un coite tantot par un alutre, il ne s'y produira aucune différence de parties.

Pour que la reptation rende compte d'une diflierence tignumentaire, il laut que la face sur latuelle elle se fiat soit prealablement determinee, sinon l'animal rampant indiflëremment sur toutes ses falces, rien ue détruilat l'homugeneité des téguments.

On pourrait dire que la situation du corps par rapport au milieu est determine par l'organisation intèrieure en l'absence de differenciation externe, et que celle-ci peut done s'expliquer par la difference des actions que l'organisme subit sur ses differentes faces. Mais outre que bien de's organi-

(1) Princ. de Biologie. vol II p. $447-448 \S 311$. 
sations sont indifferentes a la situation du corps, il y aurait encore lieu d'examiner jusqu'a quel point l'organisation interne ne suppose pas elle-mème que la situation normale du corjss par rapport au milieu est déjà déterminée, ce qui ne pourrait itre, dans ce cas, que par la diffèrenciation des téguments.

'instabilité el'homogène ta persistance de la force.

Le principe de l'instabilité de l'homogène étunt établi, Spencer le rattache au principe de la persistance de la force, en faisant remarquer encore une fois que cette instabilité est due à l'action des forces tant intérieures qu'extérieures.

Celles-ci, en effet, auront en général une action résultante différente pour les diffërentes parties de l'homogìne. En consérfuence, l'action de ces forces tendra a transformer diversement les parties, puis les subdivisions des parties transformées, et produira ainsi une heterogeneité plus ou moins grande dans l'agrégat.

Ce raisonnement ne contient aucune idée nouvelle, et s'il parait en lui mème concluant, on ne voit pas d'autre part en quoi la persistance de la force est nécessaire pour obtenir le risultat signalé. Evidemment, aucune modification ne serait obtenue si la force se trouvait annullée, mais les variations qu'elle subirait et qui seraient contraires atu principe de la persistance, n'empècheraient pas que son action ne fìt destructive de l'homogénéité. Si donc l'instabilité de l'homogène est une conséquence de la prersistance do la force, elle n'a cependant pas avec ce principe une connexion très ètroite.

a multiplication des effots.
Dans ce qui preceide, nous avons considiere une première canse gencrale de dillierenciation des parties auparavant smmblatiles d'un agremat : cest celle qui ronsiste dans la situation diflidrente de cees parties par mapport it une foree incomplexe ot dans laction diflerronte quadles subissent 
ronséquemment. Il y a une autre eause dont l'efticacité est mène plus considèrahle : c'est la multiformitè qu'acquiert la force elle-meme dans les phénomenes quelle détermine. Cette multiformite résulte de la difference des milieux dans lesquels la force produit ses rfiets, - ch prenant cette formule dans son sens le plus generial.

In rayon de lumière tombant sur une surface metallique polie est réfléchi en partie et en frartie absorbes sous forme de chaleur; la partie réllechie passant all travers d'un prisme en verre est dévice et décomprosée en rayons de lumière colorée; cenx-ci, lregrus sur me playue photographique, decomposent plus on moins les sels d'argent. Cet exemple suffit pour l'intelligences du principe que Spencer apprelle la multiplicution des eflets.

Il decèle évilemment une caluse d'évolution divargente. Un agregat compose de milieux differents atant exposi it l'action d'une force, subira des moditications diverses dans ses différentes parties. Spencer fait remarquar que celle différenciation angmente, de sa nature, suivant une progression gémetrique. Lat force qui a ete transmise sous des formes differentes aux parties de l'agringat, constitue sous chacune de ces formes une nouvelle somre d'activite, wt par conséquent de dillerenciation analogute.

irmation On diveloppe inductivement cestle theorice en montrant ctive et ication a cori de que dans l'evolution de la nébulesuse lat gravibation mo-

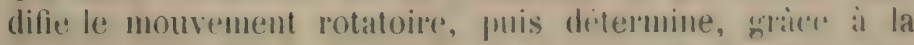
condensalion de la matiener, la chalene, la lumiere, des mouvements de convertion, des phrinompones ehimiques, et cela i diferents deurres ef sous diflirentes formes dans les diverses parties du systène.

Ainsi encorr, te refroidissement du globe produit des eflets varies at une: structure de folus en flus complexe.

Dans le's organismes, nous voyous une impression, surtout $\therefore$ le est forte, allecter simultanément et différemmen 
les dillërents organes : les nerfs, les muscles, la circulation, les fonctions sensitives.

Une modification géographique importante modifiera l'allure des phénomp̀nes météorologiques et aura sur la faune rt la flore des eflets divers, non seulement d'après les esperces, mais encore suivant l'endroit qu'elles habitent et la perturbation plus ou moins considerable qu'ont subie les conditions de leur existence. Le résultat sera non seulement une modification, mais encore une différenciation des espèces préexistentes.

II est manifeste que les évenements exercent une action très dillèrente sur l'àme des individus suivant leur degré d’intelligence, les comnaissances qu'ils possèdent et les autres conditions subjectives qui les caractérisent.

Les reactions infiniment multiples qui se produisent dans la societie, il la suite, par expmple, d'une mesure légitative ou d'un changement politique, sont une démonstration de l'influence comploxe produite sur l'evolution sociale par une cause simple.

Lf: principe (que nous exposons est susceptible d'une justitication a priori. Lorsqu'une forea agit sur des milieux diflerents, l'ollet qu'elle ydetermine - et qui n'est autre qu'une forme quelcongue d'énergie - s'y combine nécessairement are les forces prexistantes earacteristiques : d'où Irs risultats diférents. Cette conclusion est diduite, non pas précisement de la persistance de la forer, mais plutiot de l'existronere dorers aracteristiques des milieru. Cen'est que

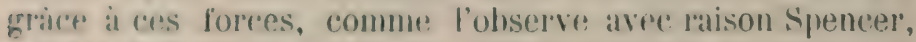
que les differences des milirux alfectent notee conscirnce et peuvent nous ètre connues.

Il n'est pas, ce noms semble, inutile de distinguep ici dous manieres dont peat se produire la multiplication des ellets.

Parfois ellre sera simplement une: transformalion épuivalent-

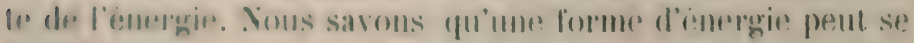
transformer en une antre forme qualconque. Dispersere dans

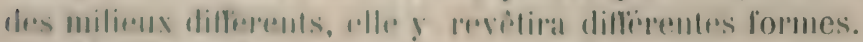

Yais une foree proul encore produire des ellits varies en 
mettant en liberté de l'éneigie potentielle, ou plus generalement, en excitant l'activite d'autres forces.

Tant que la temprematnre reste basse, le contenu d'un oruf de poule ne se distingue grutre de celui d'un couf de canard ou d'un autre oiseau quelconque. Mais des qu'on les soumet a la temprerature d'incubation, les forces latentes dans le germe entrent en jeu et produisent les oiseaux diflèrents que nous connaissons.

Il va sans dire que si dans le premier cas les eflets multiformes peuvent ètre attribués sans restriction à leur cause determinante, puisfu'ils n'en sont qu'une transformation equivalente, il n'en est phus de mime dans le second.

rergence. Les deux principues tres simples que nous venons de faire connaitre, si l'on retheduit à la multiplicite desforces dont les eflets se superposent et se combinem, font prévoir une énorme varieté d'eflets. On concoit dés lors l'extrime complexite de l'ivolution dans tous les ordres de choses et comment, en particulier, la faune et lat flore en sont arrivers à presenter une variets de formes qui déconcerte l'intelligence.

Il est cependant inevitable qu'il se, produise parfois des phènomènes de convergenre, e'est-i-dire des modifications reproduisant plus ou moins une situation précédente, ou encore, des procès d'évolution indépendants amenant des résultats analogues.

Dans les phénomènes phỵsiques la chose est évidente: les formes d'énergie sont en nombre restreint, de sorte? qu'elles se reproduisent mutuellement. Les naturalistes signalent également l'intléchissentent de certaines formes vers le type ancestral par une similitude d'adaptation: tels les mammifères marins qui se rapprochent des poissons par la forme de leur corps, par leur's membres et leur queue transformée en nageoires; ou bien encore des séries de formes plus ou moins parrallèles, comme celles des chevaux de l'Ancien et du Youveau Moncle. 
Cette restriction à la loi de différenciation est explicitement adınise par Spencer dans sa réponse à T. E. Cliffe Leslie. " $\mathrm{Si}$, dit-il, pour des organismes d'ailleurs diffërents se produisent des conditions semblables auxquelles certains de leurs organes se trourent soumis, ces parties auront une tendance à la similitude. ") (1) Il en donne plusieurs exemples. Ainsi dans l'embranchement des mollusques nous voỵons un développement du système nerveux qui aboutit chez les grands céphalopodes à une organisation comportant de grands centres nerveux, analogue à celle qu'on rencontre chez les vertébrés. L'évolution parallèle du système vasculaire chez les mollusques et chez les articulés aboutit de part et d'autre à la constitution d'un organe central de propulsion. La configuration semblable des yeux chez les mollusques supérieurs et chez les vertébrés est encore le résultat d'un phénomène de convergence.

Ces phénomènes ne contredisent pas en réalité la loi de dillërenciation: ce ne sont pas des cas d'homogénéité qui persistent ou d'hétérogénéite qui tendent à disparaitre; ce sont des coïncidences ou mieux des parallélismes plus ou moins parfaits dans des procès d'évolution d'ailleur's illdépendants.

Lorsqu'une force agit sur un agrégat elle modifie d'une façon plus ou moins analogue les parties de même nature et produit en général des effets différents sur les parties de nature différente. En particulier, lorsqu'il s'agit d'une force qui produit du mouvement visible, celui-ci sera communique sous differentes formes à differents mobiles. Lat manitre dont les objets se comportent sous l'action du vent suivant leur masse, leur densite ou leur contigumation ast un exmple simple de cette proposition. Elle permet de se rendre compte d'un caractère de l'évolution gue nous avous recounu : la délinition progressive des parties dillérenciées.

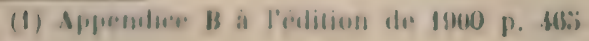


En effet, les forces qui agissent sur l'agrexat tendent à accentuer la difference pntre les parties, et s'il s'arit de forces qui déterminent des déplacements visibles, elles tendent à séparer les élements de nature diliernte.

Parmi les expmples apportés en confirmation de ce principe de la ségréyation, nous notons la séparation des sels dissous au moyen de la rristalisations. Il faut donc, dans la pensée de spencer, étendre la signitication du principe non seulement aux forces interienres de l'agrégat, mais encore aux forces caractristiques de ses elements.

Nous ne pourous pas approuver que spencer rattache à la loi de ségrégation les mouvements diflérents conçus par des parties de meme nature sons l'action de diflérentes forces.

Dans ces cas, en elfot, la situation des parties pent seule expliquer l'intluence dillerente gu'elles subissent. Ce phénomene rentre dons dans la caltegorie de crux qui sont la raison de l'instabilite de l'homogrène. Tous an

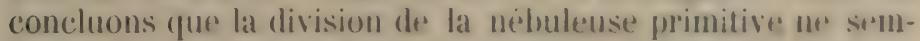
ble pas convenablement choisie comme exrmple de ségrégrution. Cello-ci suppose: une diflerence de nature: dalls les flarties de l'agrégrat.

Au contrare les formations greologiques nous fournissent des applications nombreuses do ce principe. Jans toutes les formations sédimentaires, pent-on dire, lat force micanique de l'eau a séparé les éléments suivant leur masse et lent densite. Les accidents de terrain causés par l'érosion qui n'entame que lentement les roches dures, tandis qu'elle emporte facilement les roches molles, l'action dissolvante sélective qu'exerce l'rau dans les terrains, le remstrment du lit des cours d'eau ut de leurs ver'sants sont encore des ségrégations qui s'opèrent sur toute la surlace des continents.

Spencer signale dans le regne organique la reduction des extrémites des membres qui caracterise l'évolution des ongules perissodactyles et artiodactyles. (On pourait à la rigueur voir un exemple de semrrigation dans le développement d'un ou deux doigts qu'accompragur la disparition progressive des autres, quoique cette manière 
d'envisager le phénomène soulève des objections. Mais on ne roit pas comment la soudure des métacarpiens dans l'os canon, ou la soudure des vertébres dans certaines parties de la rolonne vertébrale peuvent être apportees comme exemples d'une telle loi.

Linterpretation de la stabilité de l'espèce par la sélection naturelle, gràce i laquelle disparaissent les individus qui s'écartent d'une manière défavorable du ț̣pe normal, contient sans doute une part de vérité, mais qui semble peu importante. La stabilité de l'espèce est très relative et la suppression des individus inaptes par la selection naturelle n'en est certainement pas le facteur principal. spencer eùt pu, avec plus de raison, signaler la sèlection naturelle comme un procès de ségrégation, en tant que facteur d'érolution organique. Des différences individuelles ettant produites dans les ìtres d'une espèce (instabilité de l'homogène) la sélection naturelle favorise la multiplication des individus les plus aptes à l'existence et extermine les autres.

Dans l'évolution mentale se produit d'ahord une ségrégation "inverse » des forces réunies dans les objets, graice à l'orgatnisation du système nerveux sur lequel ils agissent. C'est l'operation du classiment des connaissances expérimentiles.

Elle supuose que les impressions recues determinent des modifirations diffirentes dans les differentes parties du système nerveux.

fros conditions exterieures aussi bien que les sympathies naturelles maintiennent separies les races humaines, de minae que les aptitudes naturelles et surtout l'éducation maintipnnent separens les classes sociales.

L.e mincipe de lia ségregation en tant que distinct des drax precedonts semble avoir une portee beaucoup moins enendue. Spmenere te rattache au principe de la persistance de la force, en tant que cedui-ci proclame qu'une difterence antre les effets ne preut provenir que diune diflérence dans les forres on dans les whjets sur lesquels elles agissent. A notre point rue corte remarque n'a plas d'importance. 
apitula-

tion.

Nous arons dans ce premiel paragraple, itudie les theories de Spencer sans insister sur les drveloppentents ronsidérables qu’il donne a leur véritication indurtive dans les diflèrents ordres do choses. Ces applications sont presque toujours interessantes. On peut leur repnocher d'être parfois chargées de détails inutiles et à quelques unes d'itre un peu forcées; mais dans l'pnsemble olles constituent une contirmation solide et tris itrmlue des considrations theriques. Le lecteur retirena la plus grande utilité de la lecture des pages que spencer y consacre. Nous n'en avons rapporté que re qui nous semblait nécessare pour l'intelligence des principes et surtout nous avons tiche de les completer par quelques remargutes ou applications nouvelles. Yous pouvons mainternant jeter un conj) d'uril d'ensemble sur cet essai d'interpletation de l'evolution. Yous avons recomnu à celle-ci ces caratclires : c'est d'abord d'itre un proces d'integration tant des parties que de l'rensemble ; cost ensuite d'itre dans tous les cas d'évolution proprement dite un proces de difherenciation croissante; e'est encorre d'citre une délinition el rntin une coordination progressive des parties.

Nous arons vu comment spencer au moyen des principes de l'instabilite de l'homogène ot de lat multiplication des eflets rend rompte du second de ces caractipes et nous avons reconnu que ces principes ont une portée très generale. D'autre part on ne roit pas quel autre principe gentral on pourrait invoquer en cette matiere.

Nous avons vu aussi que le principe de la ségréyntion explique, an moins dans certains cas, la délimitation de plus en plus nette des parties.

A cel égard donc, l'interprétation de l'évolution comme phenomère genéral paraitra satisfiasante.

Mais il est étonnant que Spencer ne se soit pas occupé dans cette partie de son ouvrage du procès d'intégration qu'il déclare ètre principal, quoique, comme il le dit lui-mème, 
il n'en eût pas d'abord reconnu la véritable nature. (1) La redistribution primaire, comme il l'appelle, affecte aussi bien les parties que le tout. C'est elle qui est la cause générale de la détinition progressive des parties.

Quoique tout progrès d'intégration d'une partie ne soit pas nécessairement un progrès de délimitation, cependant tout progrès dans la nettete des limites est un progrès d'intégration, de sorte que l'intégration complète comprend une délimitation précise.

C'est aussi au procès d'intégration qu'il faut rattacher la coordination croissante des parties, laquelle contribue à augmenter l'unité de l'agrégat.

Spencel lui-mème, dans l'étude inductive de la ségrégation, cite plusien's fois, comme exemple de délimitation progressive, des phénomènes où il semble impossible de voir autre chose qu'un progre's d'integration. Il n’apparait mullement, par exemple, que la soudure des vertebres ou des métacarpiens délimite phus nettement ces os, ni que dans la societé les associations professionnelles délimitent plus nettement les classes de citoyens ou elles sont établies.

II suffira donc, pour interpreter la delimitation progressive des parties, de concevoir le phénomène de l'integration dans toute sa genniralite, ce qui permet d'y rattacher la coordination croissante des parties dans le tout, et l'on pourra considerer la ségrégation comme se rattachant à l'integration des parties.

L'integration etant une marche vors l'unite est foujours domince par un principe d'uniti. Co principe est dillerent dans les difterentes existences materielles. Il est important de. lo recherehre el, si possible, d'en expliquer l'orignine.

bans l'owolution du systiome solaire, c'est lat gravita-

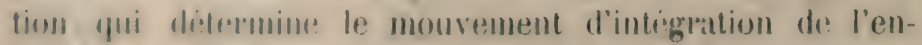

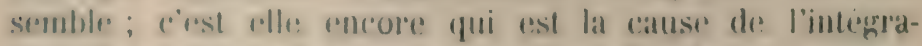

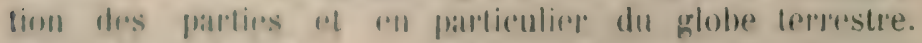
loppuion yui exigo un principe vital pour lexplication

1) P. P. p. $3601120-$ F. P. p. 271 . 
de la vis organique a précisément pour fondement la nécessité d'un principe d'unité pour l'évolution organiquer individuelle. L'ivolution des etres vivants en tant qu'elle consiste dins une multiplication d'ètres semblables a pour principe d'unite l'hérédité. L'intinct social des hommmes est la cause fondumentale de l'intiggration progressive des societes, quoique d'antres callses secundaires, commor la crainte des mnenis du dehors ou du dedans, ou l'artion

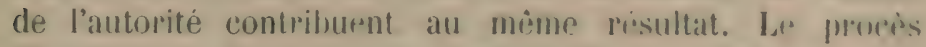
d'intégration d'une amsre industristle ou artistiyure doit son uniti au hut que poursuit son auteur.

Tous ces principes d'unite representent de réritables forres en tant qu'ils determinent des redistrilutions de matiere. Nous avons distingue plas haut des categorips grimerales de proces esolutifs dapres les forces qui dreferminent l'intrugration de l'argrigat, c'est-it-dire la realistribution primare. Cofte consideration est des plus importantes et il n'y a point de dente que lat nature des forers qui sunt la cause du procès fondamental me duive étre considereers comme caracterisant chaque espece d'rivolution. Il y a la un point de vare qui a ate negrligre par sprencer. II ne s'ocempe an elfiet de rendre compte ques des la differenciation ef de la définition yui sont des procis secondaires. Yous avons tiche de combler cette lacume dans la mesure ou l'exige la therorie génerale. L'étule de l'évolution oruanigue à latquelle nous allons consacrel les pages suivantes nous donnera l'occasion d'étudier l'hrédité et de reconnaitre l'existence d'autres facteurs internes d'ésolution dont il n'est pas toujours possible de priciser le role parce qu'ils sont imparfaitement connus. Mais on concoit qu'agissant dans une direction diterminee, ils constituent des prineipes d'unitr pour les serries des formes oruniques qui descendent les unes des antres, et quiples contribuent ainsi non seulement a l'integration des individus, mais encore à l'unite du procès évolutif des steries. 


\section{\$I. LES CALSES DE L'ÉVOLLTIOX ORGANIQLE}

Les causes d'évolution particulières. - L'évolution organique. Ontogénie et phylogénie. - Les causes d'évolution ontogénique. - La sélection naturelle. - L'adaptation et l'héréditè des caractères aquis. - Les tendances internes d'évolution. - Théorie de De Vries.

Les causes évolution rticulières.

Les considerations que nous avons faites dans le paragraphe pricédent, si elles suffisent dans une certaine mesure pour expliquer les caractrires sénèrax, communs a toute evolution quelconque, n'ont pas et ue peurent pas aroir pour but de rendre compte des procès particuliers d'évolution qui se poursuivent dans les dillerents

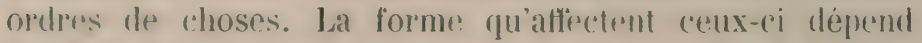
en effer des éléments qui y prennent part, de lour siluation initiale, de la nature et de l'intronsite des forces yui y agrisstent at de leur distribution daus le tempss rot et dans l'espace.

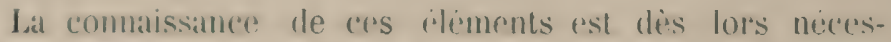
saire lorsqu'il s'agit d'étudirer, non plus l'evolution dans sa conerption la plus générale, mais un proce's d'ivolu-

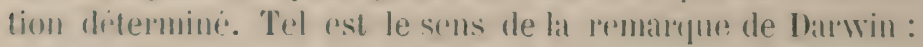
"Lorsqu'on se rapporte, dit-il, a lat premiere androre de? lit vie, all moment où les ettees organises, comme on pent le croire, presentaient la structure la plus simple, on pose la question: Comment las premniers pas onl-ils elr laits dans le proces de la dillerenciation des parties? H. Spencer pepondmit pue les organismes unicellubares arri-

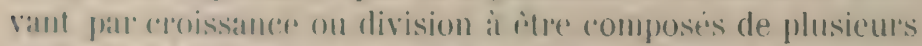
crollules, s'allachamt à une surface quelconque comme support, subiront laation de sa loi, d’apres lapurelle ders unites humbeness dordre quelconque sont ditlemenciees en propor-

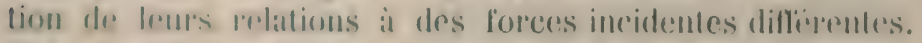
Mais enmum nous n'atons pas de faits frour nous muiclere,

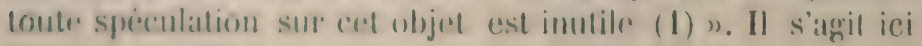


d'un cas particulier pour l'explication clupuel la connaissance de la loi greneialde rist insulfisinte.

les caractires gentratux de levolution romme telle sont peu complexes, tandis que sourent lat forme sous layuelle elle est readisér de fait l'est extrimement. Ainsi, la simplici-

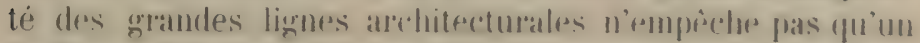
edditier s'ollire en realite nue grande. remplication de details. Lat differenciation de l'homogime selat d'antint plus artive et d'autant plus multiformes que seront plus nombreuses ot plus

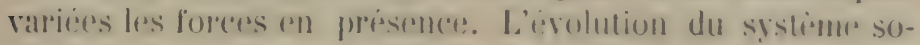

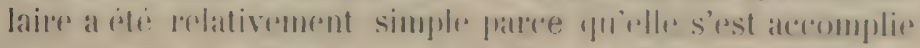
sous l'adton predominante d'une finere uniq̨ue : la gratrition.

L'écolution de lat Trare dins largurlle ont joure un roile,

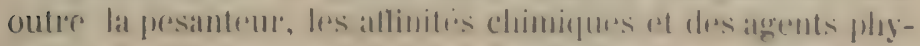

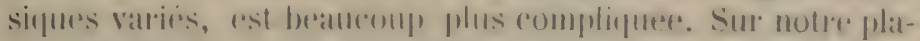

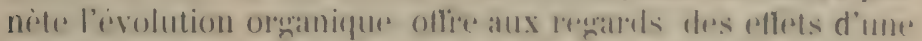

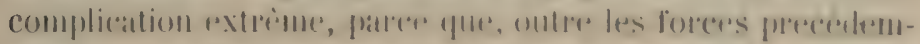

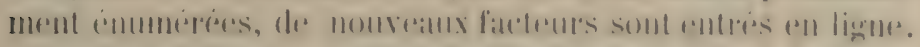

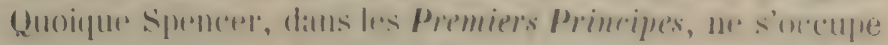
pas en frarticuliere de l'ivolution organiquen, nous allonis

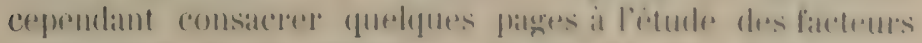
particuliers de ce poctes, at cantse de son importance prefonderante. Lat distinction que nous arons laite molre les causes generales devolution of les causes panticulienes se trouvera ainsi contirmite; et mons anrons en mème temps roceasion de rencontrer certaines opinions relatives

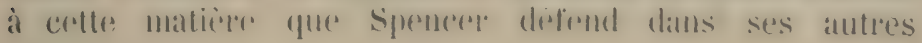
ouvrages.

volution anique

Nous avons dija lat voir brièvement comment la loi d'exolution s'applicgue atux itres vivants, suit qu'on les considère dans lrur ensemble, scrit qu'on rmvisage l'évolution d'une forme en particulier, soit entin qu'il s'agisse du deverlopement d'un individu. L'érolution d'ensemble du règne vivant est la somme des irolutions particulieres. Il n'y a done en réalite que deux procés à envisager: 
l'évolution de la forme et l'évolution de l'individu. Encore ces deux procès ne sont-ils pas completement distincts puisque l'évolution de la forme, comme nous allons le voir, n'est que la suite des changements que subit l'érolution des individus dans les générations qui se succèdent.

atogénie et Précisons d'abord ce qu'on entend par évolution de hylogénie. l'individu (ontogénie) et évolution de la forme (phylogénie). On appelle géneralement individu vivant toute portion de matière organisée, organicquement continue, ayant une vie propre at distincte. Yous donnons le nom de forme vivante i l'ensemble des caractires que possédent tous les individus d'un groupe sỵstématique (classé sous une mème denomination nar les naturalistes) considérés en relation avec les caractères possédes par leurs ancètres.

Lappliration de ces definitions aux eas concrets doune lien at certaines difficultes d'ordre secondaire que nous pouvons négliger ici. Mais une observation nécessaire est la suivante: lne forme vivante est une chose abstraite (uin ensemble de caractires). Elle peut l'ètre plus ou moins suivant yu'elle est réalisée dans un groupe plus ou moins large: ainsi la forme vertébré est plus abstraite, plus generrale que la forme mammifère, celle-ci est plus gènérale que la forme primate etc. Ces groupes plus ou moins larges portent le nom d'embranchement, classe, ordre etc. jusquaux variètés ou races. En outre, si l'on considère une forme en tant qu'elle se moditie, elle pourra appartenir successirement a plusieurs groupes dillérents.

L'ontogenie est l'ensemble des états par lesquels passe l'individu depuis le premier moment de son existence juscun'i l'age adulte, c'est-ì-dire jusqu'au moment oi tous les organes ont le dexeloppement nicessaire à leur fonctimmment normal. Dans toutes les catergories d'itres visants, l'ontogrinie commence par le stade cuf qui ne comprend qu'me soule cellule. Séanmoins des individus mulficellulaires perturent premile naissanes par simple fractionnement d"un individu précedent. Ainsi les plantes se multuplient par houtures. Dans cee cas il peut se 
faire que tous les organes soient présents dans chacune des parties de la division, ou bien que la partie ia laquelle manquent certains organes les reproduise: ainsi les boutures mises en terre produisent des racines. Certains biologistes considèrent ces parties, mème après leur séparation, comme ne constituant qu'un seul individu et cette maniere de les envisager oflire des avantages a certains points de vue. Mais il est preférable de les considérer comme des individus distincts, conformement a notre détinition. II faut remarquer seulement que ces individus obtenus par division n'ont pats accompli pour leur compte, du moins pass entièrement, l'érolution ontogénique et qu'ainsi, rigonreusement frartant, ils ne constituent tous ensemble qu'une gremeration.

La multiplication des individus par division n'est pas possible che\% les animax suprerieurs: l'integration y est trop avancie pour que les parties detachrees soient capable's de vive isolément. Ils ne se propagent donce que far ovule, et la condition normale du developpement de l'orule est qu'il soil frecondis par la fusion arec une cellule mile ou spermatozoïle. Cheo les plantess et chez les animaux infirieurs les deux modes de mulliplication coexistent. Les organismes micellulaires se multiplient par division spontane on antiticielle, le hourgeonnement n'etant qu'une forme particulière de division (1).

Lat phỵlogenie est l'ensemble des etats par lespuels a passe une forme jusqu'a son etat artuel. Cers stades suciessil's ont ete realises par des individus vivants dont parfois on a retrouve les restes et qui ont ete les anceitres des individus qui représentent le stade actuct. Cette detinition suppose la theorie transformiste; mais dans ces termes génélaux, il n'est pas un naturaliste qui me l'adme'tte aujourd'hui.

Il est certain que la plyglogenies a lisu d'une fagon divergente, c'est a dire que si on suit en la remontant la ligne des ancetress dans des groupe's anjourd'hui très dilleirents, on rencontre sur ces lignes des groupes de moins en moins

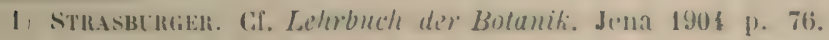


différents et jarfois on aboutit à un seul groupe. Par exemple, il est admis par les naturalistes que les lignes des ancètres des pigeons à queue de palon, des pigeons culbutants, des pigeons capucins, etc., aboutissent toutes au groupe des ramiers ou pigeons sauvages. De même, en suivant les lignes des ancètres des différents groupes de mammifìres modernes on ahoutit à des mammilères de l'époque éocène qui sont beaucoup plus ressemblants entre eux que ne le sont les mammifères virant aujourl'hui. Il est bien entendu que quand on trace la descendance a travers des formes fossiles, les lignes sont loin d'ètre complètes. Elles sont plutot marquees par des jalons que par des traits continus et somvent elles sont on honne partie hypothétiques. On admet aussi que les lignes d'ancetres en convergeant dans le passe aloulissent toujoms timalement it un seul groupe d'individus trés ressemblants all point qu’il n’y a pas lieu d’y distinguer plusienrs groupes systematiques; et que, d'une firon grenemale, cos lignes so rencontrent a une eppoque d'autant plus rapurochée quilles partent de groupes actuels moins differents.

Celte: loi de disergance rest sommiso a certaines excep)tions qui comslituent les phrmomines de comergence ot dont nous arons dieja parle. Lese ligmes dascendance de deux groupres assez semblables 0.1 wllant du moins des

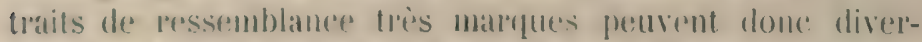
grep or aboutir a dese grompes flus dissemblables. Par exemple, les haleines ont probablement comme ancibres des mamilines terrestres plus dillërents des poissons qui

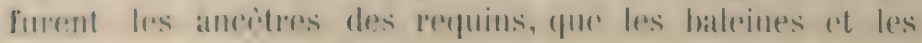
requins ne diflirent entre eux.

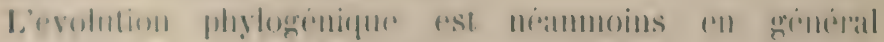

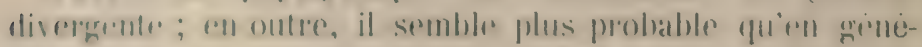

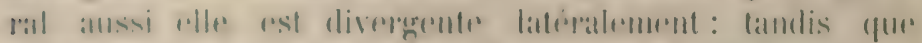

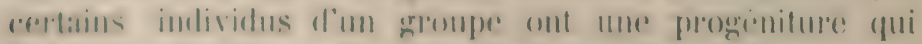

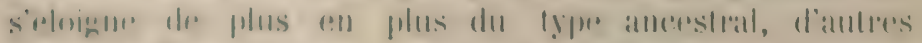

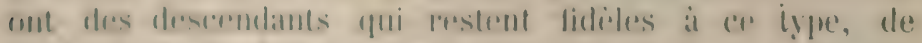

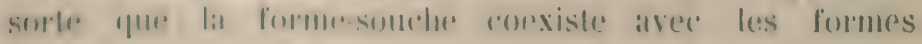


dérivees. Ainsi le ramier coexiste alre lis dillirentes races de pigeons domestiques.

Une forme vivante peut displaraitre, soit farce qu'elle a été détruite, soit parer qu'rlle s'est modifice ; e'est à dires: il se pent que tous les individus d'un grompe menrent sans laisser de descendants, on hiem yutaucun de leurs descendants ne reste tidple au type ancestral. Parmi les animaux vivant aujourd'hui, il n'y a pas de descrendants des grands reptiles marins de l'epoque secondaire. Cars lormes ont été detruites. An contraire, si les probuscidirns lortiaires ont disparu, les élephants moderness sont sans doute lenrs descendants.

Si l'on tient comple de: l'immensu variele des lommes vivantes qui sont toutes issues d'un momlure publiallement tres restreint de formes primitives, on so comvaime

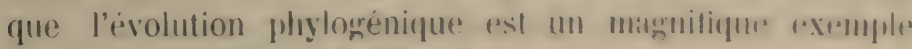
de la loi de differenciation.

uses de Le facteur priacipal de l'rvolution individuclle est in-

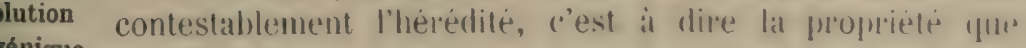
Jénique.

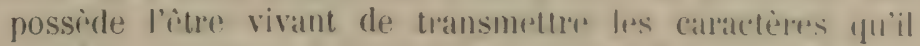
possède lui-mème à l'individu alupuel il domme naissance. Lorsque le caractipe transmis so retrome chez laseondant direct on l'appelle hereditiore dans le serls strict; s'il ne se trouve que clue les ascendants éloignis, on lui donne le nom d'atarique. Dans ce dernier cats, on dit que les ascendants intermédiares, oil te caractere napparait pas, le possinlent virtuellement ou it liatal latent.

Les lois de l'hérédite sont encore en grande partie ignorées. On connait rependant d'une manière precise les mode de transmission, apres croisement, de certains caldactères distinctifs des races ou variettis phutot que des especes, et auxpuess on a donné le nom de canticteres mendéliens, parce que les lois qui gouvernent leur transmission ont 
élé découvertes et publiées en 18 fiti par G. Mendel, (1) abbé hénédictin de Brünn. Ces lois sont asser simples lorsqu'on considere le cas de deux caractères opposés qui se combinent par croisement. Il nous sultira pour les faire comprendre de citer un exemple concret.

1. Mendel croise un Pisum à tige longue avec un Pisum a tige courte. Les descendants de ce croisement possèdent tous une tige longue. Appelons ( $\mathrm{L}, \mathrm{C}$ ) I les individus de cette première génération.

2. Les $(\mathbf{I}, \mathbf{G})^{1}$ sont soumis à la fécondation directe (de charque plante par elle-même) et les graines sont semées. Yendel constate cu'il apparait approximativement, de Pisum à tige courte et ${ }_{4}^{3}$ à tige longue.

De ces deux faits Mendel conclut que si tous les pois de lat première génération possident une tige longue, c'est que le caractère tige longue l'emporte sur le caractère tige courte. Il appelle le premier du nom de caractire dominant, le second du nom de caractire récessif. (Loi de prévalence).

3. Les individus de la seconde génération sont encore soumis à la fécondation directe. Ceux à tige courte (ap)pelons-les ( 2 ) domment exclusivement des descendants a tige courte. Parmi ceux i tige longue, un tier's (appellonsles $\mathrm{L}^{2}$ ) donne uniquement des descendants à tige longue. Las deux autres tirrs, $\left(\mathrm{I}, C_{6}\right)^{2}$, domnent des descendants à lige courte et des descendants a tige longue dans la proportion de 1 it 3 ( $\frac{1}{4}$ à tige courte, $\frac{3}{4}$ a tige longue).

4. A la quatrieme generation on constate que tous les petitsfils dres fiz sont a lige courte, tous les petits-lils de's $\mathrm{L}_{2}$ sont à tige longue.

Quant atux petits-tils des (I, C) 2 , on constates de monvesulu que les pois a tige combe dr la troisiome grencration, C\%, nont domni que des descmulants it

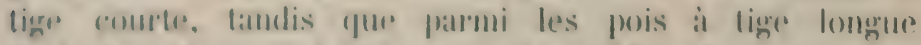

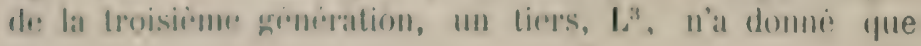

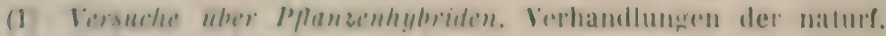
Vereiness. Briann. 1 sim 
des liges longues, less deux autres tiers, (I, C. $)^{3}$, ont donne ; de liges courtes ot: dre tiges longues.

Et ainsi de suite.

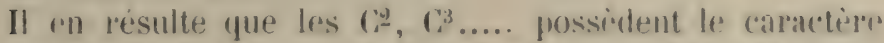
recessif C à l'état de pureté; les $12^{2}, 13^{3}$... prossèdent lo caractere dominant $\mathrm{I}$ a l'ital de purete ; tandis que les (I. C) ', $(\text { I. C. })^{2},(\text { L C C })^{3}$.... possiedent le camactère I a l'atal manifesté et le caractère $\mathrm{C}$ à l'état latent.

Par conséquent, il se fait à chaque grenération qui suit la premirie, unr dissoriation dess carationes dans la moportion \&, L, $\frac{1}{4}$ C, $\frac{2}{4}$ LC. (Loi de dissociation.)

Mendel explique cette seconde loi en admottant yure les caractires sont dissocies dans les cellules reproduc-

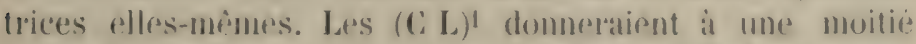
de leur pollen le caractere $C$, a l'autre moitir le calare-

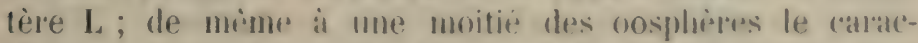
time C, a l'autre moitie le caracliere L. Cela elant, il! ama qualte combinaisons possibles lors de la foroudation: CC, LL, CL, LC. Les drux dernieres sont épuivalentes. On atuat done trois sortes de produits, possidant le rat-

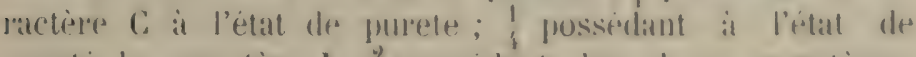

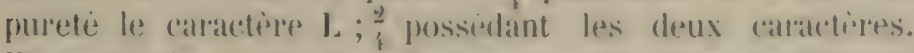
En vertu de: la loi de prevalence ces derniers possederont

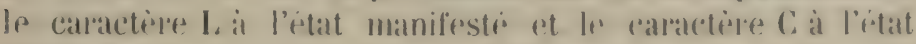
latent. Cest precisement cre que l'ubservalion bous apprond.

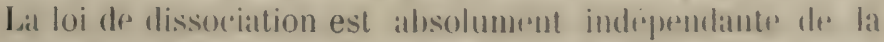
loi de frridominante; elle s'appliqure exaloment anx rallatelieres opposes dont ancun n'est predominant, mais dont lat combinatison fournit un caratetere intromediaite. (1)

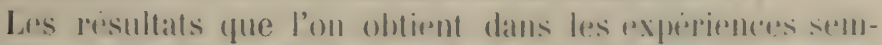
bathles at cenlles que nous venons de lapprorter, sunt bealleoup

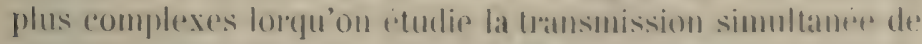
phusienrs caractipes: la couleur des tleurs, la forme des feruil-

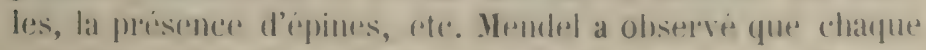
(atratetere se thansmort independamment des antres (loi de l'indéprendance.)

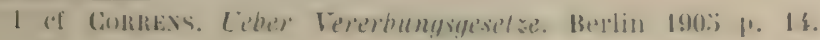


Díja Mendel avait commencé l'étude de la véritication de ses lois pour le règne animal. Depuis lors, d'autres biologistes se sont consacrés à cette tàche avec succès.

Lne autre question importante au sujet de l'hérédité est celle de la transmission des caractères acquis par opposition aux caractères congénitaux. Yous en parlons plus loin.

Indépendanment de l'hérédité, l'organisme possède un pouvoir autoformateur. C'est le mérite de W. Roux d'avoir' attire l'attention sur l'importance de ce facteur. Il ne peut à aucun degré remplacer l'héredité, ni l'expliquer. (1)

La serrie des formes qu'un organisme parcourt, depuis le premier moment ou il existe, se trouve donc virtuellement déterminée dans la cellule-øuf. Les circonstances extirlieures dans lesquelles l'oxut et plus tard l'organisme va se developper contribueront a lui communiquer certains caractires individuels, mais la structure spécitique, avec fous ses ditails. se deploie independamment des circonstunces, pourvu que celles-ci soient favorables d'une fason ginnirale. Ces circonstances sont les conditions nécessaires pour que les tendances héréditaires puissent agir. Parfois aussi clles determinent le mode d'action de ces tendances. Le tronc du lierre, par exeinple, pousse des racines absorbantes sur les parties qui touchent le sol et des crampons sur les parties qui s'appaient au mur. Or, seules les circonstances extérieures déterminent quelle est la partio du trone qui tonche le sol, et rien n'empiche de changer la disposition de la plante el d'obtemir ainsi des racines sur la pantie qui, auparavant, ne donnait que des campons et vice-versa.

Cinst une faute de raisonnement commise par certains phiysologistes, que de sappuyer sur des laits semblables pour attribure l'évolution de l'utre vivant aux circonstances exterienures. "Une telle conclusion, dit aver rason Wrisulann, me repose que sur une confusion d'iders...

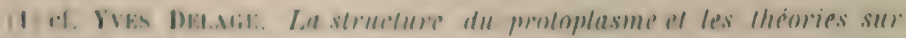

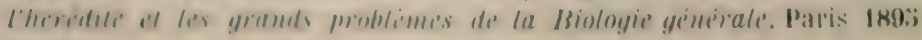
p. $733 \%$. 
Si un pliénomene ne se produit que sous certaines conditions, il n'en résulte pas cependant que les conditions soient aussi la cause du phenomène. " (1) Tout au plus pourrait-on soutenir qu'elles 'n sunt les causes partielle's, quoique leur part de causalité doive ille considerée comme: très petite et n'atteigne en rien la structure sprecilique de l'organisme. Le type spécilique etant done virtuellement determines dans l'individu, des le premier moment de son existence, ce n'est pas lá qu’il s'agrit d'an expliquer la genese, mais bien dans l'évolution de la forme qui se poursuit à traver's les grinéations successives sous l'mupire de la loi d'heriedité. C'est ce qu'alfirme implicitement spencer, lorsque, faisant a l'évolution individuelle des oranismes superieurs l'application de la loi de l'instabilite de l'homogène, il dit : "dans les germes des organismes superrieurs, les metamorphoses dues immeidiatement a l'instabilite de l'homogine sont vite masifués flar celles qui tirent leur origine du type héréditaire ».(2)

Lévolution individuelle d'un organisme quelconque, soit par exemple, d'un homme, est grourernée par l'hériclité, nou seulement quant i ses caracteres specitiques, mais également quant a ses caractires de race, en prenant ce mot dans sa signification la plus restreinte. Plusieurs caractires strictement individuels provent même dépendre de cre fucteur, en ce sens qu'ils résultent d'une combinaison des caracteres ancestraux.

Le ròle préponderant que joue dans les premiers phénomenes de l'évolution individuelle les chromosomes du noyau des cellules reproductrices, servent de base à l'opinion d'après laquelle ils contiennent les principes determinants de l'ontogenie, quels qu'ils soient d'ailleurs, ou du moins y exercent l'iufluence principale.

Nous avons examine plus haut (Ch. II, \$s) l’hypothèse de Spencer qui assimile l'évolution individuellè à la formation

(1) Essais sur l'hérélite et la sélection naturelle. Trat. II: VARtciss. Paris, 1892, p. 317-ว18.

(2) P. P., p. 443̈, § $132,-$ F. P., p. 339. 
d'un cristal. C'est la théorie dles unités physiologiques. Weismann a montré le peu de probabilité de cette conception mème au point de rue purement mécaniste. (1) Elle ne peut donc pas être assimilée à la théorie de Weismann, pas plus qu'à celle de Nïgeli ou d'autres biologistes, comme le voudrait Saleeby (2).

On donne le nom de Pangénèse à une théorie adoptée par Darwin et d'après laquelle les cellules reproductrices contiendraient des éléments - gemmules - empruntés constamment à toutes les cellules du corps et portant des déterminations caractéristiques de chacune. Darwin considerait cette hypothèse plutòt comme un moyen de représenter l'hérédité d'une faron concrète que comme une théorie proprement dite.

Parmi les biologistes modernes, aucun peut-itre ne s'est autant appliqué à élaborer une théorie de l'héridité que Wrismann. Pour lui, les chromosomes sont composés généralement de plusieurs groupements appelés ides et dont char'un contient tout l'ensemble des particules représentatives de l'organisme. Les ides sont eux-mèmes composés d'un grand nombre de déterminants. A chaque partie du corps isoliment et heréditairement moditiable correspond un déterminant particulier. (3) Cette conception peut invoquer en sa faveur des arguments probables que Weismann developpe et qui trudent surtout à démontrer que les différentes parties de l'organisme correspondent a difterentes parties du plasma germinatif. Yous ne savons rien au sujet de la manière dont les déterminants produisent les organes qui leur corresfrendent. (4) Itapres Weismann il laut a priori exclure toute intre lorece que les proprietes physico-chimiques de's atomes. (ii) Nous arons dejij dit que heancoup de biologistes repoussent ces vues exclusives.

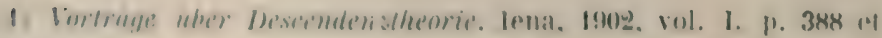
$3983,8 \pi$.

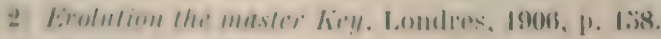

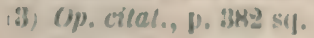

(1) Ibid, vol, II, [1. is et 1.

(8) Ibud, D. 38\%. 
L'héridité, comme canse de l'évolution individurlle, comprend en realite, on vient de le voir, un grand nombre de facteurs. Quelle que soit l'idée qu'on s'en liasse, il faut admettre que d'une facon ou d'une autre les intluences des parents et des anceitres sont representeses dans l'embryon et agissent sur son developpement. Cette multiplicite do facteurs est en harmonie avec la complexite de l'érolution organique dans l'indivilu et nous trouvons ainsi contirmée la remarpue que nous avons faite plus haut.

L'hérédite nous permet aussi d'interprriter d'une manierr satisfaisante ce qu'on a appele la loi biogénétique. On designme sous ce nom le lítit admis par durlyues hiologistes, de la reproduction an racenourci de la phỵlogénie diuns l'ontogerenie: l'individu, disent-ils, avant d'itre adulte, parrout tous less stades par lesquels a passe la forme dans les grimerations meredentes. Le developpement embryonnaire reproduirait, d'apres cela, les phases que les ancitres de l'indiridu ont réalisées à l'ètat adulte.

Lat phylogenie et l'ontogenie partront l'une at l'autre d'une serule crellule et aboutissent an meme point, puispue lat forme nexiste que dans les individus. Cone certaine ressemblantep

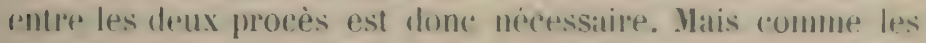
conditions dams lespuelles its saccomplissent sont tout it fiat differentes, on ne roit pas pourquoi les stades intermediaires seratent les mimes de part ef d'antre. Aussi la toi biogenenttique congrue de cette fagon soulèv-terlle de graves objections et a-t-elle èté fortement battue en brèche.

II semble bien plus aationnel de considirer les phases rmbryonnares, non pas comme des vestiges de phases anderstrales adultes, mais comme ha reproduction de phasses. ancestrales embryonnaires elles-memes. Nons anrions ainsi une simple application de la loi d'hérédite, admettant toutes les restrictions qui s'imposent à l'héréditi elle-mime. En d'antres termes, si dans l'emblryon des formes supurieneses on remeontre des organes qui se trourent dans létat adulte des formes infereveures plus anciermes, r'est parre que les embryons de res formes inlérimures possident certains organes quiolles conservernt it l'etat adulte, tandis 
qu'ils disparaissent dans les formes plus élevées. Ce qui revient à dire que nous sommes en présence d'une sinilitude dans le développement individuel chez les diffèrents groupes, similitude qui a sa raison d'ètre dans une commune origine. (1)

L'ontogénie n'est pas d'ailleurs uniquement sous la dépendance de l'hérédite, mais bien de l'ensemble des lois physiologiques. Ces lois etant générales déterminent nécessairement des ressemblances dans le développement embryologique, sans qu'il soit nécessaire de recourir à une parente. Il est malhemreusement impossible de préciser cette remarque. La question des relations entre l'ontogénie et la phỵlngenie est done loin d'ètre élucidée. Mais ces relations existent et il est plus que probable que l'hérídité n'y est pas étrangère. (2)

Certains caracteres individuels sont açuis par l'individu ¿ mesure qu’il se developpe, qui dependent des circonstances : le milieu, la famille, la societe au sein desquels il vit, l'activité que lui-même déploie etc. exerent sur son organisme une influence considirable et profonde.

Persome ne prétend qu'il est facile de distinguer ce qu'un homme posside par herédite et ce qu'il a acquis durant son existence, les tendances congenitales et les habitudes contractées, la part des dispositions naturelles et du travail personnel. Au point de vue yui nous occupe, il y a entre ces deux soptes d'elements une dilference capitale: les uns, élements lifréditaires, préexistent virtuellement dans l'ovule microsropirgue d'où cet homme est sorti; les autres, déments acquis, se sont formés pendant l'existence.

lievolution individuelle est la resultante de ces deux catégories de lacteurs : d’une part les impressions recureillies du drohors of la tramsformation que l'organisme subit sous leur andion, d'autre fart le type ancestral tor qu'il est transmis pal

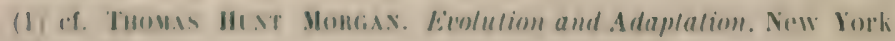
19383 p. $76 \mathrm{sq}$.

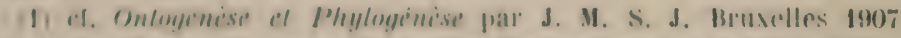

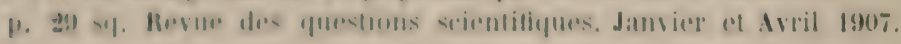


la génération. Celui-ci est donné implicitement diss l'orignine de l'individu, celles là se produisent à mesure flue sa vie se déploie.

ogénie ou Occupons-nous maintrnant des causes de l'évolution olution de la forme. Encore ici nous avons incontestablement forme. aflaire a la fois à des facteurs internes et extermes. Il ne peut pas y aroir de controverse a ce sujet. Dans tous les cas - et ils sont nombreux - vu les organismes s'adaptent is des milieux dilnerents, il y a action de fincteurs externes et reaction de facteurs internes. Le polygomum amphibium mend derux formes diflérentes, suirant qu'il est cultive datns l'eatu ou en terrain sec. Lorsique less animaux, les chevaux et les chiens, par exemple, sont transportes dans les pays froids ils se couvrent d'une toison épaisse; dans certaines contries très chatules, au contraire, les moutons ne sont pats recourerts de laine. (1) Quelle que soit l'interpretation que l'on dome de ces faits, ils appartiennent a l'évolution de la forme dans le se'ts que nous avons defini et, dans une certaine mesure, ils dependent de l'activiti? des organismes eux-mìmes.

Lat question est de sarvoir quels sont les facteurs internes et externes qui agissent et quelle est la mature et la portée de l'influence qu'ils exercent.

lection Darwin et Walline, ce dernier surtent, attachent la turelle. plus grande importancer à lat sélection nuturelle. On entend par lit le prociss on vertu duquel, gratce surtout it la lutte pour l'existence qui existe à un haut degre principalement entre organismes habitant un mème: milieu, recherchant la mème nourriture et ayant les mèmes ennemis, les plus aptes a vive sont conservés at se propagent

(1) P. Kropotkix a publie dans vimethenth Centur!y lonl vol. II

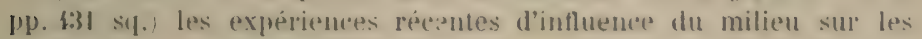
végétaux et les animaux. 
au dépens des moins aptes. Darwin a mis en lumière l'efticacite de ce facteur dans son ouvragé sur l'Origine des espèces et nous ne pensons jas qu'un seul biologiste nie qu'il n'ait eu dans l'exolution des lormes un ròle plus ou moins important.

De nombreux oiseaux périssent de froid en hiver. Ce sont évidemment coeteris paribus ceux qui sont le mieux couverts ou dont l'instinct leur fait trouver de chauds abris qui seront conserrés et par conséquent se multiplieront, tandis que périssent ceux qui sont moins bien protigés. En temps de disette, les animaux les plus forts ou les plus adroits s'emparent de la nourriture au depens dres autres. Dans une foret très dense, les arbres les plus rhases s'approprient la lumiere, tandis que des plantes plus petites sont condammées à perrir.

Mais on n’est plus d’accord dès qu’il s'agit de détinir exacfrment le rolde et l'importance de la selection maturelle. gumelques remarques spront utiles pour liare comprendre l'etat de la question.

I. Livolution de ha forme consiste dans une serie de stades dilferents et componto l'arequisition de caractipes nouveaux positifs ou negatifs. Or, la solection naturelle qui est un facfertr rexterne tend a expliquer la conservation des organismes posserdant un caractere nouvaul utile. au depens de ceox qui ne le possident pas, mass alle ne fournit ancum dement pour rxplipuer l'origine du caractire nouveau.

batroin fait remarguer que la communamed de forme rorxiste toujours avere des dillerences individuelles. Chanue raraction do la formes, meme dans les gromes les plus restremts, pepmesente tume moyeme antour dre lanpulle

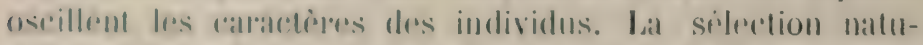
rolle consiste a lavoriser la propagation des particularites

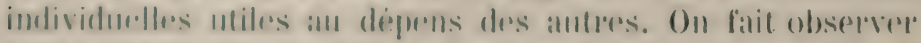

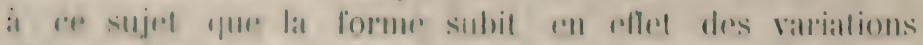

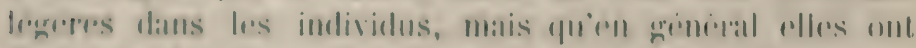

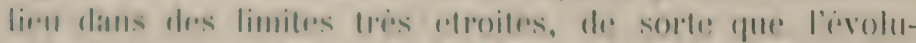

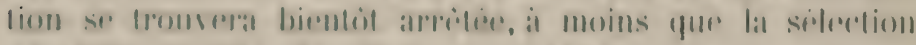

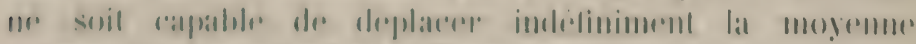
antour de lamgurlle les dillierences individurlles se pro- 
duisent. C'est ce que Darwin suppose, mais cela est loin d'etre démontro. Less experiences qur l'on a faites tendent plutiot a prouver le contraire. En outre, les variations individuelles doivent avoir une cause distincte de: la sélection maturelle. Damwin se recommat impuissant at en determiner l'origine. Tout as qu'on prent dire, iscrit-il, "cest fu'plle's se rattachent beancoup) plus etroitement a la constitution de l'organisme qui varie qu'a la mature des circonstances aluxquelles il a eti soumis." (1)

Pour spencere, les variations individuelles, yui pall leur alcemmulation assinent l'évolution de lat forme, ne sont qu'une conséquence de l'hripeditis. II suppose que les camactiones adaphatilis acquis par les individus se transmettent a leurs descendants, ol que les valdiations individuelles ne sont que le resultat de la combinatson dre rescalractiores, suivant les lois complexes de loheredite.

L'influence complexe de l'heredite est incontestable dins les vardations des formes virantres. Les cillacteres des palrents et des ancitres chant transmis a divers degres par la gremeraltion, sulfisent pour expliquar que chayue individu posside mor physionomie propme qui promet de le distinguer de tous

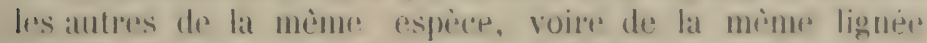
ou de lat meme portede. Jans ce dernier cats notamment, il y aura toujours de petites diffienenes entre les ovules et entre les spermatozoïles ou les anthisozoïdes; can mon seoulement les conditions dans lesquelles ils se developpent ne sont jambis rigoureusement identiques, mais en outre, les influences hereditaires qui existent dhez les parents ne s'exprent pas e'n proportion exictronent egalle daus tous les éléments reproducteurs.

Il fut encore, four les especess superieures, tenir comple des variations dues au croisement de formes legerement dillerentes. On satit qute des individus réalisant des formes tris dillirentes sont intersteriles ou ne possident qu'une liecondite restreinte. Mais des formes peu dilhirentes, appartenant, par exemple, à ce que l'on appelle des varietes

(1) The descent of Man. Londres. 1871 vol. 1 p. 154. 
d'une mène rspèce, provent ètre parfaitement interfèconcles et avoir une progéniture dont les facultes de reproduction ne soient pas altereses. Ainsi peuvent se croiser la plupart des races humaines. Les individus issus de ces croisements possident pirfois des caractères intrumediares entre les caractères dres parents; mais cela n’arrice pas toujours. Certains caractères opposés ne se combinent pas de manire à donner une movenne, mais se retrouvent tels quols, tantot l'un, tantit l'autre, suivant les lois de Mendel. L'hybridation ('st donc un facterur important de moditication de la forme.

Tel est le róle incontestable de l'he'ridite. Mais on ne peut pas en deduire que toutes les variations individuelles en sont une manifestation. L'opinion de spencer postule la transmission des ramaliores alduis qui est loin d'elre

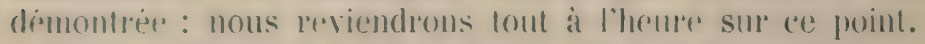

Ensuite, l'heredite consiote fessentiellement dans une ressemblance antro les descendants et les ascendants. Pond

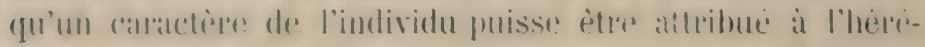
dite, il latul yu’il ait rexiste chez 111 de ses ancetres ou du moins qu'il puisse itre considere comme resultant de

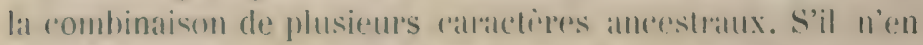

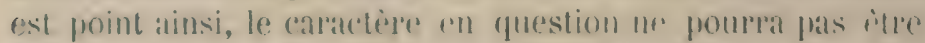

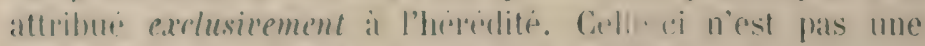
loi alsoblur, on, du moins, cefle loi est wllument complexe que daus bien dese (as nous ne parvenoms pas a en decourrir

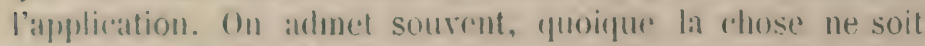

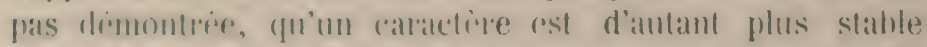

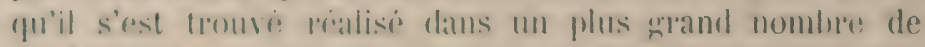

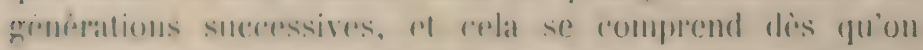

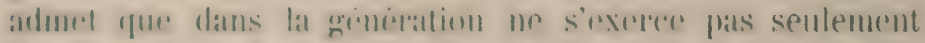

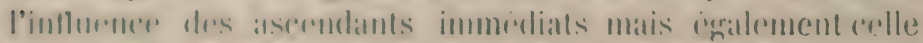

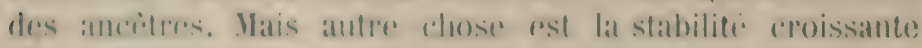

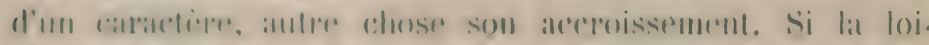

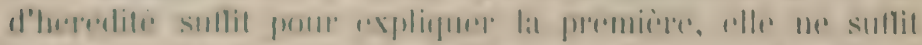

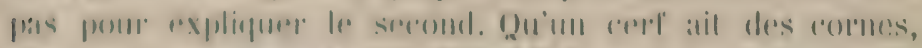

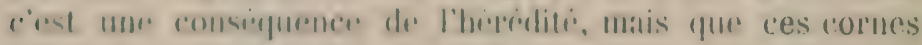

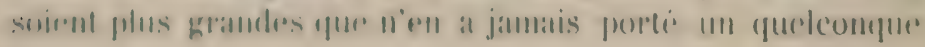


que de ses ascendants, celat sela un caractipe acquis pendant la vire, ou bien tur variation spontanée, mais ce ne sera pas une consénurmore dro l'héredité yui est essentiellement une ressemblance.

A plus forte raison ne pourat-on trourme dans liheredité la raison de caracteres alsoblument nowvenux. Dans bien des cas, l'hypothrse de l'atroutisition parr l'usage ou le non-usage devara citre eartere ol il ne restera que celle d'une variation spontimes: indrependante de l'hériditi et de l'adaptation. Spencer se laisse mantestement influencer en conte inatione parr des ideses throriques a miori. Lat

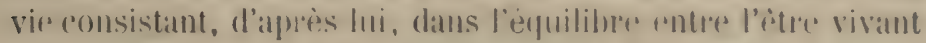
et le milieu. semals les changements du miliru penvent

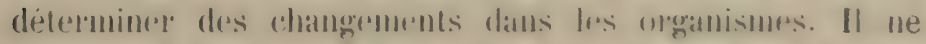
doune dis lors pas d'antre argument prom retablir sal doc:-

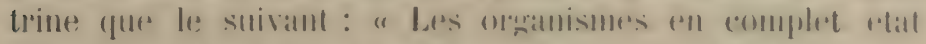

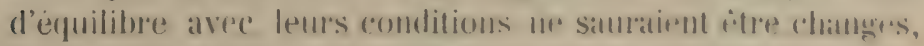

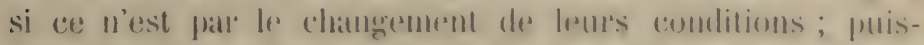

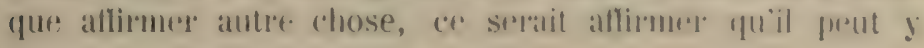
avoir un effet sans cause. » (1)

Comme nous le dirons plus limel, il n'y a pat dre maximum

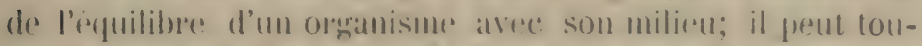
jour's s'y adapter flus parfatement; an untre, il freut s'y

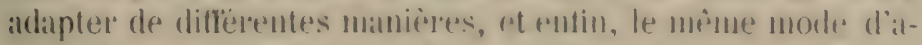
daptation se roncilie alves des valriations de structure indil-

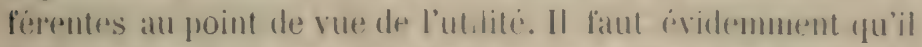

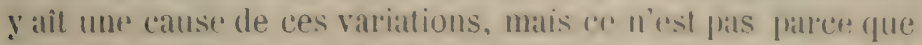

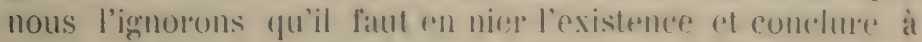
la non-cxistence de ses elfets.

Weismann qui considere la silertion haturelle eomme itant par excellence le principe d'évolution, remarque aves: raison que les individus adulles n'étant en somma que lo develop)pement des cellutes reproductrices, toute sirlection, pour idte eflicace, doit s'exereer sur celles-ci. Fous rerrous en outre que, d'apres ce satrant, les moditications que subit l'organisme pendant la vie individuelle ne se tramsmettent pas a ses

(1) Princ. de Biol., p. $330 \$ 91$ 
descendants; de sorte que l'origine de tous les caractires hereditaires nouveaux doit se trourer dans une moditication des cellules reproductrices. Weismann admet que le plasma germinatif peut être modifié par des circonstances extérieures. Mais en dehors de ces changements peu importants, les Variations des cellules reproductrices ont d'après lui ıne double cause : la combinaison de caractères par la rejroduction sexuelle (1) (amphimixis) et la lutte pour l'existence entre les déterminants.

Nous avons dejic ru quelles sont les limites qui s’imposent d'elles-mèmes à l'efficacité des combinatisons héréditaires pour la production des caracteres individuels. Quant à la competition qui existerait entre les délerminants et aux effets qui pourraient en résulter, c'est une hypothise? yui n'a ancume base experimentale, et en outre, de l'areu de Weismann lui-mème, d'alpres celte conception, les variations individuelles se produisent au hasard : "Lorsque, dit-il, on pose la fuestion : comment nait pour la premirope fos une variation de la majorité des déteminants dans un certain sens? on peut assigner deux ("anses. lat premieres est le hasard, la seconde sont des intluences rjui impriment un mime changenent aus determinants d'une certaine catégorie dans tous les ides. Nous verrons que lis influences climateriques sont un exemple du serond cas; mats les chamgements produits pall le hasard sont les plus importants, parce qu'ils sont le fondement at la point de dipart pour le process de sélection dorde supurieur, la siolection persomelle (nilurelle). Il pert paraitre invaisemblable que des phenomenes si importants reposent en derniore analyse sur le hasalrd; mais si l'on reflechit qu’il n'y a que derux diretions pour less variations, le plus of le moins, on doit admettre

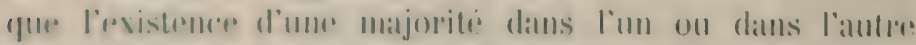

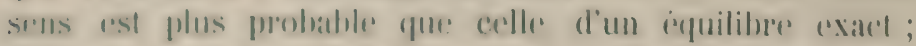

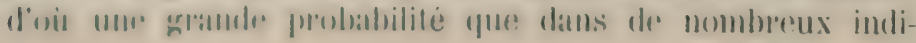

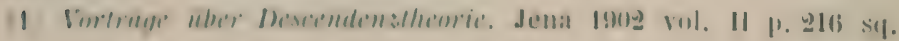


vidus du groupe, les variations en plus ou en moins d'un déterminant A l'emportent. ” (1)

La rifponse de Weismann a l'oljection qu’il se fait à lui-même est nulle. Chaque déterminant ne peut variep que dans deux sens. Soit. Mais il y a un nombre enorme de déterminunts dans chatqus ide; tous peuvent variers. Comment se fait-il que les valriations de l'organisme sont harmonieuses, c'est a dire alfectent simulamement et d'ume manière convenable tontes les parties d'un système? Comment surtunt les variations hamonipuses sp réalisentelles dans la grinèse des caractères non adaptatifs, quu? Weismann admet, (2) alor's gue le seol frincipe d'ordire, la sélection naturelle, n'a alucume action? Comment, citant domneres la multitude dess ides dans changes cosllules, la multitude des cellules reproductrices, les gromérations qui

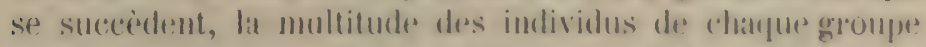
et les ceroisements, fres variations an flus of ent moins ne: se compensint-elles pas, d'antant plus que Wrosmann reconnait l'alssince de valent siolectiommenle des variations

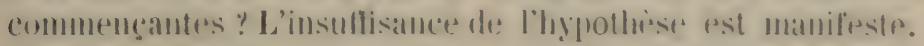

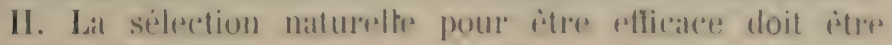

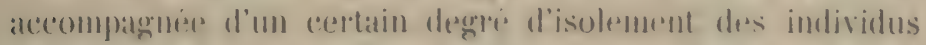

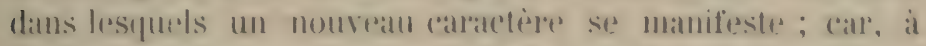
moins de supposere gure tous les atuters sont immesdiatement extermines, ce yui sera idvemment liexception, les croisements altront bientit rasison de lat viriation qui

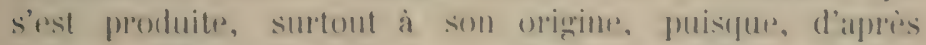

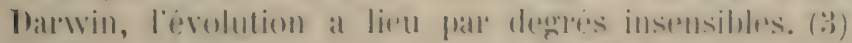

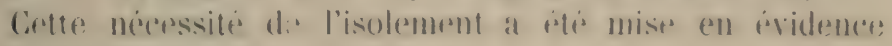
par. Romanes après Gulick. (4)

III. La sélection nalumedle "xphigues fout all plus la con-

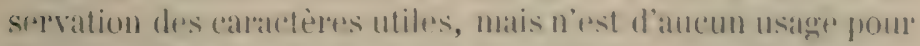

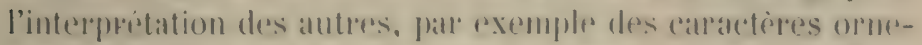

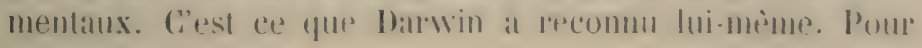

1) Ibid p. 118-1.49

(2) Ibid. P. 149.

(3) Origin of Species, Londres 18039 p. 289.

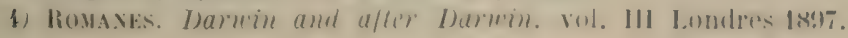


combler cette lacune il a recours à la sélection sexuelle. Weismann admet également ce facteur auquel cependant les biologistes attachent généralement peu d'importance. son application soulève des objections nombreuses et Darwin lui-mème reconnait qu'il ne suffit pas à combler les lacunes que laisse la sélection naturelle. (1)

IV. Nous ne pouvons pas accorder à la sélection naturelle, comme le voudrait Spencer, la valeur d'un principe a priori, en ce sens qu'on doive lui attribuer toute transformation d'une forme virante qui ne s'explique pas par une adaptation directe.

" Yous renons de voir, dit-il, que l'équilibration indirecte (l'adiutation de l'espèce au milieu par sélection naturelle) fait tout ce que l'équilibration directe (l'adaptation individuelle) ne peut faire. On ne saurait trop insister sur cette conclusion, que tous les procédés par lesquels les organismes sont riadaptes a leur milieu sans cesse changeant, doivent itre diun gremre ou de l'autre. A l'appui de cette conclusion, nous navons pas seulement le principe universel quiun changement de tout ordete tend à l'éguilibre, mais nous avons aussi le principe universel dans le monde oratnique, que la vio est la conservation d'un équilibre molile entre les actions internes at les externes, c'est it dire unt adaptation continuelle des actions internes aux externes, on la conservation d'une correspondance entre les forees anxpuedles un organisme est sommis et celles qu'il développe lui-même.

"En effrel, si la ronservation de? la vie est la conservalim de ant infuilibe mobile, il en risulte que les changoments qui fromellent a tule espoce de vive sous des

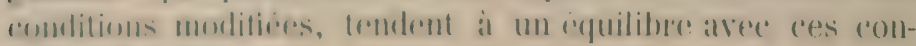
ditions modiliees.

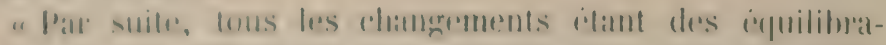

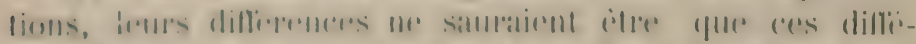

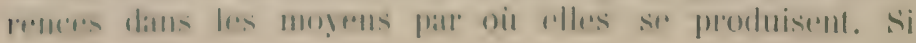

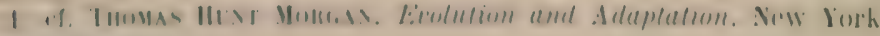
$1003\}$ p. $213 \div 4$. 
elles ne sont fas produites directement, il faut qu'elles le soient indirectement. Donc a priori nous pourons ètre certains que toutes les methodes de morlitication qui ne rentrent pas dans la classe des equilibrations directes, doivent rentrer dans celle des equiliblations indirectes. L'examen des taits contime cette conclusion ". (1)

En admettant que tout changement tend a l'é(juilibre, il n'en suit mullement que tout changement d'un organisme tend à cet isquilibre particulier yui consiste dans l'aptitude à vivre où il se trouve.

Yous n’avons fas admis la definition de la vie sur laypuelle s’appuie la raisonnement de Spencer. Pour qu’il fùt dimonstratil, il faudrait que la vie fùt entièrement detinie par l'adap)tation aux circonstances axterieures. Deses qu'il est possible de concevoir cotte adaptation de diflerentes mantieres, dis qu'un mime degré d'atap̧tation perut etre réalise sous ditlirentes formes, on congoit pue des changements se froduisent

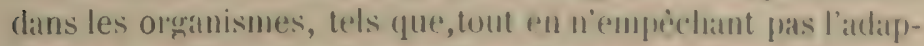
tation au milien, ils n'y contribuent pas ou ne lit rendent pats meilleure et ne soient compris ni dans l'équilibation directe ni dans l'équilibation indirecte. Cess changerments nes se fixeront pas par siledtion naturelle; mais lien ure permet d'altirmer a priori que l'intervention de cefactent est nerassitre.

Certes, les faits sont loin dr dommpr raison a sprencer. Les organismes, nous l'avons dit tout à l'hrule, possèdent en grand nombere des calactions anxipuels il nous est impossible d'assignere un ròle d'adaptation. Sipencer ne se préocerupe l'ailleurs nullement de le laire ressurtir. Il allime " priori l'intervention de la siolection maturelle des que l'adaptation directe fatit defaut. Or, Darwin s'est parfaitement rendu comp)te de l'insulfisance de la stelection naturelle, mème aidese de la lransmission des caractères arrquis durant la vie individuelle. Il y a lui-meme ajoute les lois de croissance (laues of grouth) yu’il ne précise pas autrement, la selectionsexurlle et des caulses inconnues (unlnown causes).

(1) Princ. de Biol. vol I p วั60-ð61 § 168

(2) Romanes a rassemblé les lextes de Datrwin dans Daruin and after Darwin vol. II pp. 3, 6, 321, 329 ete. 
V. On peut, si l'on veut, domner le nom d'adaptation à l'acquisition tes caracteres par l'action de la selection maturelle, puisque, ces caractères étant utiles, la forme virante dans laquelle ils se fixent devient flus apte à vire dans son milies. Mais il faut remarquer que la selpetion naturelle étant un facteur rxterieur aux organismes qu'elle transformo, celte adaptation n'est pas le filit de l'etre vivant. La forme, par hypothèse, produit des variations quelconques; la silection naturelle conserve calles qui sont utiles au dépens des autres. C'est donc elle qui adapte la lorme ot non flas la forme qui s'adapte elle-même.

sprencre, il est vai, ramenr les variations de la forme ¿ la combinaison, gràce a l'herédite, des caractères acquis pall les ancetres au moyen d'une adaptation proprement dite. Mais nous arons dejà rejoté cette interprétation comme insulfisante. Spencer lui-mimo ne se préoceupe gruere d'y rester lidlèle dans les ras particuliers, puisyu’il ne laisse gas d'imvoquer la solection naturelle chatulue fois que l'adaptation directre rest insultisante a rendre comple d'une moditication qui se produit diuns la forme.

On navanes pats la puestion en appelant adaptation ou épuilibation indirecte l'évolution par sélection naturelle. Le nom ne fail pien à la close. Si le mimetisme: sert as soustraire certains animatus it l'altention de leurs

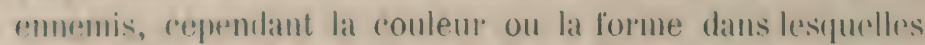
il consiste ne sont determines ni par le milien, ni par les animatux yu’il trompe, ni, du moins en greneral, pret

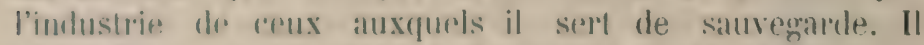
n'y a dome pas icei daction do milien, ni de reaction de

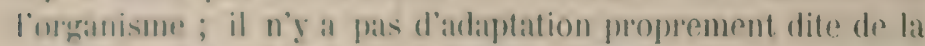

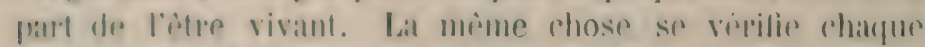
fois yue levolution est due a la seileretion nature lle. Dans

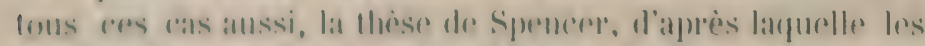

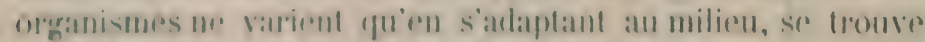

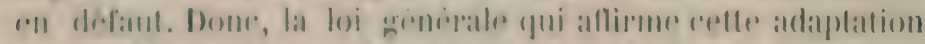

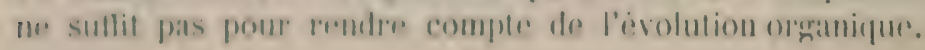


tation et Lu siecond facterur qui a eti mopose pour expliquer édité des l'évolution des formes virantes, céest la transmission heréacteres ditaire des canacteres acrpuis par l'action directe des rirconstances exteriemess sur les itres vivants at pall lat ratetion de ceux-ci - indépendamment de lat selection naturelle.

Laissant de cote les moditioations delatorables, felles que l'affaiblissement des ronstilutions dans les reprions malsaines, farce qu'plles se paltachent plutcit alu proces de dissolution, il est utile de distingmer, dans cette catis-

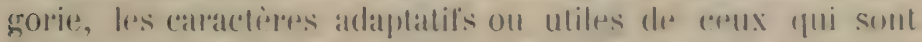
indiffipents. Il sembie qu'un climat chand pel lumineus developpes la colonation des lissus supertiriels des orgatnismes : les races hmmaines qui habitent les pays tropi"ans ont regulierement te trint plus foncre que corlles pui dabitent les reggions temprepres on frobles; les animanx

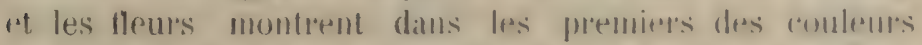
plus brillantes que dans les secomeles. (Dn me veil pas rell

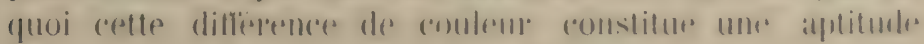
plus gramde a vires dams res climats. An contraire, la

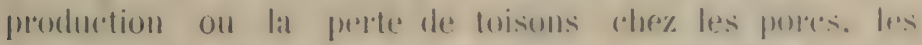

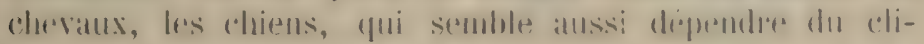

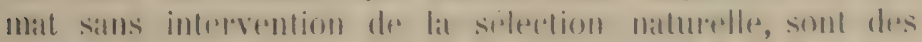

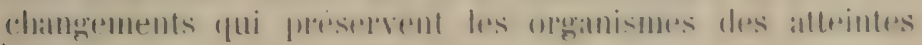
du froid ou de l'excès de chaleur.

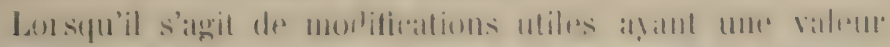

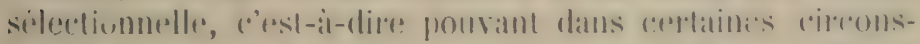

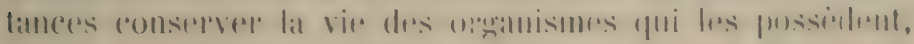

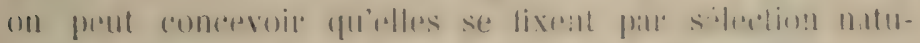

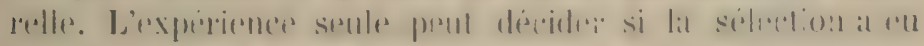

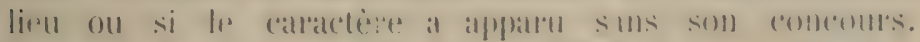

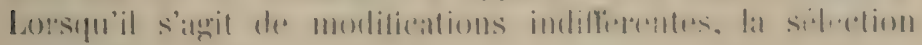
maturelle nentre plus en ligne de compte.

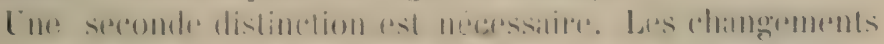

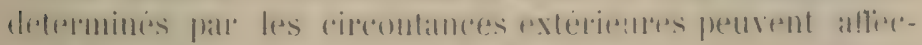

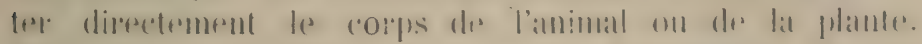
Wrism:mn lent domme lo nom de somalomemes (1). Ils

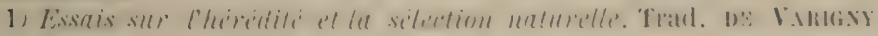
Paris 1892 p. 3037 
peuvent au contraire affecter directement le plasma germinatif (les cellules reproductrices) et avoir leur répercussion dans l'organisme à partir de la génération suivante. Weismann les désigne sous le nom de blastogènes. (1)

L'école de Weismann admet la transmission héréditaire des caractères blastogènes acquis par l'influence du milieu. Lui-mème range dans cette catégorie certaines différences qui séparent les variétés locales et qu'on peut parlois reproduire artiticiellement par l'élévation de la température, etc. (2) Ce facteur d'érolution est absolument distinct de la sélection naturelle et il ne faut pas en méconnaitre l'importance. La sélection naturelle n'est done pas suffisante à tout daus l'évolution organique, et le titre choisi flar Weismann dans les articles - dont nous parlerons bientot - de The Contemporary Revieu (The allsulficiency of natural selection) n'est pas exact.

Il n'est pas toujours facile de dicider si un changement est somatogène ou blastorène. Rien mème n'empeche a priori yu'une moditication ne se produise en mème temps et dans le mime sens sur le corps de l'individu et sur le plasma germinatif, surtout lorsqu'il s'agit de changements dans lesquels l'organisme est plutot passif, comme ceux qui dispendent de l'intluence du climat ou de la nourriture. Dans ce cas, la therie de Weismamn permeltrait d'expliquer yu'un caractère qui apparait des que les organismes sp trouvent dans certaines conditions, saccentue far tule action prolonger? sur les generations successives. (1) prent concevoir que la faculte dadaptation manifestee par les individus appartient egralement a l'esprece comme telle of s'expre par les organes mopagateurs de l'espèce, cest a dire far les cellules reproductrices. Lion comprendrail adosi le carmelere adaptatif de certains changements

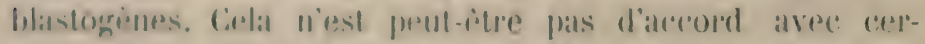
latmes ideres philosophigues, mais celat saccorde fort bien

(1) Ibid.

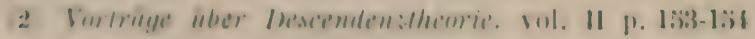


avec d'autres. Il faut donc en cette matiere se laisser guider par les faits et non par des idies priconcues.

La remarque précédente ne doit pas nous empecher de leconnaitre qu'il y 2 une catégorie importante de changements individuels n'ayant, de par leur nature, atcune l'slation avec les cellules reproductrices. Ce sont d'aloord les moditications de l'organisme qui r'isullent de l'activité déployée par les organes. Chez les animaux, le sirge de ces modifications est surtout le systime nerveux (mémoire, expérience individuelle, habitudes ou habiletés acquises par l'exercice), ou le systime musculaire (déreloppement des muscles qui travaillent). D'autres parties du corjos peurent également se moditier par l'usage : par exemple, la peau qui s'épatissit dans les parties du c'orps plus exposiess au frottement, les os qu'on roit croitre en volume dans les membres qui déploient de grrands eflorts.

Ce sont ensuite certaines modilications generalement caractérisées par une diminution de volume atrophie) l'esultant de l'absence d'usage de certains organes. Cet eflirt est très visibledans les muscles qui subissent une reduction considerable lorqu'ils ne sont plus employés.

Il faut encore ranger daus celte categorie les changements de structure par lesquels l'organisme s'adapte aux rorphs avec lesquels il est en contact ou plus géneraldment aux circonstances environnantes. Ainsi les racines et les luanches de l'arbre contomment les resistances, se dirigent vers la lumière, l'air pur, l'humidité. Enlin signalons les lésions organiques et less conséquences qui en résultent : circatrices etc.

La transmission héréditaire des caracteres acquis par l'usage ou le non usage des organes, c'est ce qu'on appelle le facteur d'évolution lantrokien. Il joue en effet un ròle prépondérant dans la théorie évolutionniste. de Lamarck.

Nous avons déjä dit que la transmission héréditare des adaptations somatogènes, de quelque nature qu'elles soient, est la base. de la doctrine de spencer sur l'évolution des formes vivantes. 
Pour l'établir, il invoque la loi de la multiplication des eflets. "Si un organisme A, dit-il, se trouve, en vertu d'une habitude ou d'une condition particulière de sa vie, modifié sous la forme A', il en résulte inévitablement que toutes les fonctions de $\mathbf{A}$, la fonction reproductrice comprise, doivent differer à quelque degré des fonctions de A. Un organisme étant une combinaison de parties qui jouent ruthmiquement un ròle dans la constitution d'un équilibre mobile, il est impossible de changer l'action et la structure de l'une quelconque de ses parties, sillns causer des changements d'action et de structure dans toutes les autres; c'est ainsi qu'aucun membre du système solaire ne saurait ètre modilié dans sa course ou dans sat masse, sans produire des réarrangements dans toute l'etendue du strstime solaire. ») (1)

Comme spencer l'observe lui-mème, cette argumentation n'indique qu'obscurement que la modification du rujeton doit se produire dans le mène sens que celle subie par le parent. Guoique les dillërentes parties de lorganisme soient ftroitement solidaires, il ne suit pas de lit que toute espece de modification à laquelle une partie se trouve soumise ait nécessairement une répercussion appreciable: dans toutes les autres, et surtout, on ne peut pas conclure que les organes generateurs seront alfectis de maniere a reproduire la moditication dans le's descendants. Yous pouvons donc considérer te raisomement de spencer comme une contimation throrique de lexperience yui semble plutot favomable is la these de la Iransmission des caracteres acyuis. Mais il nous est impossi-

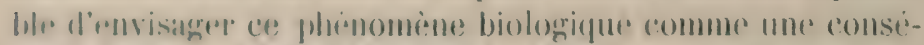
quence quion peut logingument deduire du principe de lis multipliealion des eflets ou de la persistance de la force. Les renerves que nous faisons sont indiquens ici part spencer luimeme, comme noms venoms de le dire, mais aillemes il est beancoup plus aflirmatif. "Si toutes le's parties d'un organis-

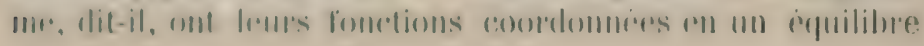

11) Princ. de Biol. Vol. I p. 310 \&4. 
mobile, tel que chague partic influrnep rontinuellement toutes les autres parties, et ne puiso inde alléree sans donner le branle it des changrements dans toutes les autress, e'est it dire si la limite du changroment est l'établissement d'une harmonir completo dans lous les mouvements, moléculaires at autres, de toutes les palties, il fant qu'au nomble des partiess moditises, molécularirment on autrement, il y ait colles qui emetlent les gremes des nouveaux organismes. Les molecules des germes quelles produisent doivent tendre toujours it contormer les mouvements, et pate consépuent less arrangements de leurs élements, anx forces molemalaress de l'organisme entime ; pt si cert agregat de forces molinalaires est moditio daus sia distribution fatl un changement local de structuar, les. molecules des geremes doivent subir graduelloment des chamgements dans les mouvements ae les alrangements

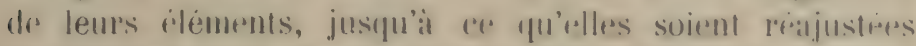
a l'angregal des foreses moleculaires. En ellet, sombenir

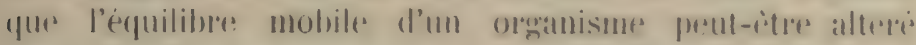

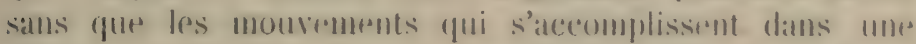

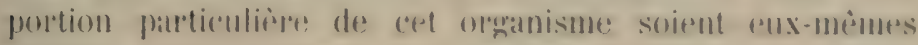

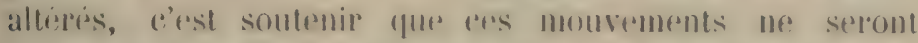
pas effectes par une modilication de: lat distribution dres

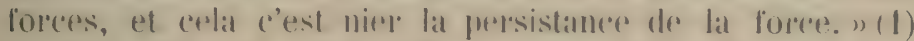

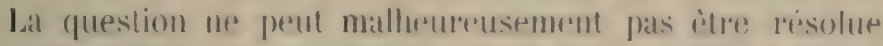
fratr des primejpes atussi simples. Salls doutr, puisyue toutes les parties de l'oramisme agrissent les unes sur les autres, il est impossible que l'une d'olles solt modifior sans que les autres sem ressentent. Mais la yuestion est dre salvoir

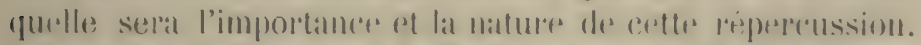
A la rigurur, coutes les parties les plus pelites de l'Lnivers

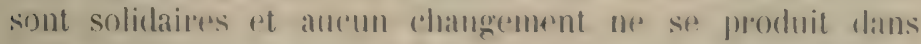
ume partie quelconque qui the se transmelte sous quelque forme à toutes les antres. Con solichatritio plus etroite existe, far exemple, entre tons les hommes. Il selait (et) perdant absurde den conclure qu'une moditioation subies

(1) Princ. de Biol. vol II p. 1:i:-1:38 \$311. 
par l'un deux doit se transmettre pour cela a tous les autres.

Il ne suffit pas, pour attribuer un phénomène à une cause déterminée, de prouver que l'activité de cette cause s'étend à cr phénomène; il faut encore que le sens et l'intensité de son action soient en rapport avec l'eliet qu'on veut lui attribuer. Le défaut que nous signalons ici dépare fréquemment les explications de spencer. Dans la question presente, il est manifeste. Personne ne niera que les changements subis par l'individu n'affectent de quelque manièrr tous ses organes et, par conséquent, la fonction reproductrice. Mais la question est précisément de savoir quelle est l'importance et la nature de cette influence et si elle consiste a transmettre aux descendants les earactères acquis pendant la vie de l'individu.

Wrismann nie la transmission héreditaire des caractires somatogines. La théorie sur l'isolement du plasma germinatif des cellules reproductrices est, jusqu'i présent du moins, trop lyppothétique, pour que la conclusion qu'il en tire doive ètre considérée comme ayant de ce chef une grande probabilite. La question est surlout de savoir si l'évolution doit être interprétée, au moins en partie, au moỵen de la transmission heréditare des caractieres de crotte sorte; ensuite, si l'on peut apporter des faits d'expérience en faveur de cette transmission. Lat question a éte liscutée, surtout au point de vue le l'évolution, entre spencel, Wrismann et Romanes dans The Contemporary heview de 1893 a 1896 . Les articles de Syencer sont de ferrier 1893, mars 1893, decembre 1893 "1 octolne 18:14; cexax de Weismann de septembre 1893,

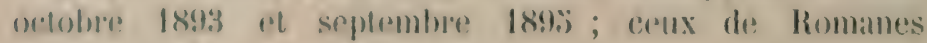
d'avril 1893 et de juillet 1893.

spremeer s'appuies sur cortains linits, par esemple, lat dillepene do sensibilito tactile des diflepentes rérions du

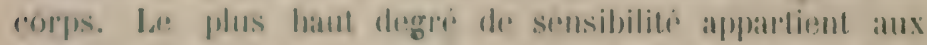
parties qui frar lenr presilon sont le plus frequemment

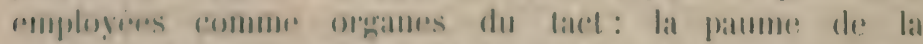

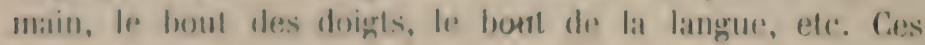


faits, d'apris lui, sont inexplicables par la sielection naturelle, parce que cette distribution particulière de la sensibiliti tactile ne confire aucun arantage a l'individu qui la possède, tandis qu'ils sont aisement explicables par la transmission des caractires acquis, l'augmentation de la sensibilité étant le resultat de l'exercier. Spencer nip également que la solection naturnlle muisse expliqum les structures qui requirient le diveloppement harmonieux d'un grand nombre d'organes clementaires. Ainsi l'accorosssement des cornes de l'élan suppose un développement simultane des muscles, temdons, merts, vaisseanx du cou de cet animal. Or, il pst incroyable que les variations simultanés de tous ces opganes se moduisent d'une fatgon larmoniruse, sans rien qui rende compte de cette co-idaptittion. La transuission des (anactimes acquis (*omble cefte lacume: a mesure que less cornes se divplopplent, le tratvial plus grand qu'elles imposidnt ans ditlispents oryanes da cou fortitie cors orranes dalls la mesure requise.

Spencer conteste lat sultisance do la sélection naturelle pour expliqupr tous les diveloppements des organrs ou des fonctions, de mrime yu’il repousses l'rxplication de la régression d'une partir par la seule cessation de la sélection naturelle (panmixia).

Weismann nie qu’il soit miressaire des recompir à l'héridite des ranactères somatogiones pour expliquer l'evolution des structures complexes on co-adaptations. De telles structures se sont developpess igalement dans les formes steriles des colonies de fourmis, ouvrieness ou soldats, ou il ne ne peut pas etre question dre transmission héreditaire de caracteres acquis far les individus. Ensuite, l'apparition et la disparition se produisent aussi loren pour les caracteres passifs, par exrmple la couleur, les tegmments, que pour les caracteres actifs. Ide mème, lon constate la formation d'organes complexes dans les parties chitineuses des insectes qui ne se developprnt pats par l'exercice, et le developpement harmonienx l'instincts qui n'ont qu'une seule fois l'ocalsion d'itres exercis dans la vie de l'individu. Dans tous cers cas, l'évolution 
n'est pas due a la transmission des caracteres somatogrenes. L'ivolution se congoit done sans ce facteur et il faut d'autant moins l'admettre que, théoriquement, son existence est peu probalıle. Quant à expliquer dans chaque cas comment lat sélection naturelle a produit les caractires que possèdent les ètres vivants, nous en sommes evidemment incapables. Il faut admettre néanmoins ques l'évolution doit lui itre attribuee, parce qu'ancume autre explication n'est adınissible.

Romanes prend ume position jusqu'à un certain point intermediaire entre? Spencer et Weismann. II maintient la valeur de l'explication donnée par ce dernicr pour la disparition des caractères devenus inutiles; it reconnait l'insuffisance des preuves qu'on invorue pour établir la transmission des ralatetères somatogènes, mais il insiste d'autre part sur l'insuffisance de la sélection maturelle, el conclut que si la transmission des caractères somatomines est démontrée fausse, il faudra admettre pour l'evolution d'antres facterurs. "si, dit-il, an mop̣en

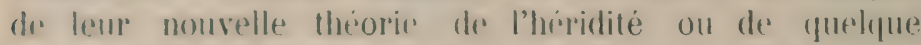
antre maniere, les mo-Ibarwinistes parvenatent a montrep l’imp̧ossibilite de la transmission des caratères acquis far l’usige, je setras enclin a adopher l'ophinion a'Asat

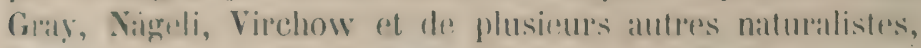

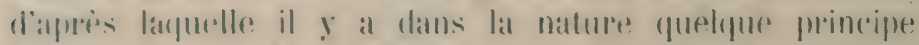
encore inconnu de moditications adaptatives, qui à present

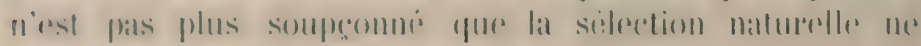
l'était il y a un demi-siecle. "n (1)

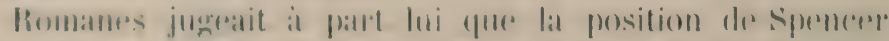

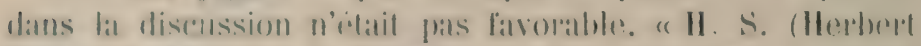

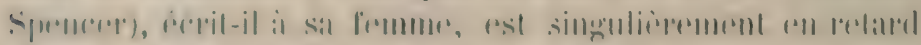

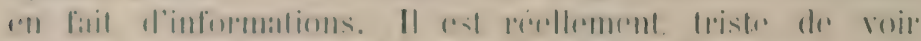

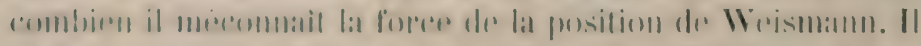

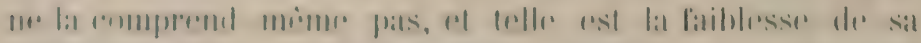

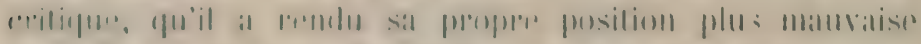

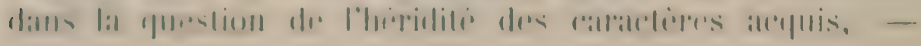

(i) The Conlamprary Reviem. Avril 1w9:3 p. 8it. 
crest a dire la base de tout son systimo de philosophie syntéthique. » (1)

La controverso se termina par une note de spencrep

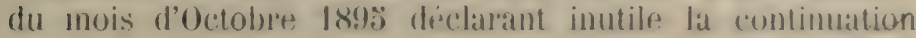
de la discussion, al constatant qu'aucune réponst n'avait ité donnce at son principal angument (les dillèrents degres de sensibilitis tactila dans les dillementes rengions die la peau).

An point de rue experimental la question de la transmission des caractiness somalogenes est moins lacile i

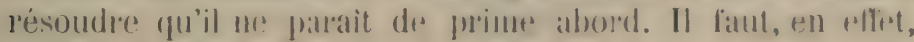
que la modilication oraninipue dont on rest prouser la thansmission ait dete determinire par des causes qui nont fas pu angir directement sur les cellules reproductrions

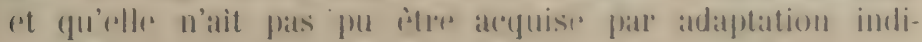

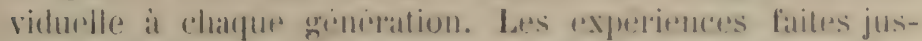

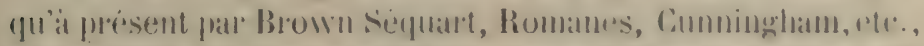

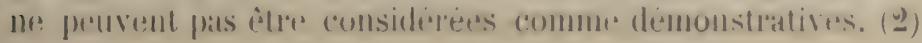

Lat quration agritere entre spencer et Wresmann lla donce

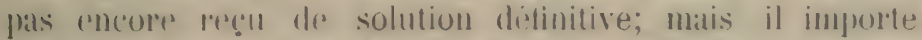
d'insistre sur une conclusion qui en resulte et gu'indigue dijji Romanes Weismamn repousse lat transmission des calatetires somatogines et conclut a lat sulfisance universelle (all-sufficiency) de la siblection natmelle, parce quil est impossible dadmeltere diatres faterums, notamment des tendunces internes grouveruant l'évolution des formes. spencer de son còté, convitined de l'insulbisance de lat sélection naturelle, s'itponic sur la meme prélendur impossibilite d'admettre des tendances évolutives internes, pour contirmer sa throrie de la tamsmission des caracteres somalogines.

(1) Life and Le'lors of G. J. Romanes. Lumbers 1902 p. 323.

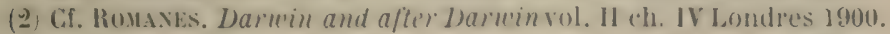

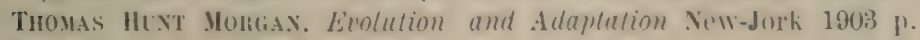
250 sq. 
Il resulte de lia, que l'admission de ces tendances ferait disparaitre la plupart des diflicultés qui sont considérées comme insolubles par l'un des deux adversaires. L'évolution harmonieuse des co-adaptions inexplicables pour Weismann et qui, d'autre part, se produit également dans des cas où I'herédite des caracteres somatogènes ne joue aucun róle, aurait trouvé son explication. En d'autres termes, on peut adopter a la fois l'opinion de Weismann et celle de Spencer daus ce (qu'elles ont de négatif : d'une part l'insuffisance de lat sélection naturelle, d'autre part la non-existence d'une transmission hèréditaire des caractères somatogènes. Si Ion ne comprend pas comment les différents degrés de sensibilite tactile ont eté prorluits dans les différentes rixrions du corjes frar la sélection naturelle, d'autre part, il n'est pas du tout évident que l'explication proposce par spencer est adtequate. Elle ne rend certainement pas comple de lat difference très grande de sensibilite qui rexiste entre le front et les paupières, du peu de semsiLiliti du genou etc. be mème c'est un fait que certaines moditirations ro-adaptatives, far exemple la moditication des races par silfection artificielle, se produisent sans l'intervention de ladaptation individuelle (1).

If II. faudrait naturellement recourir aux tendances spontannes derolution que dans la mesure où la sélection naturelle serait dimontrie insuffisante. Beancoup de hioloristes pronsent qu'elle lest dans une large proportion. II est rlair aussi qu'une conception anssi vague que colle yni correspond aux mots: tendances internes dérolution, in. frent pass ètre consideree comme deflinitive.

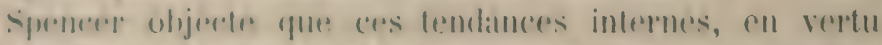
despuelles naitrait une structure comportant une co-iddaptation des parties, implingurnt lat fintilite, exest-it-dire la

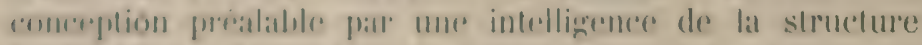

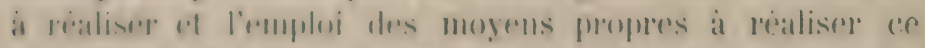
but. Tue telle idtere contredit sat philosophie mecaniste

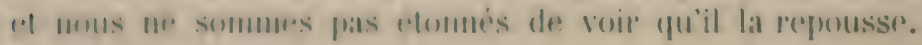

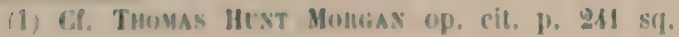


"Supposer, dit-il, qu'ils (les changements) se produisent ainsi, e'est supposer que la lin prescerite est reconnue quelque part et que les changroments sont, pas a pas, simultanement proportionnes is lachervement de rette fin; c'est supposer une production premedite en rue de ces changements. Dans ce cas alor's, il nous faut revenir en partie a l'hypothese primitive; at si nous to faisons en partie, nous pourons tout alussi bien le faire tout-it-lait; - nous pouvons tout aussi bien retourmer franchement a la theorie des creations spreciales. " Ciest tout ce que dit Spencer et ce n'est pas assez.

Wrismitmin ne veut pas qur le naturaliste almette des explications qui invoguent l'intervention du Createur pour supplies a l'insulfisance des forces de la Nature. (1) Yous sommes eggalement de cet avis, pourvil qu'il ne s'agisses

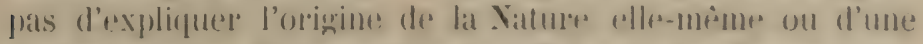
de ses parties. Nous salvons en effiet que les forces naturelles nont pas Ie pourvir createntr. Mais lat Nature etant supposie constituie, le: naturaliste doit chercher la raison dretre des phenomines maturels dans l'Lnivers lui-mẻme. Cette rellexion n'exclut atucunement l'hypothese de tendances intermes qui coopererelatent a l'irolution. Wrismann lui-meme admet que lérolution individuelle est prédetrerminee en grande partie datns less ceflules reproductrices. Pourquoi ne pourrions-nous pas admettre que l'evolution

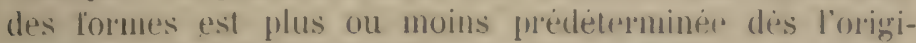
ne de la vie? Celat n'exclut pas lat cooperration necessairm de's autres lacteur's d'evolution, notamment de la selection maturelle et de l'intluence du milieu. Yous ne risquons donc pas d'instaurer l'harmonie préétablie. Il apprartient au maturaliste de chercher a connaitre res tendances originelles d'une manière manière précise et de demèter dans l'evolution la part de chacun de's facteur's qqui ! contribuent.

C'est un prejuge de croire qu'il ne peut pas y avoir d'autres forces naturelles que celles manifesties par la

(1) The Contemporary Review Sept. $189 \mathrm{~s}$ p. 4 İ̀. 
matière inorganique. Si les forces vitales sont irréductibles aux foress physico-chimiques, ce qu'il est en tout cas nicessaire d'admettre pour la vie psychique, il faut de toute nécessité admettre un Créateur à son origine, do mème qu'i l'origine de l'Lniver's materiel. Mais la vie: étant supposee exister sous ses formes essentirlles, c'est incontestablement dans les ètres vivants qu'il faut cherpcher l'explication des phénomenes vilaux.

II y a une forte présomption en faveur de l'existence de tendances internes d'érolution. Si nous admetlons que d'un groupe d'individus tres semblables entre eux sont issus, dans la suite des temps, les formes intiniment valriers qui pexplent le monde aujomml'hui, il parait bien dithicile d'ardmettre que l'action du milieu, qui n'o!fre pas en somme une très grander variste, el la sélection naturelle - qui ne tient comple qua: d'un seul point de vue: l'arantage dans la lutte pour l'rexistrence - sont les deux steules causes d'une exolution si compliequee. Notons encorr une fois atree Huga be Vries (1) que la selection ne produit pas les formes, mais sadement conserve los unes au déprus des autres, de sorte qu'elle n'est d'ancun usage pour explipue: lem o:igine.

Bon mombre de biolonistes ont etr latppes virmment de l'insulfisance de la sélection naturelle et des l'influencedo miliru pour expliquer l'evolution; et ext prexisement ar yui less a amemes a admettre les tendancess internes dont nous parlons. (2)

Il nest pas possible dexaminer ici en detail les dillirontes tentatives qui ont ete fates gour donner une expression procise a la thropire qui considipe l'evolution comme

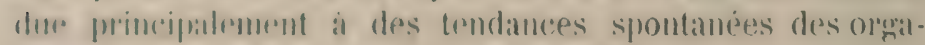

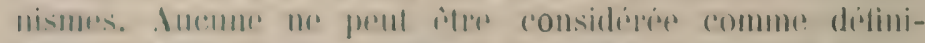

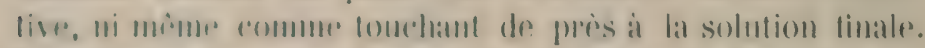

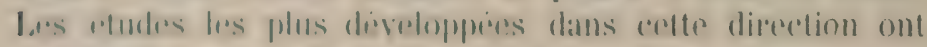

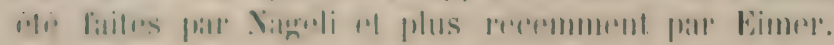

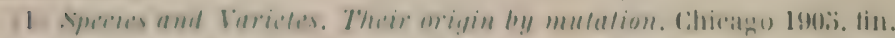

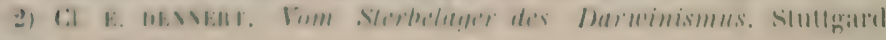
18K03, p. $98 \mathrm{s!}$. 
Yägeli distingue d'une part la perfection intrinsiapue des. êtres vivants consistant principalement dans la division du travail et le degré plus élevé d'oryanisation, d'autre part l'aptitude plus ou moins grande des individus à conserver leur vip dans te milieu ou ils se trouvent. Cietre aptitude a un haut degre est compratible avec n'importe

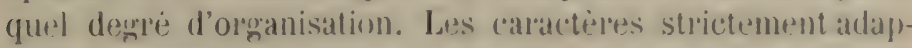
latifs forment la derniere categorie ; leur existence a pour (anse, d'apris lui, les intlurners rextriemes ret la sidnetion naturelle, tandis que le degre de pertection organigure pat dî à unes tendance intermos (vertollommungs-princip). (1)

Eimer a cherehre, principalement par libude: des paphilJons, a decourrir les lois intermes de l'rolution. Voici comment elles sont résumés par Demmert (2): 1. loi gimerale des dessins: Les ligrnes longitudinales sr mansforment en taches, fes lignes transwersales en fointes continurs. 2. Loi des moditiations leneales a direction deterlmine : De noureanx dessins progresseno d'arant en arriere fet de baut en bas on inversesment ; les anciras disparaissent de la même facon. 3. Loi de lat prépourlelance masculine: Gemeralement les miles devancent less

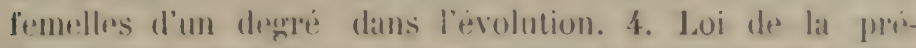
pondiance de l'age: Las nouvelles proprietes se manifestent a l'ige adulte, al l'ejogue de plus grande vigurar. 5. Loi de l'evolution ondulatoire: Pendant la formation de l'individu une série de changements farcourt le corps de l'animal dans une direction determinere li. Loi des évolutions parallèles indipendantes: Des directions evolutives semblables agissent sur des formes non allieres et produisent des phenomenes de convergence. 7. Loi de l'évolution ineigalement avancee : Des caractieres dillërents de: la mime forme perovent se développer à dillerents dregres ot dans des directions diflerentes. $\times$. Loi de l'évolution unilatirale: Les descendants ne representent pas un melange parfiat des proprietes de leurs parents, mais

(1) Cl. Thomas huvt Mokgav op, cit. p. 32i sil.

(2) Op. cil. p. 36 . 
se rapprochent darantage de l'un d'eux. 9. Loi de la regression: les tendances evolutives perwent se retourner et revenir à leur point de départ. 10. Loi de l'arrèt de l'évolution: Souvent l'évolution s'arrête longtemps à la mème phase.

(On peut lattacher aux tendances internes les facteurs téléologiques d'evolution admis par J. Ward: la tendance a la "conservation propre " et la "sélection hédonique, » f'est at dire le plaisir. (1) Nous arons ru que Darwin lui-mene admellait des lois de croissance independantes de tout antre facteur d'évolution. Il entendait par la surtout la loi de corretation, en vertu de laquelle cerlains orga nes varient toujours simultanément.

Weismann a attanue vivement les tendances internes dans Studien sirr Descendenstehre (Isiti) et plus récermment

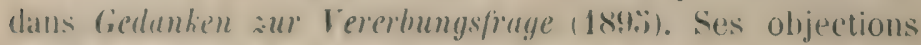

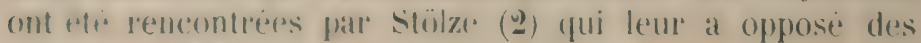
reponses a toutre avis thes salisfaisantes.

La malume des tendances internes reste tonjours fort olscore, mais leme existence nous palait extremement probable.

Théorie de De Vries.

Celle probabiliti se trouve acerue frat limportance de phos en plus gramde altribue fall les biologristes anx

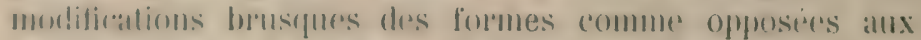

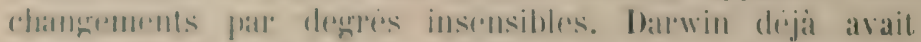

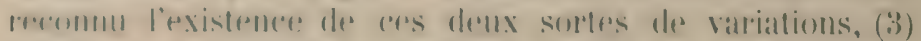

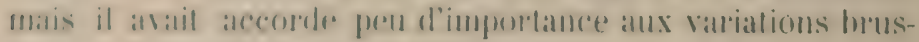

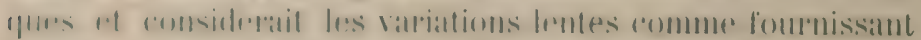

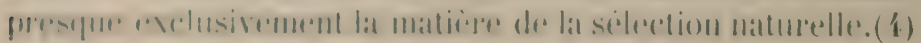

(1) Paluralism and Apmesticism vol. I p. $280 \mathrm{~s}(\mathrm{f}$.

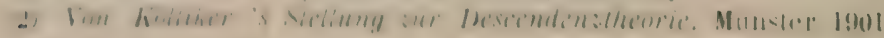
p). 84 s.?.

(3) Juscent of man p. Ilit.

(1) Origin of spuscies 11. wath. 
Lat realite des variationi bruspues of leur influence sur.

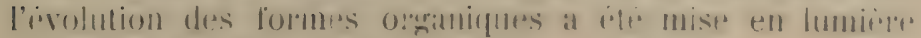

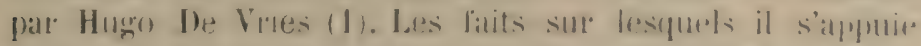

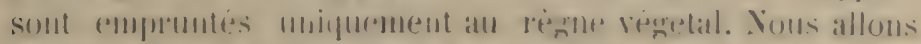
expresep son systeme dans ses grambes ligmes.

Latissant de robe les monlilications qui resultent de. l'hỵ-

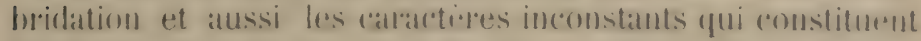

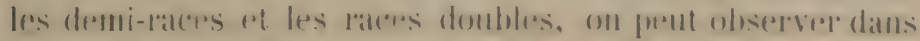

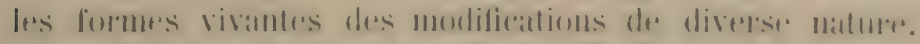
I) 'abord les formes forestollent dans les individus des dilli-

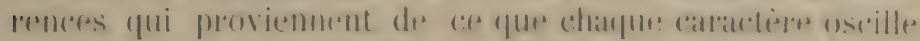

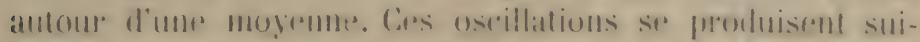

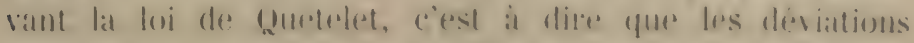

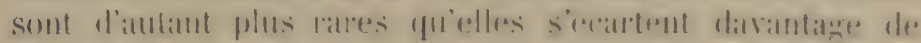

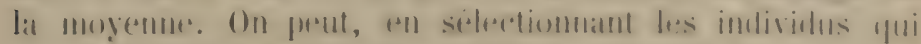

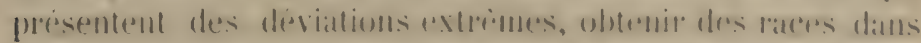

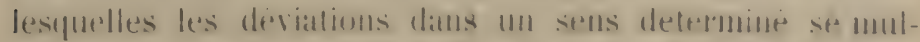

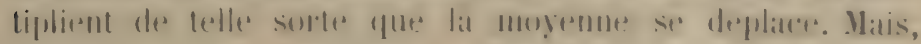

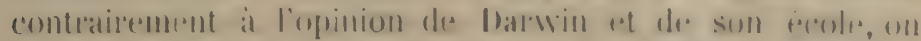

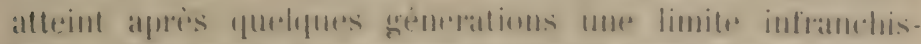

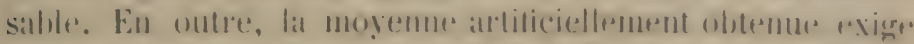

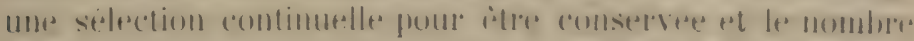

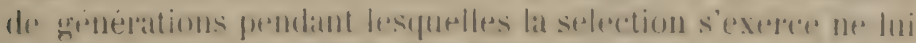

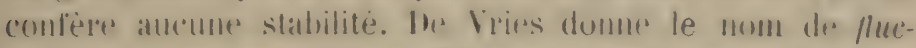

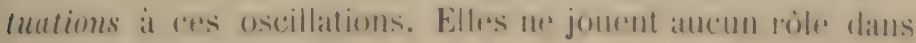
l’évolution des espèces.

Les formes vivantes subissent des dhaggrements phos importants qui portent le nom de mutations. Tandis yue les tluctuations foresemtent tous les denress intermediatpes entre les fertremess, les mutations romstiluent des

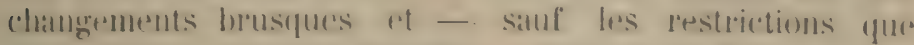
nous alloms indiguer bientrit - elles sont fixess ot se

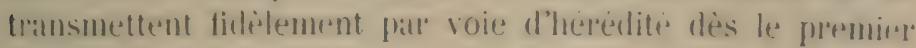
moment de leur apparition.

Lets mutations sont do deux sortes. (2) Les unes con-

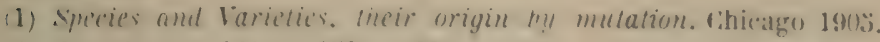

(2) Op. cil. pp. $141-192-224-439$. 
sistent dans l'acquisition de caractires nouveaux qui ne se rencontrent pas climz les ancetres, ni dans les formes voisines. Elles aflectent plus ou moins tous les organes de la plante. Elles sont un pas en avant et réalisent un nouveau type, c'est a dire un: nouvelle espèce vétrétale. De Vries donne à ces espèces le nom d'espèces élémentaires pour les distinguer des espèces systématiques (groupements plus ou moins artificiels arloptés par les botanistes et contenant généralement plusieurs espèces élémentaires). Ces espèces élementaires constituent les anneaux de la chaine de l'évolution.

D'autres mutations domnent lieu à des variétés, soit régressives, soit ataviques. Les premières naissent par la disparition d'un caractire de la forme-souche : poils, épines, coloration, ete. Ces caracteres, neanmoins, deviennent latents, ot ne disparaissent jas en géneral d'une fascon definitive. Ians certaines circonstances ils peuvent reparaitre en tout ou en partie. Ce phenomène donne naissance. aux varietes atariques caracterisees par l'apparition d'un caractire qui n'rst pas reellement nouveau ef qui de fait se petrouve souvent dans des groupes etroitement alliés. (1)

Lexistence des caracteres latents qui peuvent ètre flus ou moins nombreux ef reparaitre plus ou moins complètement, explique aussi l'existence des demi-races et des lacos doubles dans lesquelles des formes dillérentes apparaissent. Trlles sont les plantes qui moditient considrablement leurs organes suivant qu'elles vivent dans l'air ou dans l'eau, qu'elles croissent sous un climat sec ou humide. (2)

lors mutations obrissent anx lois suivantes:

I. Las expeces ciementaires apparaissent soudainement, sans transformations intermédiaires.

II. Les nouvelles formes se: detarhent lateralement de la forme dont elles sont issures. Certte derniope continue

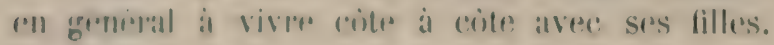

(i) Op. cit. pp. $219-291-218-219-630$.

(2) p. $14 \%$ 
III. Les nouwelles especes possident d'rmbline teur constance parfaite.

IV. Quelques unes des nomvelles lignites sont evidrum-

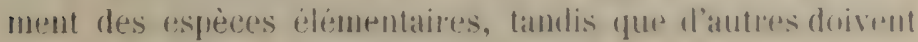
ètre considérées conme des variètès.

V. Les resperes nourelles sont produitres daus un gramd nombre d'individus.

VI. Les mutations nout rien de communn aver: less fluc-

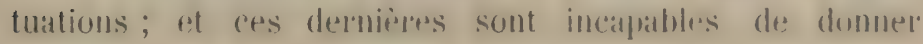
de nouvelles formes stables.

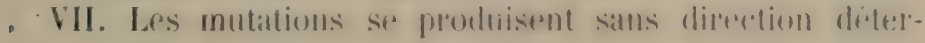
minée. (1)

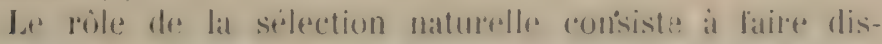
pareatre les formes yui sont inaples on yui somt les moins

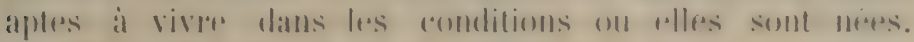

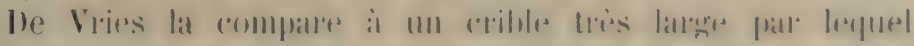

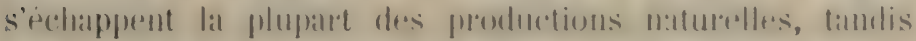

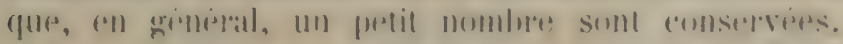

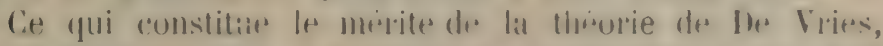

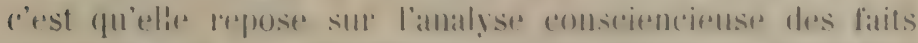
observes fall lui, notamment des mutations de Genotherd

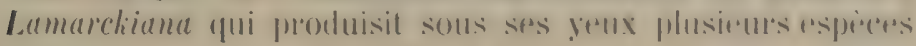

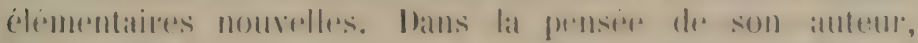

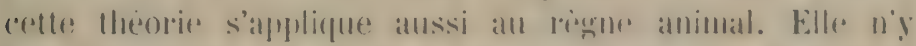

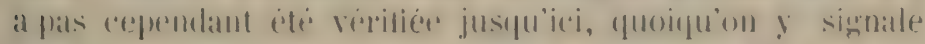

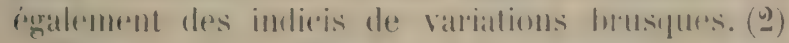

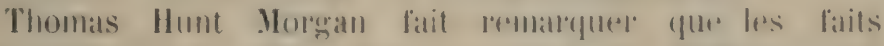

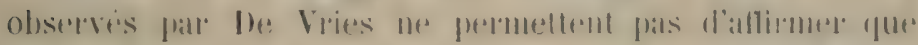

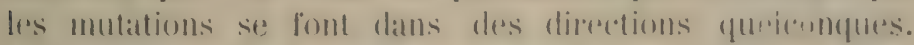

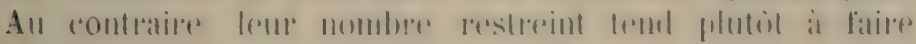
adinettre la conclusion opposie. (3)

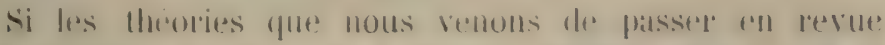

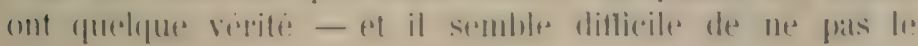

(1) Pp. $588-570$.

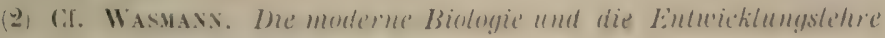
Fribourg en Brisegau 1904 p. 237.

(3) Op. cit. p. 292. 
leconnaitre, - il faut en conclure que l'évolution des formes vivantes ne doit pas itte concue comme n'etant qu'une adaptation continuelle aux circonstances extrereures, ainsi que le roudrait sperncer, mais quau contraire elle? est largement independante de ces cirronstances et gouvernere principalement far dres tendances internes.

Yous avons ansi signale les causes certaines ou probables de l'ovolulion organiques. La conformite de cette folution avec les lois gienerales a ete montres atu commencement du chapitre précedent. Elles est done soumise, de meme que l'évolution dres corps inorganiques, des societiss humaines, des institutions, des langues. des

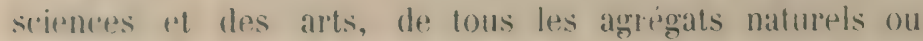
artiticiels at des lois particulières qui ne contredistnt pas

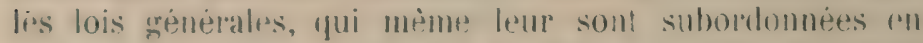
('p) sens que leurs alkets s'y conforment, mais gui n'rn

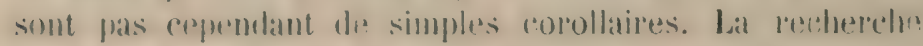
de aes lois particulieres est lobjet des sciencess sperdales:

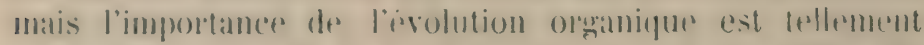
preponderante qu'il atitit impossible de me rien dire de ses lois propres, mime dans une etude ginerale.

\section{\$ III. - IA SELECTON NATLRELLE COMME FACTEUR GÉNÉRAL D'ÉVOLUTION.}

Analyse de la sélection naturelle. - La sẻlection naturelle dans le monde inorganique. - La sélection naturelle dans l'évolution individuelle. - La sélection naturelle dans l'ordre sorial. - Insuffisance essentielle de la sélection naturelle.

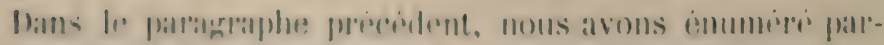

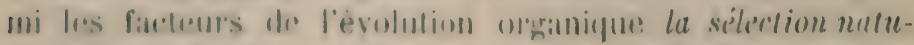

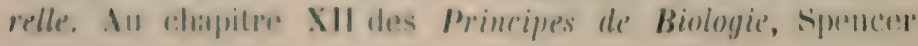


expose son rlicatite, mais th meme? frmps il fait roir, au moins en partie, son insuflisance.

Trop sousent on confond lat selection naturelle arec

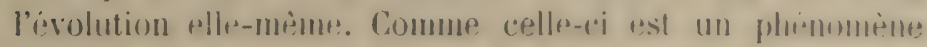

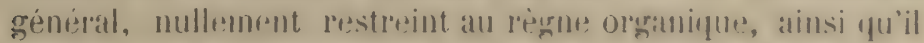
résulte de la presente itule, l'on a parfois attribure il la

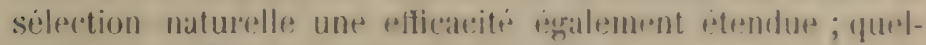
ques uns mème en ont fait la cause uniugue et universolle de toute fovolution. Cor (fur mons avons dit pour lestreindres son roles dans levolution des aspreces virantes sulfit pour ecarter cetter concerption outrese. Masis il me sera pas inutile dexaminere si l'influenere de la setection natupelle doit itre limiter a l'évolution organdigue, on si, au

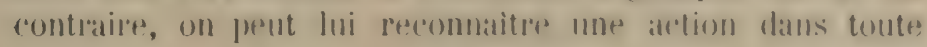
evolution et la rangere, par (a)nsteyurent, parmi les causes générales de ce procès.

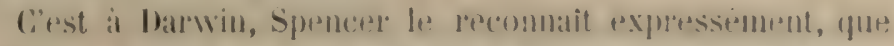
revient l'honneser d'avoir montré l'imprortance de las sielesetion maturelle dans le developpement des ètres vivants. "Lat survie des plus aptes, dit-il, yue j’ai chroche a exprimel en termes de méanique, est ce qur. Darwin a apprels sélection naturelle ou conservation des ranes favorisens dans la lutte pour la vie. Ene operation de ces genpese sedèle

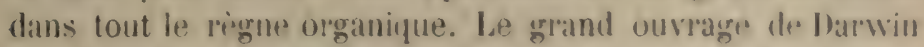
sur l'origine des Esperes l'a proure de maniere a satisfaire presque tous Irs naturalistes. ") (1) Ceppendant, màme dans la derniere sdition des Premiers principes, la sidection naturelle ne tigure pas parmi les causes grenrirales de l'evolution. Sprencer se contente de la signales comme une interprotation particulière du principes d'apress lequel le monvement se fait suivant la ligne de moindre resistance. "Au point de vue dynamique, dit-il, la selertion naturelle implique des changements le long des lignes de moindre résistance. Ia multiplication d'une espèce de plante ou d'animal dans les localités qui lui sont favorables, est une croissance au point ou les forces antagonistes sont moindres

(1) Princ. de Biol. vol. I p. $339 \& 16 \%$ 
qu'ailleurs. La conservation des variétés qui réussissent mieux que leur voisines dans la lutte avec les conditions ambiantes est la continuation du mouvement vital dans les directions vi les obstacles qui la barrent sont le plus fiacilement éludés. » (1)

Ailleurs, spencer considère la sélection naturelle comme un cas particulier de ségrégation : "Par l'opération de la sélection naturelle, dit-il, charue espèce se débarrasse d'une manière incessante des individus qui s'écartent du type commur par des déformations qui les rendent impropres à se pliep aux conditions de leur existenee ; les individus qui les composent restent done à peu près semblables. Les circonstances au milieu desquelles une espèce est exposie, elaut, ainsi que nous l'arons vu, une combinaison compliquee d. forres incidentes, et les membres de l'espèce ayant parmi enx quelques individus qui different plus que d'ordinaire de la structure moyenne requise pour en supporter l'action, il an rrisulte que res forces stparent incessamment de la masse de l'esprece les individus divergents, et conservent par cefle selection l'uniformite du reste, e'est it dires l'intignrite de l'espèce. ». (2)

Qunoinue spencer, dans les passages que nous venons de citer, envisage la selection comme restreinte all règne organipue, neanmoins le fait qu'il la ramène aux lois universelles ot qu’il lui accorde ainsi une place dans la theorie generale de l'rvolution, montres qu’il ne serait pas ćloigne de lui attri-

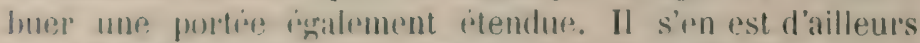
servi explicitement pour expliquer soit l'évolution des socieliss, solt l'ovolution des organismes partiels qui se developfwent an sein des socirtis, soit l'évolution de l'homme qui

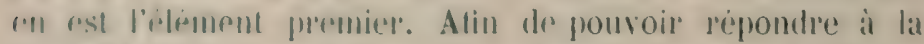
yuestion gut mous nous sommes posere, nous froms l'analyse de lat selection naturelle darwindemne; nous examinerons

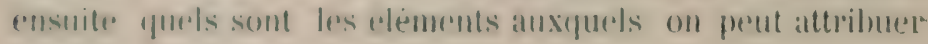
une signifleation générale.

(1) P. P. P. $251-$ P. P. $190 \% 78$.

(2) P. P. P. $207-$ F. P. $382 \& 106$. 
e de la L'evolution des esperes par selection naturells comprend d'abord des cilements négatils : a) la destruction directe ou indirecte (au moyen de ladaparement des dho-es necessaires a la vie) des ormanismes plus faibles par les plus forts; b) Ia destruction des organismes non adaptes an milien fal les forees du milieu; c) la mort des organismes qui naissent inaptes à rexercer les fonctions essentiefles à leentretien do la vie; (l) on pent ajouter, en èlargissant lat conception, la nonfroduction des ormanismes dont l'existence implique queldpue? impossibilité.

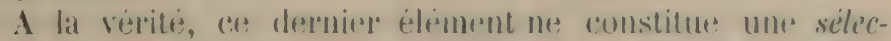
tion que dans un sems laès impropnre. Celltr-ci, "ln eflet,

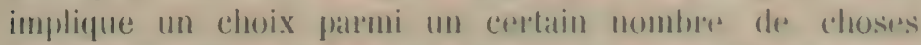
existantes dont les unes sont conservies, tandis pue les

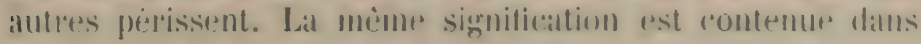
les mots: la survieance des plus aptes. Capmendant il existe

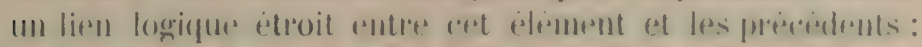
levolution des formes vivantes est limites le la mionte

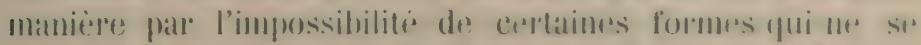

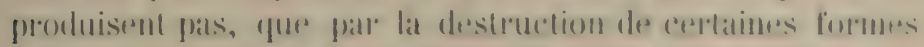

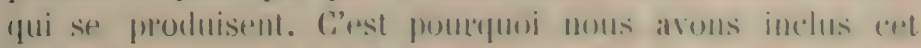

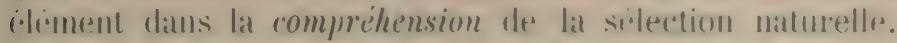

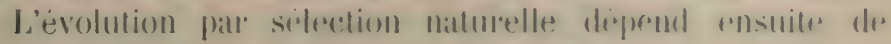
facteurs positifs: a) lat variation eles formes vivantes; b)

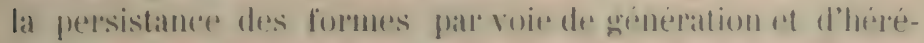
dité; (c) la multiplieation des individus representant les

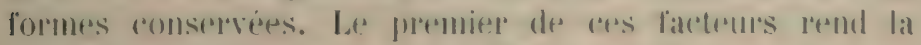
selection possible rn criant les differences; le second assure

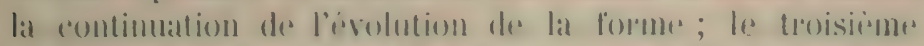

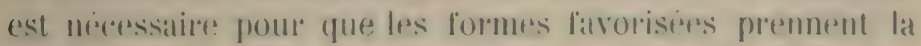
placer dess formes disfarues el pour que la selection trouve matière à s'exercer.

Dans un ordre de choses queleonque, l'swolution pourat soleromplir sous l'empire de la seleretion matmelle dans lat mesure ou les farteurs negatifs at positil's que nous arons énumeres s'y rencontrent. 
La sẻlection naturelle dans le monde inorganique.

Dans le monde inorganique, les agreigats ne possident pas la propriete de se multiplier; ils ne possedent pas davantagre le pouvoir de se perpétuer par voie de géneration et d'heredite. Il est vrai que, dans une certaine mesure, ils suppléent à l'absence de grinération par les frolongement de l'existence individuelle. Yais lorsque celle-ci prend fin, l'évolution est interrompue, et elle ne se poursuit pas, comme che\% les ètres vivants, dans la descendance.

Les agregats inorganiques sont généralement fort stables; leur variabilite est petite. Le proces d'evolution aboutit en général assez vite chez eux à l'équilibre, et souvent leur intégration s'accomplit pendant un temps trop court frour donner lieu a l'evolution composée. Les facteurs positif's qu'implique l'évolution par selection naturelle font donc, en géneral, presque complètement défaut dans le règne inorganique.

L. premier facterur négalif n'est autre chose que la lutte po:n la vie lans son acception la plus rigoureuse. La vie n'existe pas dans le monde inorganique. On dira done, d'une façon plus generale: la lutte pour l'existence. Mais une telle lutte existe-t-elfe entre les agregats de cente categorie? Y at-t-il des cas ou il se produit entre? eux une veritable competition pour l'rxistence? Dans les phrnomènes chimiques, lorsqu'un corps en détruit un antre, il est, en general, lui-mène detruit par ce dermier. si les ferments font exception a cette loi, leur action no ressimble cependant en rien a une lutte pour l'existence. On pourrait peut-fitre imaginer des cas ou certains agreigats absorbent des forces physiques nécessaires a lexistence dautres agregats qui se trouvent ainsi condammes a pritr. Mais le caratere exceptionnel de ces phemomimes, sils existent, leur enteve tout interet an point de vule des theories générales.

Il reste donc, en derniome analyse, la destruction des

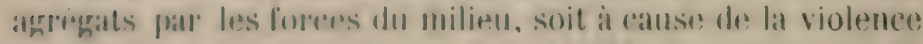

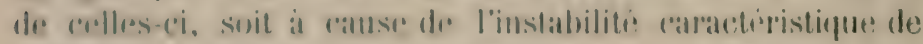

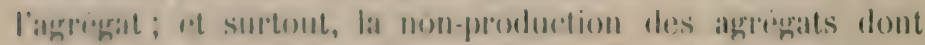




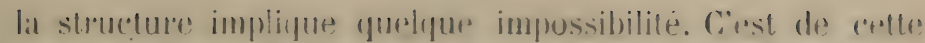

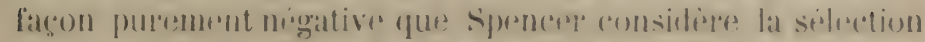
maturelle dans les passideres des premiers principes que nous avons eilés.

guels que soient less itres qui se produsent, il laut quils soient aptess a exister, et plus ils y sont apper, plus

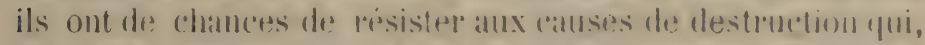

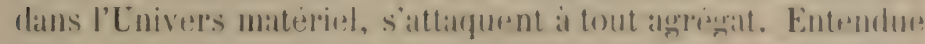

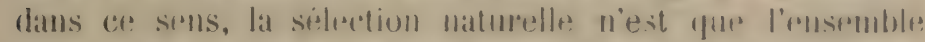

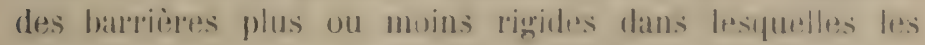
forces do la mature resterment les structures materiolles.

Il y a quelqur trmps, (i. H. Darwin, le fils du gramul

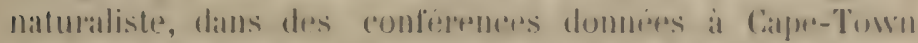

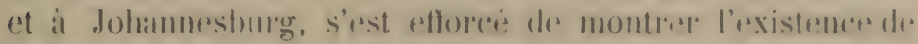

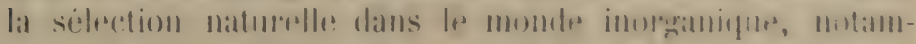

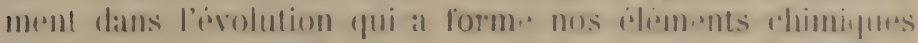

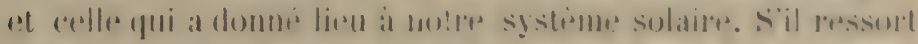

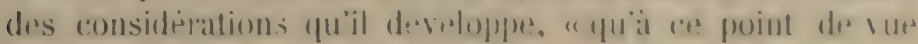

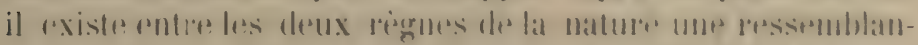

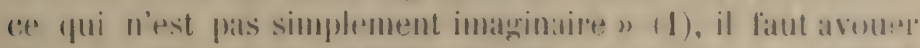

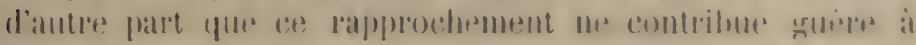

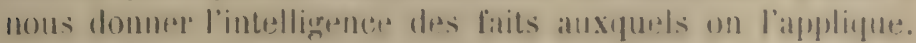

"lomagine\%, dit-il, un soleil autoul dupuel si ment ent

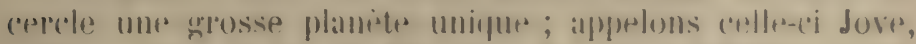

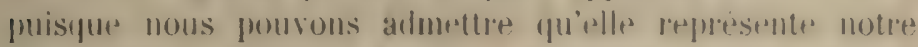
plus grande planite, dupitere. Supposez ensuits quoune

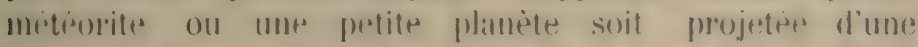

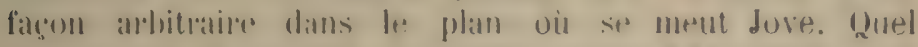

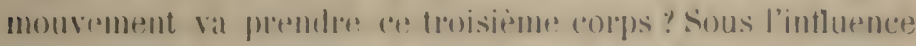
des attractions combinies du soleil et de dore, la metrorite drepira une orhite d'une complexite extraordinaire; tantcit elle irat lentement at unre grande distances du soleril ot de Jove, tantit elle: marehera a une grande vitesser, en pass-

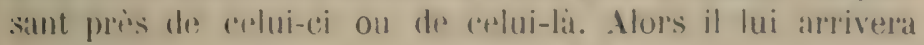
d'achapper a matute catastrophe ; mais il virndrad un monent ou une collision sera inévitable. La carrière de la metrorite

(1) Reproduit dans Ciel el Terre, 1 janv. 1205, p. 517 
sera terminer par alsorption, probablement de la part du soleil.

"Supposons qu'au lieu d'une métiorite ou petite planitte, il y en ait des centaines, se mowrant, au début, dans toutes soites de directions. Comme rlles sont toutes fort petites, leur attraction mutuelle s:ra insigniliante, et chacune dellesse mourra commesi elle n'etait en présence que du soleil et de Jove. La plupart des méterorites seront absorbies par fo soleil, et la minorite entrera en collision avec Jove.

"si nous recherchons quelle sera la durée d'une métiorite donnee, nous constatons qu'elle dipend de la direcetion et de la ritesse initiales, et que, par des dispositions convenables, la catastrophe tinale peut ètre reculée aussi loin que nous voulons. En la reculant infiniment loin, nuus arrive:"ons à la conception d'une métérite rani se meut de facon a ne jamais entrer on collision avec aucui des autres corps.

"II y a done des orbites perpetuelles, dans lesquelles unre meteorite ou une petite planete preut se mouroir iternedlement sans collision. Mais lorsigue nous avons trouse une telle orbite, il nous reste encore i vo le si un eart tris lenger est destine at devenir de plus en plus grand, pour amener entin une collision avede le soleil

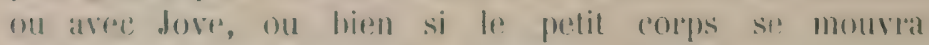

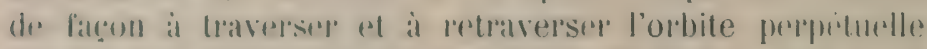

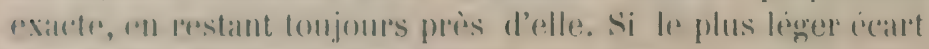
angmenter aree le tomps, lonbite est instiblite; si, atu con-

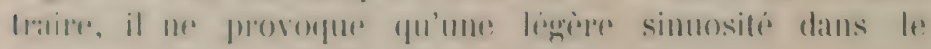
chemin a parcourir, l'orbite est stable.

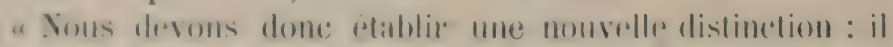

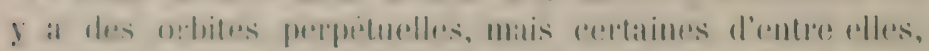

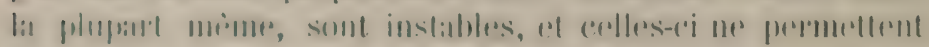

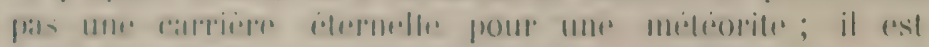

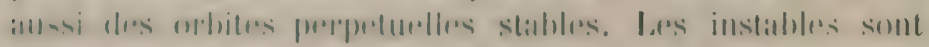

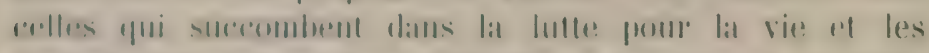

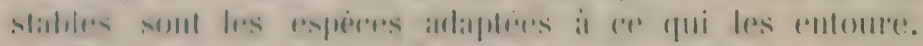

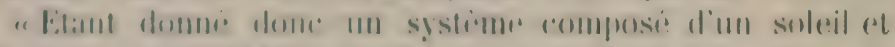

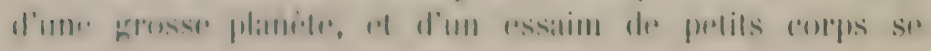


mourant dalls tou es sortes de directions, te solesil ot la planete grandiront par alcerodssement, absorbant peu à peu la poussiere ot les decombres du systime; il ne

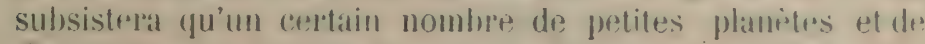
satellites Se monvant dans des orbites determines. Ia résultat final serát un systime plantaire birn ordomné, dans lequel les direrses orbites seront disposions suivant une loi bien définie. »

D'une manioms analogue, la sélection naturesle est invo-

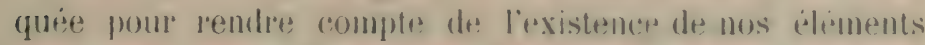
chimigues, dont les atomes, pellse-t-on, sont comploses

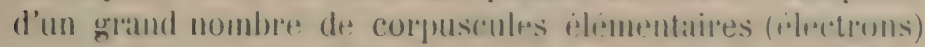
qui gravitent autour d'un centre. "I () a constatis, dit G.

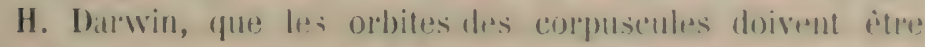
arrangees de rertaines facons bien detromineres pour yur? les mouvements soient duralohes. Ponr ume disension d"un

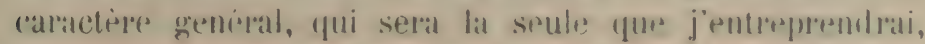

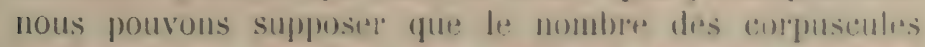
du spstime soit fixe, et nous pouvons admettre qum des nombres déterminés de corpuscultes sont capaldes des'associer en systimes stables de types deatinis.

"Lne intiniti: de syistemes, plus ou muins stables, sunt possibles. Let's corpuscules, dans un systeme, pesuvent accomplir des milliers de révolutions dans lemrs orbites avant quapraparaisse l'instabilite; un atome peut durer longtemps sous une certaine forme, si l'on isalue fro trmpes an millionnirmes de seconde; mais il doit tinalement se rompre, et lps corpuscules doivent se disperser ou se ranger dans un nouvel ordre, apris l'expulsion d'une partie d'entre eux. Nous sommes donc amenés à supposer que les divers aliments chimiques sont formes de systimes de corpuscules qui ont, par leur stabilité, réussi dans la lutte pour la vie. ” (2)

L'existence de la multitude des météorites ou des petites plantes a orbites instables, de mème que colle d'atomes ¿ structure fragile est une hypothise à peu près gratuite. si

(1) Ibid. 16 mars 1906 pp. 4 \%

(2) Ibid. 16 janv. 1906 p p. 341-312. 


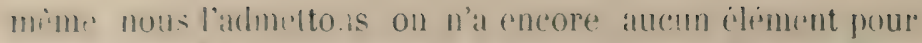
expliquer: 1" l'existence des structures qualles qu'elles

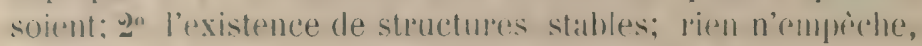

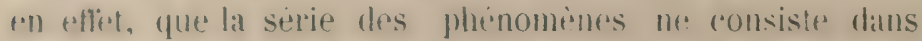
une sione indélinir: de structures instahles; 3n l'uxistence de reptaines structures stables de prefirence a d'autres.

Si nous supposons que les forces de la mature ont determini d'emble les stmetures stables que nous comnaissons - hypothese aussi rasasemblahle que la precedente - il n'y en a alcun choix et, par conseguent, allemes setection naturelle dans le sens propre du mot.

Aristole attribue quelque part aux anciens materialistes colte conception : que: d'innombrables combinaisons d'atomes scibunt formees far hasard, senles les combinaisons stables

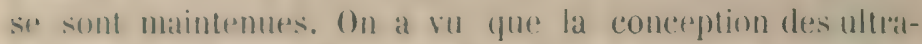
darwinistes modermes ne va gruipe plus loin. I conside er

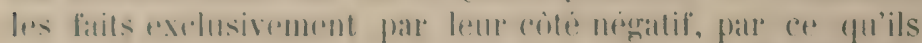

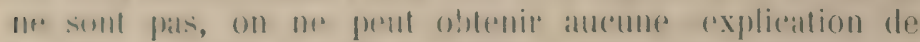
la forme quöils revelent. Toute structure determiners

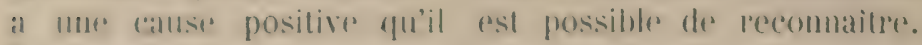
Lompossibilite d'un certain nombere d'antres structures

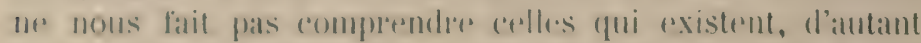

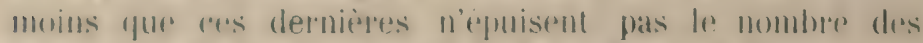
strueturess possibles. Le hatsald n'est pats unte caluse, mats

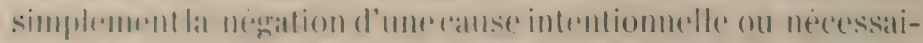

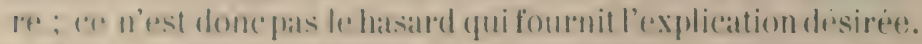

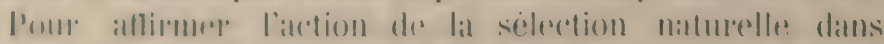

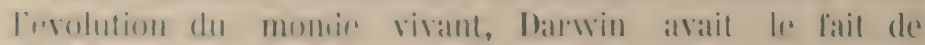
la dispartion d’un gramd nombere de formes dont les lestes

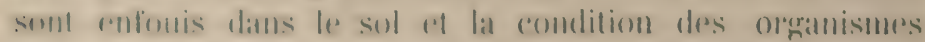

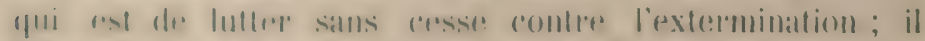

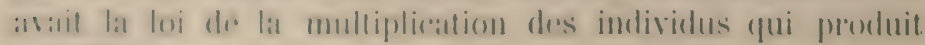

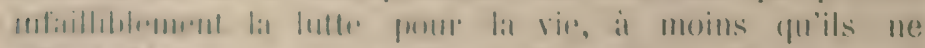

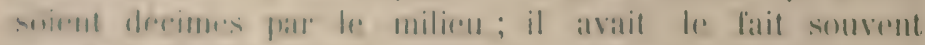

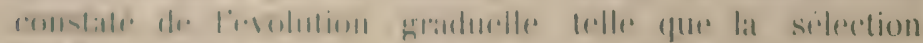

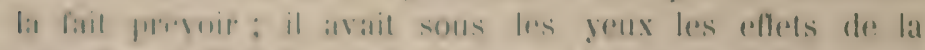

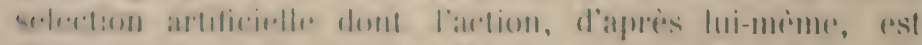

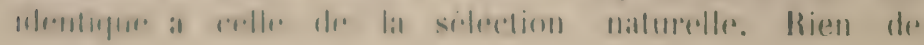




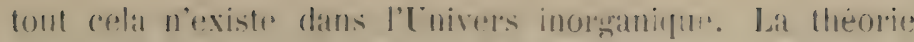

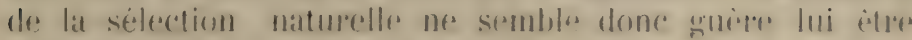

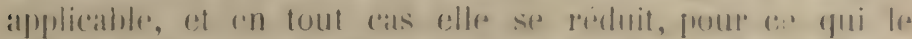

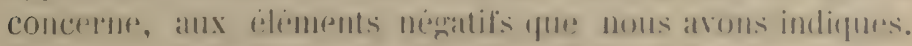

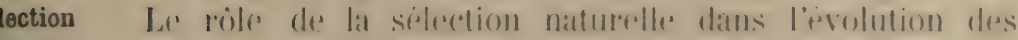
irelle

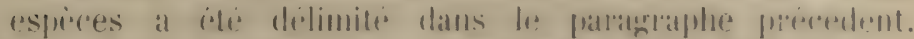
Nous n'y povendrons pas. W. Rous s'est domme prour

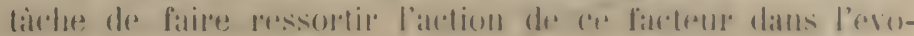

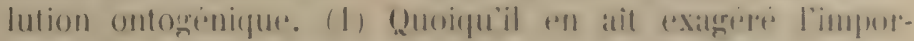

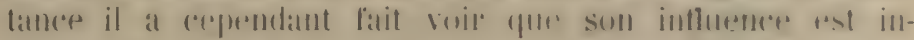
rontestahle. Roux considere sucersivement lat lutte des

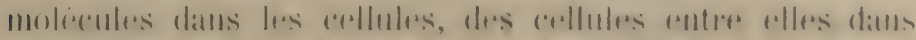

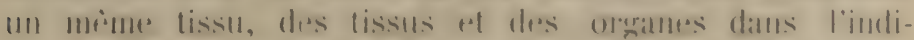

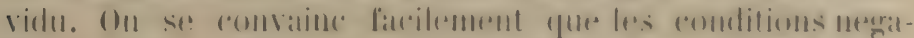

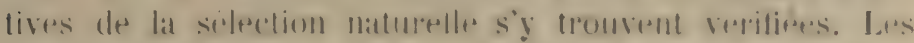

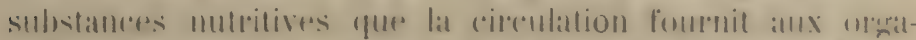
nes, lissus, cellules, molecules, pour se constituer ou se

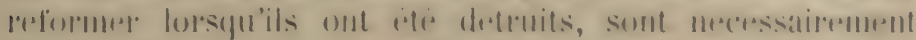

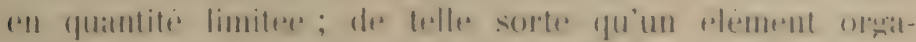

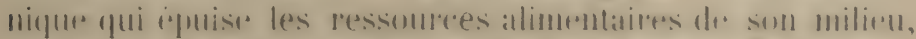

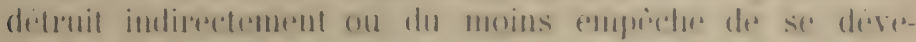

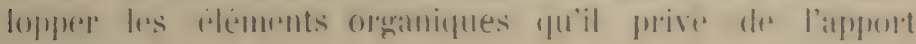
nécessaire.

Daus la pluprant dess as aussi, la plare dout disposent

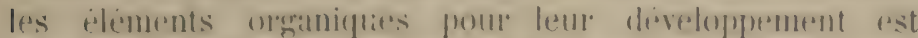

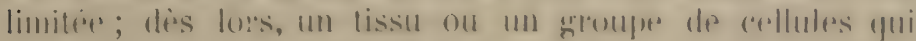
eroissent atdivement emprobent les antres moins adives

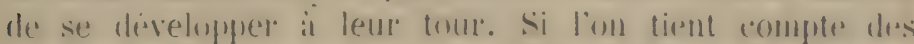
fonctions quacomplissent les detres vivants, des mourr-

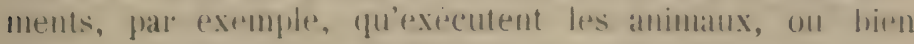

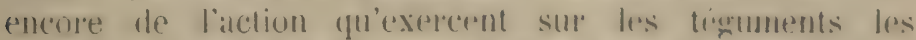

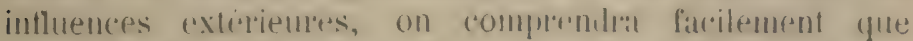

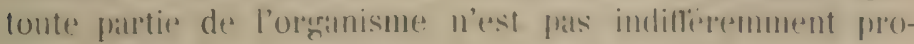

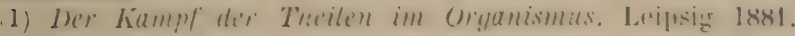


pice à l'existence d'un genre de cellules ou de tissus determine et que les élements organiques, tout comme les etres vivants eux-mèmes, sont exposés à subir l'action destructive du milieu.

Les facteurs positif's de l'erolution ne font pas non plus défiut. Les variations des éléments organiques (moleculrs, cellules, tissus ou organes) sont continuellemrnt produites par l'action des forces incidentes qui dilfierent d'une partie a l'autre. Non pas que l'action de ces forces sulfise pour expliquer la structure qui s'engendre sous leur influence; mais les diflèrences de développement ont comme cause excitante la diffërence des stimulants. Ciest ce quion a appelti laction morphogène des excitations fimctionnelles. Elle peut être considéréts soit (ommme determinant les variations des elements organigues, sojt comme favorisant la multiplieation de certans éléments au depens des autres.

Les élrments orgamifues jouissent aussi du pouroir des saccroiter ou de se multiplire. Si l'on se contente d'envisager les eflets de la lutte entre les parties dans l'orgranismes individuel, cetle multiplication des cellules de mrine: struclures représtontera la transmission héréditaire dans la plyglogenie. Mas si lon admet que les caracteres acquis dans cotte autoformation de l'organisme gràce à lat lutte des parties peuvent eux-memes se transmettre par voir d'hridite, les eflets de cefte lutte se continueront a tratrers les grenéations successives, controles sans cesse par la sifrection naturelle qui supprime les variations nuisibles. (1)

Yous avons deja litil olserver que la lutte entre les farties ne perut pas remplacer l'hereditio dans le développeranrut ontureniçur. Ia choses est évidente, si l'on songe a l'ardieverment de la structres specitique qui s'accomplit formban la, vir uterine oil l'artion des agents excitateurs

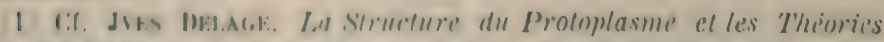

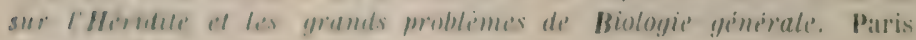
149\%, p. 721 . 
est réduite au minimum ot où, pour les cellules, les causes de variabilite independantes de l'heriditi sont peu importantes. Le lacteur mis en lumiere far W. Roux est au contraire indispensable pour l'intelligence de l'evolution strictement individuelle, c'est à dire non hèréditiaire.

lection arelle l'ordre cial.

Examinons te ròle de la sélection naturelle dans l'evolution sociale. Losqu'elles sont prospères, les societes humaines, de meme que les ruches d'abeilles, essaiment, cest à dire qu'elles possident une froprieté analogue à ce qu'est la multiplication des individus dans le règne organtique. Mais mor diflërence importante a notre point de vue entre la génération qui produit anes nonvelle plante on un nouvel animal al la colonisation qui produit une nowvelle societr, e'est que lat loi d'heredite qui produit lat lessemblances des descendants arec leurs patrents est bien plus rigoureuse dans le monde organique yue dans lordere sucial. Sans duute, il y a des chances pour que les colonies copient plus ou moins tidleltantent les institutions de la mere-patrios ; mais l'histoire des colonies espragnoles, anglaises, et dans l'antiquité conlles drs colonies grorouges nous appremel yur, de firt, il pxiste soment de gramdes differentes dans la forme du gouvernement et dans tous les antres éliments de l'evolution sociale? entre les cites-filles at lat metropoles. Lá colonisation n'issule donc pas la continnation de l'évolution garaluelle par silection naturelle comme le fait la génération des ètres vivants.

D'autre part, la doree des societes et leur variabilité sont sultisintes pour que, a cet egrard, la seilection trouve matiene a sexcercer pendant leur evolution.

La lutle pour l'existence entre les societés humaines existe incontestablement, soit sous forme de guerre, soit sous forme de concurrence industrielle et commerciale. Outre les entreprises auxque'les alles sont m luutte de la part de leurs voisines, les sociétes humaines ont a lutter, surtout 
dans leurs phases primitives, contre le milieu: le climat, le manyue de nourriture et mème les animaux saurages. Toutes renferment en elles-mèmes des causes d'instabilite plus ou moins redoutahles. Enfin, certains types d'organisation sociale, telle la république de Platon, sont irréalisables.

II faut done admettre que la sélection naturelle exerce tinn influence rérlle sur l'évolution sociale en conservant les sociétis les plus aptes à vivre au dépens des autres.

Yous renons de parler de l'évolution de la société comme telle; on peut se demander aussi quelle sera l'intluence de la sietection naturelle sur le développement de l'homme au sein de la societé. Que la lutle pour l'existence entre les individus exerce frar selection une influence sur l'evolution du tyge: humain, e'est ee que probablement tout le monde admettra. Hais la question mist de saroir si lat stelection obtenue de: celte maniere est la grande loi de progress. spencepe (1) pretend limiter la bienfaisance par la preocenpation de un pas contredire la loi naturelle, e'n vertu de

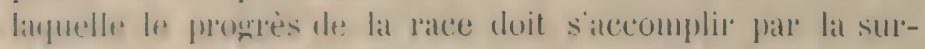
viramce des plus aptes. Co resultat sera alleint, d'apress lui, si on laisse lat lutte pour lexistence se poursuive, tandis yu'il sera compromis si l'on aide le's incapables et les dejgenérés à survivre et à se multiplier.

Rromarquons rol passant que s'il n'y a pas de liberté, lat tendanee en vertu de lauguelle l'homme a pitie des miserables et les aide, est aussi hien une loi maturelle que

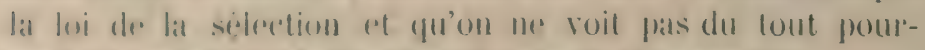

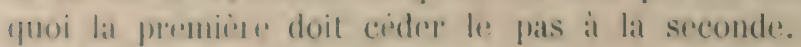

Latissant colit, il n'est pats dilficile de se ronvainere que lat loi de la survivanere, dans la societe humaine, we vise pas tonjours - tant s'en fant - les plus aptes. Lass individus d'une esprice andmale, visant dans des conditions ingale's

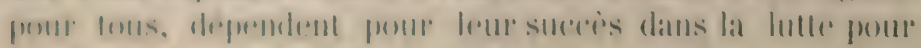

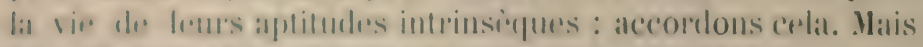

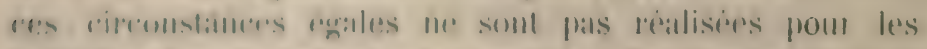
individus humains dlans nos societies; le succies danns la

(i) Bicnfaisance posilive, ch. VII \& 458. 


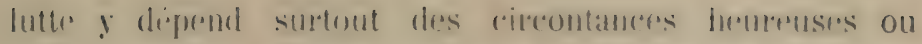

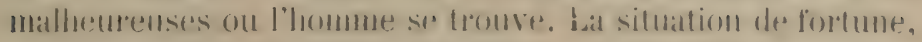

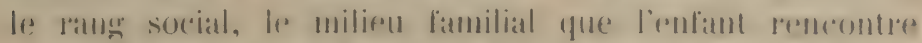

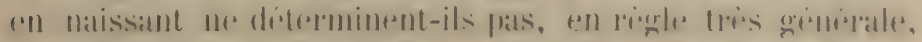

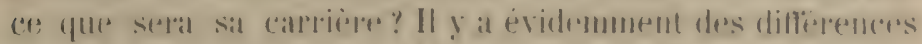
frovernant des aptitudes individurlles, ot lon woit pardois

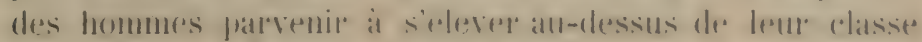

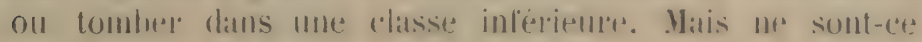
pas des exceptions?

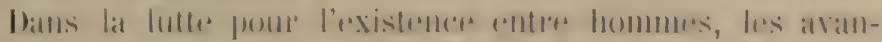

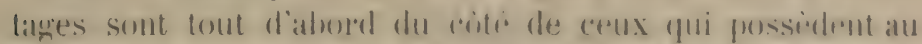

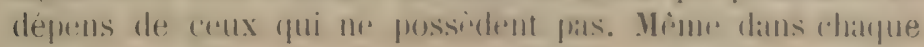

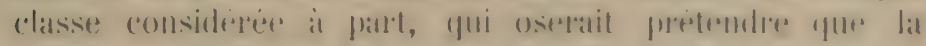
victuire appartient toujours aux plus intolligents ed aux plus

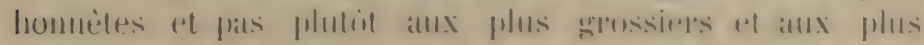

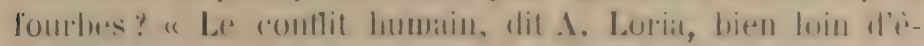

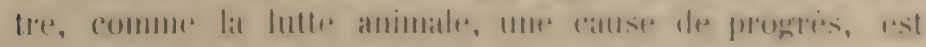

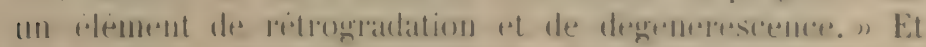

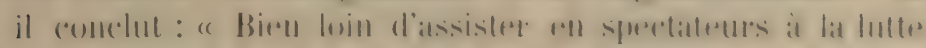

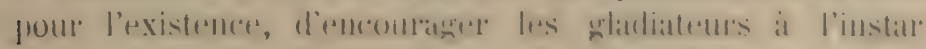

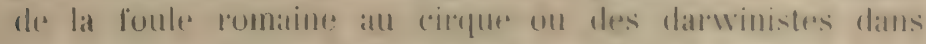

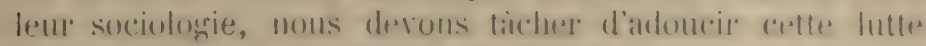

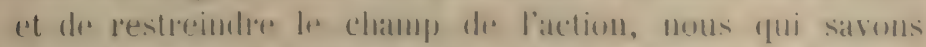

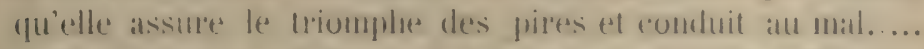

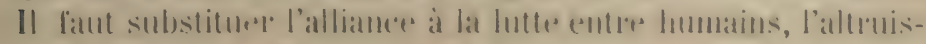

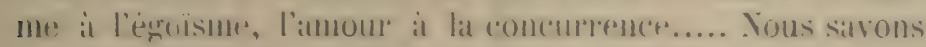

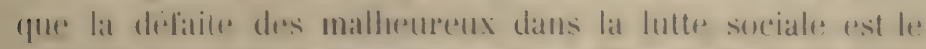
risultat der milien exenomigue et non d'une inferiorite natu-

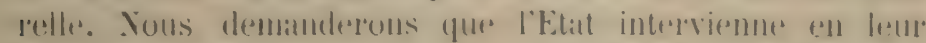
favent et allinute all moins les cruatutes dont ils sont les innocentes victimes.» (1)

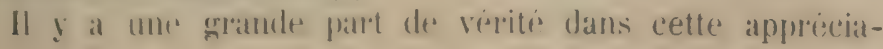

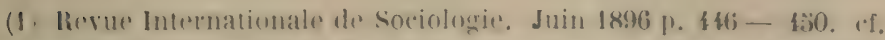

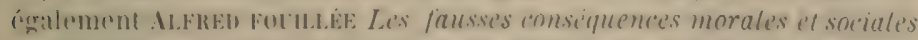

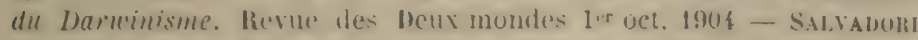

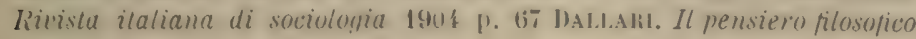
di $H$. Spencer. Turin 1904 p. 36. 
tion, assez pour aftìmer que l'évolution progressive de l'homme au sein de la société n'a pas ou du moins n'a guère pour facteur la sélection naturelle.

On ne rifute pas cette critique en faisant remarquer avec Saleeby (1) que la formule spencérienne de la selection naturelle aftirme la survivance du plus apte et non pas du meilleur. Il n'en est pas moins rai que la marche générale de l'évolution organique a été progressive al que ce progrès est consideré par Darwin et par spencer comme dù en grande partie, par d’autres comme dû uniquement à la sélection naturelle. Il est certain aussi que, d'ajprès cux. la marche générale de l'humanité sous l'action de la sélection naturelle doit-ètre progressive et aboutir à la multiplication non seulement des plus aptes a vivre, mais aussi, en general, des plus intelligrents et des plus moraux. Or, c'est précisément l'insuffisance de cette conception que nous avons montrie, lorsiqu'on l'applique a lia societe dans laquelle nous vivons.

Insuffisance ssentielle de la sélection naturelle.
Atin d'apprecier exactement l'influence de la sélection naturelle sul les différentes formes d'évolution, il faut se rappeler que, par sa nature meme, elle favorise exclusivement la diveloppement des caractères qui ont une importance décisive dans la lutte pour l'existence ou dans la survivance des plus aptes. Flle est donc incapable - comme nous l'avons dejà fait remarquer à propos de l'exulution orranique - d'expliquer le développement des caractioes qui nont pas d'utilite selectionnelle, cent a dire qui mrenfiremt pas a l'agregat une probabilité effective plus grande de survie.

En outre, dis y gion admet l'existrnce de facteurs d'evolution andres nume la sianction maturealle, il est possible que crotains ranactiress utiles soient fixis et developpes indepren-

(1) op, cil, p. $2 \times \pm$. 
damment de cette derniere. Dar exemple, on admettra sans difficulte que le progrès scientilique confire il une nation des avantages dans la lutte pour l'existence sur celles qui en sont mivées, soit dans la gurerre, soit dams la concurrence industrielle. Cependant persoune n'altribuera raisonnableInent à cette circonstance le progrè̀s scientitique qui a ite réalise dans les nations europremes pendant les dernieres siècles. Ce n'est pas flar la survivance des nations les plus savantes que refte evolution s'est produite. Cor n'est pas davantage par une selection qui se serait exerere all sein de chatgue peruples an faveur des hommes de science. La science ne se transmet pas par roie d'hriedite, ni meime,

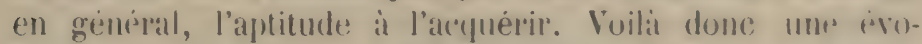
lution (qui aurait pu - alssolument parlant - se realisere

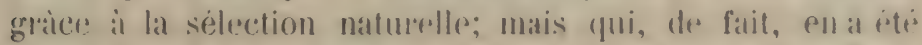
inclependante. Il faut done protestere contre le masonnement simpliste qui consiste a conchure dre l'utulitio d'un caralelore at son developpement par siededion maturelles.

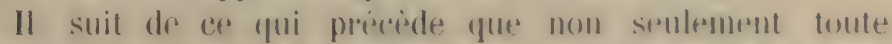
evolution n'est pats attrobable a co fitcteur, mais encore

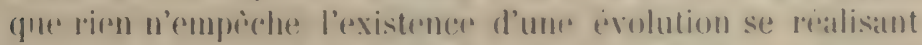
dans un sens oppose a celui que la selection natturelle

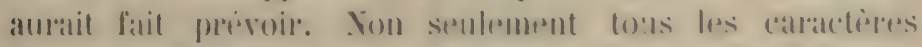

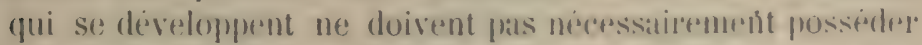

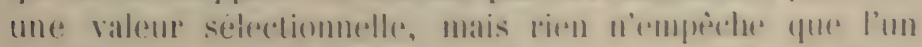
ou l'autres ne soit nuisible an posint de rue de lat lutte gum l'existeme. Il sullit gute les agrégats dams lespgurls

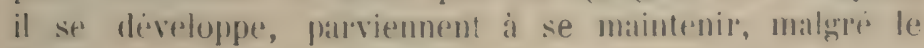

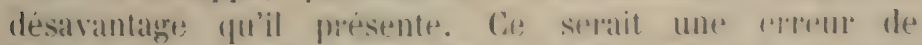
s'imagrincer quer l'appatrit on d'une inleriorite dan: la lutte pour l'rexistence entraine nécessairment et sans retard lextermination des itres aho lespurls cotte inlérionite existe. En attendant qu'ils suecombent, le camadelèe desalvantageux peut avoir tout le loisir d'evoluer. Ln tel aractiere

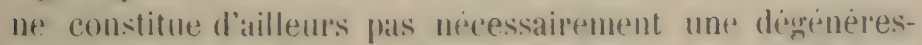
cence, pas plus qu'un carantère avantageux dans la lutte pour la vie ne constitue nécessairement un progrès au 
point do vue de la perfection intrinsèque de l'stre qui l'accuiert.

Les rétlexions que nous avons faites sur le rile de la sélection naturelle dans les formes principales d'évolution, seront facilement appliqueres par le lecteur aux autres formes salls qu'il soit necessitre d'y insister. Nous fourons conclure ugue la siflection naturelle, étant un facteur complexe, expree d'une facon ou d'une autre son intluence datls toute évolution; jamais cependant elle nest le fircteur uninue et souvent son influence est très secondaire. 
R:HAPTRE VI.

\section{\$ I L'EOLHLIBRE ET LA DISSOLLTTON}

L'équilibre absolu et l'équilibre mobile. - L’équilibre et l'évolution. - L'équilibre et l'adaptation. - Formes variées d'équilibre mobile. - La loi d'équilibre a priori. Relation entre l'évolution et la dissolution. - Etude inductive de la dissolution. - Dernier probleme.

Il nous fant maintenant repmendere les Premiers Principes, et apres avoir étudie l'erolution proprement dite. nous occuper du procès de dissolution.

[uilibre iolu et uilibre obile.

L'irvolution, d'apmes Spencer, aboutit necrssairement it l'équilibre, que ip soit te repos absolu ou absence de? mouvement, ou biren la stabilités du mouvement, cer qu'il appelle l'équilibre mobile. Il 'll donne comme exemple la phase du mouvement d'une toupie ou, dressée sur sa pointe, ellp tourme aver rapidité sans changra de place ni balancer.

Dins l'enuilitre mobile il y a toujours quelque chose? d'immobile: soit le centre antour duyuel s'accomplissent les mouvenents, soit la situation moyenne dont ils s'éartent alternativement de pallt ot d'antre, soit une relation quelconque qu'ils présentent à un éément invariable, comme l'adaptation de la vie a une situation détermine'e. 
Le repos dont il s'aunit n'est que l'absence de mouvement visible, car le mouvement invisible existe inévitablement ct constitue lui-mème, lorsqu'il n'augmente ni ne diminue, une situation d'équilibre mobile. En outre, ce qu'on considere comme repos n'est souvent que l'absence d'un certain mouvement visible, la perte de tout mouvement de cette sorte n'itant - du moins actuellement - jamais réalisè dans la nature.

II suit de li, - et ici nous nous écartons de la manière de, roir de spencer - que la situation d'érquilibre n'exclut par la continuation de l'évolution. Equilibre, par soi, dit absence de clangement, tandis que évolution, par soi, dit (hangement; a cette igrard ces deux situations sont incompatibles. Mais comme l'équilibre, mème celıi qu'on apprelle: absolu, n'est jamais que relatif - ainsi que nous venons de le dire - et comme un être peut ne pas subir dre changements à un point de vue et en subir, au conIratire, a d'autres, rien n'empèche que l'évolution d'un agrergat ne se poursuive lorsqu'on $y$ a constate à certains intards more situation d'erguilabre. Tout re que l'on peut dire, cest que son ivolution est arretée dans la mesure oit l'requilibre existe. On verra aussi par la suite que l'on considere souvent comme itant en equilibre une situation qui ne se moditie que tris lentement.

Comme preuve generirale de sa thise, Spencer signale la condance de tout mouvement visible ou de toute canse dre mouvement visible à se dissiper, c'est a dire à dispamatre pour itre remplace par du mouvement invisible uniformement ripandu. Cette consideration très grinerale montre hiren que l'lnivers, dans son enspmble, tend vers

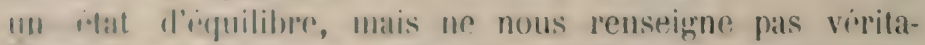

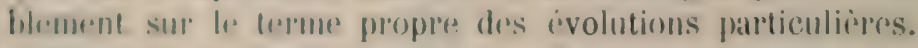

Dans l'roblulion des angremals inorganiques, commo les

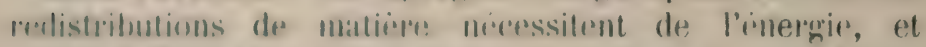

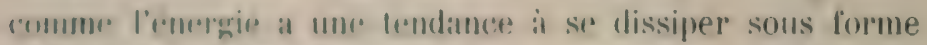
moleculatire intransformable en mouvement visible, le

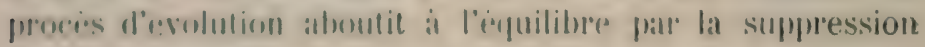
the ce mouverment. 
Mais dans les cas d'erolution naturelle ou le procès d'intigration procure a l'agriagat l'enerergie necessalire pour compenser les pertes, par exemple chez l'animal yui vit gràce à la nourriture qu'il absorbe, la mime consépuence ne s'impose jas. Il est vrai que l'incruge transformable disparaissant da longue dans l'mivers, l'animal luimême serait en tous ras rnveloppe dans Is repos universel, mais ce n'est point lia, ni rn thiorie ni en fait, l'ahoutissement de la forme particulière d'ivolution qu'il réalise.

L'organisme vivant, lorsqu'il ast arrive à l'àge adulte, présente un cas d'équilibre mobile. Spencepe le qualitie de dépendant, parce que son existence dépend de l'enerurie qu’il regoit du dehors; landis qu'il appelle indéprendunt

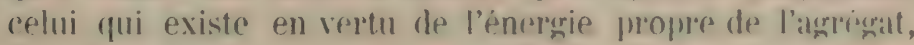
par exmule, la situation acturlle du systeme solatere.

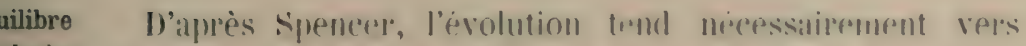

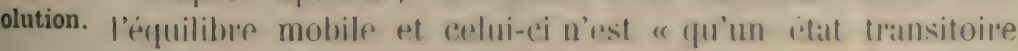
destine à aloutir à l'éfuilibre complet, ) (1) cesst à dire an repos. Ia premiere partier de cette atrimution est demontrie de lat manière solvante: "Le fait que nous devions surtout remantuer, cest que, comme conséquence de la loi d'ingulibres dejai posee. l'ivolution de tont agrégat doit marcher juspu'd t'e que l'éguilibre mobile soit ritahli, moisque, ainsi qur nous l'arons vu, l'excres de forese que l'agrengat posside dans une direction doit, en drifitive, so depenser à vancre les resistances an dhangement dans cette direction, ne laissant apres lui que les mouvements qui se compensent muturallement, de maniere a former un équilibre mobile. Quant a l'etat de structure qur l'agringat acyuiert on meme temps, il faut évidemment qu'il prisente un arrangement de forces qui contrebalance toutes les forces pal' lesifuelles l'agrégat

(1) P. P. P. ระอ $\& 170-$ F. P. p. 39อ. 
est sollicite. Tant quil restr une foree rom excos dans une direction, qu'elle soit rexerée par l'agrecent sur les partirs qui l'entourent, ou par res partirs sur l'agrigat, l'repuilibre nexiste pas et jar conséquent la distribulion de matière doit continuer. Il en résulte clur la limite de l'hrlérograneite vers laquelle tout agrengat mogresse, cest la formation d'autant de specialisations et de combinaisons te parties qu'il y a de forces spredaliseses et combines à équilibrer. ” (1)

Pour que ce raisonnement tienne, il faut: l" que l'énelgrie qui est dépensée à vancere les resistances au changement dans une direction ne soit pas continuellement remjlacrep pall l'inergie venue du dehor's; go que les forces areclesfurelles l'etat dr structure est en harmonie, e'est à dire lesfor-

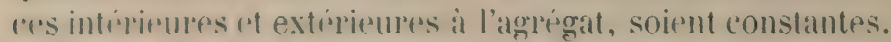

si lat première de ces derax conditions n'est pas réalisiee, l'evolution prut mancher indétintivement dans un sens destermine, en si la seconde fait defaut, la pedistribution de

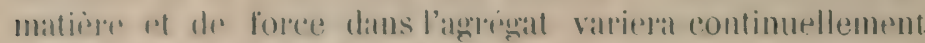
et l'on n'aura jamais la stabilitis du mouvement reguise pour l'équilibre mobile.

Les considerations inductives permettront de mirux comprendere lat portere de cers remareques trop alsomates.

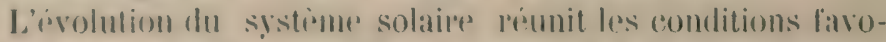

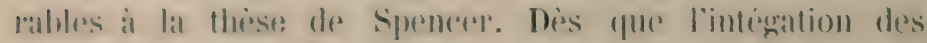

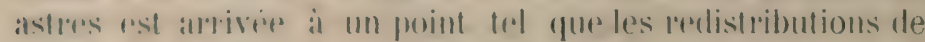

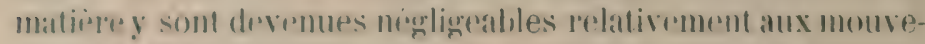

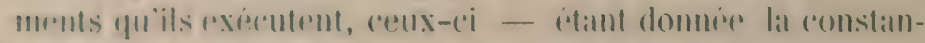
c. Ure lat loi d'attraction - ne subiront plus d'autres chan-

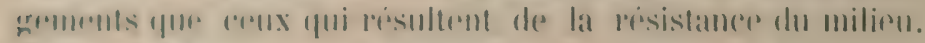

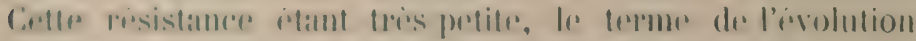

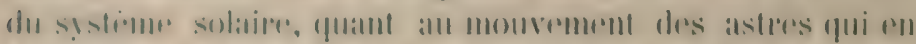

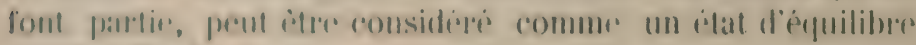

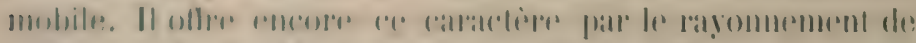

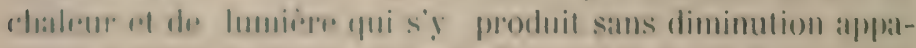

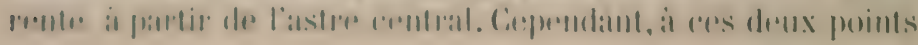

(1) p. P. p. $898170-$ F. P. P. 831:3. 
de: vure, l'bal d'enpilibre est transituire : la résistanef du

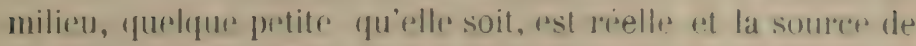
la chaleur solatre n'est pas incpuisable; les mouvements vi-

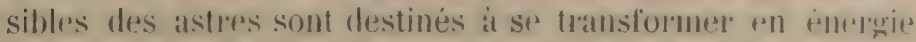

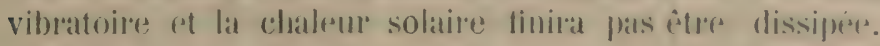

Lál terre présente dams sá forme une situation d'iapuilibre ahsolu, si l'on fait abstaction des changements superliciels - peu considerables lalativement al sal masse - qui sont

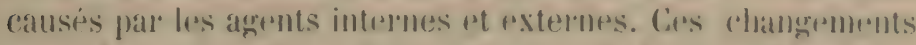

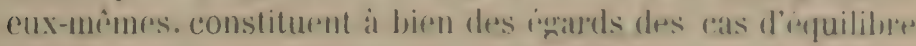
mobile: : tels, la malrolu de la tempresature, la circolation

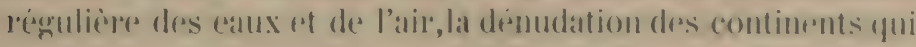

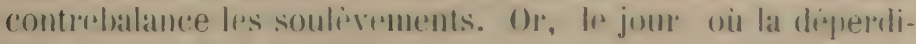

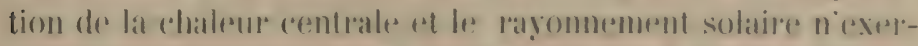

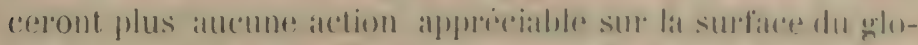

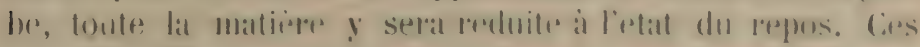

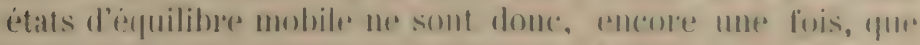

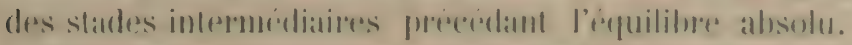

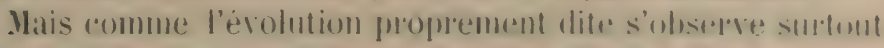

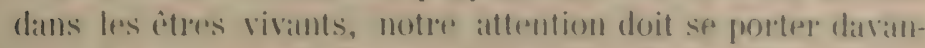

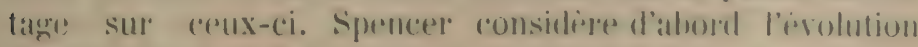

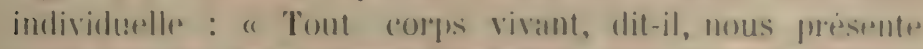

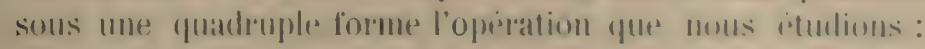
a dangue instant dans le balamerment dess forees meand-

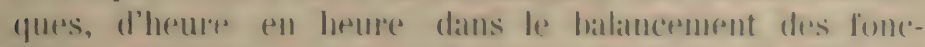
tions, d'annere en annie dalls les changements d'itat qui compensent les changements de (ondition, el timalement dans l'arret complet du mourement vital, dams lat mort. " (1)

Latissons de cote four Ir moment l'apquilibere qui existe

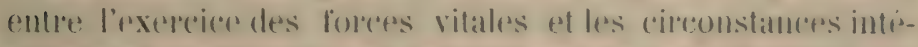

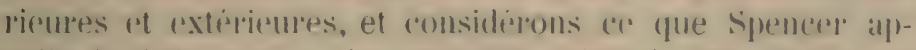

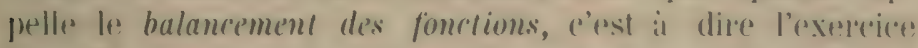
des fonctions vitales dans l'organisme adulte gràce at

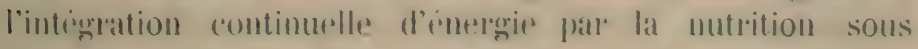
toutes ses formes. Nous y trouvons un bon "xemple d'equilibre mobile : la structure no se moditie pas d'une fagron

(1) P. P. p. $833 \$ 17 \%$ - F. P. p. 402 . 
appreciable, les pertes de matire sont sensiblement compensérs par les gains, l'usure des organes est réparée et la drepense d'énergie est contrebalancée continuellement flar l'absorption de l'énergie extérieure.

Mais si l'on so demande dans quw sens cette situation est le résultat de l'évolution, la riponse qui s'impose est yu'plle dépend presque entièrement de l'héridité et dans une faible mesure des circonstances particulières dans lesquelles s'est développé l'individu. Or, comme dépendant de l'hérédité, c'est à dire comme stade d'évolution de la forme à travers les generations successives, la forme adulte n'est plas flus un starle terminal que toutes celles qui l'ont prééélée : elle rst le stade actuel Ir l'rvolution dans une certaine dipection, mais on n'a ancune lasison de la considerer romme: un aboutissement. las forme adulte n'a cer caractere ques pour l'individu et commme celle n’a pas sa rasison d'ritre dans l'évolution individurlle, il n’y a point de raison de la considerer comme: un trime vers lepuel mache le procès évolutif.

II stmble plus juste de considerer l'blat d'equilibre de faulultre commo: la coüncidence de l'ivolution individuelle avec: rivolution de la forme. En d'antres termes, l'épuilibre "xiste dies que l'individu a atterint le state de divoloppesmont acturllement réalise par l'sspece. Dès lors, l'otat adulte comme tol. e'est à dire comme situation d'épuilibre, n’est pass determinc par l'évolution individurlle. Yous dirons ilome que relle evolution presente dans l'etal adulte

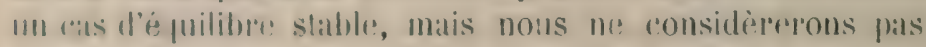

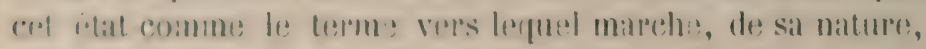
rente: évolution).

In examen atlentif ne nous permet pas non plus de

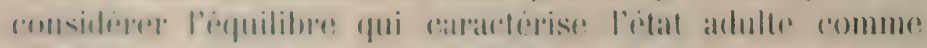

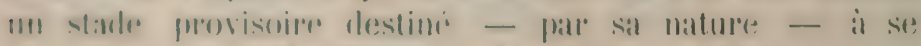

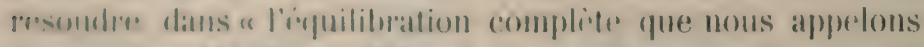

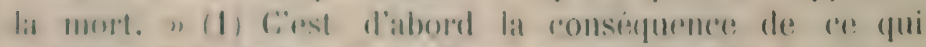

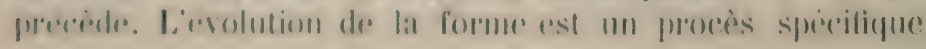

(1) P. P. p. 828 || 171. 
qui se pournuit à travers les génerations sucessives, tandis que la mort est essentiollement un phemompinte individuel. Il est v'ai qu'on parte parfois de la mont d'unce respecere, mais ce n'est la qu'une expression tigures qui signitie la disprarition de l'espece par la mort de: tous les individus.

Il He semble mêmu pas qüil faille considerere la mort comme l'ahoutissement natures de l'évolution individuelle. A priori, rien n'empèche que l'équililner mobile de l'age adulte n'ait - saluf accident - une duree infinir. En d'autres termes, si d'une part tout organisme est exposis a subir une mort violente ou accidentelle, d’autre part, la mort naturelle far dérépitule ne parait pas etre a priori un aboutissement fatal. Rirn n'empiche - absolument pardant - que les fonctions nulditives, qui, dans une large mesure, contrebalancent l'usure

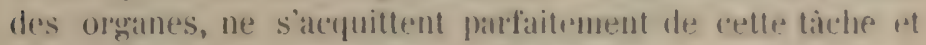
ne maintipnnent par considfuent l'organisme daus une situation toujours émalement aptes à l'exercice de l'atctivité vitalu.

Wrismamn fait rematrquer avec raison quen realite les olganismes monocellulaires sont immortels. Ils peurent évidemment pripir de mort accidente!les, mais en dehor's de cette hypothese, ils se multiplient et se propagent indetiniment par seissipariti on far hourgeonnement. L'individu, si l'on reut, cesse d'exister lorsqu'il se divise, mais il continue a virre dans chacun de's deux organismes nouveaux, et, surtout, cette multiplication n'entraine la destruction d'aucune partice de matipre virante. "En ríalite, dit Weismann, on ne peut parler ici de mort! Où est donc: lo calavre? qu'est-ce? qui est mort? Rien nts meurt, mais le corps de l'animal se sipgare en deux fragments a peu près égaux, de nature à peu press similaire, dont chacun ressemble absolument à l'animal parent et dont chacun, comme celui-ci, continue à vive pour se diviser plus tard de nouvealı en deux moitiés. » (1)

(1) Eisais sur l'héridile et la sélection naturelle. Trad. de VARIG.NY Paris, 1892 p. 23. 
Spencer a altapue cette maniere dis roo de Weismann

t. dans The Contemporary Review i Mai 1893) (1). On n’a pas. pretend-il, le droit d'alfirmer l'incxistence de la mort naturelle chez les protozoaires, II pour.ait se farre quapris

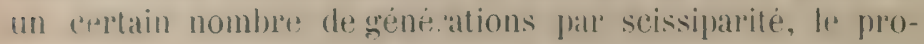
cis de conjugaison fùt nécessaire pour la conservation de la vie ot de la multipliation. (2) Cela semble résulter pn elfel de certaines expeniences de Mampas. D'antres experiences montrent cependant que cette necessite du proce's de conjugaison n'existe pas et qu'on peut, sans clle, obtenir lit continuation indefinie de la seissiparite, pourvu yquon varie de temps en temps le milieu dans lequnl les organismes vivent. (3)

I) aillemen, eomme Wrismann lobserve dams sa rejonse

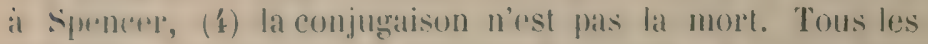
individus sont exalement propres a l'oprerer il reste done

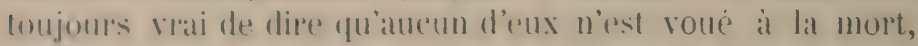

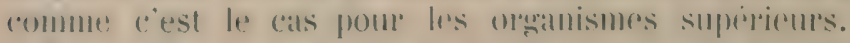

Mrime ditns ces derniers, si l'on atdmet les theories de. Wrismann, la mort naturelle n'existe qure pour les collules somatipurs, bandis que les cellules reproductrices antiment a vive en se divisant inderiniment comme les idres monocellulatres. Lat mort de l'animal ou de la planle entraine avere elle, il est vai, lat mort des cellules reproductricess qui se trourent à ce moment dans le corps de l'individu, mais éest pour elle: un cas de mort aldedentelle.

Eulin Wrismamn prestend que lat mort est, dans les orgat-

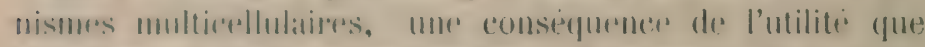

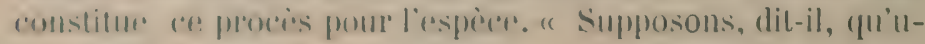

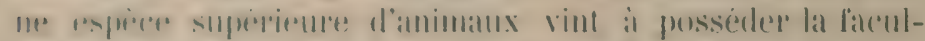

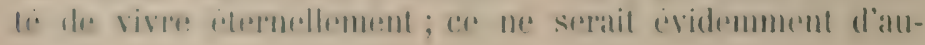

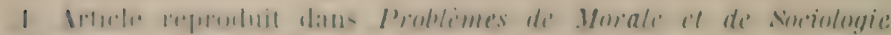

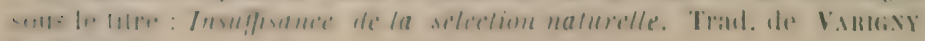
Paris 1 \&!!

12) Contemp. Rev. Mai 1893 p. 747.

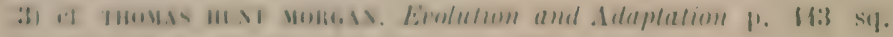

(i) The Contemporary Reviens. Oct. 1 s813 p. (801. 


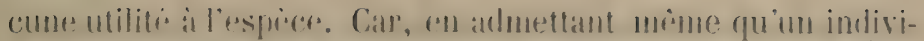

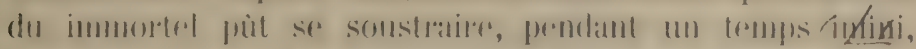
a tous les hasateds qui prourlatent detruire directement son

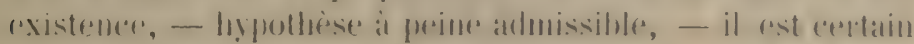

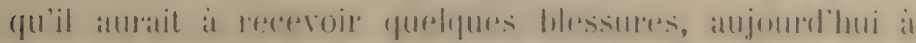

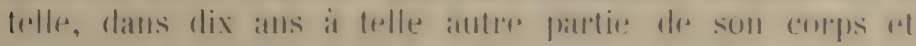

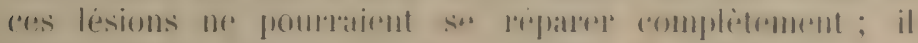

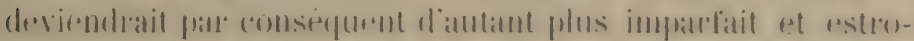

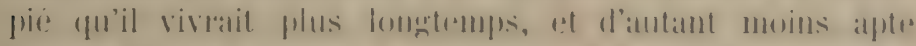

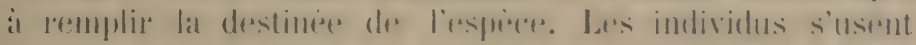

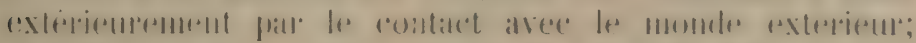

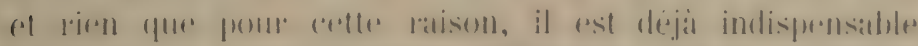

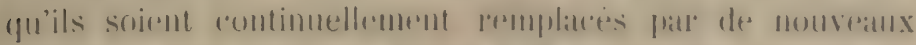

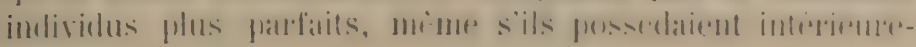
ment la faculté de vivre éternellement.

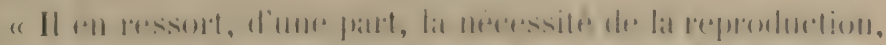

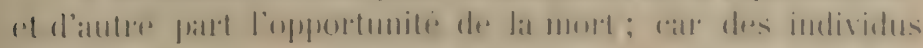

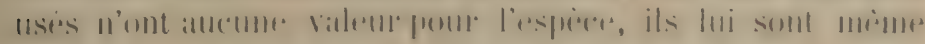

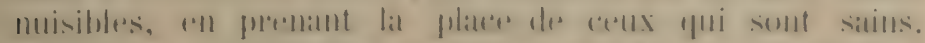

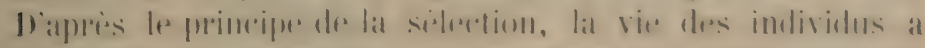

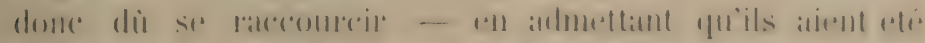

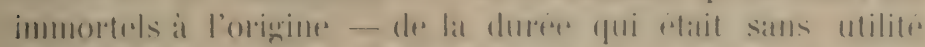

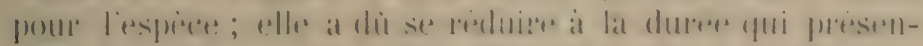

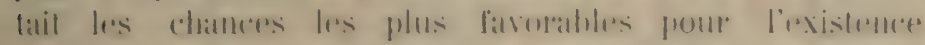

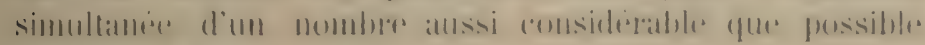
d'individus sains. ») (1)

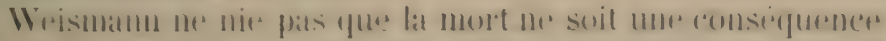

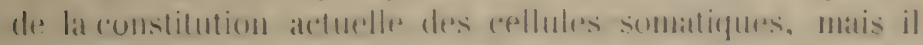

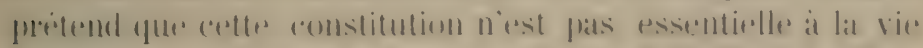

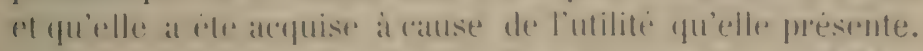

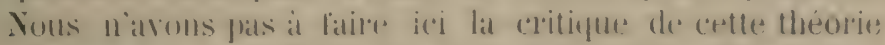

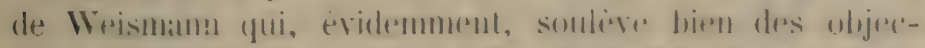

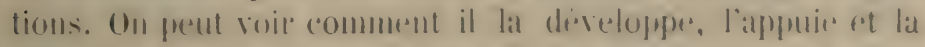
defrond, dans les deox premiers Essais du volume qur nous citons. Ce qui mous interessite cerest lat conchusion yui

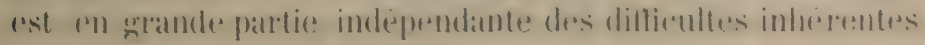

(1) op. cit. (Essais etc) p. 21. 
¿ l'ensemble de la doctrine et qui s'appuie sur une base expersmentale solide : "Comme la mort elle-même, la durée plus ou moins longure de la vie est une athaire d'adaptation; la mort viest pas un attribut essentiel de la substance vivante, elle n'est pas non plus liée nécessairement a la reproduction, elle n'en est pas une conséquence nécessaire. "(1)

Lorsqu'on songe à l'àge énorme de certains arbres, on peut se demander si la vie de beaucoup de plantes n'est pas, de sa nature, indetinie, les individus ctant d'ailleurs sujets a perir plus ou moins tòt par mort accidentelle. Il est certain, d'autre part, que chez les animaux supripieurs, une durée approximative de la vie est un caractère spécilique, de telle sorte que pour chaque espèce ou même pour chaque race, il y a une durée de vie qui n'est guere dipassece et qui est normalement atteinte. Mais cela ne sulfit pas pour que nous considérions la mort comme le trrme nécessiare de l'evolution vilale, ni comm? exigfe. par la " loi d'équilibre. »

Nous savons que Spencer trouve une application de celte l'adaptation. loi dans l'adaption de l'organisme aux conditions dans lesquelles il vit. II y a, sous ce rapport, équilibre pour aulant que ces conditions sont stables ; cal si elles changent, il n'y a plus équilibre, mais an contraire de nouvelles renlistributions en vole d'une adaptation nouvelle.

La pouvoir d'adaptation des organismes aux circonstancres est limile; les conditions compatibles avec la vie sont mame comprises dans des limites assez etroites. Mais, dams ars limites, fadaptation pst évidente et elle se malnifeste anssi birn dans l'espreere que dans les individus; -

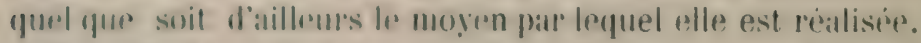

Cepromdant certaines ofoservations s'imposent ici. D'abord

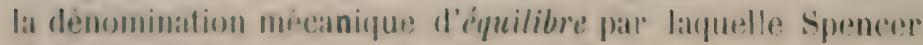

(1) Ibid p. 110 . 
désigne l'adlaptation peut s'admettre pourvu qu'elle n'impliques ancune affirmation concernant la nature des forces que l'organisme: met en jeu. Line suspension it la Cardan qui supporte la boussole d'um navire modifie la disposition de ses pieces dapries les mouvements qui hui sont imprimés, de manière a maintenir toujours la boussiste: horizontate. De mème, l'organisme: des vertithes riggle les oxpdations internes dans les conditions changeantes, de manière a maintenir constante la tempriature du corps. Mais cette analogie d'activiti - un peu rague d'aillents n'entraine pas l'identiti des forces misiss en jeu.

Ensuite, l'andaptation an milieu me doit pas se concevoir comme une situation rigoureusement determinese, tellement que sat realisition soit incompatible alver tont changement ultipienr de structure. Cesest en partant de cette ider fansse que spencer en arrive à ponvisiger l'adaptation parfaite an milien comme 1 in stade terminal de l'evolution de la formen.

"Nons arons vu, dit-il, que la limite de l'heterogeneite est atteinte quand l'ispuilibre d'un agregat devient complet, que: lat redistribution de matiste ne peut continuer que tant qu'il persiste mon mouvement non équilibre. Tons anous vu que, par suite, les arrangements lerminaux de structure doivent itre tels qu'ils puissent opposer des forces antagonistes equivalentes a toutes les forces qui agissent sur l'agrégat. Or, que suppose $41 \mathrm{n}$ agreggat organtique dont l'équiline est de reux que nous apprelons mobile? Nous avons vu que le maintien drun equilibre mobile exign lat production habituelle de forces internes correspondant en nombre, direction at intensité aux forces externes inciflentes, c'est a dire autant de fonctions internes, isolées ou combinees qu'il y a d'actions extirieures a contrebalancer.

"Mais les fonctions sont les corrriatils des organes; l'intensité des fonctions est, toute's choses ingales, corrélative an rolume des organes; ot les combinaisons de fonctions sont correlatives anx connexions des organes. Il en risulte ynu la complexité de structure qui accomprange l'équilibre fonctionnel, peut se détinir : un etat dans lequel il y a autant de parties specialisées qu'il en faut pour yu'elles puissent, 
separement ou conjointement, contrebalancel les forces séparécs ou conjointes au milieu desquelles existe l'organisme. Telle est la limite de l'héterogeneité oryanique dont l'hormme s'est plus approché que toute autre (réature. » (1)

C.e raisonnement repose sur la conception de la vie que nous avons déjà critiquée, et d'après laquelte " toutes les actions vitales consideries non pas séparement, mais ensemble, ont pour but timal le balancement de certaines opérations extérieures par certaines operations interieures. ”

Laissant de coté ce que celte délinition a d'impallait, constatons que les opérations extérieures peurent ètre " halancees s) de: mille façons diflerentes, puisque nous royons les organismes les plus divers vire dans le mème milien et sy adapter chatum de sa lacon. Do ce que la vie est un mode d'activité et s'exeree, comme toute activite, sur des objats, - dre ce gute, de's lors, clle presinte nécessairement des polations avec ces objets of notamment avee les ubjots exterieurs, il ne latut pas conclule que sa nature est entiperment determinie par ces objets, comme ume force est determines en intensite ef en direction par la condition qu'elle doit fatre écquilitne it me forre dommée.

Mime si l'on admetlait que la selertion est le seoul firreleur d'evolution organique et que les organismes varient toujours dans le: sems d'une meillemere adaptation au

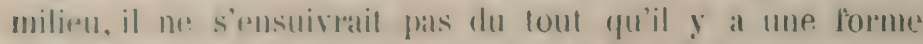
d'adaptation limite qui est determince par le milieu. Qu'un organisme soil plus ou moins apte a pourvoir a ses hesoins, at saisir sil nourriture, à se délemdre contre sas emmenis, yur ses organes soirnt miens disposes pour les fonctions qui lui sont devolues, que la mutrition y soit meil-

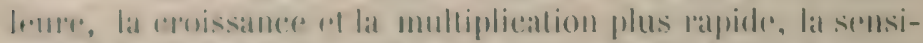
bilite (s'il s'argit d'animaux) plus fine, la mobilite plus grande: are sumb des romblitous qui, considereses isolement on dans

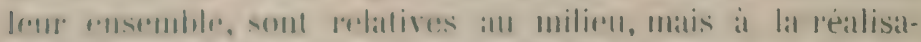

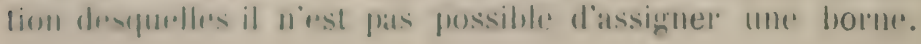

1) P. P p. is38 8 173 - F P. p. 40\%.

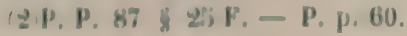




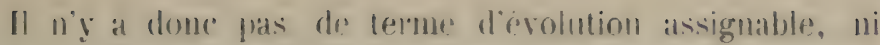

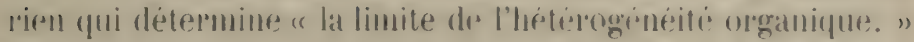
Et sous ef lapport, encore une fois, il n'apparait pas que l'ivolution tende vers un etat d'ayuilibue.

s'il lallait admettre la conception de sprencer, d'apris laquelle les organismes presintent une situation d'aquilibre rigoureux entre les fores plastiques et le milieu ambiant, il faudrait en conclure que l'ovolution est entierentent determinfo gar les chamgerments de ce milien. Elle consisterait uniquement en ('foci : l'equilihre n'existant plus, les forces plastiques entront on jen et monlitirnt longat-

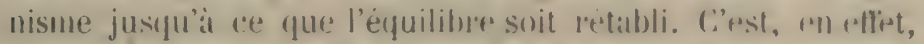
ce que spenter enseigne : " Tums tromsons, dit-il, gutes la progression levolutiont prisulte non d'une tendaners sperciale anx corjes vivants, mais d'un eflit mopern gentral

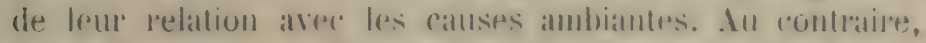

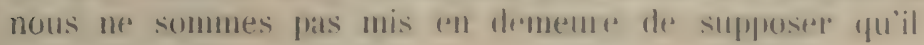
existe dans les organismes ma frenchant primitif qui les porte is se developper rontinumllement sous des formes plus hetrominges; nous royons quine aptitude all derroloppement nat des atdions rt des reatctions molde les organismes et leurs milienx valrables. Entin nous ronommassons que l'existemer d'ume cause de developprament suppose la non-production do dreselopprement aux endroits

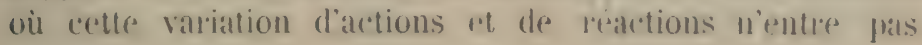
en jeu » (1).

Yous n'avons gatrde de nier l'importance des chamger ments dn milien an point de vue de l'ivolution, ni de contester que l'évolution de l'espren tout comme celle de l'individu exigr des conditions fitromables, matis de lat a considerer les variations du milien "l l'ativite qu'plles determinent dans les organismes comme les sfuls fitctems de l'érolution, il y a loin. Yous venons de voir que la thérite sur laupulle s'appuie spencru pour l'allirmer manque d'une base solide.

Entin il n'est pas inntile de le ripéter encore: rien

(1) Princ, de Biol. vol. I, p. 322 \$ $15 \%$. 
ne nous autorise a altimer que toute ivolution est allaptative, meme dans le sens restreint que nous venons d'exposer'. A còté de l'érolution plussiologique qu'on peut considérer comme telle et des moditications de structure? qui s'y rattachent directement ou indirectement. il y a la genèse des particularités purement morphologinues pour lesquelles il est impossible d'imaginer un role adaptatif. Romanes rapporte qu'arant examine un a un les caractères spécitiques se rapportant à la coloration dans toutes les espèces de certains genres d'oiseaux et de mammifères, il a trouve que le nombre des caractères auxquels il est impossible d'attribuer une utilité ou une relation quelconque au milieu diprasse de beaucoup le nombre de ceux qui apparaissent comme des adaptations. (1) Largument n'est pas peremptoire, parce qu'on peut tonjours objecter notre jenorance, mais il n'est pas sans valeur et il montre quil faut etre sévèr a l'egard des considerations thériques sur lesquelles repose la thise dre sipencer.

Formes variées d'équilibre.

Ln ilement strictement limiti par les conditions extéricures, c'est le nombre d'individus appartenant à un groupe d'organismes. L'espace yu'ils occupent est restreint et, par conséquent, les ressources qui sont il leur disposition.

En outre, le mème espace est occupri simultamement frar d'autres groupes qui leu font la gurere d'une facon direste ou indirecte. Lorsqu'unt: espece se multiplie, ello comprond apres peu de temps le nombre d’individus que les circontances comportent et il sélablit un écpuilibre entre sa force de moparation et les obstacles positifs (ennemis) of nisatifs (manque de ressomrors) qu'rlle rencontre.

Ladaphation an milion se firit che\% less andman principat-

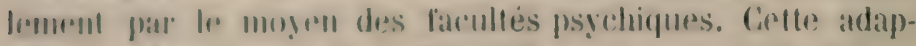

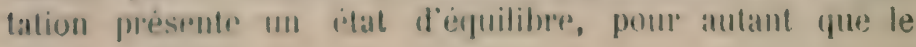

(1) Daruin and afler Darwin, vol. II, p. 17:, 
milien est stable, (omme nous l'arons dit anparabant. ()n

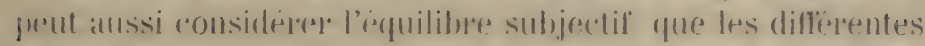

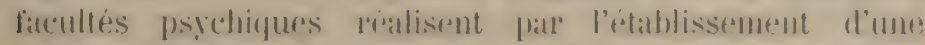

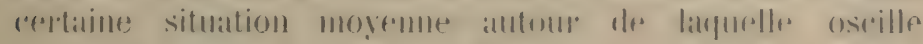

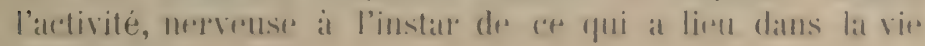
ol:ranique.

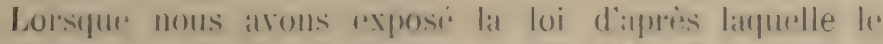

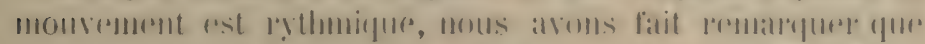

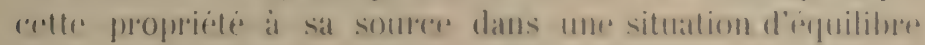

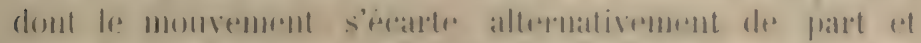

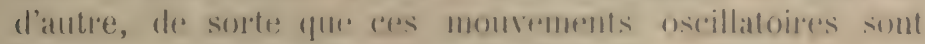

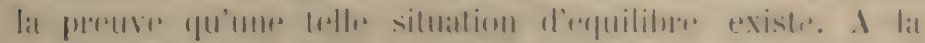

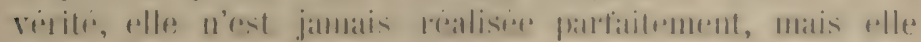

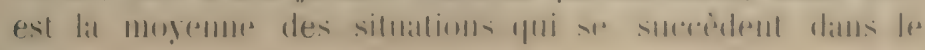
fait.

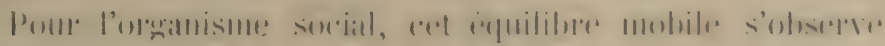

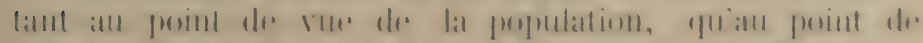

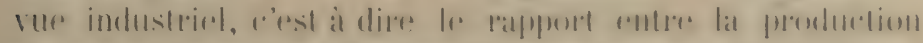

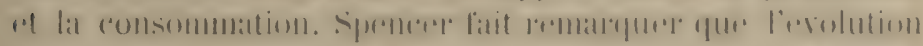

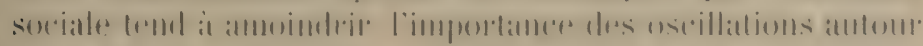

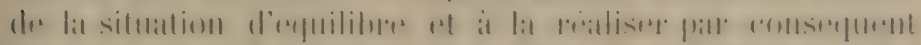

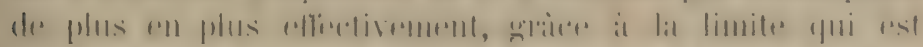

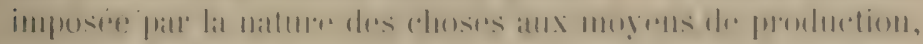

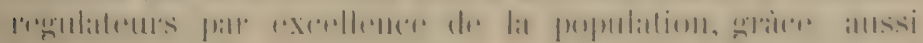

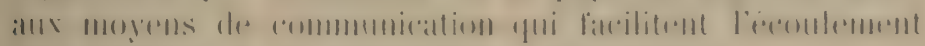

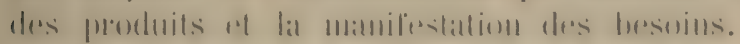

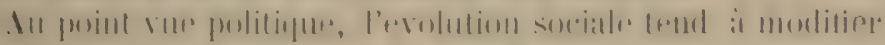

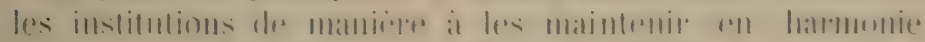

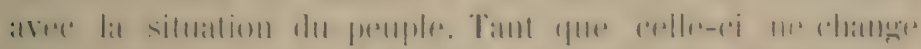

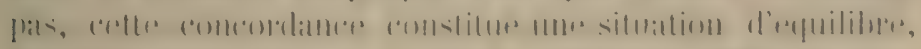

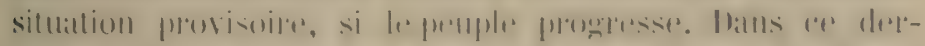

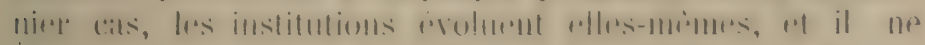

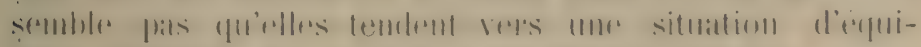

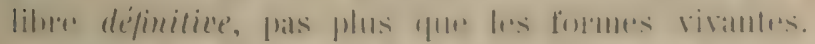

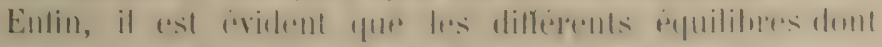

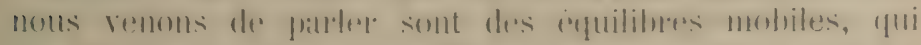

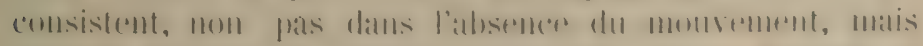

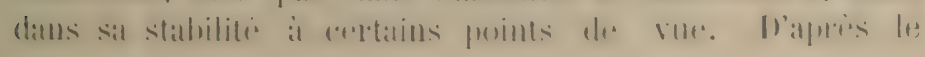


principe spencer, cet equilibre mobile ne serait qu'une: étape vers l'équilibre ahsolu. Yous ne voyons ancun moyen d'interpreter cette loi ('n l'appliquant soit aux societés, soit à l'érolution de la vie psychique. It faudrait commencer par délinir an quoi consiste pour celleci ou pour celle-lá l'équilibre absolu, ef nous arouons ne pas concevoir une telle détinition.

La loi d'équilibre a priori.
Lat dimonstration a priori de la loi déquilibre est tire par spencer de la deperdition fatale du monvement visible: " des somstrantions prepetuelles, "ausées par la communication du mouvement au milieu resistant, doivent népsairement metter fin au mouvement des corjs dans

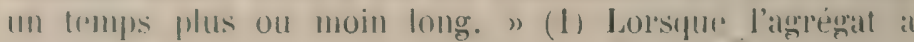
jerlu lout momventent visible, c'est l'équilibre absolu; lenpulihre mobile sorablit lorspue des divers monvements

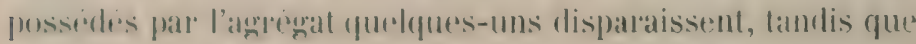
les antres contimunt. " Dans lout agregat animé de: divers mouvements, conx qui sont les plus faibles et qui rementrent la plus grande resistance se dissipent relative-

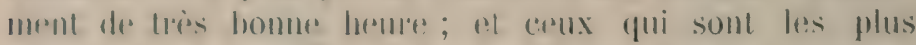
forts et qui rencontrent tr moins de résistance se conservent longtumps: c'rst amsi qum se lomment les equilibres mobiles deprondants of les équilibres mobiles independants. "

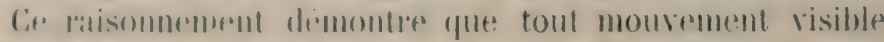
doil dimalement disparatere of que toute evolution serat, en lin de rompte, emseloppree dans cette destruction du momvement. It montre aussi que, dans certaines circons-

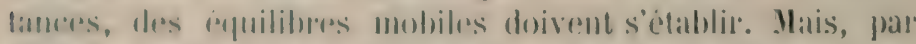

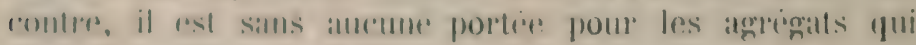

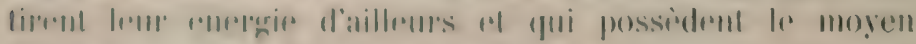

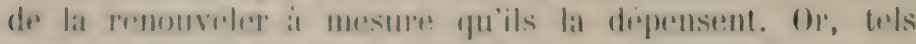

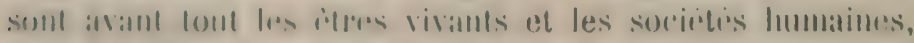

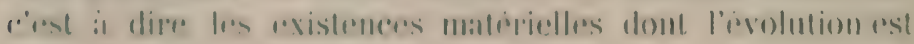

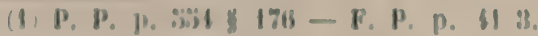


de beancoup la plus intiressante. Il est viai que ces existences elles-mimes réalisent souvent de l'ume ou de l'autre faron des situations d'ónuilibre, mais l'explication

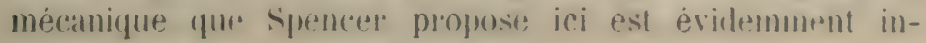
suffisante pour en rendre compte.

On prut se demander s’il ne serait pas phus juste dro considerer l'éfuilibre absolu comme, un phénomene caracléristique des aurrégats inorganiques, at l'équilibre mohilr. comme une chose propre aux itres vivants individurs ou collectil's.

Less fremiers, en ellet, n'ityant regulip'rment pas les mogen de puiser dans le milien l'imeregre qu'ils depensent, aboutissent nicessarement at lat perte de tout mouvement visible. si, le fait, l'émergip leur est constamment fourne de l'exteriener, commes le solepil putretient le monvement

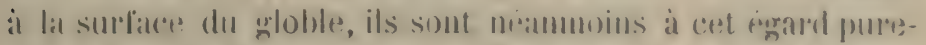

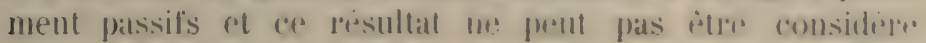
comme "aratereristiqute de leme nature. Et si dans d'antres cas, comme pour les systiemes astronomiques, la dreperdition du momerment est très lente a caluse de la faible resistance quil rencontre, de sortre que la situation reste sen-

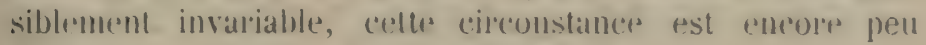
importante an point de vue theoriques.

Les ifles virants, all rontraire, quoique sujets a mourir, prisentent néanmoins un renouvellement continuel d'energire qu'ils resureillent dans le milieu gràce à leur activite prome. Les resistances qui determiment le repos absolu dans les agregats inolganiquess se trouvent dans l'agregat luimeme on dans le milien. Quand la chaudiere cesse de fommir de la valpur à la machine, celle-ci continue a marcher prondant furlque temps, puis elle s'arrite à cause dos frottements de ses organes. Les mouvements des planètes, all contraire, subissent une diminution très petite a rause de la resistance du milieu dans lequel elles se meuvent.

De mime, les ètres vivants rialisent l'équilibre mobile en sadaptant soit aux circonstances exterieures soit aux circonstamés intérieures. Les animaux et les plantes règlent 
leurs fonctions d'après le milieu ou elles vivent. Les socirlés s'oryanisent suivant les besoins de leur's membres. De tolles adaptations peurent ètre spontanees, comme dans les exemples que nous venons de citer, ou simplement imposices par les circonstances, comme la multiplication limitre d'une esperce. Dans ce dernier cas, notamment, l'é(uilibre ne leeut pas ètre considéré comme le résultat naturel de l'évolution.

En résumé, il resulte de l'exposé que nous venons de laire et des critiques que nous avons formulées, que: spencer, à notre avis, consoit d'une faron difecturuse les resations do l'asuilibre aver l'évolution.

Jous renons de rappeler que tout agregat, par lá mème

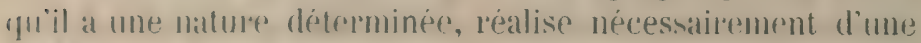
faron on d'une antre me situation d'expuilibes stable.

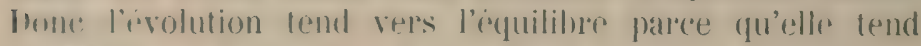

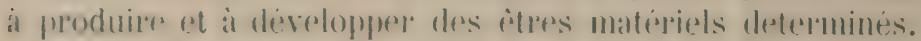

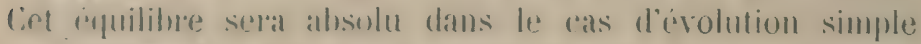
oil lintrgation finate ne componte pas de mourements introtems visibles; il serat an rontraire mobile dans le

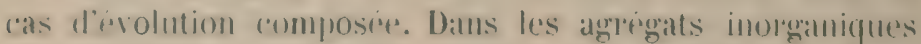

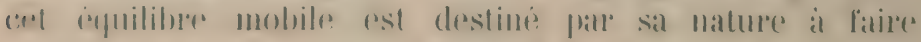

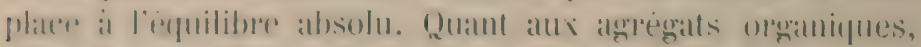

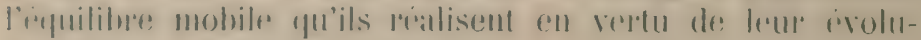
lion propre, determine simplement leur individualite, mais

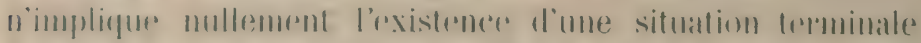

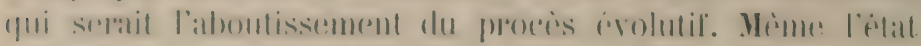

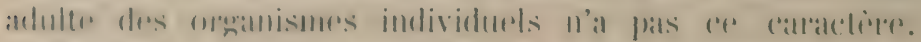

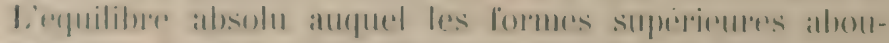

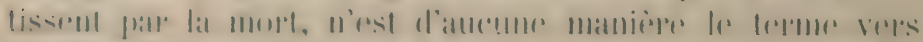

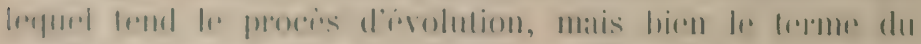

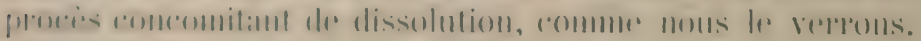

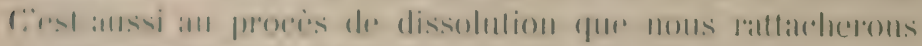

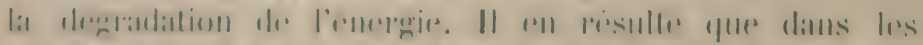

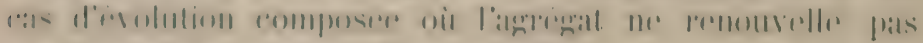

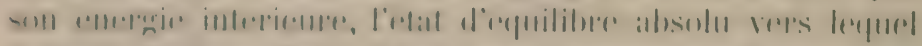

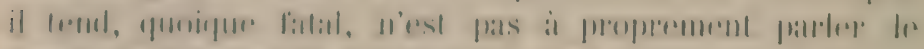

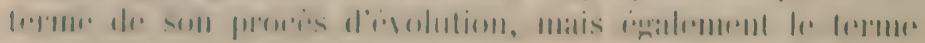


du proris de dissolution fall dresintegration du monvement. Sotons, molin, fue l'on pent observer dans l'évolution,

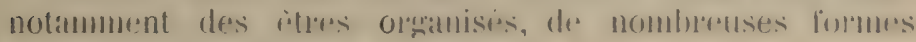
secondaires d'équilibre mobile.

ation

l'èvoluet la Intion.

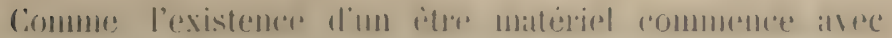

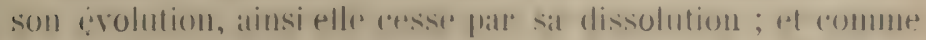

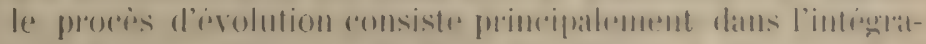

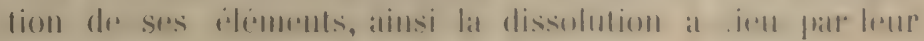

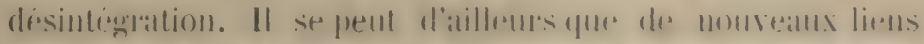

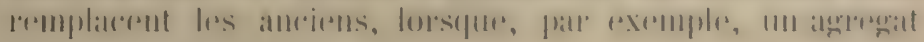
est absorbé par un autre.

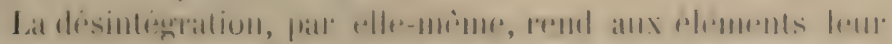

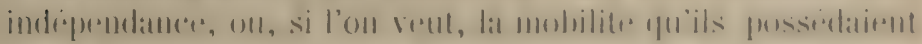

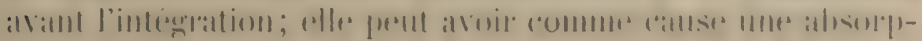

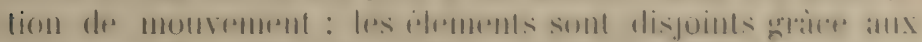

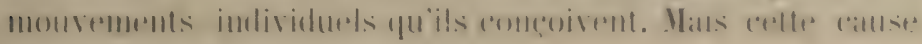
de dissolution n'est pas la seule.

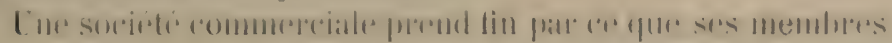

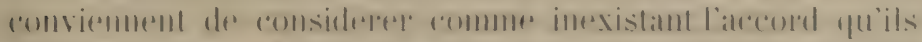

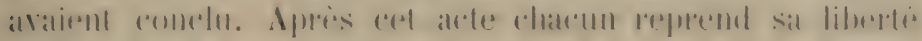

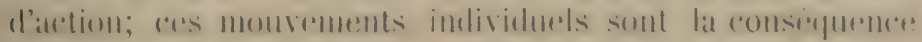

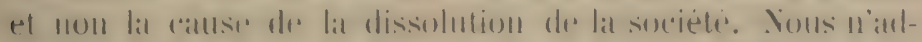

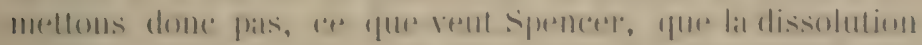

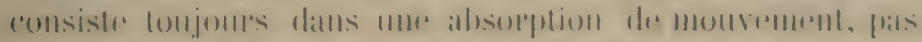

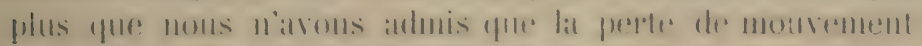

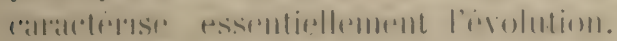

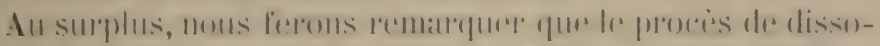

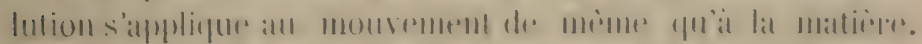

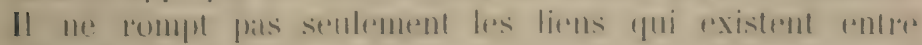

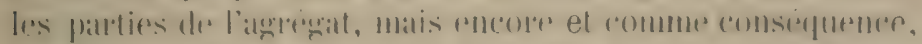
ilr detromine me desintegrattion du monsement. si mons

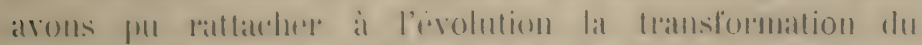
du mombement invisible des particules an momement

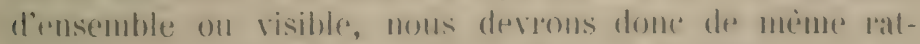
lander an foreces de dissolution lat transformation inverse, 
cest a dire la degradation de l'énergie ou accroissmment dre l'entropie dont nous arons déjà eu plusieurs fois l'occasion de parler.

si le systeme solaire marche vers une situation ou tout Ir. mouvement visible sera converti en chaleur, c'est qualors loules les planters seront reunies detinitivement au soleil at ne formeront plus qu'une masse unique. Ce sera l'integration le la matière constituant le système, mais ce stra aussi la fin du système lui-même et des astres parriculier's, par la perte de leur inclividualité. Pour les corps qui constituent le șsteme solaire, ce sera la dissolution aussi bien au foint de vue de leur existence propre que de leurs mouvements.

D'une facon genérale, dans tous les agregats, un relarlemment dans les liens qui unissent les parties sera accompagne d'une diminution dans la cohesion de leurs mouvements. Il ne faut pas confondre la desintegration du monrmunt arec sat diférenciation. Celle-ei appartient au proces progressif el est parfiatement compatible avec une condinalion et une rohésion croissantes. Lorsqu’il s'agit des agregats sociatu, if ne faut pas non plus se figurer que loule conordination des mouvements doive être necessairement resglese par la loi et déteminé par la contrante. 1.0 progres moral des individus peut etre assuré dans une largo mesure par la coordination spontanée de lems mouverments es, disis lors, permettee l'existence d'une liberli: flus grandre, sans que la coliesion de l'ensemble s'en trouve amoindrie.

Labsorplion du mourement gui detranine partois la disso-

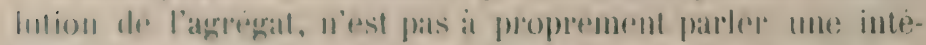

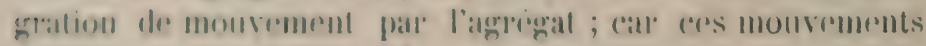

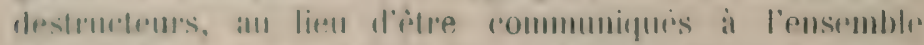
romme lols on aux parties an vure du tout, aflectent alu contraire les parlies prises isolemont of lemdent a les séparer.

Lat dissolution est determinte par toute circonstance qui

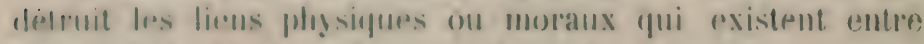

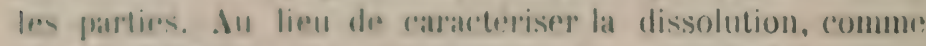

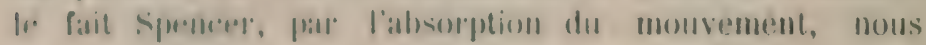


proferons lat définir par la drosintegration du monrement accompagnant la désintroglation de lat matière, almsi flue nous venons de l'expliquer.

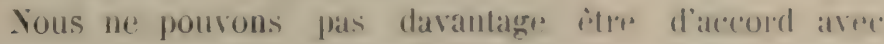

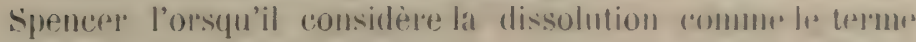
vers lequel tend l'arolution el encores moins lorspu'il l'en-

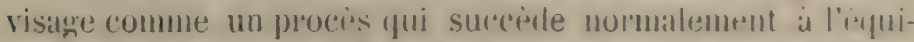
libre. Il n'est pas trop diflicile de dembler les proccupa-

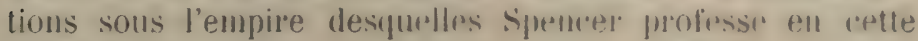
matiere des opinions yui sont, comme nous fo remons,

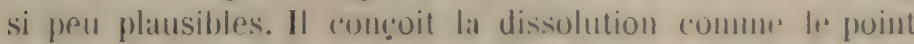
de départ d'une évolution nouvelle; et, lat comsiderant alors

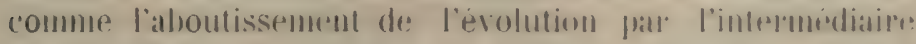

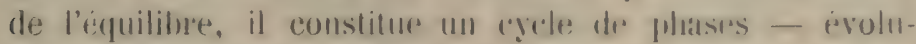

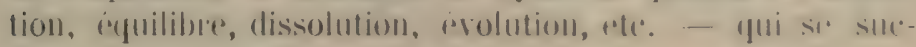
cèdent fatalement et à l'infini.

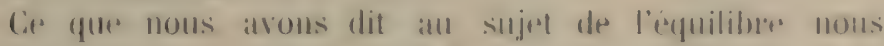

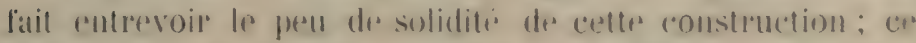

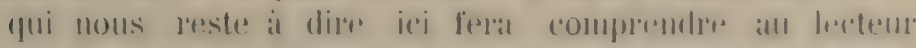

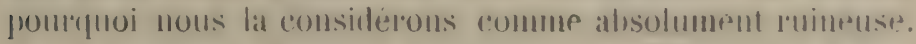

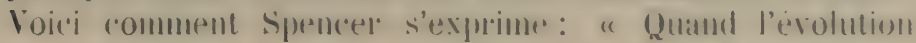

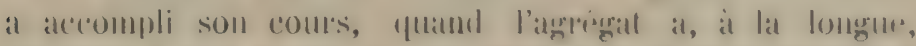

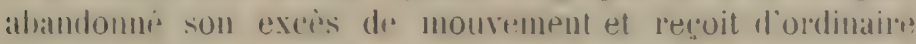
de son milien atutant yuril fered, quand il at atteint cert

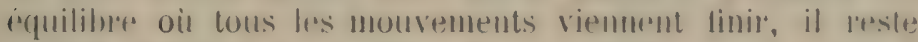

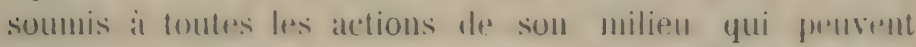
accoitre lat fuantite de mourement quil contient, et yui, dams le coms du fomps, dommeront assurement a ses parlies, doune maniepre lente on subite, un "xcès de

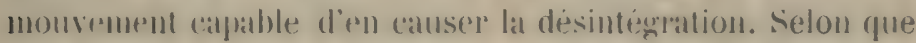

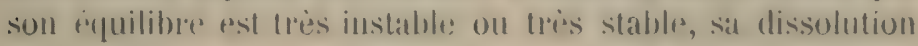

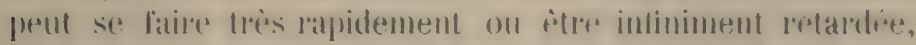

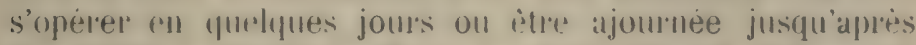

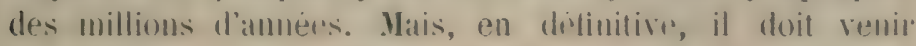

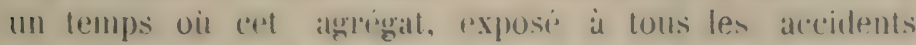
qui dépendent non seulement des ubjerts de son roisinage immriat, mais dou morers partout en monvement, perian 
surul oil all compagnie des agregats emvironnants far lat décomposition de ces parties. "(1)

Dans les lignes precedentes spencer considerer la dissoIntion comme te fatit du milien agissant sur l'agregat qui est arrive a l'épuilibre après aroir acheve son prolufion. Yous arons dejaj fait olsserver qu'il n'y a aucums raison d'assigner un terme il tonte évolution, par exemple, a lirrolution sociale on a l'evolution organique de l'esprece. Evidemment dess procès peurent itre arretés par des cimonstantes exterintmes, mais dre lrum mature ils sont indetinis.

En untre, l'équilibre qui strait précurseur de la dissolution est, d'apres spencere, l'equilitne absolu. Or, sente l'evolulion des atmes inorganiques qui nout pas de source exti-

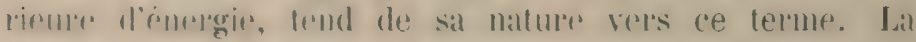

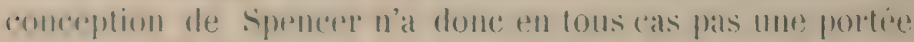

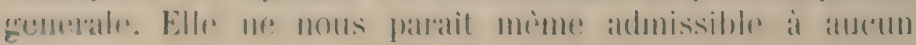
degrè.

Fin altiel, la dissolution par le lait du milien, mon sembement

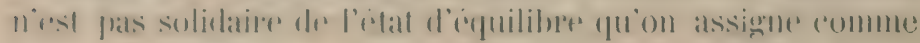

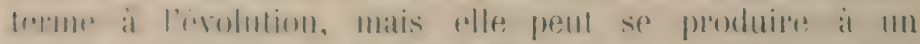

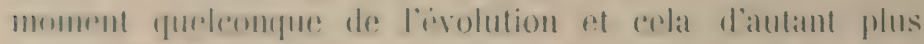

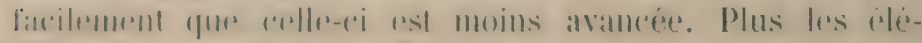

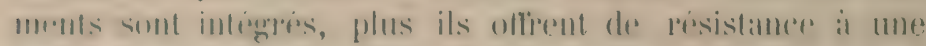

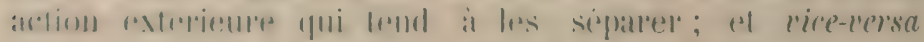

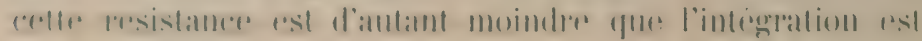
moins complete.

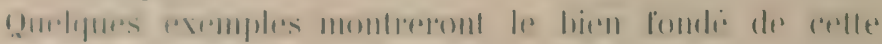

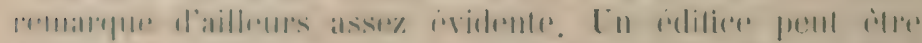

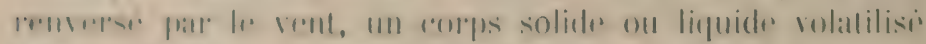

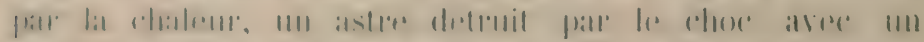

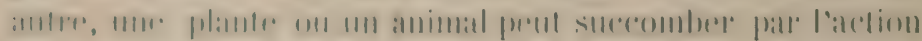

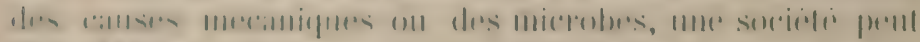

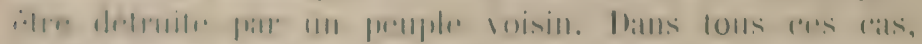

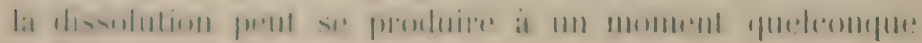
de l'existence de cers agrengats.

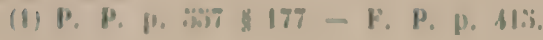


En outre, an editice on construction est remperse plus facilement pal l'unragan; Is liquides moins intiogres que fr solide, se volatise plus aisement; la dissipation de la

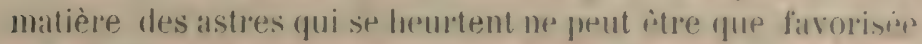
pal lat chaleur quils pussident alvant te choc; un orgatnisme tris jeune, regretal ou amimal, stroombe plus facilement aux blessures et aux maladless; un peuple maissant est plus aisément détruit.

Que si l'on objecte? la canlucite des organismes virux, sociamx ou individuels, nous rejondrons que centre condition daus laquelle ils se trouvent, tout rommes lat mort à lacpuelle elle alooutit, asst un ellet de la dissolution, et

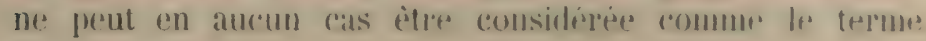
de l'évolution.

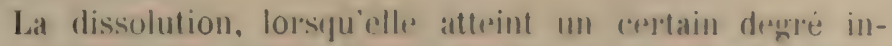

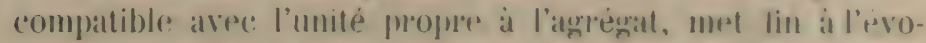
lution, ou, en tous cas - si l'evolution est terminter alvant qu'elle: n'ait eu lieu - elle met lin à l'existence qua l'exolution avait fait naitre. C'est en cor stens semlement que ces demx procies sont comelatifs : ils deferminent le commencement el la fin des atress matreriels. Mais nous apoyons gu'il faut les considiper comme alsolument independants it tous les autres points vue a notamment fat les causes ou Irs circonstamees qui les determinent. Iat contirmation de cette maniere de roir risultera do l'etude inductive du sujet.

Spencer envisare d'abord la dissolution des sociétís. Elle consiste dans la désorganisation, c'est à dire dans la destruction plus ou moins complète des organes sociaux. L'armée est dispersée ou licenciér ou en révolte contre ses chefs; ceux qui étaient investis du pouroir central ont disparu ou sont dépossélés; les chambres législatives sont dissoutes; le pouroir exécutif est impuissant. Ces efferts. plus ou moins complètement réalisés, peurent avoir pour motif une invasion étrangère on une révolution intestine, 
l'une el l'autre pouvant itre detominces pall des causes variées.

On sittend à re que spencer mette en lumière ici fue la dissolution de lat socicti est l'aboutissement de son erolution. De fait, il ne s'y emploie grueres. Voici tout ('f qu'il dit a ce sujet : " Le Japon nous offere un bon exemple de la manire dont ces disintrgmations sont susceptibles d'ètre mises au jour dans une societé qui atteint la limite du développement du type auquel elle appartient, et atteint un atat d'enuilibre mobile. »(1) Et uin peu plus loin: "Là mème où une societé parvenue à l'apogie du developpement que permettait le caractère de ses unitrs, commence a déperir et entre en deadener, la dissolution mogressive qui s'y manileste est ancore de memr nature. ) (2) Ces passages montrent que spencere supunso la thrse, mais n'en apportent aluculle contimation.

Fu realite, toutre organisition est exposer à des emmemi.

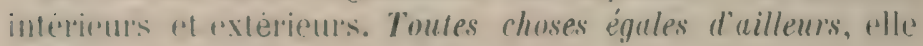
resistera d'autant mienx a lem action qu'elle est plus solide, rest i dire mirux intrigrerer. Lu grand dangerer pour un organtisme social ce'est de ne plus itre en larmonie atre le siluation et les aspirations do peuple, mais ce fait "si le resultat, non pas de l'evolution, mais plutot

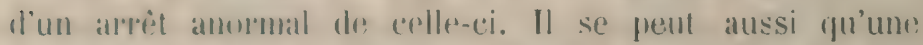
organisation sociale deviemne cadupue, farce qu eble s'est laisse movalir pall les abus; mais encore une lois re resultat lia rien de eommun ares l'erolution. Elle est an contraire le losultat do l'action destructive quexerent dijit

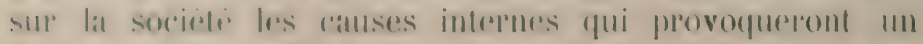

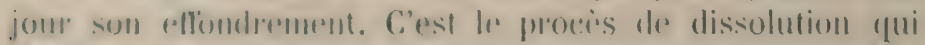

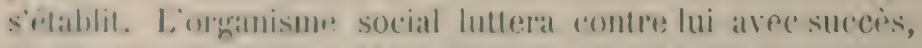

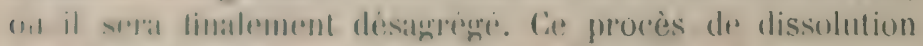
entrase levolution el s'il l'emporte, tinit, non serulement

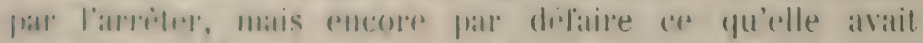

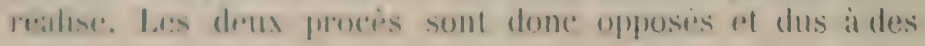

(1) P. P. p. shis $178-$ I. P. p. 417

(4) P. P. 10. $\sin$ of $178-$ F. P. P. 117 
canses indipendantrs, bien ytur l'un soil le prolongement naturel de l'autre.

Lat méme ronchusion est annemese par la consideration des organismes vivants. Remarquions d'aborel que leur verpitable dissolution est lat mort, et non pas la disperesion de leurs abrments qui ampire parlosis apres. A lat mort l'atre vivant n'existe flus, l'unitio qui le canacteriso a

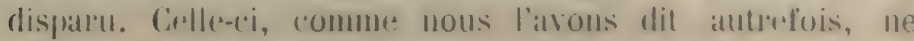
comsiste pas dams la juxtaposition des allomes, mais bien

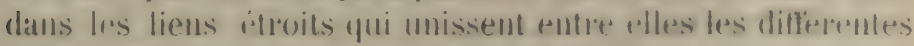

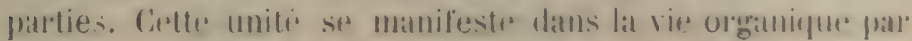
l'intime sulondination at courdinallion des orgatles, mais

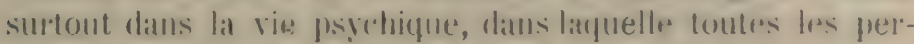

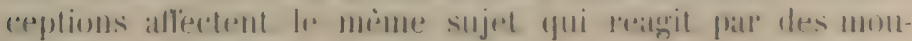
vements appropriés de l'ensemble.

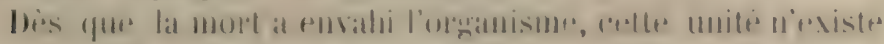

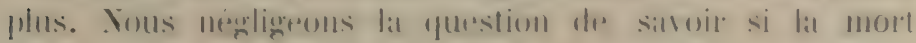

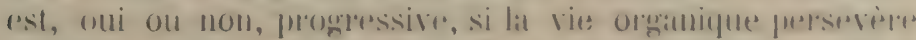

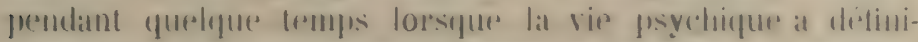

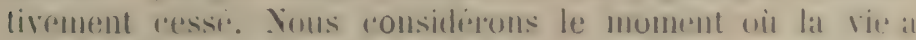
pris tin el nous disoms que dis a momment les dilfrentes

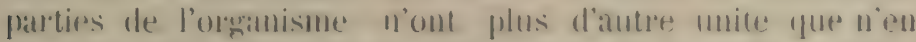
possedent des corps loruts queleompues justaposes on mallit-

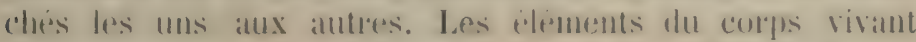

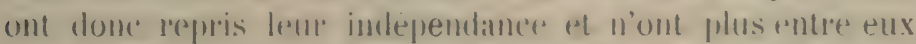

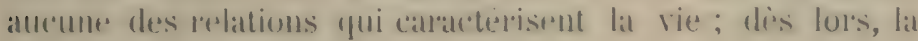
dissolution est accomplie.

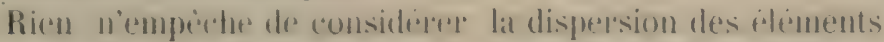

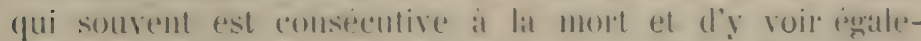

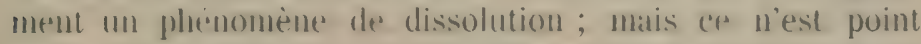
la dissolution d'un etre vivant, c'est la dissulution d'un reorjes iborganigue alsolument parreiltr, dans sal natume ef dans ses resultats, it colle que prot présenter un agrigat de

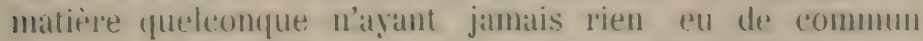
avece la vies. Dès lors, les considerations que lait syencere sur les conditions qui lavorisent un entratent la decomposition des caddires n’offrent pour la question acturlle aucun intérèt. 
Cela itant posé, il est d'abord evident qu'un organisme peut mourir de mort violente ou par suite de maladie à un moment quelcourque de son évolution ef que la mort n'a done avec cette érolution aucun rapport necessaire. Cous pensons qu'elle n'en a point d'autre? que de l'entratver plus ou moins et que pour le reste, les proces d'isrolution et de dissolution sont, ici encore, entièrement indejendants. La chose est évidente pour les cas ct daus la mesure ou la mort est due a l'action des causes extérieures accidentelles. La même interpretation s'impose, lorsquon la considère comme la consérquence des causes internes ou normales.

En eflet, l'solution individuelle aboutit a une situation d'rounilibre lorsqu'elle a rejoint le stade de l'erolution attrint

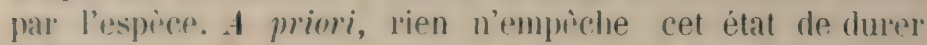
indéliniment. Pour comprendre qu'il prend tin, il faut tenir compte d'un procès de dissolution qui, en l'áalité, prend naissance en mime temps que l'individu. Cette dissolution va en progressant, d’abord paree que l'organisme est incapalile de reparer completement les avaries qu'il subit inévitablement de's le commencement de l'existence, ensuite parce qu'il flimine imparfaitement les matières imutil's qui s'y introduisent ou les matériaux qui ont été deja utilises el qu'on appelle les residus organiques. Ainsi, d'une frart, les organes s'usent par des lesions plus ou moins notables qui vont s'accumulant; lautle part, les tissus s'encombrent de: matieres incrustantes on dégrnèées. II arrive un moment où l'oroganisme est délinitivement incalpable drexereer les fonctions vitales essentielles et ou l'estre vivant cesse d'exister.

Gue l'on considire of que nous venons do dire comme prohit par la lutfre des forres inorganicues contre les foress vilales, ou d'une antre facon quelcompue, ce qui mous parat incombestable, rest qune la dissolution est un proces paralliele a levolution of qui mest independant.

Il n'est dene: bas admissible quon les considere comme des phromomenes alternatifs, se succidant, de par leur nalume, l'un a l'aulle el constitumut ainsi un cycle indelini. 
Quant a la dissolution des agregenats inorganiques : des corpes liquides ou sulides, de la Ture ou d'un astre: qundromplue, mous en atrons dit asse\% pour montrer quinlle

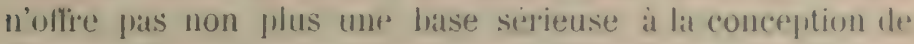
sprencer. Cess itres tendent vers l'inguilibre stable, mais leur dissolution est un proces absolument indipendant de leur integration.

De: ae qu'il n'y a pas entre les proces d'trolution et de dissolution lat comexion que: spencer inagine, il ne suit

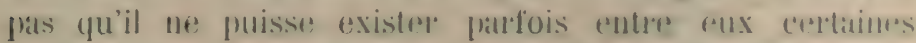
relations qu’il est interessant dexamismer, molamment dans

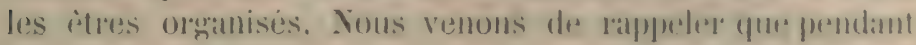

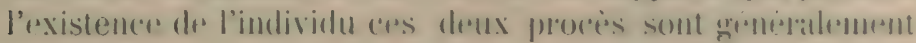

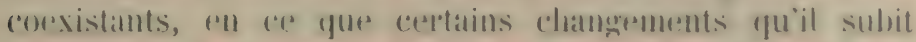

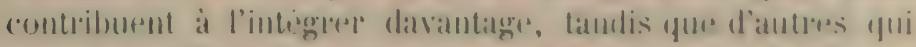
s'y ancomplissent simultanement poursuivent ou preparrat

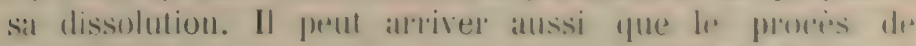

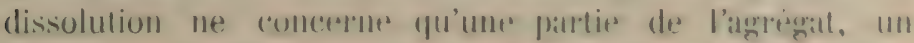
organte de l'individu ou de la lorme vivantr, salls compromettre l'existence de l'ensemble.

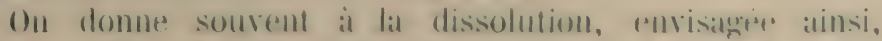
surtout dans la forme, fer nom de régression ou d'érolution

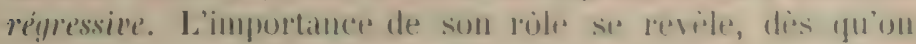

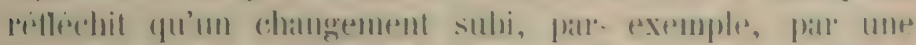

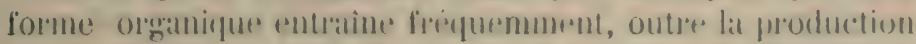

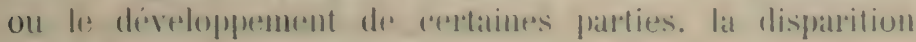
ou lamoindrissement de certaines autres.

Alin de mieux nous rendre comple du calactime de levolution regressive dans les itles vivants, considerons dillprentes circonstances dams lespuelles elfo so produit.

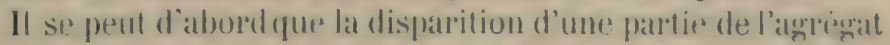
scit simplement la consépuencer du developpenent d'un.

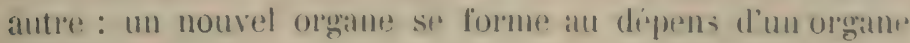
préexistant.

Le cas le plus simple consiste dans la transformation

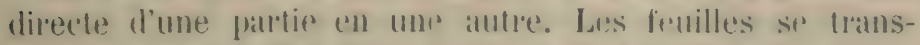
forment en less differents organtes de la them, les membres se flansforment en nagreoires, la vessie notatoire se 
transforme en poumon etc. C'est le mème phénomène qui est à la fois progressif et régressif d'après le point de vue auquel on se place. Toutefois l'un des deux caractères sera considéré comme prédominant, suivant que l'organe nouveau est plus ou moins élevé en organisation que celui qu'il remplace. D'autres fois, la disparation de certains organes est impliquée dans un procès d'intégration : ainsi l'intégrégation des vertèbres, ou bien la soudure des os dans les extrémités des membres de certains animaux entraine la disparition des articulations et des organes accessoires.

Ln second cas à considérer, c'est la régression d'orgatnes devenus inutiles, soit que lemr fonction ait eté dévolue à d'autres organes, soit que cette fonction ait cessé d'exister.

Telle la disparition des branchies chez les animaux à respiration abrienne, des stomates dans les fenilles des plantes aquatiques, la regression des dents des baleines, l'atrophie des organes de la vur ('hez les animaux qui Jabitrnt les cavernes ou les abîmes océaniques. Il n'est. pas toujours facile de décider si c'est la cessation de la fonction qui a determine la remression de l'organe, ou bien si c'est la disparation de l'organe qui a eu comme consé(quence la suppression de la fonction. Les ailes de l'autruche, du ('istor, des pingonins sont-elles riduites parce qur les anceitres de ces oiseaux ont renonce au vol? Les membres des orvets sont-ils rudimentaires farce que ces animatux se sont habitues a se trainer sur te sol? Ou bien dans les deux cas faut-il adopter l'hypothèse contraire?

Lorsque la fonction qui a disparu n'etait plus utile pt. gue l'organf n'btait done plus d'aucun usage, on jugerat

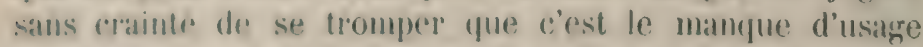
dre lorgante ons son mancue d'utilitio qui a determine sa disparition. Dans re cas, le phenomene de rompression ne

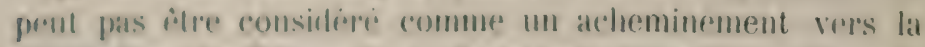

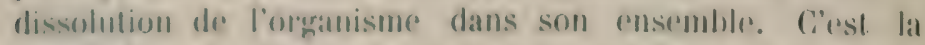
rempression do lomenane, mais non pas la regression de l'organisme.

Il faudrail r'l juger antrements s'il s'agrissail d'une fonction 
(ividemment utile. Dans ce as on me pourat pxpliquer sá cessation que comme une conséquence de latrophie de l'organe correspondaut, ret cette regression surait un pas en arriere pour l'organisme tout entirer.

Yous rangerons donc dans mo troisimme ratengrie les displaritions d'organes yui ne s'expliquent pas frar la disparition prealabe de la fonction. De telles regoressions sont nombreuses dans l'existence des individus et on doit les considierer comme priparant la dissolution linale. Mais existent-rolles aussi dans l'évolution des formes?

Mrime les partisans de l'pllicarile universidles dro lal

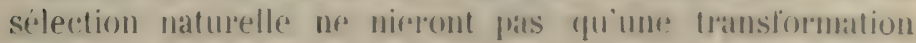

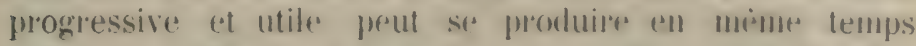
qu'une modilication rogressive at muisible, surtout soil y

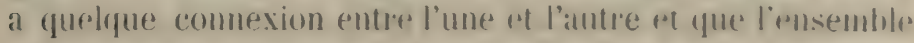
des deux caracteres constitue un avantagre. Ils admettront ixgalement qu'une transformation peut etre favorable is l'organisme dans les circonstances particulieres oit il se tromve, quoigue, alu point de vate de la preffertion intrinsèque de la forme, elle constitue un recul. Lue laille très grande, des organes tres complexess ot far suite tries deliclats peuvent itre, daus certains cas, des desavantages dans lat lutte pour la vie.

si l'on n'admet pas que la selection naturelle doit rendre compte de tous les changements des formes vivantes, on ne verra pas de difficulti a adopter la conception de Ine Vrirs que nous avous rapportie plus haut au sujet de la maissance des variutes, et l’on se fera ainsi une idée plus complète de la simultanéitr des procés d'évolution et de dissolution dans les organismes. "L'avancement gènéral la de nature vivante, dit cet auteur, dépend de l'évolution progressive. Dans les difërentes parties du riggne regretal et mime dans les diffërentes familles, cette progression a lieu suivant des lignes difierentes. Il en résulte une divergence croissante entre les groupes. Chaque pas est un progrès et bien des pas en arant ont dù être faits pour que des plantes florifères naissent des algues monocellulaires les plus simples. 
"Vais en pelation et en comexion fres intime avec ce progris, on rencontre l'évolution régressive. Elle est également universolle; peut-êtres ne fait-elle jamais defatut. Il ne s'est pas accompli de grands changements sans que, d'une part, des qualités nourelles aient èté acquises, el d'autre part, des qualités soient devenues latentes. Pantout ces riggressions s'observent. Les genres polypétales pyrolu, ledum et monotropa parmi les bruyeres șmpetalns en sont un exemple remar(uable. Toute l'évolution des monocotylédones a partir des ordres inferieurs de dicotyledones implique la perte apparente de la croissance du cambium et de beaucoup d'antres qualités. Dans l'ordre des arrölizes depuis l'acorus calamus avec ses fleurs petites mais complites, jusqu'aux lentilles d'eau (lemna) presque entiopentent reshlutes, on pent tracer une ligne ininterrompur d'atapes intermediaires montrant partout la coexisLence de levolution mogressive at rigressire. ") (1)

On freut conceroil d'apres cela qu'une serrie de phénomenes rogressifs, surtout s’ils alfir:tent les organes de reproduction, amine la disparition de la forme, comme l'érolution régressive de l’individu ahoutit à sa mont. Ciest un fitit yure des formes nombreuses se sont elrintes pendant les perioles geologigues sans laisser de descendants. Sans doute, crs disparitions peruent s'itre produites parce que les cireonstanres pxlipieures de climat ou de nourriture ont bti moditiés, ou farce que ces organismes ont suceombe dans la lulfo pour l'existence contre des noureaux-venus. Darwin nadme pas fuon ait recours at une autre explication. Creperdant, rien ne mous autorise a aflirmer qure cos causes out agri daus tous les cas at pue jamais la destruction

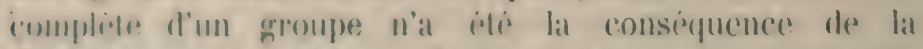
degenemserence grimerale de tous ses representants. On

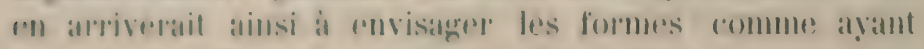
mone duroe desistence limilioe de? mime yur les indi-

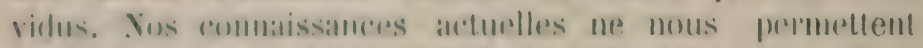

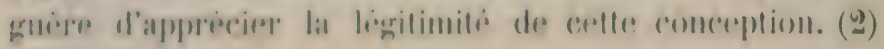

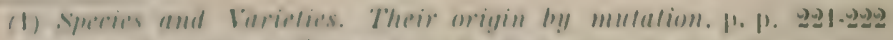

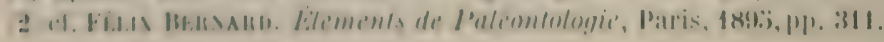




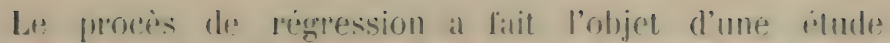

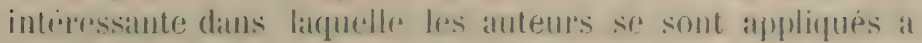

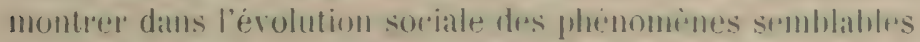

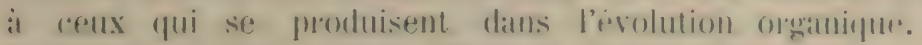

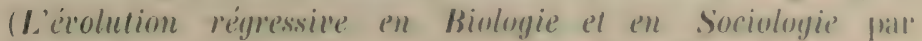

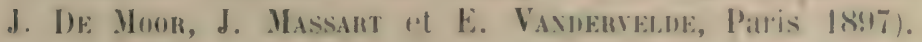

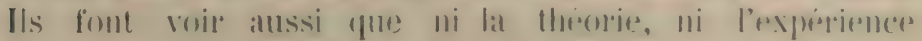

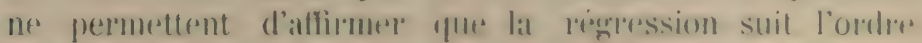
inverse de l'évolution progressive. (1)

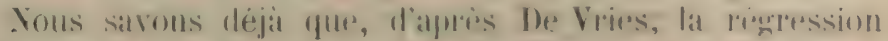

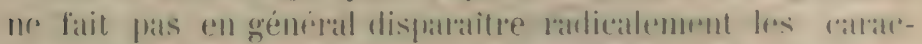

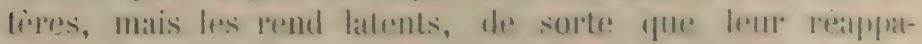

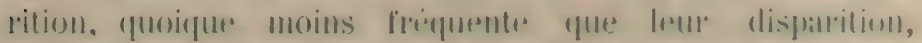

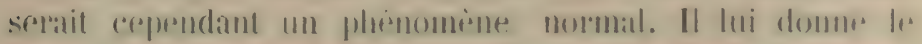

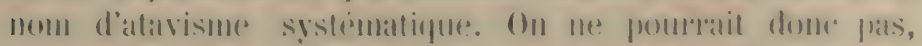

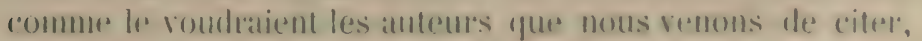

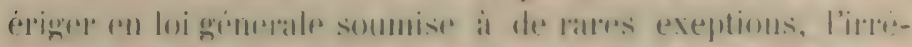

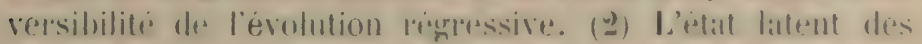

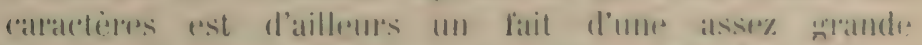

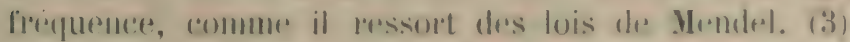

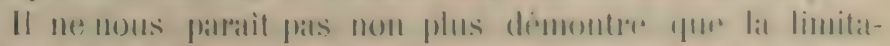

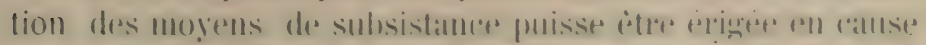

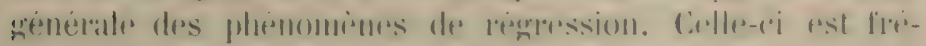

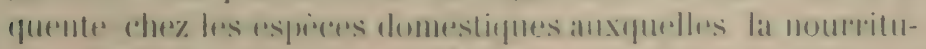

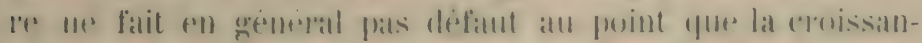

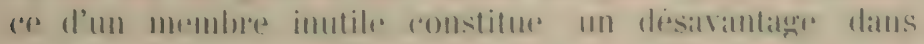

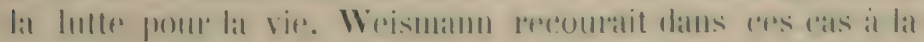

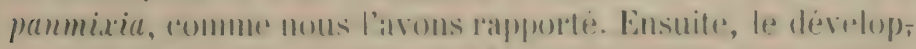

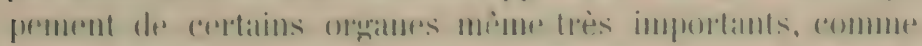

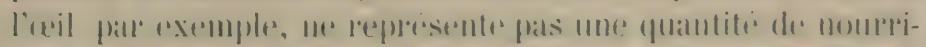

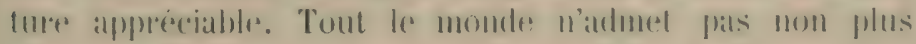

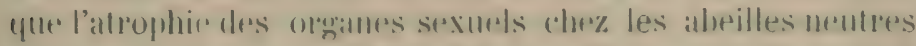

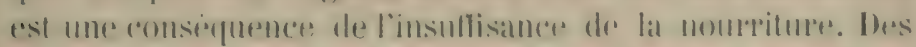

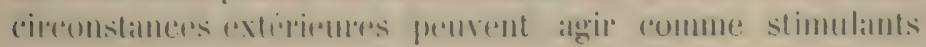

(1) p. 16; st.

(2) p. $211 \mathrm{sil}$.

(3) cf. DE VhIEs op. eit. p. 216 su. 
sans ètre des causes véritabes, et de fait, les différences qui sejarent les insectes neutres des formes sexuées ne se reduisent pas à l'atrophic des oryanes reproducteurs. Entin les auteurs admettent eux-mèmes des cas d'atrophie nombreux dont il est impossible dassigner la cause. (1)

Dernier problème.

Il restu à résoudle un dernier problème. "L'évolution dans son ensemble, dit spencer, aussi bien que l'evolution dans ses détails, marche-t-elle vers le repos complet? L'itat de privation absolue de mourement, appelé mort, yui termine l'ivolution dans les corps organiques, est-il le type de la mort univer'selle au sein de laquelle l'évolution universelie tend it s'engloutir? Entin, devons-nous considerer comme la tin des choses un espace infini peuplé We soleils etrints, vonés a l'immobilite eternelle?

"A cette question speculative, il ne peut y aroir qu'une répronse spreculative. Celle qu'on peut hasarder doit ètre considere moins comme unt reponse positive, que comme une objection a la conclusion qui pretendrait que le resultat prodhain est le resultat detinitif. Si, poussant a l'extreme l'argement que l'esolution doit aboutir a un inquilibre ou repos complet, le lecteur conclut que, quoifu'il puisse arriver de contraire, la mort universelle continnera indétiniment, it est legitime d'indiques comment en poussant l'argument encore plus loin, nous sommes combluits it inferer une nouvelle vir universelle. "(2)

Voici lat conception que suencer propose : toutes les inciles de notre systeme sideral etant soumises a la forex de gravitation, il faut quil $y$ ait un monvement d'ensiemble qui rapproche ress astres of linisse par les precipiter les uns sur les antres. Ce choe riduira la matiore des etoiles

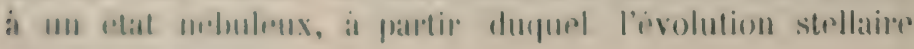
poura recommencerp.

(1) p. 271 84.

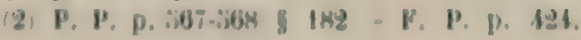


"Il faut pourtant, ajonte spencer, indiquer une condition essentielle de l'entirer accomplissement de ce résultat; je veux dire que la quantité de mourement moléculaire. rayonnée dans l'espace par chaque étoile, tandis qu'elle se forme au sein de la matière difluse, ou bien ne doit pas s'echapper de notre systeme sideral, ou doit ètre compensée par une quantité égale de mouvenent moléculaires envoyé dans notre systrme sideral par les autres parties de l'espace. En d'autres termes, si notre point de depart est la quantité de mouvement moléculaire que suppose l'état nebuleux de la matiere de notre systeme sideral, il résulte de la persistance de la force que si cette matière subit la redistribution qui constitue l'évolution, la fuantiti de mouvement moléculaire abandonné durant l'intégration de chaque masse, plus la quantite de mourement moléculaire abandonnies durant l'integration de toutes less masses, doit sullire à les réduire de nouveau da la même forme nébuleuse. $»$ (1)

Jans l'hypothese adoptés ici par Spencer, le mouvement molérulaire réjandu daus notre systeme stellaire, augmenté de l'energie que representent les mouvements visibles des astres, suffira en effet pour réduire la matiere stellaire à l'état nibuleux qui a servi de point de départ, mais seulement à condition que er mouvement moleculaire soit communiqué intégralement à la nouvelle nébuleuse au moment ou elle est produite. Or, c'est ici que la théorie de spencer est en défaut. Au moment ou les étoiles tombent les unes sur les autres, leurs mouvements visibles se transforment en une quantité de chaleur qui suflit peut-ètre pour rédure leur matière à l'état nébuleux; mais le mouvement moléculaire qu'elles ont perdu auparavant, pendant leur condensation, ne prend aucune part à cette transformation ef n'est par conséquent pas communiqué à la nébuleuse. Celle-ci contiendı donc moins de mouvement molículaire qu'elle n'en possédait lorsqu'elle a commencé la première fois à se condenser ; et à chaque nouveau

(1) P. P. p. รั73-574 § 182 
cycle elle on perdea davantage ; d'ou, comme aboutissement final, le repos absolu.

Que lo: mouvement perdu par le rayonnement des astres soil dfmeuré dans le système stellaire, cela n'est d'aucun secours. On prut, en effet, concevoir deux hypothèses: ou bien l'éther qui forme la trame du systime s'etend dans un pspace trés grand par rapport an volume de la nebuleuse, ou bien la nébuleuse remplit à peu près tout l'espace occupe par l'éther. Dans ce dernier as, la nébuleuse ne peut pas perdre une quantité appréciable de chaleur par rayonnement. Elle conservera sa température et, par consequent, son ètat nébuleux qui en est lat consequence. lès lors il n'y a ni condensation ni evolution possible : ce sera l'équilibre de température et la suppression de tout momrement visihle promptement rialisé. Le résultat sera le. mème si, choisissant l'hypothise d'une grande étendue dr. l'ether par rapport a la nebulense, on suppose que cette derniere resoit d'autres parties de l'espace antant de mouvement molivalaipe qu'alle ren rayonne. La concentration nest possible que si la chaleur est rayomee dans les

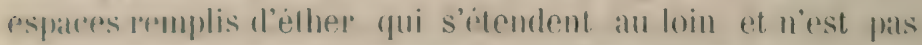

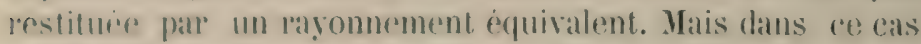
anssi lat chalem rayoune est irvémédiablement perdue pour la nébuleuse. Les respaces compris dans ses proplese limifrs anront toujours une temprimature plus revre que ceux

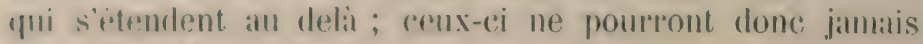

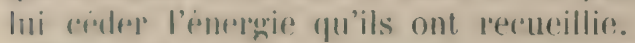

simemere admet gur: les altermatives de concentration et.

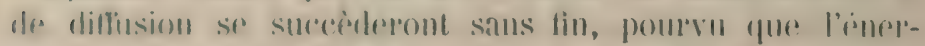

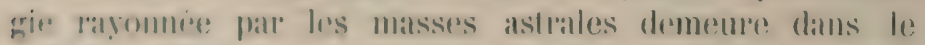
systeme stellaire, res yui "xigr qu’il soit limile ou qu’il

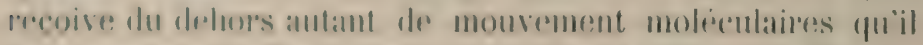

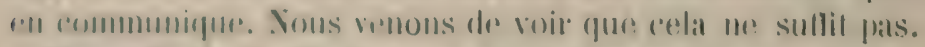

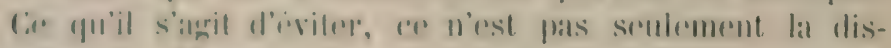

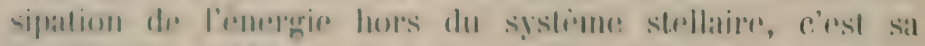
distribution muiforme daus ore ststime lui-mime. La ron-

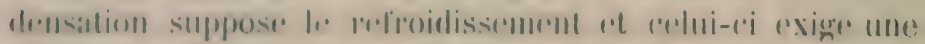

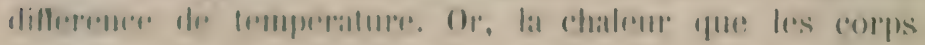


sideraux perdent jar le rayonnement vers les esplatess introstellaires ne prout jamais. Irur itro pestilur sous ancunc forme. Quelles que soient dome les altruations de

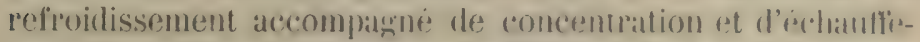

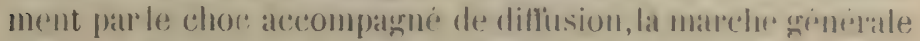
est un acheminemrent vers l'expluilihre de tempriature at l'ahsence de moturement visible. En rllet, a chatpue phatse dre diflusion, la tempriature de lat nébuleuse serat dimimure de tonte la chaleur rayomnie pendant la concentration forrcridente, en supposint mime que lout lo mentrement visi-

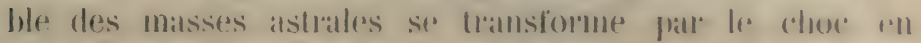

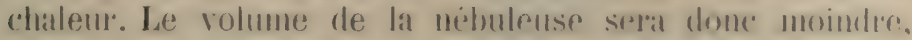

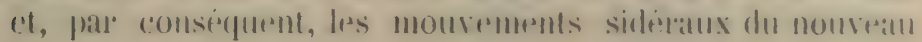
système seront réduits.

Las periodes de dillusion ef dro concentration ne constituent donce pas un rythme indelini, mais, tont an plus, un de ders

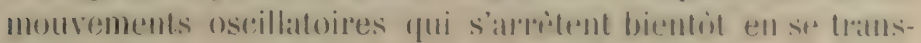

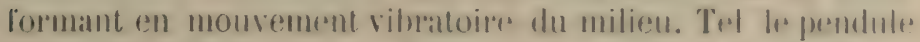
qui oscille dans l'air el dont lese creorsions ì partire de la position dépuilitore ront dimbmant petit it petit.

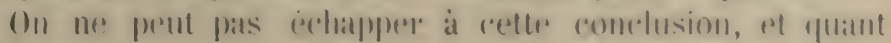

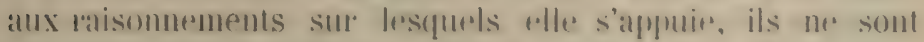

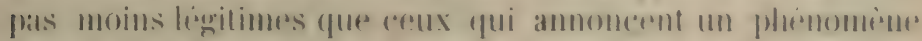

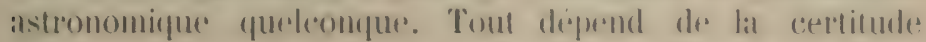
des lois ef des demnees sur lesifuelles on si based. L'immensite des espateres et des demress anxugueds ils s'appliguent ne les intirme an lieen, at nous ne complete-

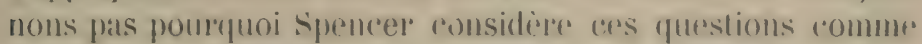
"depatssant les limites de lat spreculattion ratlommelle $)(1)$;

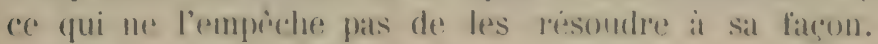

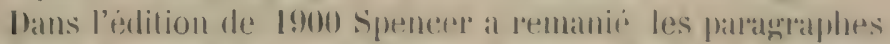
fur" nous aritiquons, mais sams y ajouter rien de nouveall

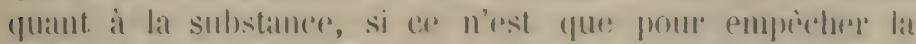

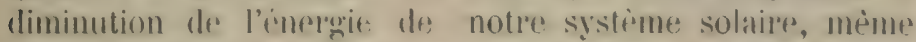
dius l'hypothese d’un ether sillns limites, il sutlit de supposer que "la tempreature de l'espratere est la mime "ll

(1) F. P. p. 131 
dehors el a l'intérieur de notre système sidéral. " (1) Nous craignons fort que cette proposition n'ait pas l'ap)probation des pliysiciens. Nous nous abstiendrons de l'examiner parce qu'elle ne modifie en rien notre conclusion.

Nous n'admettrons donc pas que l'étudél'Lnivers suggère " l'idée d'un passé durant lequel il y a eu des évolutions successives analogues à celle qui s'accomplit actuellement et d'un avenir durant lequel il se peut que des évolutions pareilles s'accomplissent successivement » surtout si l'on concoit, avec Spencer, ce passé et cet avenir comme indélinis. On accordera si l'on veut que le raisonnement fait prévoir des "renouvellements d'activite de vie » (2), toujour's est-il qu'il impose à l'esprit “ l'équilibre et la mort » comme aboutissement final.

Donc: l'érolution générale de l'lnivers matériel a eu un rommencement et aura une fin. Cette tin n'est pas la fin de l'Lnivers, c'est a dire des corps qui le constituent, mais la fin des Iransformations qu'ils subissent par l'établissement d'un état d'ŕquilibre qui consiste dans l'absence de mouvement visible et la diffusion uniforme du mouvement moléculaire, - en rertu de la loi naturelle de la dégradation de l'énergie.

\section{$\therefore$}

\section{\$ II EXAMEX DE LA SYNTHESE FINALE.}

Evolution universelle. - Alternatives indéfinies d'évolution et de dissolution. - La force comme réalité fondamentale.

Evolution Spencer lepminr pal une risapitulation. Tout en resu-

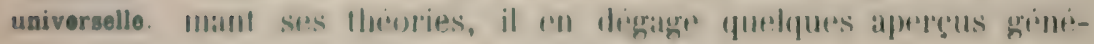
raux qui doivent ctre examines.

(f) F, P. P. 131

(2) P. P. P. 876 y 18:3 
C'est d'abord l'idee d'après laquelle toutes les evolutions particulières ne sont que des parties d'une évolution génerale. "Yous avons jusquu'ieci, dit-il, considére la loi d'évolution comme complètement vraie de tous les ordres d'existences pris a part comme ordres distincts. Mais sous cette forme, l'induction manque de l'unisersalite qu'elle acquiert quand nous considerons les divers ordres d'existences commus formant par leur ensemble un tout naturel.....

"Nous avons iterativement remarque qu'en mème templs yu'un tout se développe, il se fait tonjours une evolution des parties qui le composent ; mais nous n'avons pass observe que certle loi est enalement vade de la totalite des chuses, an tant que composere de parties depuis la plus grande juspuna la plus petite. Nous sarons que tandis qu'un agrengat plysique coherent comme le corjes lummain s'aceroît en volume et prend sal forme greneralr, dhacun des organes qui le composent fait de meme; que tandis que chaque organe grandit et devient diflerent des autres, il se fait une diflesenciation at une integration des tissus et des vaisseaux qui le composent ; et que mème les ithments de ces tissus composants s'acervissent sejarement et prenuent des structures plus distinctement heterogrines.

"Mais nous n'arons pas assez remarque que, ell partant du corps humain considere comme une particule et en s'élevant aux parties plus grandes, on voit se manifester également la simultaneité de translormation : que tandis que chaque individu sp developpe, la societe dont il est une unité insignitiantr. se développe aussi; que tandis qune: l'agregat-masse qui forme une sociéte devient plus distinctement heterogenene, la Terre dont certe societe est une partie inappreciable, le devient aussi ; que tandis que la Terre dont le volume n'est que la millionnieme partie du systeme solaire, progresse vers une structure concentrée et complexe, le sprstime solaire progresse de la mème manière, et que mème ses transformations ne sont pas autres que celles d'une partie a peine appreciable de notre système sideral, lequel a traversé en mìme temps des changements analogues. 
"Ainsi comprise, l'erolution n'est pas seulement une en principe, plle est ule en fait. II n'y a pas plusieurs métamorphoses (fui s'opèrent de fal mème manière, il y a une sulule mramorphose (qui s'avance universellement, partout oir la metamorphose contraire n'a pas commencé. »

Rienn n'empèche de considerer l'Univers comme un tout; nous ne voyons méme aucune raison a priori de nier qu'il arcomplit une ivolution d'ensemble. Celle que spencel inatgine pour notre systeme stellare ot que nous arons malpportè est anologgue à l'évolution du système solaire. s’il s'agit de tout l'ensemble des ètres corporels, on peut con(eroir yuril soit sountis a un proces d'integration de ceptte sorte, pourva toutefois qu'on ne supposf pas l'L nivers inclétini.

Mais ce n'est pas ce que spencer a en rue, dumoins primripalrment, dans lo passage rite. Son but est de nous latre considero toutes les ivolutions panticulières comme rotant dess parties d'un process d'exolution universelle.

II me sagit pas ici d'une question de mots. Si par evolulion miversello on ne voulait signifier antre chose

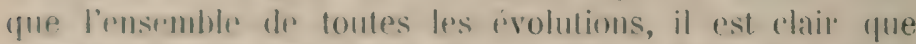

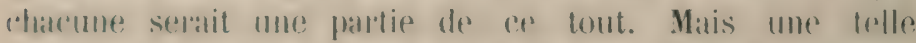
allipmation me? serait qu'ume lautologere et nous ar' pourons

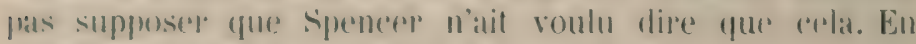
realite, it s'agit de satroir si l'ensemble de toutes lessivofulioms ast reellement une evolution. De mime qu'un en-

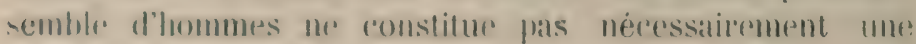

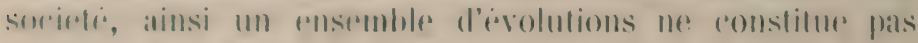
nécessatrement une évolution tolale.

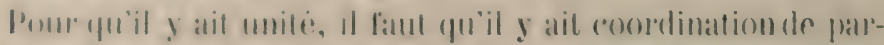

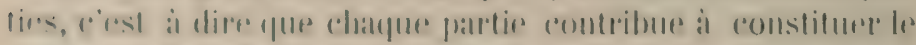

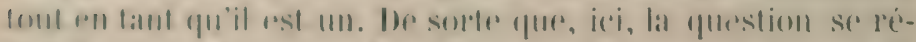

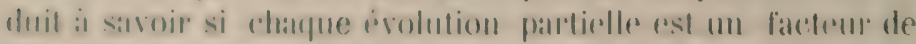

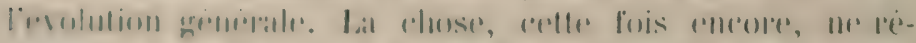

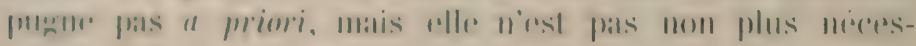
sarire. Il faut en juger d'après le fait.

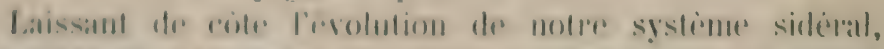

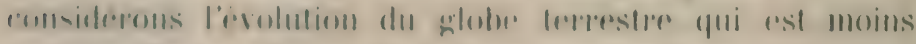

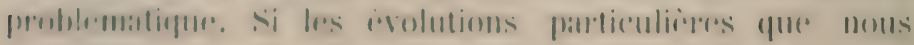


observons antour de nous font partie d'un proris gemeral, ce serat ren lant qu'elles font partie dre l'evolution der lat

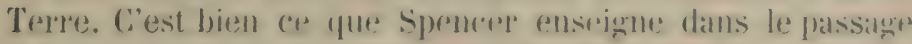

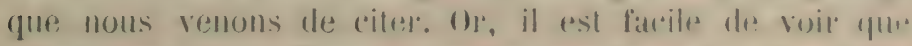

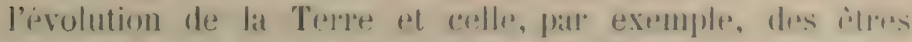
vivants soml drolx poroces alssolumment distincts et quion me peut pas ratsonnablement consicheren la secomble comme

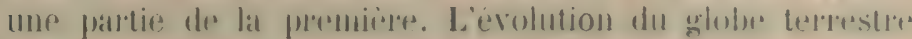
ast en somme une condensulion par refroidissomenl, lesidif-

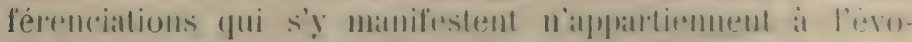

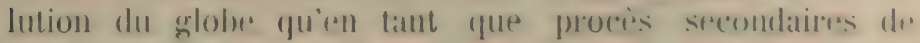

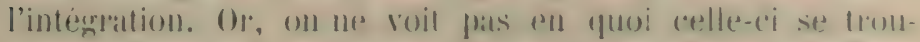

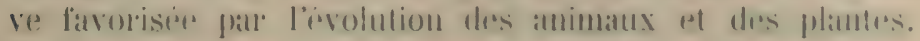

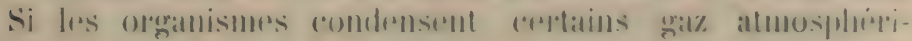
ques, ceste du moins normatement, pour les abamtommes de.

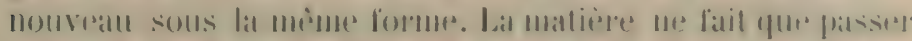

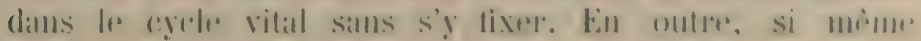

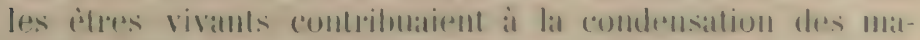

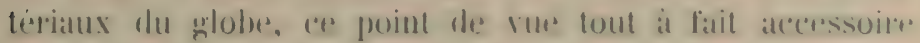

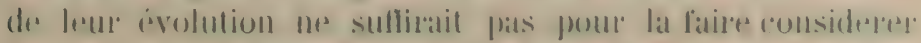

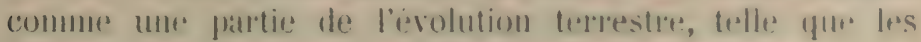

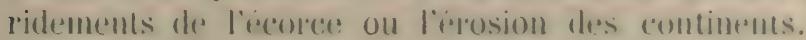

Toute evolution tire son mite du grimeiper qui lat distere-

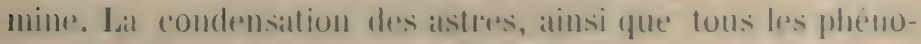

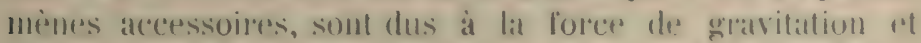

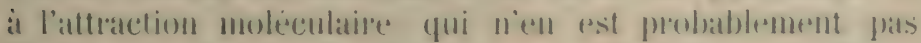
distincte ; landis que lat vie n'al que? des resations atssez vagues arec coss forees naturolles. Gue lat Terre deviemes phus

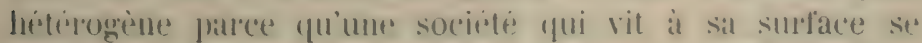

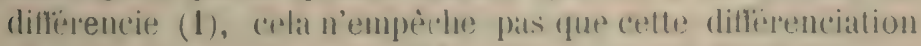

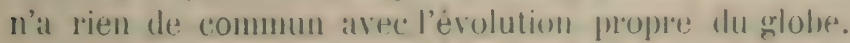

Tous sommes disposies a admettre que l'évolution de lat vie terrestre possisle une uniti reritable, ayant roll vaseinhiblement des deluts assez homogemes, et s'blant poursuivie sous l'empire des lois geneneales, de sorte quansin l'erolulion de chapue individa est resellement une partie de l'iso-

(1) P. P. P. $\$ 86$ \$ $188,-$ F. P. p. 434. 
lution d'ensemble da lapuelle il contribue pour sa part. Mais, si sur quelque autre planète des ètres vivants se sont également développés, le procès d'évolution eit-il été analogue a celui qui a été réalisé sur la Terre, nous n'en serions pas moins en présence d'une évolution absolument distincte et indépendante, et il serait illécitime de considérer ces deux évolutions comme des parties d'une mìme *rolution totale, alors qu'elles sont séparées l'une de l'autre parr l'espace et le temps et qu'elles ne concourent pas par leur nature a un effet d'ensemble.

rous repoussons done cette idée de l'Unirers accomplissant une évolution unique dont toutes les existences particulières ne seraient que des épisodes.

D'ailleurs, Spencer restreint lui-meime le caractère absolu de sa conception, lorsqu'il excepte de cla métamorphose (qui s'avance universellement) les parties de l'Lnivers où la mblamorphose rontraire a commencé. Il n'y a donc pas un procis d'évolution gémérale, puisqu'il y a des parties de l'Lnivers ou a lieu la dissolution. Ce n'est pas que ces deux procès ne puissent pas aroir entre eux des relations itroites. La mort des individus est intimement live à l'èvolution des societés, de mème que la dissolutions des cellules epithriales se lattache à la vie de l'animal. Yéanmoins les deux prochs sont distincts et leur existence constitue une dualité qui ne s'accorde pas avec l'unité privée par spencer.

" Guel fondement a-t-on, dit J.Ward, pour affirmer que l'univers (comme tol) a subi une evolution? Un homme, une nation, un continent, un systime sicleral, comme ohjets particuliers, onl charun leur histoire determine de naissance et de mort, dre progres et de dicadence, de broullat ln'ulant en de rensolidation froide ot morte. Mais eroissaner et derobsamer, ascension et declin, elévation et dégradation evolution of dissolution sont partont contrmporains. Nous

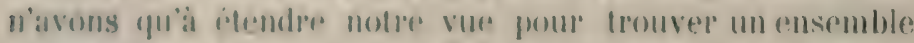
permanent deitres imdividures qui passent et qui se trourent at coutes les phases du chamerement. ) (1)

(1) Naluralism and Apuestietism.vol. I p. 191 
tives mies

ion ot lution

solutions farticulires ue sont pas les parties d'un proces de dissolution universelle.

Tous les itres matritels sont sujets a itre detruits, parce qu'ils sont comploses de parties qui pruvent ithe séparees les unes des autres par une force suflisantr. Il y a, sous ce rapport, une distinction a latre entre les agrégats dont l'equilibre est tress pen stable, comme les ètress vivants, et ceux dont l'écguilibre est trés stablr, comme certains colpes inorganipues a l'etat solide.

Les premiers sont facilement detruits et n'echapurent, en gemeral, pas tres longtemps de lat dissolution. Ils ont meme a luttre constamment contre elle: : lat mort arrive pall le fait qu'ils y strceombrolt. Les serends, all contratre, St: maintiennent pendant des preriodes illintites.

Quoigue la destruction soit, dans le serts que nous venons de dire, le sort commun des itres materiols, il n'y a pas lien de la considereg comme les resultat matumal du frocès d'evolution. Yous arons montri dans le paragraphe precedent que l'evolution du systeme solatre el ceelte evolution que spencer admet pour notre systeme stellaire, tendent l'une et l'autre vers l'équilibre définitif.

Do sorte que la conception d'ume evolution universelle sucededant a la dissolution universelle far un mouvement rythmique indélini est inacerptable a tous les points de vue. Spencep dit bien que " celte conclusion ... est un cornilaire de la loi de la persistance de la force ". (1) Mais il n'apporte ancune preuve pour établir cette attirmation.

Spencer dit encore: "si nous sommes ainsi conduit à concevoir une série d'érolutions remplissant un passé sans limite et une serie d'évolutions remplissant un avenir sans limite, nous ne pouvons plus attribuer a la creation

(1). P. P. p. $\$ 90 \$ 190$. 
visibh un commencement "t une fin dianis, on la croire isolese. Elle s'unitie arec toute existence avant ou apres, et la Foree que l'Lnivers manifeste rentre dans la meme

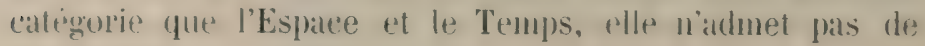
limite dans la pensée. ”(1)

Yous croyons itle justities a dire que cette idee synthétique rst en opposition arec les resultats que nous arons recueillis de l'etule de l'unires's materiel. En outre, elle sonleve, all point de: vue speculatif pur, des objections peremptoires. Yous allons les indiquel mievement pour les lecteurs qui nadmettent pas arec spencere quil est loisible d'adepter des concegutions, lors mème you par ailleurs clles sont démontrées absurdes.

lgu'um serrie soit illimitee dans l'avenir, cela n'a rien d'impossible, patree que l'avenir n'étant jamais realisé tout entier, les termes qui se succielent les mos allx atutres formeront foujours un nombre limite. Mais il n'en ast plus de: mème si on suppose une serie illimite dans le

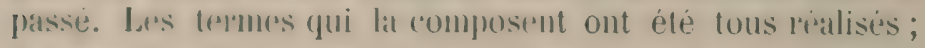
ils constiturent une multilude determinese. Or, ante multi-

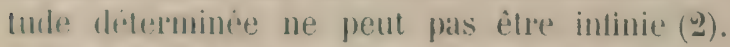

En outre, à chaqur moment il est vrai de dire qu’un evenement qui se produira seulement apres un temps intini ne: se produira jamais. On nompive jamais all bout d'un trmus intini, puisque ce tempes n'a pas de houl, c'est à dire de fin.

(1\%, si l'univers existe depuis un temps intini, certains evinements sont actuellement passies depuis un temps inliui. Can si foules les phases quo'a parrourues l'univers sont passeres depuis un tempes fini. l'univers noxiste que dreguis un tromps timi, il a en un commencement, il n'est flas éternel.

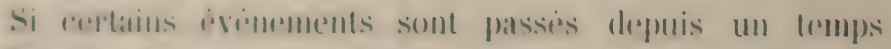

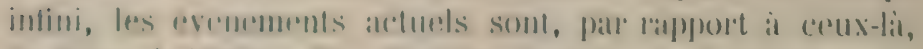

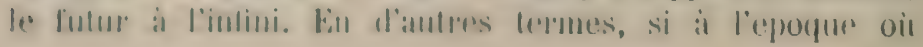

(1) P. P. P. 8590.8991 \& $191-$ F. P. \& 190, p. 142.

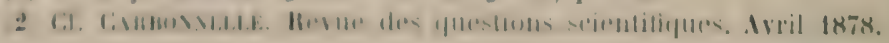




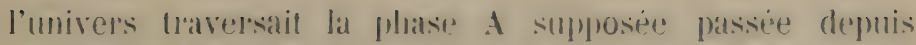
un temps inlini, on demandait apmis combien de temps se rabliserat lat phase actuelle, on devat rejpondre : appres

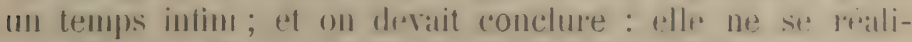

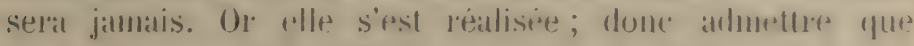
certaine phases A de l'Luivers est passioe depuis un lemples

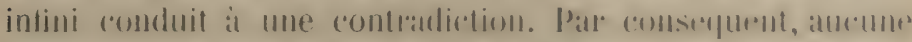

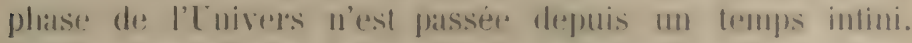

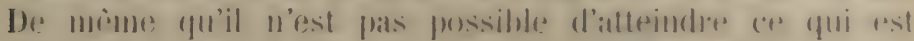

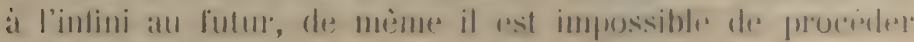
de l'intini au passé.

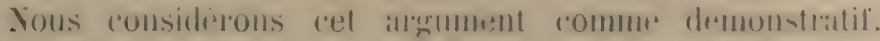

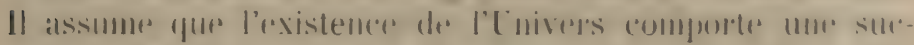

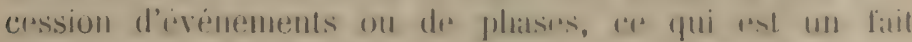

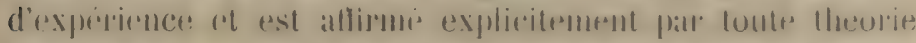

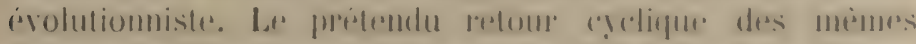

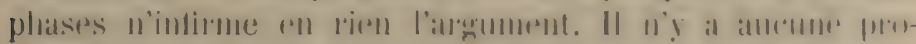

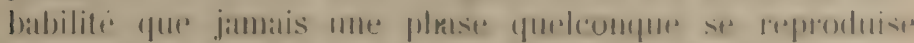

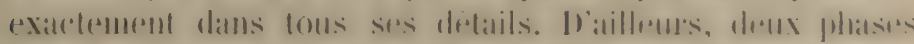

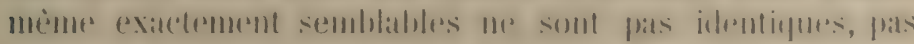

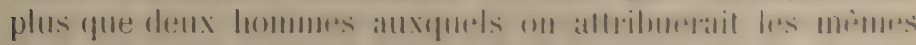

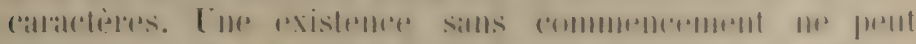

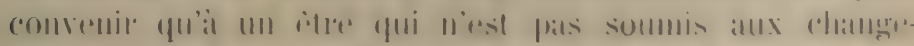

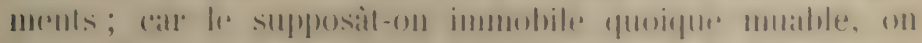

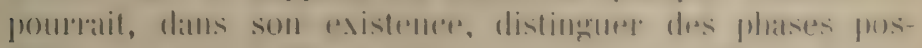

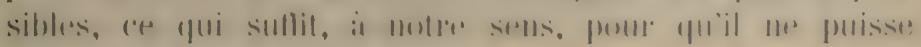
pas être sans commencement.

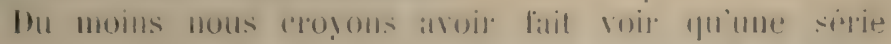

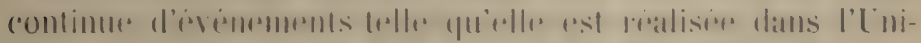

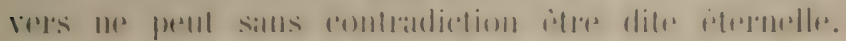

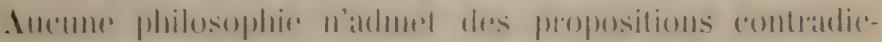

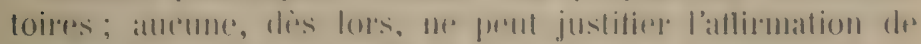

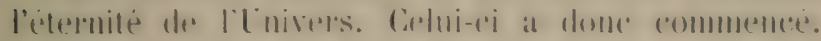

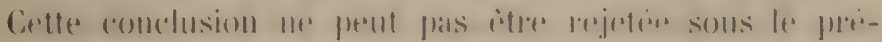

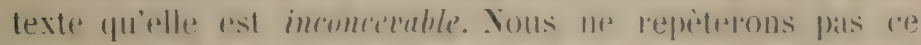

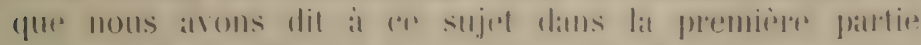

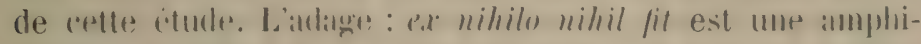

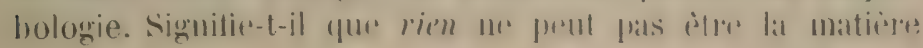


dont on fait quelque chose? Cela est bien évident; mais aussi qui l'alfirme? Veut-il dire qu'aucune chose ne peut. être produite sans quelque matière dont elle soit fabriquée? C'est bien cela qui est en question. Mais nous ne, connaissons aucune démonstration de ce prétondu principe et nous avouons ne pas en roir l'évidence.

La création n'est pas plus inconcevable que l'attraction, la communication du mouvement, la transformation de l'inergie et mille autres choses dont l'experrience nous garantit la réalité.

Las difficultes que certaines conceptions entrainent ne doivent pas nous empècher d'en reconnaitre la vérité. "Toutes les fois, dit Pascal, qu'une proposition est inconcevable, il faut en suspendre le jugement et ne pas la nier a cette marque, mais en examiner le contraire; et si on le trouve manifestement faux, on peut hardiment aftirmer la premiere, tout incomprehensible qu'elle est. ) (1)

Yous avous dejáa dit que l'espace et le temps, loin de nadmeltre aucume limite dans la pensée, sont au contraire nécessiarement conçus comme limites, pourvu qu’il s'agrisse de l'espace et du temps réels. Il en est de mème de la force el de la masse. Ou mine, ces dernières possiedent un dément, l'intensité, qui non seulement exige " priori des limites, mais dont les limites se constatent par l'expérience.

Ensuitr, si la force est illimitie - el la mème chose s'applique at la masse - comment concerrons-nous la persistance de la force, cest-it-dire "que la force conserve la mine quantigr dans le passe comme dans l'avenir." ? Ia diminution d'ume forere, par exemple de la force de Eravilation, - hypothese evidemment concevable - entranoratedle la diminution de la foree? si non. le prin-

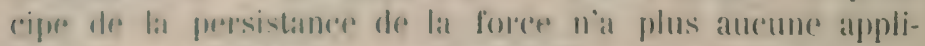
calion ans phenomines mallurels. Si oui, on hien apres

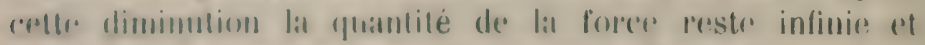

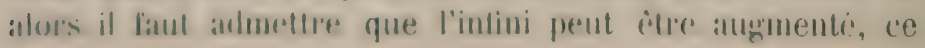

(1) Penstes. 10 Partie, art. II. Paris 1875. p. 21. 
qui est absurde farre que l'alugmentalion suppose des limites; ou bien la quantiti de la fores devient limitese par la soustration yu'on y a fate et alor's il faudrat conclure que la somme de deux quantites tinies donne l'infini.

L'étude du connaissable rejoint, d'apress spencer, la conclusion qui aflitmo l'existentere de l'Inconnatissable all fond des choses: " Yous aroms dit, dit-il, ytae la rougance en In pouvoir dont on ne peut concevoir les limites dams le temps ni dams l'espane, est létrement fondammlal de la Religion, élement qui survit a tous les change-

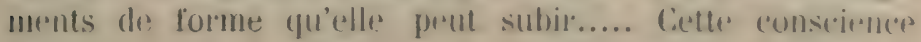

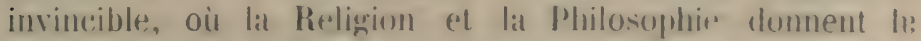
main au sens commmon, est allssi, mons latrons demumtré, cellor qui sert de base at la siremere.

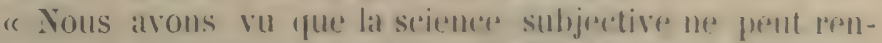

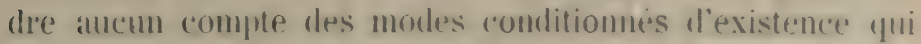
constituent la conscience, sams supposide l'existemere d'un itre inconditionne. Nous arons vil encores que la science

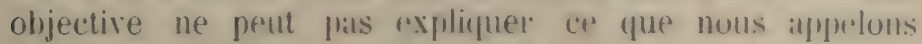
le monde exteriem, sans reacalder ses dhangements de forme comme des manifestations de yurlyue chose qui demeure donstant sums toules les lommes. C'est encomer a ce prostulat que sie ramene la synthese que nous renous d'élifier. Ia reconnaissunce d'une fore persistante, qui varie toujour's ses manifestations, mais qui conserve la mème quantité dans le passié comme dans l'avenir, nous permet seul d'interpreter chatue fuit concret, et en définitive nous sert at unitier toutes les interpritations concrètes. » (1)

L'Inconnaissable n'est donc fras antre chose que la force qui se deploie sous mille formes dans l'Lnivers.

(1) P. P. p. $392 \$ 191$. - F. P. p. 143. Speneor a moditie plus tarul la l'edaction de ec passage. mais l'idée reste la mime. 


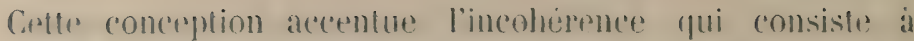
dedarep ineomnatssible une (dhose dont on alfirme l'exisferece et a lapuelle? on attribue des caractires detinis.

Yous allons reprendre brievement, en tes contirmant, les aryuments qui nous empredient de sonserite a la synthise d'après lanuelle de la persistance de la fores deriveraient toutes les lois des phimomènes matripiels.

1. Qnoiyu'on puicse amployer le mot force an singulier pour designer l'ensemble de toutes les forees phrsiques, chimigues of plụsiologiques; il ne laut pas oublier que daus l'itat actuel de nos comnaissances, elles nous apparaissont comme de nature dilferente. Certains theori-

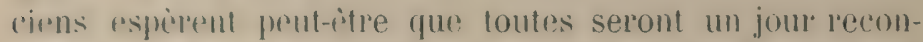
murs romme des manifestations variess d'une seule force primordiale. Mais ce n'est qu'ume vague conjecture. En tous cas il sera impossible d’idrntiliel la force vive avec fes forers yni ne consistent pas dans un mouvement. Il ny

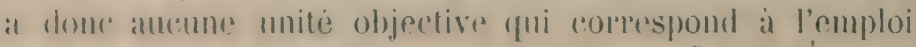
du mot force au singulier.

2. Jes forees que nous commaissons ne sont pas persistantes, mais elles varient suivant des lois ronstantes. lonu des principales de ces lois est la conservation dre l'entrugie an moins dans les monde inorganique. L'énergie ellemime n'ast bas mique ; elle se compose de deux élements

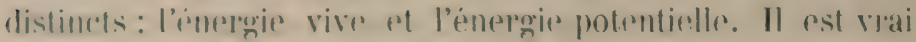

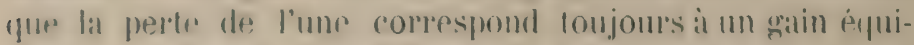
valent de l'autre. Mais, rigourensement parlant, il rest

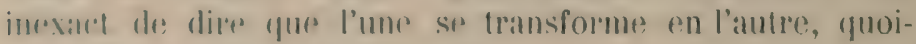
que cette expression soit reçue.

Lat pluphall des phimomines physico-chimigums mes sont

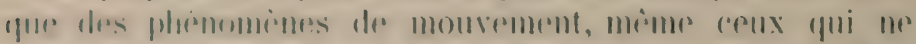

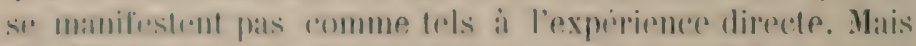

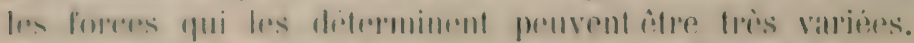
Tumles sonl sommises a la loi de la consenvation de l'o-

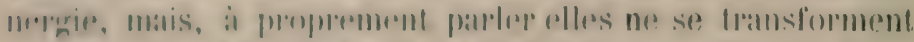

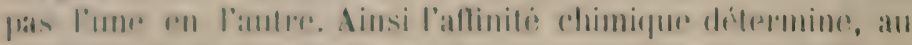

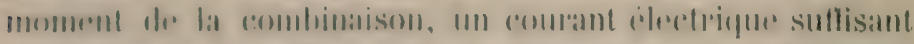

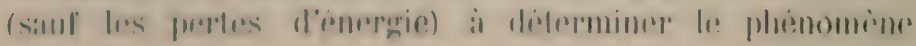




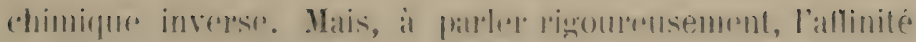
chimique ne se transforme muliament rin counant alectrique.

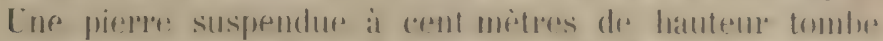

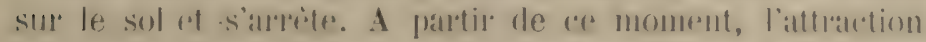
lerrestre existra anssi bien du'angaravant, mais lat situation du mobile n'est plus telle que cotle force puisse produire

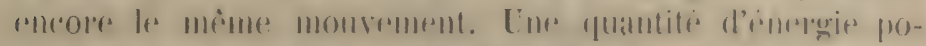

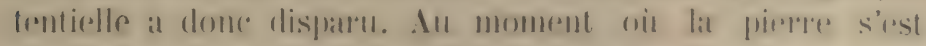

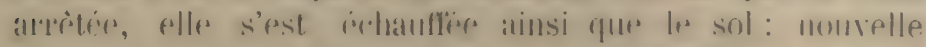

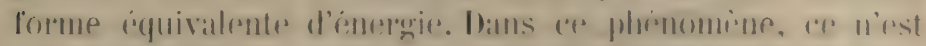

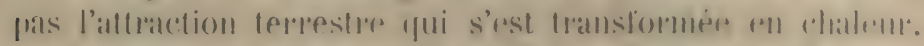

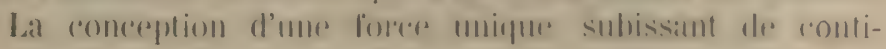

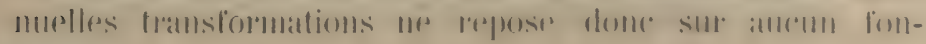
dement.

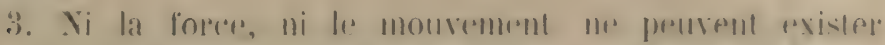

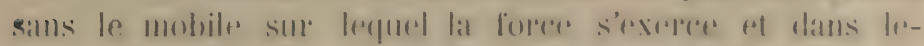

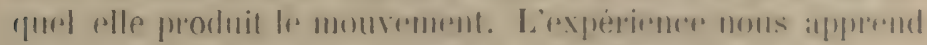

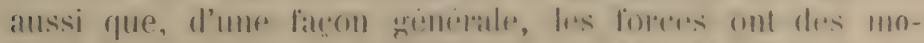

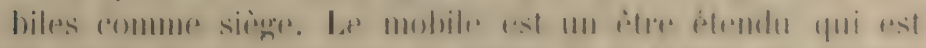

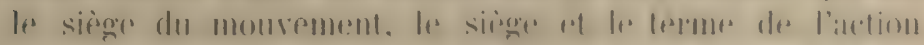

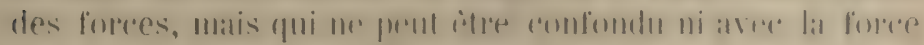

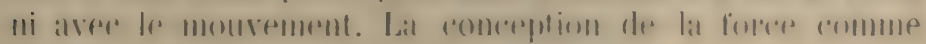
réalité unique n'est done pas admissible.

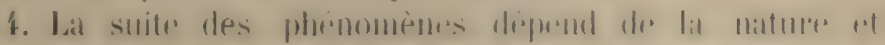

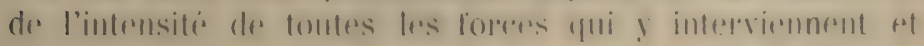

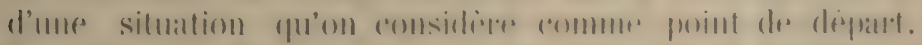

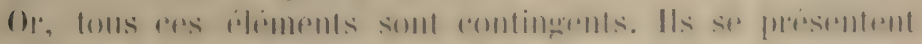

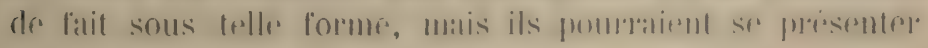

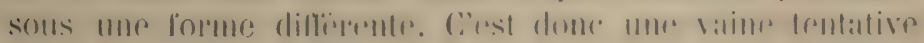

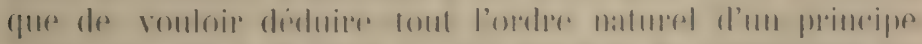

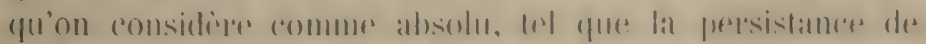

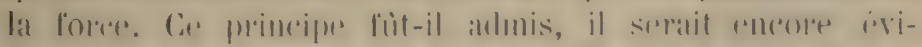

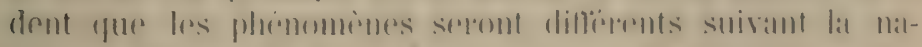

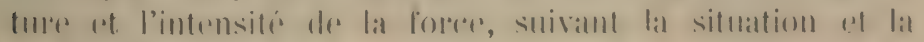
masse des corps sur lesquels elle agit.

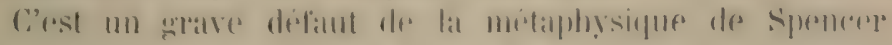

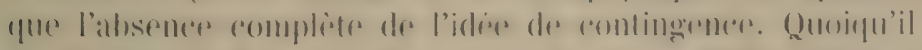

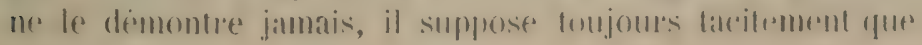


tout est nécessaire. Un principe unique s'impose, d'apris lui, à l'intelligence et de ce principe tout découle par une consequence inevitable; la loi d'evolution n'est une partie de la philosophie que si on démontre qu'elle est une conclusion nécessaire du premier principe et il en est de mème de toute autre vérité.

Il est vrai que Spencer part de certaines domées: le moi el le non moi, l'espace, le temps, la matière, le mourement, la force. Mais outre que ces donnees sont insulfisantes comme point de depart d'une conception détorminée de l'Univers, Spencer les considire comme des manifestations de l'Absolı et n'envisage jamais l'hỵpolhèst? de leur contingence.

En outre, pour lui, la force est le " principe des prin(ripess), la matière et le mourrment n'étant que des manifestations de force, l'espace et le temps les conditions de ces manifestations; (1) et la persistance de la foree? est la seule raison fondamentale de tous les phénomenes. Tous moyons aroir le droit d'attirmer que cette synthesp n'est qu'une illusion.

sprocer termine mo protestant quon ne peut pas légitimmornt dire dre sa philosophie qu'elle est materialiste. Yous or revendrons pas sur aetle question sur laquelle nums nous sommes sultisimment explique plus haut.

(1) P. P. [1. 1798 8 $800 .-$ P. P. p. 18:2. 
(IIIPITRE VII.

Les origines de la Philosophie des Premiers Principes.

Récapitulation. - Appréciations.

rigines Quelles sont les origines de la philosophie de: Spencent? la Nous n'avons i repondre à cette question qu'en ce qui sophie remiers concerne les Premiers principes. Le penseur anglitis ne reconnaissait pas volontiers avoir emprunti ses ideres anx autres; ancune critique ne le blessait plus vivement que celle-là. Il faut reconnaitre d'ailleur's, comme nous l'avons fait observer, que sa philosophie est largement originale. Mais ou est le prenseur isole? ?ui peut se vanter d'echapper à l'intluencr des théories règnantes, ou même, le ne pas itre profondément inthuencer par elles?

Nous avons déjà eu l'occasion de dire comment la théorie de l'Incomaisable témoigne de l'influence exercée par la critique kantiemne sur les spéculations modernes. "C'est Kant, dit A. Férro, qui traça véritablement la voie : Comte, a Spencer, à la philosophie de tout le XIXe siècle. " (1) La quintessence de la théorie de l'Inconnais-

(1. La critica della conoseensa in $E$. hant et $H$. Spencer. Savone. 1900 p. 39 
Sable se trouve dans le chapitre qui traite de la relativité de $n o s$ connaissances. Spencer s'y appuie sur Hamilton et Mansel. "De mème, dit F. Aveling, que Mansel se servit des principes etahlis par Hamilton, ainsi Hamilton fut largement influence par la doctrine de kant. Il laut donc assigner au systeme de hant une relation historigute arec les conclusions de spencer sur la relativité de la combaissance et de l'Inconnaissable. " (1) Si syencer a cite de preférence Hamilton et Mansel, ce n'est fas, grobahlement, que ces deux philosophes aient eu sur sus idees une influence plus grande que d'autres le la mème école, mais c'est, sans doute, fatrece que l'un w l'antre, tout en ensrignant l'impossibilité pour la raison d'attrindre l'Inlini, admettent cependant son existrnce comme: objer de la revélation et de la foi. Spencer a fretendu egalement faire considerer l'Inconnaissable conme objet de Rerligion. Il s'fearte de Hamilton et de Mansel en

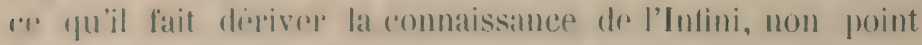

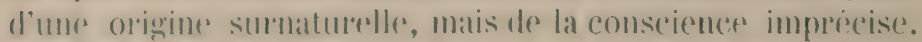
En cela - puispu’il admet an sommo l'existence d'une realite fondamentale indipendante de nos rommatsanees il se rappurocle davantage de Kant que de l'école idcialistr. L'intluence du philosophe de kornigrsbereg sur sprencer se home all point yur nous venons dre signalere. Co n'est fas a kant que spencer a emprunte le mocanicisme, ni la théorie de l'évolution.

(I) a signale allssi dans les Premiers Principes l'inlluencer

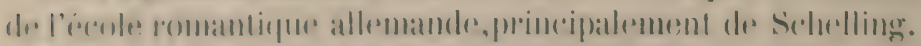

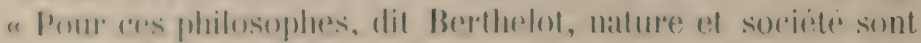

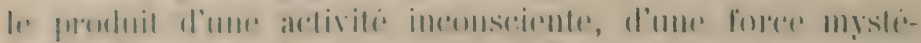

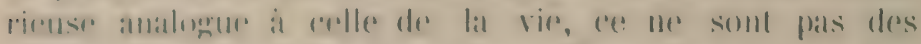

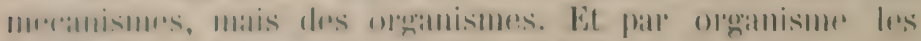

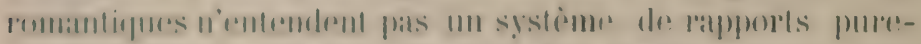

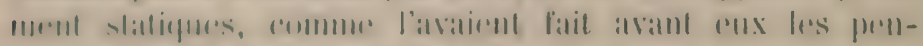

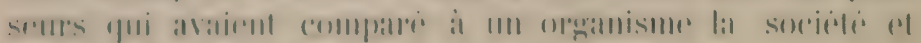

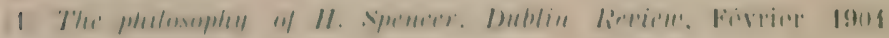
I. 93 
la nature, un Platon, fart rexmple, dialls l'antipuitio, un Hobbes dans les tempes modermes; ils entemdent pald lat

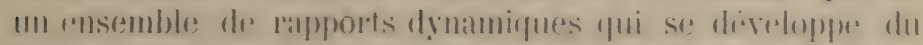

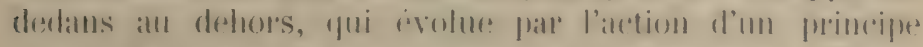

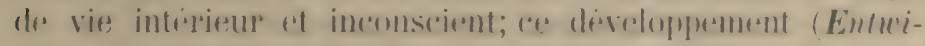

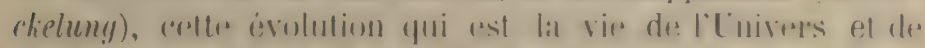

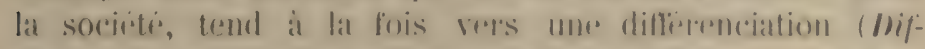

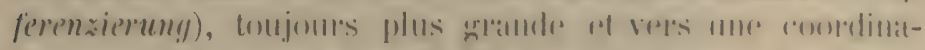

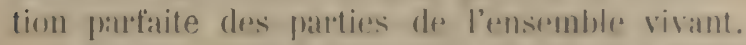

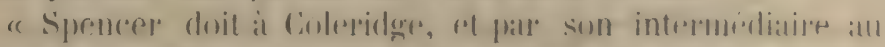
romantisme allemand, alve les appressions metmes de vire

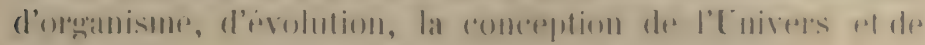

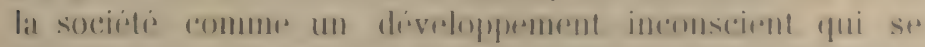

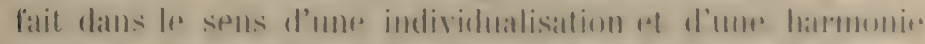

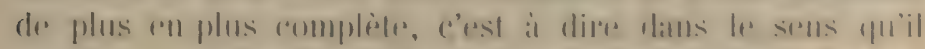

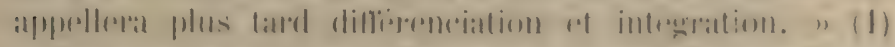

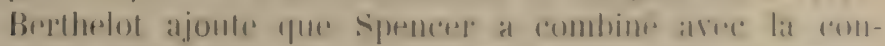

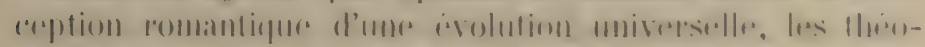

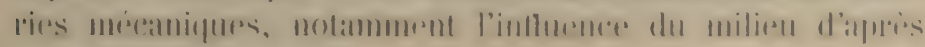
les idies de Iamarck.

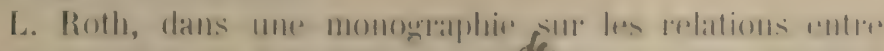

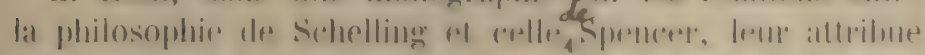

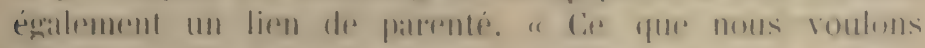

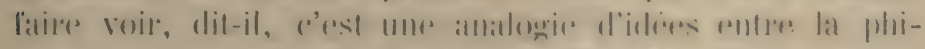

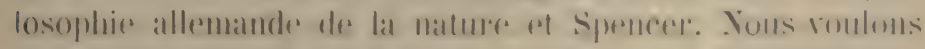

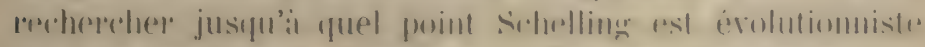

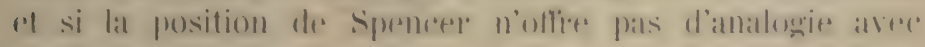
celle de schelling.

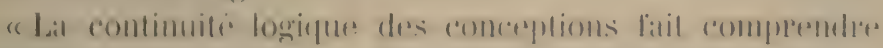

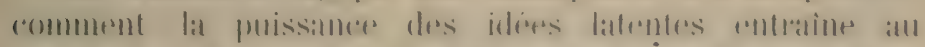

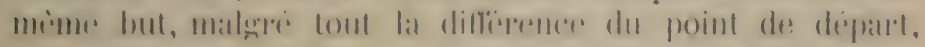

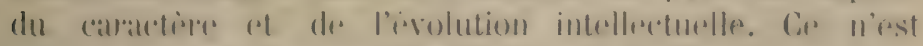

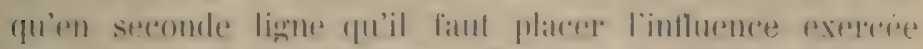

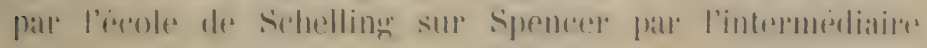
de C. E. von Baer et de Coleridge.

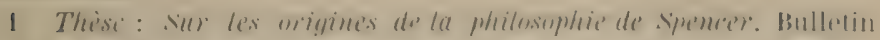
de la Société française de Philosophie, 1904 p. 9\& 
"La pensée de Spencer a ressenti rette influence à une époque ou il était préoccupé de ce qu'il y a d'essentié clans le concept de dévelopjement. Elle fut en même temps un pas en avant pour l'idée d'évolution. Spencer dans son second ouvrage répond à la question de "la nature du développenent " ou du progres avec CorelidgeSchelling: Le developpement consiste dans la tendance à l'individualisation. Plus tard aussi, dans une seconde plase distinctive de sia pensée, l'étude des icrits de von Bael hàta le progrès de la conception. ") (1)

I. Roth signale igalement des analogies cutre la théorie de la connaissance chez les deux philosophes. Nous avons dejal dit que la doctrine de l'Inconnaissable se rattache all Kantismo. Il est vai que Spencer, comme Schplling, (2) combat le realisme an cherchant it faire voir des contradictions dans l'hypothèse de l'existence en soi des objuts de notre experience; mais cette méthode de raisonnement se retrouve également chry Kant. D'autre part, l'idéalismo transcendental qui affirme l'identité absolue du moi ot du non-moi et supprime la distinction entre la réaliti of comnaissance (3) est inconnu à spencer. Nous avons vu que la manière dont il établit la distinction fondamentale du moi et du non-moi est purement empirique. A cost égard donc, la dilférence entre sa philosophice el celle de sidulling ou de Hégel rst radicale.

spencer se rattache plus reelloment a schelling par la conceptiou d'une évolution génèale. Celui-ci conceoit en elfet lat nature entièe comme un organisme vivant dont la vie moverselle se manifreste dans les formes les plus variars en developpenunts gradues, - dans lequel l'Absolu st difliremair en objots particuliers, pour les reprendre et les matieg dams son inlinite. (f) II est valisemblable que

(1) Seluelling und Spencer. Burne 1901 p. p. 7-8

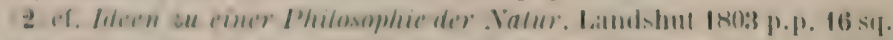

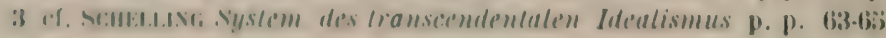
el passim

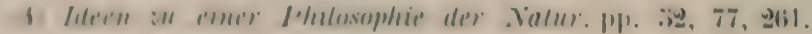


Spencer dans la gieneralisation de la therre évolutire a éte influenci indirectement par ces conceptions monistigurs. Nous devons remarquele cependant que l'idér d'une evolution universelle se prisente foul autrement che\% lui fur chez Schrlling. Pour celui-ci, l'évolution ginérale est la conséfuence ou mieux la simple expression der lidlablisme trauscendental. L'absolu ideal et l'absolu rexel sont unre sinle? et mème chose. "labsolu est III acte de connaissance? eternel, dit Schelling. Les choses an soi sont les idters

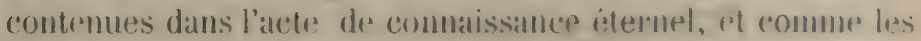
idées sont une seule iltée daus l'Absolı, loutes les choses ont vrament au fond un seul thre, e'sest a dire l'atres du

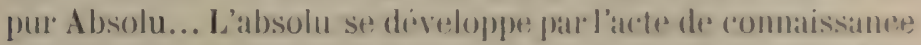

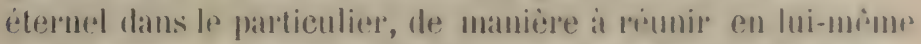
Ir tini par lat formation (Einbildung) de son intinite dans le tini.... Le: colé réel de l'andivite ietermelle (de l'Absulu) se manifeste daus la Vature : la Nature ens soi ou la Matme eternetle est l'esprit lurine daus l'otgiet, l'ktre divin introduit dams la formor.... tandis que la Salture qui appratrat est la lormation de l'Etre dans la formes so manifestant comme telle, c'est a dire dans le particulier. ") (1)

Che\% siprencer, an contraire, l'evolulion universelles applatratit comme la spouthese des fovolutions particulienes qui ont

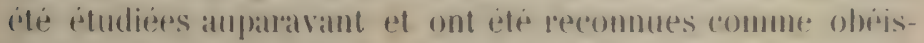

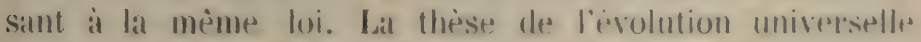

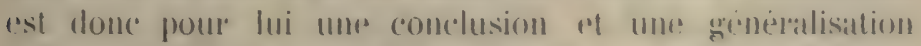

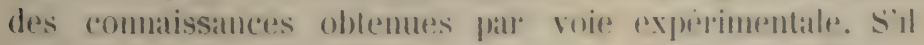
est vari, commer le dit Berthedot, que lat loi de dillérentociation el d'integration évouge le souvenig des lhèses,

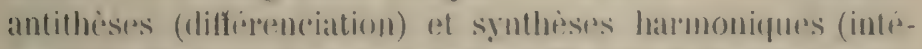
gration) dont nous parle Schelling, (2) il faut avouer cependant que lanalogice est assez lointaine et que less fondements sur lespuels repose la loi d'évolution de spencer sont fout dilhrents des spécolations transcendentales

(1) Ibid. pp. $67-76$.

(2) Ibid. p. 97 . 
l'où Schelling a tiro sal formule. Dans sa réponse a Martinean, spencer a lui-meme combatu l'érolution concue à la lason de Schelling ou de Hergel. (1)

Avant l'applarition de l'Origine des Espèces de Darwin (18:8), spencer avait a plusirurs reprises rexpose l'ulés de l'érolulion dles respeces organiques, notamment en $18: 37$ dans Prongess, its lause and cuuses at daus des articles fublibes lat meme anne dans Vutional Review et dans Westminster lieviene. II y insistr surtout sur l'inportance de l'action du milieu. Ce n'est donc pas à Darwin que spencer a emprunte la these de l'évolution des espères, ot son roble n'a pas consisti, comme on le dit parfois, a étendre à d'autres domaines la decomverte laite par Darwin dans le monde organique. (2)

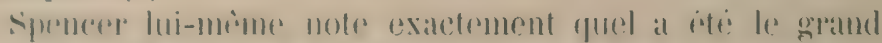

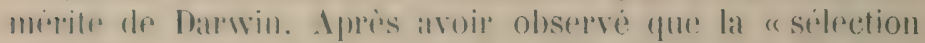

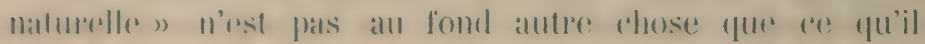

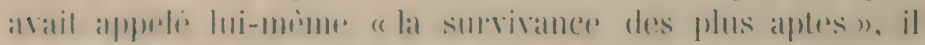

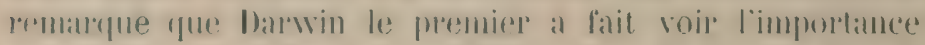

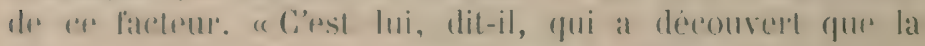

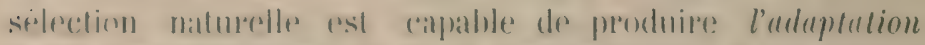

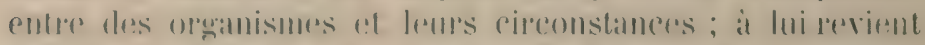

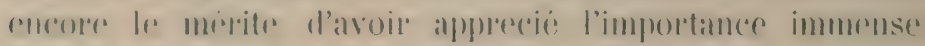

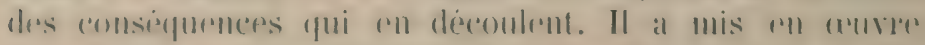
1112. masse imorme de lats pour en laire sortir lat de-

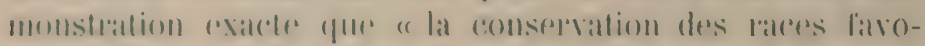
risiers dalls la lulle pour la vire $)$ est une rallse sills

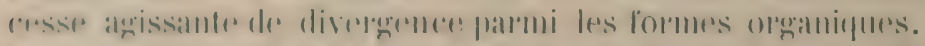
II a suivi les resultalts compliegures de lopreation de la

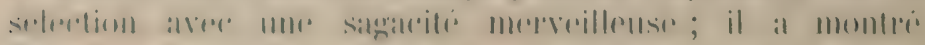

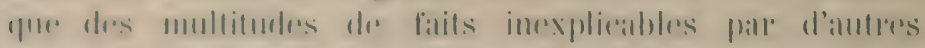

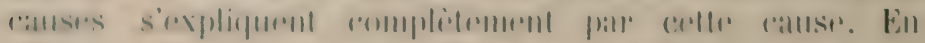

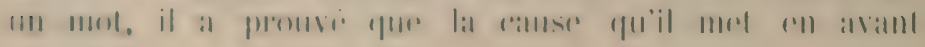

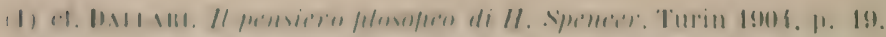

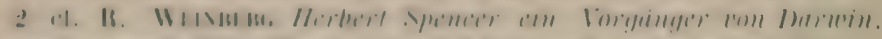

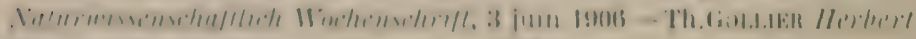

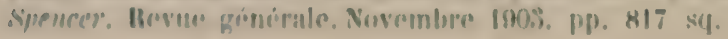




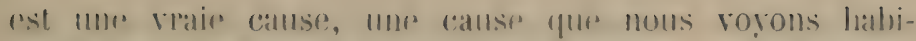
tuellement all action, 14 que les resultats quon peut en

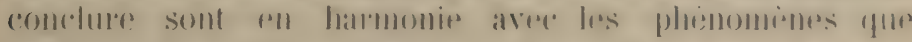

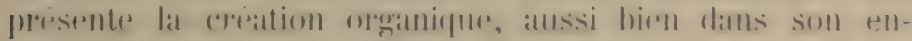
semble que dans ses détails. » (1)

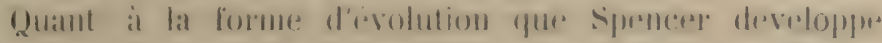

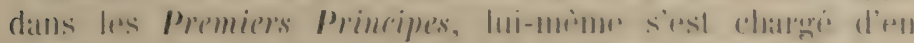

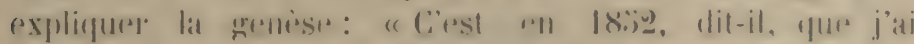

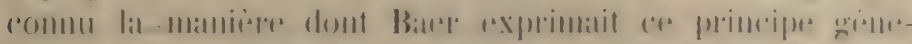

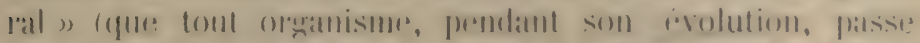

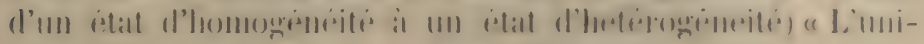
versabilitie des la loi a toujours ele pour noi un postu-

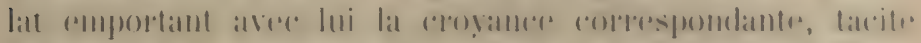

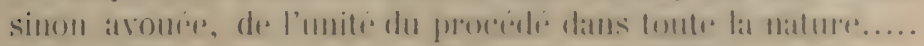

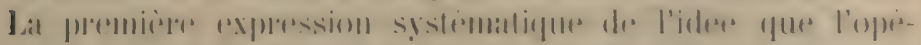

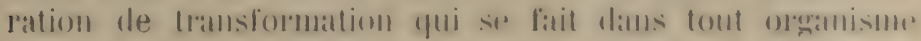

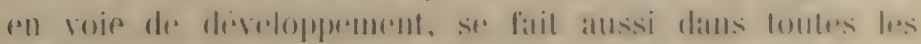

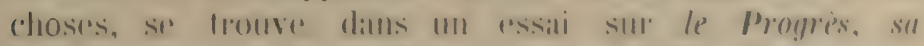
loi el sa rause, que jai jublie diuns The Westminster

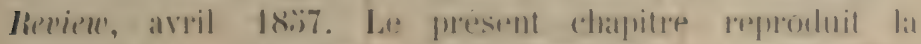

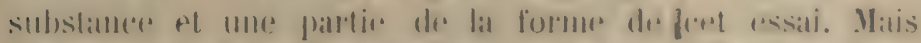

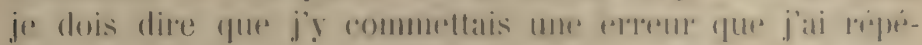

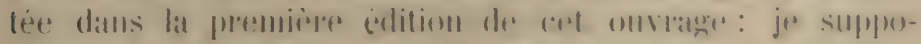

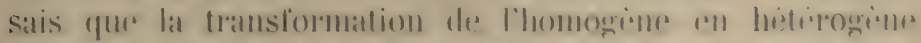

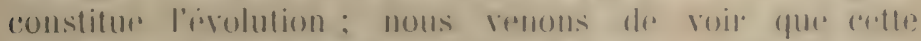
transformation ionstitur la redistribution serendaire pui

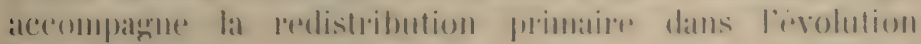

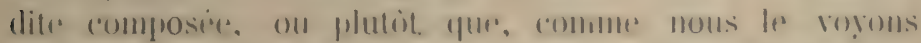

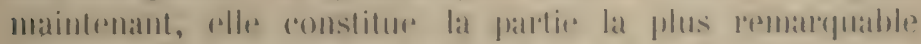

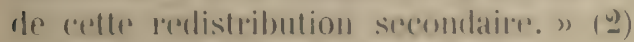

En lanit cet anticle de The Westminster Revieu (3) (ontient dioja la plupart des ideres matresses des Premiers

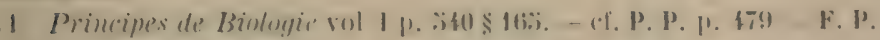
p. 362. note.

(2) P. P. p.p. 383)-360- F. P. p. 270, nute.

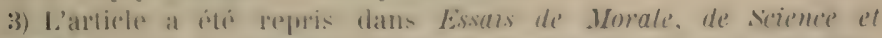

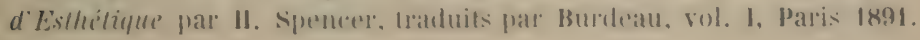


Principrs. Spencer part de la notion de progrès dans lis corps vivants : transformation de l'homogene en heterogène (Goethe, Wolff; ron Batr). Cette conception se grénéralise d'elle-mème. L'auteur l'applique aux phénomènes rosmogoniques, géologiques, biologiques, sociaux, au langage, aux sciences, aux arts.

Quelle est la cause de cette diflérenciation croissante? C'est que tout agent produit plus d'un effet, ou mieux : que tout changement engendre plusieurs changements. La complexiti des effets d'un agent est proportionnelle it la complexite du milieu sur lequel il exerce son activite. Dans une note, Spencer indique le principe de l'instabilité de l'homogène.

Le progrès pst l'effet d'une bienlaisante necessité. En disant cela nous n’atteignons pas la cause métaphỵsique, substintielle du progries. Au contraire, par nos études nous royous de mieux en mieux que cette cause est inaccessible. Ainsi la seience fortitie la Religion.

Cettr dernière riflexion dont le développement ne prend gurie que deux pages est devenue la premiere partie des Premiers Principes. Yous avons vu comment, dans la seconde partie, s'est complete le concept du progries ou de l'évolution ot la comnaissance de ses causes.

Sfrencers a iscrit en I8tif et publie a part en 1884 quelques pages pour contredire ceux qui le presentent comme un disciple de Comtr. (1) Dans d'autres endroits de ses ourrages (2) il proteste contre conte maniore de voir, el l'on doit recommatre qu’i envisager l'ensemble de la doctrine dr. Sprencere, colle diest pas fondere. Cependant ir passage

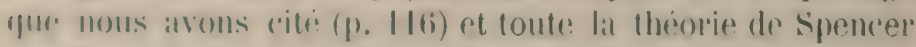
sur la presendure ipuration des doctrines relighiruses par la srience oltire une incontestable analogie avec la doctrine

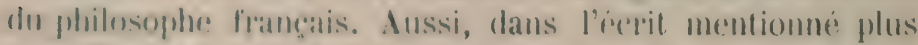

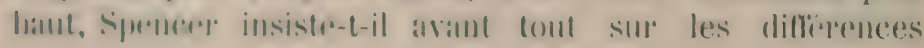

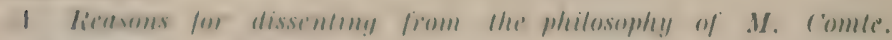
domadreas ISE6.

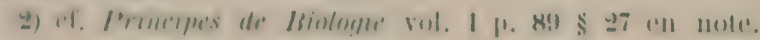


(qui le siparent de Comtre dans la question du progress des connaissances humaines.

On sait que d'apres Comte, les commaissances des l'humanite ont patsse par trois etats dillerents: l'état théologique dans lexpel l'homme attribue les phenomenes a l'interventtion d'rtres suprerents, de divinites, nombreust's d'abord,

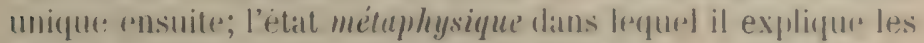
faits en invoquant des canses de plus en plus universolles; 'mlin l'bat scientifique ou positif dans lefpued il se contrente?

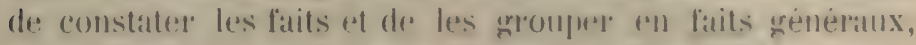
con nighligeant complètement, comme "inacoessible en vide de sens pour mols n, la recherclur des callses soit premières soit finales.

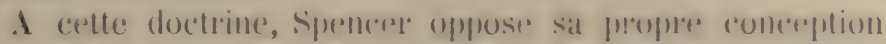

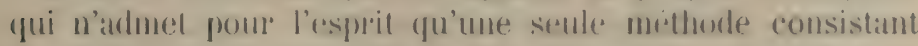

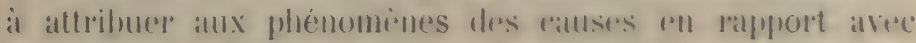
le degré de generalisation que lexperience a realise :

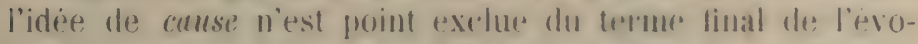
lution intellectuelle el la methode scientitigute, ant lim de remplatere les concerptions religiruses, fes regoint all roul-

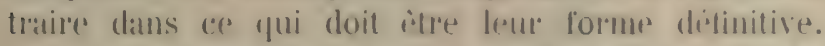

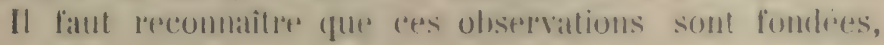
e't qu'il ne prent pas ible question de conlonder complètement les deux doctrines. Il n'en est pas moins vad gue Spencere lui-mène elablit une opposition motre les conceptetions religieuses, felles quidles pxistent de fatit, et les conreptions stientiligues; yur aetle opposition consiste, d'après lui, daus lat substilution dess causes malumolles at des agents persomnels pour l'explicaltion des phenomeness, ce qui n'rest fas autre chose an fond que le promplacement de 'etat theologinue pill l'etat metaphlysingue. Et si spencere maintient a la fois la liggitimite des conceptions leeligiensers et desconceptions scientitipues, c'est at condition que le: Dien unipute de la Théologio et la causa universelle et dernière de la science soient l'Inconnaissuble; ce quai, dans lat rigueur des termes, signitic yur l'un et l'autre s'evanouissent an monent ou l'esprit croit lessaisir.

Il y a donc, semble-t-il, entre le's idees de Comte et 
celles de spencertr, au sujet de l'avolution historigurs de l'inlelligrence lumaine, plus d'analogie que er dernier ne reut le recombitre. Il faut arouer néanmoins que la ressemblance n'est pas complète, et que, même dans la pre-

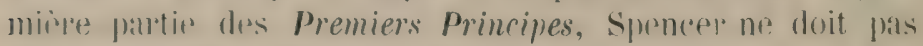
cittre consideré comme un disciple de Comte.

Le philosophe francais, fui lait profession de navoir jamais lu ni kant ni Hégel, (1) nensergene nulle part la doctrine de la pure pelativiti de nos connaissances qui aboulit, comme nous l'avons dit, à l'idralisme' ef rérluit tontes nos idés à n'être que des phémomènes purement subjectils. Il repousse " le panthëisme témbreux dont se ghoritient si étrangement, surtout en Allemagne, tant le profonds métiphrisiciens (2) n at qui ne prout être que l'idialisme transcendental.

Tandis que spencer no veut pas du réalisme grossier, gui consiste a almeftme l'existence objectior des choses dipedentent prercurs par l'experience tells qu'elle est

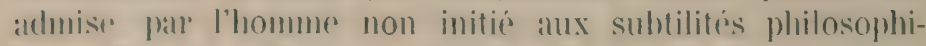
gues. Comte grofesse, an contraire, que "lo virital)le esprit philosophique consiste uniquement en une simple? rextension midhodigue du bon sens vulgaire it tous les sujuts arcessibles a la raison humaine. ") (3) L'Incommaissuble n'rest donc pas pour Comte une réaliti fondamentale, incomaissable paree qur absolue, dont nous pereevons vaguenent et irresistiblement l'existence, mais que nons

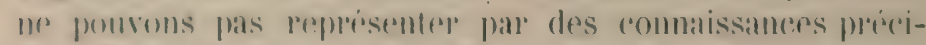
sirs. Corst simplement tout l'ensemble des doctrines reli-

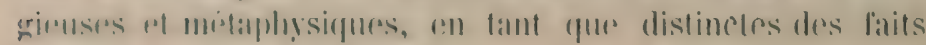
constatables par l'observation.

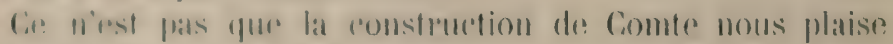

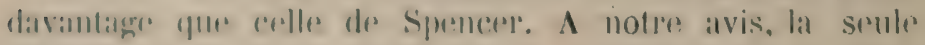

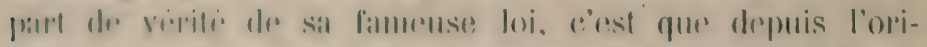

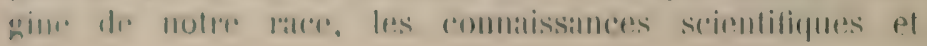

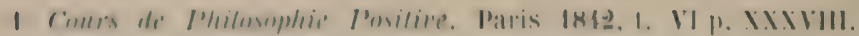

(2) Ihid t, V. D. 34.

(3) Ibiul, L, V1, D. (E)io. 
lesprit scientiligues ont its, d'une maniere générales, m progressant; que, dès lors, les hommes ont reconmu arroc une evidence rat une exactitude croissantes les lois auxquelles obéssent les phénomines matériels. Mais, comment ce progris contredit la conception d'appres laquelle l'l'nivers est, en dernière analyse, l'expresision d'un acte lihres de l'Etre Intini, ce qui est l'essence de la doctrine theologique, on jourduoi il est illegitime de se servir des

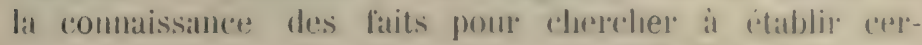
tatines conclusions sur la nature des ropps qui an sont Io thésitre, e'est are que Comte ne montres nulle fallt. Les

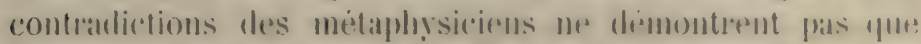

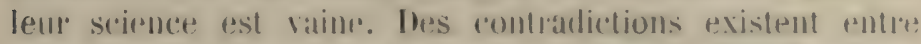

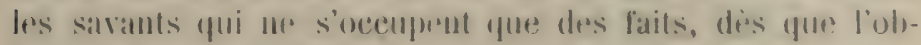
servation en est difficile on yue les mogrens d'informattion ne donnent pas une entière évidence.

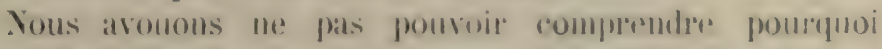

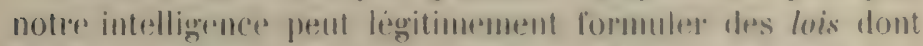
la portere dipasse toujours loblservation proporement dite

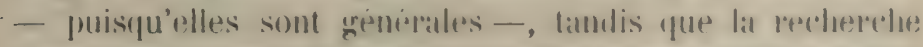

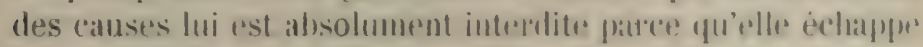

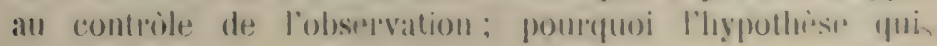

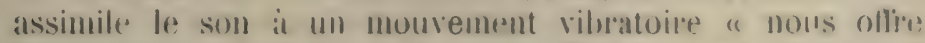
l'expression exacte d'une evidente realite" ", (1) landis quere l'hypothese yui considion lat lumieres comme consistant, elle: atssi, dans des momrements ondulatoires d'm fluide est rangero parmi les chimeres metaplnsigfurs, commes visant a devoimp lat " nature intime of le mode rssentiel dr: produrtion des phénumisness n. (2) Comment labllirmation

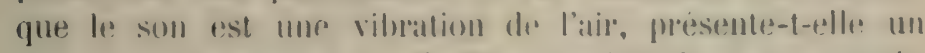
fait et se rattiche-tedle a lestat positif de notre esprit,

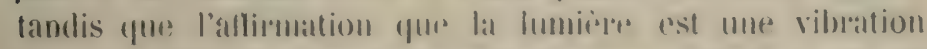

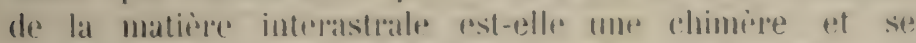
rattache-t-rlle a l'etal metajplysigue de notre intelligeneres?

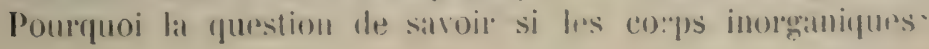

(1) Ibid. 1. H. p. H6.1.

(2) Ibit. t. 11, P. 501 . 
et organiques different de nature est-elle " un reste d'influence des habitudes théologiques et metaphysiques » (1) landisque les chimistes en affimmant " que les substances different aussi bien par la proportion que par la nature le lours principes constitutils ") (2) sont parfaitement dans les bornes de la science positive?

La loi de Comte est une conception arbitraire et incohérente et ce que spencer en a adopté dans sit thérie sur lopposition entre la science et la Religion ne vaut pas nieux, comme nous l'avons vu ailleurs.

La fartie vaiment originale de la philosophie spencérienne - malgré les influences étrangèes dont clle porte les traces - e'est la doctrine de l'évolution. On preut allirmer quelle est tout à fait indépendante de la philosophir positiviste. Comle ignorait l'evolution organique des espreps. Dans la discussion entre Lamarck el Guvier il tenait pour or dernirr. " (On ne saurait gruère douter, dit-il, surtout d'apres la lumineuse argomentation de Cuvier. que les esperes ne demeurent aussi, par low nature, essentiellement fixes, it travers toutes les variations exterieures compratibles avee leur existence ... (3) Nous pourons disormats... regander comme demontrip la discontinuiti. néressaire de la grande serpie biologique. ") (4)

Plus tard il ferit: "Pleinement apprecece, cette troisirme loi biologigue " (d'alpres laguelle tout itre vivant

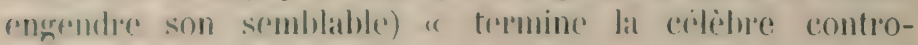
verse, encore essentielloment pendante, sur la perpetuiti des espreces. Cart, une lelle loi, assurant l'herédite oldy-

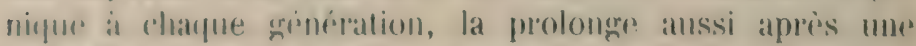
suceresion momwelle. Elle consiste, at lond. at maintente spombanement l'intergrite du type, yun que soit le nomber does transmissions... Lophinion de l'inslabilite des resprees

(1) Shid, 1. I, P. 73.

(2) T. 13, p. 117. C'est nous qui soulignons.

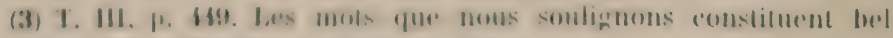
of bien une affirmation méthaphysique.

(4) Ibid. p. frig. C'ost nous qui soulignons. 
ast une dangrepesse imanation du matrialismo cosmologrifue, d'apmes une irmationnelle exagriation de la reation vilale des milienx inertes qui n'a jambis ete bien congue. » (1).

L'évolution organique des individus n'a pas altire laallention de Combe. Il n'a décrit le phenominte rolutil que dans l'ordre sorial. Dans celui-ci il admet comme Simentere une tendance generale au remplacement du type militaire par le

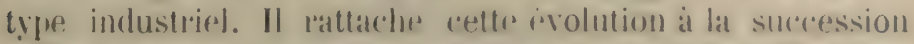
des trois etats, theologrigure, mituphysique et positil. (2) Centre interpratation est aussi freu salisfotisante que lat loi des trois

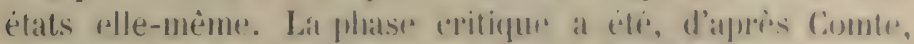

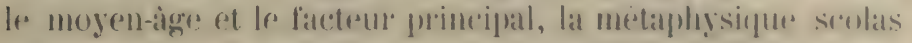

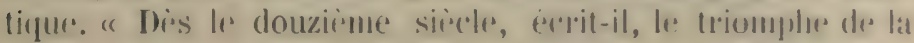

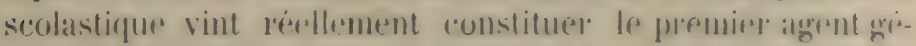
neral de la desorganisation radicale de lat puissintee of de

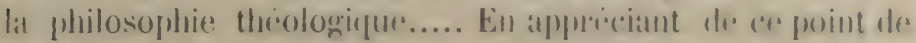

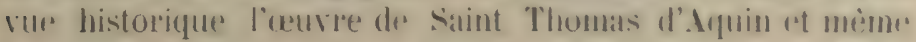

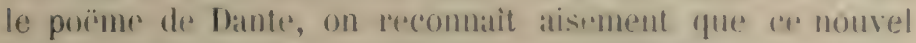
('sprif mitaphysiqur avait alors essentipllement anvahi toute l'itude intellectuelle ot molale de l'lomme indivi-

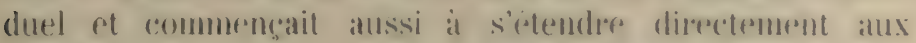

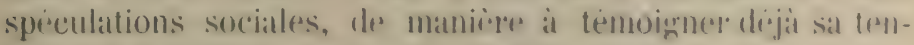
dance inevitable a allianchir delinitivenent la raison hu-

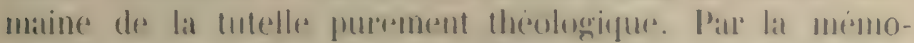
rable canonisation du gramul docteur scolatstipue, d’ailleurs

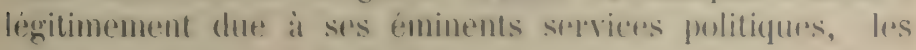
papes montraient at la lois leur propresentranement invo-

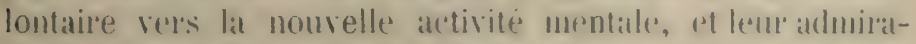
be prudenes a s'imorporer, antint que possible, fout ce qui ne leur alait pas manilestrment hostile. "guoiquil en soit, le caractire antithrologrique d'unr telle melaphysique ne dut longtemps se manifestor que par la direction plus

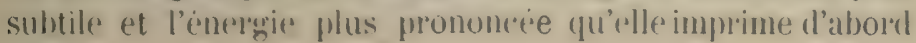

1) systeme de Politigue I'ositice. Hoisieme idlition, Paris. 1. 1. p. 392 .

2. Cours de philosophic Positive 1. I. Physique Sociale. 
¿l'esprit de schisme "t d'heresie.... Mais les grandes luttes décisives du quatorzieme et du quinzième sirele contre la puissance enropéenne des papes et contre la suprematie ecclesiastique do siege Pontifieal, vimrent enfin procurer spontanement une large el durable applicattion sociale i ce nouvel esprit philosophique, qui, ayant déja atteint la pleine maturite speculative dont il était susceptible, dut desormais tendre surtout a prendre aux débats politiques une participation croissante, qui, par sa nature, ne pouvait itre que de plus en plus négative envers l'ancienne organisation spirituelle, et mênr', par une consiegurnce involontaire, ultericurement dissolvante pour le pouroir temporel correspondant, dont elle avait d'abord tant secondé le système d'envahissement universel. " (1)

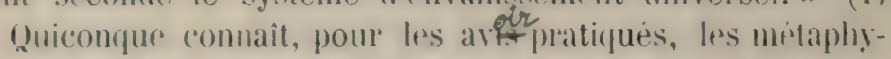
sicions du moven-ighe ne lira pas sans etonnement cette appréciation et se dira que la philosophie positive ne prémunit pas contre les spreculations fimtaisistes. Sinencep nat nullement suivi Comte dans cette manière d'envisager l'évolution sociale.

La loi unique do l'évolution humaine est, d'après Combe, celle-ei : "Lhomme devient de plus en plus religieux. "(2)

Lat religion, d'apress lui, r'st " caracterisée par l'état dr pleine harmonie propre à l'existence humaine, tant collertive qu'individuelle, quand toutes ses parties quelconyues sont dignement coordonnies.... Lat religion constitur donc pour l'ame un consensus normal exactement comparable: a celui de lat santi envers le corps. D'après l'intime solidaritie entre le moral of le physicque, te rapmorhement des deux élats généraux pourrait même s'moudre jusqüa concevoir le second embrassé par lo: premier." (3) Le progrès religienx de Comte n'a, on le voit, renen de commun aves laurond entre la Religion et

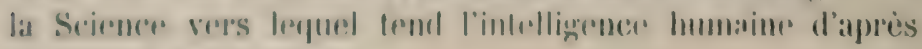
la theorie spencerienne.

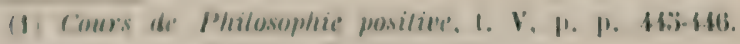

(2) Nystime de Politinue Positive 1. 111 1). 10.

(3) Ibid, 1. 11 p. 8. 
L'our le philosophe fiameatis, la loi d'erolution de l'huma-

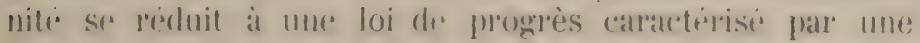

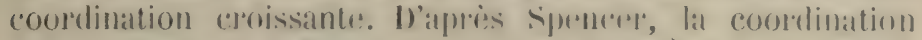

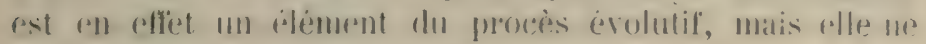
le constitue pas à elle seule.

Comb n'at jamais concen l'évolution enomme un frocos's

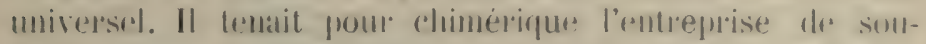
medtre tous les plomomenes a unf loi ginerale. (1) Hatr-

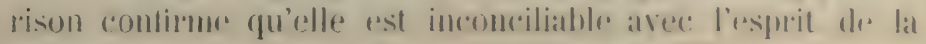
philosophie Comtiste. (2)

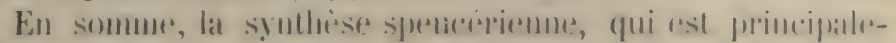

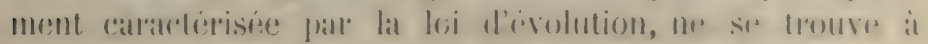

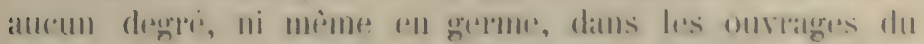
fomdateme de la Religgion de l'Humante. Spencere avait dome

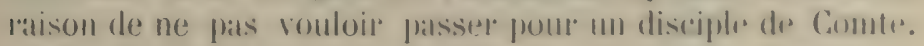

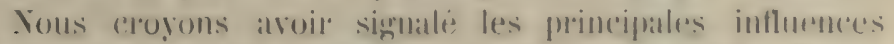

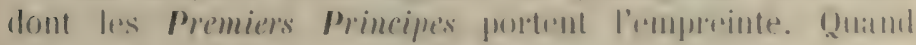

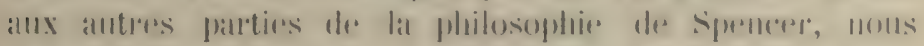

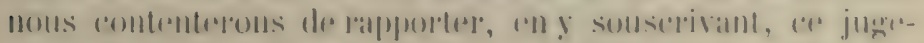
ment sommatre de Rene Worms : "spremere at stlici ilsant

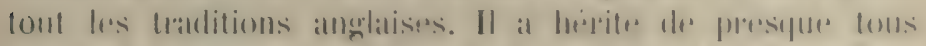

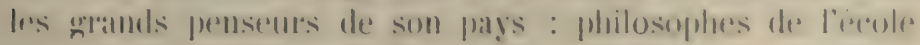

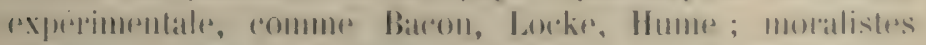

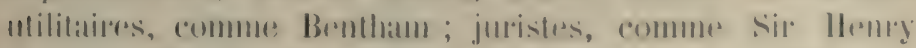

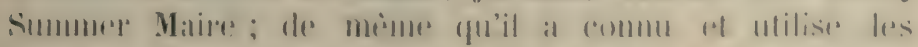

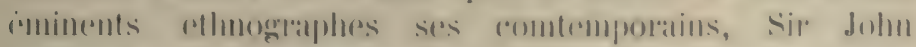

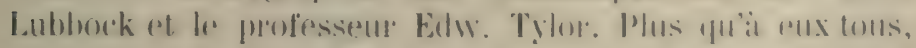

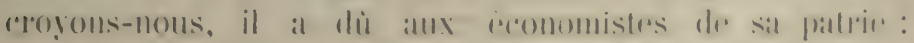

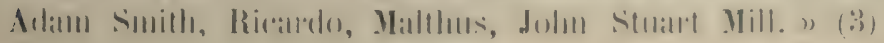

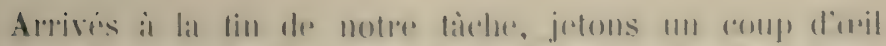

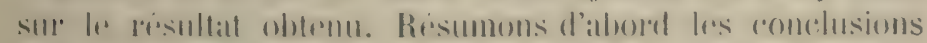

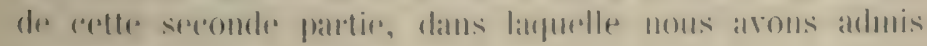

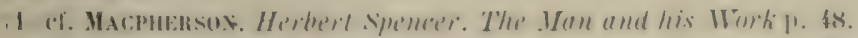

(2) The Herbert spencer Lecture p. 2 \&.

(3) Journal des liconomistes. Janvier-Xars 1904 p. 103. 
la plupart des idés de spencer, tout en tachant de les anender ; les passages qui y sont soulignes sont crux qui contredisent sa doctrine ou la complètent.

L'évolution, dans sa signilication la plus litrge, est 111 procis anyuel sont soumis aussi bien le sujet pensant, le moi, yur les oljets extérieurs, te non-moi. Comme phenomène grineral, alle consiste dams une rodistribution do matière at de mouvement soms l'action de forces. La matière présente comme caractères essentiels l'étendue et la masse qui, aver la force et mouvement, constiluent quatre éléments irréductibles de l'Lnivers matériel.

L'évolution so déploie dans l'espace et dans le temps.

Les forcess sont purement mécaniques dans la nature inorganique; la vie organique est caractérisée par des forces plastiques; In vie sensible et la vie intellectuelle par des forres psychiques. Ces rois catégories de torces sont irreductibles. La considération des forres permet de distinguer plusients ratégories d'énolutions. Les forces, à lexception de lu volonté libre, agissent suivant des lois fatales e't constantes.

lievolution est sommise a) aux fois génerales du monIr malriptel: conservation de la masse des corps, - laansformalsilité et ronversition de la somme de léneryje ater restriction possible (et en tous cas pe'u importante) due à lie mulliplication des forces plastiques of psychiques, dirretion du mowsement suivant la résultunte des forres, sonerent suivant la moindre résistance ou la plus forte Iraction, - caractène rythmique (lu mouvement; b) aux lois spéciales de l'évolution:

I. Loi de l’introgation. Livolution propurement dite (pro-

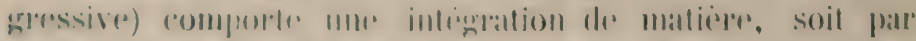

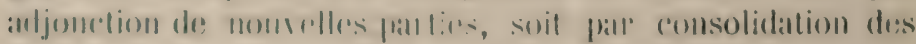

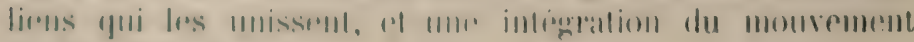
intripleor de l'agregrat.

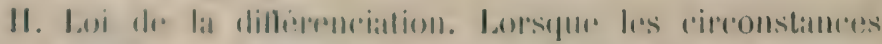

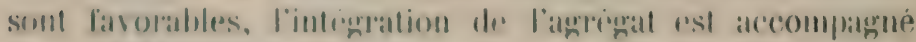

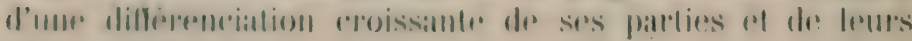
mouverments.

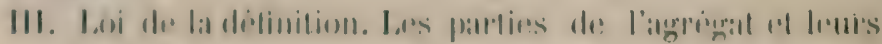


mouvements tendent a se delimiter de, plus en plus rexartement.

IV. Loi de la courdination. A mesurreque les parties se dillienenciont et se limitent, elles tendent at noner entre nless des relations de flus en plus etroites. It en resulte unr coordination croissante de leurs mompments.

V. Loi de la dissolution. Les itres matririols en mime trmps qu'ils se développent, subissent un procis simultani ef oppose de destruction qui finit par les finire disparatre. Ces proces de dissolution comporte lit desintegration de la mattiore et du mouvement.

I. Loi de la relation entre l'érolutiom et lu dissolution. L'Pvolution des agréguls organiques est de sa nuture indéfinie; ni l'équilibre, ni surtout la dissolution ne doinent ètre considerés comme l'aboutissement vers lequel tondruit, far luimème, le procès d'évolution.

VI. Loi de la dégradation de l'énergrie. Lat" momement subit une desintegration constante par la transformation grat-

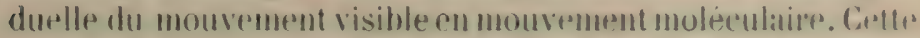

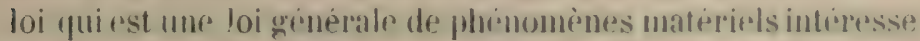
erprendant spécialement l'évolution, esn epe quelle indigue que l'ensemble des évolutions de l'niters a eu un rommencement et aura - naturellement - une fin.

Lat formule grenerale de l'ivolution qui resulte de cess lois se verilie diuns les dillorents ordres de choses, dans les unes parce qu'elles sont matérielles, duns les autres parce qu? tout en étant immatérielles. elles dépendent d'une fucon ou d'une autre des redistributions de matière et de mouvement.

Le proces evolutil a des auses gendriales ef des causes particuliores à chapure gentre d'évolution. P'armi les premieres il laut signaler l'instabiliti de l'homogime et la multiplication des eflets qui diterminent la diffrenciation, la siegrégation qui sert i interpréter la definition dus parties, les forres par les quelles se manifeste l'unité de l'ngrégat et qui produisent l'intégration et la coordination des parties, la sélection. nuturelle, facteur complexe, yui d'une fason ou d'une antre jone. un rôle dans toute évolution, de manière, en général, à favoriser le progrès. Recherchant les causes particulieres de l'évolution 
orinanique, nous avons roleve l'hérédité et l'atavisme, la transmission probable des caracteres acquis, l'adaptation aux circonstances, les mulations el d'autres phénomènes dépendunt de fucteur's internes imparfaitement connus, mais probablement prépondérants.

Dans les points que nous venons d'énumérer, nous avons adopté en grande partie les idees de Spencer. Parfois nous arons cherche à les préciser, à les contirmer ou al les corriger. Le lecteur aura jugé si nous avons rte henrux dans ces retonches que nous arons apportées a l'ceurle du maitre. Yous espérons qu’il partagera notre adhesion à sa vériti substantielle et qu'il jugera avec nous qu'rlle fournit de l'tnivers matieriel une conception juste, interressante et féconde.

Au contraire nous arous repousse d'un hout a l'autre la theorie de l'Inconnuisuble of nous resperons egalement yur. le lecteur jugeral suffisante la rifutation que nous en avons faite.

L'homme place en face de l'Lnivers doit resonder le probleme de son origine, non seulement a cause de l'intirrit spreculatif qu'il prosente, mais encore parce yuil a hesoin de rethe solution pour ordonner sa vie. Il n'est fas permis d'y inhapper par le pretexte que toutes les riponses froposies soulivent des ohjections, on que notre intelligence ne sullit pas a cotte tache. Les diflirolles que presentr la solution spritualiste ne sont pas insurmontables, ef les theories en vertu despurelles on

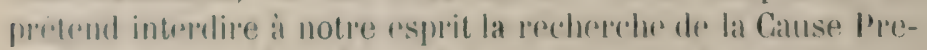

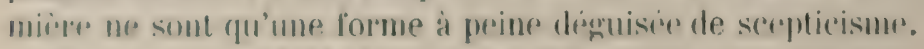

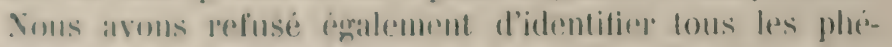
nominnes qui se poduisent dams les rtles rorporels. T'out

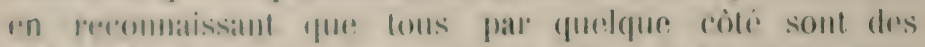

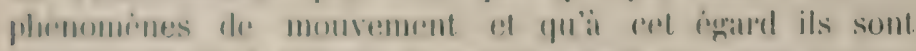

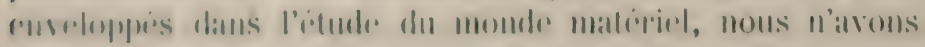

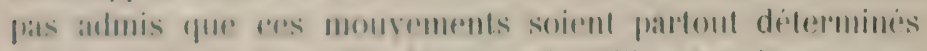

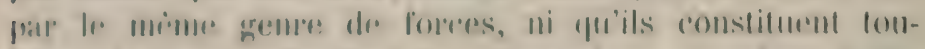

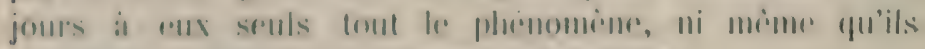

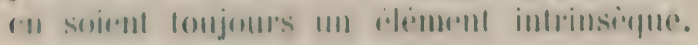


L'érolution, dans laquelle dhaune phase at sal raison

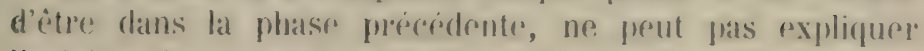
l'origines des choses radicalement mouvellos. Si, comme nous l'avons admis, la vie organirpue est irreductiblo aus foreses physiconchimiques, l'evolution des corps inoreminiques est incapable de produire lat vire Ite memes, purisque manifestement la sensibiliti ne peut fas se réduire a des déplacements d'atumes, une ivolution qui ne comprend rien de plus me perut pas expliputer l'origime de la vie sonsitive. Et puisquas colle-ci ast continese dans des commaissances combertes do choses mathrielles pt dalls les mouvements qu'elles délerminent, l'evolution de la sensibilite ne pent pas itre la ladison sullisante de l'appla-

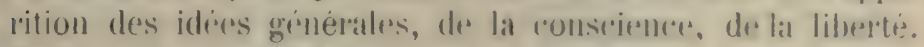
Entin toute irolution suppose des dommens dre lixistrmes desquelles il faut rendre compte.

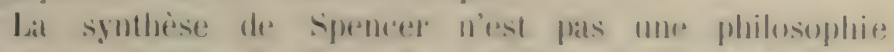

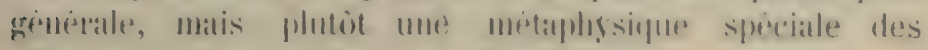

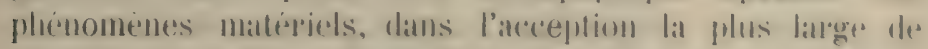

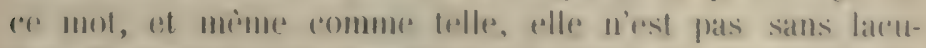

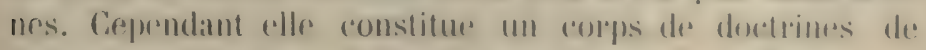

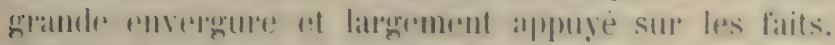

Ce que mous avons admis dans lixuler de spencere an

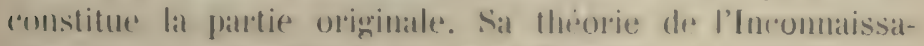

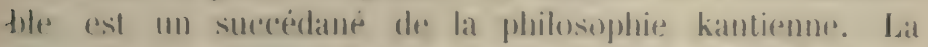
thesere yni ansidere toul les phemomenes commus subsfantieblement identigues at refuse de voir dans latetivitio psichique autre chose que les monvements de la matiege

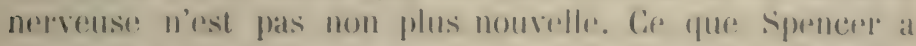
temte le puremier, e'est more etude dre l'irolution conside-

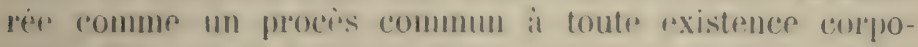
relle. En rela il at remssi daus ane lange mesure et il a ainsi ajoute a la philosophir un chappitre interessint et d'une importance incontestable.

ations Iat théorie de l'Inconnaissable ue lo mécanismo outré ont emperelse sombant de rexonnaitre la fart de verite que rontient la sponhrise spencripienne et ont fait qu'elle a éte 
jugee parfois arec une sévérite trop absolue par les philosophes spiritualistes. Il en a ite ainsi en Angleterre, au témoignage de Hemri Michel. (1) Il faut reconnaître d'ailleurs que les critiques de l'oure de spencer contiennent en général une grande part de vérité. Citons en quelques-unes.

Fr. Aveling, dans Inblin Review, tont en rendant hommage it la grandeur de l'aruvre, la trouve essentiellement fausse dans la théorie de l'Incommaissable, l'évolution, la doctrine morale. (2)

Renouvier dont nous arons signalé les principales mitịues croit que la postérité refusera de ranger la doctrine de spencer parmi les grandes constructions philosophiques. (3)

Le P. Roure, dans les Etudes Religieuses, signale l'insuffisance de la doctrine évolutionniste. " On nous promet, dit-il, lat comnaissance des réalite's objectives et on nous fait l'histoire de leurs changements. Nous avons lieu de declarer que nous ne somimes pas satisfaits. Répondre que tout change, se n'est pas promer qu'il n'existe, meme en apparence, que des changements, ou quon me peut connaitre que fo changement. ") (4)

C'est la critique que fait egalement Harrison. "si le monverment dévolution et de dissolution a travers des phases alternantes de diflérenciation et d'intregation, dit-il, est la clef maitresse de toute science, alors la science est simploment la loi dos procies do changement. Mais les lois drs stabilité, de permaneme, sont également tisenlielles of dominantes; de fait elles precedent les lois de changement. Dommant aux termes leme viribable portin

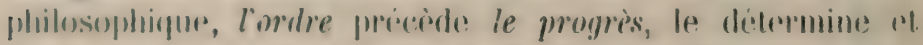

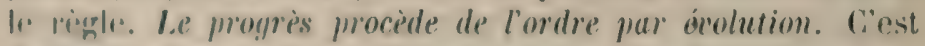

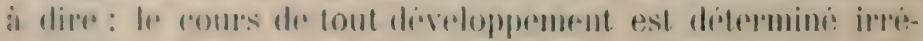

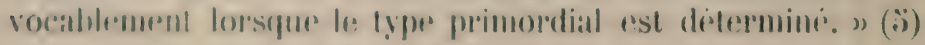

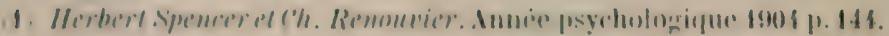

(2) The Philosophy of $\boldsymbol{H}$. Spencer. Febr. 1904.

(3) Op. cit. Ix\&s, vol. II, p. 3ixis.

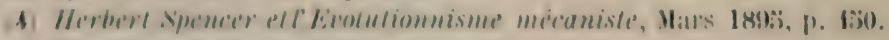

(3) The Hetrert Spencer Lecture, p. 19. 
Cette observation est justr jusqüa un certain point. s’il y a des choses qui changent, celat meme suppose qu’il

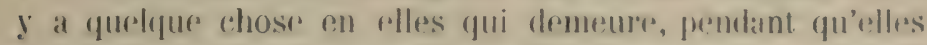
changent, entre te moment ou elles commencent at exister et le moment où plles linissont. Mais rneoles est-il rrai de dim que les choses matryefles thlles qu'olles se pros-

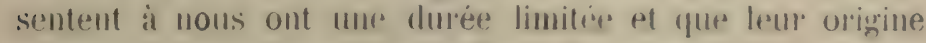

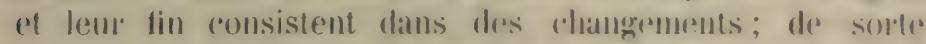
que ce qu'il y a dre permanrat ron rolles, daus les limites do leur durée, est le terme d'un changerment. Lat connaissance

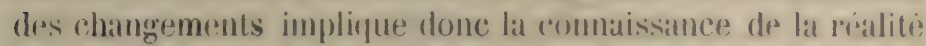
des choses. Ainsi, lat conmaissance des chamgements qui

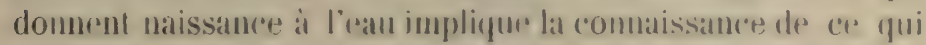

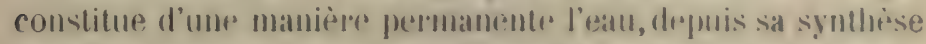

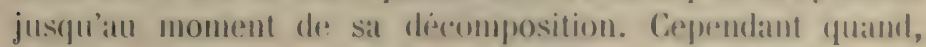
dans la nature, une chose materiolle resse d'exister, ollo:

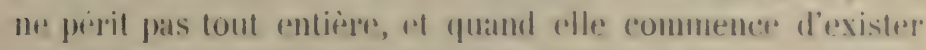

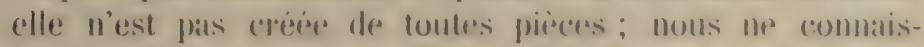

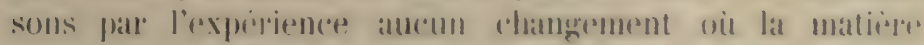

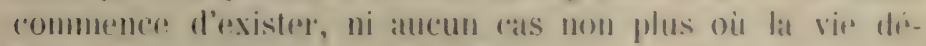
Jule. Lal combaissance de's changements nemtraine donc

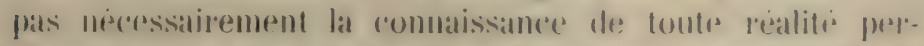
mamente - meme abstraction late des l'imperfection des molece commaissance des changements - mais elle pent y contribure. Il litut en conclure fure, comme nous l'allons fait observer à phosiems peprises, la philosophie me peut fas comsister ditns la semle throrie de l'érolution el que

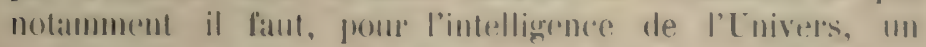
on plusients points de depant - siluations initiales ou types primordianx - a partir destpuels l'evolution s'ateromplit conformément à ses lois générales.

Le. P. Roure aritique engalement la foi d'évolution formulew par spencer" : Dans l'evolution des seiences ef des ants, dit-il, comme dams rella des sociétés, nous voyons un progrès dans la coordination of la subordination des parties; mais ("ust

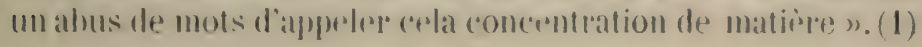

(I) Ibid. p. 453 
II nadmet pas davantage la " dissipation du mourement ". Fons avons longuement examine nous-memes ees points de la doctrine spencerienne; nous n'y reviendrons donc pas.

C'rst egallement le méanicisme ladical que Proost reproche principalement it spencer dans les excellents articles qüil consacra di sa philosophlie dies 1878. (1) Il fait remarpquer aussi, et juge sívèrement le manqur fréquent de précision de cette meranique. "En matière de mecantque at de thermo-dynamique, dit-il, lat terminologie vagure et parfois inexacte du savant anglais permet de roore qu'il ren est absolument au point où se trouvent beaucoup do salvants modernes qui ne sont pas mathemaliciens. Ils s'imaginent comprendre clairement ce (qu'ils no lont en réaliti qu'entrevoir très imparfaitement. ") (2) lroost rend d'aillems justice au mérite de spencer, lant en re qui concerne l'ilee d'évolution que pour le caractère emcyclopredique de son génie. "II est une chose, dit-il, füon ne peut denier au philosophe angrais... c'est une immense erudition qui ne se borne pas a des rommaissimeres approfondies de l'économie politique ol des scionnes psichologigues, mais qui rmbrasse le vaste domaine des scioneres de la nature, depuis la plọsigue, la chimie, la grenloger ret l'astronomir. jusqu'aux dimirentes branches de lat biologrer, la zoologire, la botanique, l'monbroblogire of lamatomire compromer, la physiologie, la pathologie, ote. ") (3)

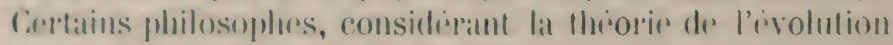
creranipure comme te point dre dipart de la philosophire

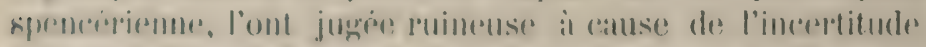

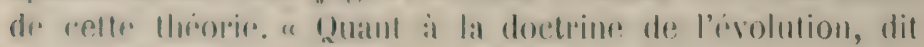

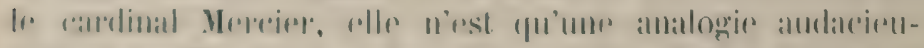

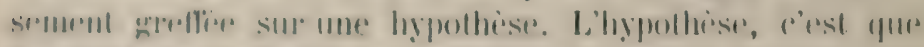

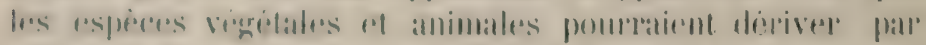

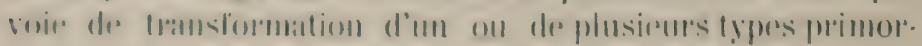

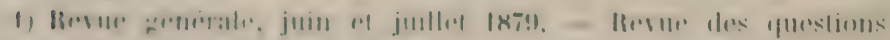
serentificunes, danvior, Avris 1878.

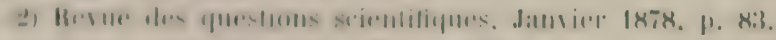

(3) Rovue griperale, Juin 1879, 1). 8343. 
dianx, en vertu de la sélection naturelle, ou, en trimes plus explicites, sous l'influeno' avantageusement combinire du milien, de la survivanere des plus aptes dans la lute? pour la vire, ret de l'herridite. L'analogice consiste à chargir indéliniment l'hypothese tansformiste at a l'appliquer a tous les faits olservahles, deponis la formation des mondes strolaires, du systeme solaire, dre notre glober jus-

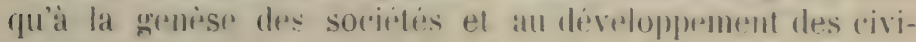
lisations. Tout le mondre conviemdra qu’il n'y a dans cette vaste conception ni scirnere proprerment ditre ni véritable philosophie. ") (1)

La Civilta Catholica trouve Exaldement que less bases de

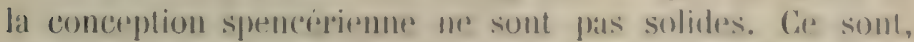

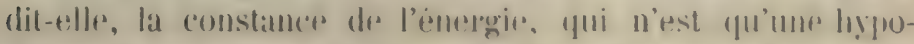

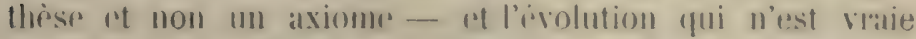

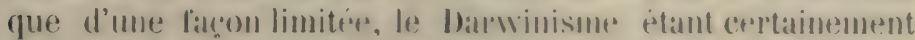

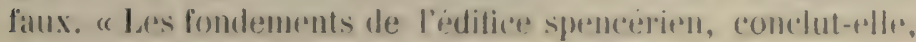
ne sont pas inchranlables, mais beatucoup des ses parties sont belles, fortes et en harmonie arece d'antres igalement belles et fortes. I eut-il jamais, d'aillemes, depuis Thales jusqu’i nos jours, un philosophre qui ait éleres un éditioes philosophique parfait dans toules ses partirs? \# (2)

Larticle de la Civiltà fut critique par l'Inità Catolica (3) comme trop élogreux pour sprencer. Mais la Revues romainte maintint son appréciation. (4)

L'évolution dans sun acereption la plus gimerale est un fait. Reste a roir si la sucression des faunes et des tlores est un phenomene d'évolution. Conla revient à demander si elle est un phénomène naturol. Lat question etant posie dans ces termes et abslaction faite de tonte theorie particulière sur les facteurs de cette evolution, la reponse a faire ne semble plus guère doutruse il linporne atuelle. Nous souselivons volontirers an jugement de J. Yverach,

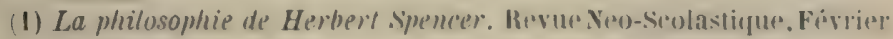
1898 , p. 28.

(2) 1904 p. 104

(3) 19 Janvier 1904

(4) 6 Fêvrier 1904. 
dins The Critical Review. (1) "Yous avons toujours ressenti et nous ressentons profondément aujourd'hui combien ce lut un matheur que la grande et féconde theorie de l'evolution ait eti associete chez ses principaux auteurs ot expositeurs arec des doctrines mitaphysiques et autres qui nont aucune liaison arec elle. L'agnosticisme at l'évolution sont indépendauts l'un de l'autre.... Nous rrogrations que l'aspect agnostique de la philosophie de spencer ait empreche de reconnaitre la valeur de la théorie de l'évolution; cal' cette théorie a suscité des travaux firconds dans presque tous les départements de la connaissance humaine. $)$

Ceux qui condamnent la tendance matérialiste de la philosophie de spencer, s'aceordent, en géncral, pour reconnatitre le caractipre grandiose de son cumre, l' " abondance incroyable d'informations et une puissance de coordination, une energie synthrtique qui met cette vaste

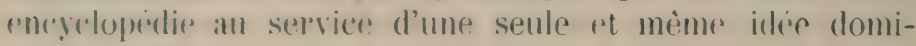
nante. » (2)

On compurend qu'en dehors de l'école spiritualiste lat synthese spencerienno a rencontre soment une admiration rnthousiasto ot sans resorve. "La philosophie de speneer. dit Gonzalez Sermano, arece son idfe maitresse de la conception liologique? dr l'Univers, constifur le plus grrand alfont de lat pensise moderne pour conciliere les sprembations idealistes ares les exigences scientitiques. ) (3)

l. Iecterur s'est ateresu que notre appreciation suit monr voir mogenne entres la condammation radicale et l'approbalion alssoluc. Nous aroyons fue l'arure de spencer "l en farticulier Les Premiers Principes renferment une

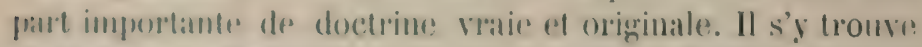

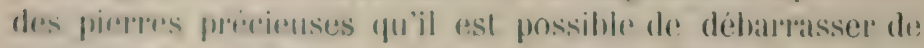

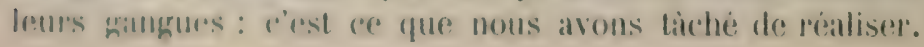

(1) 3909 , D. 101.

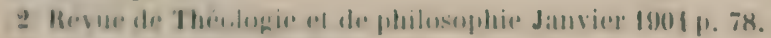

(3) La Lectura Janvier 1906 p. 90. 


\section{BIBLIOGRAPHIE}

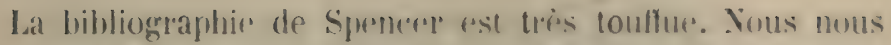
bornons ic:i a signaler d'atrord les traductions des Premiers principes, ronsuite les travaux ugui s'orempent d'unr liugon grenerale dre la philosophie sprencoriemne, antin les atudes qui se rapportent specialsment aux Premiers Principes. Yous négligrons tout ce qui a rappont aux parties spor-

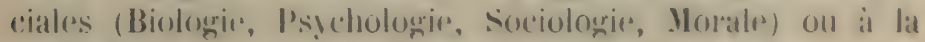
biographie du philosophe. Meme dans aes limites, notpr liste est loin d'étre complète.

Spexcen H. - I primi principi frer culä di G. Salvanon, Torino 1901.

Sireven H. - Les premiers principes. Traduction par Cazzelles. 9e édition, Paris 1904.

spexcer H. - Les premiers Principes. Tuaduit sur la b" edifion anglatise par M. Gromor, Paris 19(1).

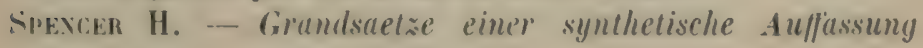
der Dinge, Stuttgart 1901.

SPENCER H. - System der synthetischen Philosophie. X rol.

Trad. B. Vetter, Stuttgart 1902.

Suevcer H. - Algimeene grondstellingen. Trad. vax Tracht.

Avelavg. Fr. - The philosophy of Herbert Spencer. The Dublin Review, April 1904. 
Bertuliot R. - Ilerbert Spencer. La grande incyclopédie, t. XXX.

Pu. B. - Ilerbert Spencer. Revue de Theologie et de Philosophie, Janvier 1904.

Bowne B. P. - The philosophy of H. Spencer. NewYork, 1874.

Coldus F. - Epitome of Synthetic Philosophy of Herbert Spencer. Londres, 1897.

Counss. F. - Epitome der synthetischen Philosophie H. Spencer 's, über'setzt von S. V. Carus.

Condsss F. - Résumé de la philosophie synthétique de

H. Spencer. Paris, 1904.

DE Roвеnт. - Auguste Comte et Ilerbert Spencer. Paris, 18!:3.

Deries J. - The philosophical work of Spencer. Plilosophical Review, Mars 1904.

IHFER E. H. - C. Herbert Spencer en $\approx$ yn tyd. Amsterdam, 190:i.

Dhlan. - Il pensiero filosofico di Spencer. Torino, 190:).

Fatial 1. - Spencer é il suo sistema filosofico. Rivista tilosotica, Janvier-Février 1904.

Gacl. V. - Ilerbert Spencer. Stuttgart, 1!(00).

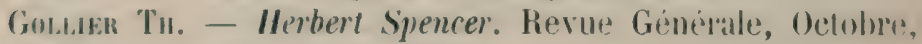
Novembre, Décembre $190 \%$.

Grocin. - An examination of the structural principles of H. Spencer 's philosophy. Oxlord 1883.

Hablix d. - La philosophie d'Herbert Spencer. Revue Scolastique, Février, Mai 1904.

Hanusos Fr. - The Ilerbert Spencer Lecture. Oxford, I!no:. Husso. W. H. - In introduction to the philosophy of Herbert Spencer, with ubiographical slietch. New-York, Istor.

liman d. - Th' philosophy of II. Spencer examined. L.ondres, 1884.

Ivracin J. - Ilerbert Spencer. Critical Roview, Mars 190)

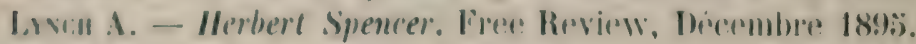
Macrususes H. - Iterbert Spenter: the mun and his work. Londres, 1900).

Mracita D. - La philosophie de Herbert spencer. Revus Yeo-Scolastique. Pévrier 1 x:8. 
Machel H. - Ilerbert Spencer et Churles Renouvier. L'année psychologique 1904.

Mishelet - II. Spencer's System der Philosophie und sein Verhälmiss zur Deutschen Philosophie. Halle 1889.

J'вost. - Les naturalistes philosophes. II. Spencer. Revole des qunestions scirntifiques, Janvier, Avril 1878.

P'roost. - La philosophie naturelle en .Ingleterre. Revur générale, Juin, Juillet, 1879.

I'srcuosis. - Our modern philosophers, Durnin, Buin and Spencer. Londres 1884.

Sibl.te C. F. - II. Spencer und Friedr. Nietssche. Vereinigung der Giegensütse auf grund einer newen These. Leipsig.

Salvaluen G. - Herberl Spencer e l'operu suu. Florence 1900).

Serravo V. - Spencer. Lerctura, Janviep 1904.

Tarozaz G. - La sintesi di II. Spencer. Revista di lilosulia, Janvier-Février 1904.

Thumisos. - Herbert Spmer. Brooklyn 1889.

Vula. - La filusofiu di Ilerbert Spencer. Rivista italiana di sociologia, septembre-Décemblse, 1!nok.

...... - llerbert spencer. La sua vilu e le sue apere.

La Civilta Catholica, Janvier 1904.

....... - A proposite di un nostro articolo intorno ad

Ilerbert spencer. La Civilta catholica, Firviep 1904.

Wansen Fiтe. - II. Spencer as a philosopher. Journal of Philusophy, Issycholugy and scientitic methods. I.

Watsox J. - Comte, Mill and Spencer; an oulline of philosophy. Glasgow 1890 .

Wевен R. Н. - Die philosophie II. Sprencer's.

Yotsc A. - Acriticul examination into the philosophy of U. Spencer. San-Franciseo 1887.

Иамвох. - Herbert Spencer. Bulugne 19104.

Zuccante. - Herhert Spencer. Vicenzal 1904.

\section{III}

Armico R. - L'Inconoscibile di II. Sprencer e il Voumeno di Kant, etc., Padoue 1901. 
Andigo R. - La dottrina spenceriana dell'Inconoscibile, Rome 1899.

Armigo R. - Opere filosofiche, vol. vil (Spencer e Kant) Padoue 1901.

Arthir W. - Religion without God an God without Religion. Agnosticism and H. H. Spencer, Londres 188:\%. Bertherot R. - These sur les origines de la philosophie de Spencer. Bulletin de: la société française de philosophie, Avril 1904.

Bınкs. - Modem physical filalism and the doctrine of Evolution, including an examination of Spencer, s First Principles.

Boxiart E. - La critique de $\boldsymbol{M}$. Renouvier et l'évolutionnisme, Lausanne 1880.

Bıtlen Bıнке J. - II. Spencer and the muster key. The Contemporary Review, Juin 1906.

Cians. P. - Kant and Spencer. I study of the fallacies of Agnosticism. Chicago 1900.

Cinser A. - La religione nella filosofia di Erb. Spencer. Rivistal di tilosotia, Janvier-Fiviriel 1908\%.

Pвнко. A. - La Ciritica della Conoscenza in E. Kante H. Spencer. Savona 1900.

Fishe J. - Cosmic Philosophy. Londres 1874.

Fiscuen E. I. - Viber das Gesetz der Entuirklung mit Burlisicht unf II. Spencer, Wirtzburng 187i.

fitz sams siremex. - The unkmowable and the unlinoun. Ninetheenth Century, Juin 1884.

liacionx E. - Die Cirundlagen der Spencerschen Philosophie. Berlin 188x.

(ikz. W. - Der Almostirismus II. Speneres 's mil Rüchsichl

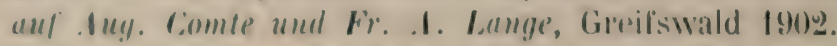

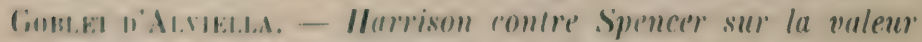

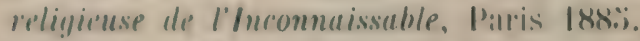

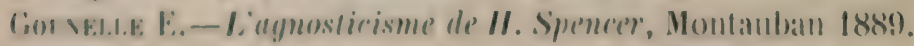

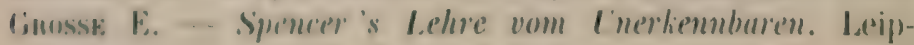
sig 1890 .

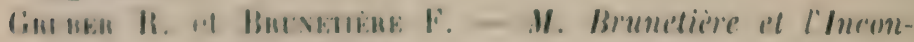


naissable de Spencer et de Comte. - Réponse an R. P. Gruber. Revue philosophique, Fisrier 19103.

Hamiton G. - The insuppressible Bool : A controvers!y between II. Spencer and $F$. IIarrison. Boston 188:3.

Joxes J. H. - Know the Truth; a critique of the Ilamillonian theory of Limitution including some strictures upon the theories of. H. Spencer. Londres 1863.

Lavget 1. - M. Ilerbert Sprencer el les études philosophiques

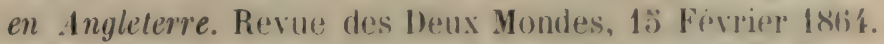

LACY W. M. - In exumination of the philosophy of the Unknowahle. Philadelphie 1883.

Ladrexs C. - L'évolution et ./. Merbert Spencer. Lyon 1884.

lacis J. - Agnosticism and Religion; an examination of spencer's Religion of Intinowable. Baltimore 18:1\%).

Magerae Th. - Agnosticism. II. Spencer and F. IIterrison. Dublin 1884.

Malcolu Githrie. - Spencer"s Inification of knowledye. Londres 1882.

Malcolm Guthre. - .". Sprencer's formula of Evolution. Londres 1879 .

Marcinesing G. - La metufisica di Erh. Spencer. Rivista di filosolia, Janvier-Février $190 \%$.

Minhaldowshi X. - Qu'est-re que le mogres? Exumen des idées de Spencer. Paris 1897.

Narmasn. A. - Spencer wider hant. Hambourerg Isx:i.

Nichoss J.-B. - Spencer 's Definition of Evolution. Tha Monist, Octobre 1902.

Pack E. - los Relativitutsprinsip in Spencer's Entwichlungslehre, Leipsig 1892.

Rotu L. - Schelling und Spenrer, Berme 1!ntug.

Paster G. S. - Spencer's Eulutionstheorie dargestellt. O. 0.1896 .

Rexorven Ch. - Examen des Premiers Principes de II. Spencer. Critique philosophicyue 188:3-86.

Rotre L. - Ilerbert Spencer et l'érolutionnisme mécaniste. Etudes religieuses, Mars 189..

Ronre. L. - Ilerbert sipencer. L'idée religiense et l'Inconnaissable. Etudes Religieuses, Avùt $180 \%$. 
Saleery C. W. - Evolution the Master-hey. Londres 1896. Simuoxs A. - Ignostic First Principles, Londres.

H. Spexcer et F. Harrisox. Ninetheenth Century 1884, Religion a Retrospect and Prospect (Spexcer, Janvier). The Ghost of Religion (Harrison, Mars). Retrogressive Religion (Śpexcer, Juillet). Agnostic metuphysics (Harrisox, Septembre). Last words ahout Agnosticism and the Religion of Humanity (Srencer, Novembre).

Sinevcer el Martiveac. - Contempolary Review 1872, Avril (Martineati). Ir Martineau on Evohution, Juin (Sibencer). Siever H. - Replies to Cristicisms. Forthnightly Review, Novembre et Décembre 1873.

Spricker G. - Spencer's Aussichten ueber das Verhältniss von Religion und Wissenschaft. Münster 188!.

Toner Prestox. - Comparison of some vieus of Spencer and Kant. Mind, Avril 1900.

Tronto. - La doltrina della conoscensa di II. Spencer, Bologne 1904.

Vindarnita. - Ilerbert spencer e l'enolutionismo. Rivista italiana di tilosolia 1897, p. 63.

Vorranamp R. - Agnosticisme van II. Spencer. Groningue 1897.

WABI J. - Naturalism and Ignosticism. Londies 19003.

Yormass E. I. - The nature and reulity of Religion. I contronersy betwen $\boldsymbol{F}$. IIarrison and II. Spencer. YewYork 188: 


\section{TABLE DES MATIERES}

Arant-Propos. p. ข้.

\section{Jre PAIRTIE}

\section{La Philosophie de l'Inconnaissable}

\section{Chaptrae 1. - Religion ot Science.}

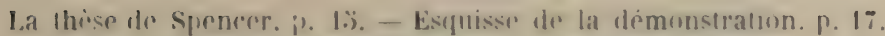
- Lobjet rommun de la Religrion et de la sedenes. 19. 17.

\section{Chapithe II. - Les idées dernières de la Religion.}

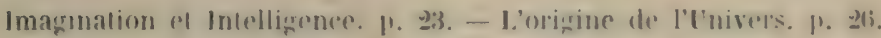
- La nature de l'Univers. p. $\mathbf{3 4}$.

(:hapithe III. -- Les idées dernières de la Science.

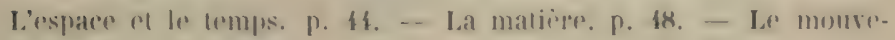
ment. p. S1. - La force. p. :8. - La conscience. p. 66.

Cinapitie IV. - La question de la relativité de toute connaissance.

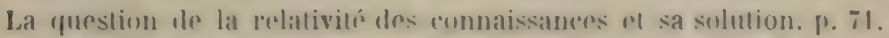
- Influene de kant. 1. 7.;. - Opinions des philesuphes. ए. is. -

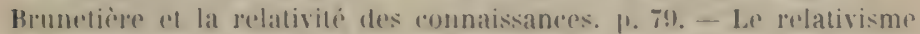

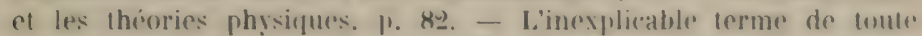

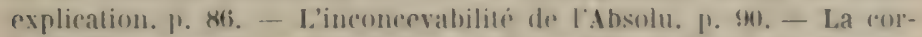

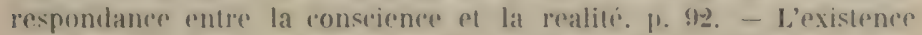
rélle du nommine. p. 9x. - L'itre abstrait al l'elre illimite. p. I01.

\section{Chapitre V. - Laccord de la Religion et de la Science.}

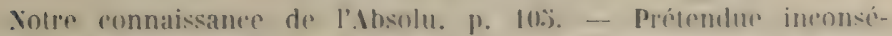

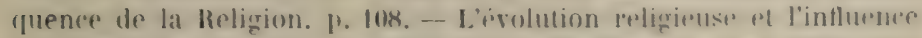
de la science. I). 110. - I'ivolutimn scientitigue at les idies religieuses. p. 113. - L'Inconnaissable. p. 120. 


\section{PARTIE}

\section{La théorie de l'Evolution.}

\section{Chapitre I. - Les données.}

Is connaissable. 1). 120. - L'ívolution objet de la philosophice. p. 132. - La vérité, p. 136. - Le moi et le non-moi. p. 139. l.espace et le temps. p. 1 11. - La matiere. p. 14.;. - Le mouvement. p. 117. - La force. p. 148.

\section{CHAPITRE. II. - Les principes.}

\$. I. L'indestrutibilite de la matière comme principe a priori.

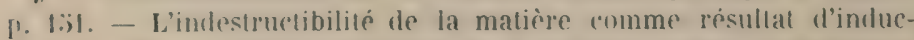

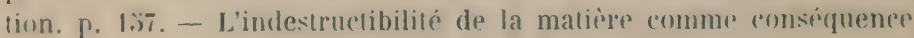
de la persistance de la force. p. 160.

$\$$ II. La consurvation du mouvement a miori et devant l'induelion. p. 163.

\$ III. La persistanee de la foree. Veritable sens de ce principe. p. 169. - Ja persistanee de la foree est-elle un poslulat? p. 1733. La force el l'ineonnaissable. p. 177.

\$IV. La consiance des lois relations entre les foreesi. p. 179.

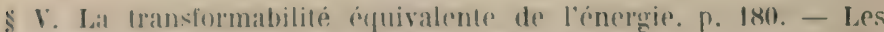

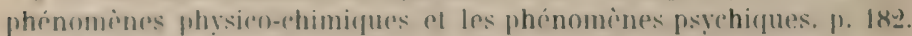
-- les phorom ines physieu-chimiogues of les phemomines oryani1fups. 1. 192. - Les phenomines sociaux. p. 199. -- I.e materia-

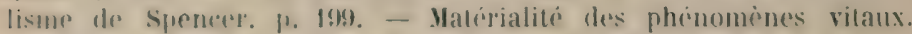
p. 20): - Etude induelive de la loi de transformation. p. zot. -

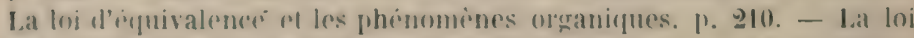

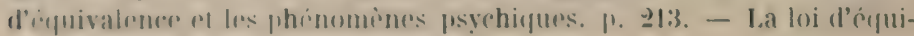
valence "t les phénomènes sociaux. p. 211.

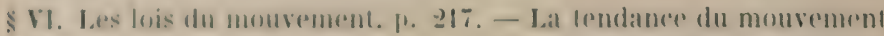

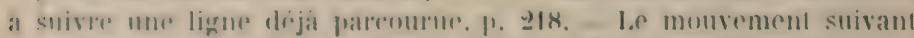

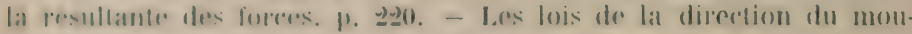
vesnent dans l'activité vilale. p. 2023.

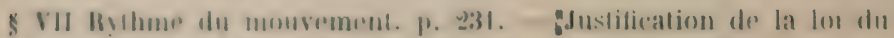

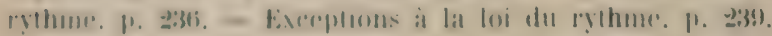

\section{Cillaptit: III. - L'évolution.}

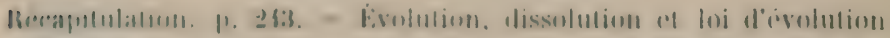

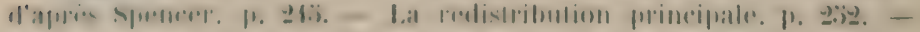

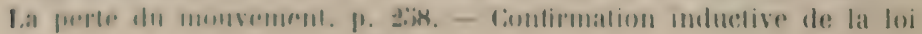




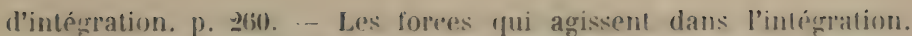

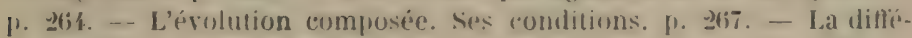
renciation. 1). 27:;. - Contirmation induetive de la loi de difleren-

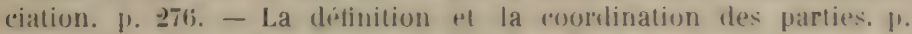

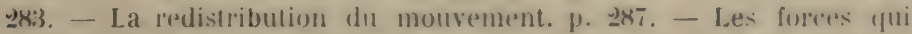

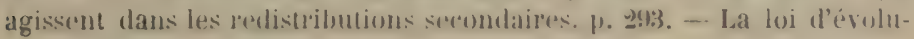
lion amendée. p. 291.

\section{Chapitia IV. - Les applications de la loi d'évolution.}

L'évolution des opranismes. 1. 2ug7. - L'évolution de la vie. p.

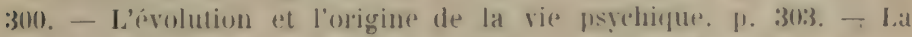

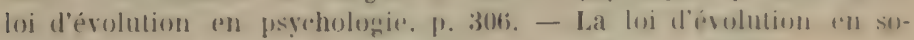

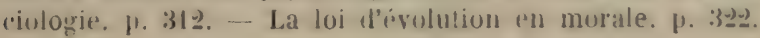

\section{Chapithe V. - Les causes de l'ovolution.}

\section{\$. Les causes générales.}

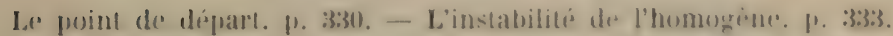

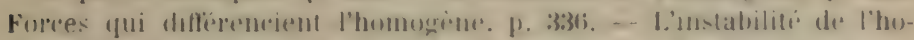

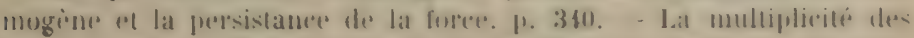

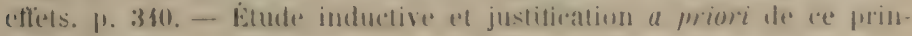

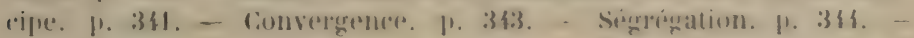
Récapitulation. p. 317.

\section{$\$$ II. Les canses de l'évolution oryanique.}

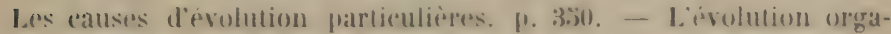

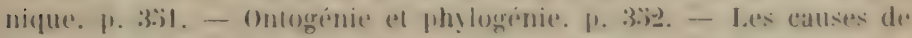

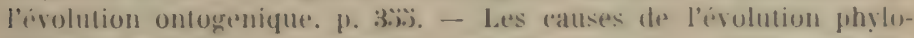

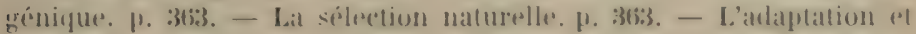
l'hiredite des caracteres atcunis. 1. 373. - - less tendances internes d'évolution. p. 381 . - La théorie de de Vries. p. 386.

\section{\$III. La splection naturelle comme facteur géniral d'solution.}

Analyse de la sélection matureslle. p. 3:133. - La sélection naturelle

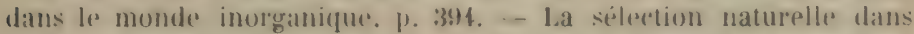

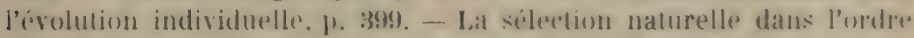
surial. p. foll. - Insuntisance essentielle de la sélection maturelle. p. 10 i.

\section{Chapitra VI. - \$1. L'équilibre el la dissolution.}

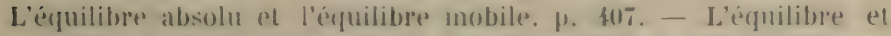

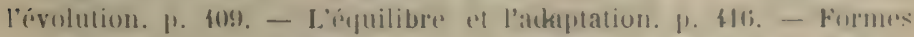

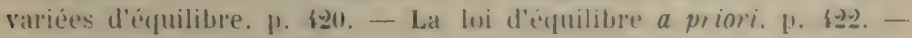




\section{$-488$}

Relation entre l'évolution al la dissolution. p. 12:3. - Etude inductive de la dissolution. p. \{29.9. - Dernier probleme. p. 138.

\$I. Examen de la synthèse finale.

Évolution universelle. 1\% \{12. - Alternatives d'évolution et de

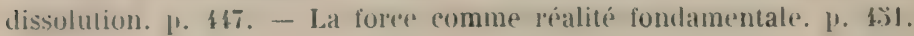

\section{Chapitre VII.}

Origines de lat philosophie des Premiers Principes. 11. 19.;. -Récapitulation. 1. 169\%. - Appréciations. 1. 173. - Biblogiraphie. p. 479. 


\section{ERRATUM}

P. 8, 1. 24, lisez: Certains principes sont, au lieu de : Certains principes ou bien sont.

P. 69, 1. 13, " : Et s'il n'est, an lieu de : Et il n'est.

P. $69,1.22$, " : peut à la fois exister et ne pas exister, ou bien ... au lieu de : peut ì la fois exister, ou bien .....

P. 71, en marge, lises: relativitè, au lieu de : validité.

P. 97, 1. 26, lises : n'implique pas, au lieu de : pas n'implique.

P. $110,1.2 \%$, " : : n'est au fond que la série, au lieu de : n'est an fond que de la série.

P. 114, dernière ligne, lises: (3), an lieu de : (1).

P. 1:2, 1. 26, lise: : Celui-ci, au tien de : Celui.

P. $157,1.3, \quad$ 1) : est une vérité, an lieu de : et une vérité.

P. 167, 1. 26. ") : hauteur, au lieu de: auteur.

P. 176, 1. 22, " : conservation, a lieu de: conversation.

P. $18: 3,1.33$, "1 : sont les faces, au lieu de: ont les faces.

P. 189, I. 24, ": : il n'y a rien, au lieu de: il n'y rien.

P. 193, I. 31, " : du monde inorganique, au lieu de : du monde organique.

P. 222, 1. 9, „ : Celle-ei, an lien de : Celui-ci.

P. $2 \%, 1.9, \quad$ " : a fait ce qu'elle, an lieu de : a fait de quelle.

P. 328, 1. 7, " : : entre les principes, au lieu de: les entre principes.

P. 338, I. 1, 1) : des forces incidentes rend comple de l'existence.

P. 408, 1. 11, „ : pas, au lien de : par.

P. 415, 1. 2, „) : indéfini, au lieu de : infini.

P. 435, 1. 22, ") : de la nature, au lieu de : la de nature.

P. 442, I. 5, "1) : l'étude de l'Univer's, au lieu de : l'étude l'Univers.

P. $457,1.22, \quad$ "I : celle de Spencer, au lieu de : celle Spencer.

P. 468, 1. 15, i) : avoir, au lieu de : avis.

Le lecteur bienveillant corrigera aisément les autres " coquilles ». 



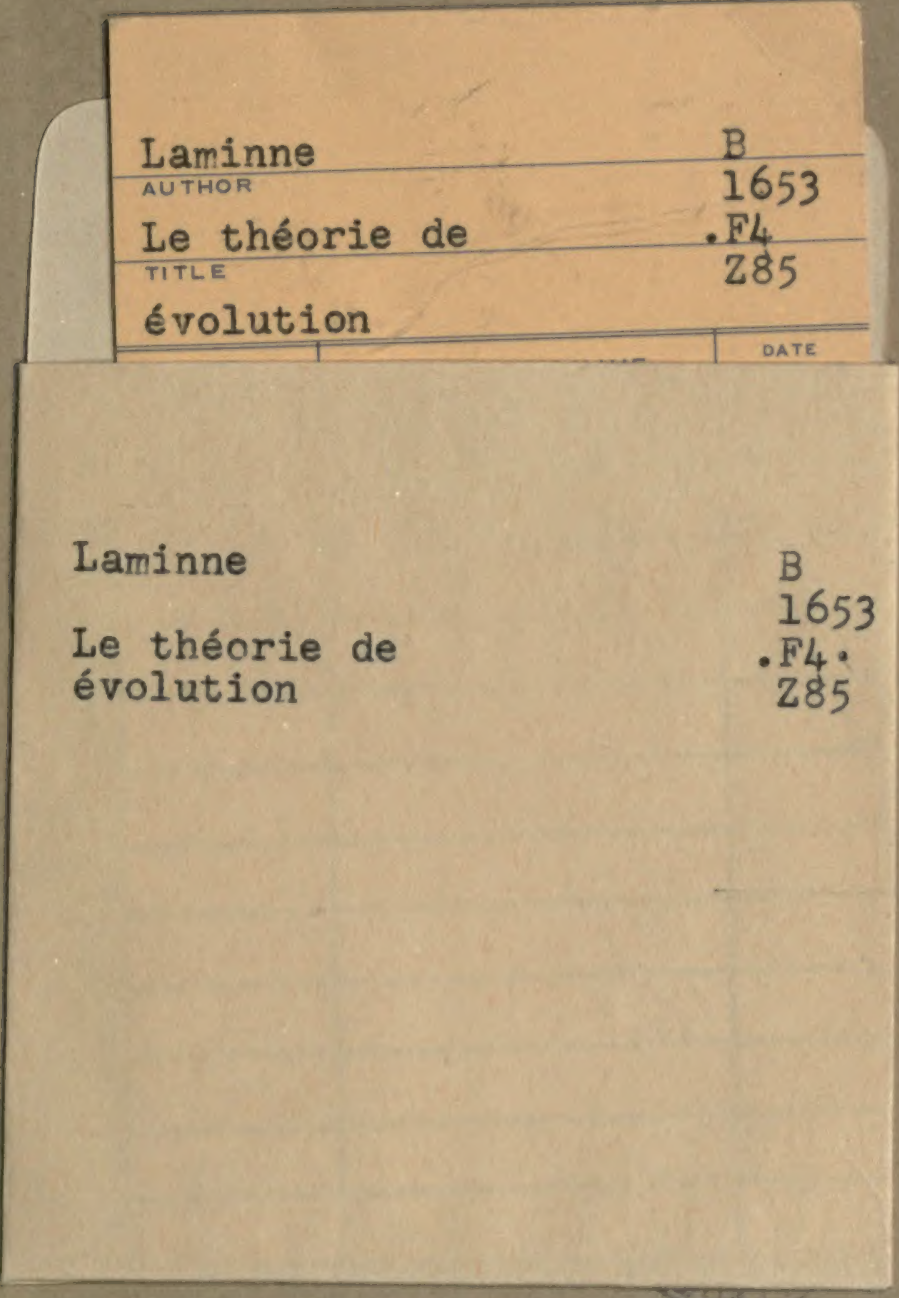


